10 year Summer Teletherm dynamics for VALLEY HEAD, AL:

$\div 274-\mathrm{A}$

(1) Sep

$>244-$

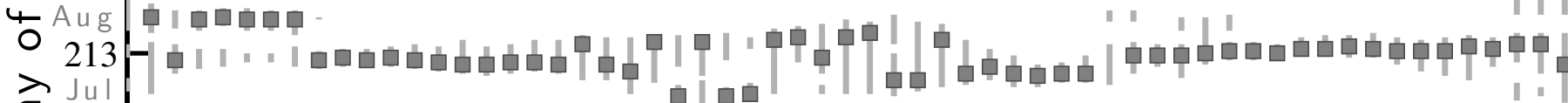

त

$\cap_{\mathrm{Jun}}^{182}$

$152-$

May L_L

$-1920$

| | |
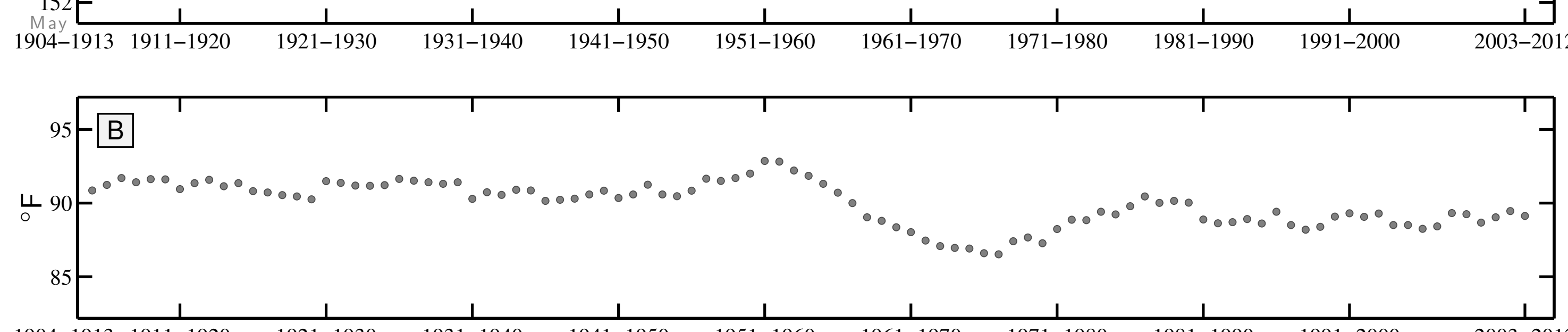

1904-1913 1911-1920

1921-1930

1931-1940

1941-1950

1951-1960

1961-1970

1971-1980

1981-1990

1991-2000

2003-2012

10 year Winter Teletherm dynamics for VALLEY HEAD, AL:

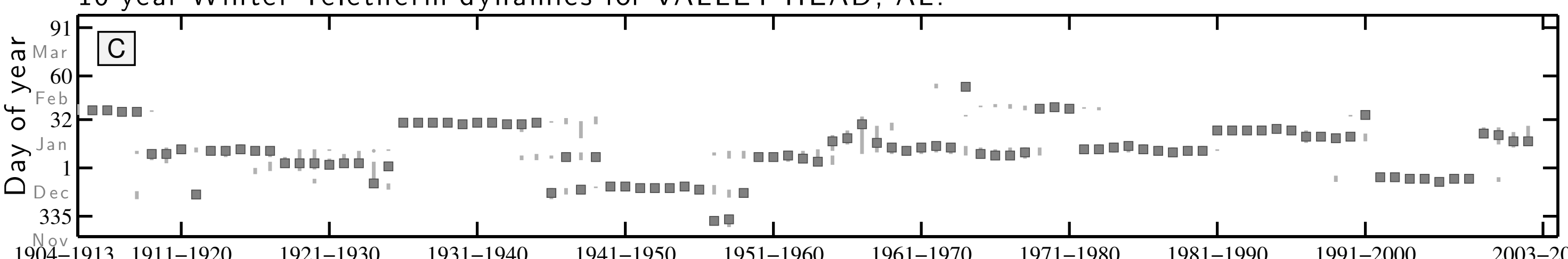

1904-1913 $1911-1920$ 1921-1930

$1931-1940$

1941-1950 1951-1960

1961-1970

1971-1980

$1981-1990$

$1991-2000$

2003-2012

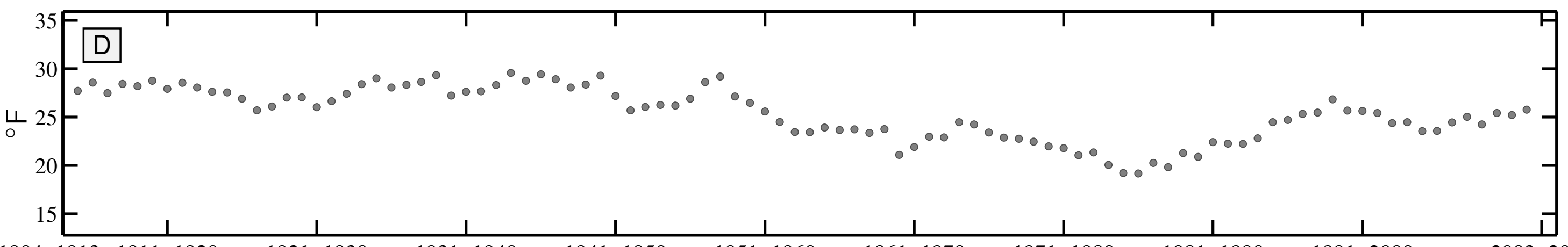



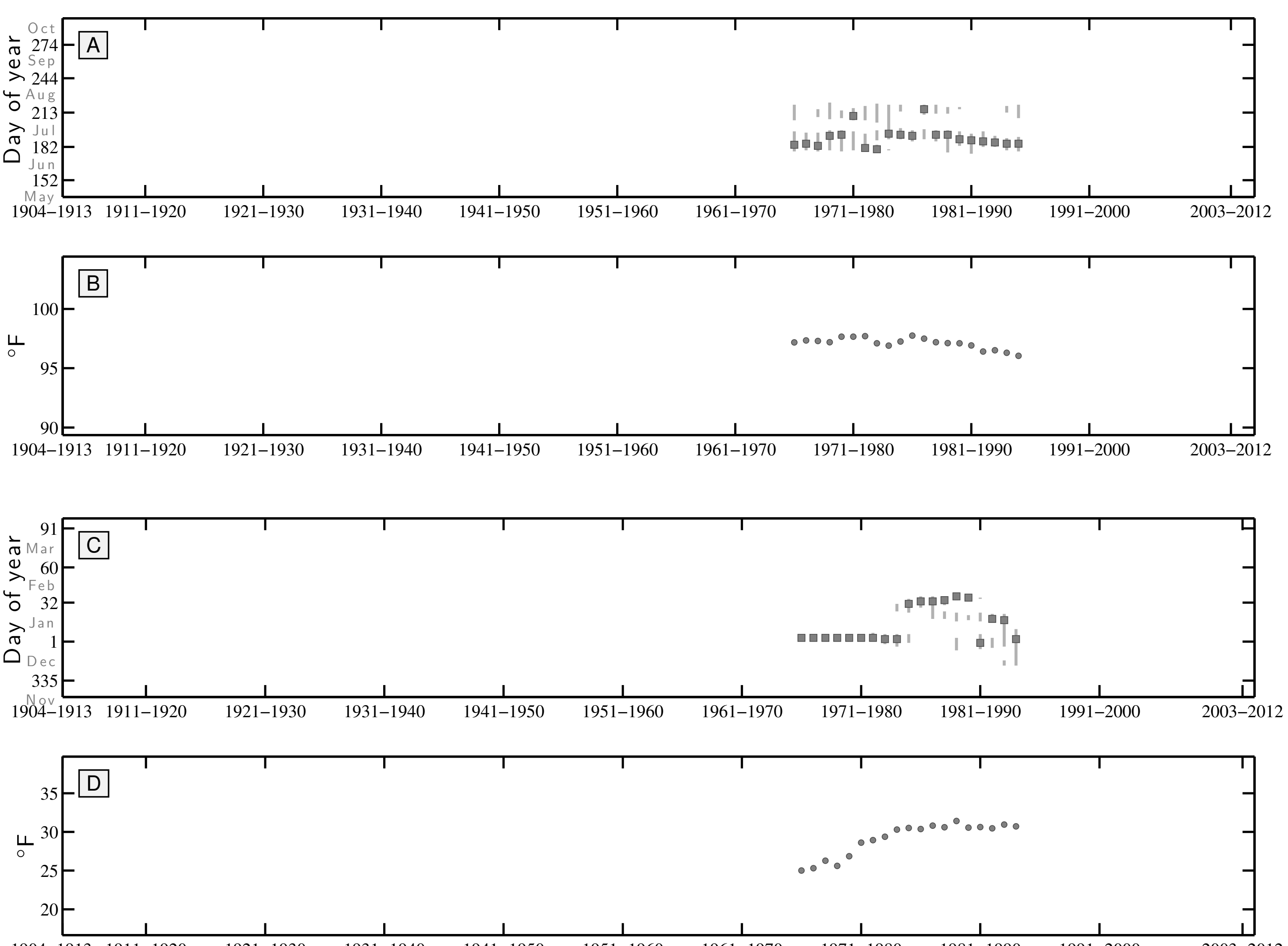

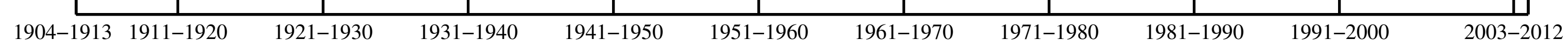


10 year Summer Teletherm dynamics for PRESCOTT, AZ:

$\div$ Oct 274

(1) Sep

$>244$

4 $\mathrm{Aug}$

$213-$

○ 182

$\bigcirc$ Jun

$152-$

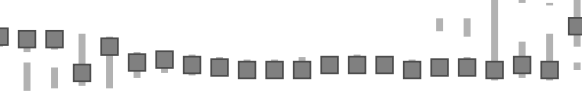

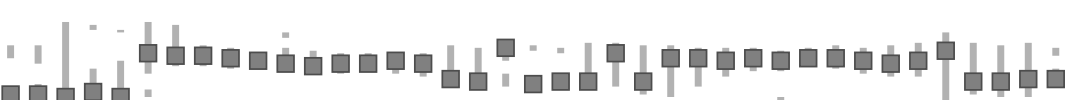

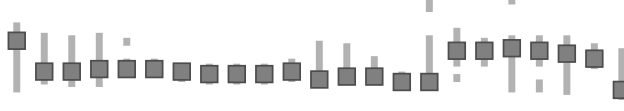

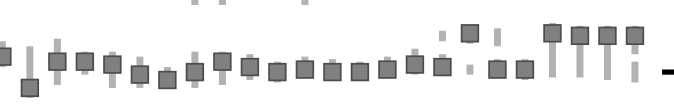
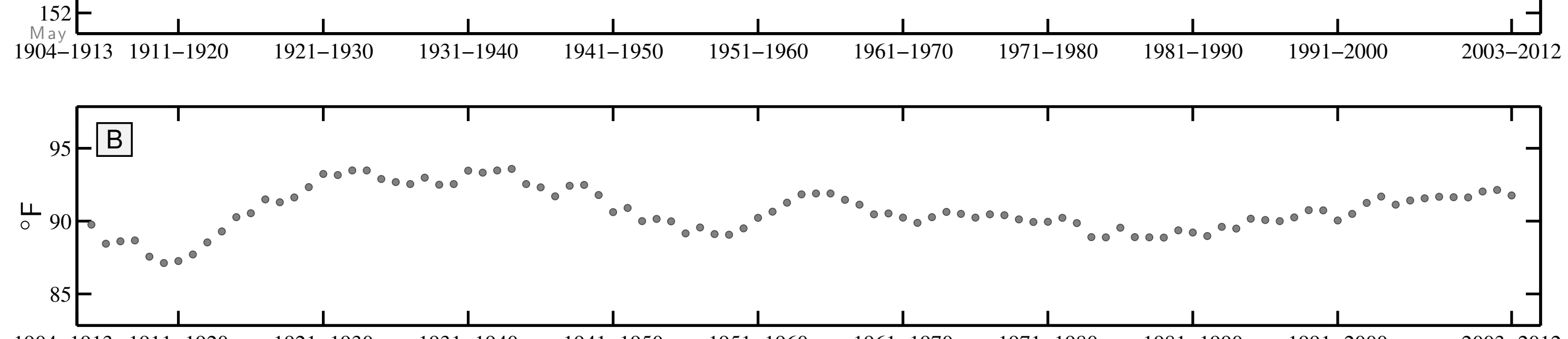

$1904-1913 \quad 1911-1920$

$1921-1930$

$1931-1940$

$1941-1950$

$1951-1960$

$1961-1970$

$1971-1980$

$1981-1990$

1991-2000

2003-2012

10 year Winter Teletherm dynamics for PRESCOTT, AZ:

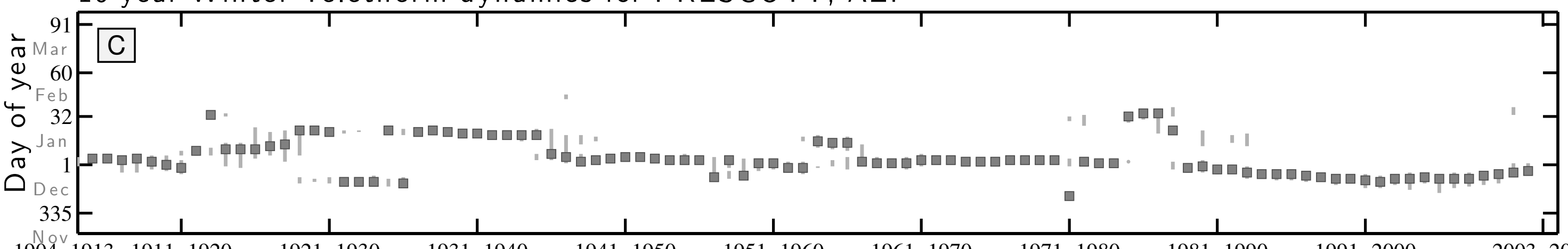

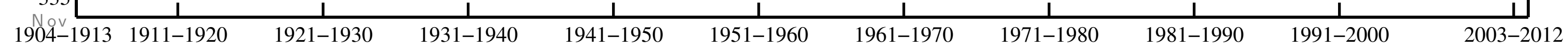

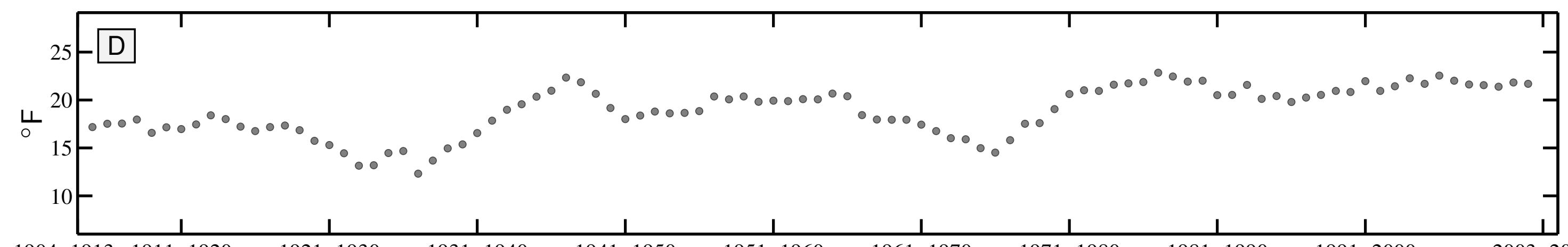


10 year Summer Teletherm dynamics for SACATON, AZ:

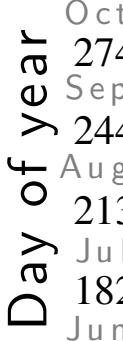
A

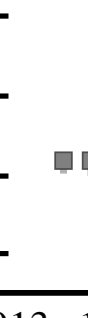

口प⿴囗十

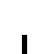

1

$\frac{1}{1921-1930}$

$\frac{1}{1931-1940}$

$1941-1950$

$\frac{1}{1951-1960}$

$\frac{1}{1961-1970}$

$1971-1980$

1981-1990

1991-2000

2003-2012

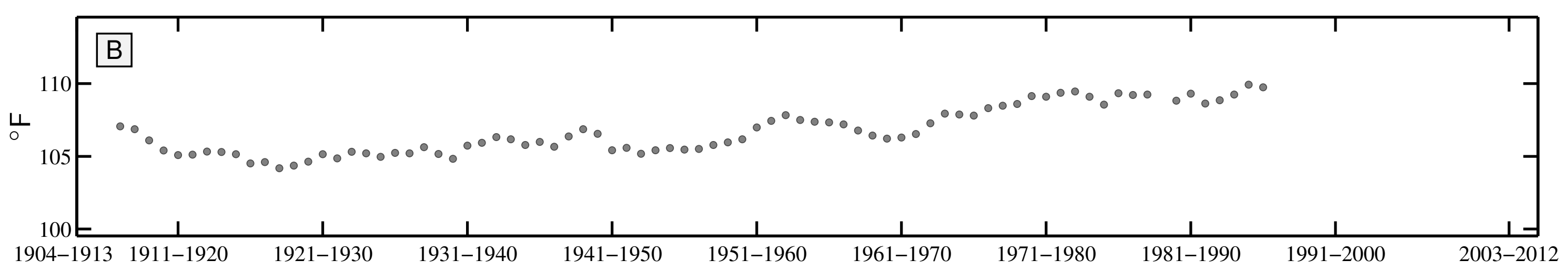

10 year Winter Teletherm dynamics for SACATON, AZ:

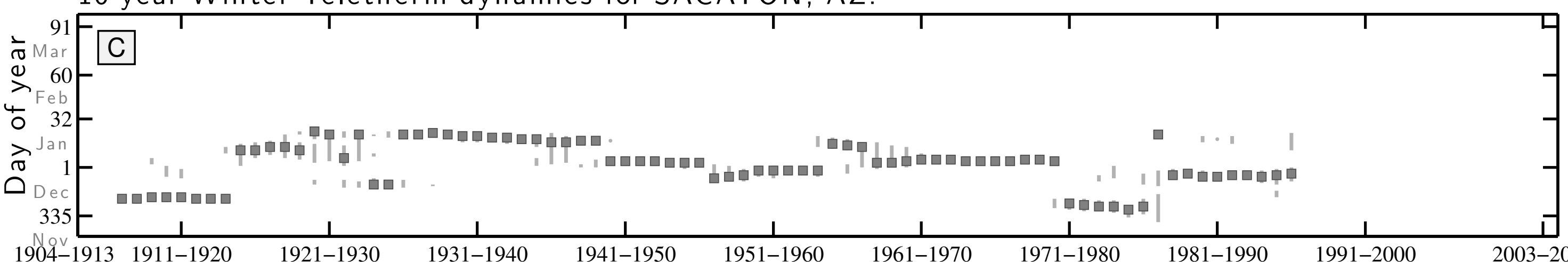

$$
\begin{array}{r}
3 \\
190
\end{array}
$$

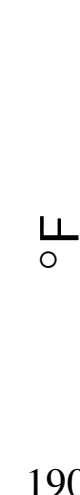

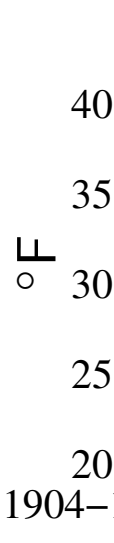
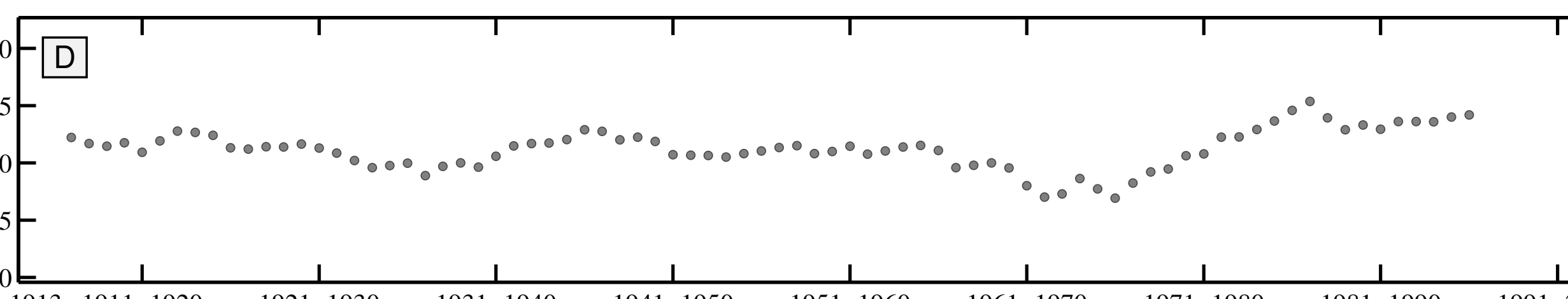

1 
10 year Summer Teletherm dynamics for SAINT JOHNS, AZ:

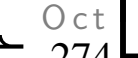

A

(1) Sep

$>244$

(1 A g

त Jul

\% $182-$

$\mathrm{J} u \mathrm{n}$

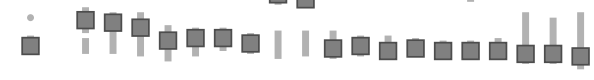

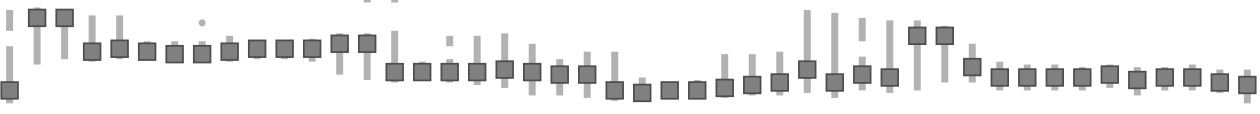

$\square$

1904-1913 1911-1920

$1921-1930$

$1931-1940$

$\frac{1}{1941-1950}$

1951-1960

1961-1970

1971-1980

$\frac{1}{191-1990}$

991-2000

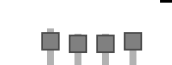

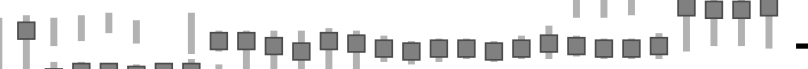

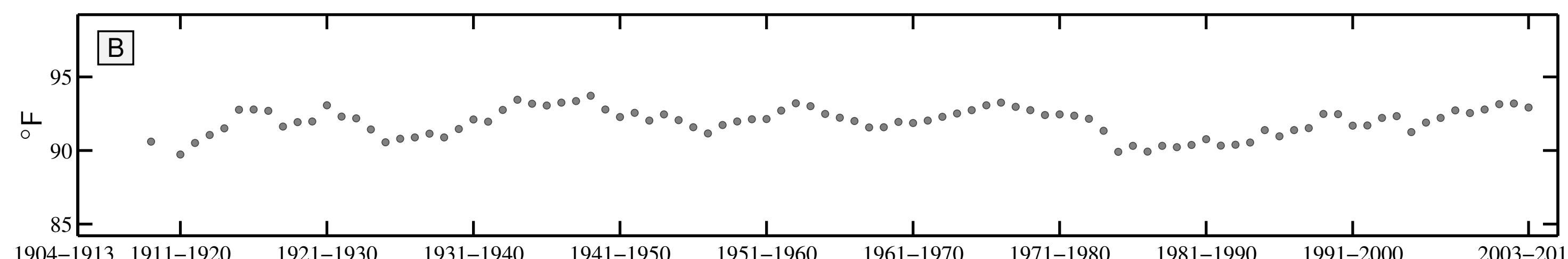

1904-1913 1911-1920

$1921-1930$

$1931-1940$

$1941-1950$

$1951-1960$

$1961-1970$

971-1980

1981-1990

991-2000

2003-2012

10 year Winter Teletherm dynamics for SAINT JOHNS, AZ:

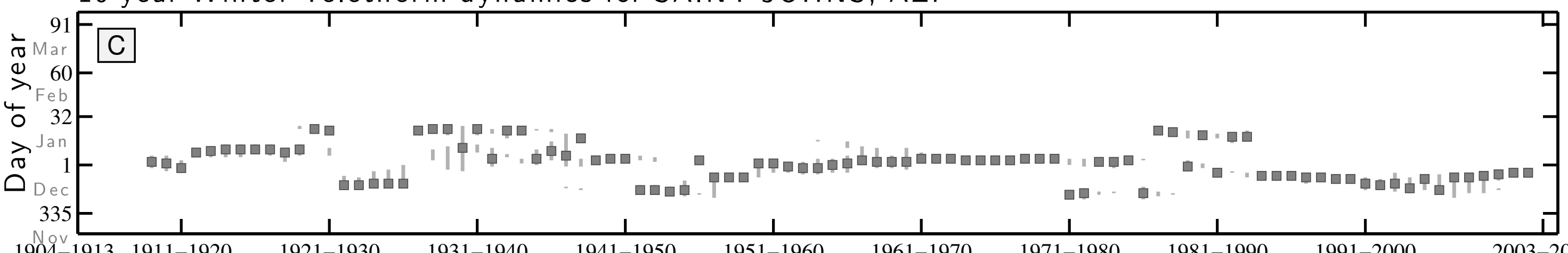

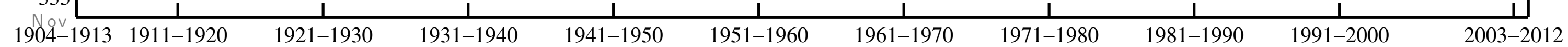

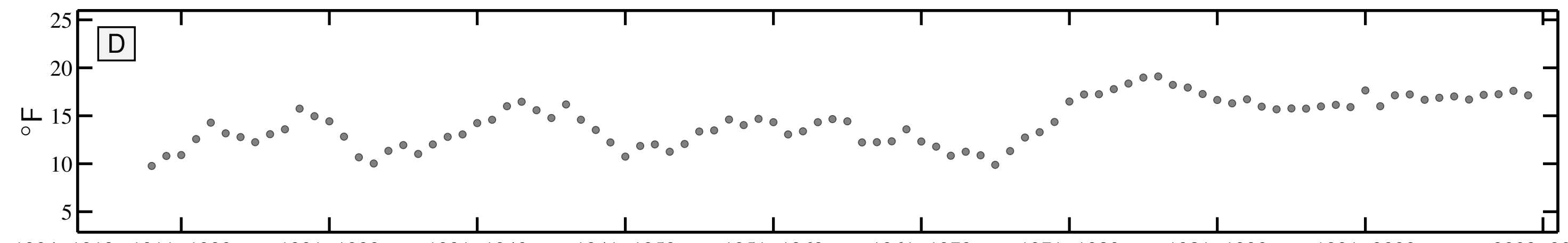


10 year Summer Teletherm dynamics for SELIGMAN, AZ:

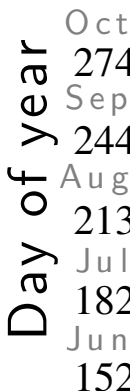
A

244

O Aug

$\gtrsim \mathrm{Jul}$

$182=$

$152-$

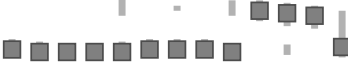

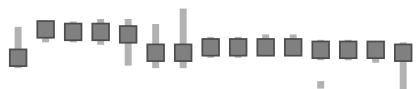

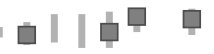

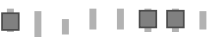

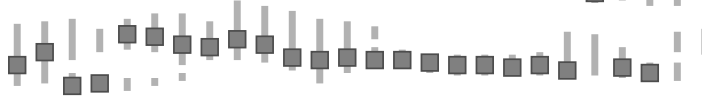

$-1960$

$1961-1970$

$1971-1980$

1981-1990

991-2000

2003-2012

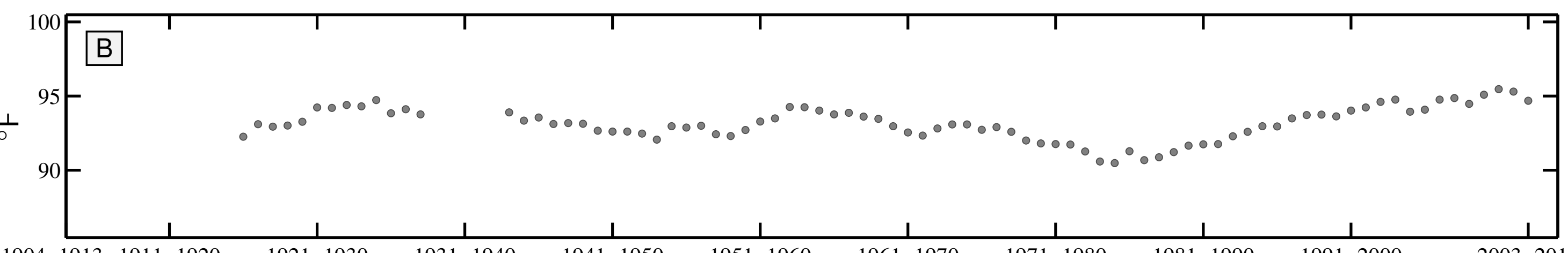

1904-1913 1911-1920

$1921-1930$

$1931-1940$

$1941-1950$

$1951-1960$

$1961-1970$

$1971-1980$

$1981-1990$

$1991-2000$

2003-2012

10 year Winter Teletherm dynamics for SELIGMAN, AZ:
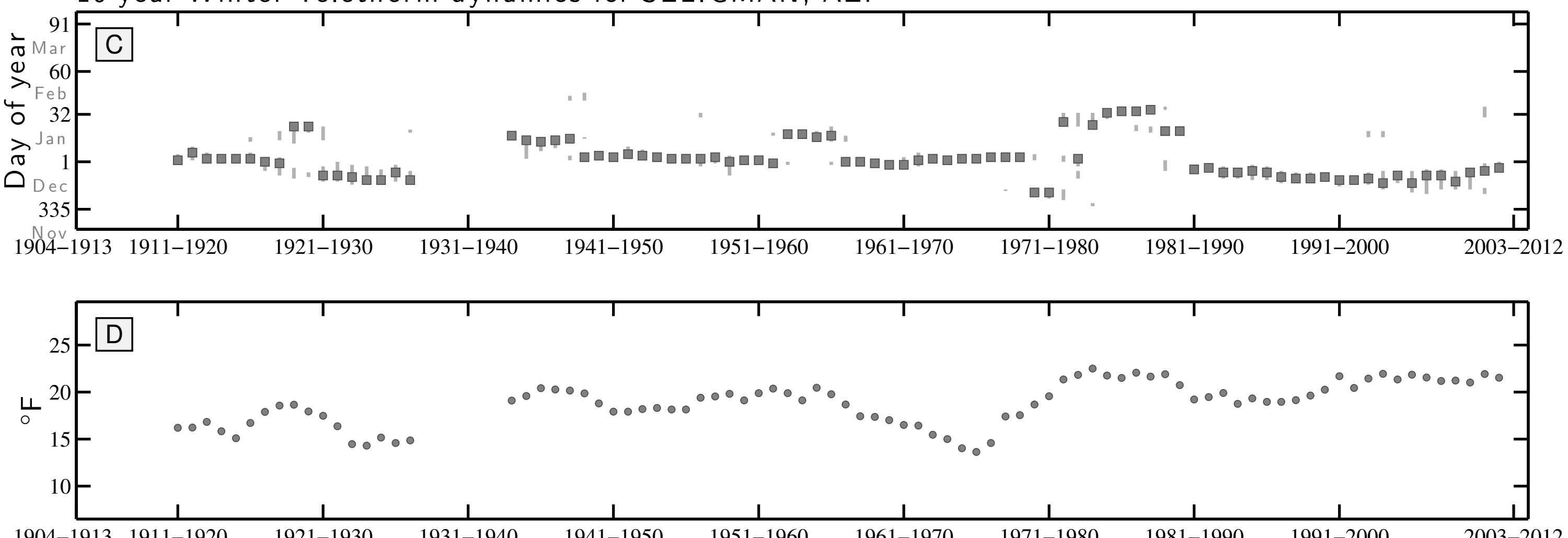
10 year Summer Teletherm dynamics for WHITERIVER 1 SW, AZ:

$\div \quad 274$

\% $274-A$

(1) Sep

$>244$

○ 213

$>\mathrm{Jul}$

ס 182

$152-$

May $\square$

1904-1913 1911-1920

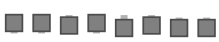

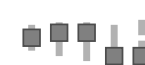

|

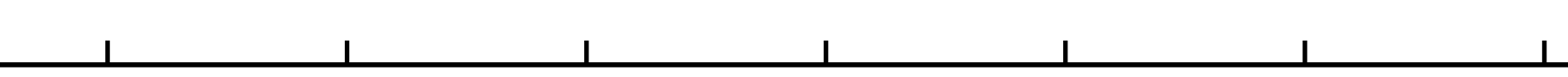

$1981-1990$

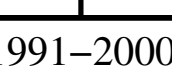

뭄ำ

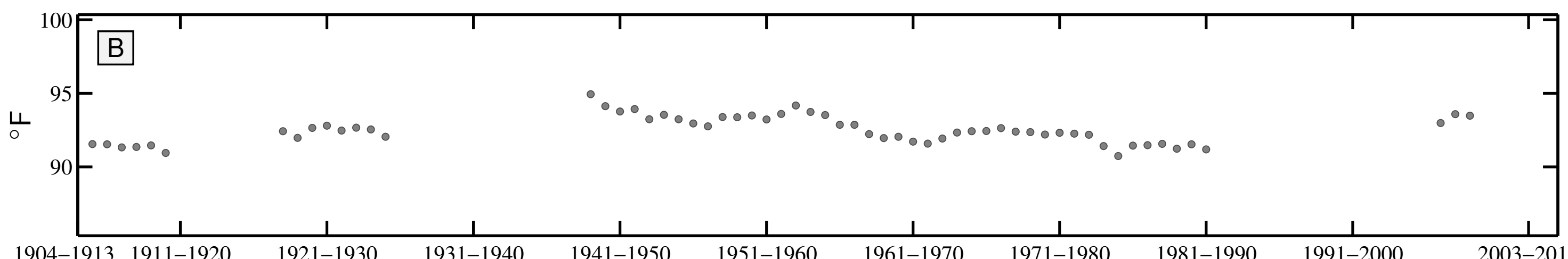

10 year Winter Teletherm dynamics for WHITERIVER 1 SW, AZ:

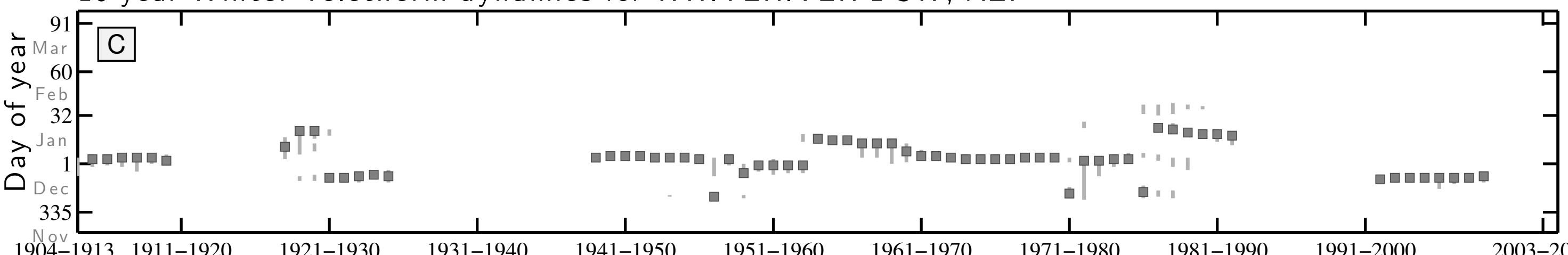

$1904-1913$ 1911-192

$1921-1930$

$1931-1940$

$1941-1950$

$1951-1960$

$1961-1970$

$1971-1980$

$1981-1990$

$1991-2000$

2003-2012

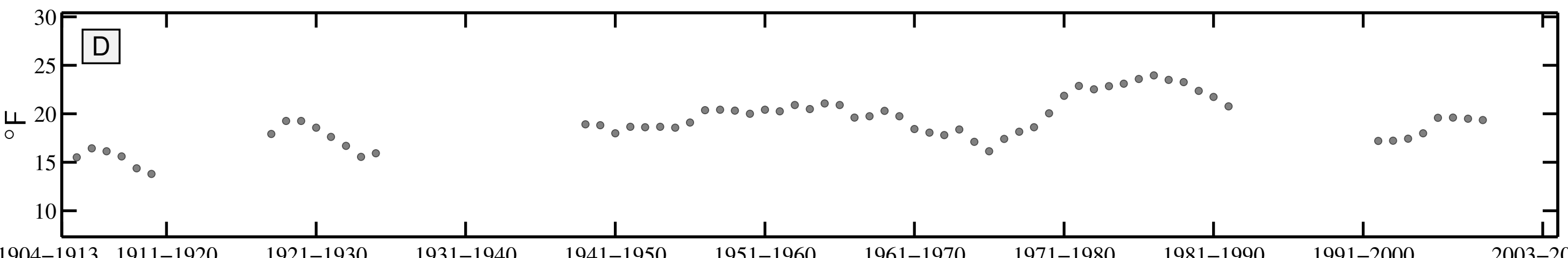


10 year Summer Teletherm dynamics for WICKENBURG, AZ:

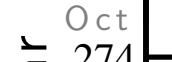

(1) Sep

$>244$

( $\mathrm{Aug}$

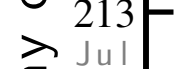

\% $182-$

$\mathrm{Jun}$

152

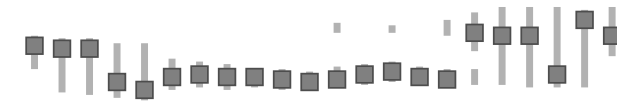

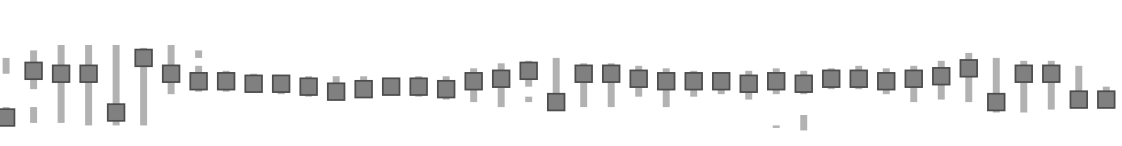

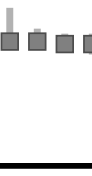

| || |

1904-1913 1911-1920

1921-1930

$1931-1940$

1941-1950

1951-1960

$1961-1970$

$1971-1980$

$1981-1990$

1991-2000

$2003-2012$

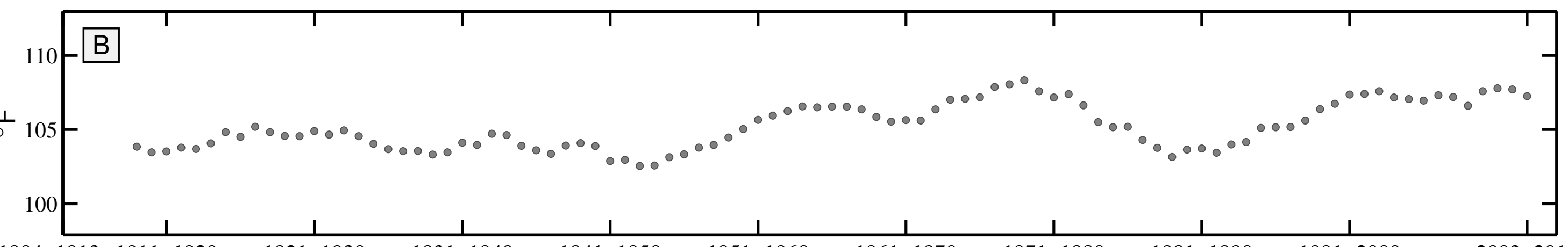

1904-1913 1911-1920

$1921-1930$

$1931-1940$

$1941-1950$

$1951-1960$

$1961-1970$

$1971-1980$

$1981-1990$

$1991-2000$

$2003-2012$

10 year Winter Teletherm dynamics for WICKENBURG, AZ:

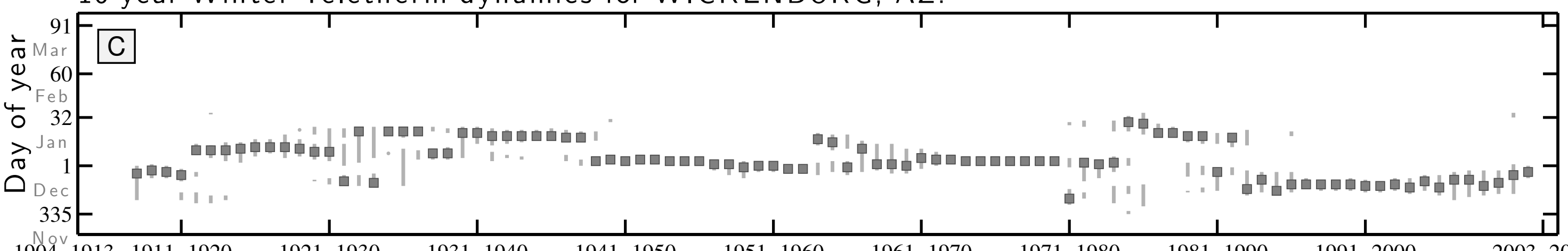

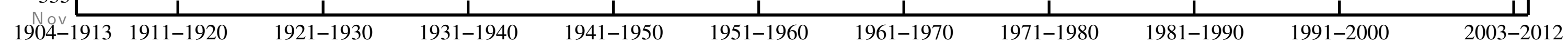

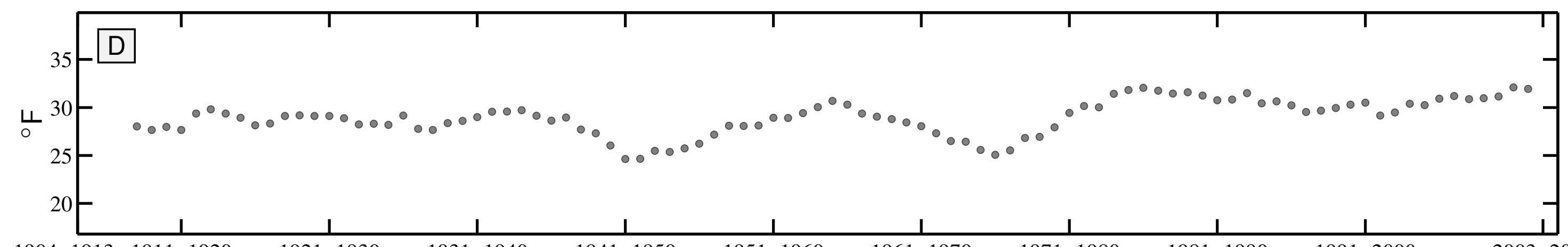


10 year Summer Teletherm dynamics for WILLIAMS, AZ:

$\stackrel{0}{2} 274-A$

$>244$

O Aug

$\underset{\mathrm{Jul}}{213}$

$\overbrace{}^{\circ} 182$

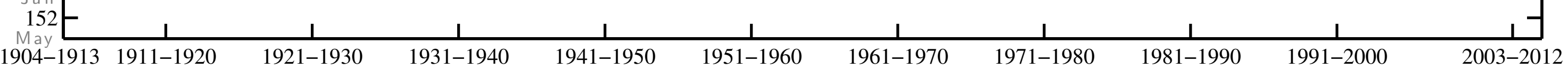

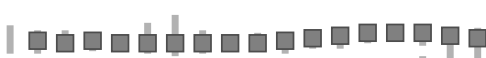

" "
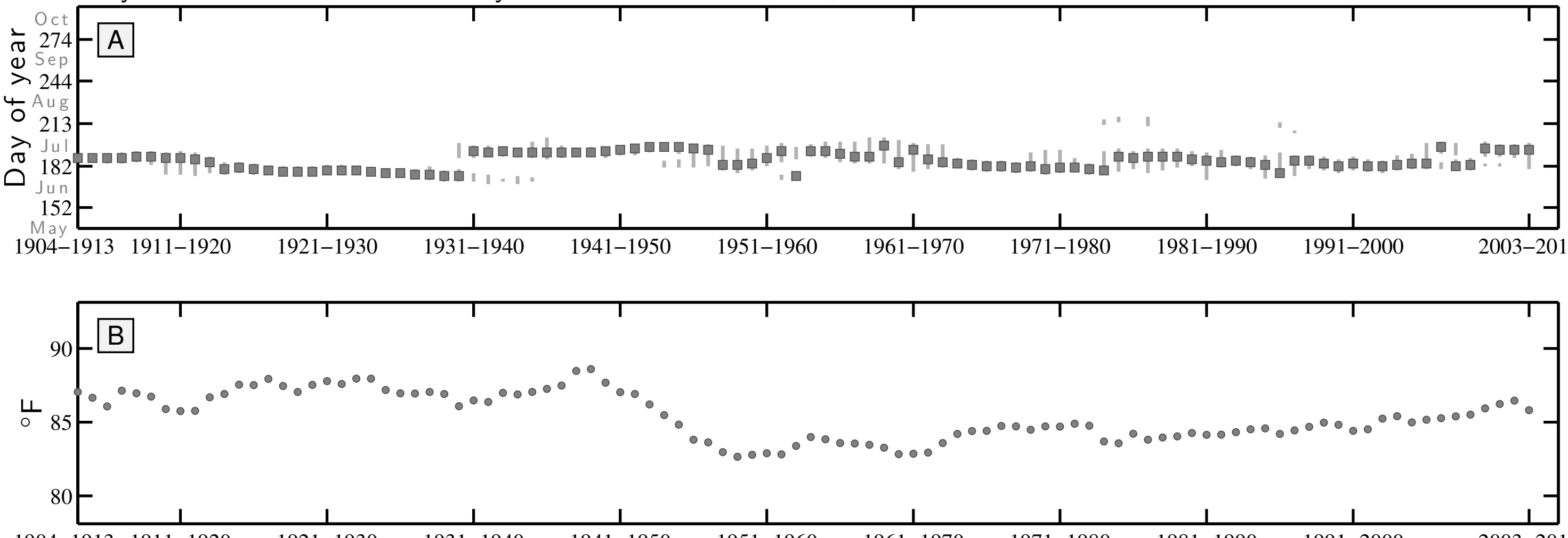

$1904-19131911-1920$

$1921-1930$

$1931-1940$

$1941-1950$

$1951-1960$

$1961-1970$

$1971-1980$

$1981-1990$

$1991-2000$

2003-2012

10 year Winter Teletherm dynamics for WILLIAMS, AZ:

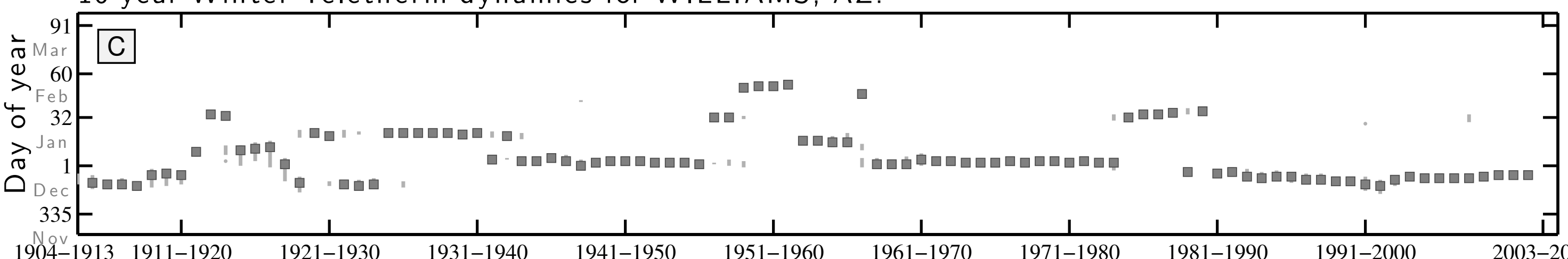

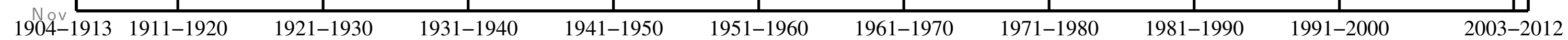

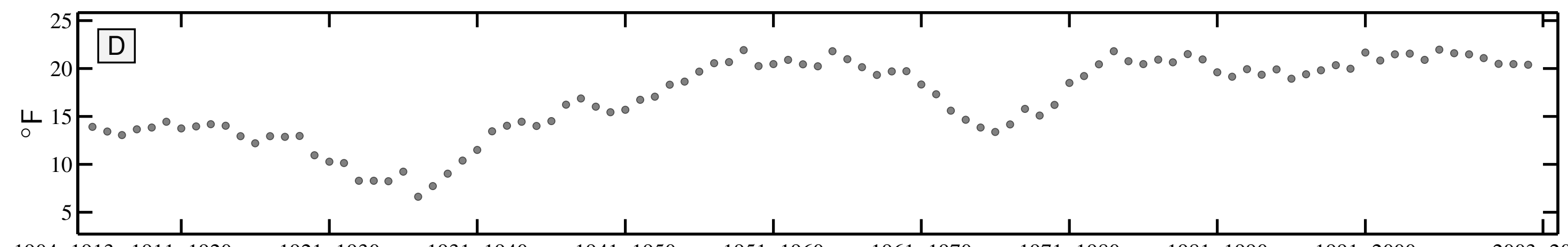


10 year Summer Teletherm dynamics for EUREKA SPRINGS 3 WNW, AR:

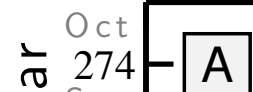

¿ Sep

$>244-$

4 Aug $\square \square \square "$ "

$\underset{0}{213} \mathrm{Jul}=$

م 182

$152-$ - I

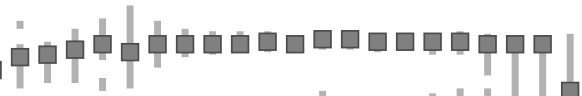

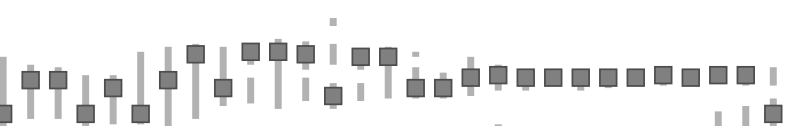

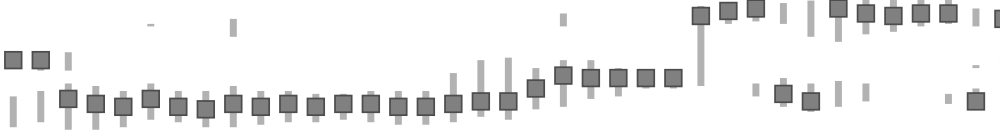

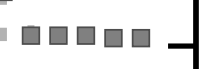

May $\square$

1904-1913 1911-1920

$1921-1930$

$1931-1940$

$\frac{1}{1941-1950}$

$\frac{1}{1951-1960}$

$\frac{1}{1-1970}$

$\frac{1}{1971-1980}$

$\frac{1}{1981-1990}$

991-2000

2003-2012

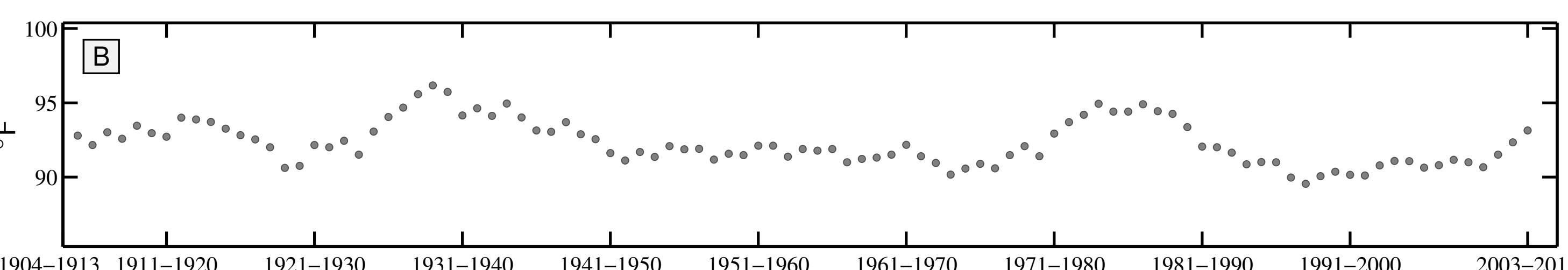

$1904-1913 \quad 1911-1920$

$1921-1930$

$1931-1940$

$1941-1950$

$1951-1960$

$1961-1970$

$1971-1980$

$1981-1990$

$1991-2000$

$2003-2012$

10 year Winter Teletherm dynamics for EUREKA SPRINGS 3 WNW, AR:

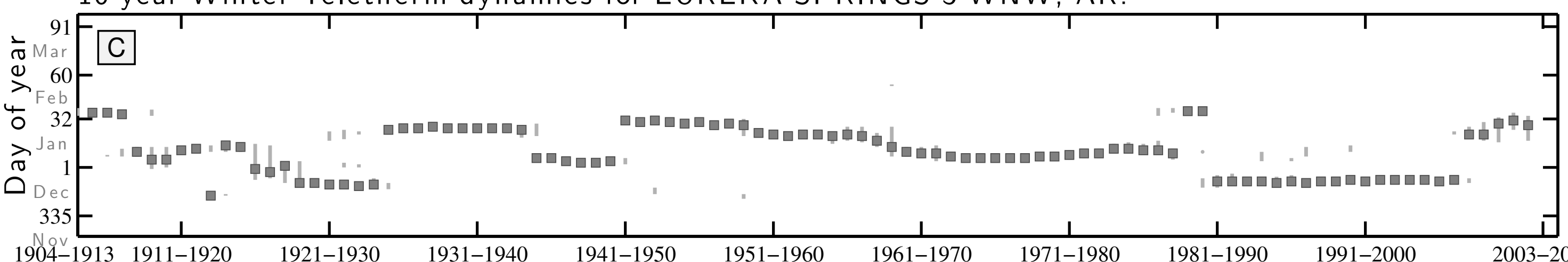

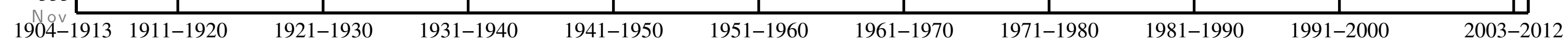

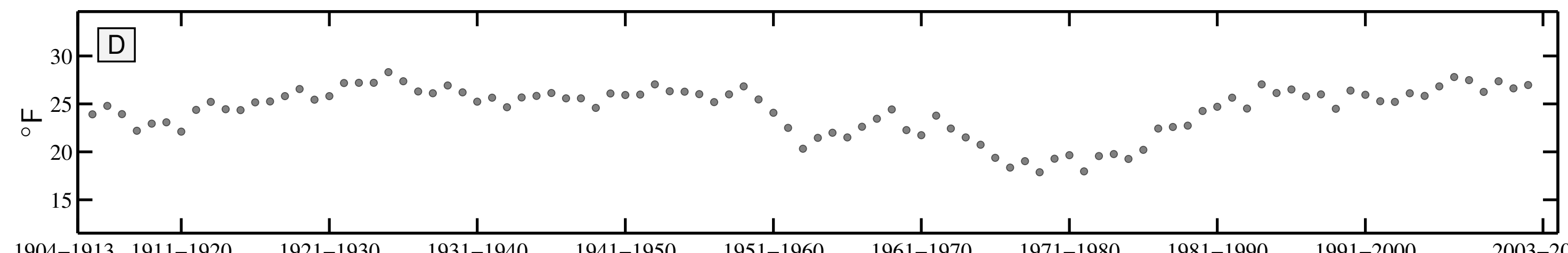


10 year Summer Teletherm dynamics for MAMMOTH SPRING, AR:

$+\mathrm{Oct}$

急 274

(1) Sep

$>244-$

O A g g

$213-$

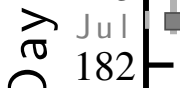

$\bigcirc \underset{J u n}{182}$

$152-$

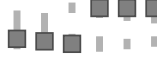

뭉

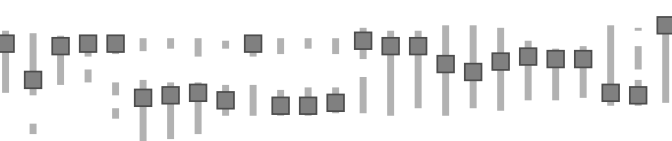

| | | || || ||

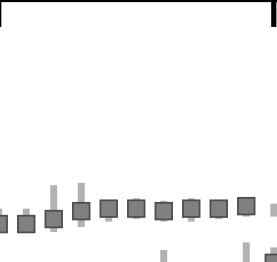

1

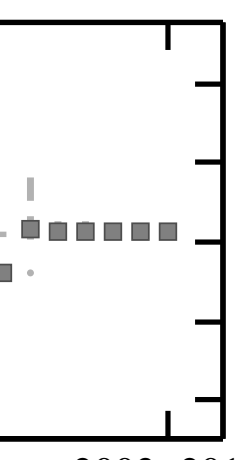

$1904-1913$ 1911-1920

$\frac{1}{1921-1930}$

1931-1940

$\frac{1}{1941-1950}$

$\frac{1}{1951-1960}$

$\frac{1}{1961-1970}$

$1971-1980$

1981-1990

$1991-2000$

2003-2012

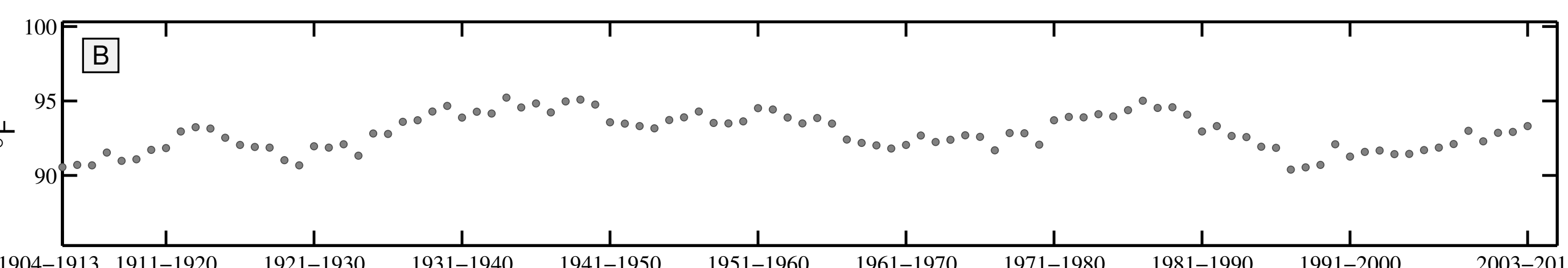

1904-1913 1911-1920

1921-1930

$1931-1940$

1941-1950

1951-1960

1961-1970

$1971-1980$

$1981-1990$

$1991-2000$

$2003-2012$

10 year Winter Teletherm dynamics for MAMMOTH SPRING, AR:

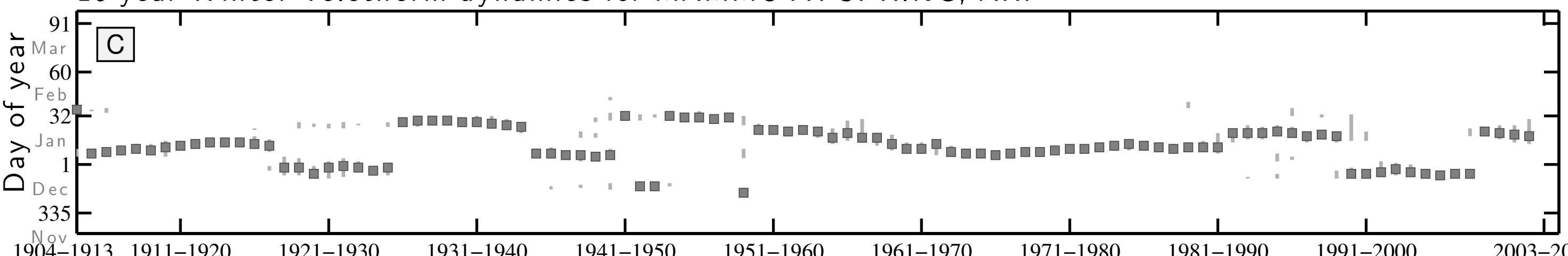

$1904-1913 \quad 1911-1920$

$1921-1930$

1931-1940

1941-1950

1951-1960

1961-1970

1971-1980

1981-1990

1991-2000

2003-2012

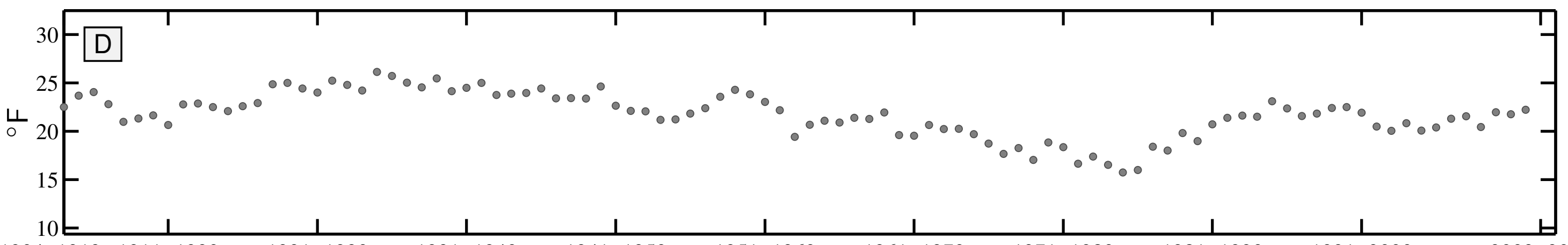


10 year Summer Teletherm dynamics for CHULA VISTA, CA:

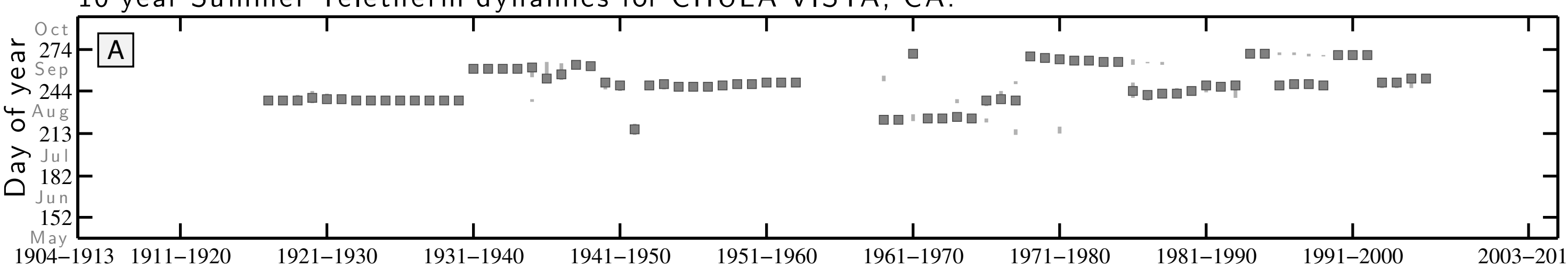

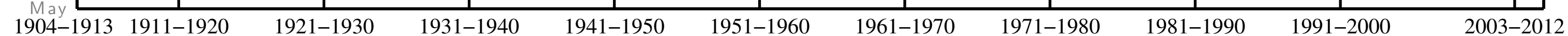

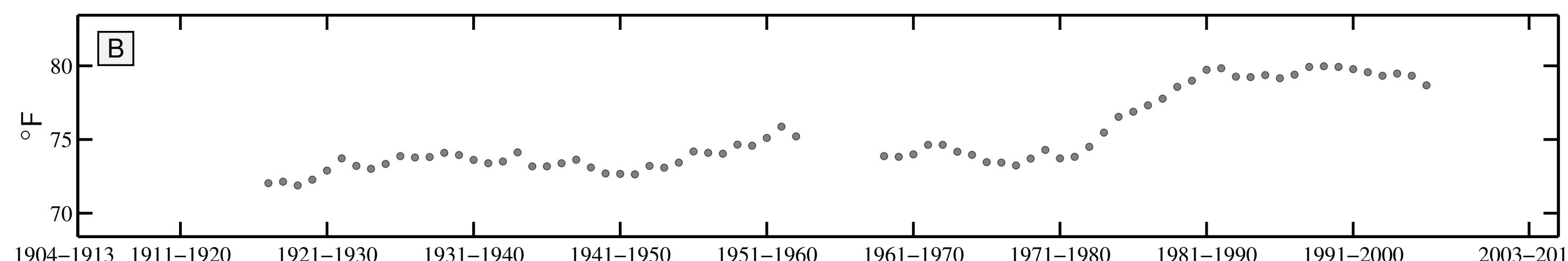

$1921-1930$

$1931-1940$

$1941-1950$

1951-1960

1961-1970

$1971-1980$

1981-1990

$1991-2000$

2003-2012

10 year Winter Teletherm dynamics for CHULA VISTA, CA:
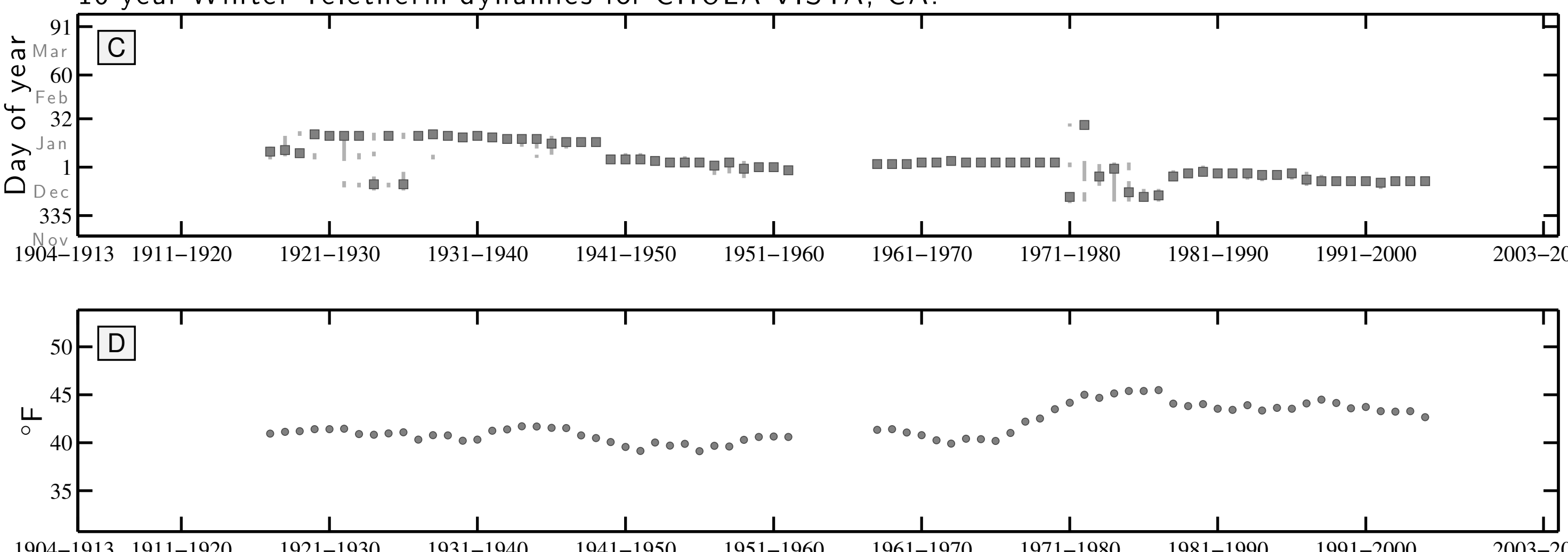
10 year Summer Teletherm dynamics for COLFAX, CA:

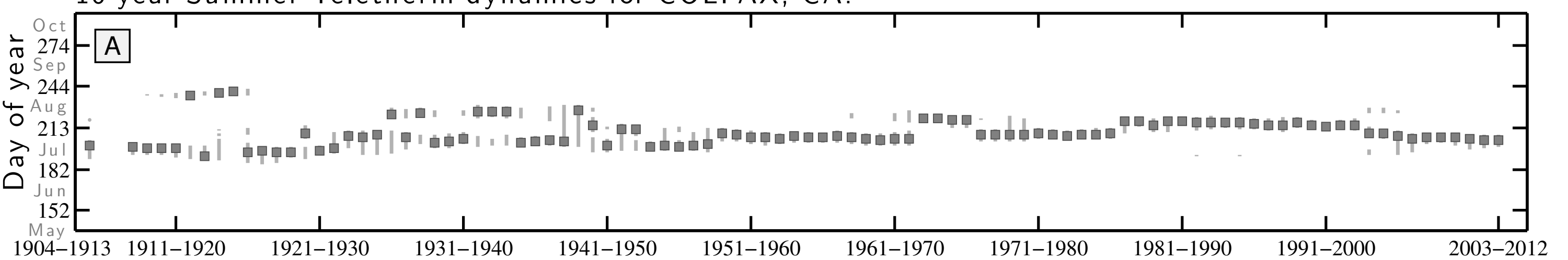

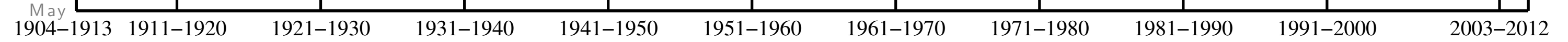
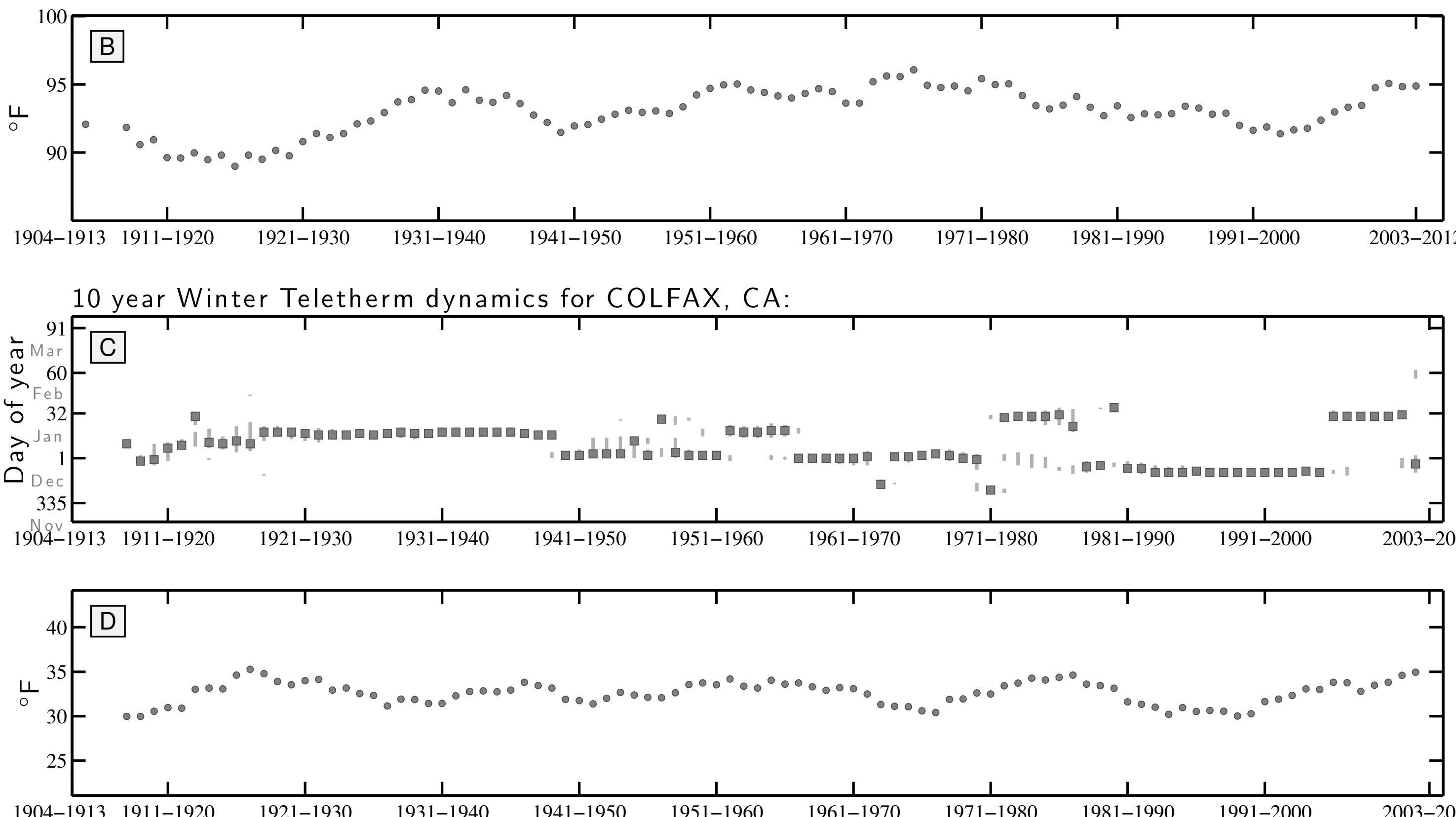

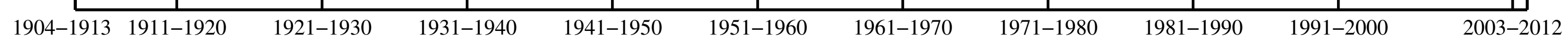


10 year Summer Teletherm dynamics for MARYSVILLE, CA:

$\div \quad 0 \mathrm{ct} A$

(1) Sep

$>244-$

O 213 -

$\gtrsim \mathrm{Jul}$

$\overbrace{}^{\circ} 182$

152

May $\square$

1904-1913 1911-1920

$\frac{1}{1921-1930}$

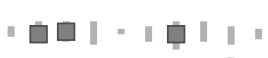

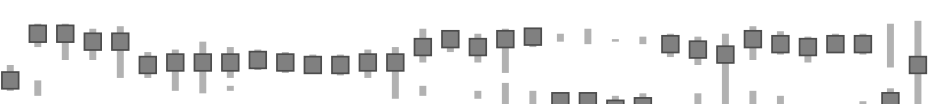

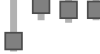

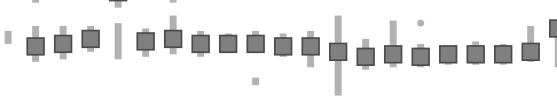

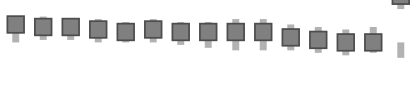

1

$\frac{1}{1951-1960}$

$1961-1970$

$\frac{1}{1971-1980}$

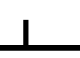

1981-1990

$1991-2000$

2003-201

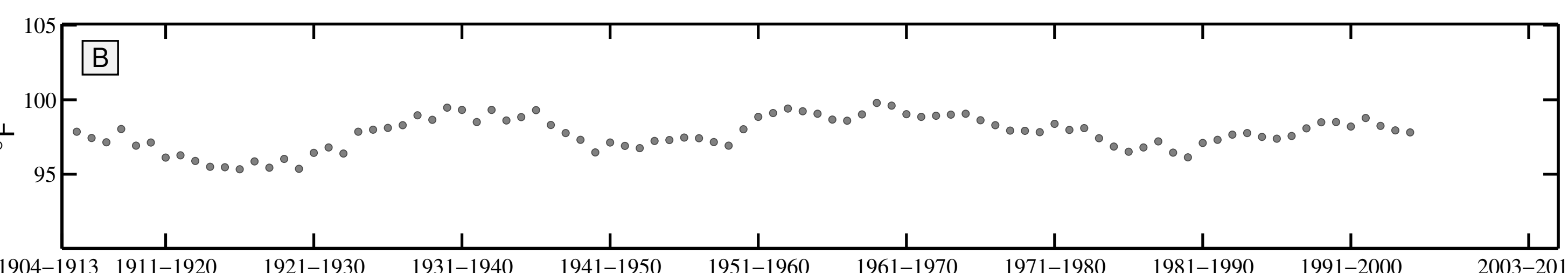

1904-1913 1911-1920

1921-1930

1931-1940

1941-1950

1951-1960

1961-1970

1971-1980

1981-1990

1991-2000

2003-2012

10 year Winter Teletherm dynamics for MARYSVILLE, CA:

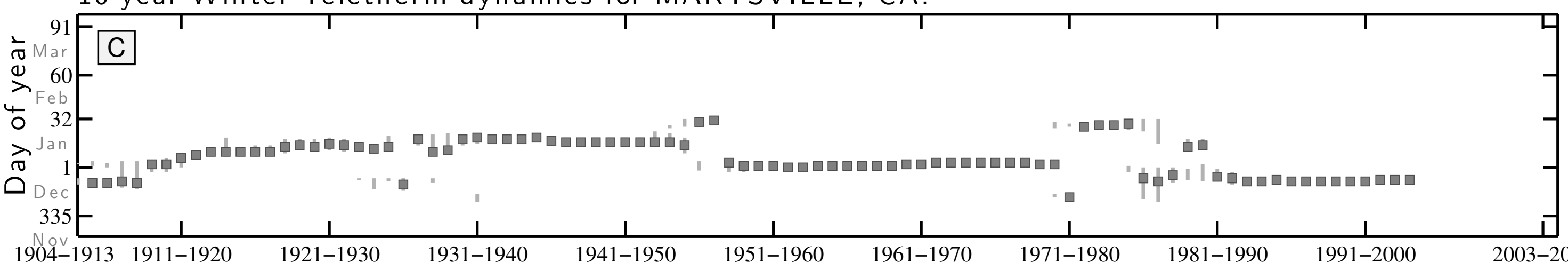

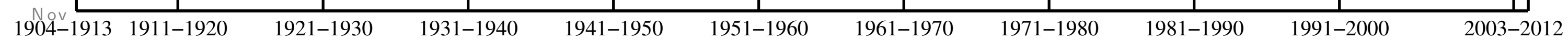

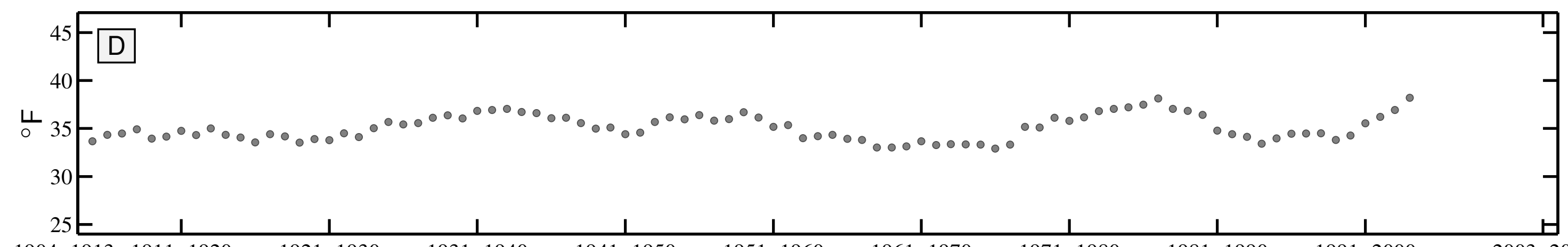


10 year Summer Teletherm dynamics for WASCO, CA:

$\div 274-\mathrm{A}$

\& Sep

$>244-$

4 $\mathrm{Aug}$

$\gtrsim \mathrm{Jul}$

$\overbrace{}^{\circ} 182$

$152-$

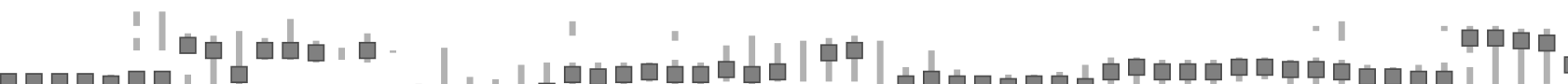

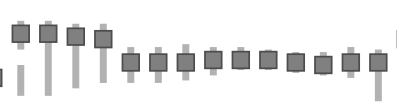

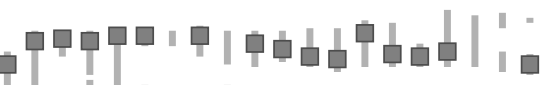
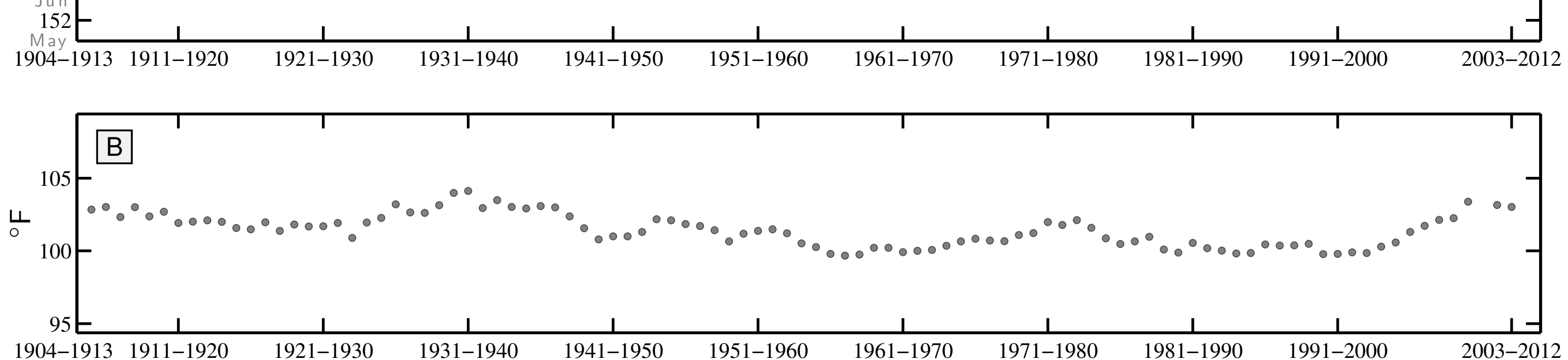

1904-1913 1911-192

$1921-1930$

$1931-1940$

$1941-1950$

$1951-1960$

$1961-1970$

$1971-1980$

$1981-1990$

$1991-2000$

2003-2012

10 year Winter Teletherm dynamics for WASCO, CA:

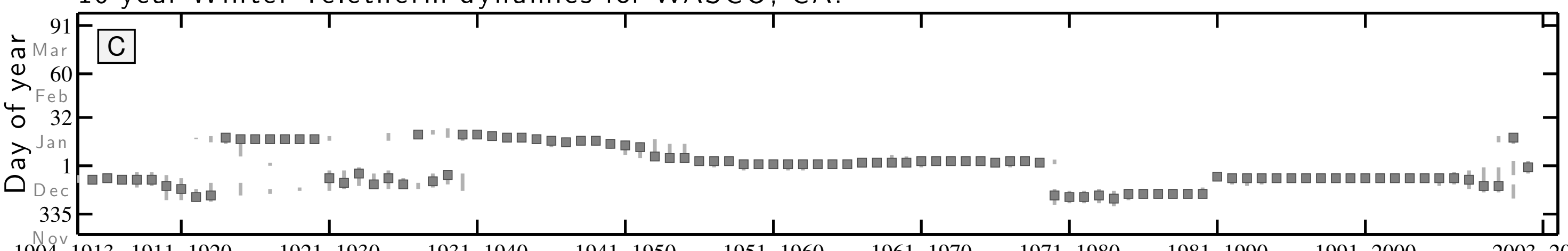

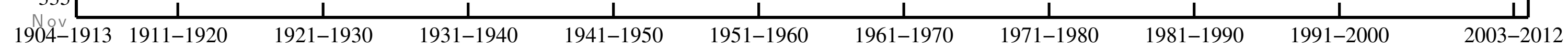

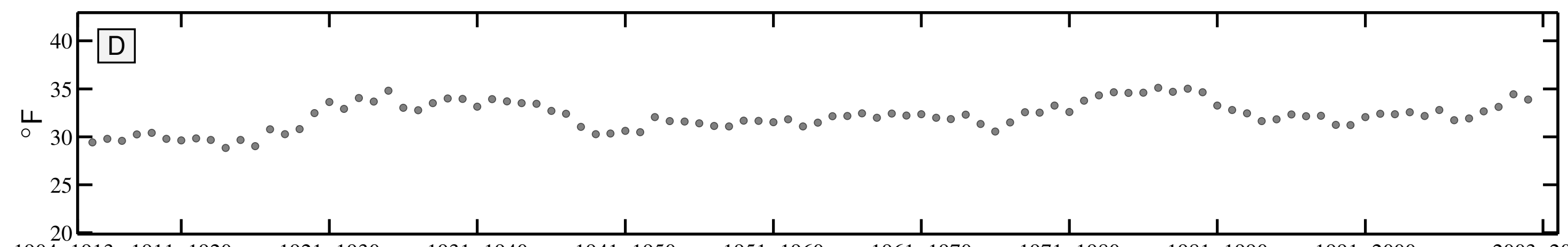


10 year Summer Teletherm dynamics for CANON CITY, CO:

$-O c t$

$\pi 274-A$

(1) Sep

$>244$

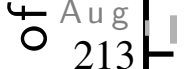

$>$ Jul

(82-

Jun

152

$1904-1913$ 1911-1920

|

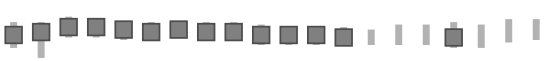

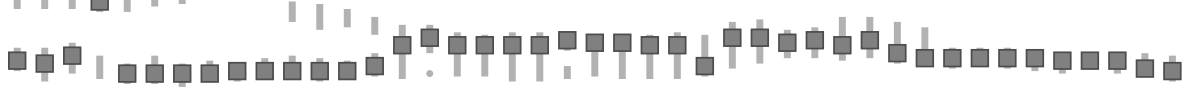

| || || प | = |

$\frac{1}{1921-1930}$

$\frac{1}{1931-1940}$

$\frac{1}{1941-1950}$

$\frac{1}{1951-1960}$

1961-1970

1971-1980

1981-1990

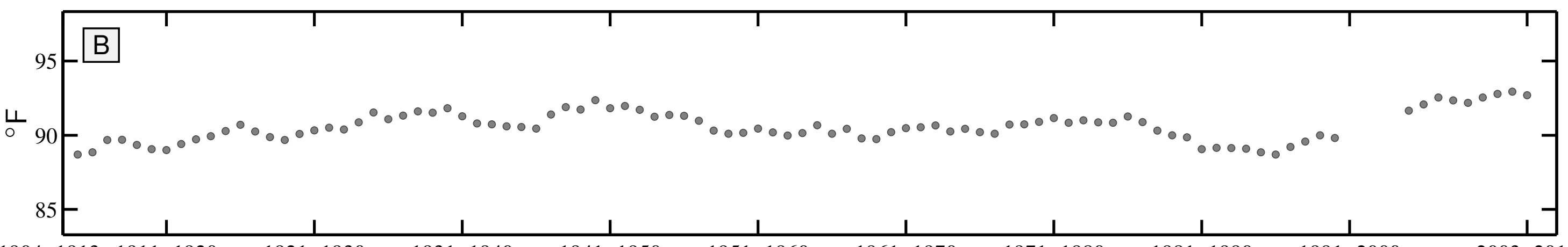

1904-1913 1911-1920

1921-1930

1931-1940

1941-1950

1951-1960

1961-1970

1971-1980

1981-1990

1991-2000

2003-2012

10 year Winter Teletherm dynamics for CANON CITY, CO:

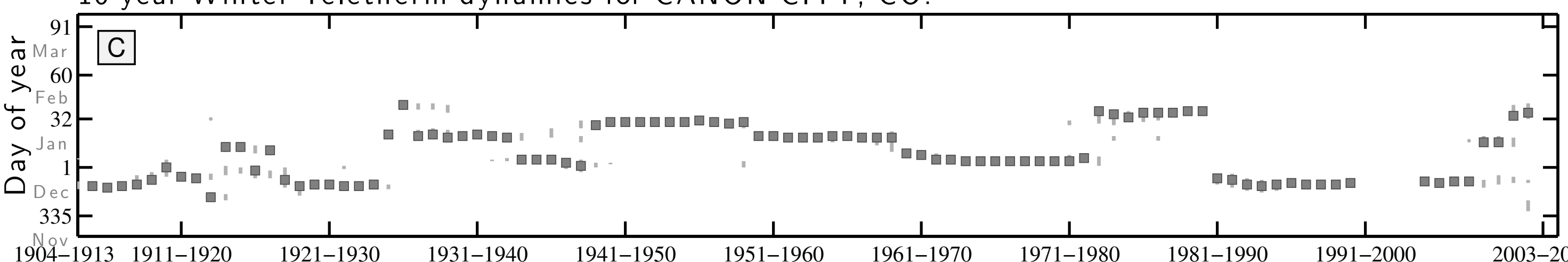

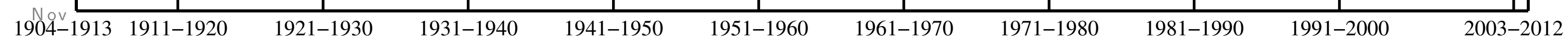

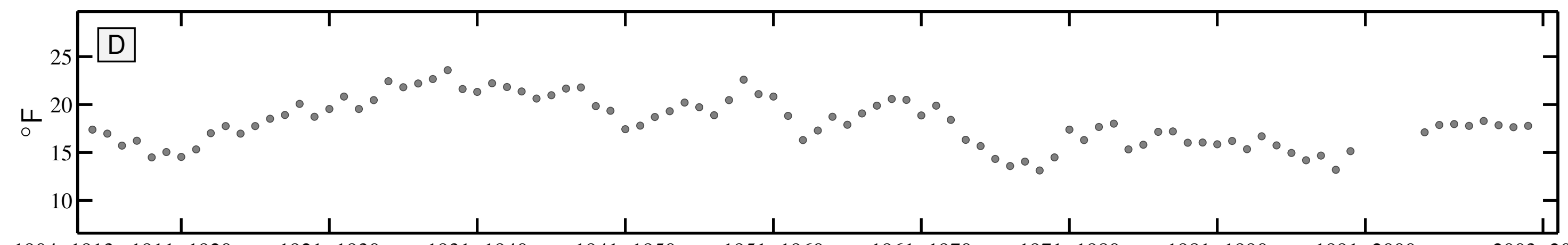


10 year Summer Teletherm dynamics for COLLBRAN, CO:

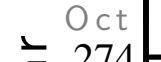

A

(1) Sep

$>244$

O $213=$

ते Jul

182

$152-$

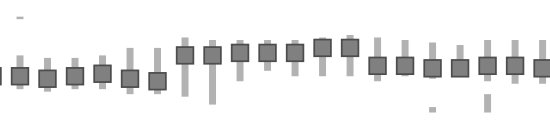

$-1920$

1921-1930

$\frac{1}{1931-1940}$

$\frac{1}{1941-1950}$

1951-1960

$\frac{1}{1961-1970}$
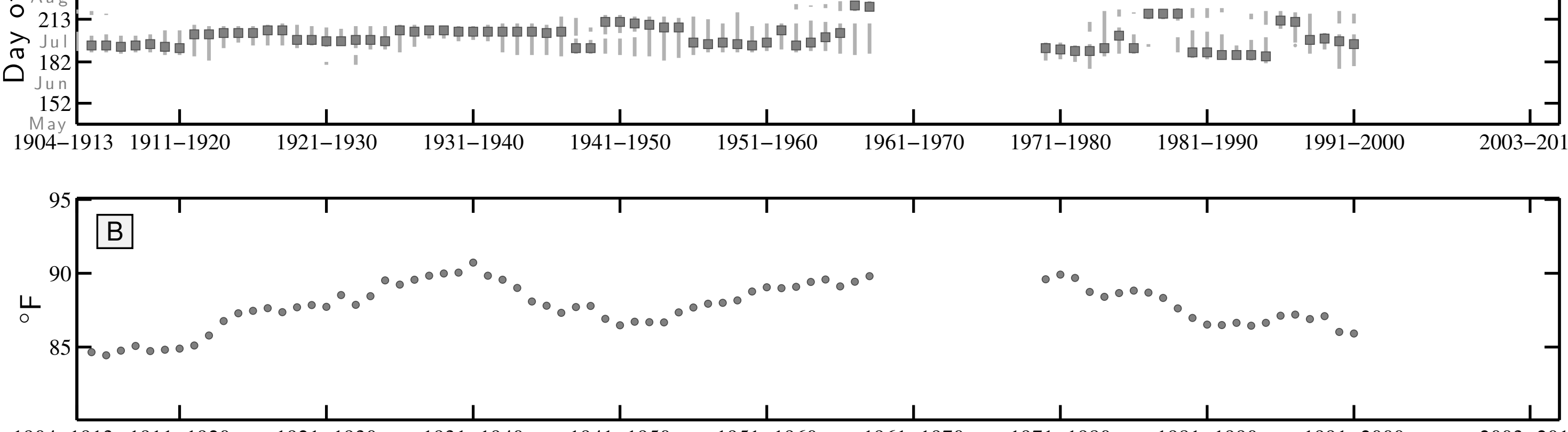

1904-1913 1911-1920

$1921-1930$

$1931-1940$

$1941-1950$

1951-1960

1961-1970

1971-1980

$1981-1990$

$1991-2000$

$2003-2012$

10 year Winter Teletherm dynamics for COLLBRAN, CO:

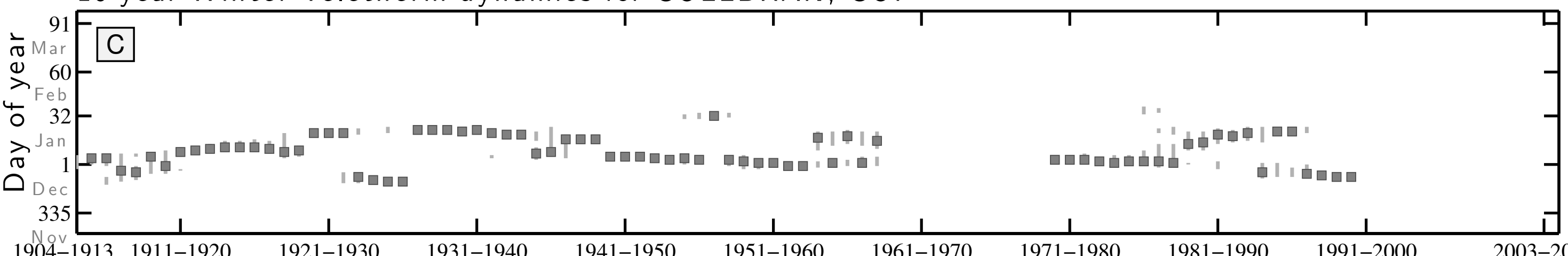

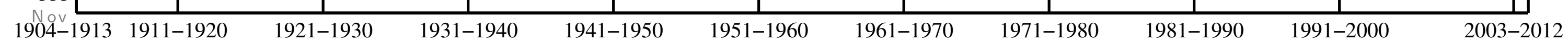

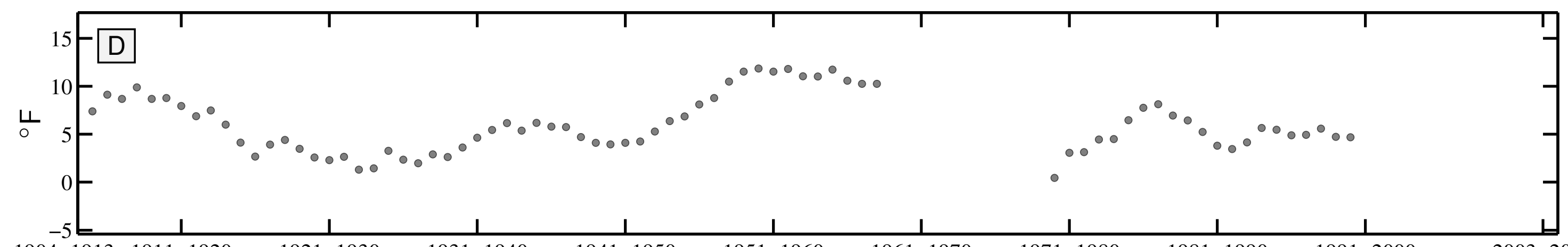


10 year Summer Teletherm dynamics for DEL NORTE 2E, CO:

$\mathrm{Oct}$

更 274

(1) Sep

$>244$

( $\mathrm{Aug}$

$>\mathrm{Jul}$

\% 182

$\mathrm{J} u \mathrm{n}$
152

152

1904-1913 1911-1920

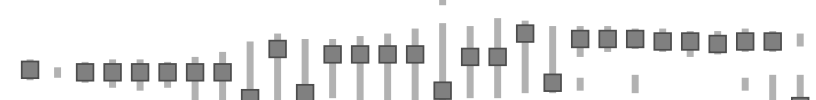

"

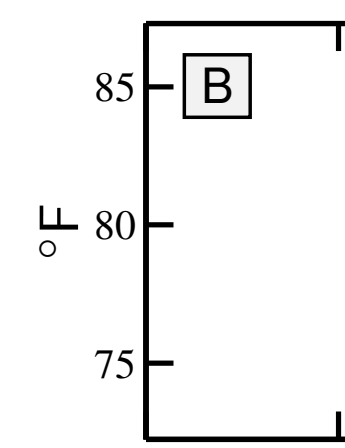

$\frac{1}{1921-1930}$

$1931-1940$

$1941-1950$

$1951-1960$

$1961-1970$

1971-1980

$\frac{1}{1981-1990}$

991-2000

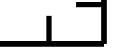

2003-2012

1904-1913 1911-1920

1921-1930

1931-1940

1941-1950

1951-1960

1961-1970

1971-1980

1981-1990

1991-2000

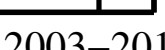

10 year Winter Teletherm dynamics for DEL NORTE 2E, CO:

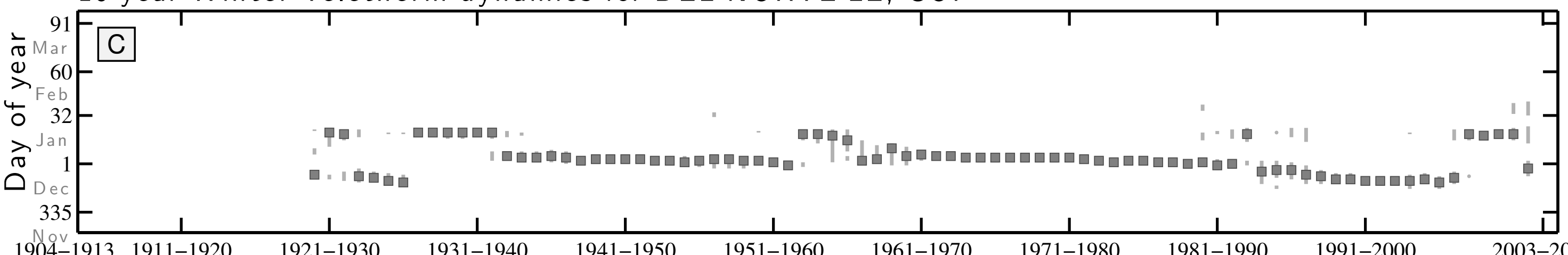

1904-1913 1911-1920

1921-1930

1931-1940

1941-1950

1951-1960

$1961-1970$

1971-1980

1981-1990

$2003-2012$

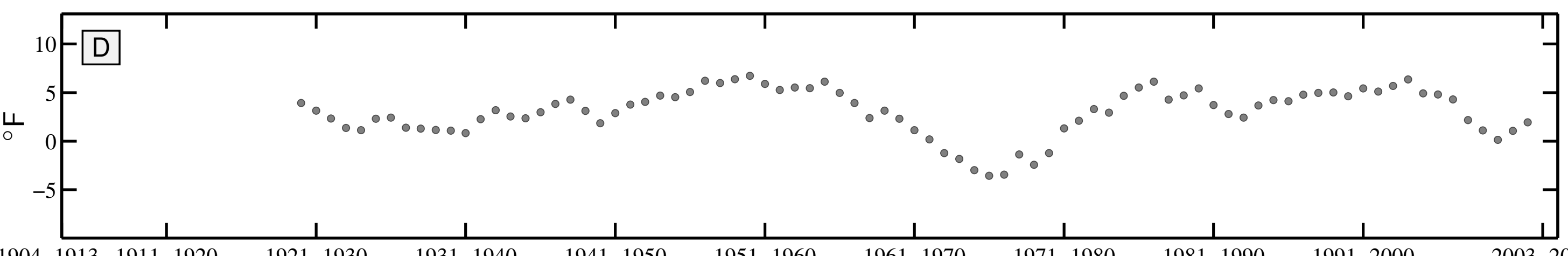


10 year Summer Teletherm dynamics for FT MORGAN, CO:

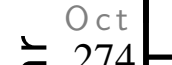

A

(1) Sep

244

213

$\gtrsim \mathrm{Jul}$

$\overbrace{}^{\circ} 182$

$152-$

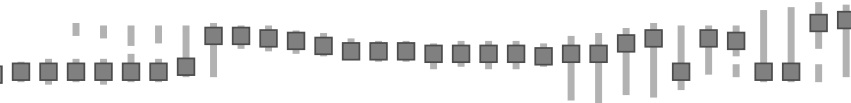

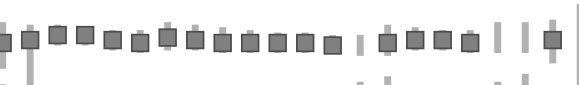

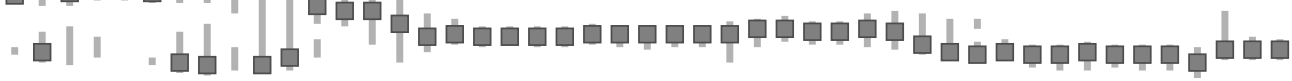

$1904-1913 \quad 1911-1920$

$1921-1930$

$1931-1940$

$\frac{1}{1941-1950}$

$1951-1960$

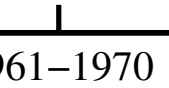

$1971-1980$

$\frac{1}{1981-1990}$

$1991-2000$

2003-2012

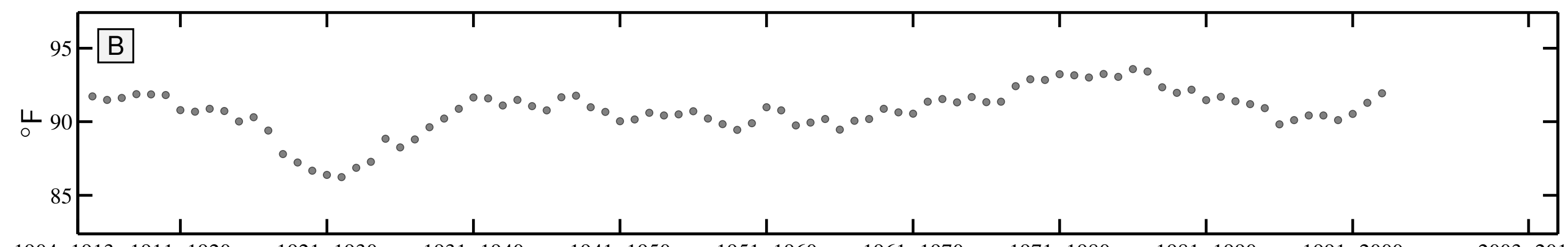

1904-1913 1911-1920

1921-1930

1931-1940

1941-1950

1951-1960

1961-1970

1971-1980

1981-1990

1991-2000

2003-2012

10 year Winter Teletherm dynamics for FT MORGAN, CO:

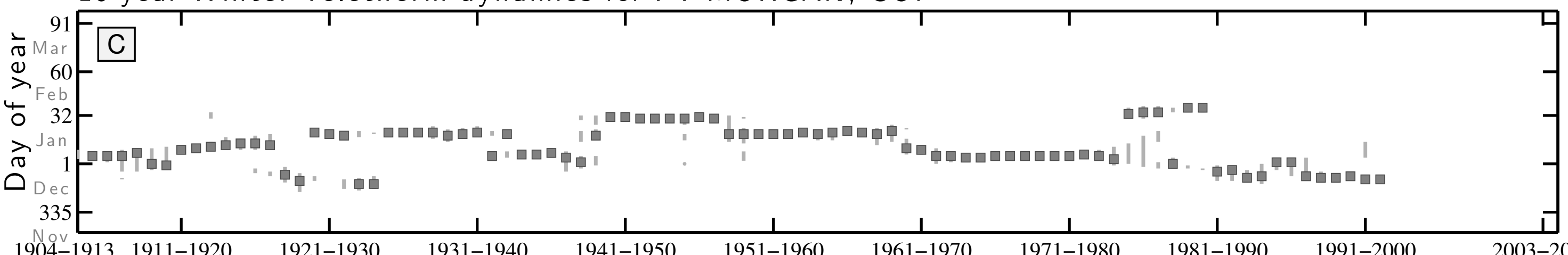

1904-1913 1911-1920

1921-1930

1931-1940

1941-1950

1951-1960

1961-1970

1971-1980

1981-1990

1991-2000

2003-2012

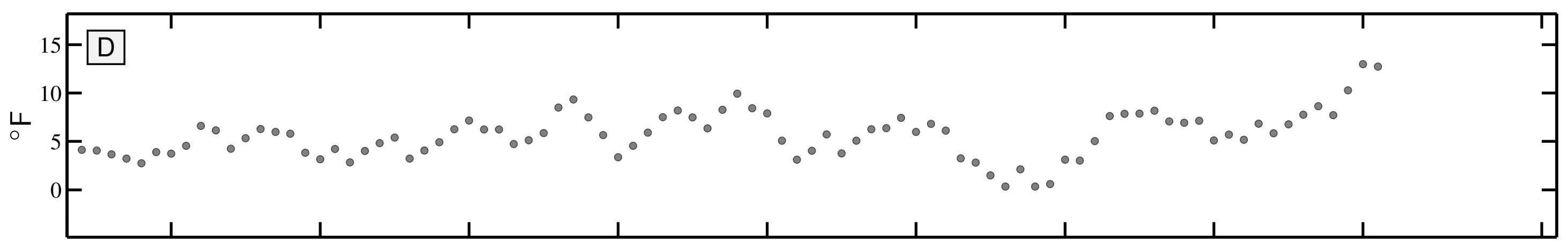


10 year Summer Teletherm dynamics for FRUITA, CO:
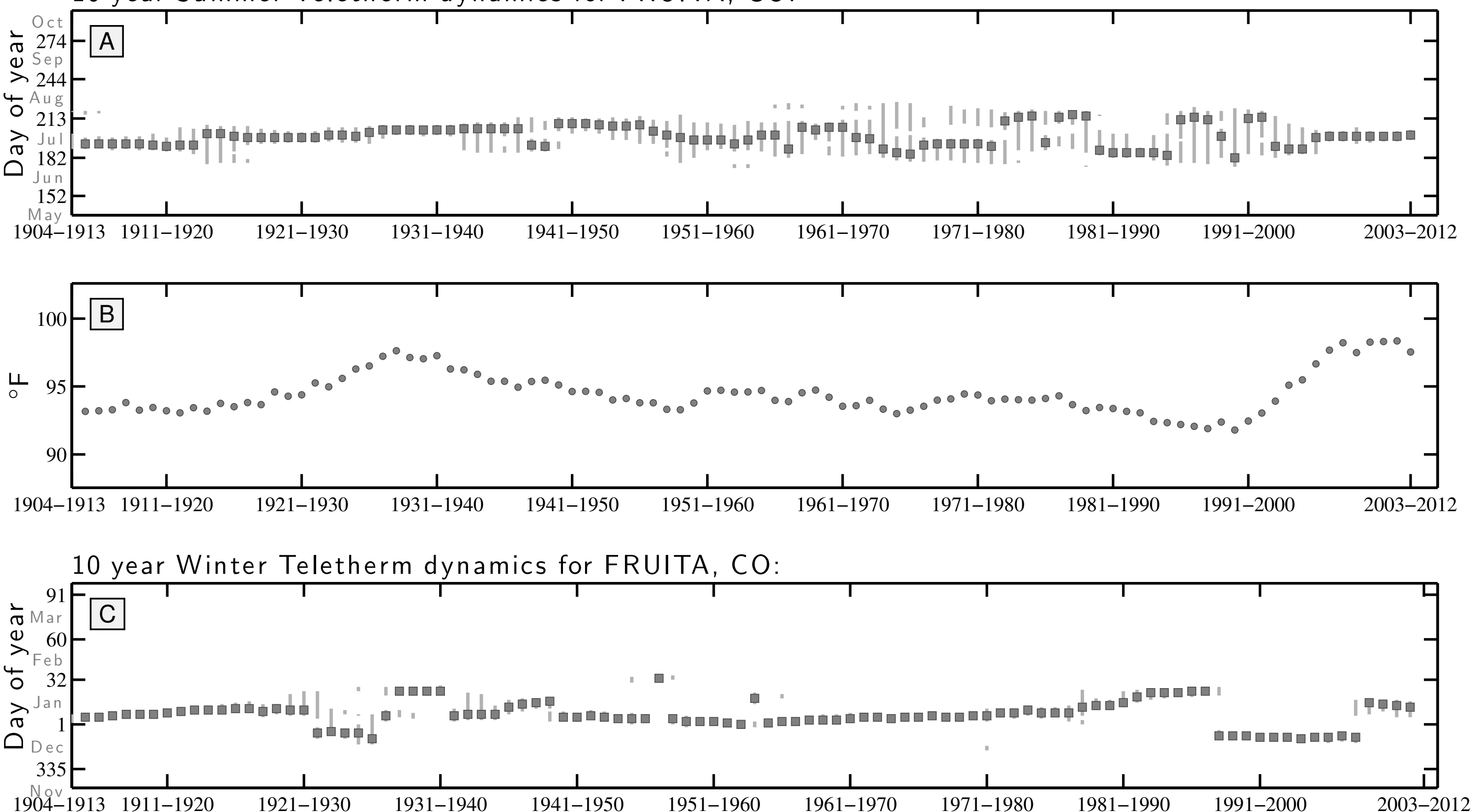

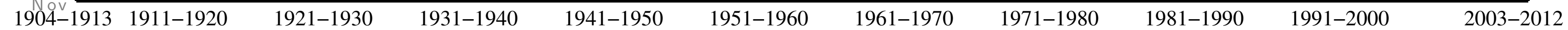

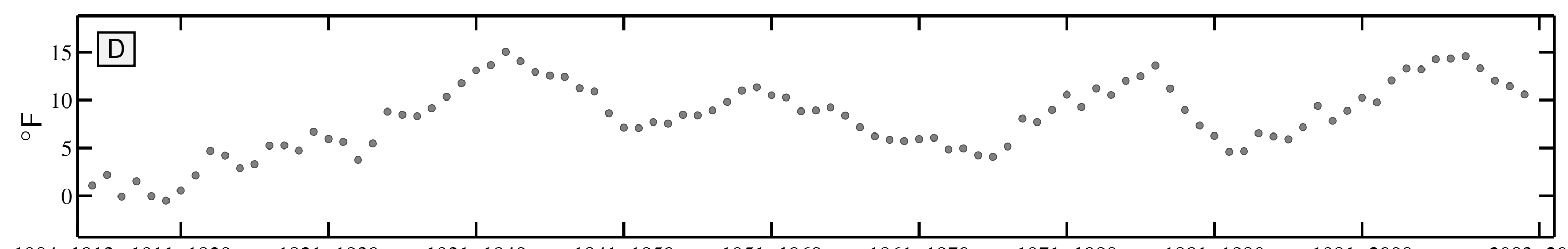

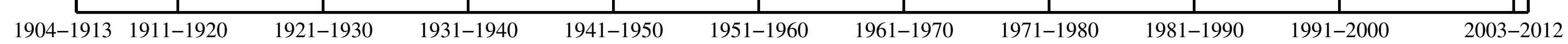


10 year Summer Teletherm dynamics for HOLLY, CO:

$+\mathrm{Oct}$

( 274

(1) Sep

$>244$

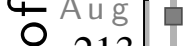

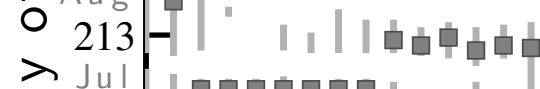

\% 182

152

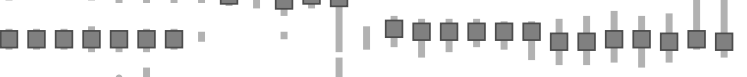

| | | ㅁㅁㅁㅁำ

ดロ

|| | | | | || -

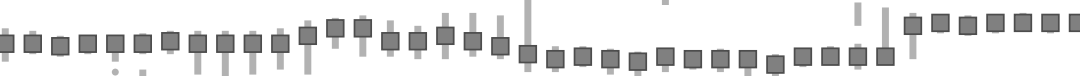

152

1

$\frac{1}{1-1930}$

$1931-1940$

$1941-1950$

$\frac{1}{1951-1960}$

$\frac{1}{1970}$

$\frac{1}{1971-1980}$

1981-1990

1991-2000

2003-2012

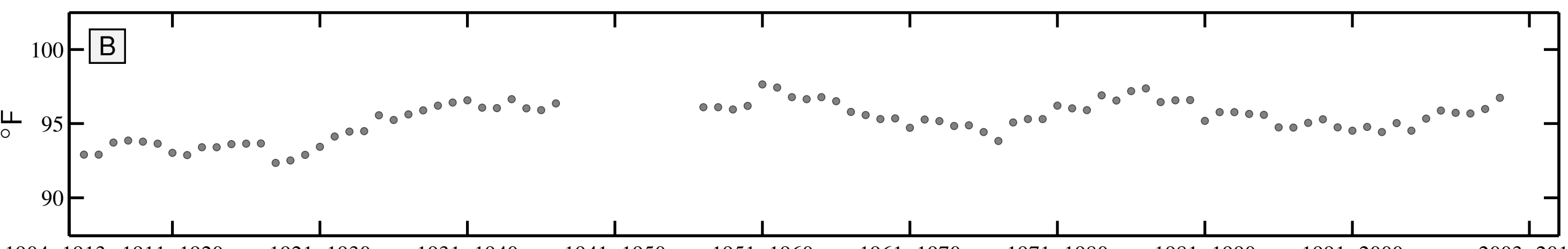

1904-1913 1911-1920

$1921-1930$

$1931-1940$

$1941-1950$

$1951-1960$

$1961-1970$

$1971-1980$

$1981-1990$

$1991-2000$

2003-2012

10 year Winter Teletherm dynamics for HOLLY, CO:

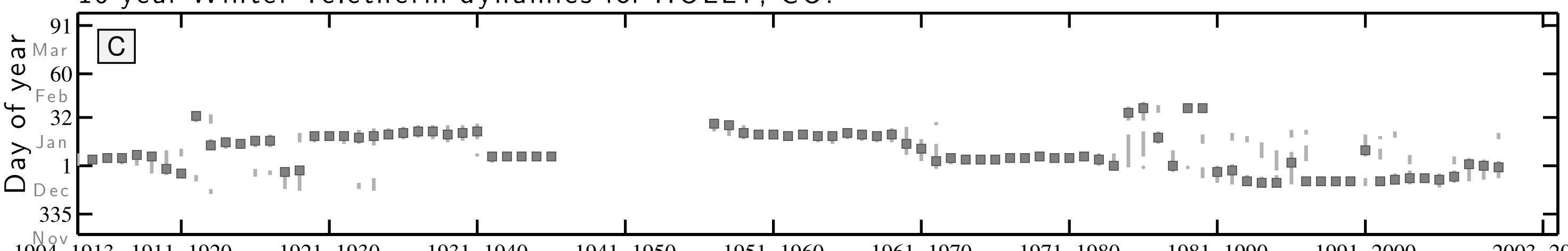

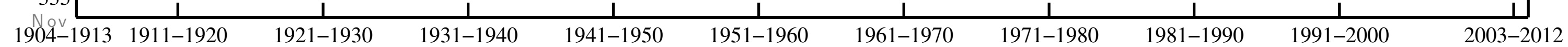

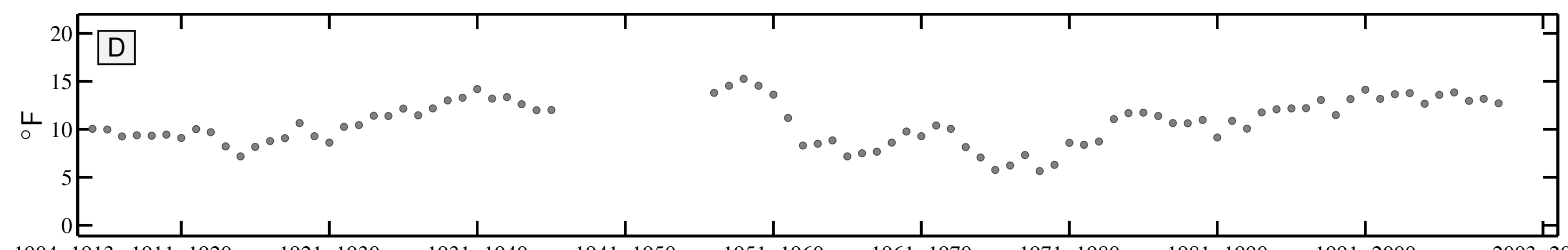


10 year Summer Teletherm dynamics for ROCKY FORD 2 SE, CO:

$\div 274-\mathrm{A}$

¿ Sep

$>244-$

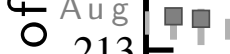

$213-1\|\|\|\| \square$

ते Jul

$\overbrace{\substack{u \\ \text { n }}}^{182}$

$152-$

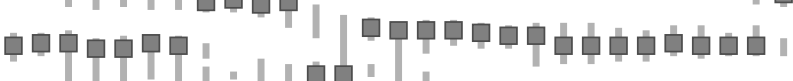

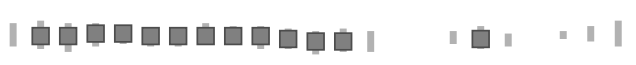

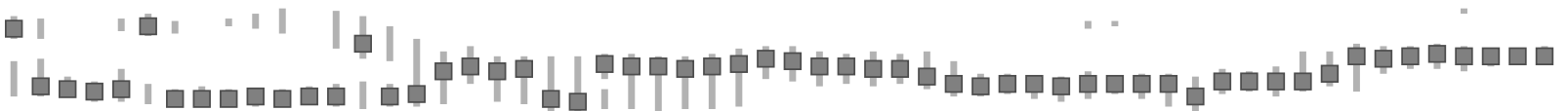

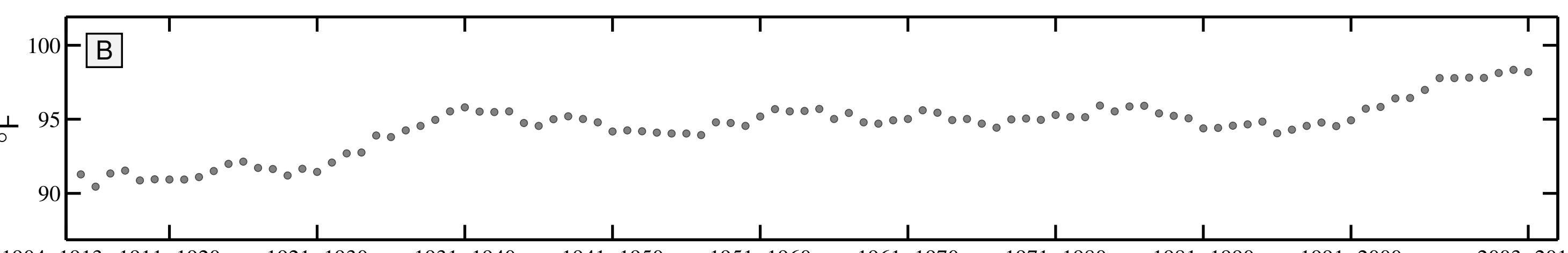

10 year Winter Teletherm dynamics for ROCKY FORD 2 SE, CO:
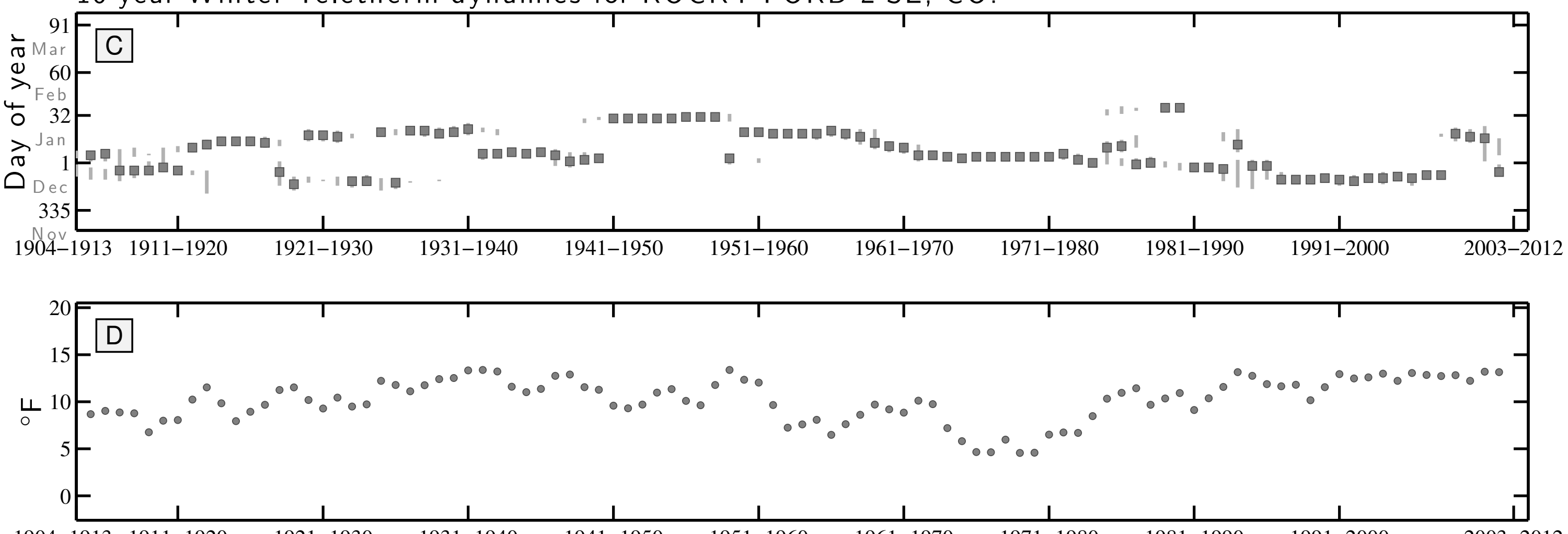
10 year Summer Teletherm dynamics for STORRS, CT:
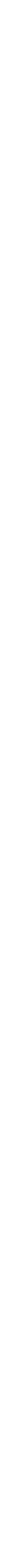

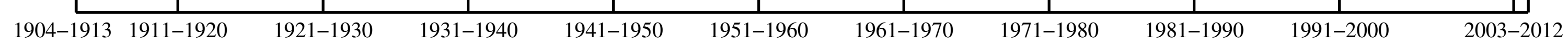


10 year Summer Teletherm dynamics for GREENWOOD 2NE, DE:
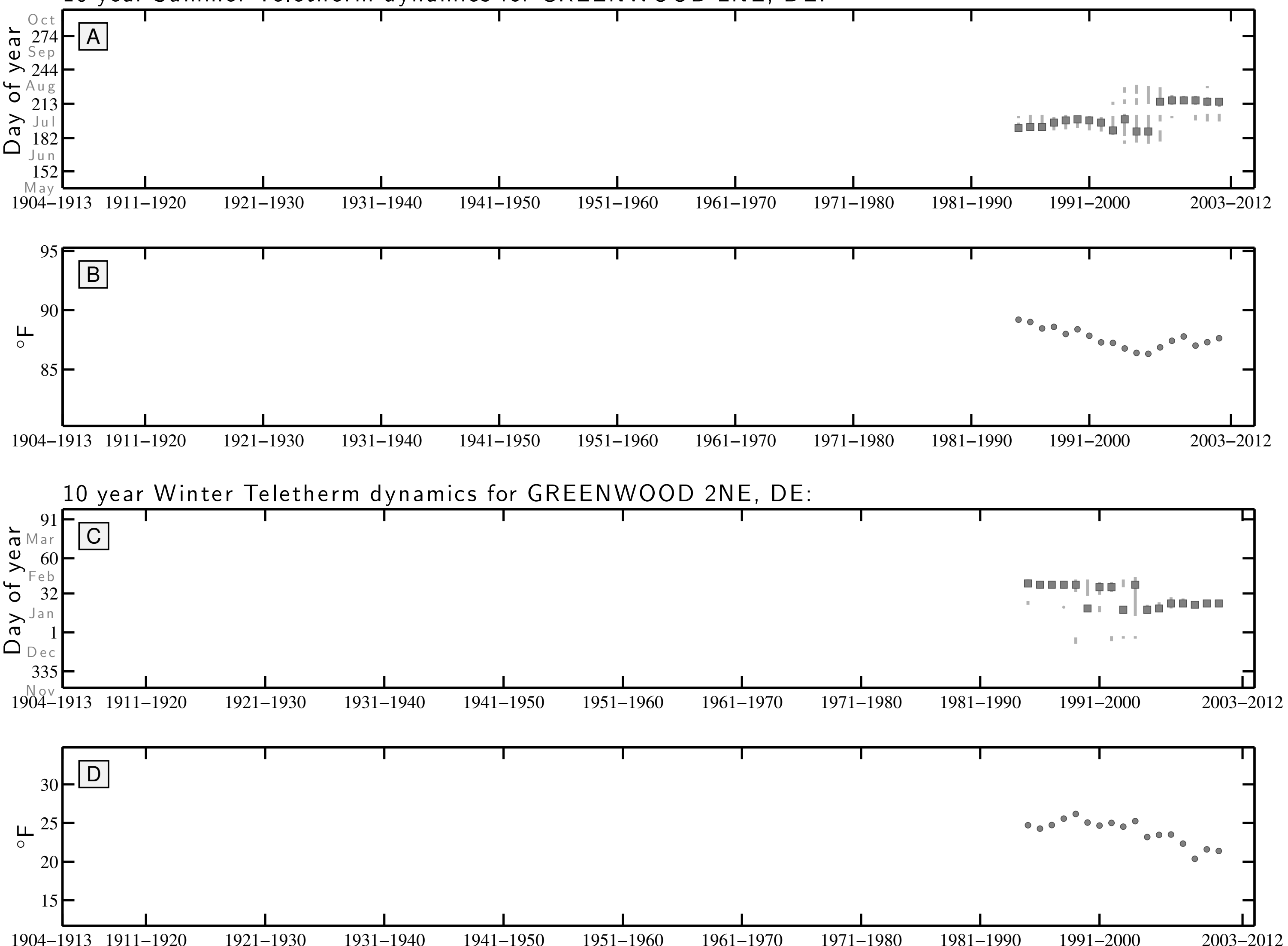
10 year Summer Teletherm dynamics for MILFORD 2 SE, DE:

$\div$ Oct 274

(1) Sep

$>244$

4 $\mathrm{Aug}$

$213-$

๙ 182

Jun
152
May

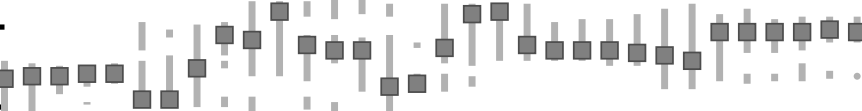

-

$-1920$

1921-1930

1931-1940

$\frac{1}{1941-1950}$

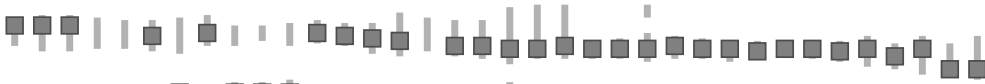
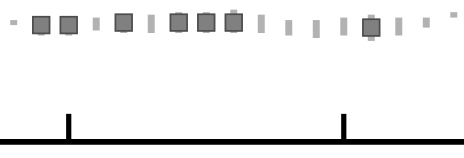

$\frac{1}{1961-1970}$

$1971-1980$

1981-1990

$1991-2000$

2003-2012

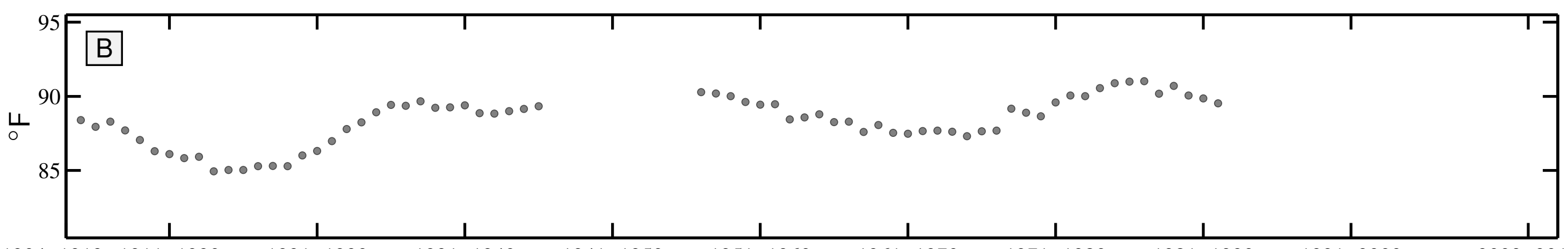

1904-1913 1911-1920

$1921-1930$

1931-1940

$1941-1950$

$1951-1960$

$1961-1970$

$1971-1980$

$1981-1990$

$1991-2000$

2003-2012

10 year Winter Teletherm dynamics for MILFORD 2 SE, DE:

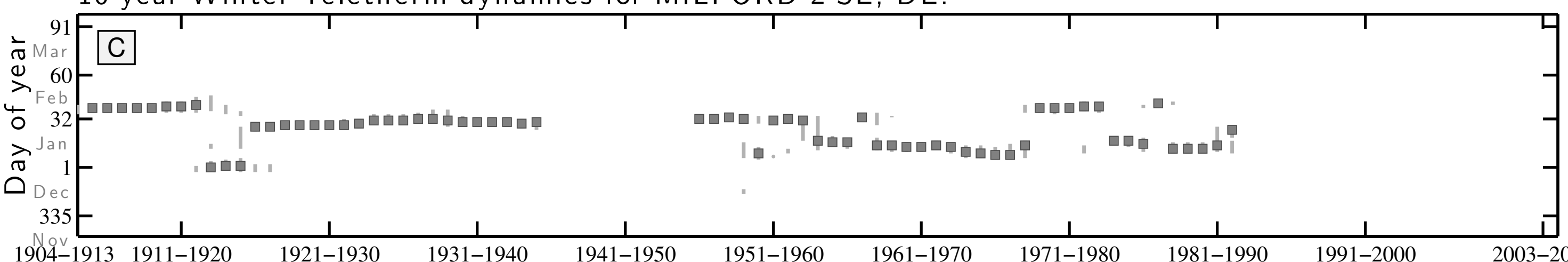

1904-1913 1911-1920

$1921-1930$

1931-1940

$1941-1950$

1951-1960

1961-1970

1971-1980

1981-1990

991-2000

003-2012

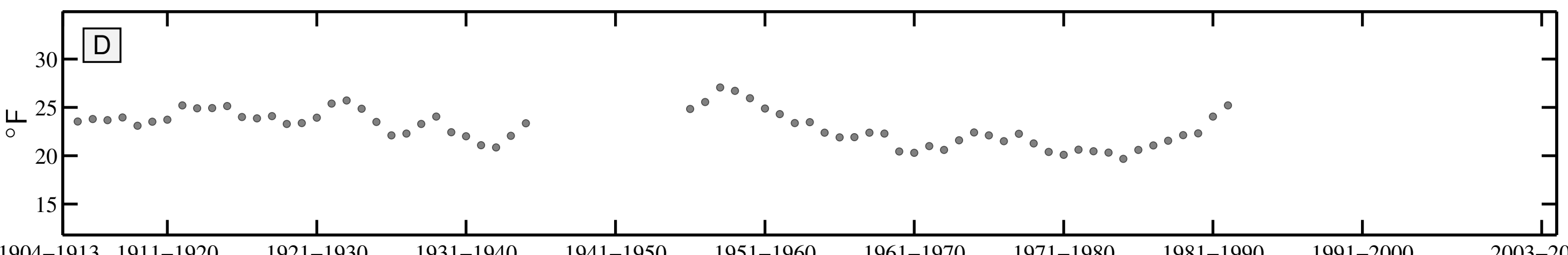


10 year Summer Teletherm dynamics for EVERGLADES, FL:
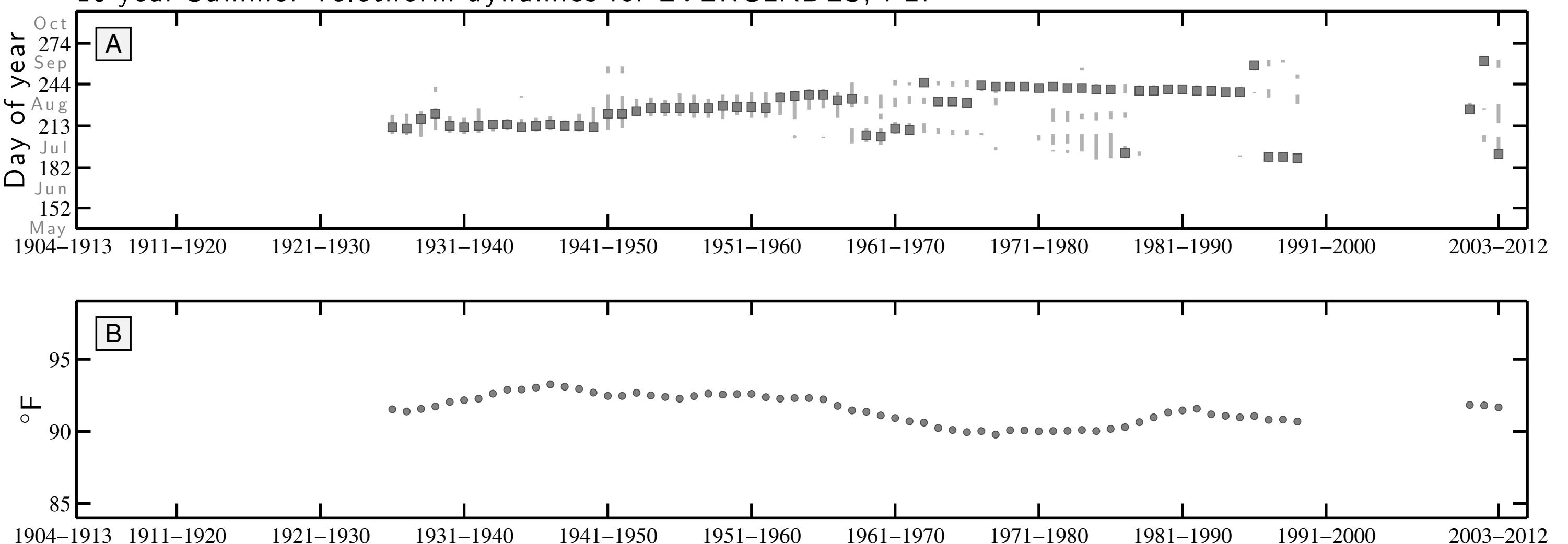

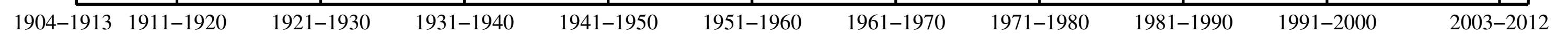

10 year Winter Teletherm dynamics for EVERGLADES, FL:

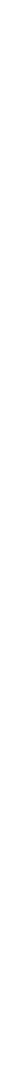


10 year Summer Teletherm dynamics for FERNANDINA BEACH, FL:

$+\mathrm{Oct}$

A

$\stackrel{2}{>} 244$

- Aug

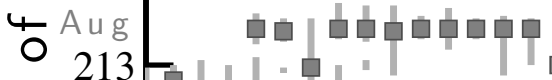

$>\mathrm{Jul}$

\% 182

$\mathrm{J} u \mathrm{n}$
152

$152-1$

I

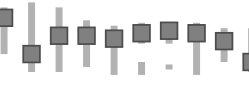

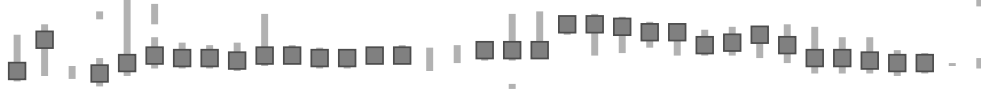

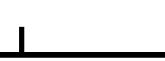

$\frac{1}{1951-1960}$

$\frac{1}{1961-1970}$

$\frac{1}{1971-1980}$

$\frac{1}{1981-1990}$

$1991-2000$

2003-2012

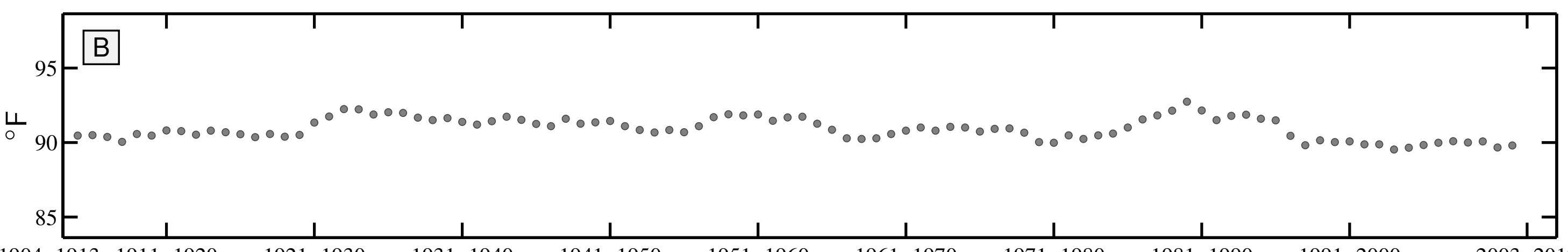

1904-1913 1911-1920

1921-1930

1931-1940

1941-1950

1951-1960

1961-1970

1971-1980

1981-1990

1991-2000

2003-2012

10 year Winter Teletherm dynamics for FERNANDINA BEACH, FL:

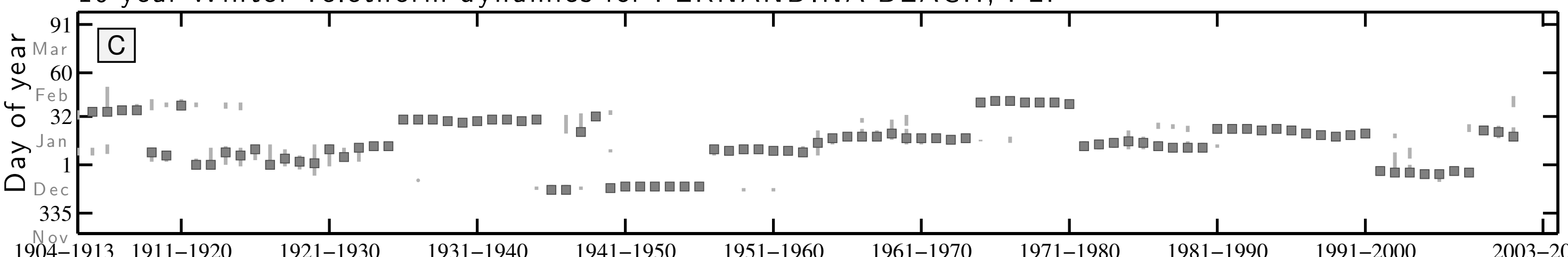

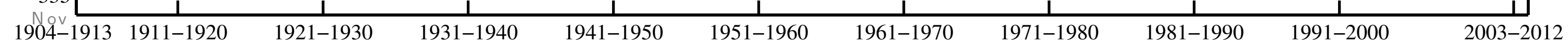

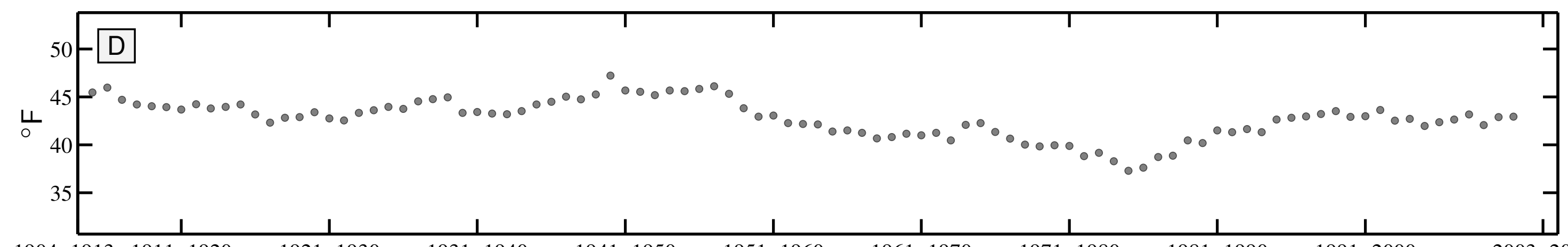


10 year Summer Teletherm dynamics for FT PIERCE, FL:

$={ }^{O} c t$

更 $274-A$

(1) Sep

$>244-$

4 A u g

$\underset{\mathrm{Jul}}{213}=$

ก $182-$

$152-$

May —

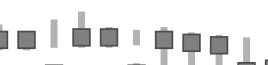

$\cdot$

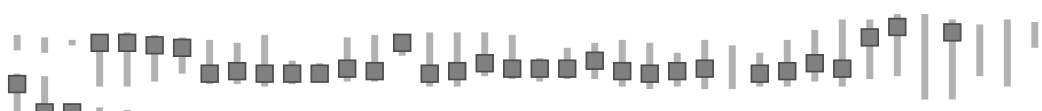

1904-1913 1911-1920

$\frac{1}{1921-1930}$

$\frac{1}{1931-1940}$

$\frac{1}{1941-1950}$

$\frac{1}{1951-1960}$

$\frac{1}{1961-1970}$

$\frac{1}{1971-1980}$

$\frac{1}{1981-1990}$

1991-2000

2003-2012

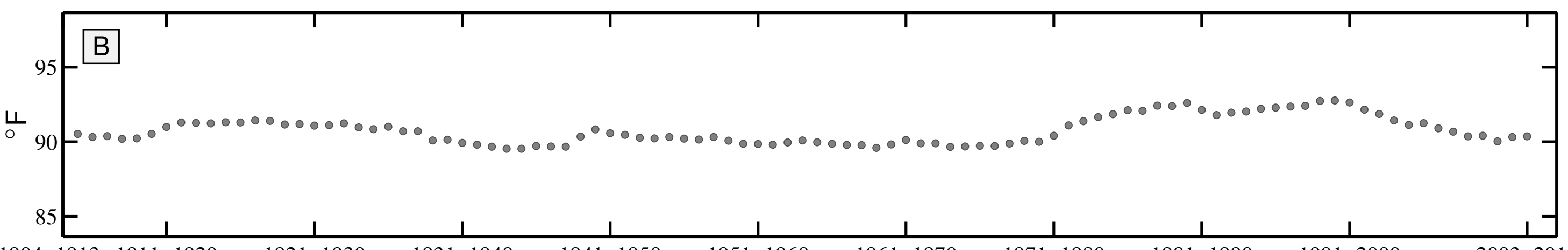

1904-1913 1911-1920

$1921-1930$

$1931-1940$

$1941-1950$

$1951-1960$

$1961-1970$

$1971-1980$

$1981-1990$

$1991-2000$

2003-2012

10 year Winter Teletherm dynamics for FT PIERCE, FL:

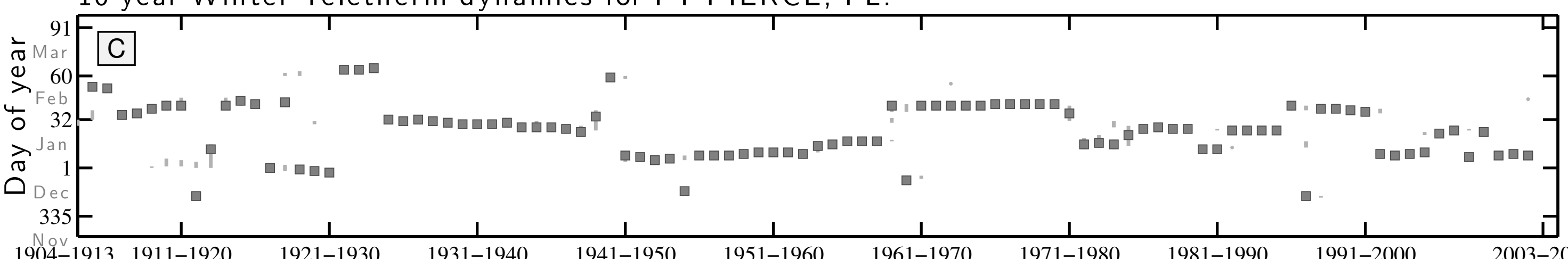

1904-1913 1911-1920

$1921-1930$

$1931-1940$

1941-1950

1951-1960

1961-1970

1971-1980

1981-1990

1991-2000

003-2012

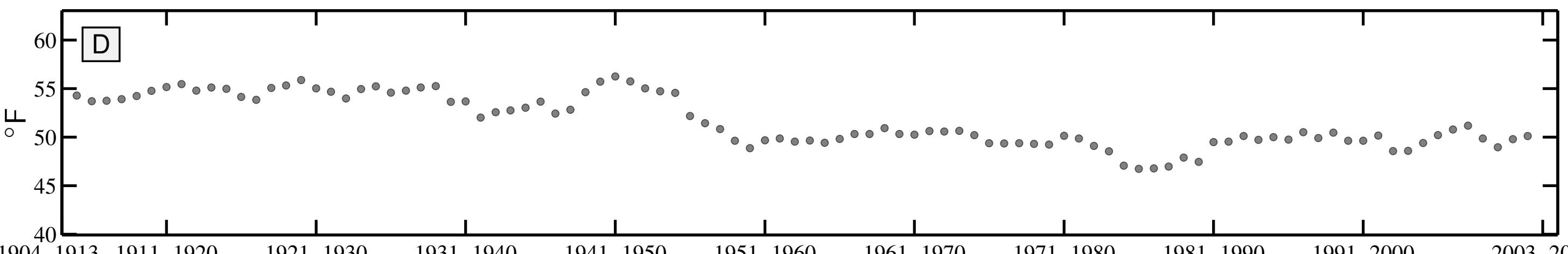


10 year Summer Teletherm dynamics for MADISON, FL:
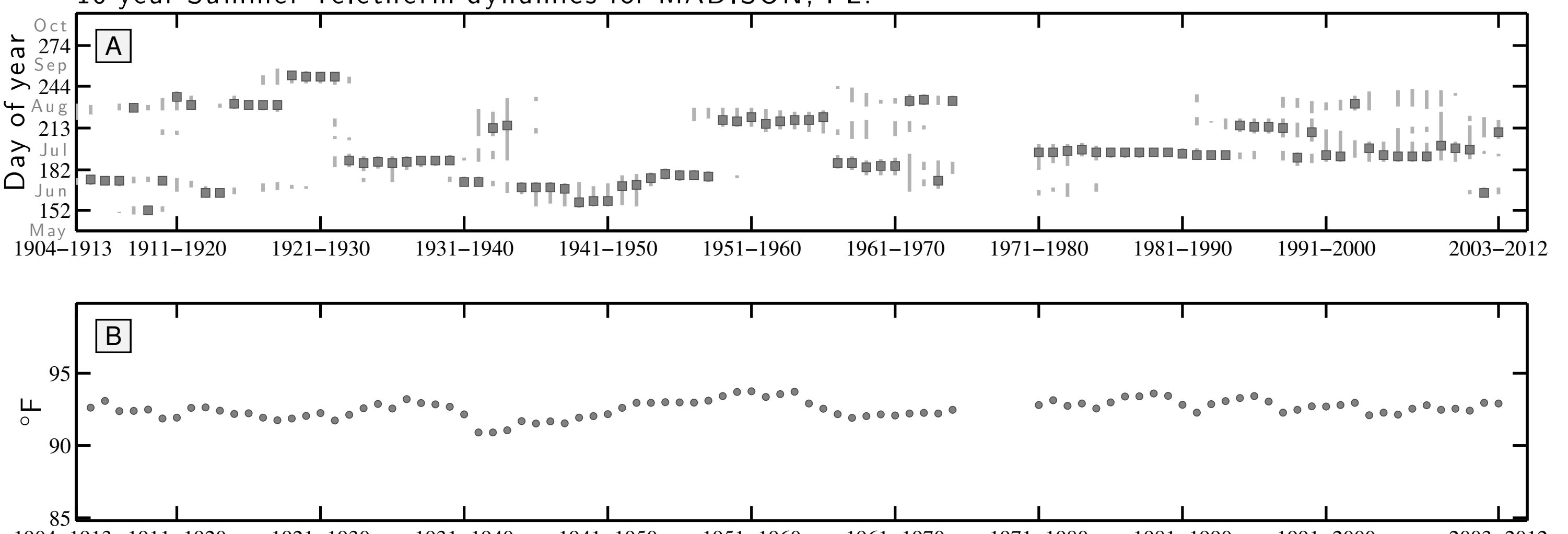

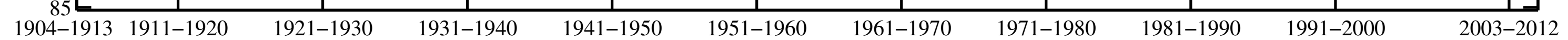

10 year Winter Teletherm dynamics for MADISON, FL:

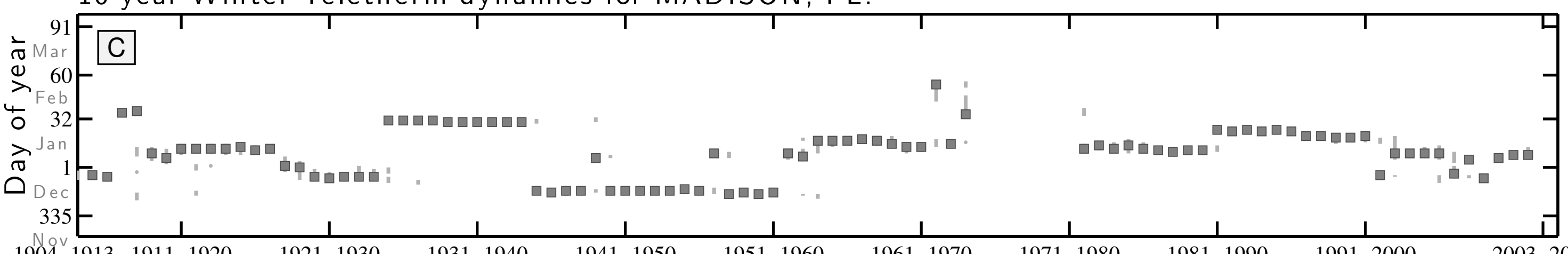

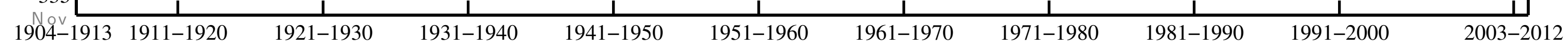

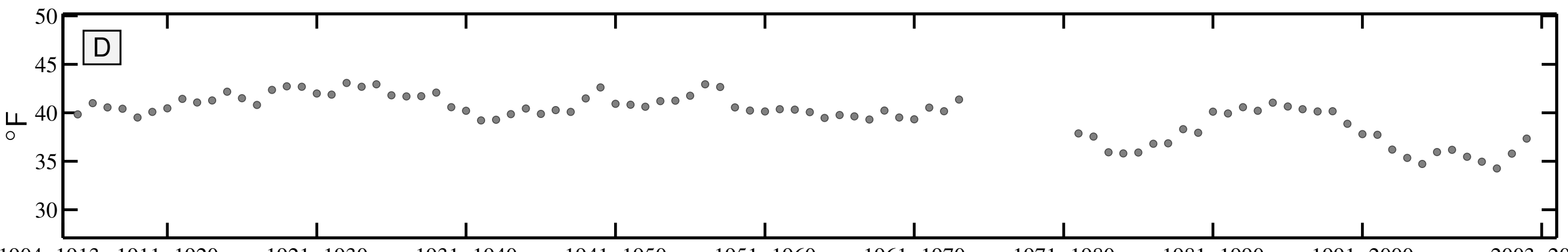

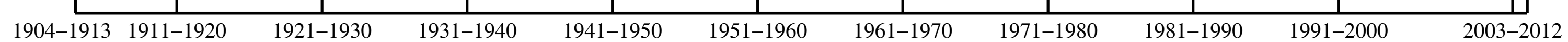


10 year Summer Teletherm dynamics for PERRINE 4W, FL:
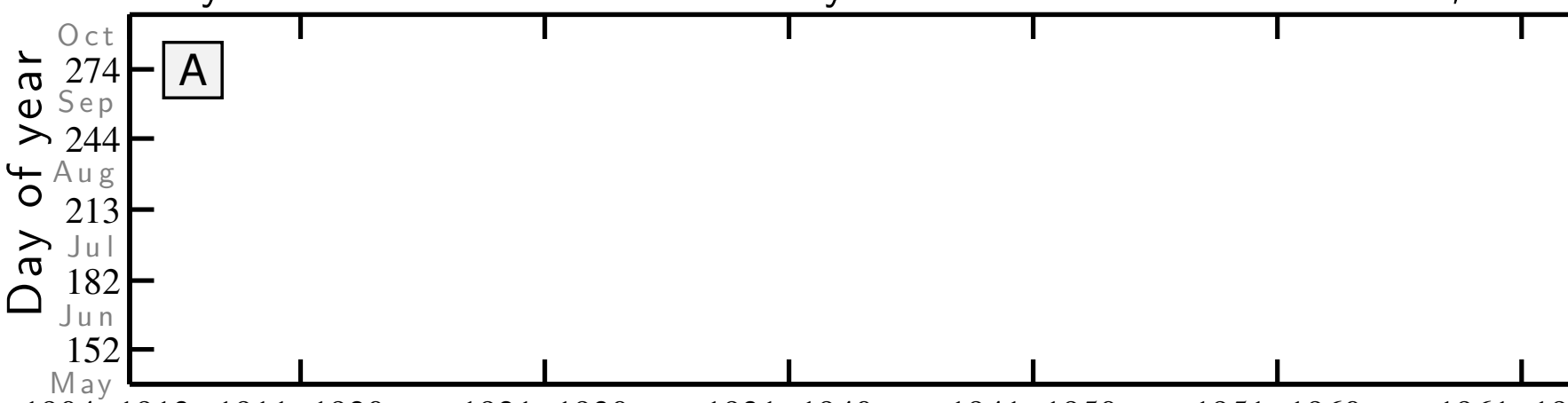

1951-1960

1961-1970
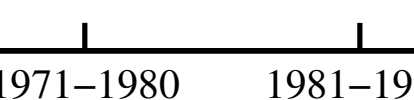

1981-1990

1991-2000
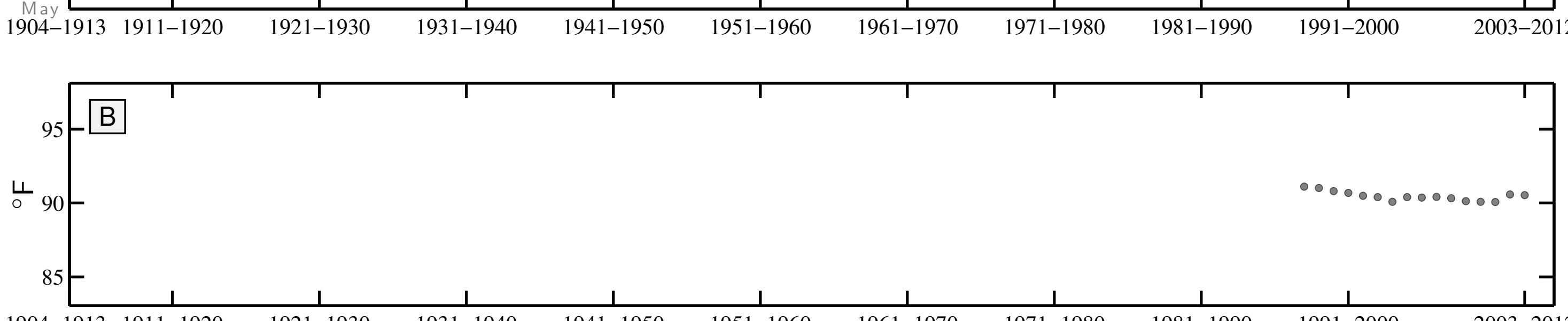

1904-1913 1911-1920

1921-1930

1931-1940

1941-1950

1951-1960

1961-1970

1971-1980

1981-1990

1991-2000

2003-2012

10 year Winter Teletherm dynamics for PERRINE 4W, FL:
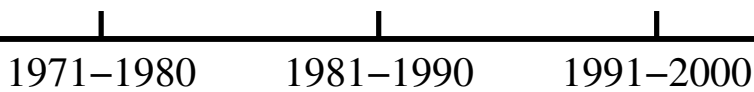

$\square \square$
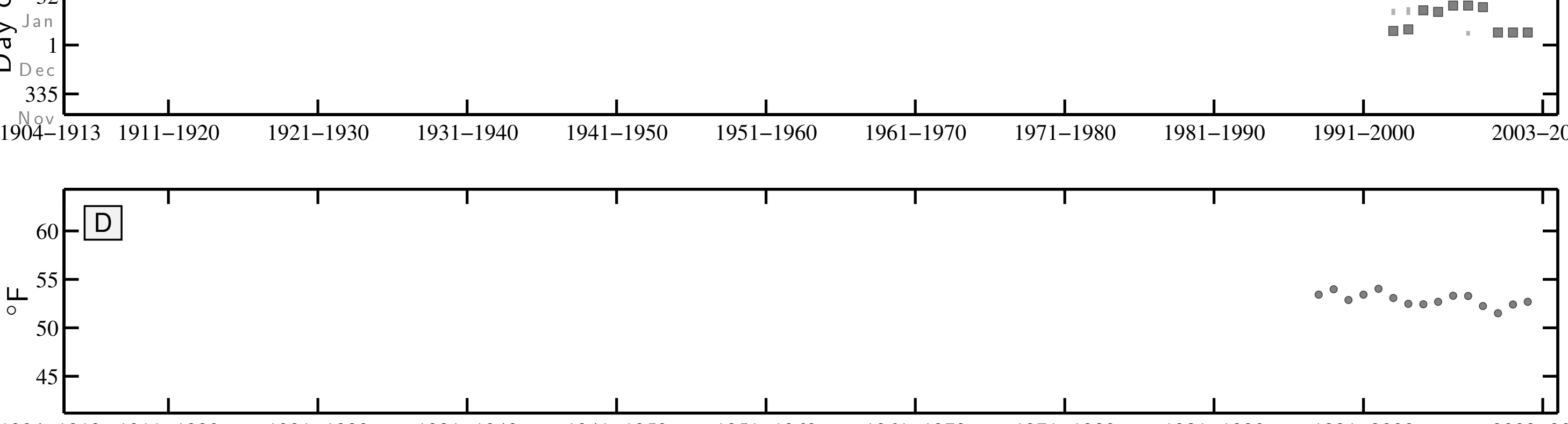
10 year Summer Teletherm dynamics for TARPON SPGS SEWAGE PL, FL:

$\div 274-A$

(1) Sep A

$>244-{ }^{\prime}$ -

4 Aug

त Jul

त 182

|

$152-$

152

$1904-1913 \quad 1911-1920$

$1921-1930$

$1931-1940$

$1941-1950$

|| || || 由中|| || 中 | || ||

| | 口曰 || " ||

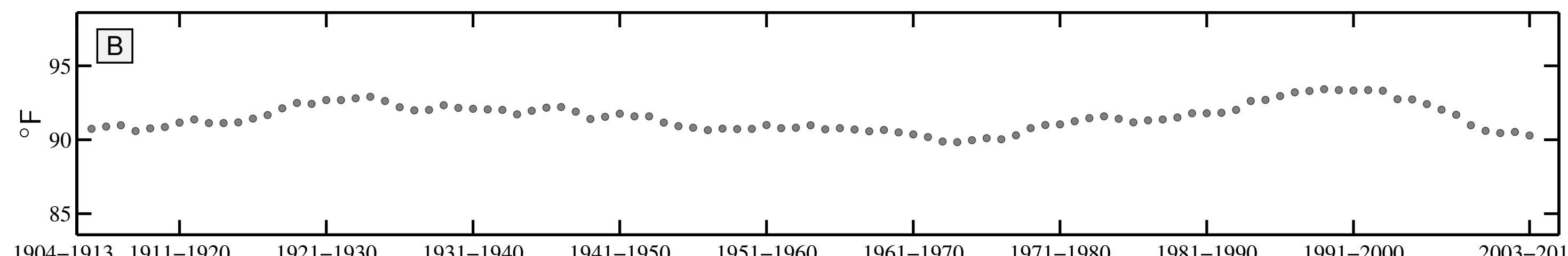

1904-1913 1911-1920

1921-1930

1931-1940

1941-1950

1951-1960

1961-1970

1971-1980

1981-1990

1991-2000

2003-2012

10 year Winter Teletherm dynamics for TARPON SPGS SEWAGE PL, FL:

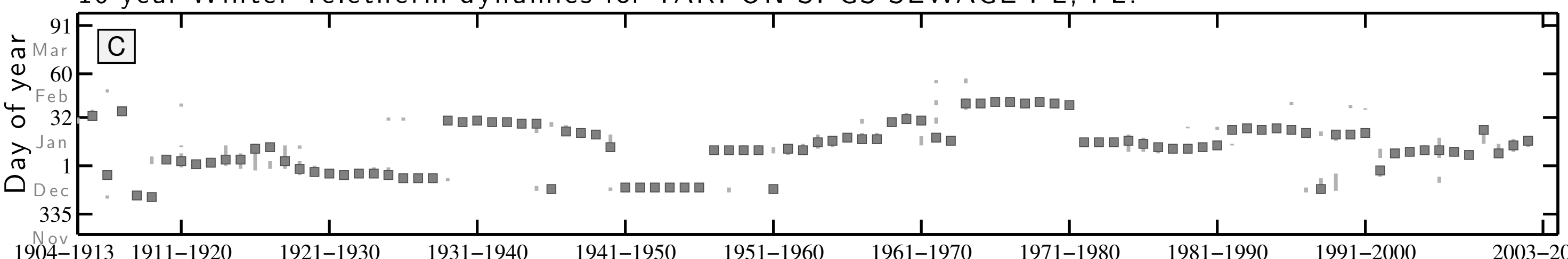

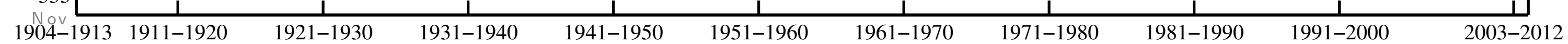

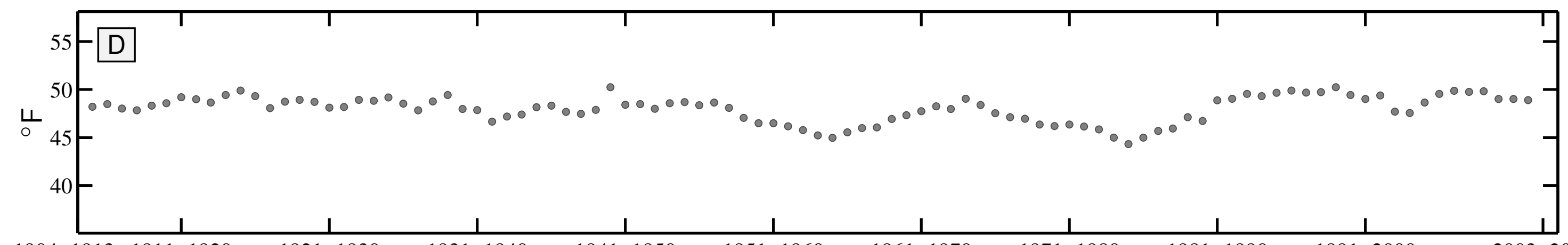


10 year Summer Teletherm dynamics for BAINBRIDGE INTL PAPER, GA:
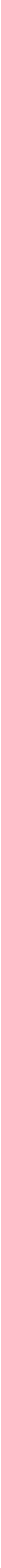

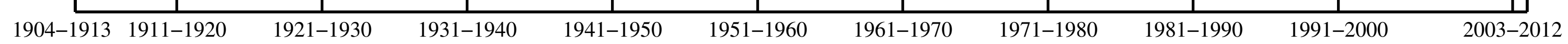


10 year Summer Teletherm dynamics for GLENNVILLE 3NW, GA:

$+\mathrm{Oct}$

(6) 274

(1) Sep

$>244-$

$4 \mathrm{Aug}$

$\gtrsim \mathrm{Jul}$

\% 182

$\bigcirc$ Jun

152

$\mathrm{May}$

1904-1913 1911-1920

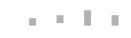

- " " | |

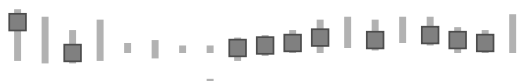

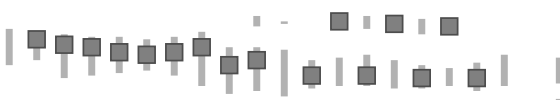

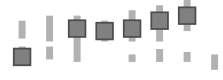

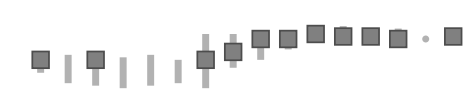

| | |

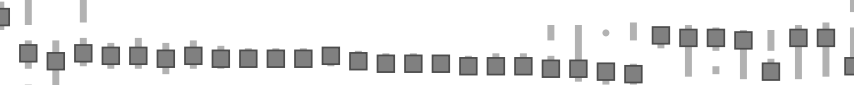

$\square$

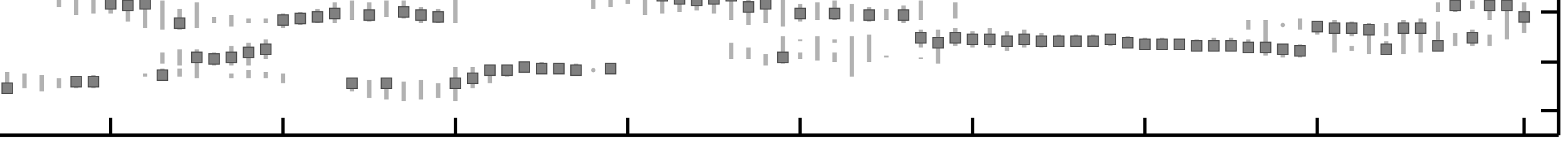

$1931-1940$

$1941-1950$

$1951-1960$

$1961-1970$

$1971-1980$

$1981-1990$

$1991-2000$

2003-2012

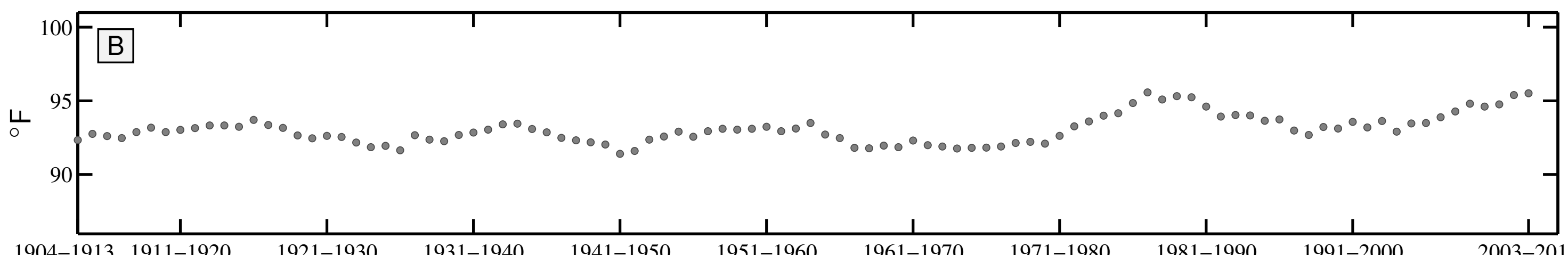

$1904-1913$ 1911-1920

1921-1930

1931-1940

$1941-1950$

$1951-1960$

1961-1970

1971-1980

2003-2012

10 year Winter Teletherm dynamics for GLENNVILLE 3NW, GA:

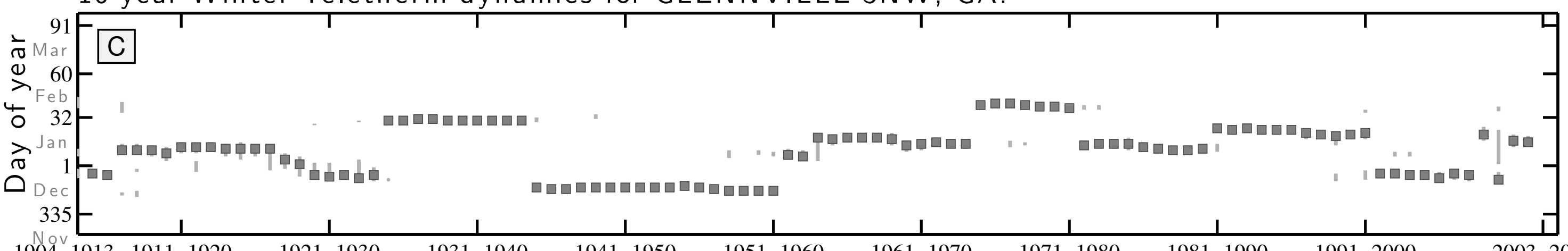

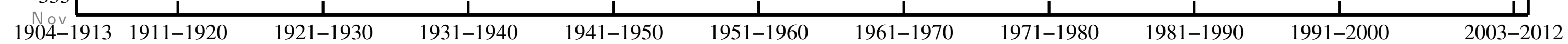

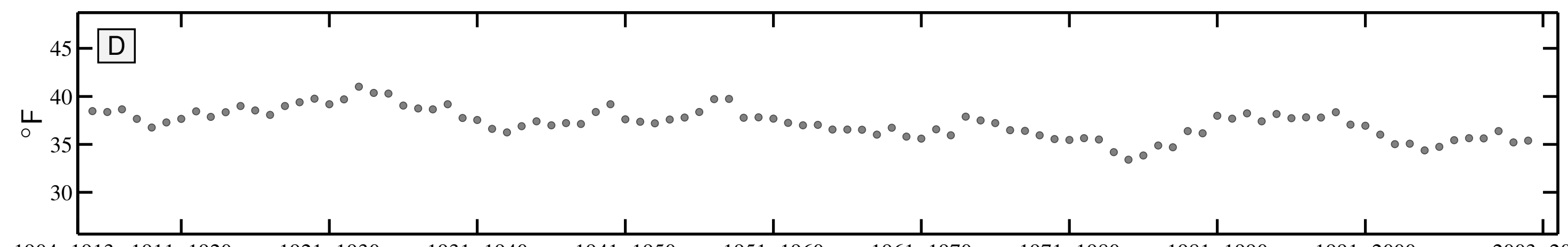


10 year Summer Teletherm dynamics for MILLEN 4 N, GA:

$+274$

o $274-A$

$\stackrel{\infty}{>} 244-$

A A g

O 213

$\gtrsim \mathrm{Jul}$

ก $182-$

$152-$

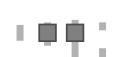

904-1913 1911-1920

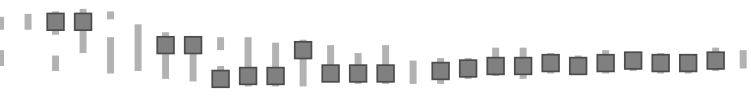
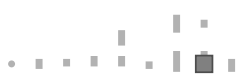

ㅁㅁロロロ

| | 무무뭄ㅁषํ

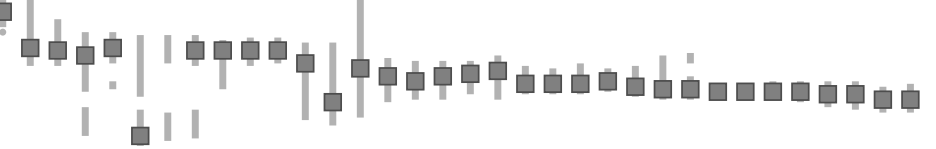

$1921-1930$

$\frac{1}{1931-1940}$

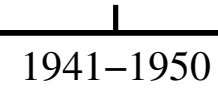

$1951-1960$

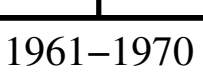

$\frac{1}{971-1980}$
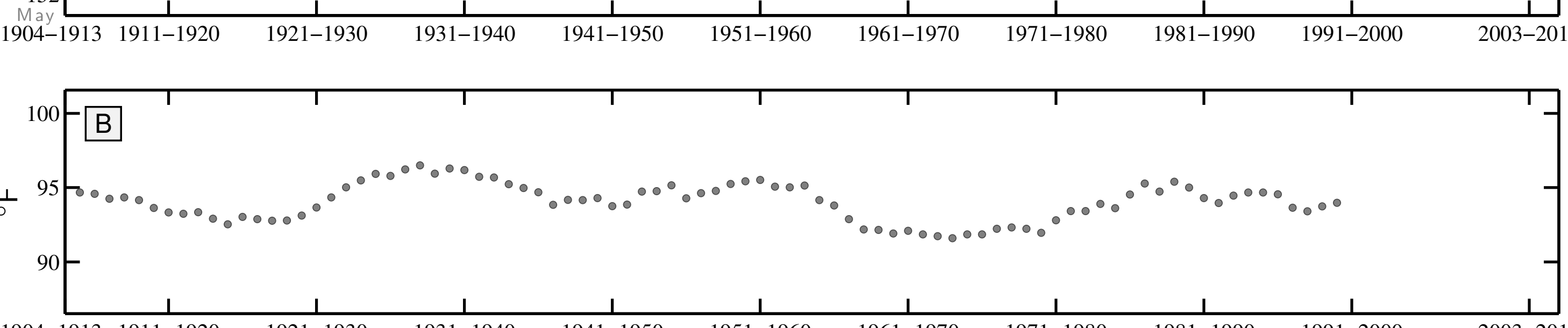

1904-1913 1911-1920

1921-1930

1931-1940

1941-1950

1951-1960

1961-1970

1971-1980

$1981-1990$

1991-2000

2003-2012

10 year Winter Teletherm dynamics for MILLEN 4 N, GA:

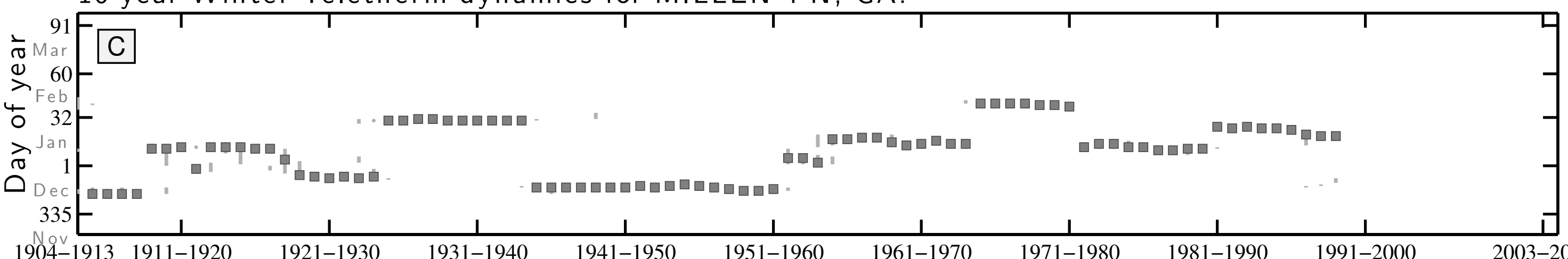

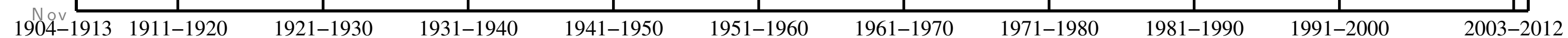

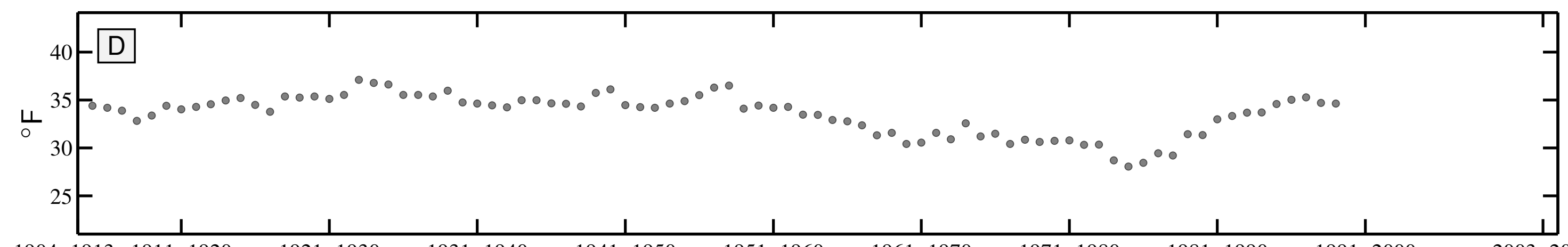


10 year Summer Teletherm dynamics for QUITMAN 2 NW, GA:

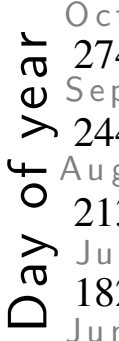
A
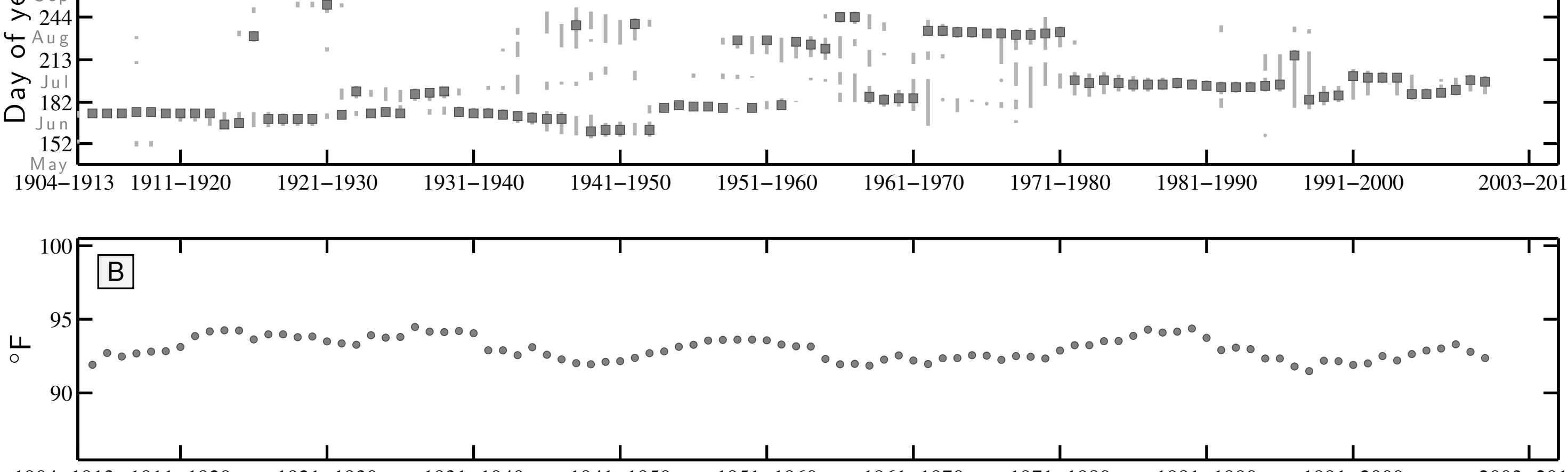

10 year Winter Teletherm dynamics for QUITMAN 2 NW, GA:

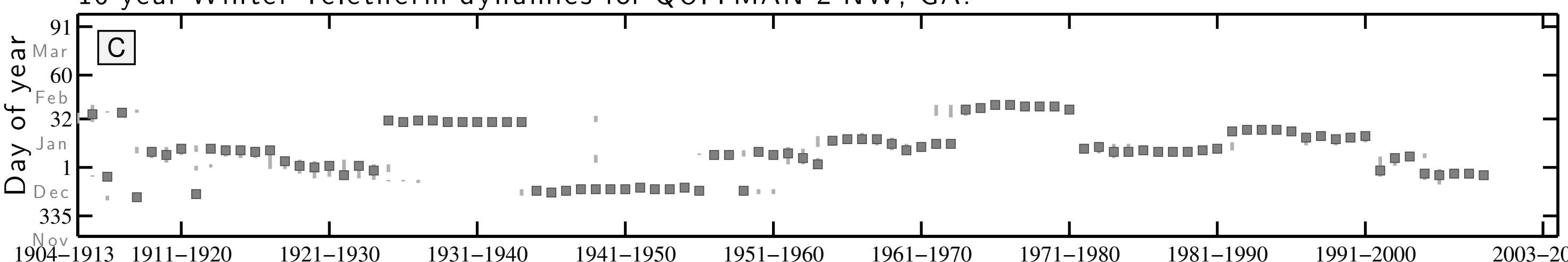

1904-1913 $1911-1920$ 1921-1930

$1931-1940$

1941-1950

$1951-1960$

1961-1970

1971-1980

$1981-1990$

1991-2000

2003-2012

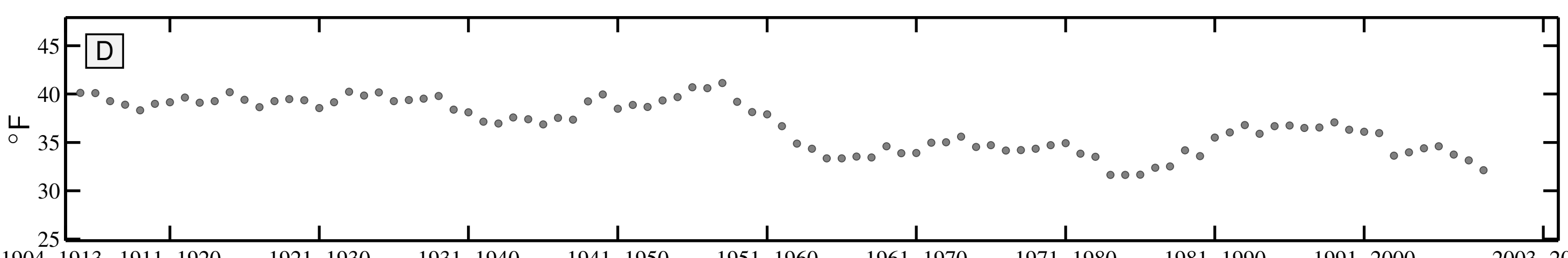


10 year Summer Teletherm dynamics for ROME, GA:

Oct

(2) 274

$\stackrel{0}{>} 244-$

4 Aug

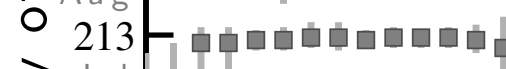

$\widehat{\sigma}_{1}$

$\overbrace{}^{\pi} 182$

$152-$

May

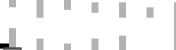

| | | | |

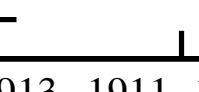

$\frac{1}{1-1920}$

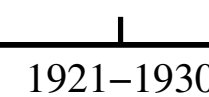

" | | | |

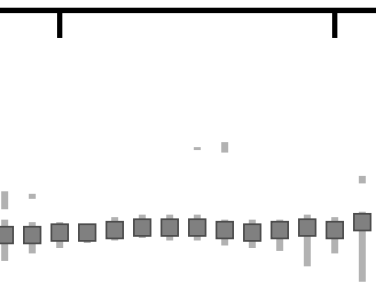

$1904-1913$

1921-1930

1931-1940

1941-1950

$\frac{1}{1951-1960}$

$\frac{1}{1961-1970}$

$1971-1980$

$1981-1990$

1991-2000

2003-201

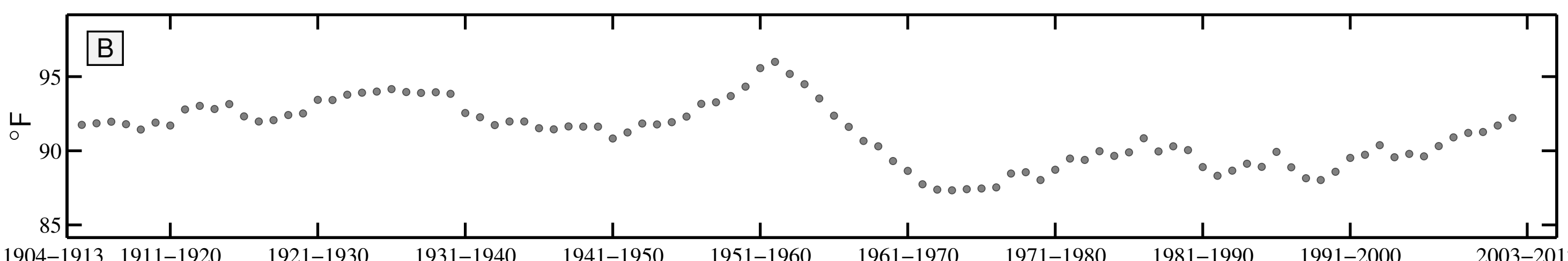

1904-1913 1911-1920

1921-1930

1931-1940

$1941-1950$

1961-1970

$1971-1980$

1981-1990

1991-2000

$2003-2012$

10 year Winter Teletherm dynamics for ROME, GA:

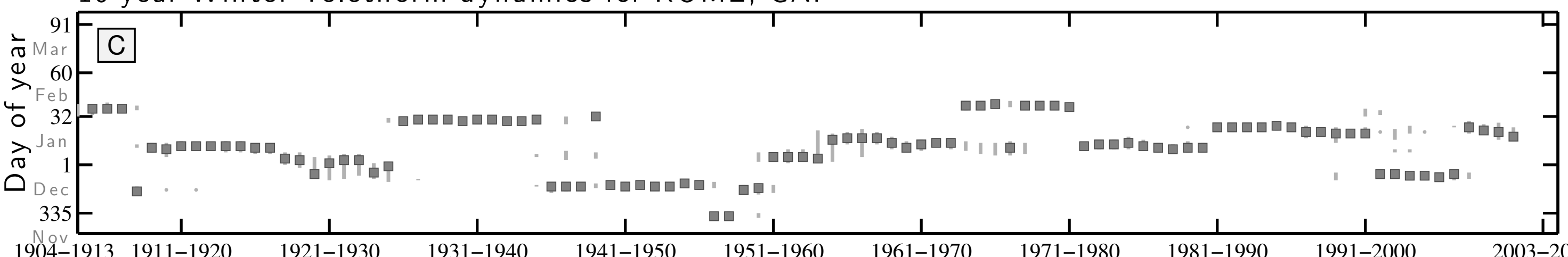

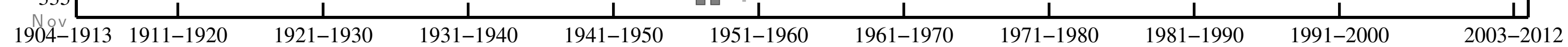

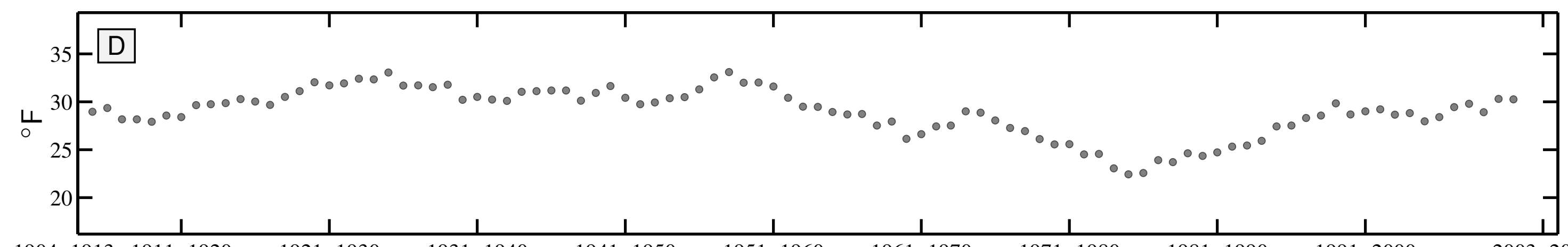


10 year Summer Teletherm dynamics for BERN, ID:
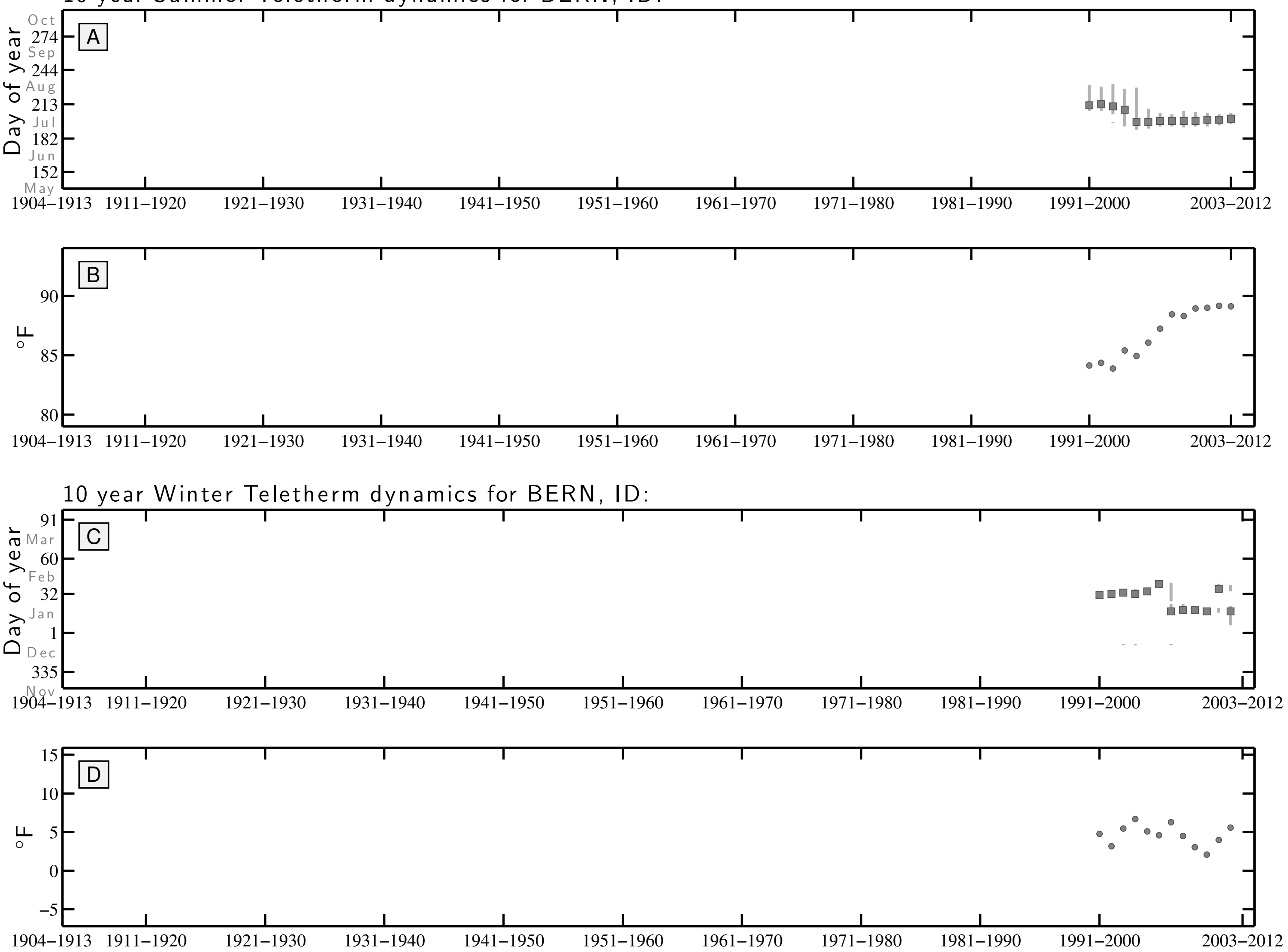
10 year Summer Teletherm dynamics for FENN RS, ID:
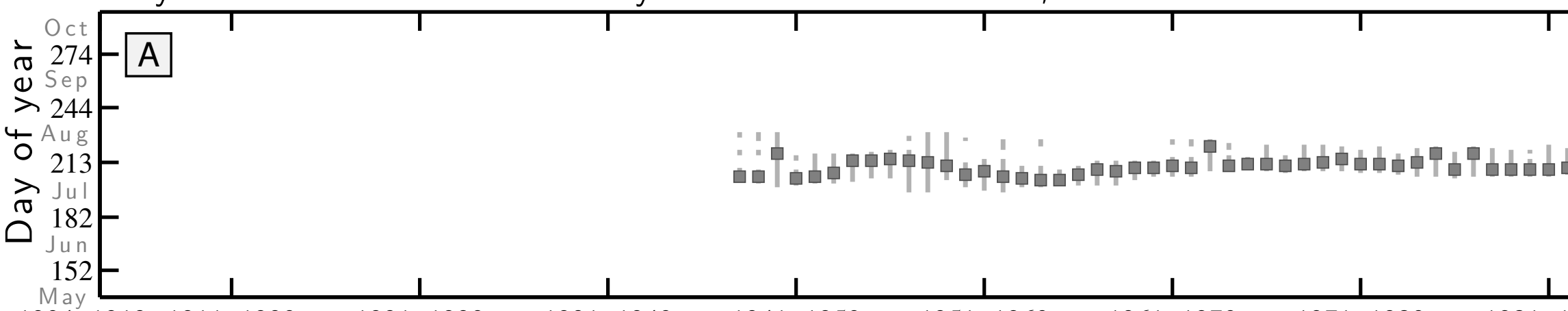

100

$B$

ㄴ

$95-$

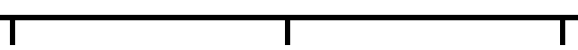

1941-1950

1951-1960

$\frac{1}{1961-1970}$

$\frac{1}{1971-1980}$

$\frac{1}{1981-1990}$

1991-2000

2003-201

1904

1904-1913 1911-1920

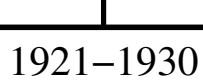

1931-1940

1941-1950

1
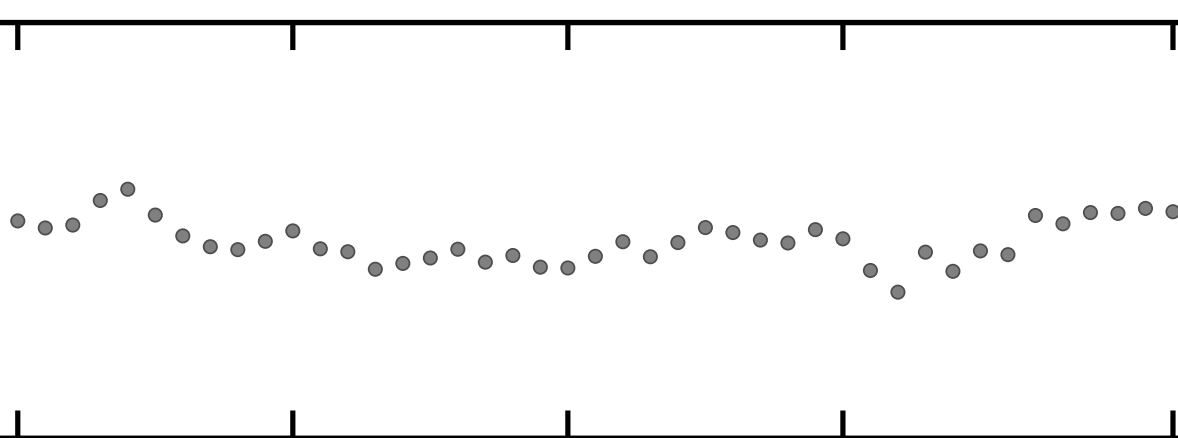

10 year Winter Teletherm dynamics for FENN RS, ID:
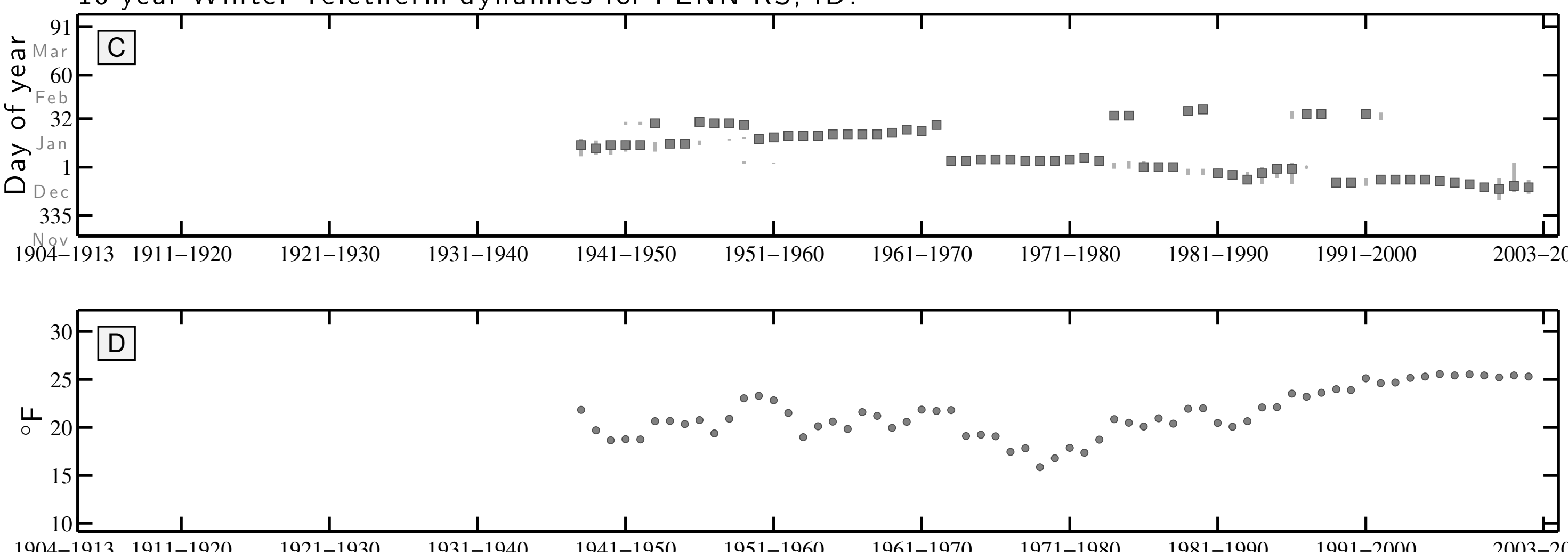
10 year Summer Teletherm dynamics for HAZELTON, ID:

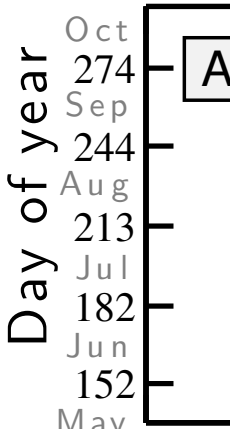
$A$

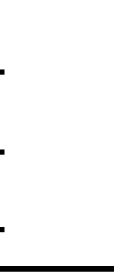

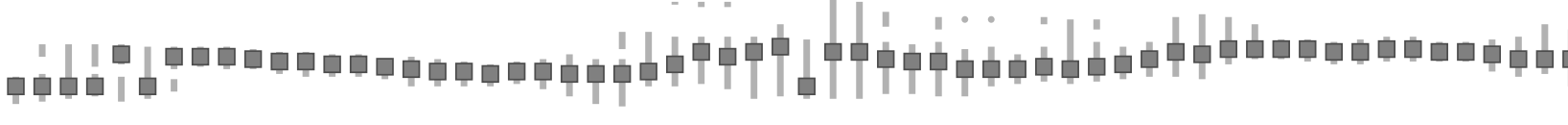
$1904-1913$ 1911-1920

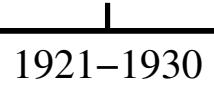

$1931-1940$

$1941-1950$

$\frac{1}{1951-1960}$

$\frac{1}{1961-1970}$

$\frac{1}{1971-1980}$

$\frac{1}{1981-1990}$

$1991-2000$

$2003-2012$

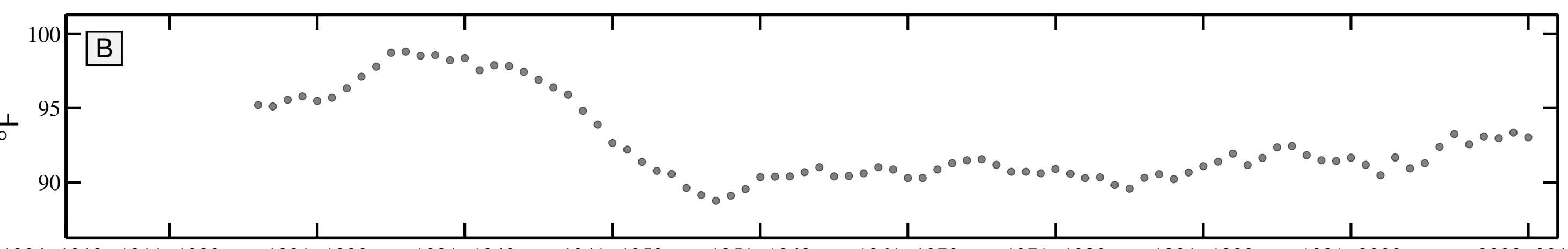

1904-1913 1911-1920

1921-1930

1931-1940

1941-1950

1951-1960

1961-1970

1971-1980

1981-1990

1991-2000

2003-2012

10 year Winter Teletherm dynamics for HAZELTON, ID:

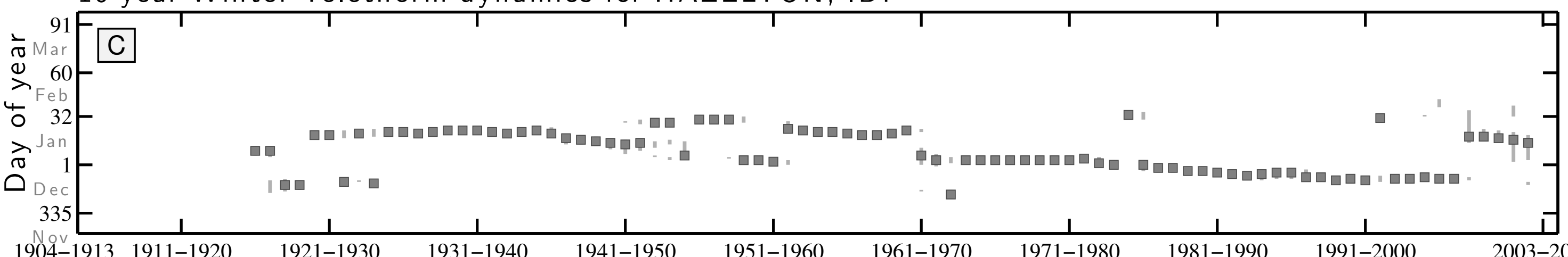

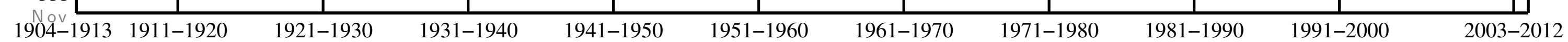

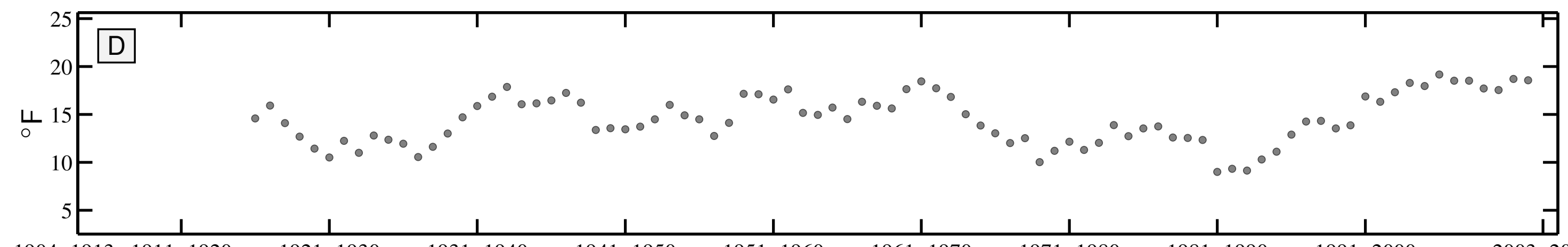


10 year Summer Teletherm dynamics for HOLLISTER, ID:

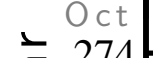

\% 274

(1) Sep

$>244-$

응

$>$ Jul

\% 182

Jun

$152-$

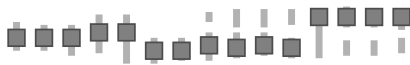

1904-1913 1911-1920

1921-1930

1931-1940

1941-1950

$\frac{1}{1951-1960}$

$\frac{1}{961-1970}$

1971-1980

1981-1990

$1991-2000$

2003-2012

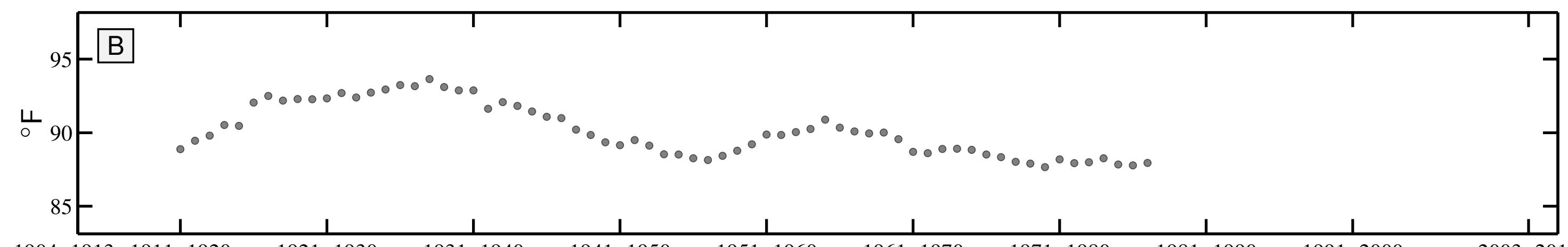

$1904-1913$ 1911-1920

$1921-1930$

$1931-1940$

$1941-1950$

$1951-1960$

$1961-1970$

$1971-1980$

$1981-1990$

$1991-2000$

$2003-2012$

10 year Winter Teletherm dynamics for HOLLISTER, ID:

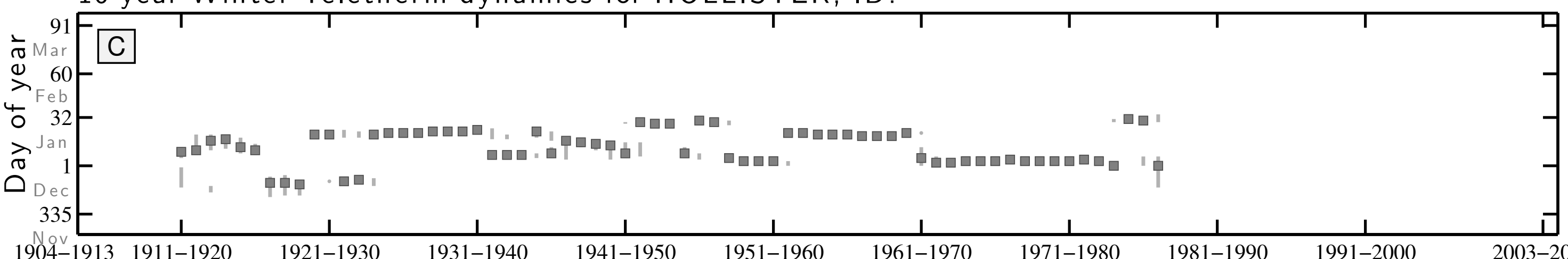

1904-1913 1911-192

$1921-1930$

$1931-1940$

1941-1950

1951-1960

1961-1970

1971-1980

1981-1990

991-2000

003-2012

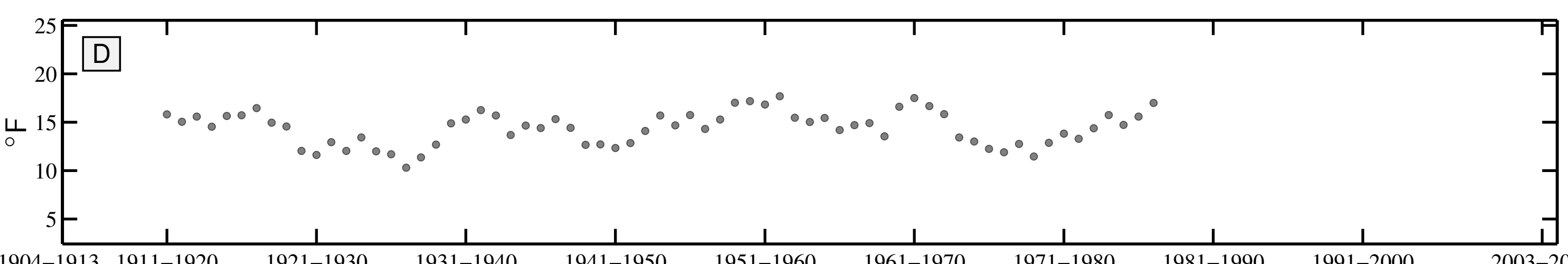


10 year Summer Teletherm dynamics for MACKAY LOST RIVER RS, ID:

$\mathrm{Oct}$

A

(1) Sep

$>244$

O

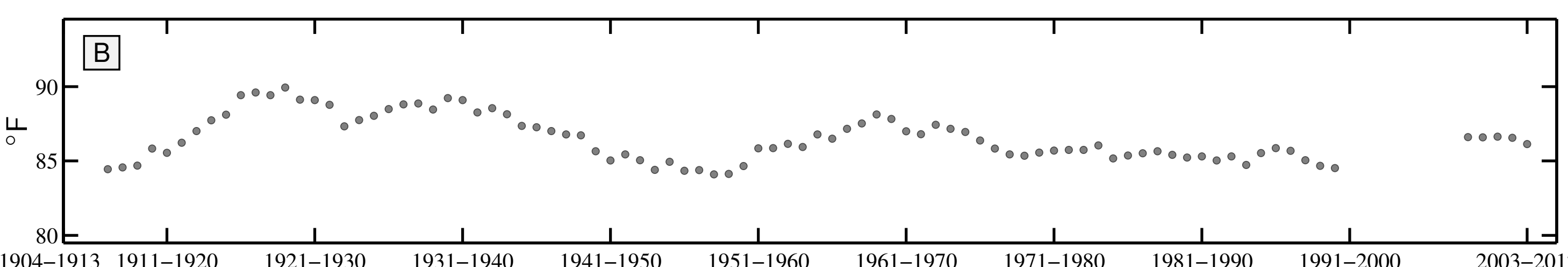

1904-1913 1911-1920

10 year Winter Teletherm dynamics for MACKAY LOST RIVER RS, ID:
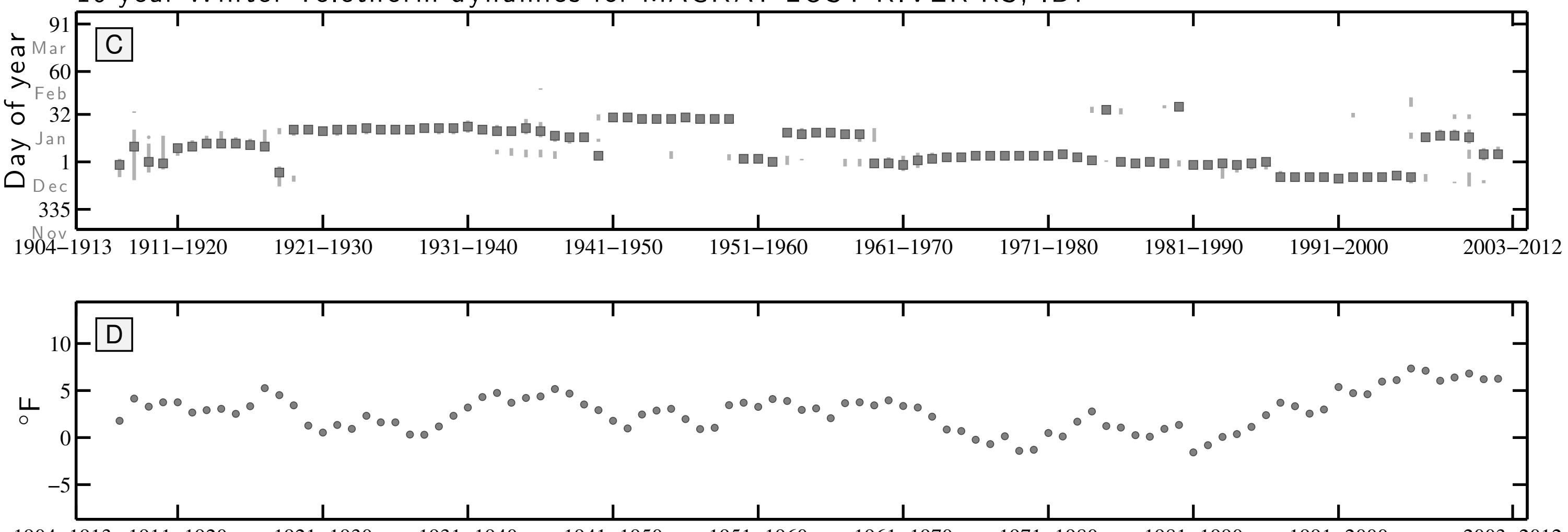
10 year Summer Teletherm dynamics for MALAD CITY, ID:
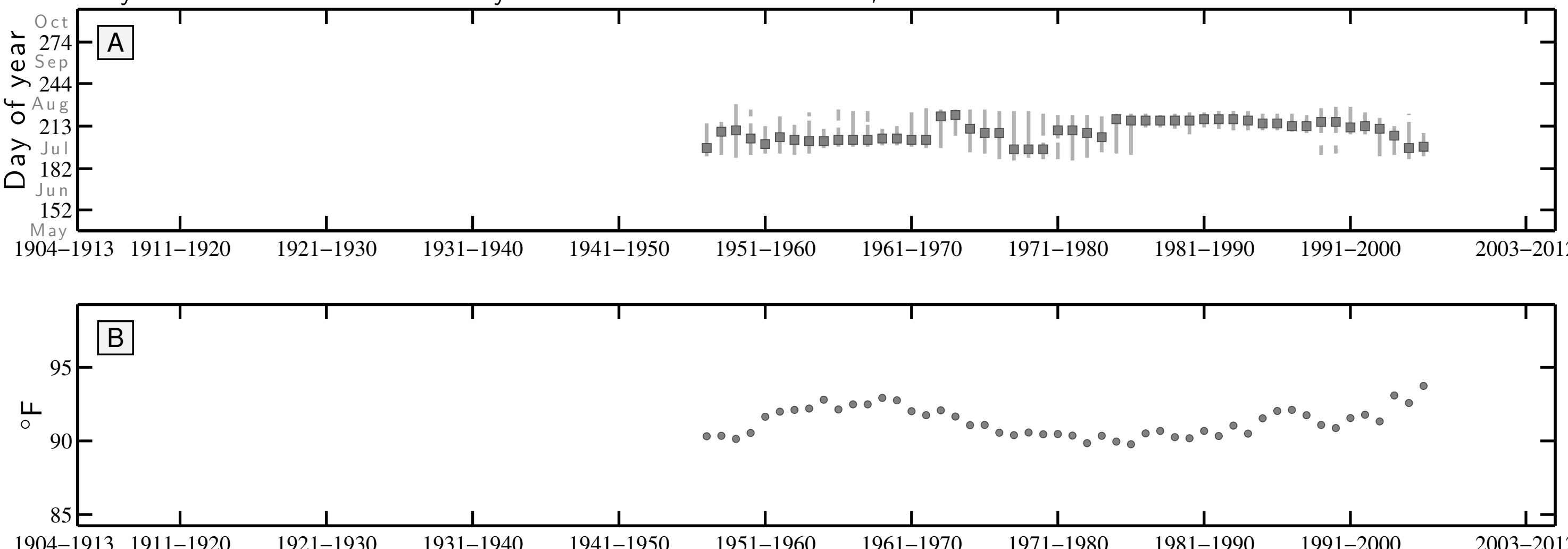

1904-1913 1911-1920

1931-1940

1951-1960

1961-1970

1971-1980

1981-1990

991-2000

2003-2012

10 year Winter Teletherm dynamics for MALAD CITY, ID:

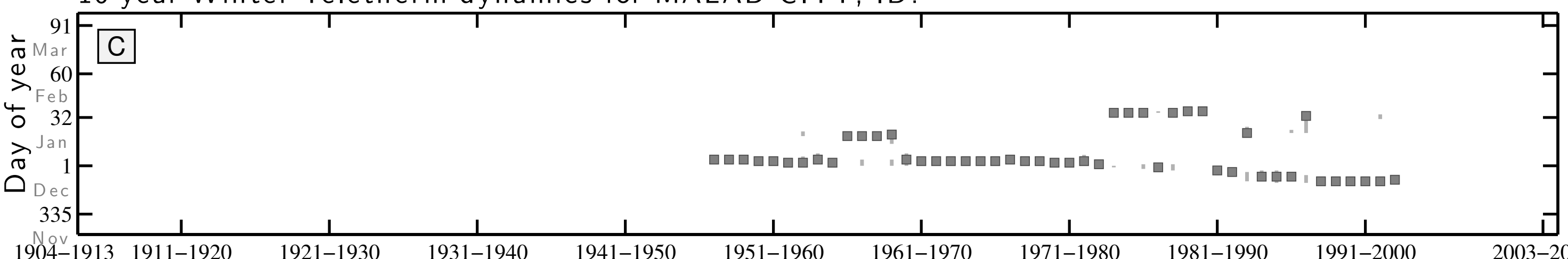

1904-1913 1911-192

1921-1930

1931-1940

1941-1950

1951-1960

1961-1970

1971-1980

981-1990

$1991-2000$

2003-2012

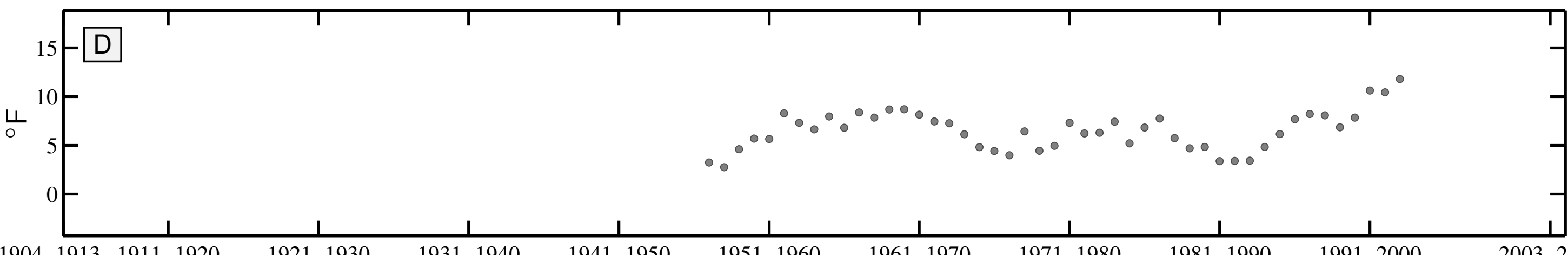


10 year Summer Teletherm dynamics for MOSCOW U OF I, ID:

$\div 274-A$

Sep

(1) Sep

244

O $213-$

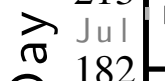

○ 182

$152-$

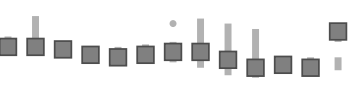

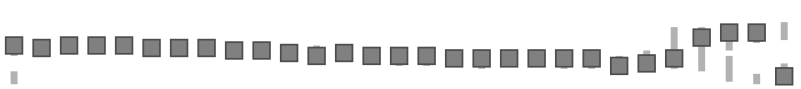

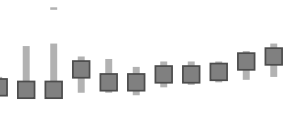

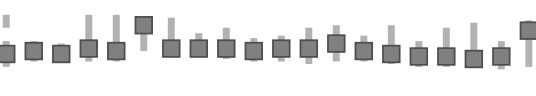

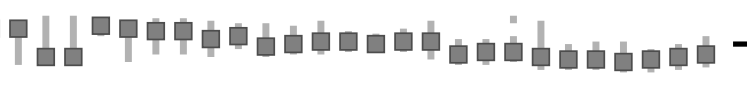

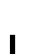

$\frac{1}{1921-1930}$

1931-1940

$\frac{1}{1941-1950}$

$\frac{1}{1951-1960}$

1961-1970

$\frac{1}{1971-1980}$

$\frac{1}{1981-1990}$

991-2000

2003-2012

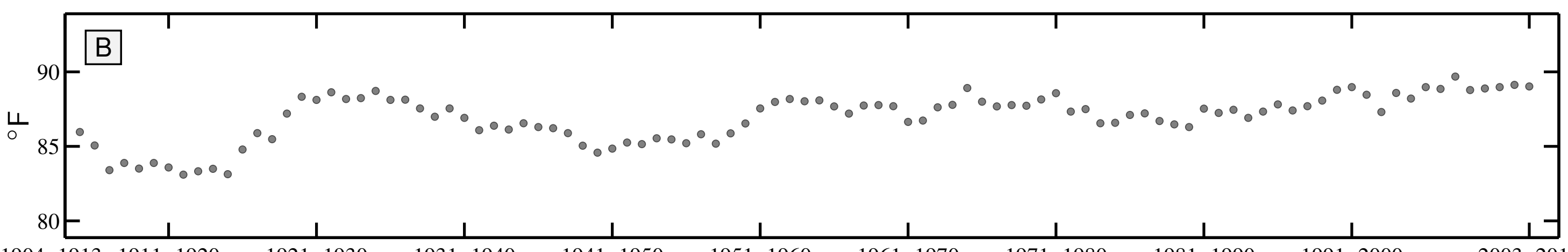

$1904-1913$ 1911-1920

$1921-1930$

$1931-1940$

$1941-1950$

$1951-1960$

$1961-1970$

$1971-1980$

$1981-1990$

$1991-2000$

$2003-2012$

10 year Winter Teletherm dynamics for MOSCOW U OF I, ID:

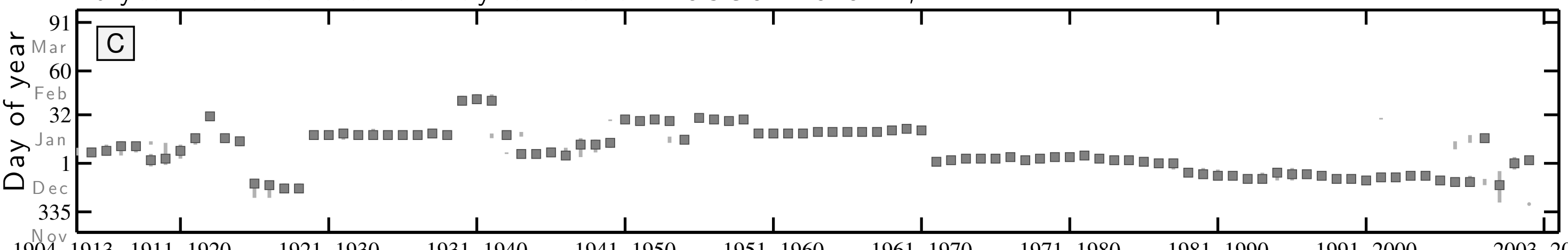

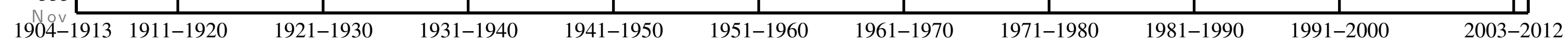

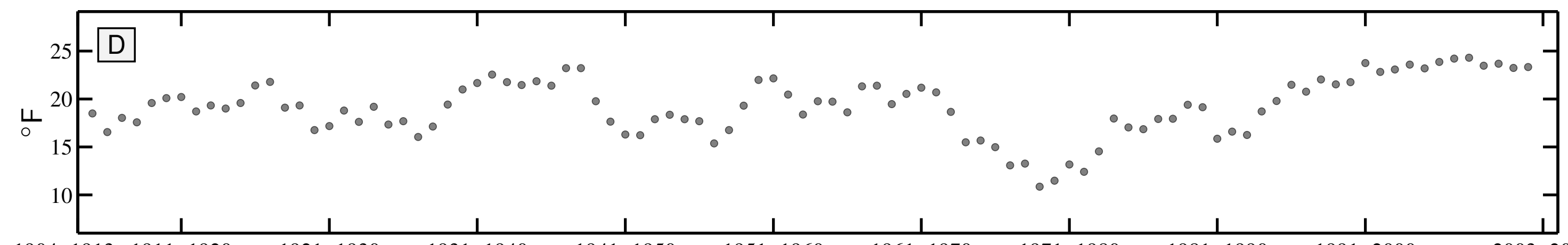


10 year Summer Teletherm dynamics for OAKLEY, ID:

$\div 274-\mathrm{A}$

$\stackrel{\text { I Sep }}{>} 244-$

4 Aug

○ 213 몌 | " |

त Jul

$\overbrace{}^{\circ} 182-$

$152-$

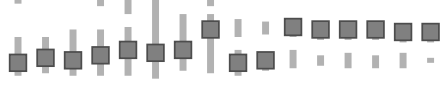

\section{- | |}

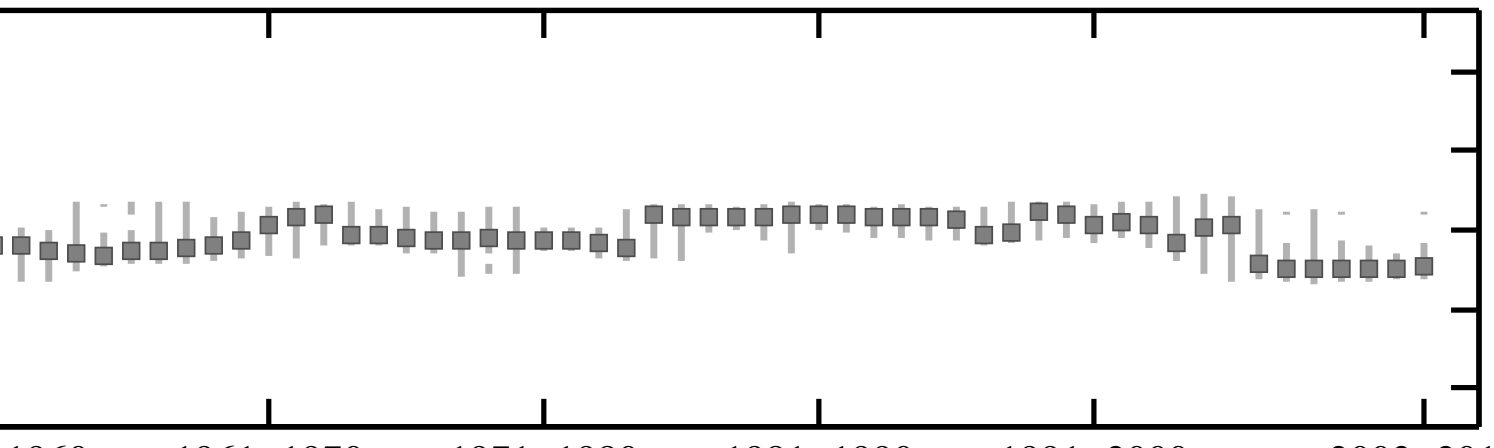

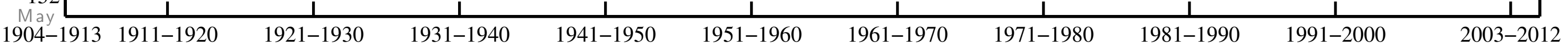

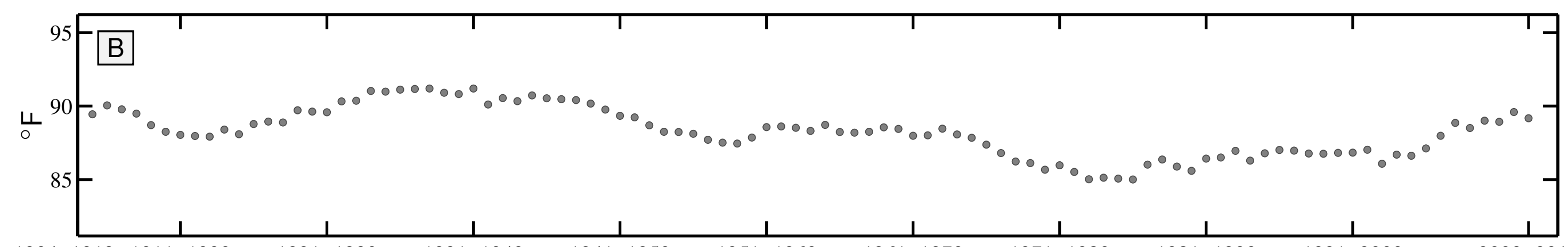

1904-1913 1911-1920

$1921-1930$

$1931-1940$

$1941-1950$

$1951-1960$

$1961-1970$

$1971-1980$

$1981-1990$

$1991-2000$

2003-2012

10 year Winter Teletherm dynamics for OAKLEY, ID:

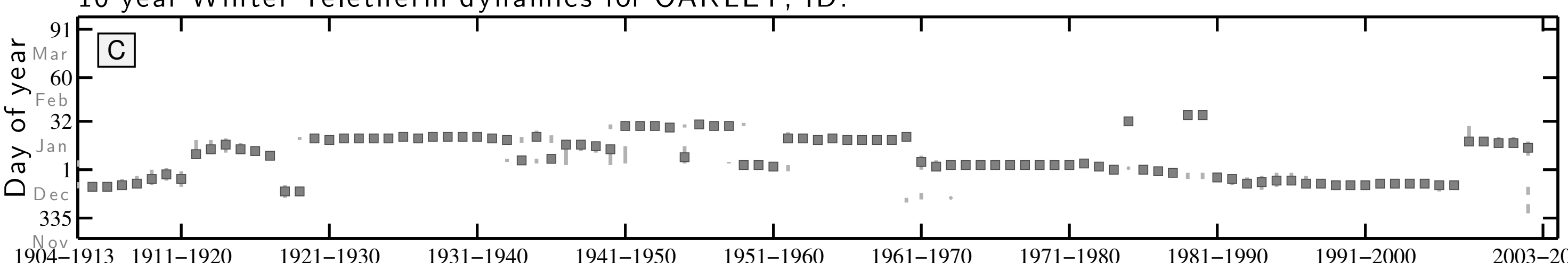

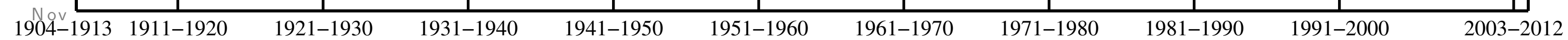

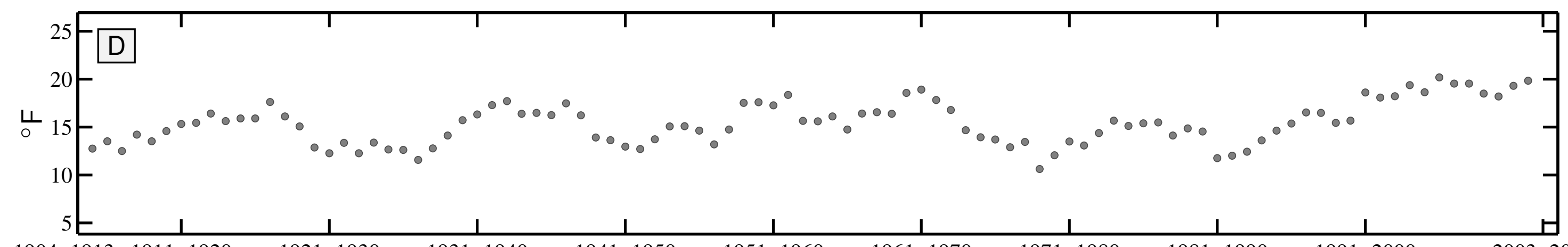


10 year Summer Teletherm dynamics for ALEDO, IL:
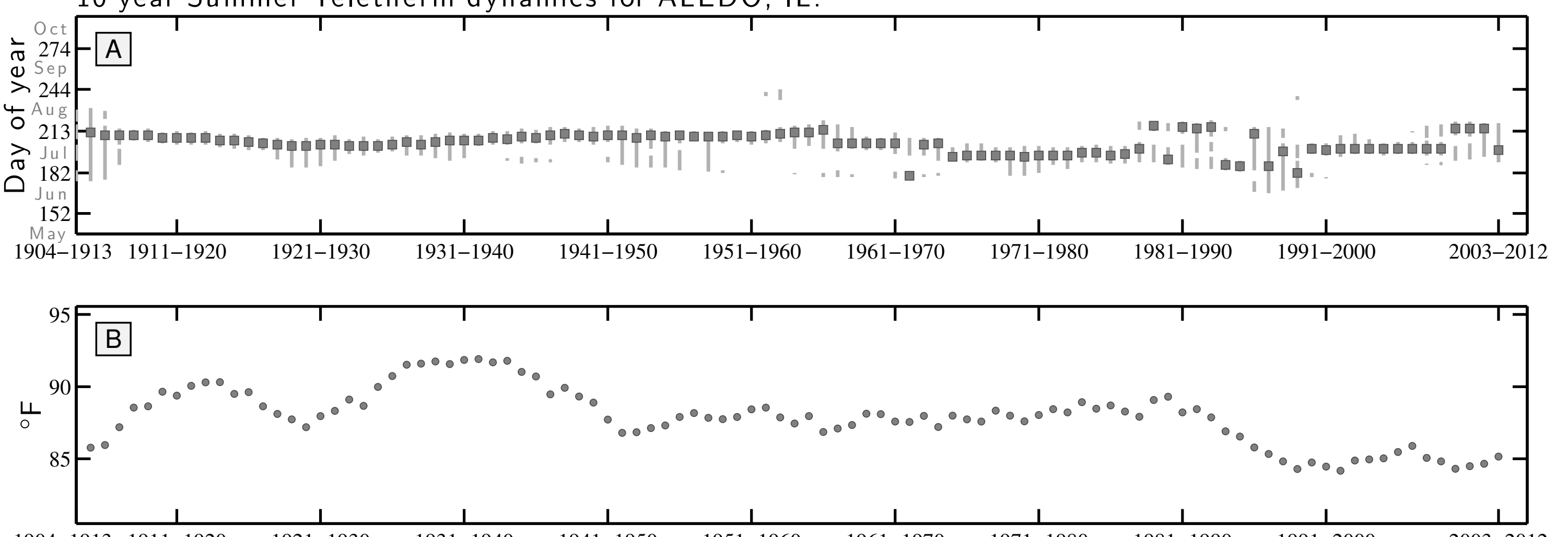

1904-1913 1911-1920

1921-1930

1931-1940

1941-1950

1951-1960

1961-1970

1971-1980

$1981-1990$

$1991-2000$

2003-2012

10 year Winter Teletherm dynamics for ALEDO, IL:

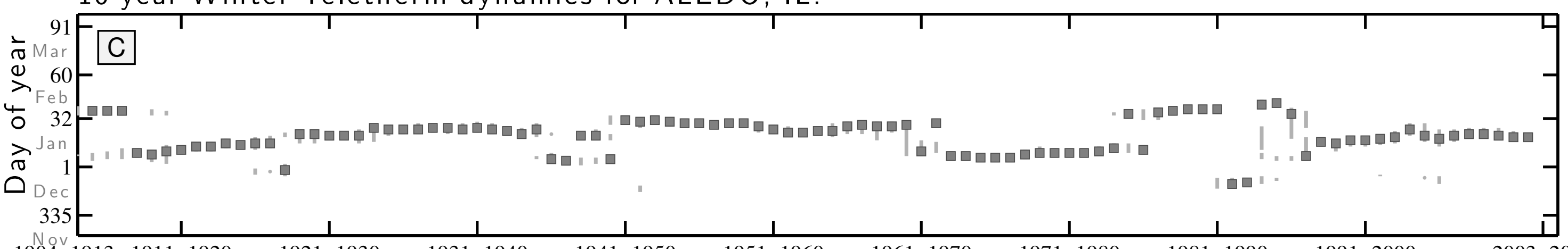

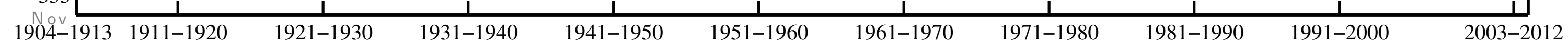

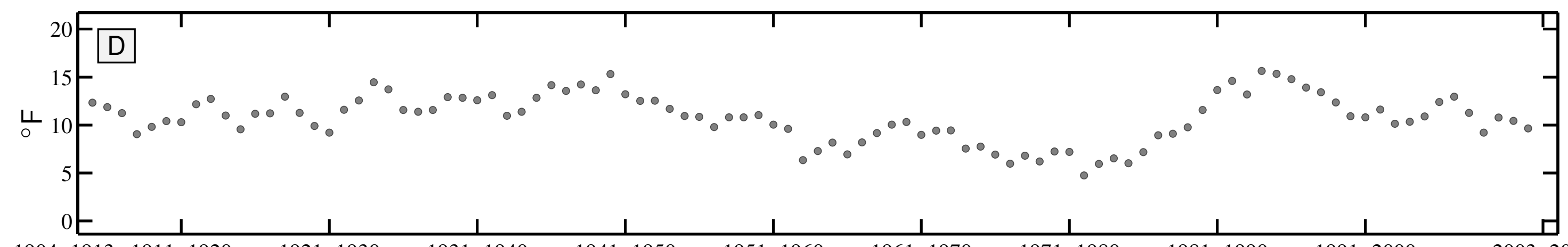


10 year Summer Teletherm dynamics for AURORA, IL:
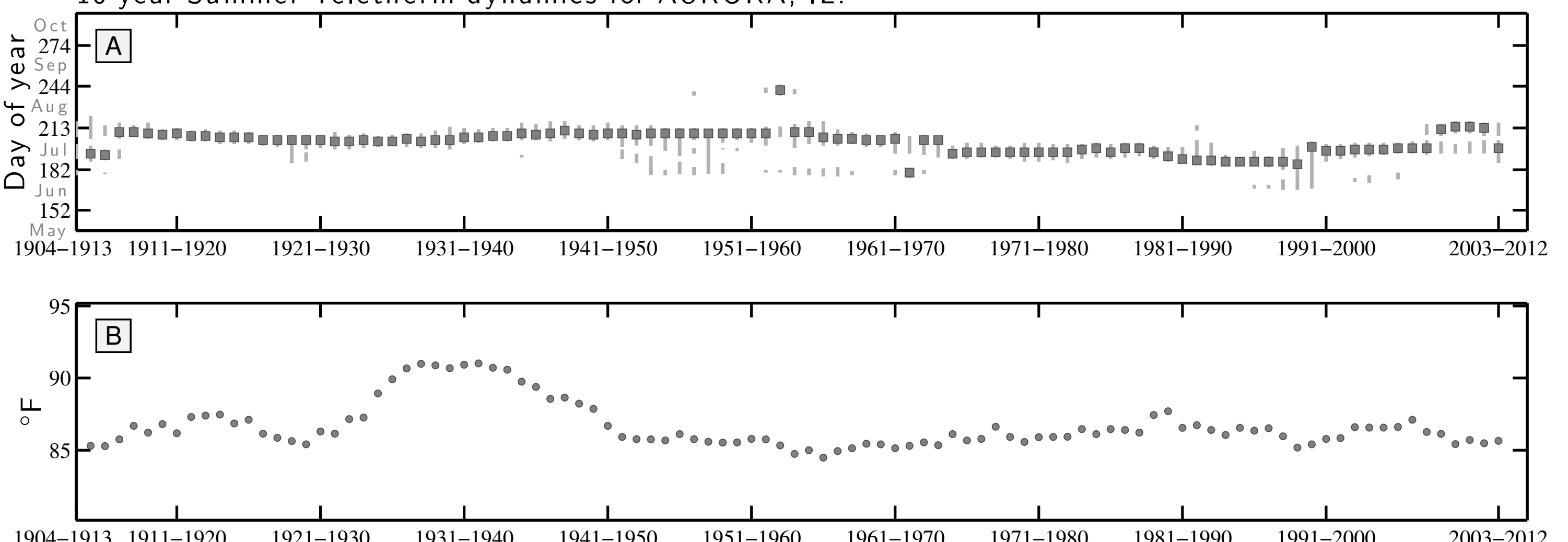

$1904-19131911-1920$

1921-1930

1931-1940

1941-1950

1951-1960

1961-1970

1971-1980

1981-1990

1991-2000

2003-2012

10 year Winter Teletherm dynamics for AURORA, IL:

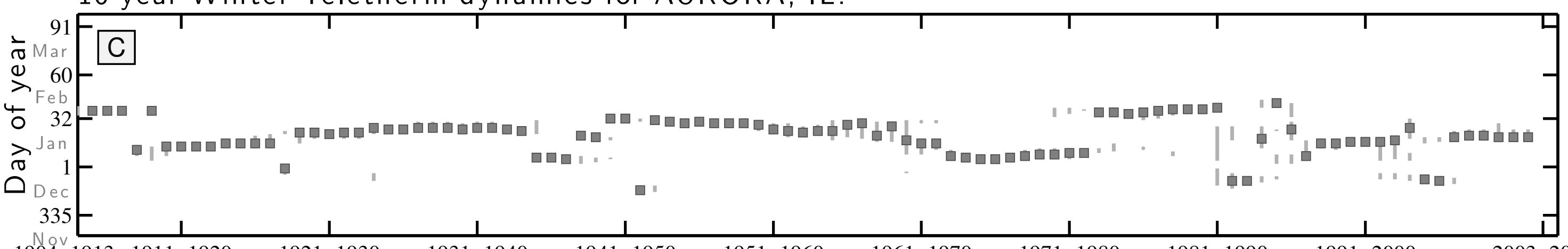

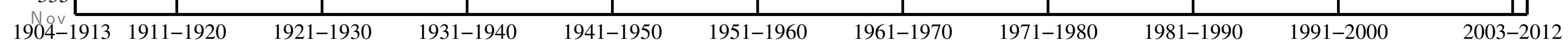

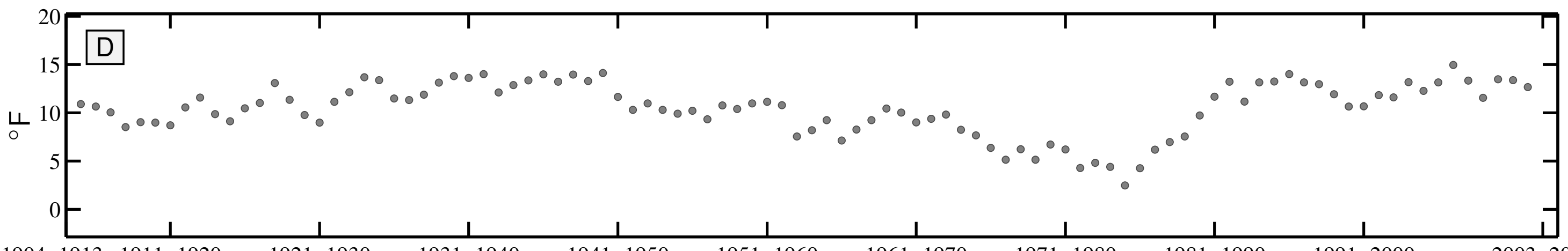

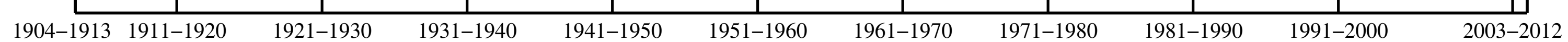


10 year Summer Teletherm dynamics for GALVA, IL:
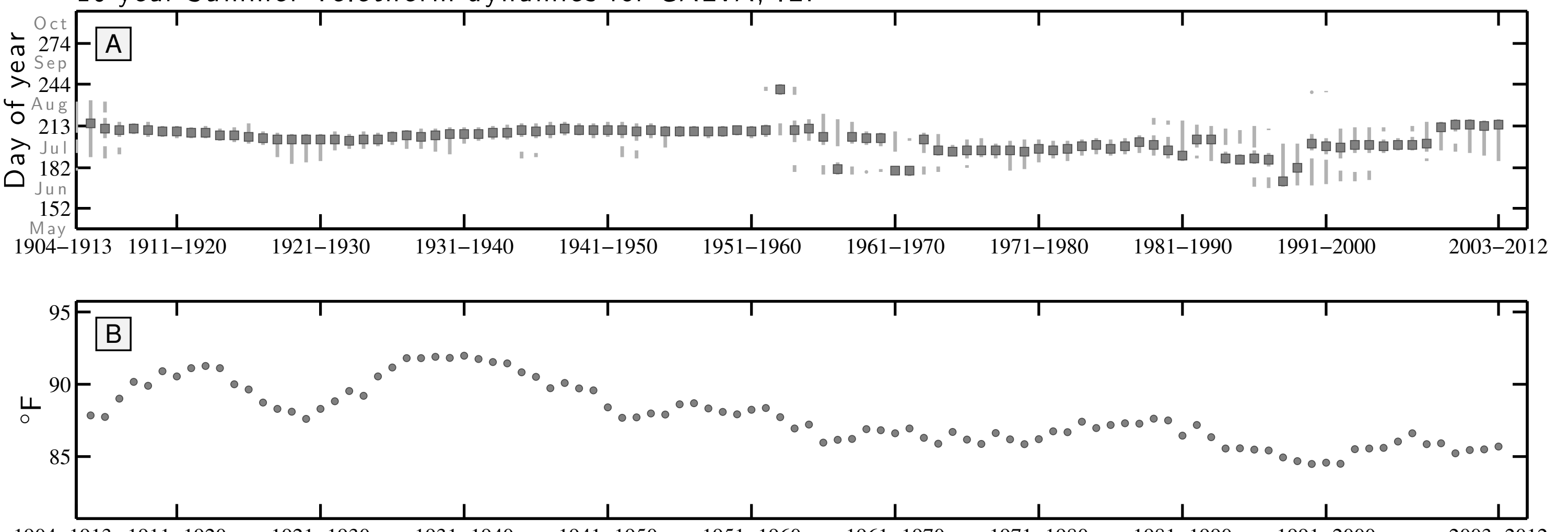

1904-1913 1911-1920

1921-1930

1931-1940

1941-1950

1951-1960

1961-1970

1971-1980

1981-1990

1991-2000

2003-2012

10 year Winter Teletherm dynamics for GALVA, IL:

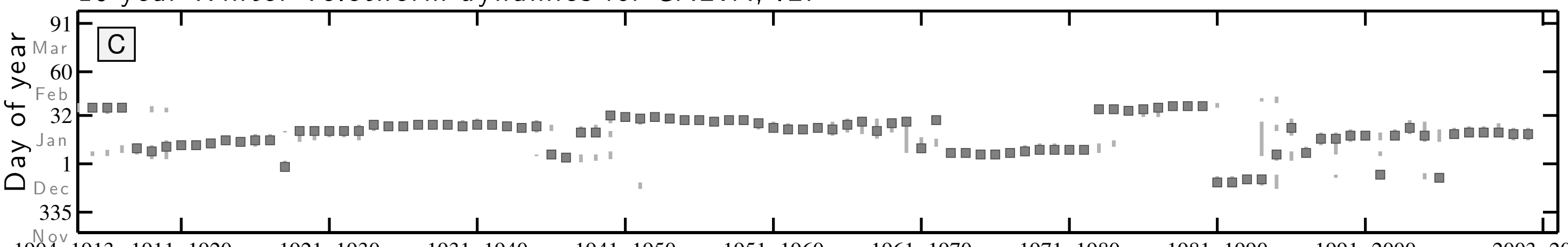

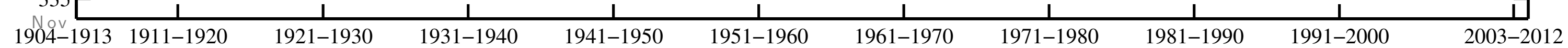

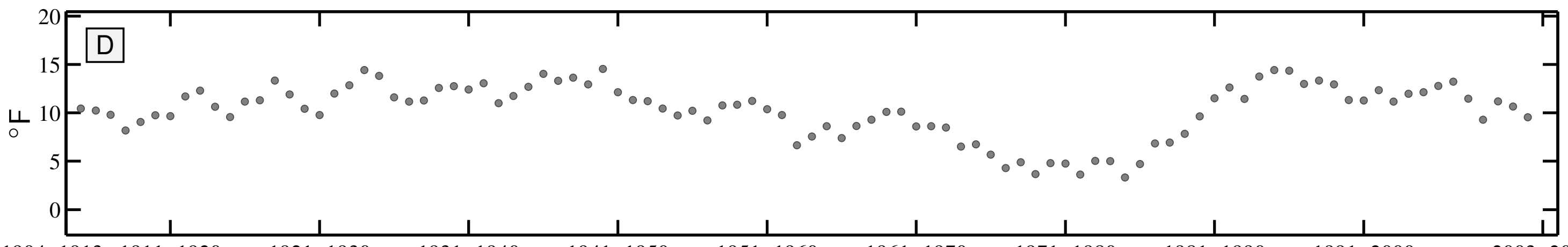


10 year Summer Teletherm dynamics for HILLSBORO, IL:

$\therefore \mathrm{Oct}$

A

(1) Sep

$>244-$

A A g

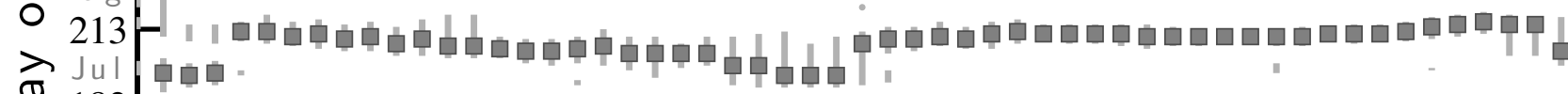

$\overbrace{}^{\circ} 182-$

$152-$

May $\square$

1904-1913 1911-1920

$\frac{1}{1921-1930}$

$\frac{1}{1931-1940}$

$\frac{1}{1941-1950}$

$\frac{1}{1951-1960}$

$\frac{1}{1961-1970}$

$\frac{1}{971-1980}$

$\frac{1}{1981-1990}$

1991-2000

2003-201

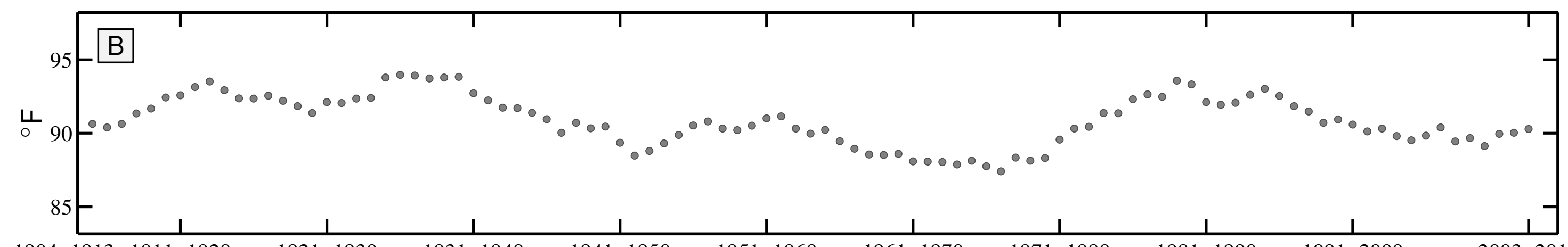

1904-1913 1911-1920

1921-1930

1931-1940

1941-1950

1951-1960

1961-1970

1971-1980

1981-1990

1991-2000

2003-2012

10 year Winter Teletherm dynamics for HILLSBORO, IL:

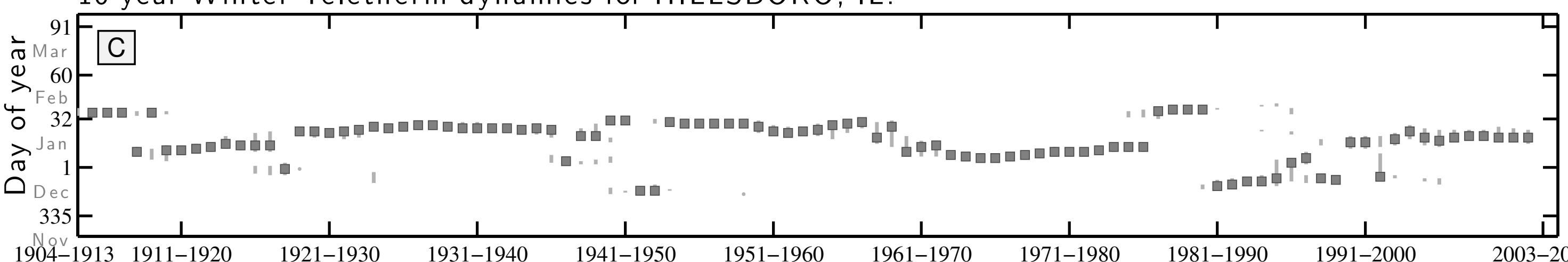

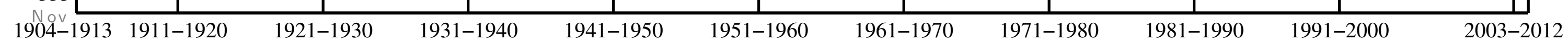

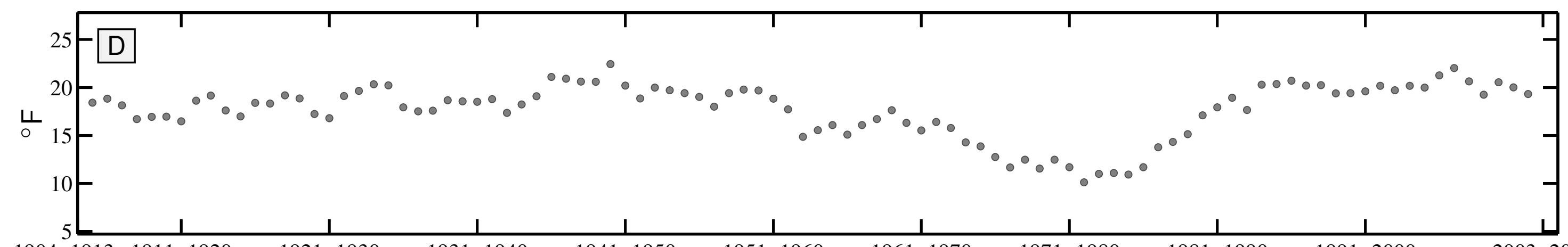


10 year Summer Teletherm dynamics for MCLEANSBORO, IL:

$\div 274$

(1) Sep

$>244-$

213

入 $\begin{gathered}213 \\ \text { Jul }\end{gathered}$

م $182-$

$152-$

ष

1904-1913 1911-1920

$\frac{1}{1921-1930}$

$\frac{1}{1931-1940}$

$\frac{1}{1941-1950}$

$\frac{1}{1951-1960}$

$\frac{1}{1961-1970}$

$\frac{1}{971-1980}$

$\frac{1}{1981-1990}$

1991-2000

2003-2012

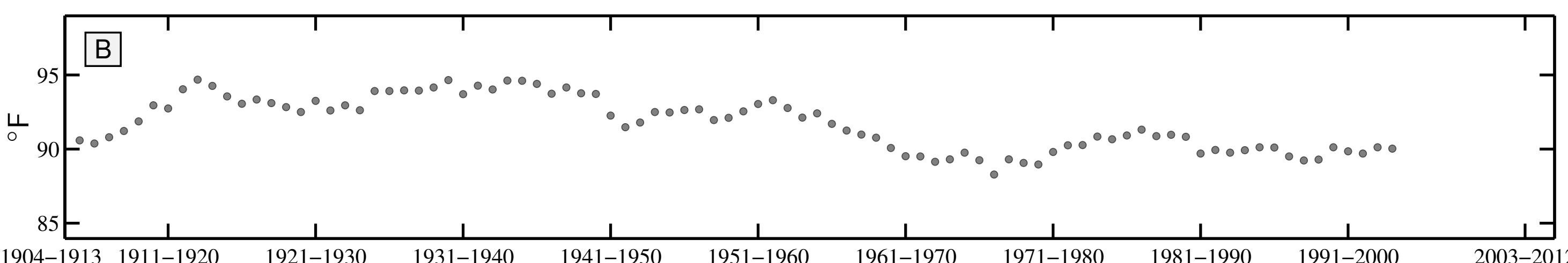

1904-1913 1911-1920

$1921-1930$

$1931-1940$

1941-1950

$1951-1960$

1961-1970

1971-1980

1981-1990

991-2000

$2003-2012$

10 year Winter Teletherm dynamics for MCLEANSBORO, IL:

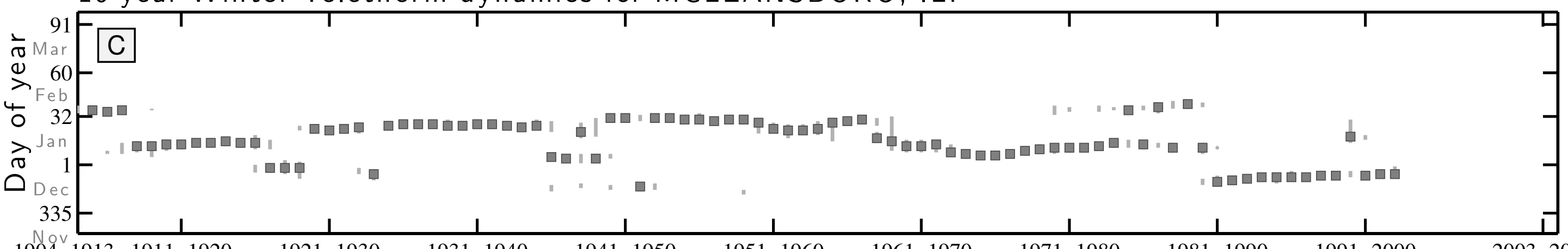

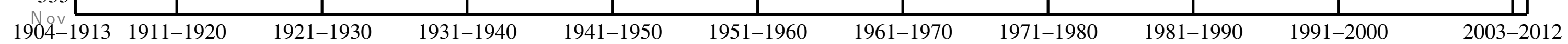

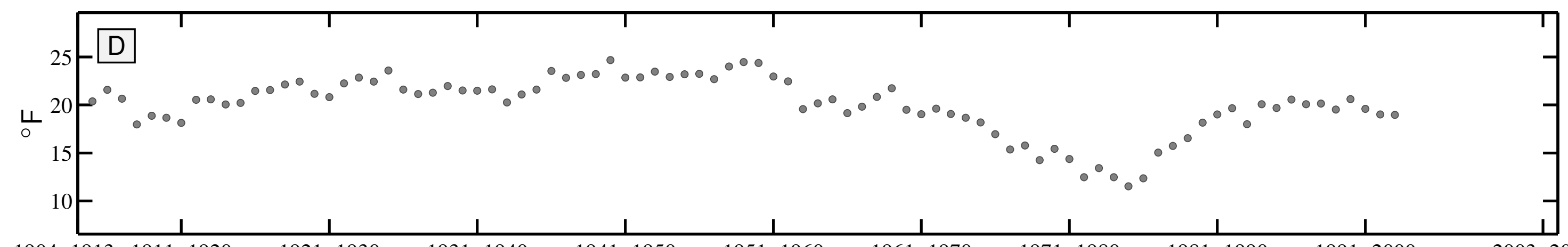


10 year Summer Teletherm dynamics for MT CARROLL, IL:

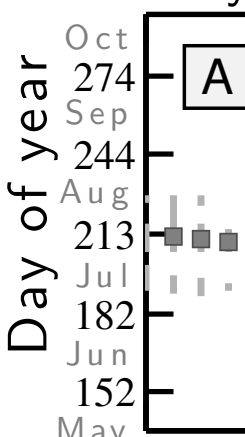
A

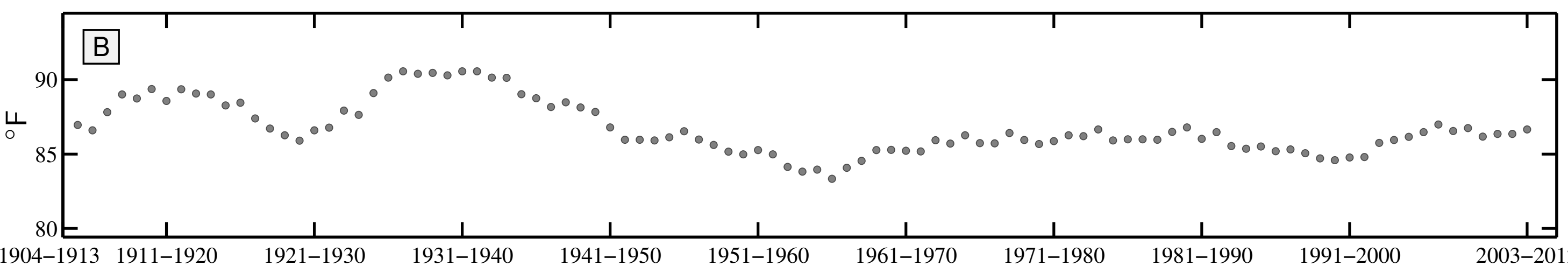

1904-1913 1911-1920

1931-1940

1941-1950

1951-1960

1961-1970

1971-1980

1981-1990

1991-2000

2003-2012

10 year Winter Teletherm dynamics for MT CARROLL, IL:

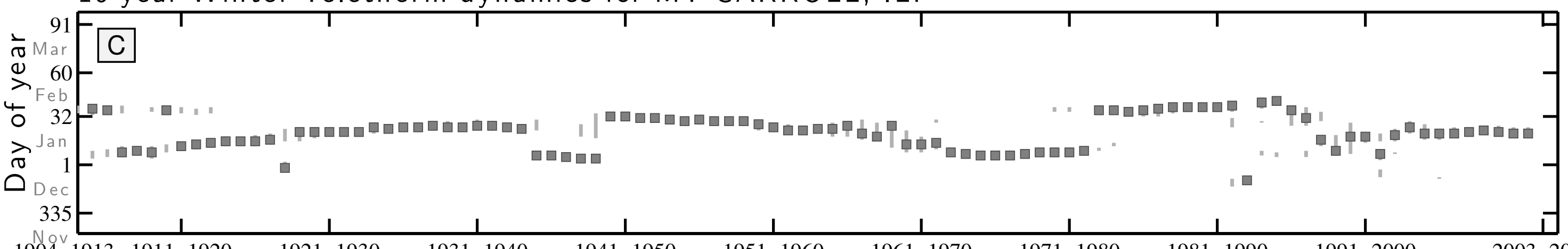

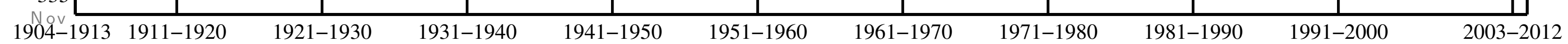

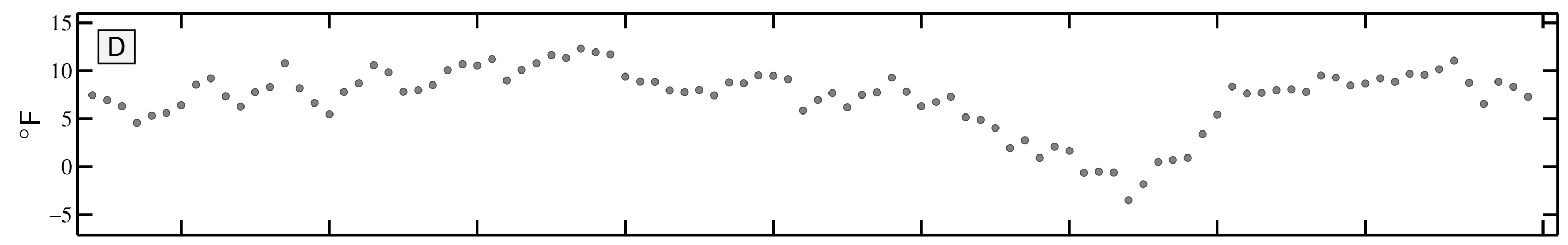


10 year Summer Teletherm dynamics for MT VERNON 3 NE, IL:

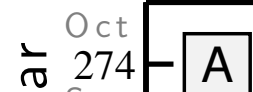

\& Sep

$>244-$

O Aug

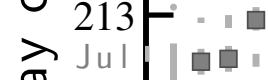

○ 182 -

$152-$

自国

May

1904-1913 1911-1920

$\frac{1}{1921-1930}$

$\frac{1}{1931-1940}$

$\frac{1}{1941-1950}$

$\frac{1}{1951-1960}$

$\frac{1}{1961-1970}$

$\frac{1}{1971-1980}$

$\frac{1}{1981-1990}$

$1991-2000$

$2003-2012$

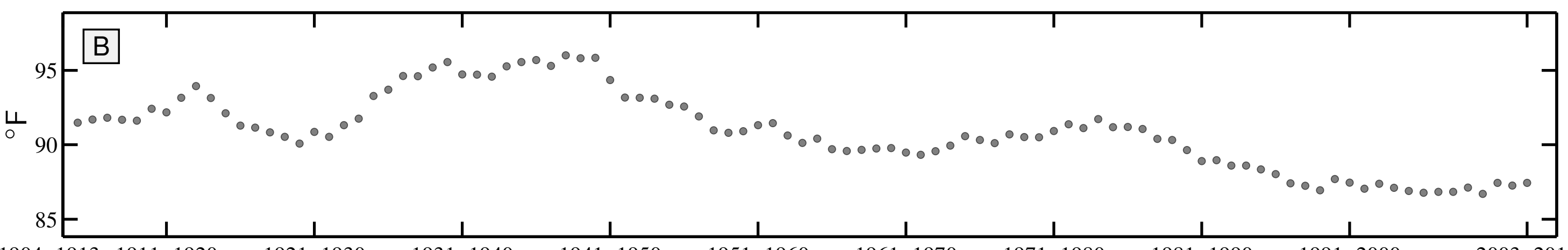

1904-1913 1911-1920

1921-1930

1931-1940

1941-1950

1951-1960

1961-1970

1971-1980

1981-1990

$1991-2000$

2003-2012

10 year Winter Teletherm dynamics for MT VERNON 3 NE, IL:

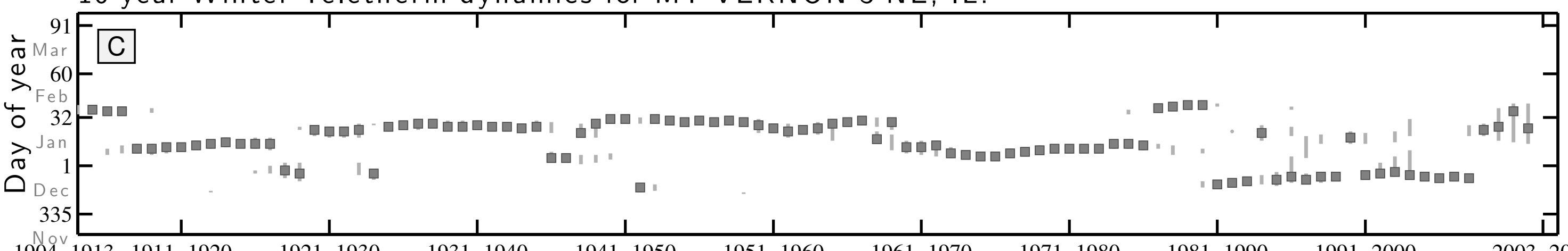

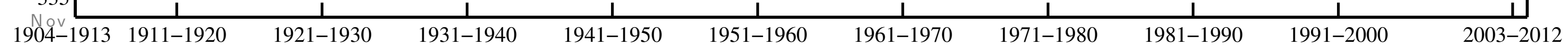

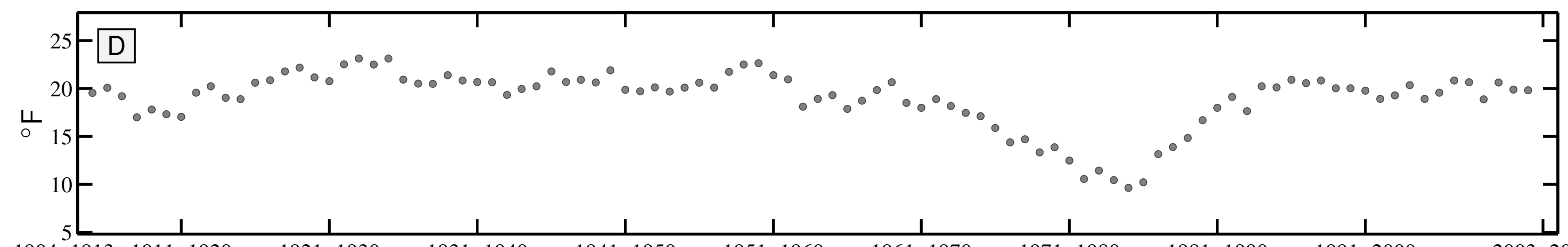

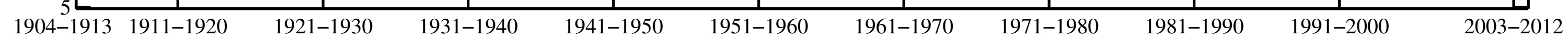


10 year Summer Teletherm dynamics for PARIS WTR WKS, IL:

$\perp$ Oct

Sep

$\stackrel{0}{>} 244$

Aug

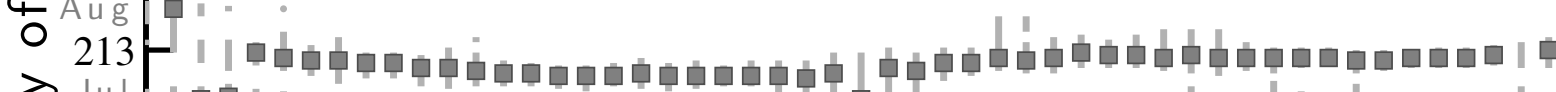

$\vec{\sigma}_{14}$

$\overbrace{}^{\circ} 182$

$152-$ | 中 由 " A

$1904-1913$ 1911-1920

$\frac{1}{1921-1930}$

$\frac{1}{1931-1940}$

$\frac{1}{1941-1950}$

1951-1960

1961-1970

1971-1980

1981-1990

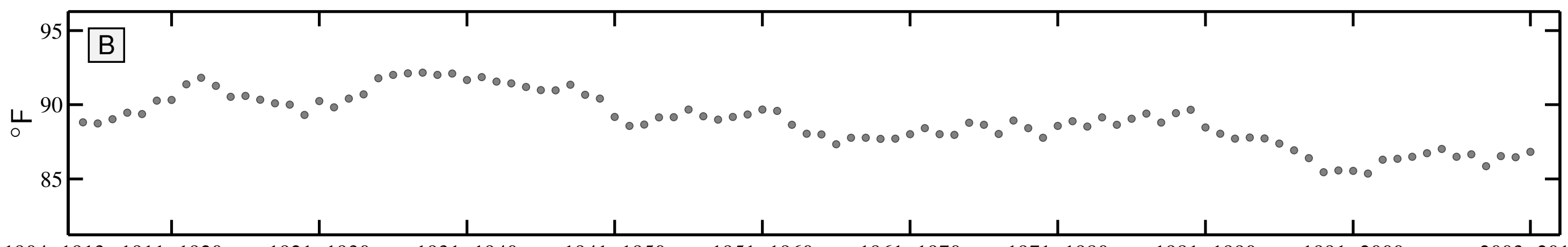

$1904-1913$ 1911-1920

$1921-1930$

$1931-1940$

$1941-1950$

$1951-1960$

$1961-1970$

$1971-1980$

$1981-1990$

$1991-2000$

$2003-2012$

10 year Winter Teletherm dynamics for PARIS WTR WKS, IL:

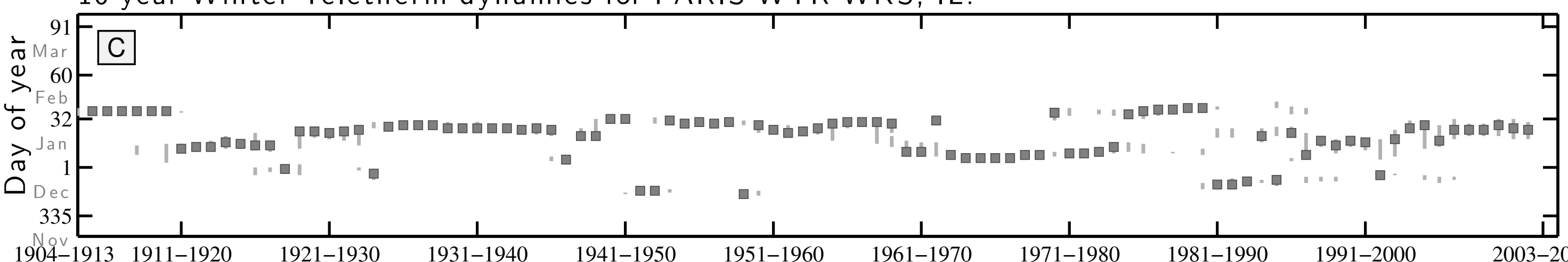

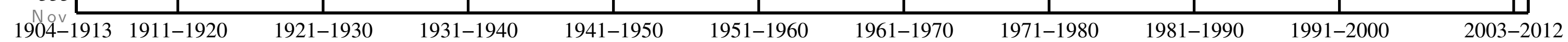

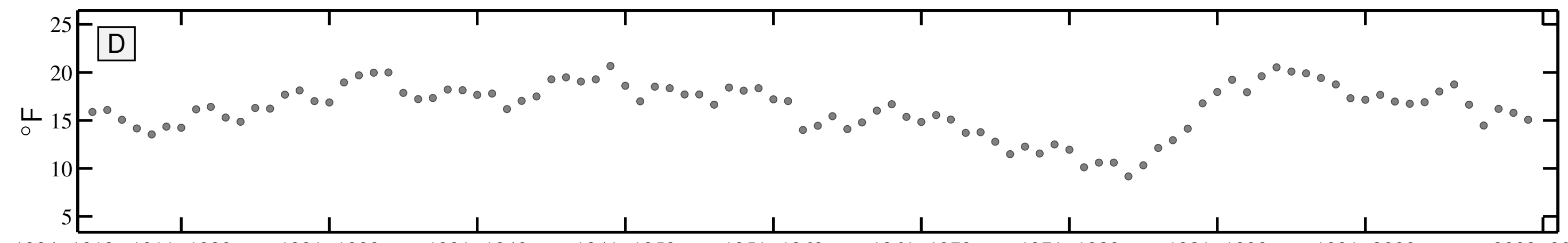


10 year Summer Teletherm dynamics for PERRY 6 NW, IL:
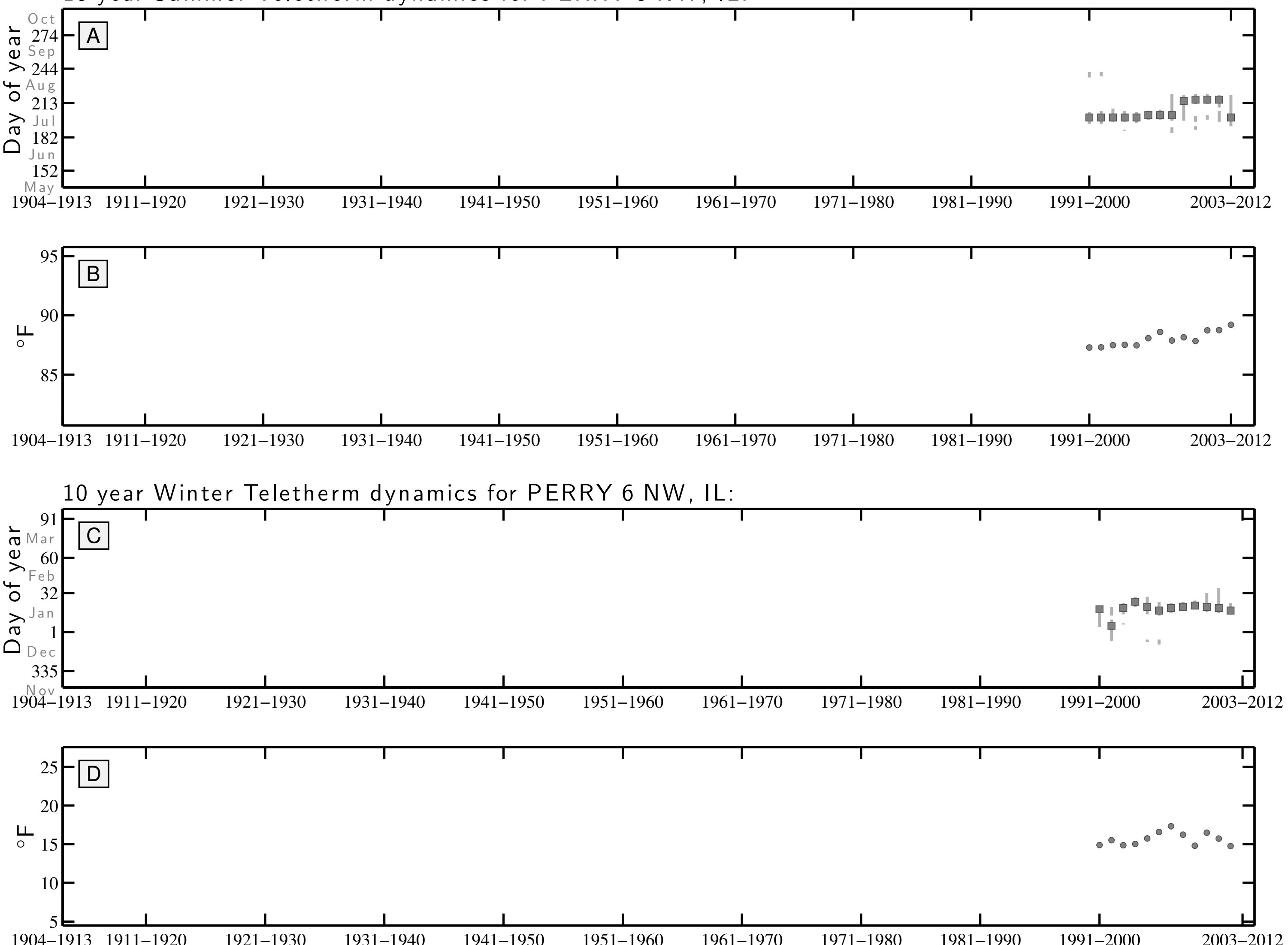

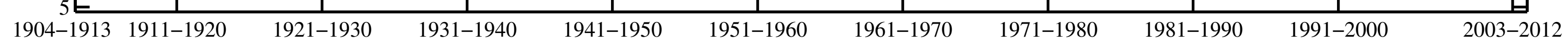


10 year Summer Teletherm dynamics for BERNE WWTP, IN:

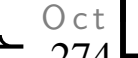

A

(1) Sep

$>244$

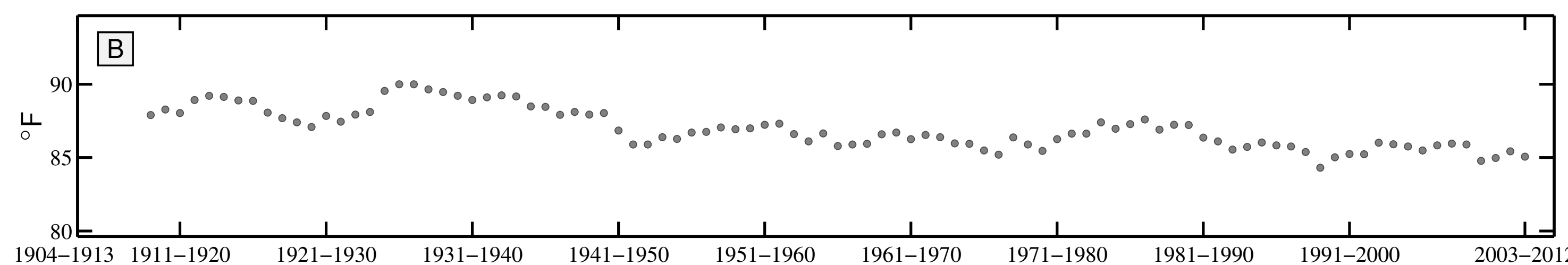

10 year Winter Teletherm dynamics for BERNE WWTP, IN:

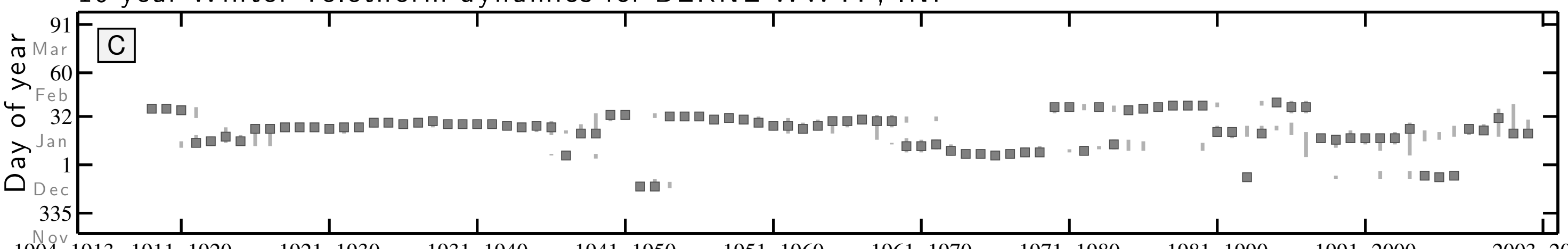

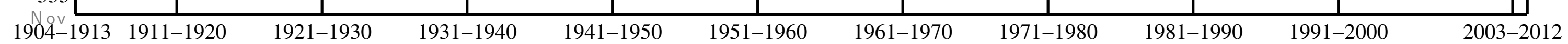

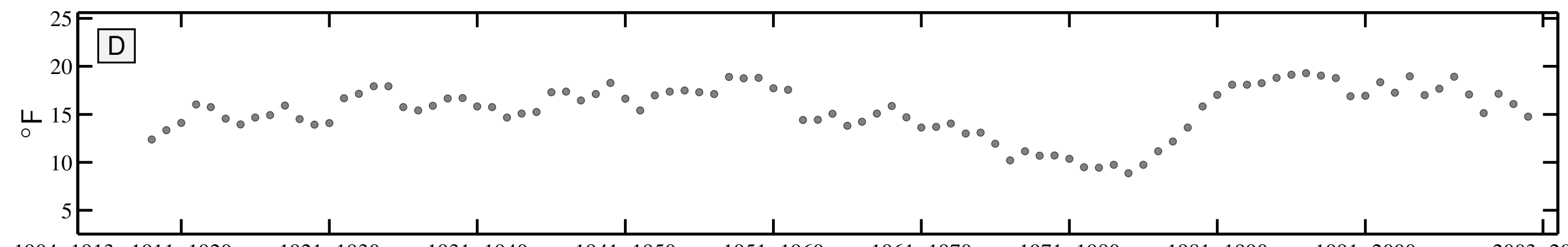


10 year Summer Teletherm dynamics for BLOOMINGTON IN UNIV, IN:

Oct

\& Sep

$>244-$

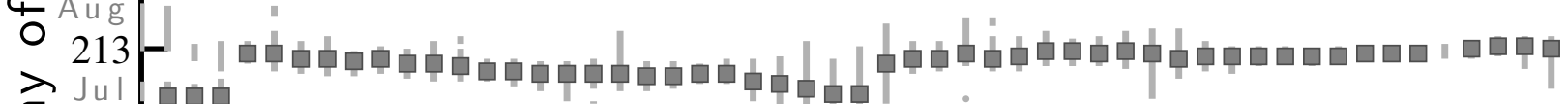

ते Jul

$\overbrace{}^{\sigma} 182=$

$152-$

May 1

1904-1913 1911-1920

$\frac{1}{1921-1930}$

$\frac{1}{1931-1940}$

$\frac{1}{1941-1950}$

$1951-1960$

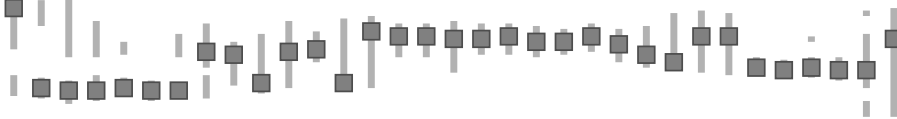

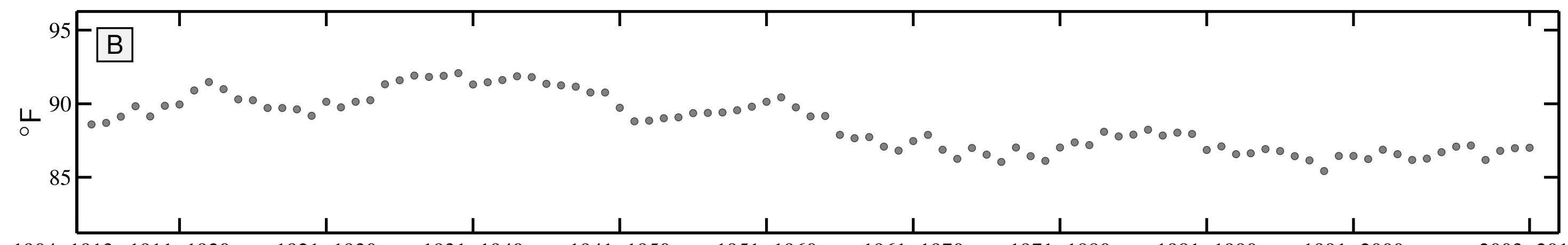

$1904-1913$ 1911-1920

$1921-1930$

$1931-1940$

$1941-1950$

$1951-1960$

$1961-1970$

$1971-1980$

$1981-1990$

$1991-2000$

$2003-2012$

10 year Winter Teletherm dynamics for BLOOMINGTON IN UNIV, IN:

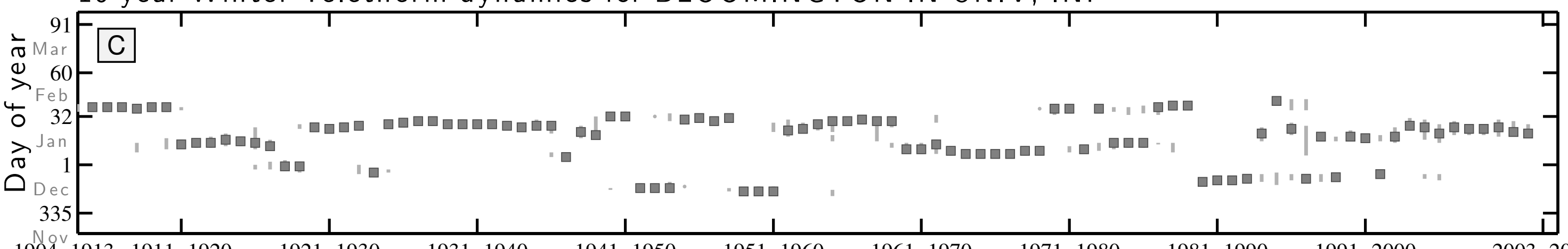

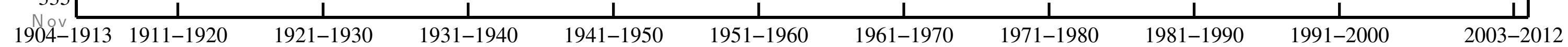

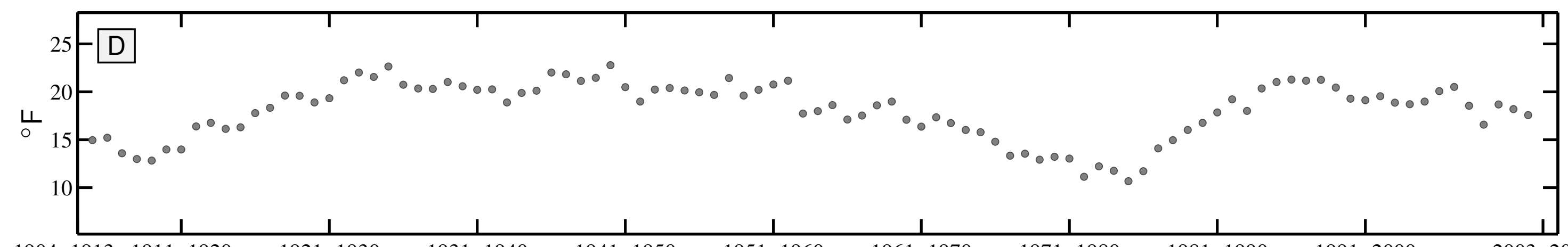


10 year Summer Teletherm dynamics for CHARLESTOWN 5 NNW, IN:

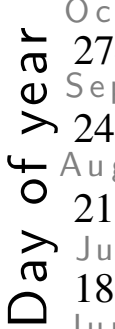
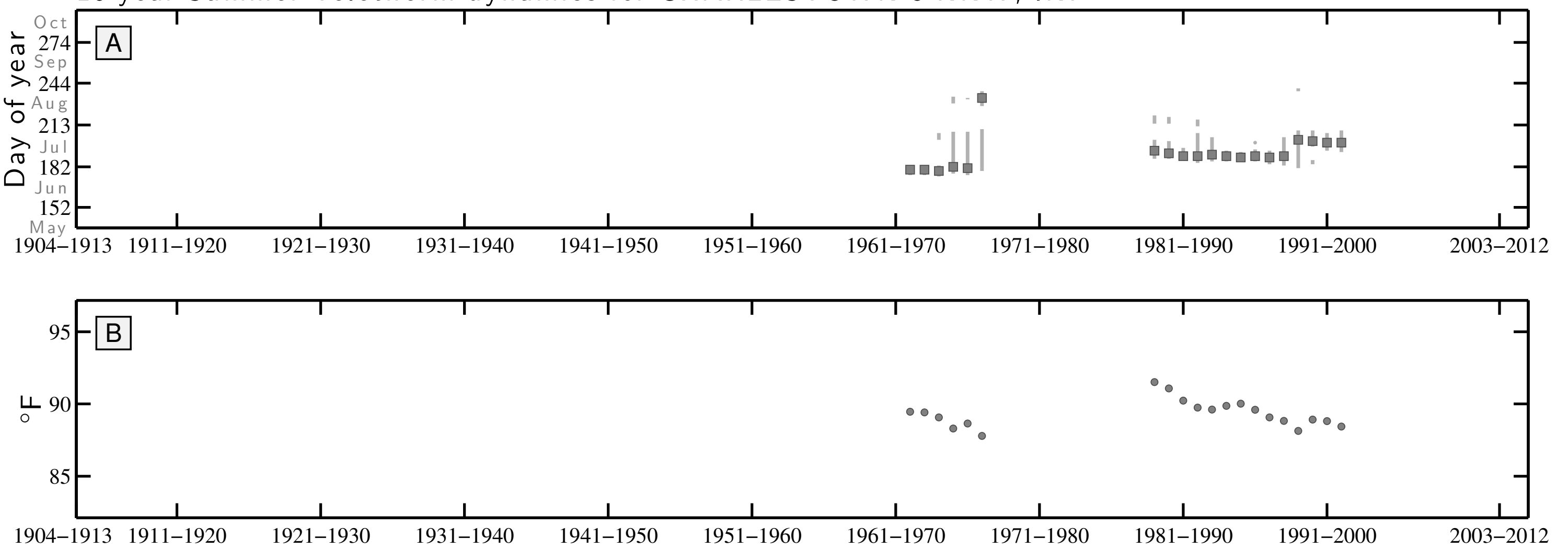

10 year Winter Teletherm dynamics for CHARLESTOWN 5 NNW, IN:
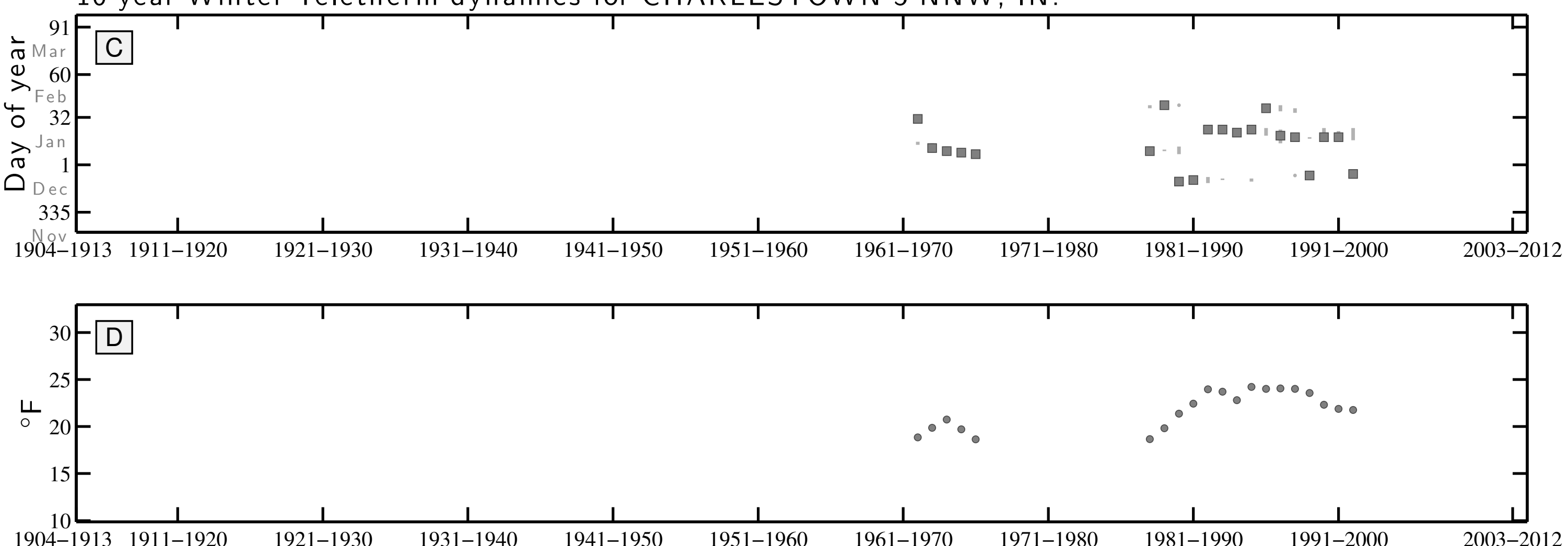
10 year Summer Teletherm dynamics for COLUMBUS, IN:

$+0 \mathrm{ct}$

(1) Sep

$>244-$

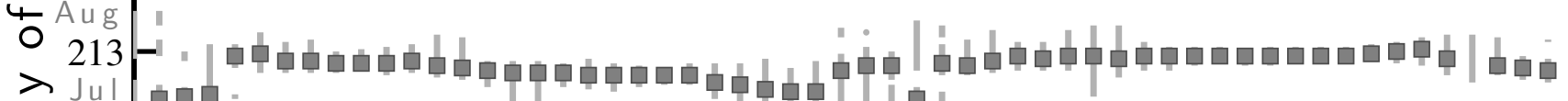

事

$\overbrace{}^{\pi} 182=$

152

1904-1913 1911-1920

$\frac{1}{1921-1930}$

$\frac{1}{1931-1940}$

$\frac{1}{1941-1950}$

$\frac{1}{1951-1960}$

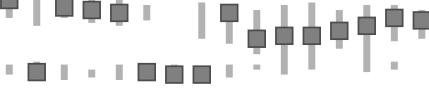

]

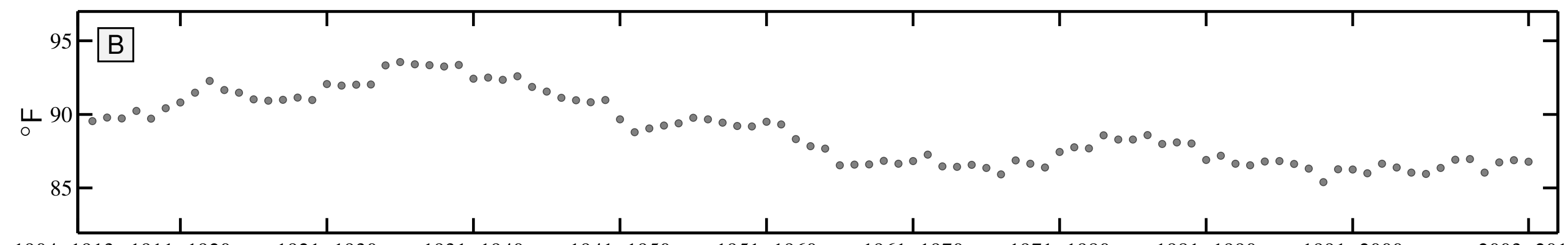

$1904-1913$ 1911-1920

$1921-1930$

$1931-1940$

$1941-1950$

$1951-1960$

$1961-1970$

$1971-1980$

$1981-1990$

$1991-2000$

2003-2012

10 year Winter Teletherm dynamics for COLUMBUS, IN:

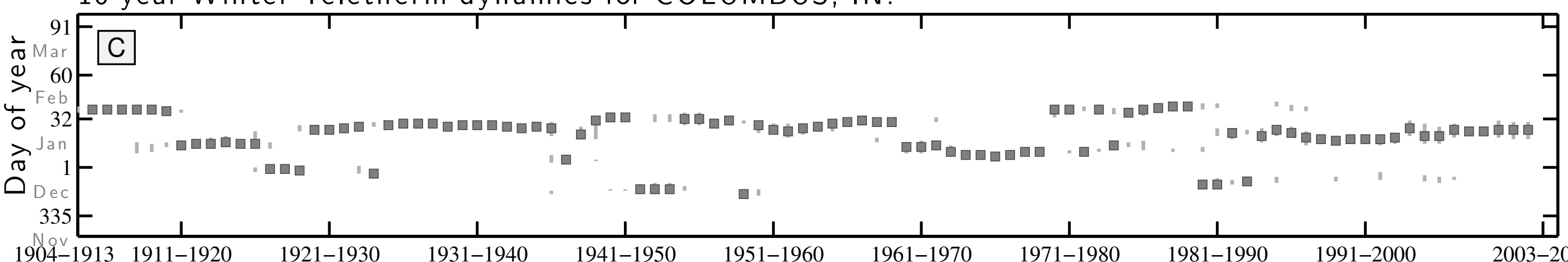

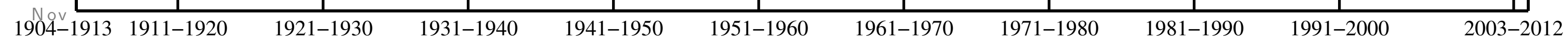

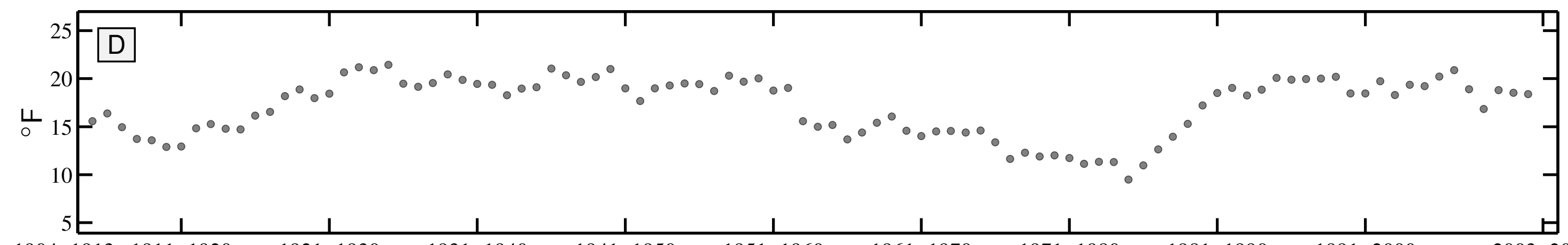


10 year Summer Teletherm dynamics for GREENFIELD, IN:

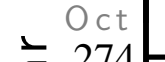

(1) Sep

$>244-$

ㅇ A g

$213=\square$

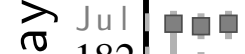

$182-$

152 -

May ए

1904-1913 1911-1920

$\frac{1}{1921-1930}$

$\frac{1}{1931-1940}$

$\frac{1}{1941-1950}$

$\frac{1}{1951-1960}$

961-1970

971-1980

$\frac{1}{1981-1990}$

1991-2000

2003-2012

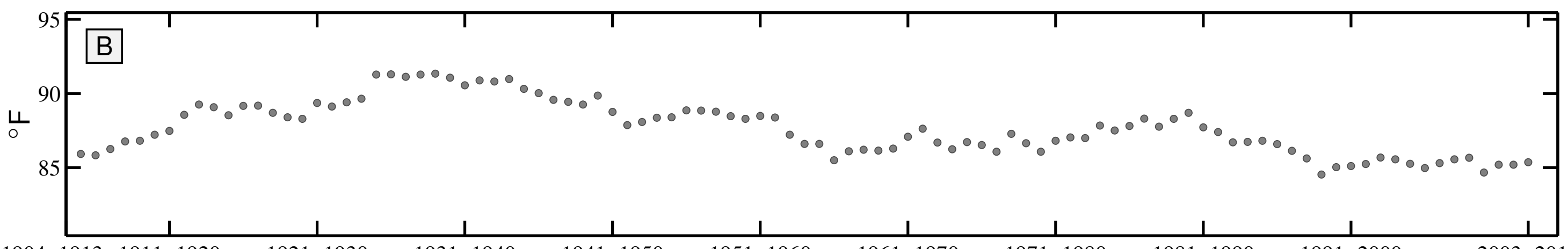

$1904-1913$ 1911-1920

$1921-1930$

$1931-1940$

$1941-1950$

$1951-1960$

$1961-1970$

$1971-1980$

$1981-1990$

$1991-2000$

2003-2012

10 year Winter Teletherm dynamics for GREENFIELD, IN:
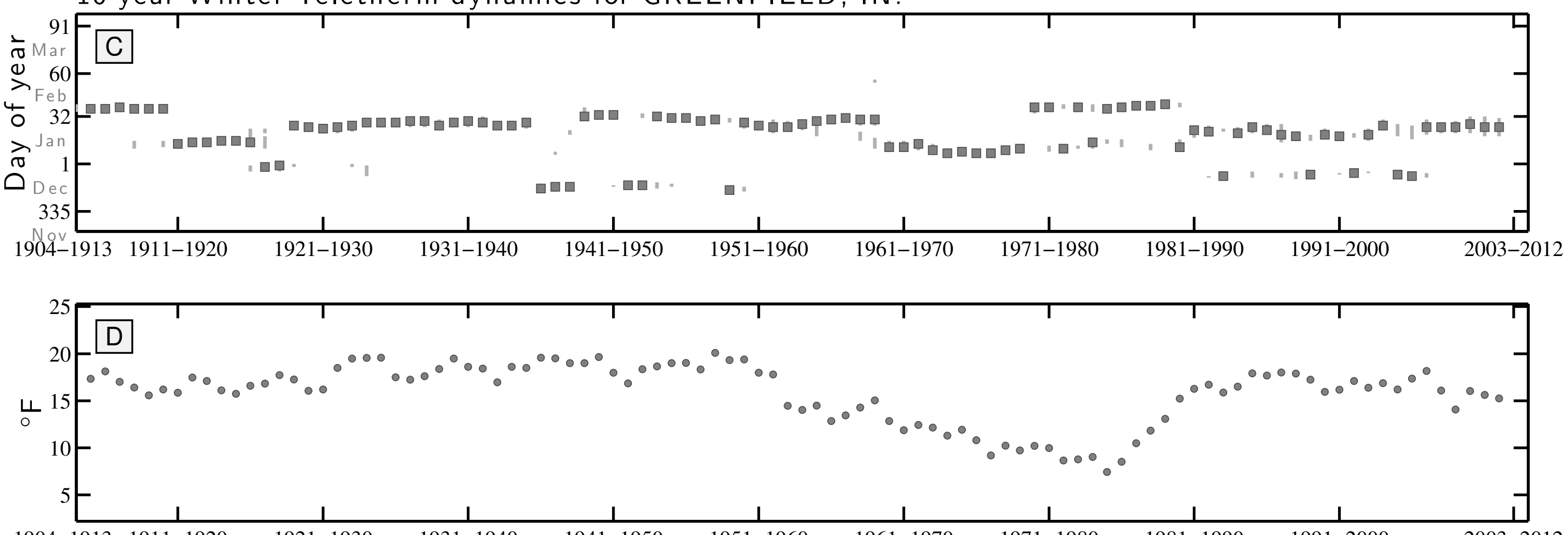
10 year Summer Teletherm dynamics for HUNTINGTON, IN:
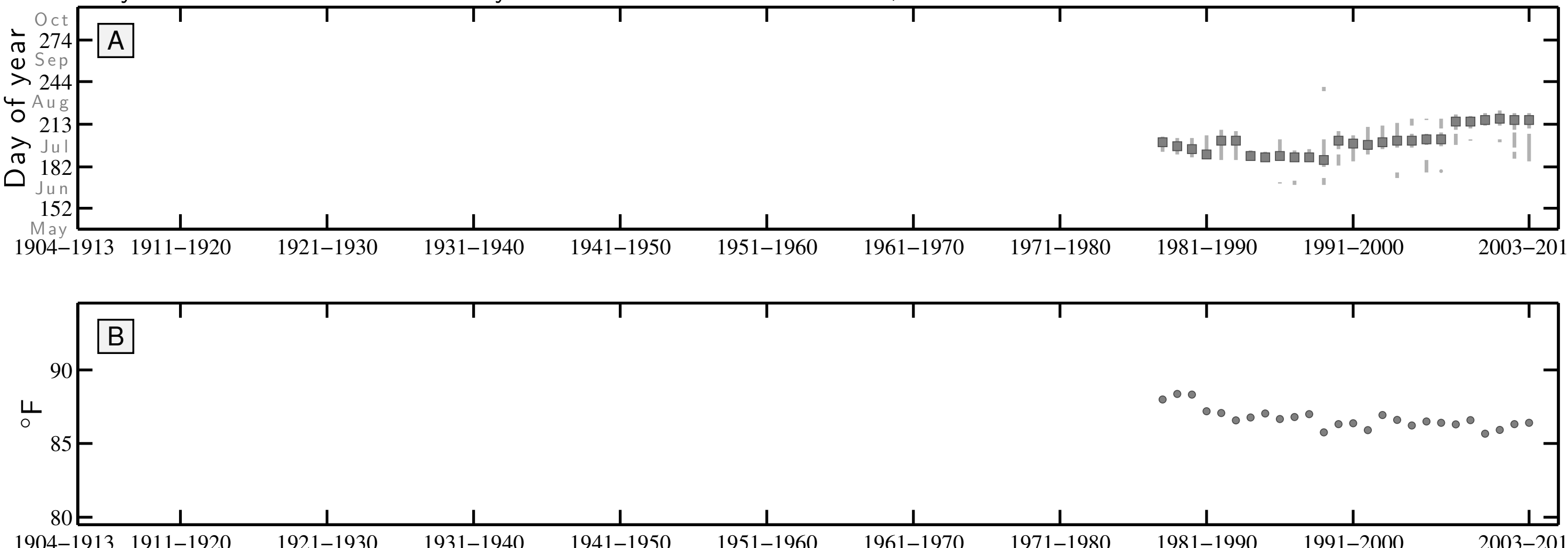

1904-1913 1911-1920

971-1980

1981-1990

991-2000

2003-2012

10 year Winter Teletherm dynamics for HUNTINGTON, IN:

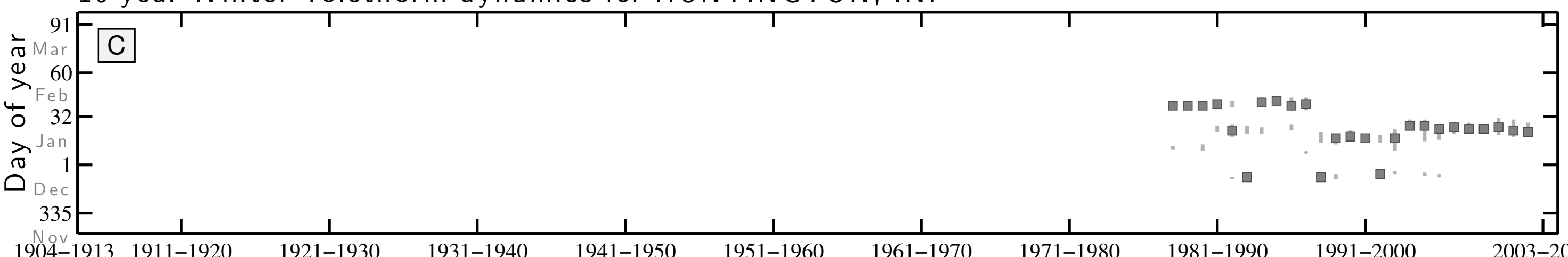

1904-1913 1911-192

1921-1930

1931-1940

1941-1950

1951-1960

1961-1970

1971-1980

1981-1990

1991-2000

2003-2012

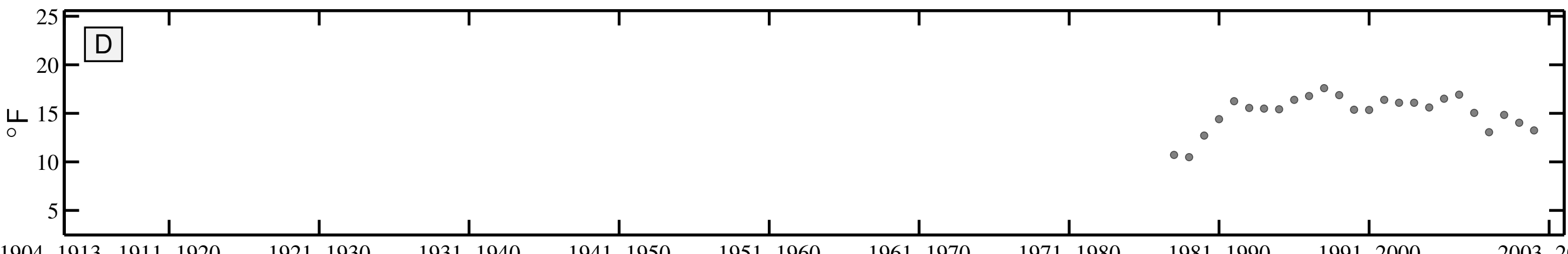


10 year Summer Teletherm dynamics for MADISON SEWAGE PLT, IN:

$\mathrm{Oct}$

( 274

(1) Sep

$>244-$

잉

$>$

○ 182

$182-$

$\mathrm{Jun}$

$152-$

May —

1904-1913 1911-1920

1921-1930

1931-1940

$\frac{1}{1941-1950}$

$\frac{1}{1951-1960}$

1961-1970

1971-1980

$\frac{1}{1981-1990}$

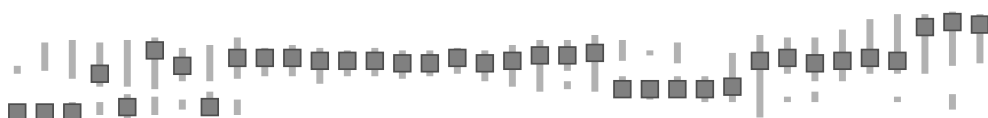

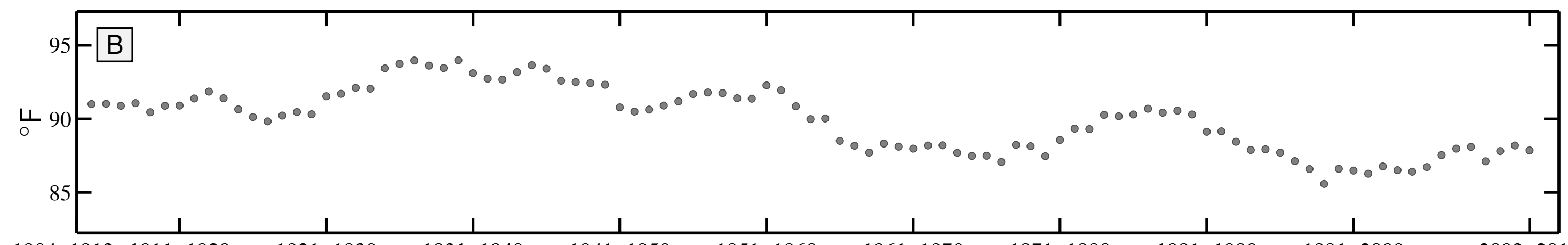

1904-1913 1911-1920

1921-1930

1931-1940

1941-1950

1951-1960

1961-1970

1971-1980

1981-1990

1991-2000

2003-2012

10 year Winter Teletherm dynamics for MADISON SEWAGE PLT, IN:

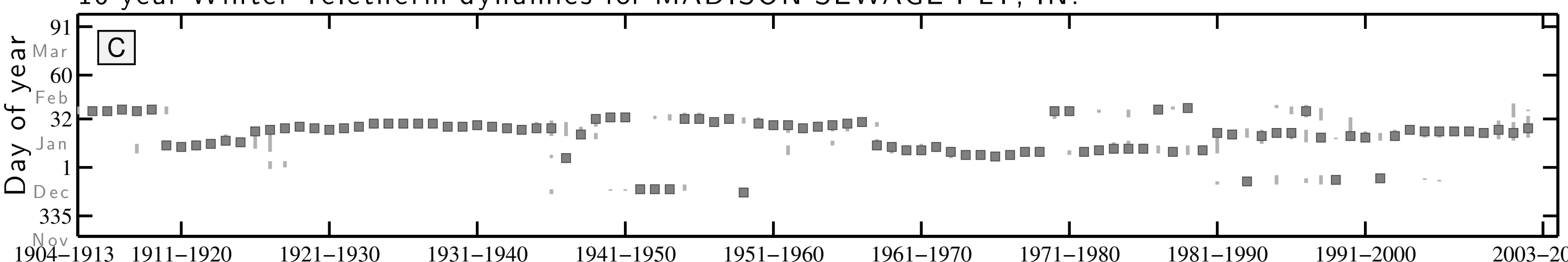

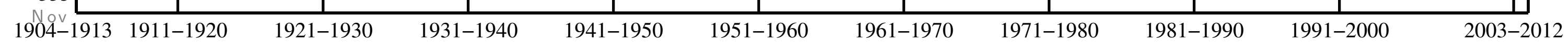

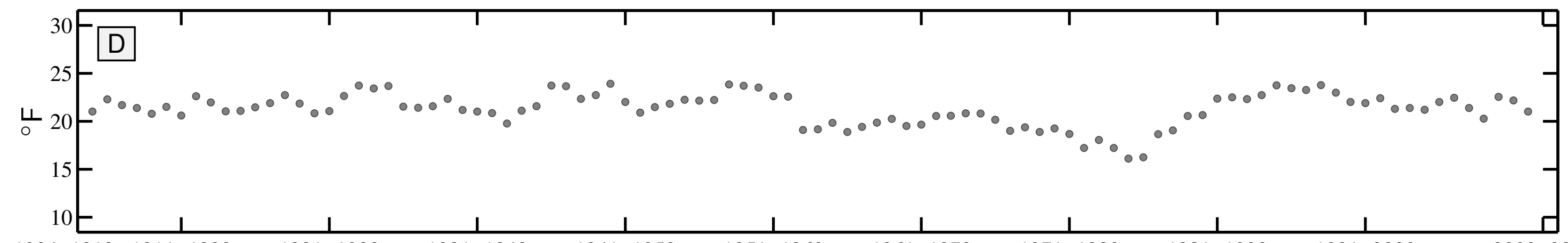


10 year Summer Teletherm dynamics for MARION $2 \mathrm{~N}$, IN:

$-O c t$

(1) Sep

$>244-$

( $\mathrm{Aug}$

$>\quad 213$

182

$\mathrm{Jun}$

$152-$

1904-1913 1911-1920

$\frac{1}{1921-1930}$

$1931-1940$

$\frac{1}{1941-1950}$

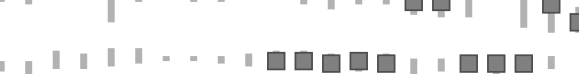

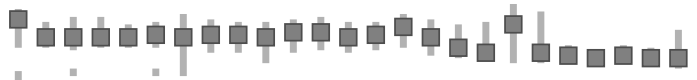

-

$1-2000$

$2003-2012$

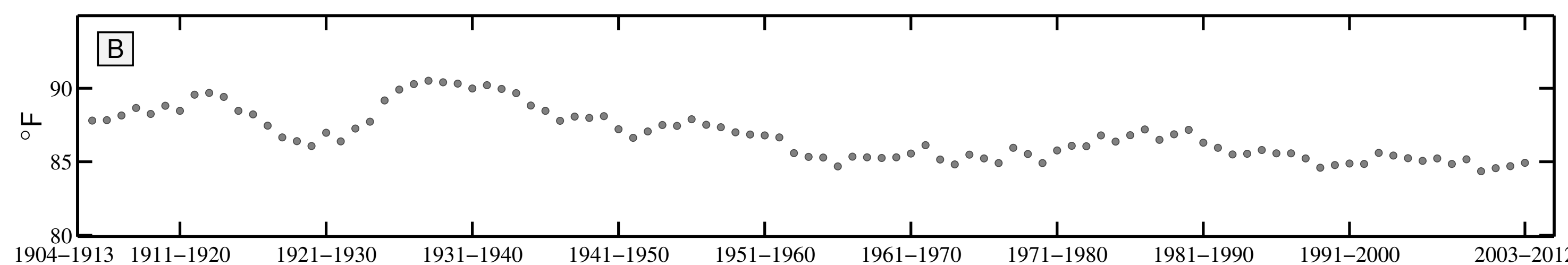

10 year Winter Teletherm dynamics for MARION 2 N, IN:

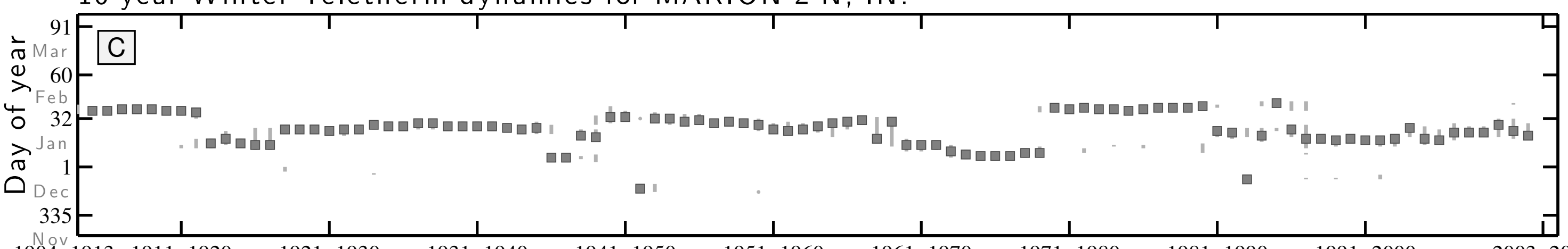

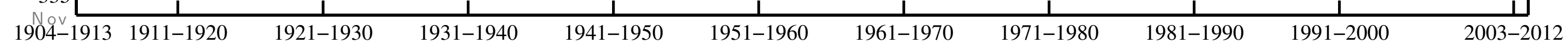

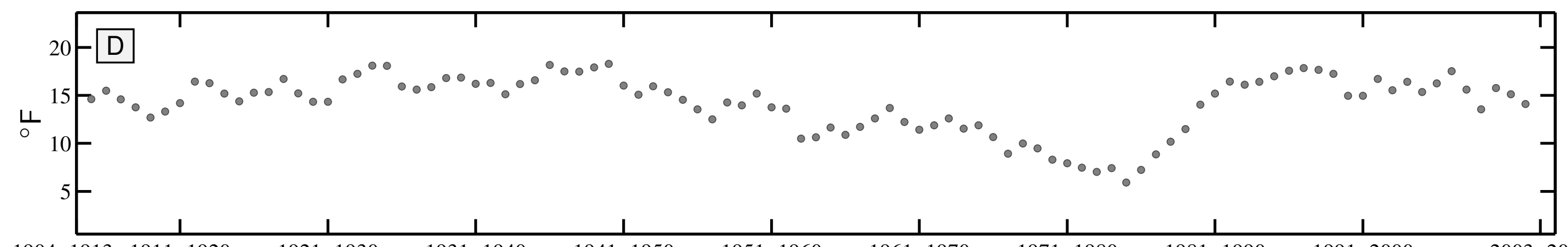

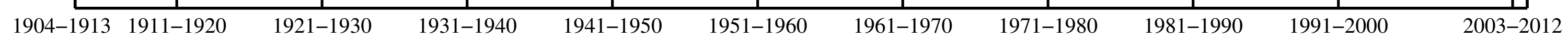


10 year Summer Teletherm dynamics for PRINCETON $1 \mathrm{~W}$, IN:

$+\mathrm{Oct}$

A

(1) Sep

4 Aug

O 213

$>\mathrm{Jul}=$

م $182-$

$152-$

ㅁ "

_

1904-1913 1911-1920

1921-1930

1931-1940

$\frac{1}{1941-1950}$

$\frac{1}{951-1960}$

961-1970

$\frac{1}{971-1980}$

$\frac{1}{1981-1990}$

1991-2000

2003-201

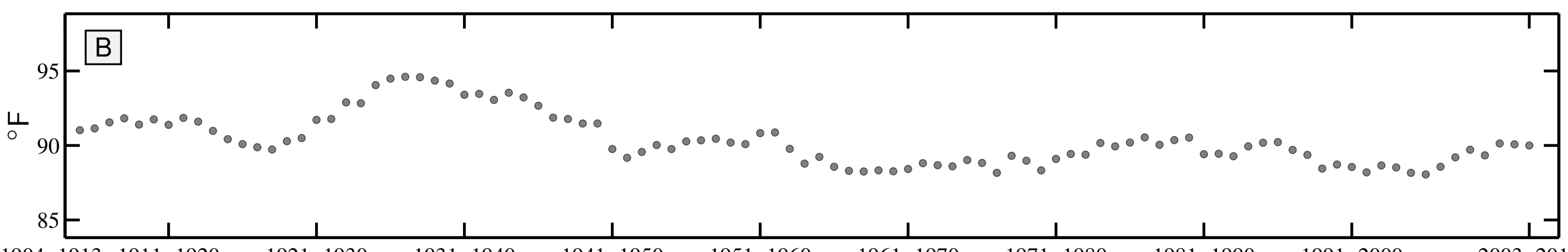

1904-1913 1911-1920

$1921-1930$

$1931-1940$

$1941-1950$

$1951-1960$

$1961-1970$

$1971-1980$

$1981-1990$

$1991-2000$

$2003-2012$

10 year Winter Teletherm dynamics for PRINCETON $1 \mathrm{~W}$, IN:

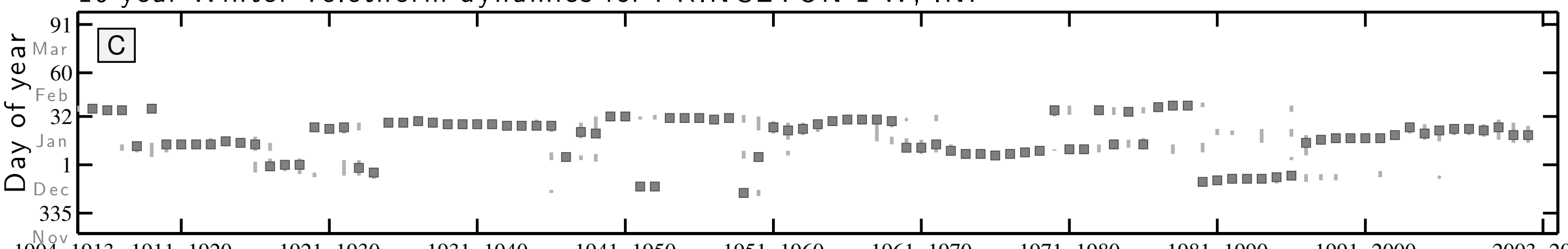

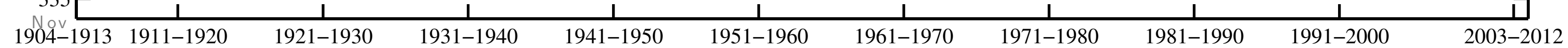

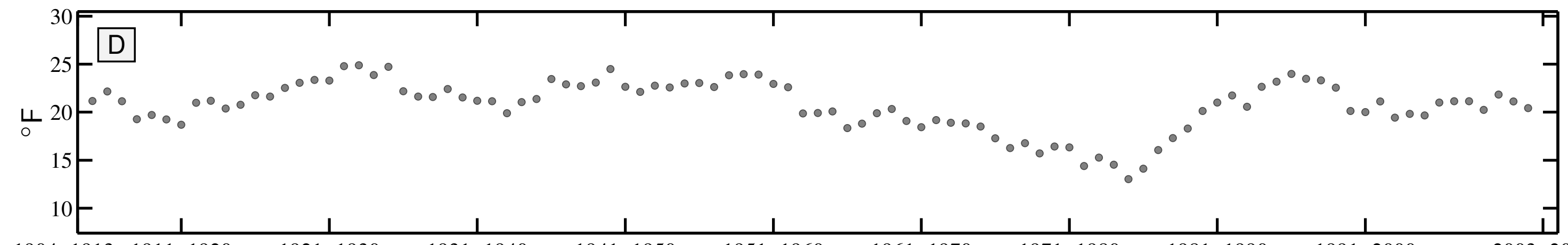


10 year Summer Teletherm dynamics for ALBIA 3 NNE, IA:

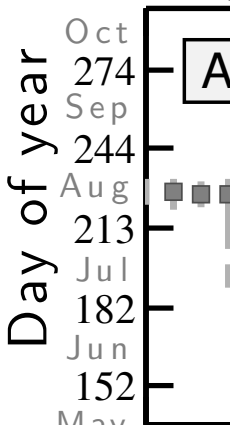
A
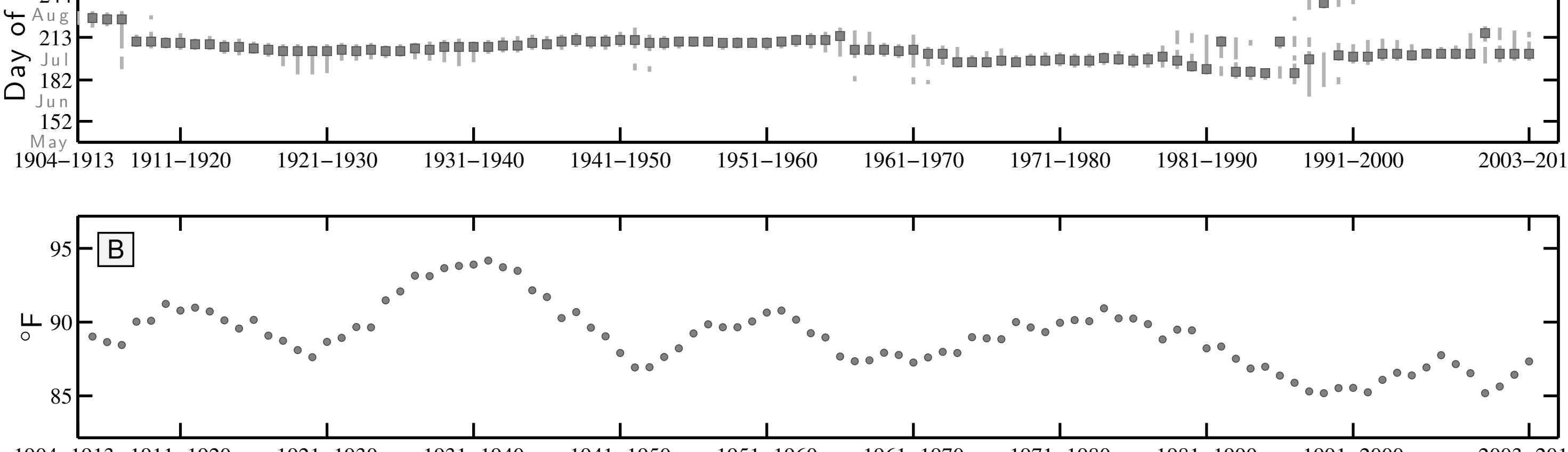

1904-1913 1911-1920 1921-1930

1931-1940

$1941-1950$

$1951-1960$

$1961-1970$

$1971-1980$

$1981-1990$

991-2000

2003-2012

10 year Winter Teletherm dynamics for ALBIA 3 NNE, IA:

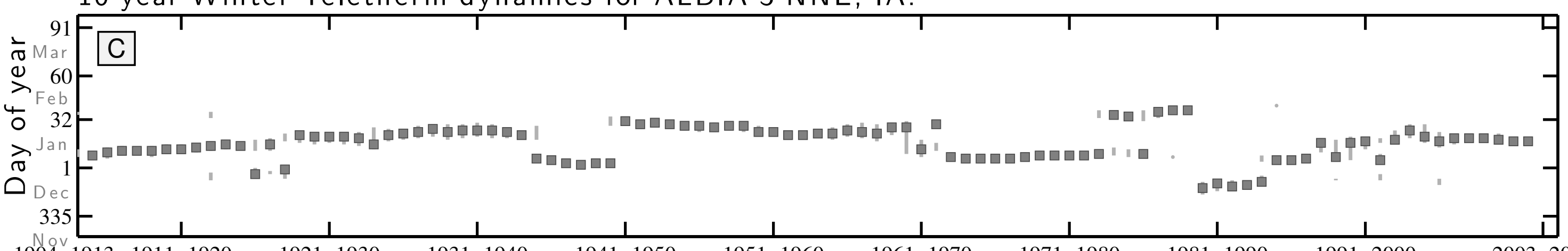

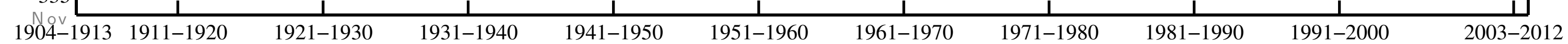

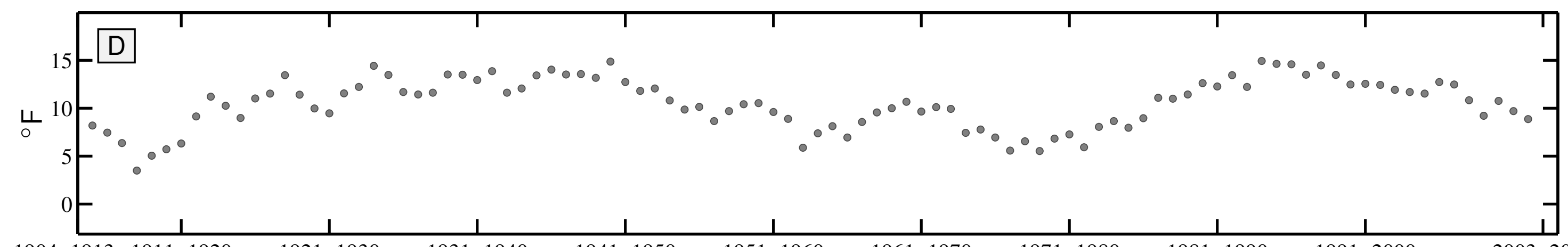


10 year Summer Teletherm dynamics for ALGONA 3 W, IA:

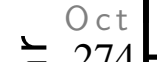

A

(1) Sep

$>244-$

4 A u g

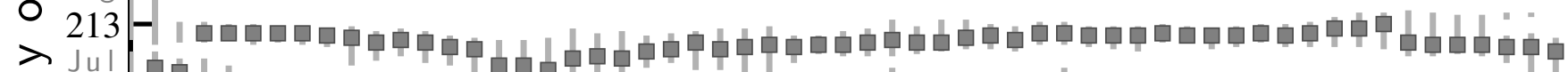

182

Jun

$152-$

May 1

$-1920$

I. = ।

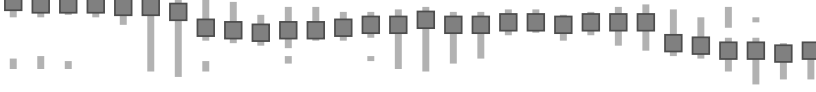

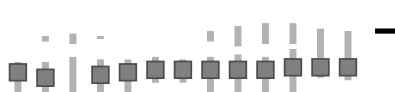
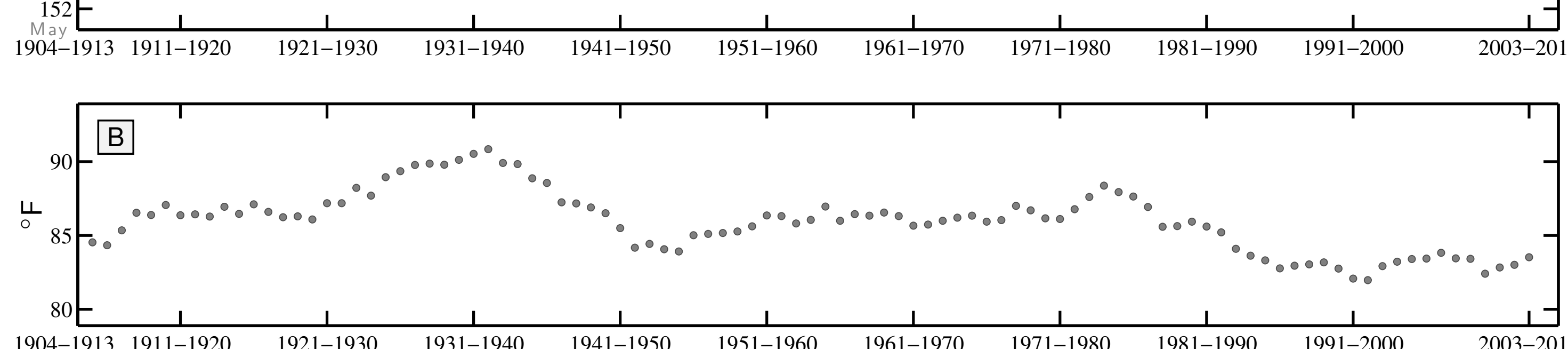

$1904-1913$ 1911-1920

$1921-1930$

$1931-1940$

$1941-1950$

$1951-1960$

$1961-1970$

$1971-1980$

$1981-1990$

991-2000

$2003-2012$

10 year Winter Teletherm dynamics for ALGONA $3 \mathrm{~W}$, IA:

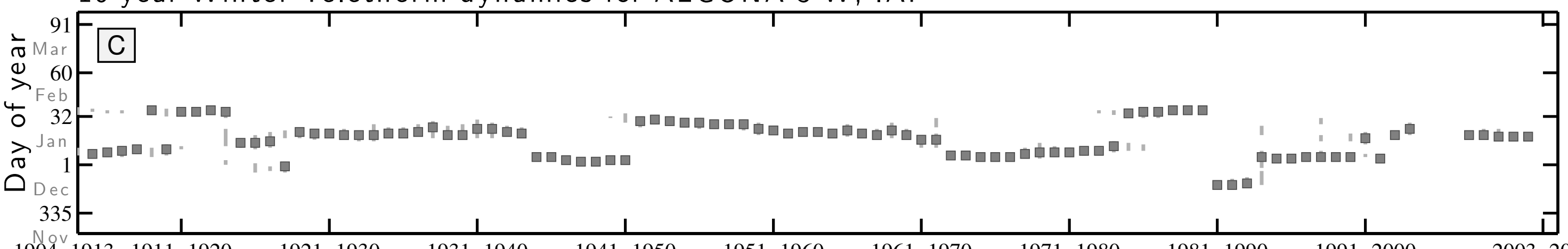

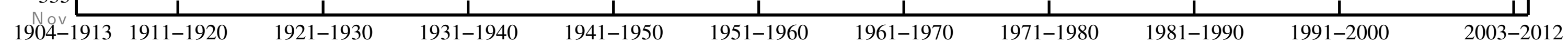

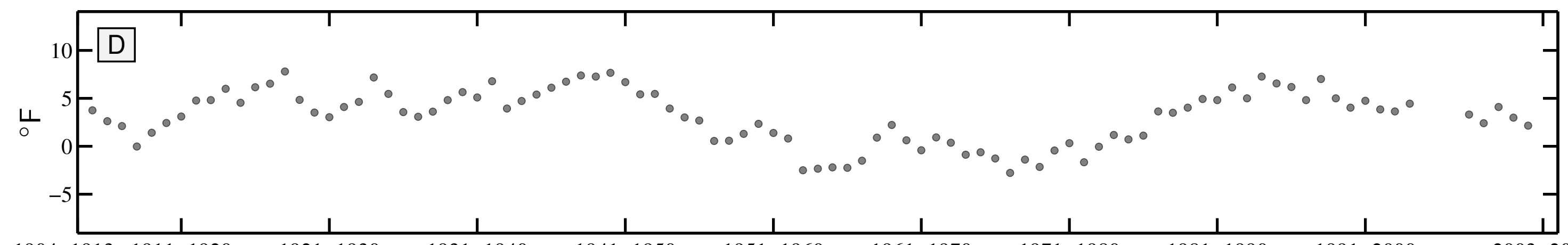



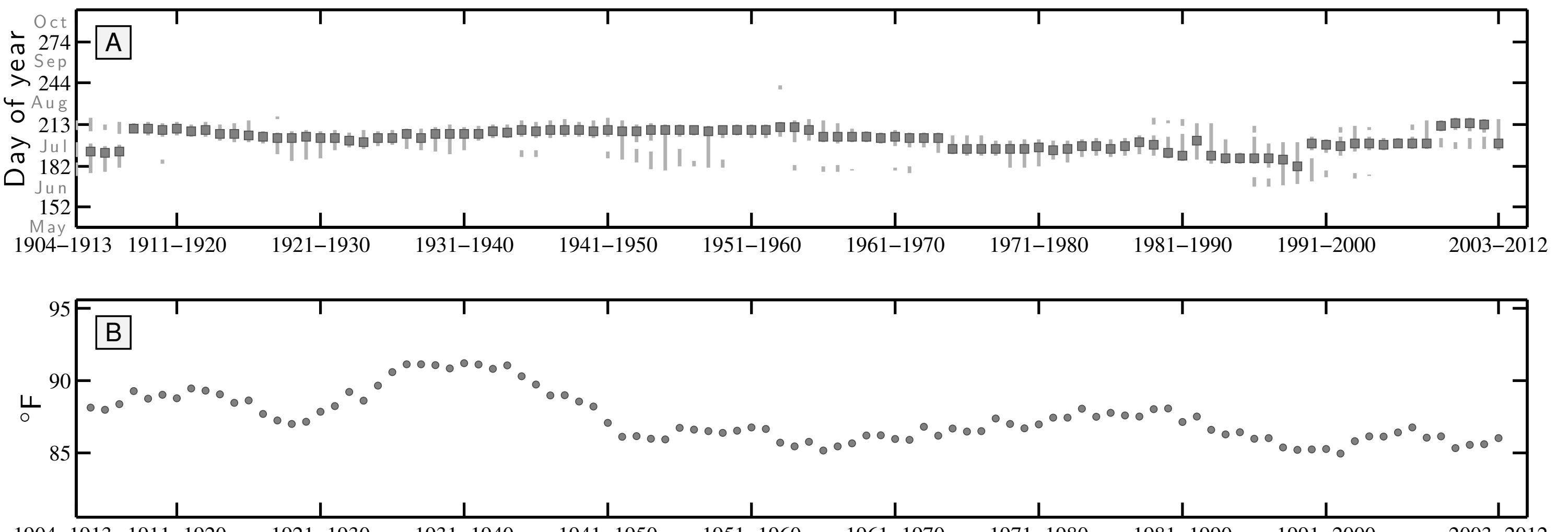

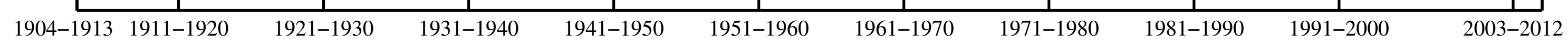
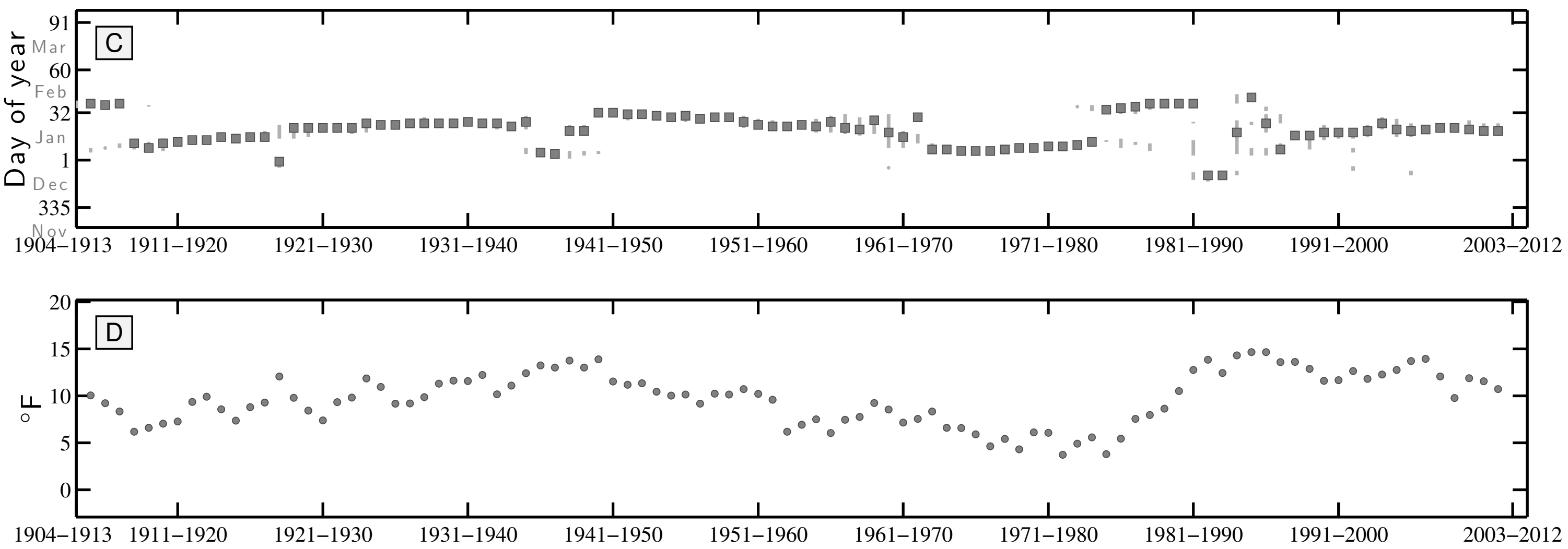

$\begin{array}{llllllllll}1904-1913 & 1911-1920 & 1921-1930 & 1931-1940 & 1941-1950 & 1951-1960 & 1961-1970 & 1971-1980 & 1981-1990 & 1991-2000\end{array}$


10 year Summer Teletherm dynamics for FAIRFIELD, IA:
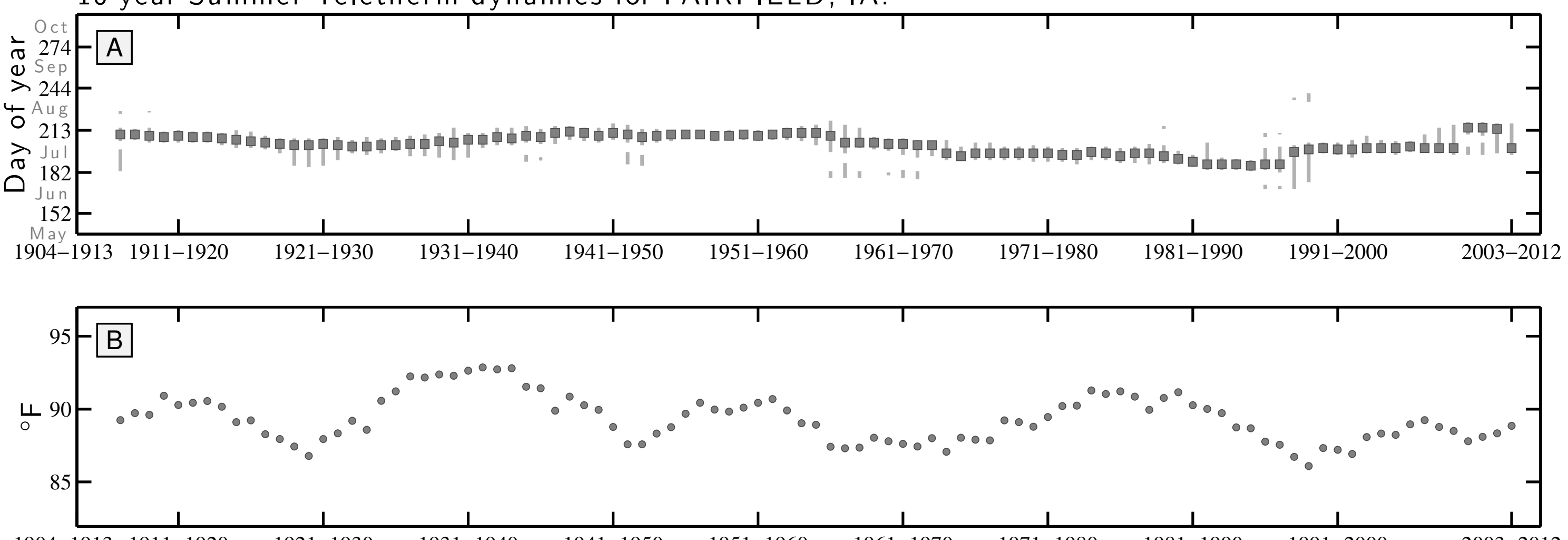

1904-1913 1911-1920

$1921-1930$

$1931-1940$

$1941-1950$

$1951-1960$

$1961-1970$

1971-1980

1981-1990

$1991-2000$

2003-2012

10 year Winter Teletherm dynamics for FAIRFIELD, IA:

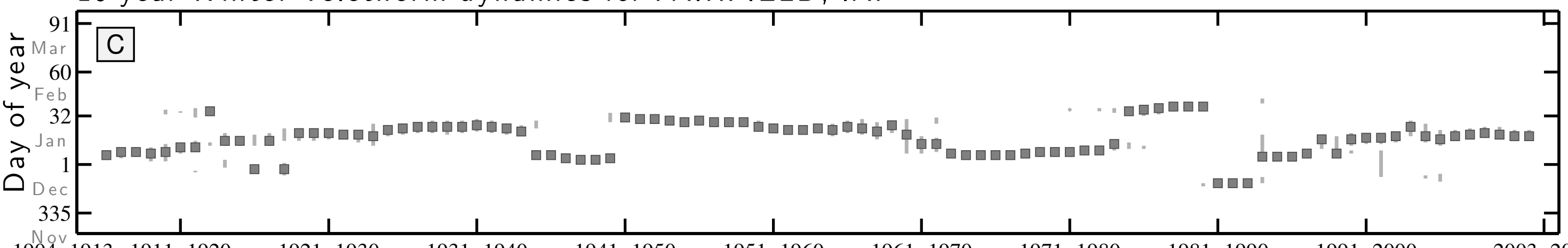

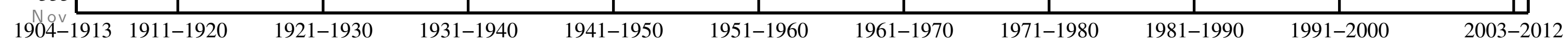

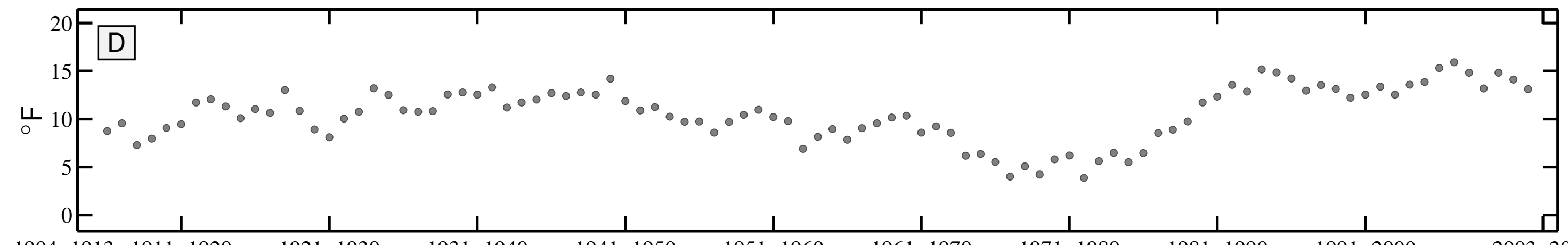


10 year Summer Teletherm dynamics for FORT DODGE 5NNW, IA:

Oct

$274-A$

(1) Sep

$>244-$

O A g

$\gtrsim \begin{gathered}213 \\ \mathrm{Jul}\end{gathered}$

$182-\square$

$\mathrm{Jun}$

$152-$

May —_

1904-1913 1911-1920

$\frac{1}{1921-1930}$

1931-1940

ن

|

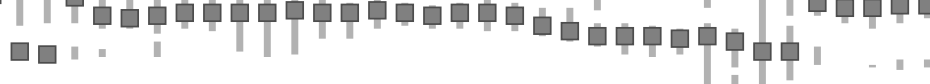

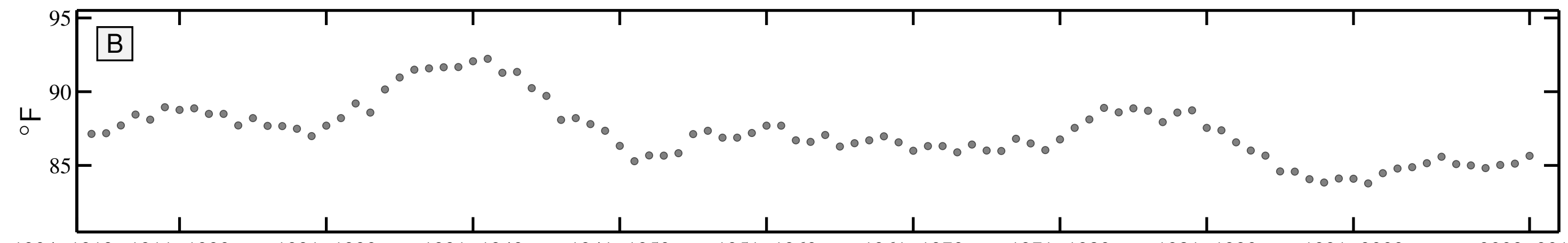

1904-1913 1911-1920

1921-1930

1931-1940

1941-1950

1951-1960

1961-1970

1971-1980

1981-1990

1991-2000

2003-2012

10 year Winter Teletherm dynamics for FORT DODGE 5NNW, IA:

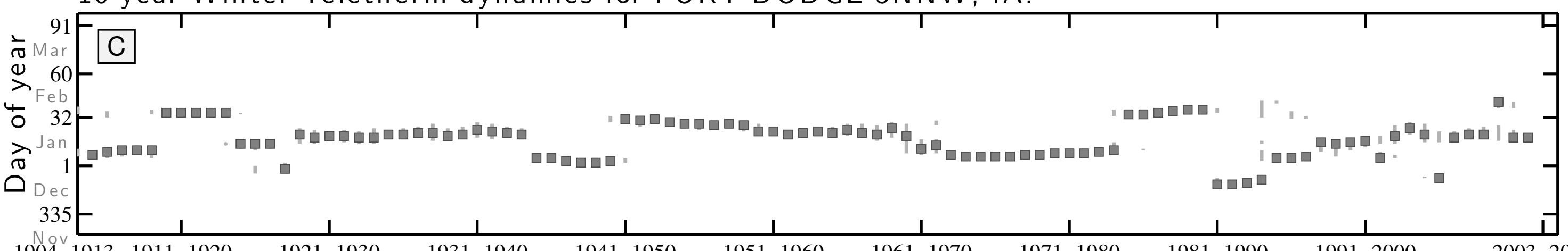

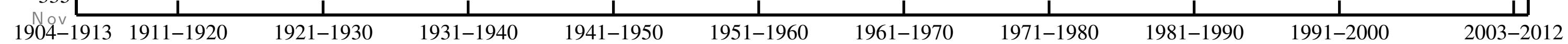

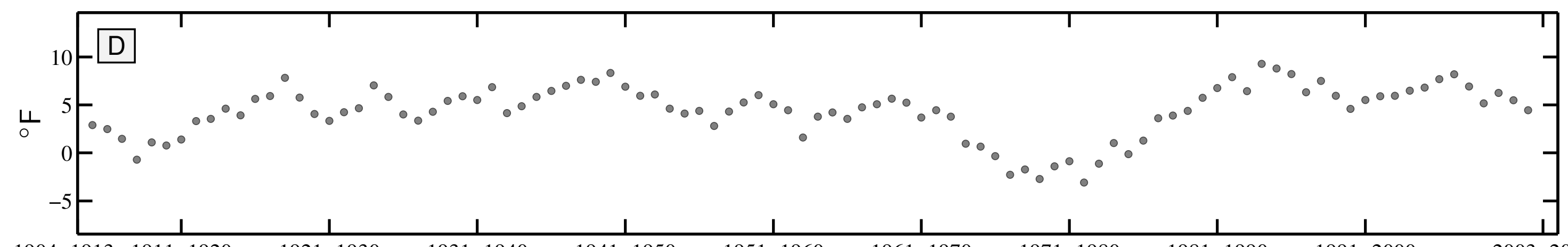


10 year Summer Teletherm dynamics for IOWA FALLS, IA:

$\div$ Oct 274

(

(1) Sep

$>244-$

4 $\mathrm{Aug}$

$\gtrsim \begin{gathered}213 \\ \mathrm{Jul}\end{gathered}$

$\overbrace{0} 182$

$152-$

May $\square$

1904-1913 1911-1920

$\frac{1}{1921-1930}$

$\frac{1}{1931-1940}$

1941-1950

1951-1960

| เ

$\frac{1}{1961-1970}$

$\frac{1}{1971-1980}$

$\frac{1}{1981-1990}$

1991-2000

2003-2012

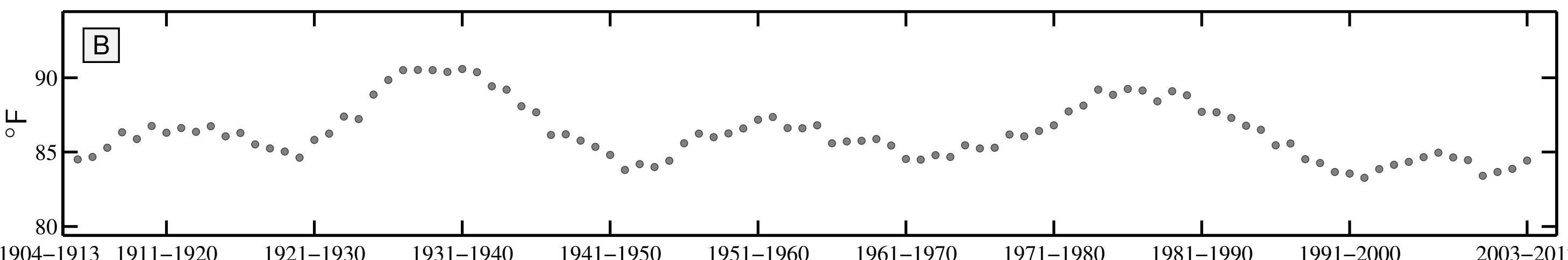

1904-1913 1911-1920

1921-1930

1931-1940

1941-1950

1951-1960

1961-1970

1971-1980

1981-1990

1991-2000

2003-2012

10 year Winter Teletherm dynamics for IOWA FALLS, IA:

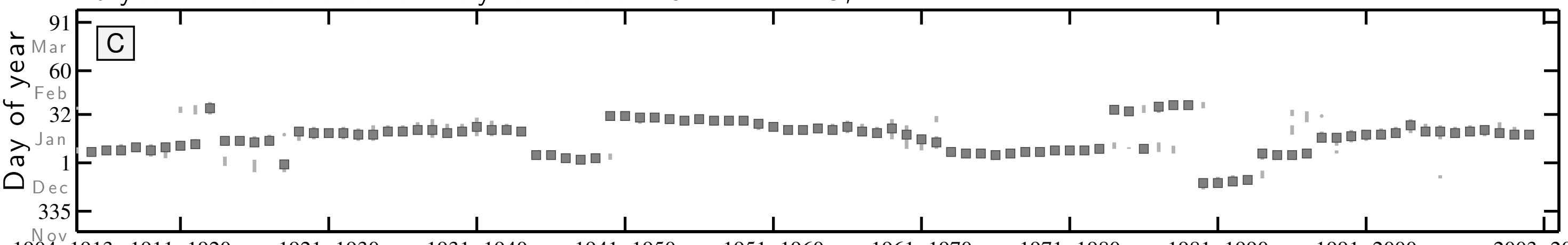

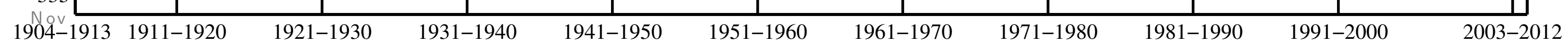

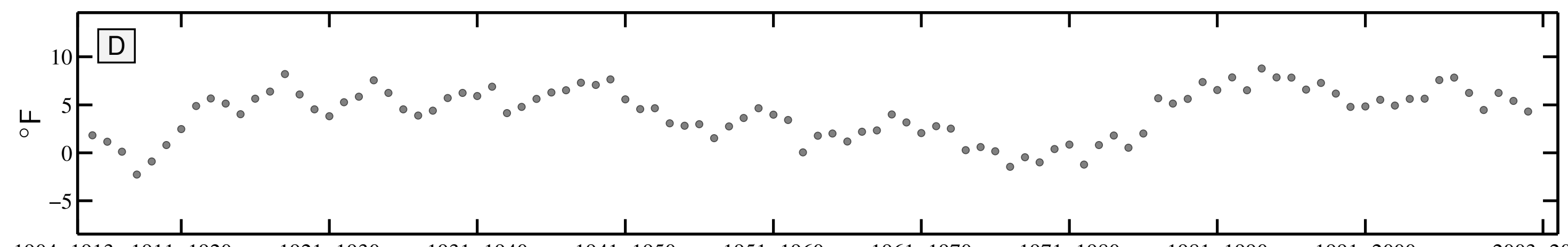


10 year Summer Teletherm dynamics for LE MARS, IA:

+ Oct

\% $274-A$

(1) Sep

$>244-$

O

$\lambda_{\mathrm{Jul}} 213$

182

Jun

May

1904-1913 1911-1920

$\frac{1}{1921-1930}$

$\frac{1}{1931-1940}$

$\frac{1}{1941-1950}$

$\frac{1}{1951-1960}$

$\frac{1}{1-1970}$

$\frac{1}{1971-1980}$

1981-1990

991-2000

2003-201

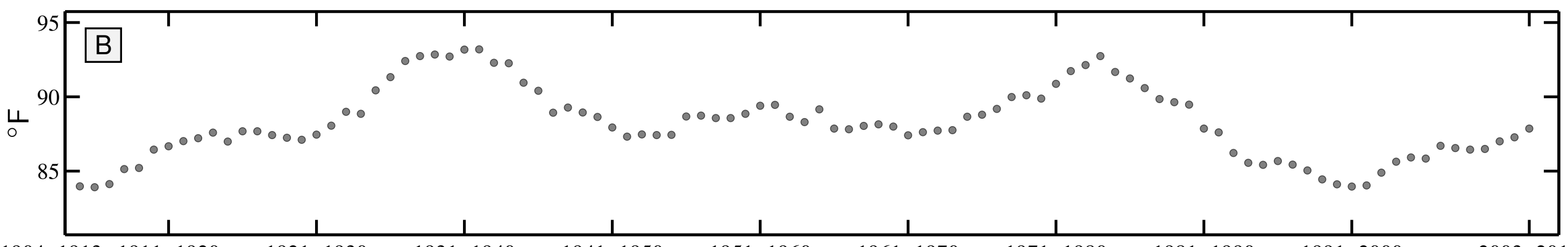

$1904-1913 \quad 1911-1920$

$1921-1930$

$1931-1940$

$1941-1950$

$1951-1960$

$1961-1970$

$1971-1980$

$1981-1990$

$1991-2000$

2003-2012

10 year Winter Teletherm dynamics for LE MARS, IA:

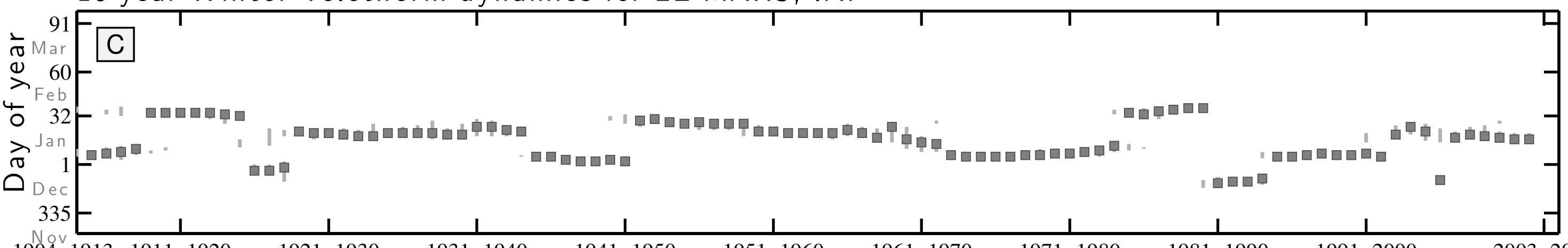

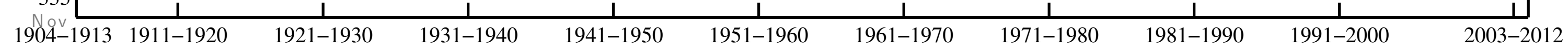

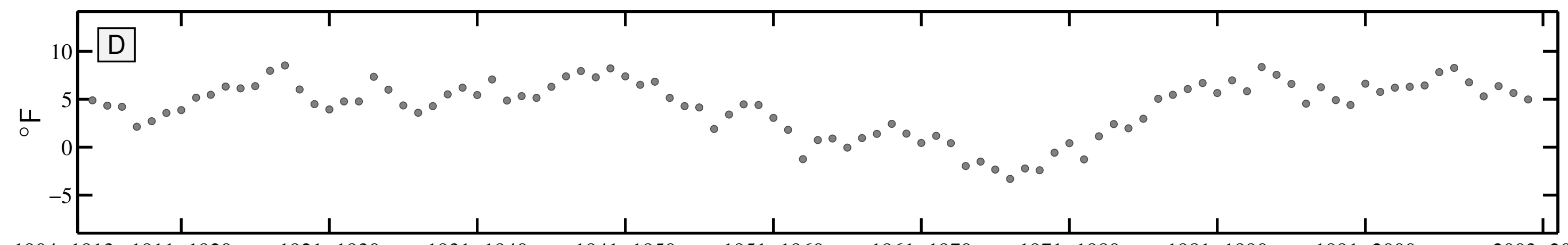


10 year Summer Teletherm dynamics for ROCKWELL CITY, IA:

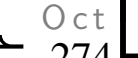

\% 274

(1) Sep

$\longrightarrow$ Aug

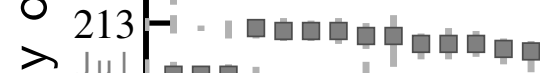

$182-1$

$152-$

May

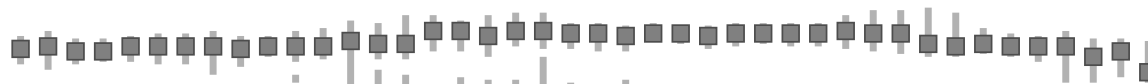
(1)

$\frac{1}{1930}$

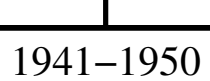

1951-1960

$\frac{1}{1961-1970}$

$\frac{1}{1971-1980}$

$\frac{1}{1981-1990}$

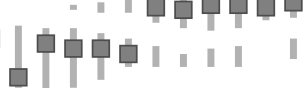

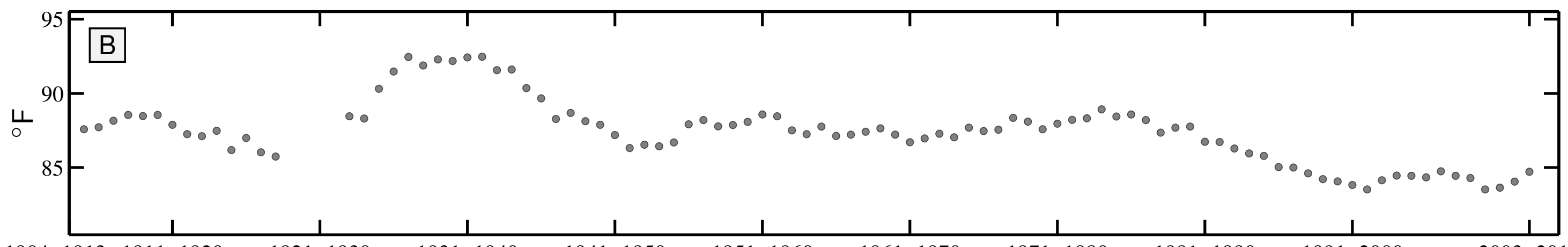

$1904-1913 \quad 1911-1920$

$1921-1930$

$1931-1940$

$1941-1950$

$1951-1960$

$1961-1970$

$1971-1980$

$1981-1990$

$1991-2000$

2003-2012

10 year Winter Teletherm dynamics for ROCKWELL CITY, IA:

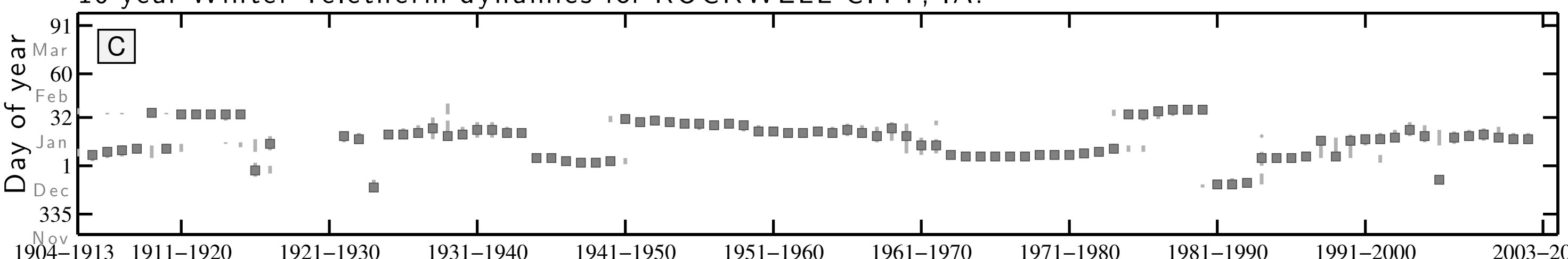

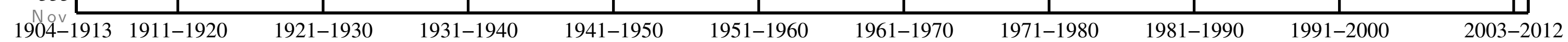

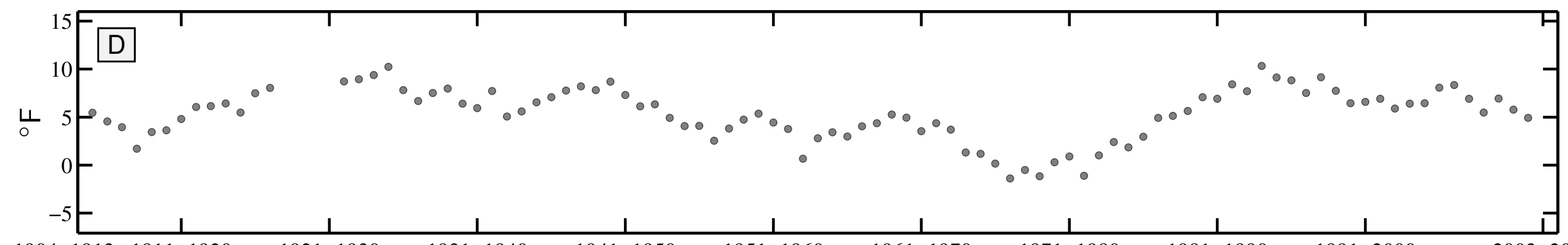


10 year Summer Teletherm dynamics for WASHINGTON, IA:

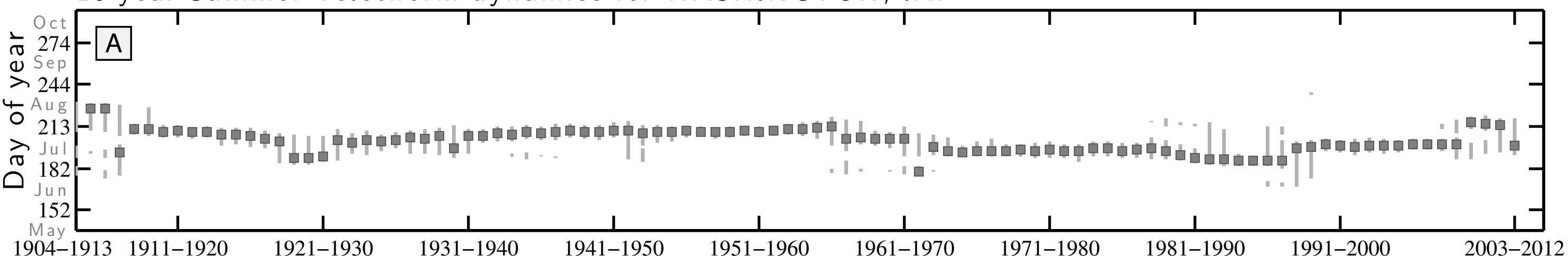

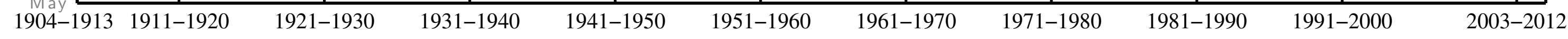

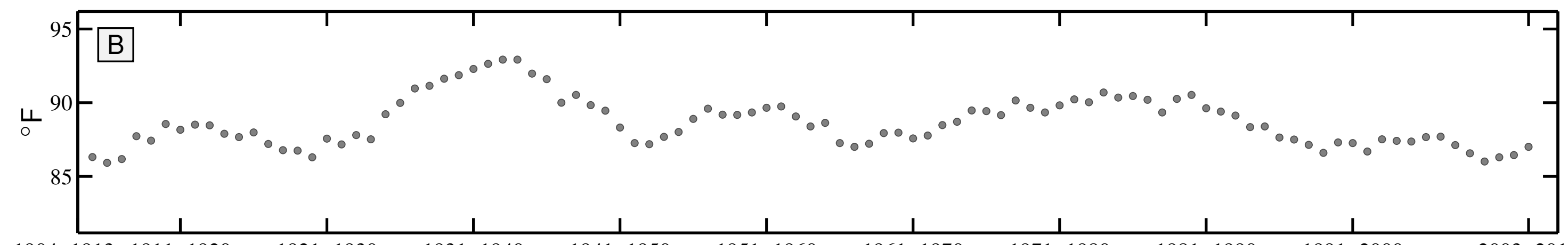

1904-1913 1911-1920

1921-1930

1931-1940

1941-1950

1951-1960

1961-1970

1971-1980

1981-1990

1991-2000

2003-2012

10 year Winter Teletherm dynamics for WASHINGTON, IA:

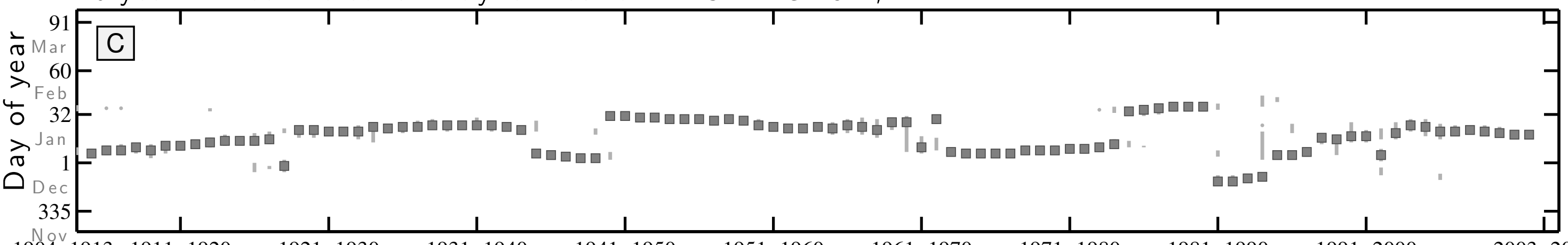

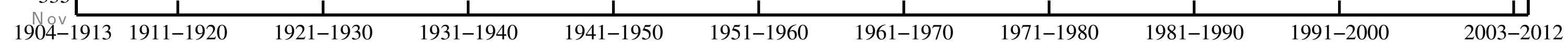

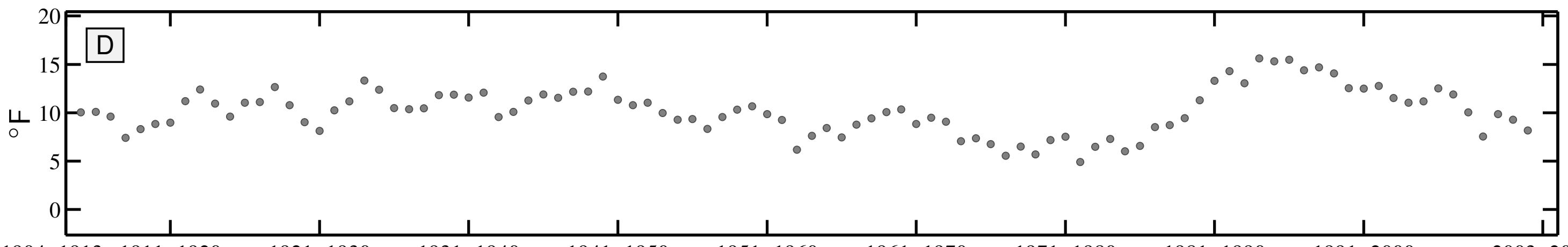


10 year Summer Teletherm dynamics for SAINT FRANCIS, KS:
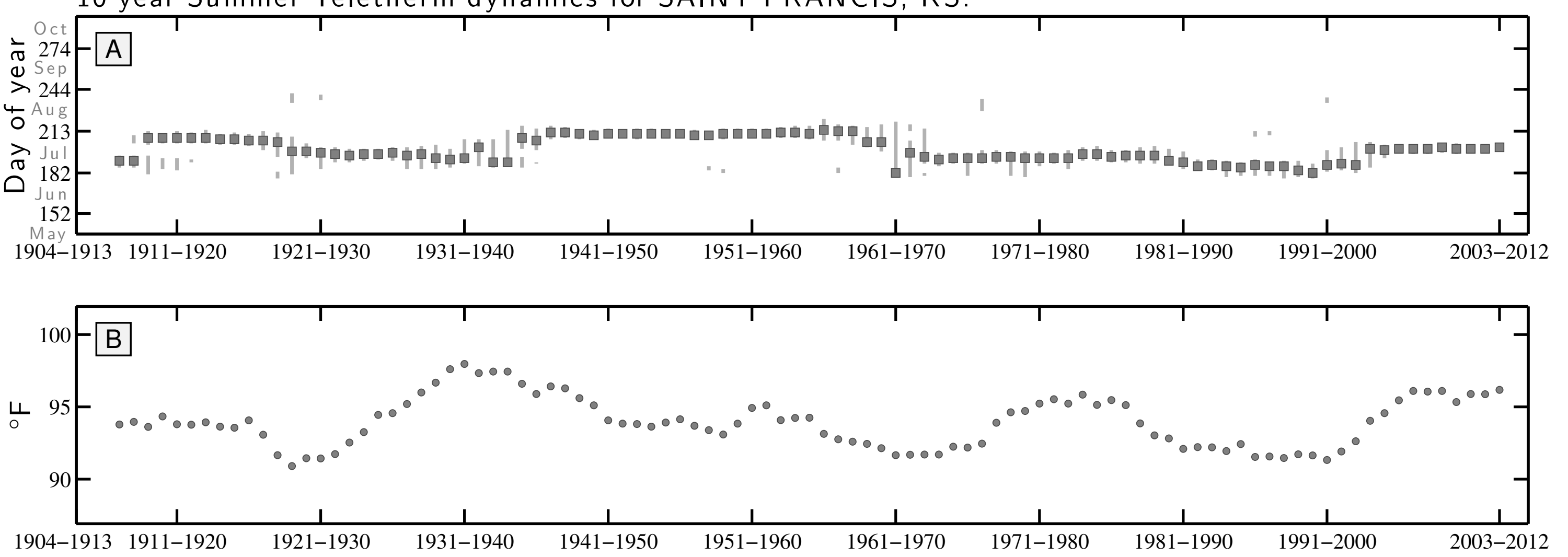

10 year Winter Teletherm dynamics for SAINT FRANCIS, KS:
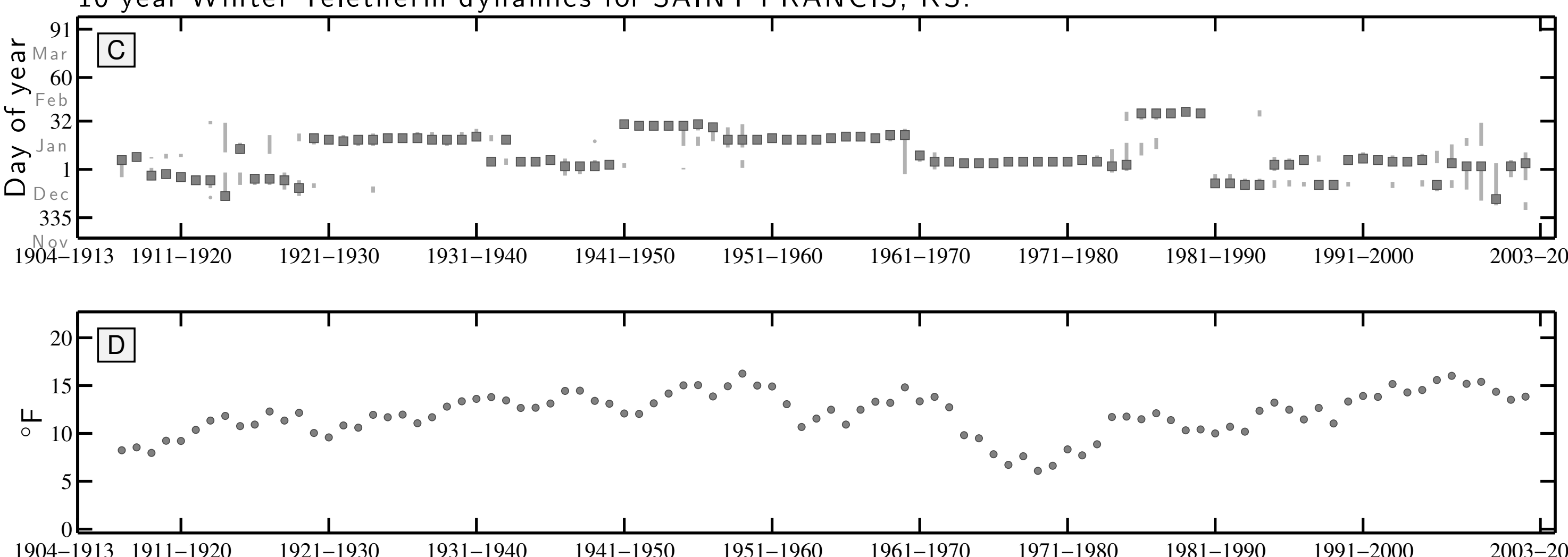

\begin{tabular}{|c|c|c|c|c|c|}
\hline $1904-1913$ 1911-1920 & $1921-1930$ & $1931-1940$ & $1941-1950$ & $1951-1960$ & $1961-1970$ \\
\hline
\end{tabular}


10 year Summer Teletherm dynamics for BEREA COLLEGE, KY:

$\div$ Oct 274

(1) Sep

$>244-$

(t)



$\mathrm{Jun}$

$152-$

草 - | I "

1

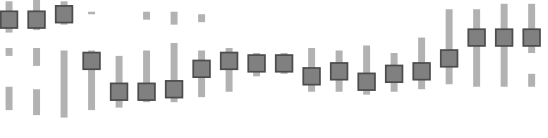

『 |

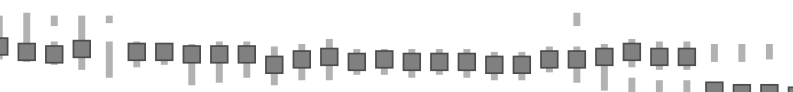

맴ㅁㅁำ

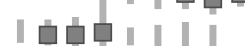

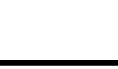

1

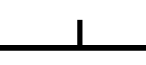

뭄|| || ||

1904-1913 1911-1920

1921-1930

1931-1940

1941-1950

1951-1960

1961-1970

$1971-1980$

$1981-1990$

1991-2000

2003-2012

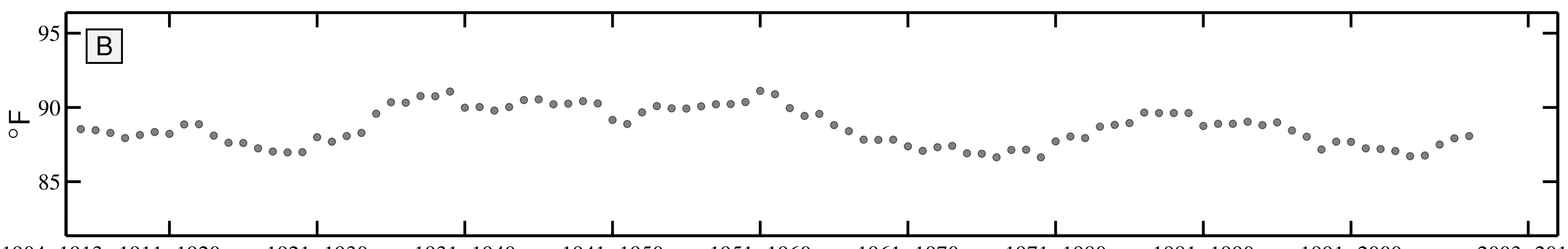

$1904-1913$ 1911-1920

$1921-1930$

$1931-1940$

$1941-1950$

$1951-1960$

$1961-1970$

$1971-1980$

$1981-1990$

$1991-2000$

$2003-2012$

10 year Winter Teletherm dynamics for BEREA COLLEGE, KY:

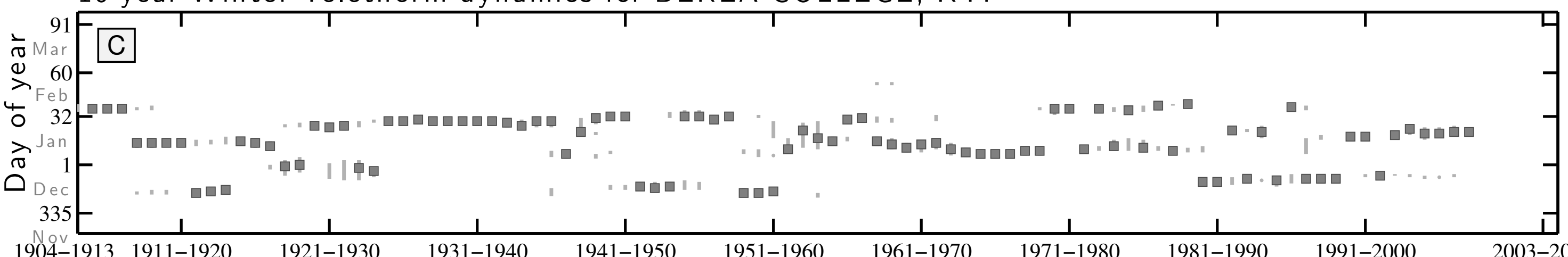

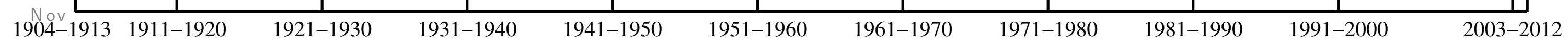

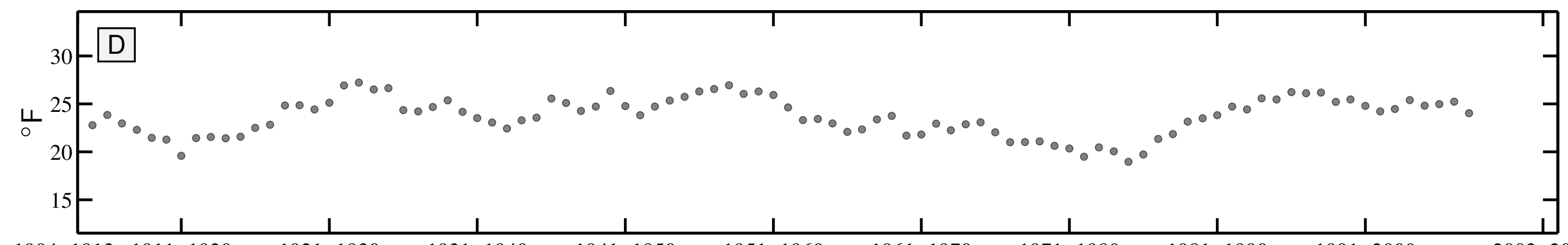

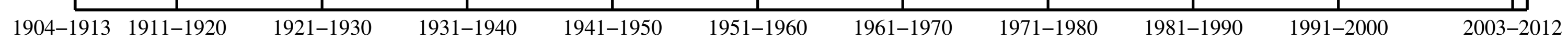


10 year Summer Teletherm dynamics for BOWLING GREEN RGNL AP, KY:

$\div \quad$ Oct 274

Sep

$\stackrel{2}{>} 244$

4 Aug $\square$ I

2 213 - 2 |

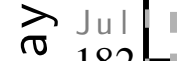

$\overbrace{}^{\circ} 182$

$152-$

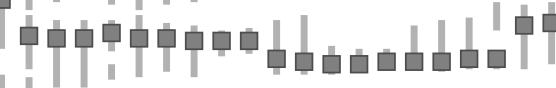

May

1904-1913 1911-1920

$\frac{1}{1921-1930}$

$1931-1940$

$\frac{1}{1941-1950}$

$\frac{1}{1951-1960}$

1961-1970

1971-1980

$\frac{1}{1981-1990}$

991-2000

2003-2012

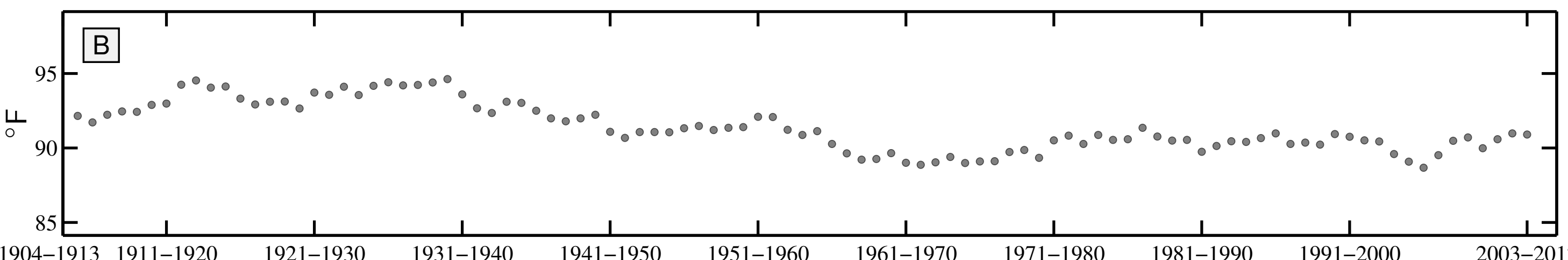

1904-1913 1911-1920

$1921-1930$

$1931-1940$

$1941-1950$

$1951-1960$

$1961-1970$

$1971-1980$

$1991-2000$

$2003-2012$

10 year Winter Teletherm dynamics for BOWLING GREEN RGNL AP, KY:

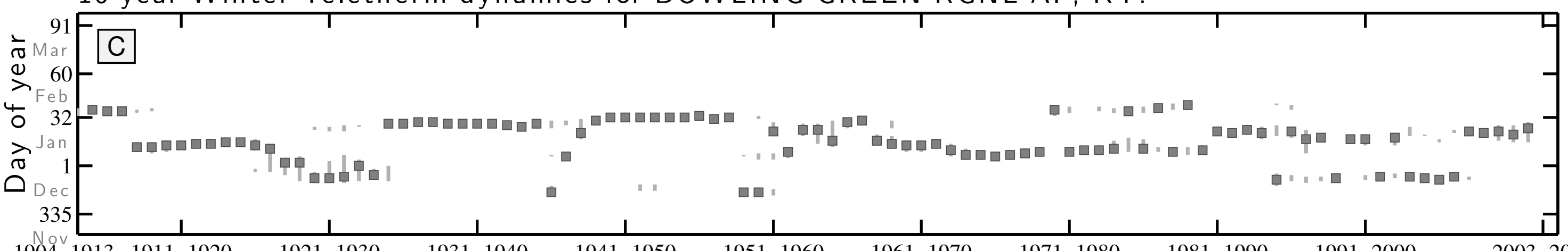

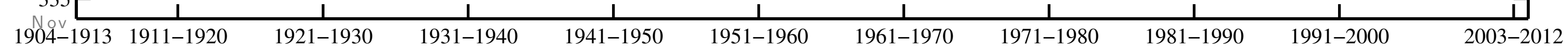

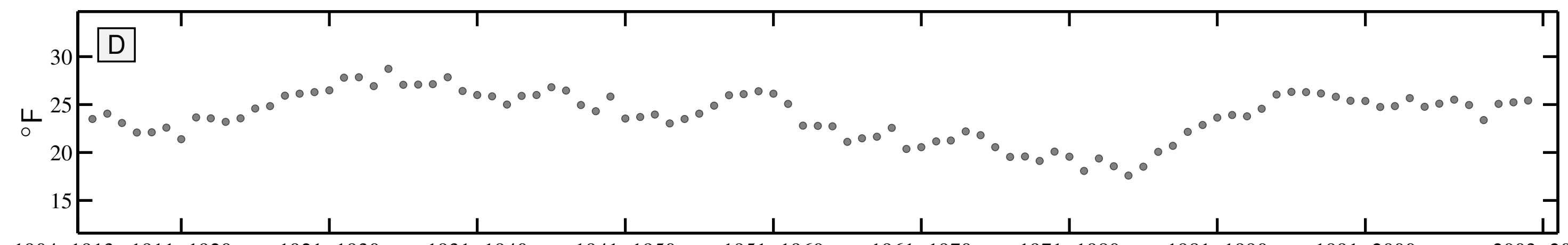


10 year Summer Teletherm dynamics for GREENSBURG, KY:

$\mathrm{Oct}$

A

(1) Sep

$>244-$

O Aug

入 Jul

$182-$

Jun
$152-$

152 ए

1904-1913 1911-1920

$1921-1930$

1931-1940

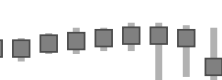

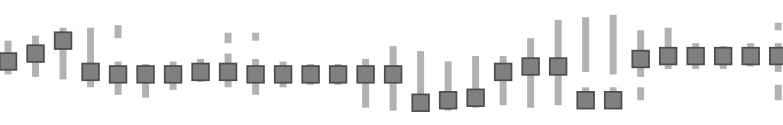

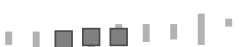

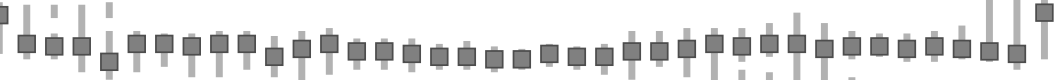

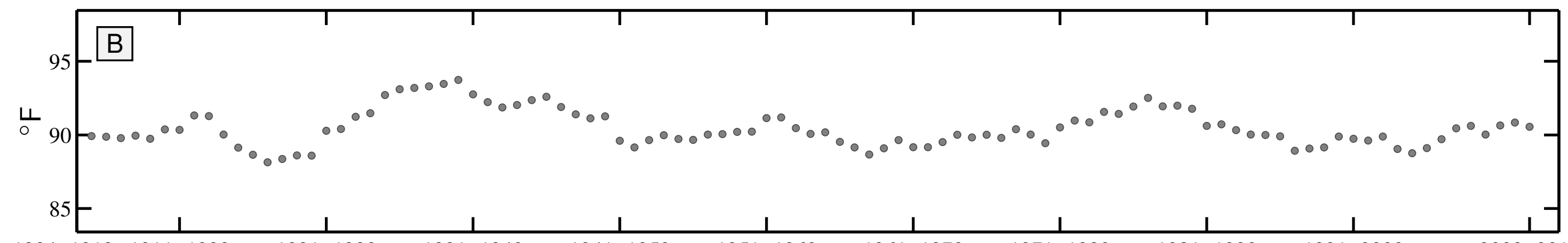

1904-1913 1911-1920

1921-1930

1931-1940

1941-1950

1951-1960

1961-1970

1971-1980

1981-1990

1991-2000

2003-2012

10 year Winter Teletherm dynamics for GREENSBURG, KY:

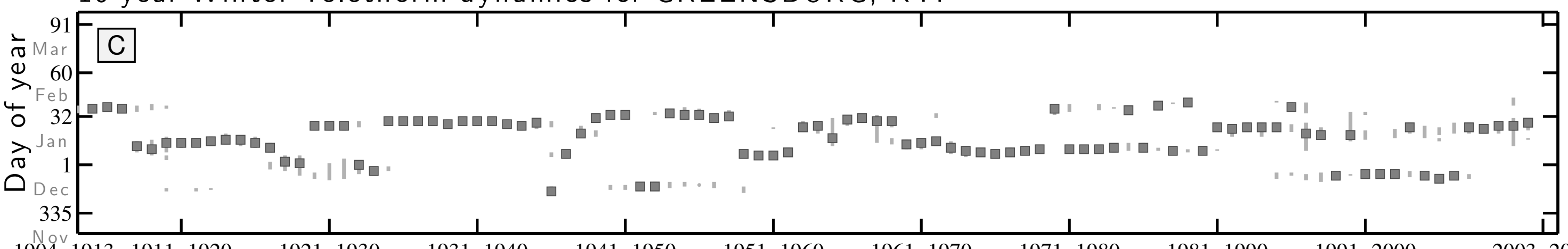

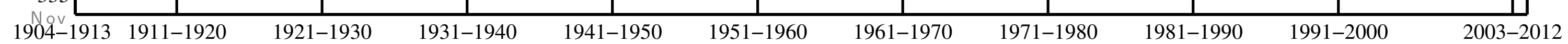

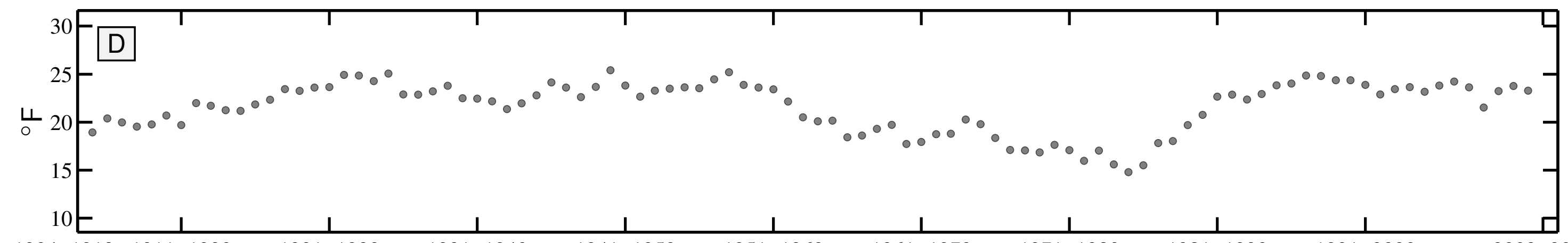


10 year Summer Teletherm dynamics for WILLIAMSBURG, KY:
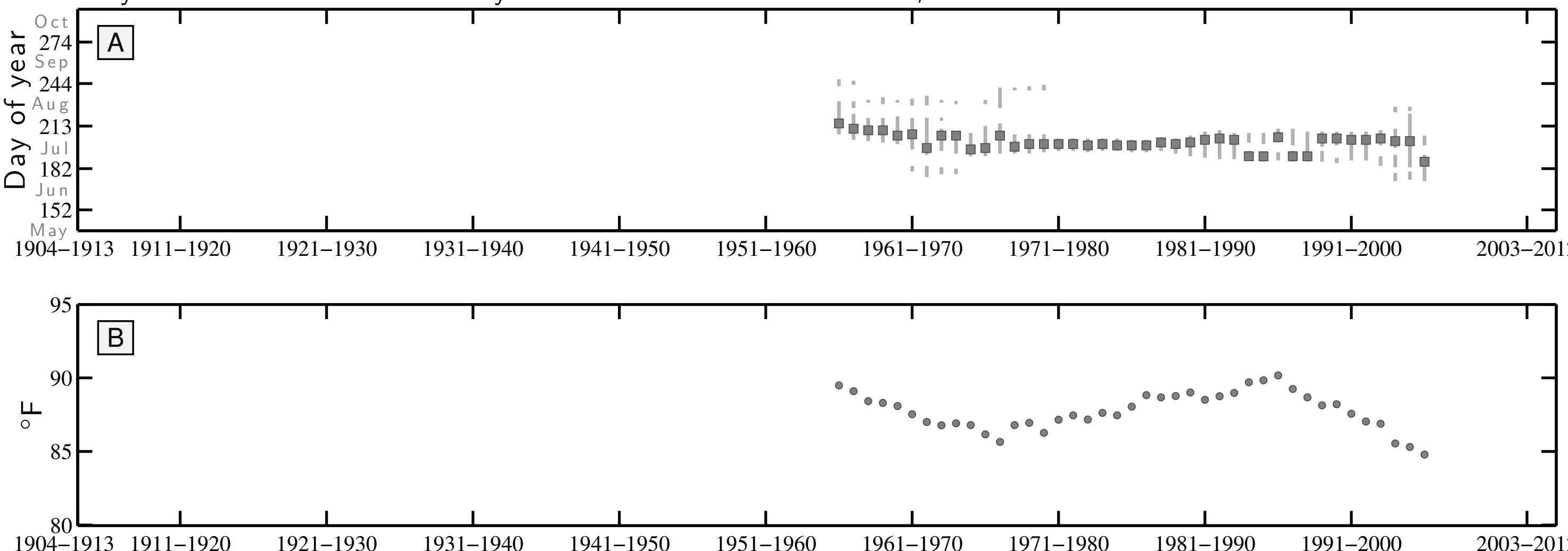

10 year Winter Teletherm dynamics for WILLIAMSBURG, KY:

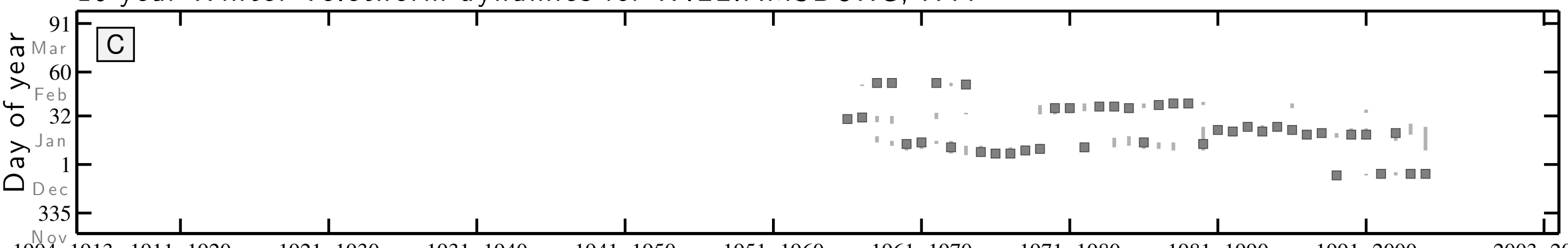

1904-1913 1911-192(

1921-1930

1931-1940

1941-1950

1951-1960

1961-1970

1971-1980

1981-1990

1991-2000

2003-2012

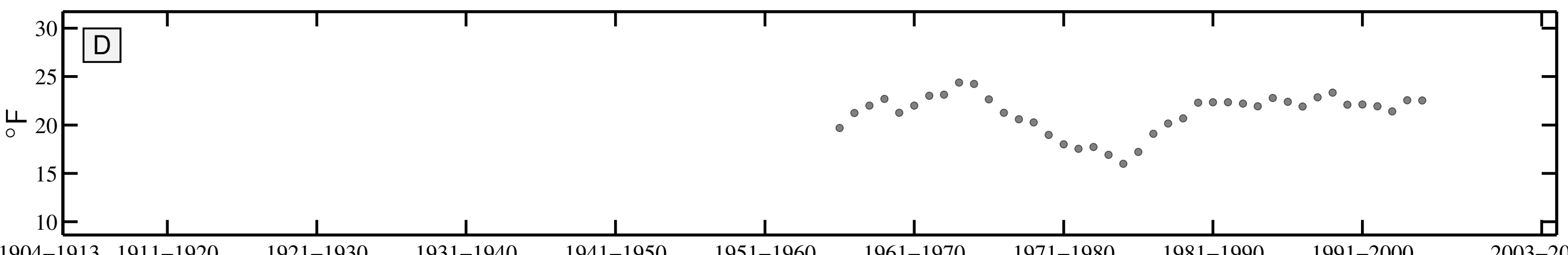


10 year Summer Teletherm dynamics for CALHOUN RSCH STN, LA:

$\div 274-A$

S Sep A

$>244-$

4 Aug

- 213

$\vec{\sigma} \mathrm{Ju}$

$182-$

152 $\square \|$

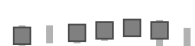

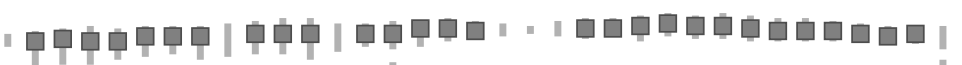

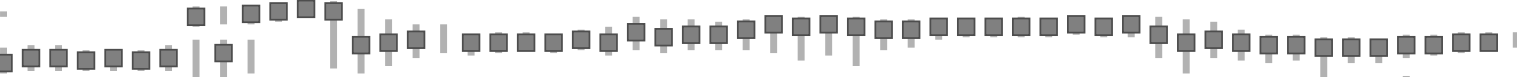

I

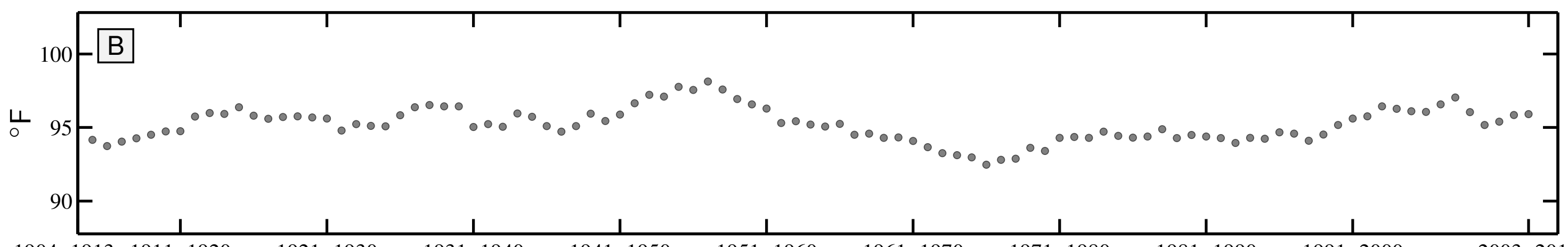

10 year Winter Teletherm dynamics for CALHOUN RSCH STN, LA:
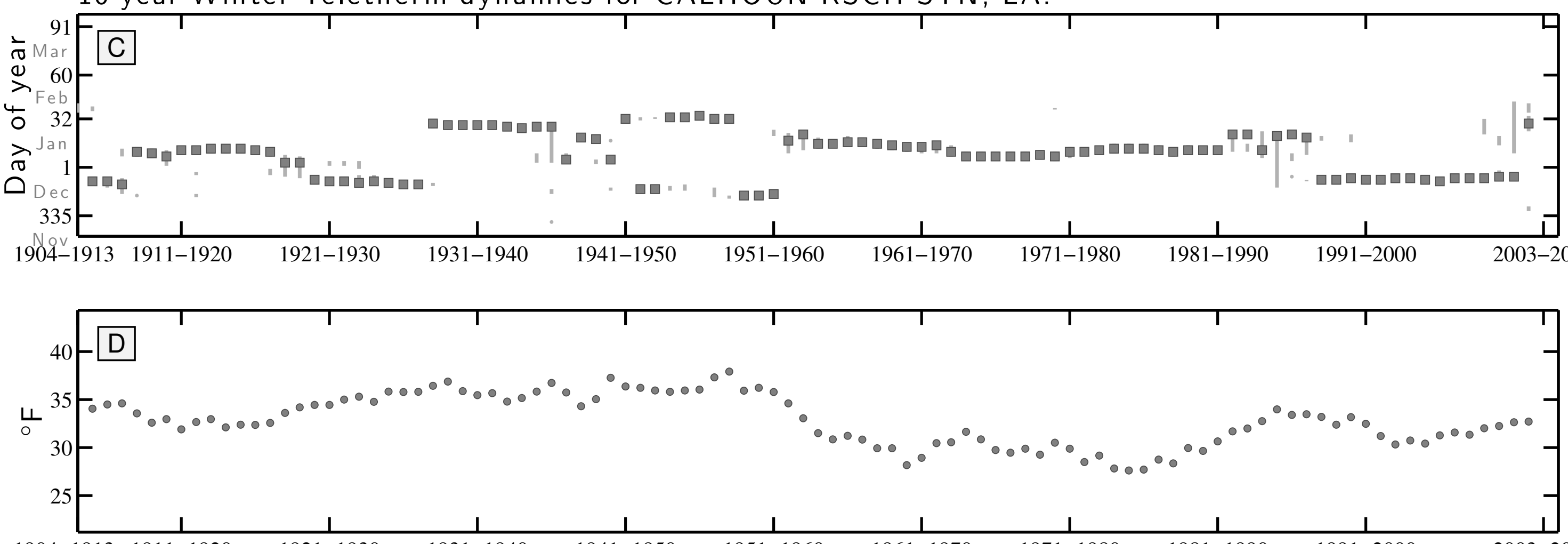
10 year Summer Teletherm dynamics for COVINGTON 4 NNW, LA:

Oct

$\stackrel{\circ}{2} 274-A$

(1) Sep A

$>244-\|$

范 Aug 213

$\rightarrow \mathrm{Jul}$

$\overbrace{}^{\circ} 182-$

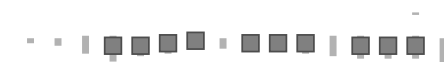

เ | | | | | |

| I |

| | | |

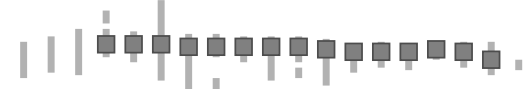

$152-$

May _ L _ _ _ _

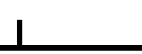

$\frac{1}{1931-1940}$

$1941-1950$

$1951-1960$

1961-1970

1971-1980

1981-1990

1991-2000

2003-201

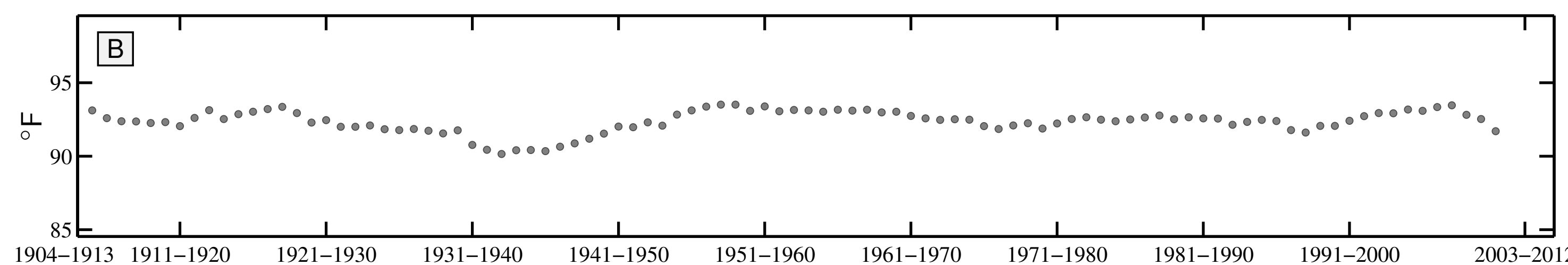

10 year Winter Teletherm dynamics for COVINGTON 4 NNW, LA:

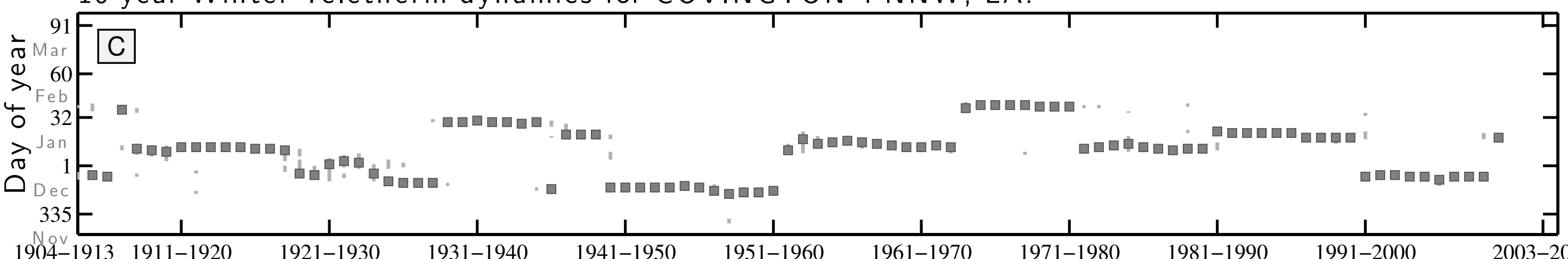

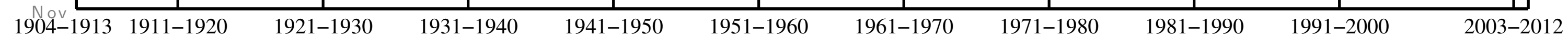

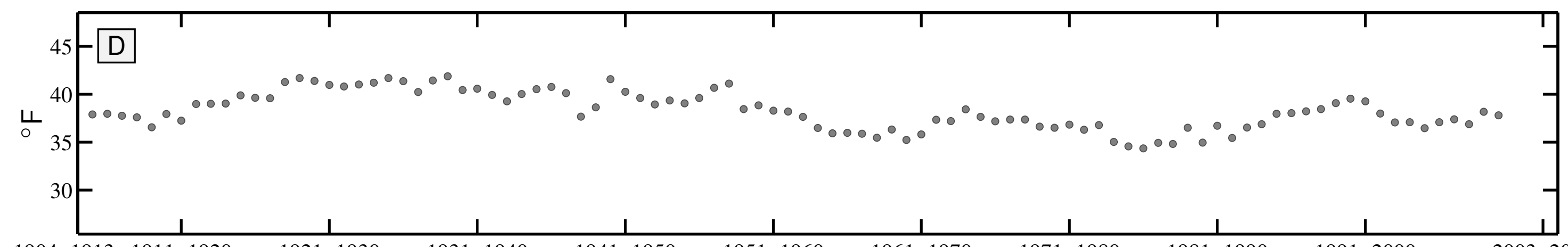


10 year Summer Teletherm dynamics for FRANKLIN 3 NW, LA:

Oct

( 274

$\stackrel{\text { U Sep }}{>} 244-\square \square \square \square$ $4 \mathrm{Aug}$ O 213 - 문

$\gtrsim \mathrm{Ju}$

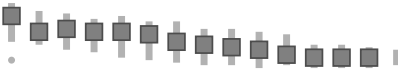

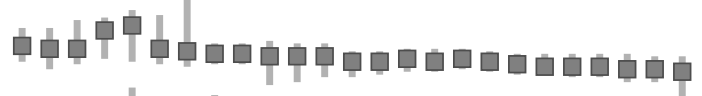

낭

$B$

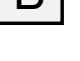

T 1 1

$\frac{1}{1941-1950}$

$1951-1960$

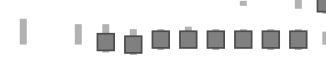

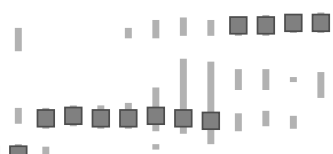

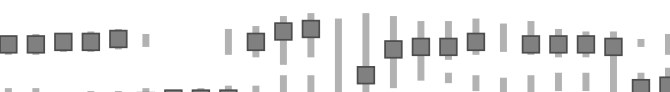

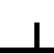

10 year Winter Teletherm dynamics for FRANKLIN 3 NW, LA:
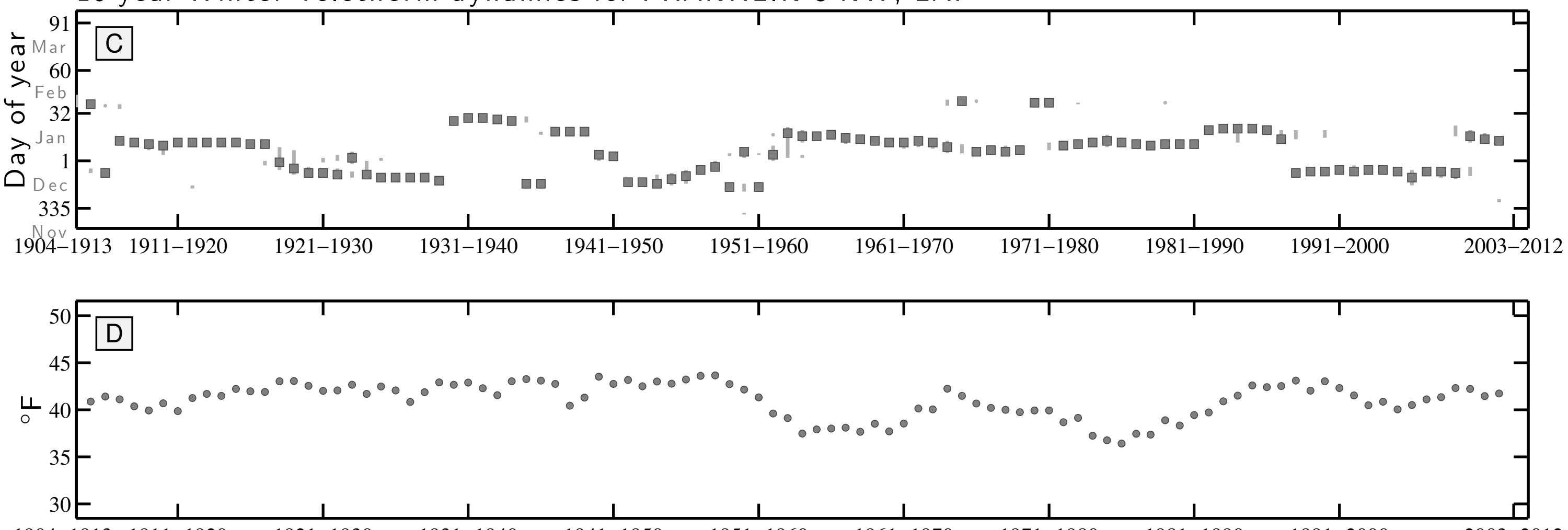
10 year Summer Teletherm dynamics for JENNINGS, LA:

274

(1)

$>244-\square \square \square$ 4 Aug

1 由
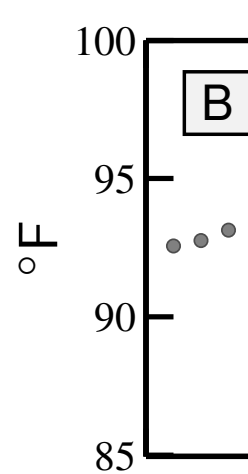

1904-1913 1911-1920

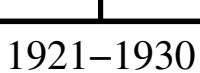

$1931-1940$

1941-1950

1951-1960

1961-1970

1971-1980

1981-1990

1991-2000

2003-2012

10 year Winter Teletherm dynamics for JENNINGS, LA:

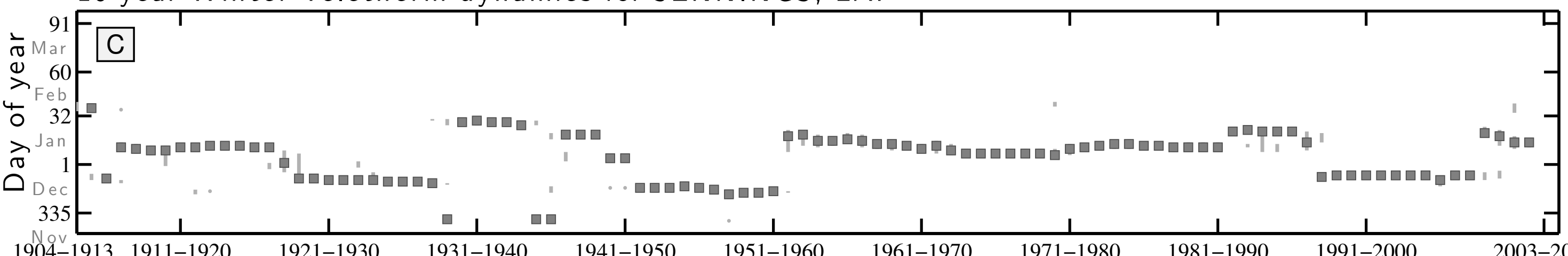

1904-1913 1911-1920

1921-1930

1931-1940

1941-1950

1951-1960

1961-1970

1971-1980

1981-1990

2003-2012

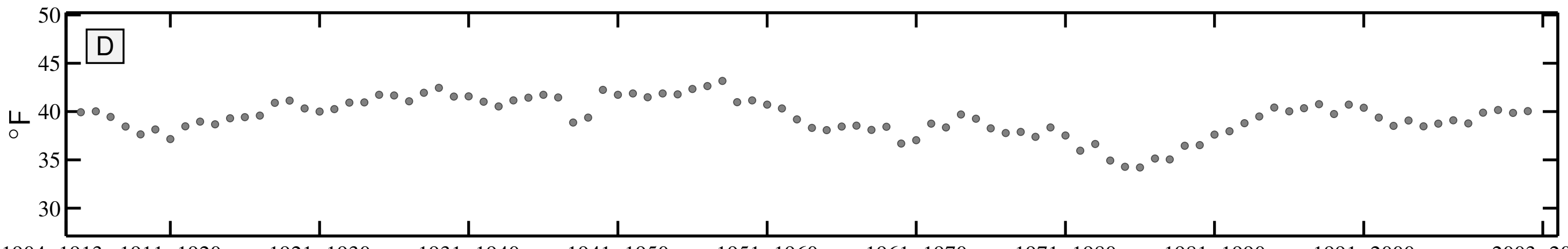


10 year Summer Teletherm dynamics for LAFAYETTE FCWOS, LA:

$\stackrel{\circ}{2} 274-\bar{A}$

o Sep

$>244-$

它 Aug

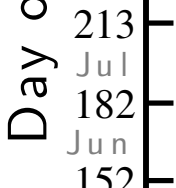

$152-$

1904-1913 1911-1920

$1921-1930$

|l| 1 ing

. | | | | | | | | |

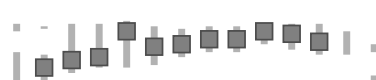

| || || || | -

中吊

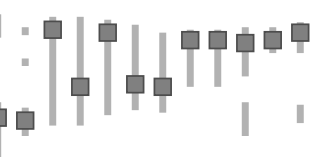

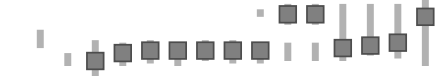

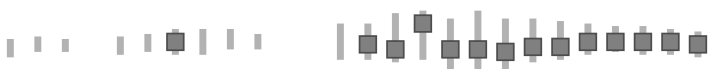

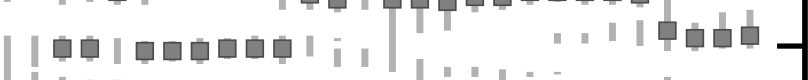

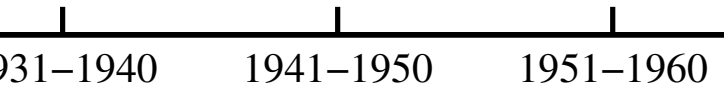

1961-1970

1971-1980

1981-1990

991-2000

2003-2012

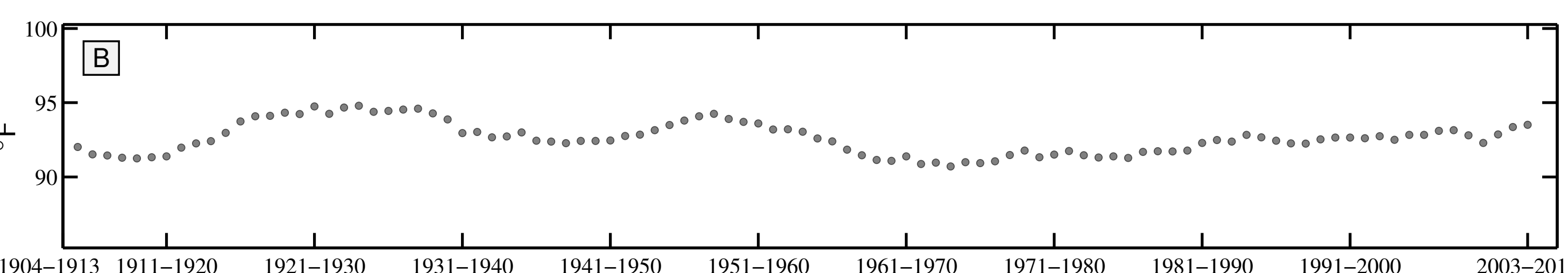

1904-1913 1911-1920

$1921-1930$

$1931-1940$

$1941-1950$

$1951-1960$

$1961-1970$

$1971-1980$

$1981-1990$

$1991-2000$

2003-2012

10 year Winter Teletherm dynamics for LAFAYETTE FCWOS, LA:

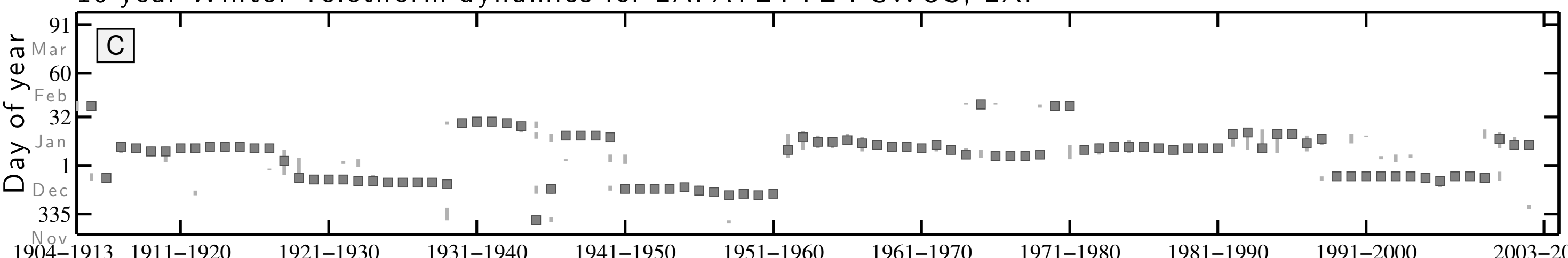

1904-1913 1911-1920

$1921-1930$

1931-1940

1941-1950

1951-1960

1961-1970

1971-1980

1981-1990

1991-2000

003-2012

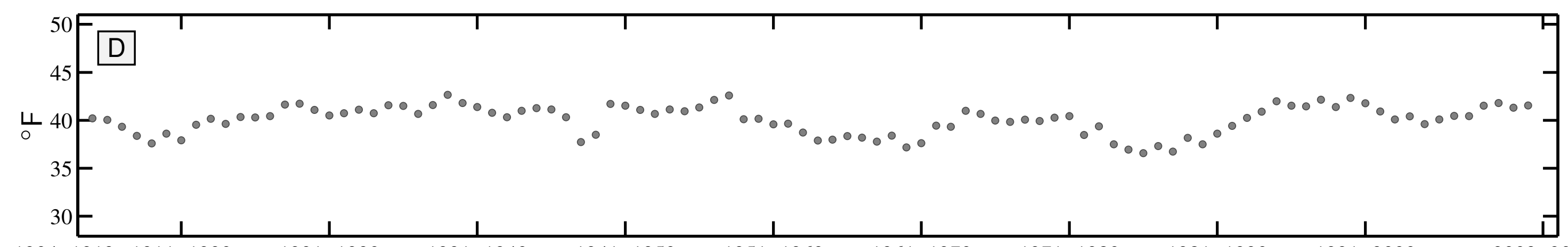


10 year Summer Teletherm dynamics for NEW ORLEANS AUDUBON, LA:

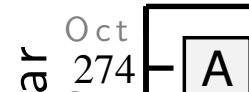

$>244-$

ᄂ Aug

○ $213-$

त $\mathrm{Ju}$

$\overbrace{}^{\circ} 182-$

$152-$

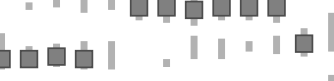

|| || || || ||

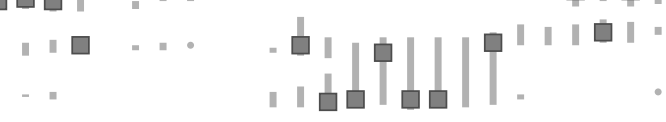

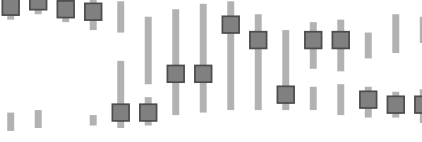

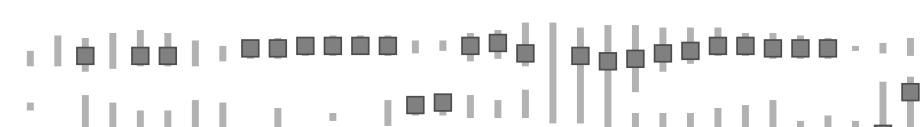

$\frac{1}{1-1920}$

$\frac{1}{1921-1930}$

$\frac{1}{1931-1940}$

$\frac{1}{1941-1950}$

$\frac{1}{1951-1960}$

$1961-1970$

$1971-1980$

$\frac{1}{981-1990}$

$991-2000$

2003-201

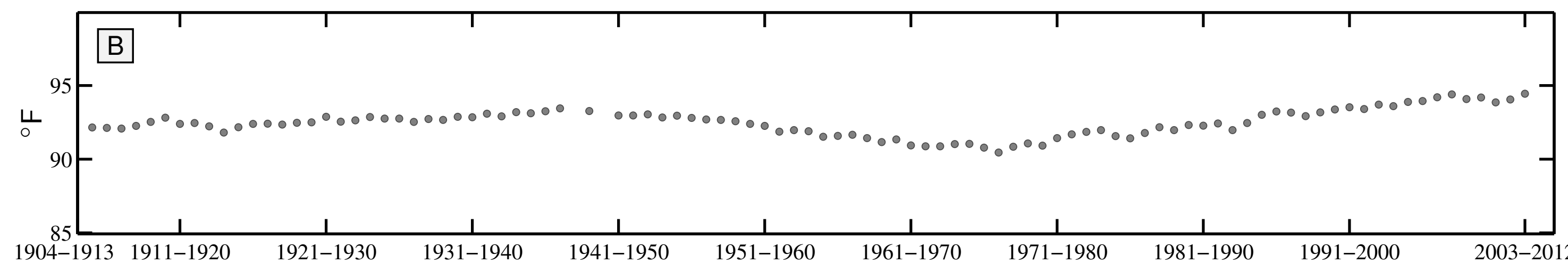

10 year Winter Teletherm dynamics for NEW ORLEANS AUDUBON, LA:

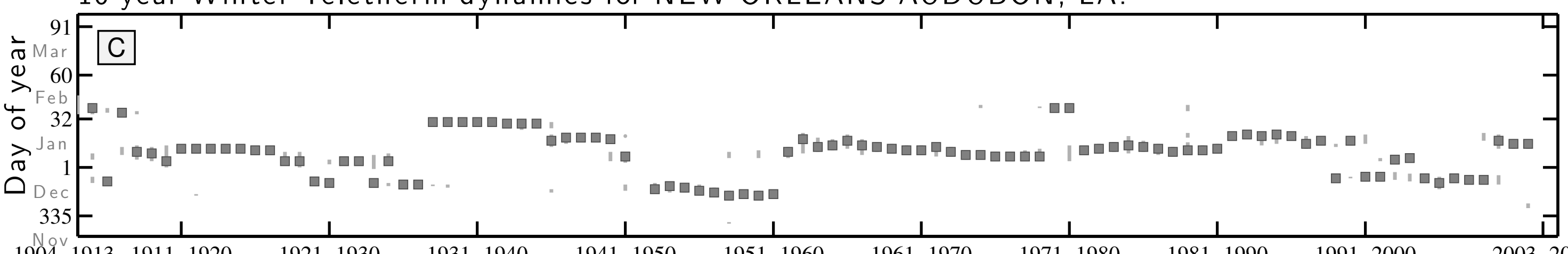

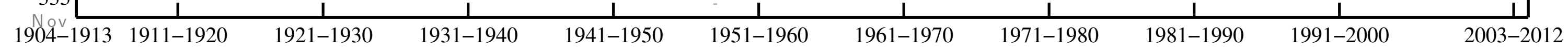

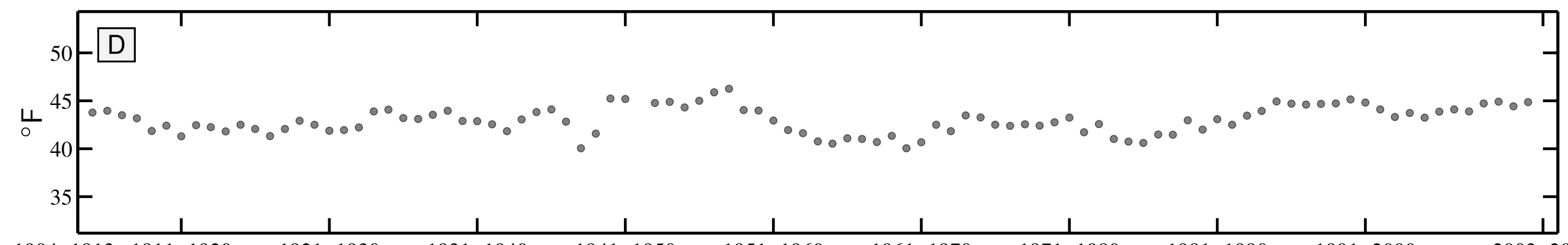


10 year Summer Teletherm dynamics for FARMINGTON, ME:

$\div$ Oct 274

(s Sep

$>244-$

4 A g g

$213-$

त 182

Jun
$152-$

1904-1913 1911-1920

1921-1930

$\frac{1}{1931-1940}$

$\frac{1}{1941-1950}$

1951-1960

1961-1970

$\frac{1}{1971-1980}$

$\frac{1}{1981-1990}$

1991-2000

2003-2012

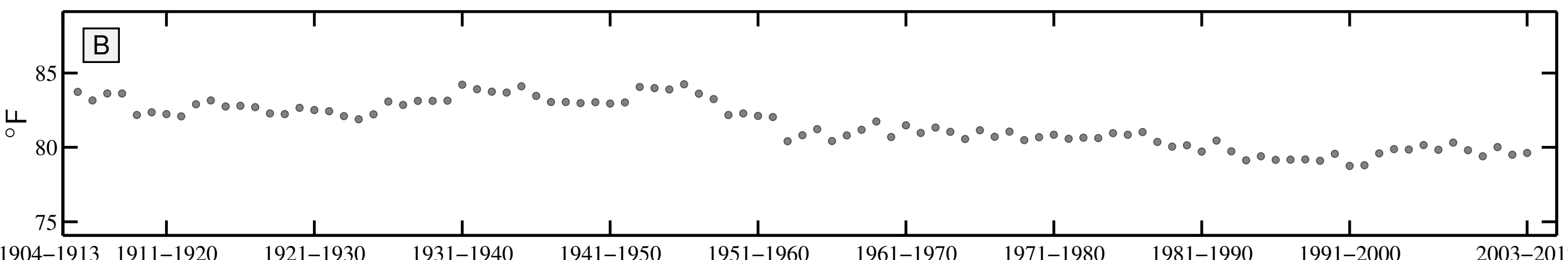

1904-1913 1911-1920

1921-1930

1931-1940

1941-1950

1951-1960

$1961-1970$

1971-1980

1981-1990

$1991-2000$

$2003-2012$

10 year Winter Teletherm dynamics for FARMINGTON, ME:

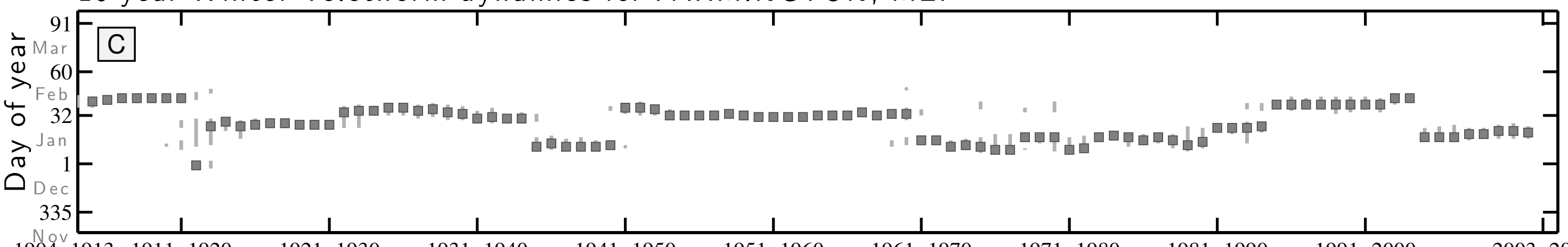

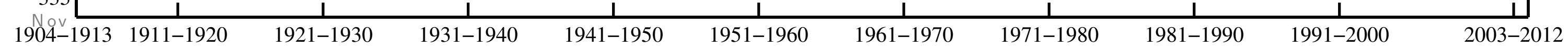

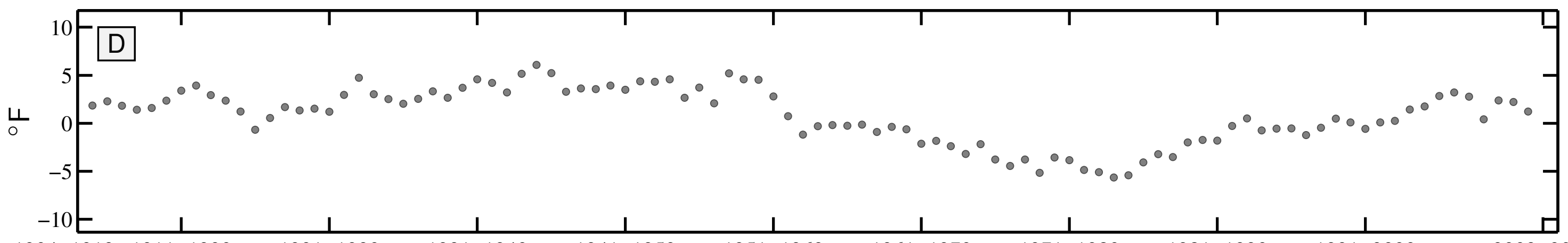


10 year Summer Teletherm dynamics for GARDINER, ME:

$\perp$ Oct

б $274-A$

(1) Sep

$>244$

${ }^{4} \mathrm{Aug}$

$213-$

$\gtrsim_{0} \mathrm{Jul}$

182

$152-$

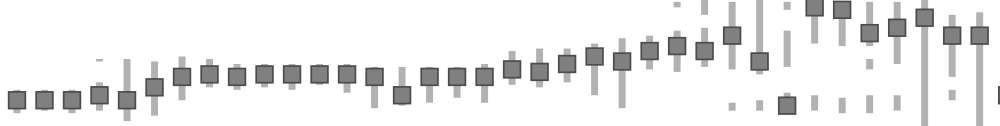

1904-1913 1911-1920

$1921-1930$

$\frac{1}{1931-1940}$

$1941-1950$

$1951-1960$

961-1970

$\frac{1}{971-1980}$

1981-1990

991-2000

2003-2012

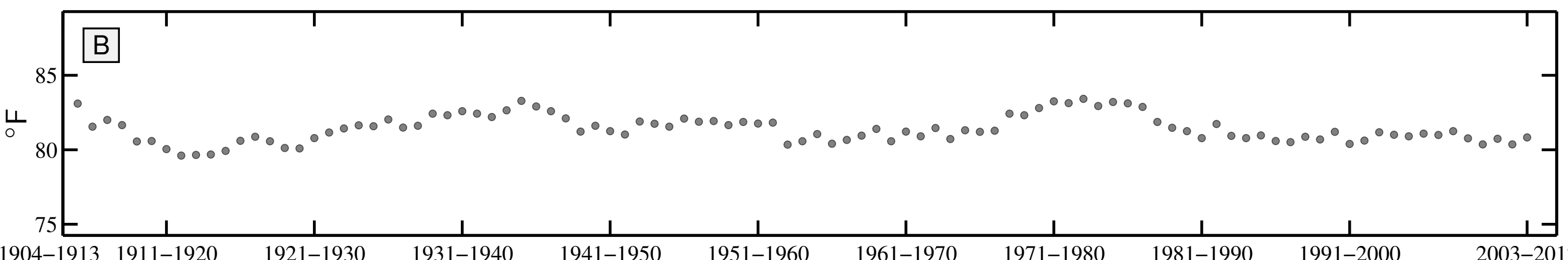

1904-1913 1911-1920

$1921-1930$

$1931-1940$

$1941-1950$

$1951-1960$

$1961-1970$

$1971-1980$

$1981-1990$

$1991-2000$

2003-2012

10 year Winter Teletherm dynamics for GARDINER, ME:
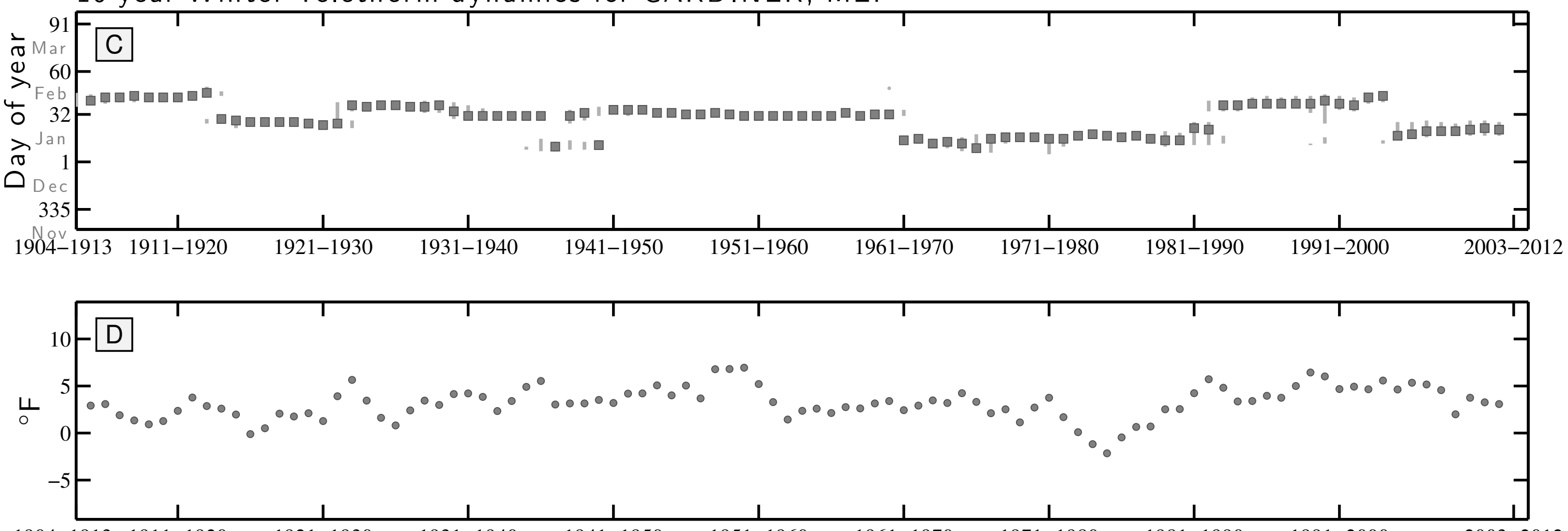
10 year Summer Teletherm dynamics for HOULTON 5N, ME:
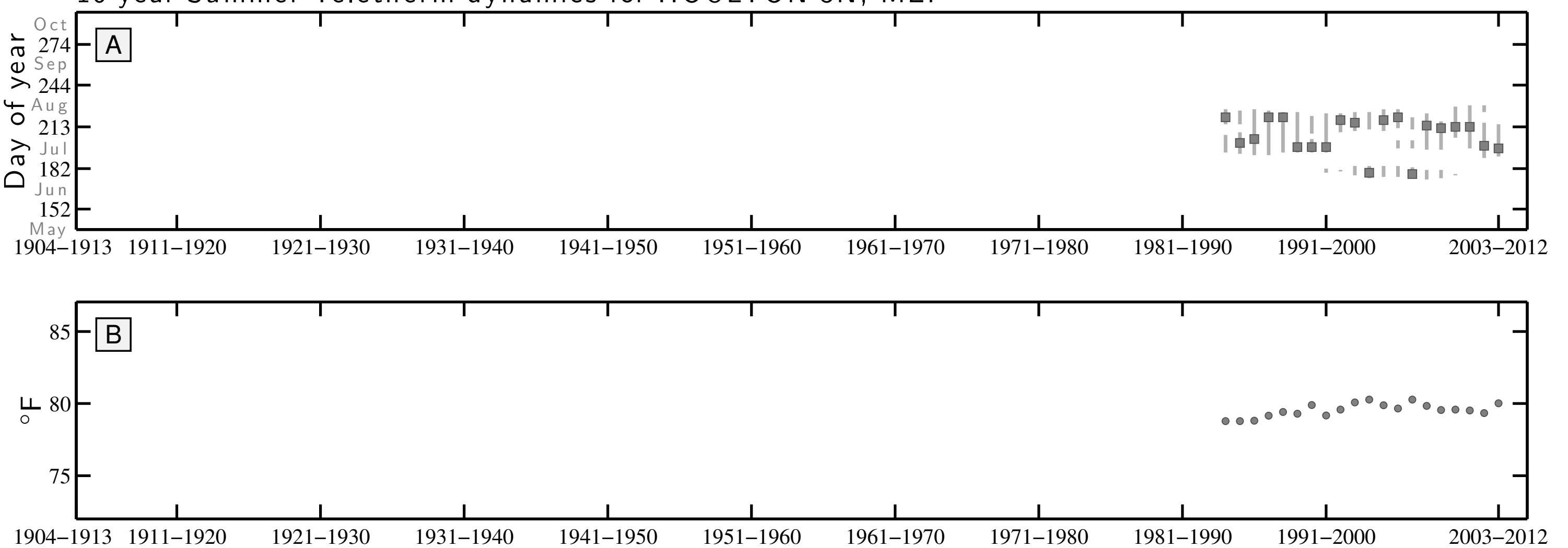

10 year Winter Teletherm dynamics for HOULTON 5N, ME:
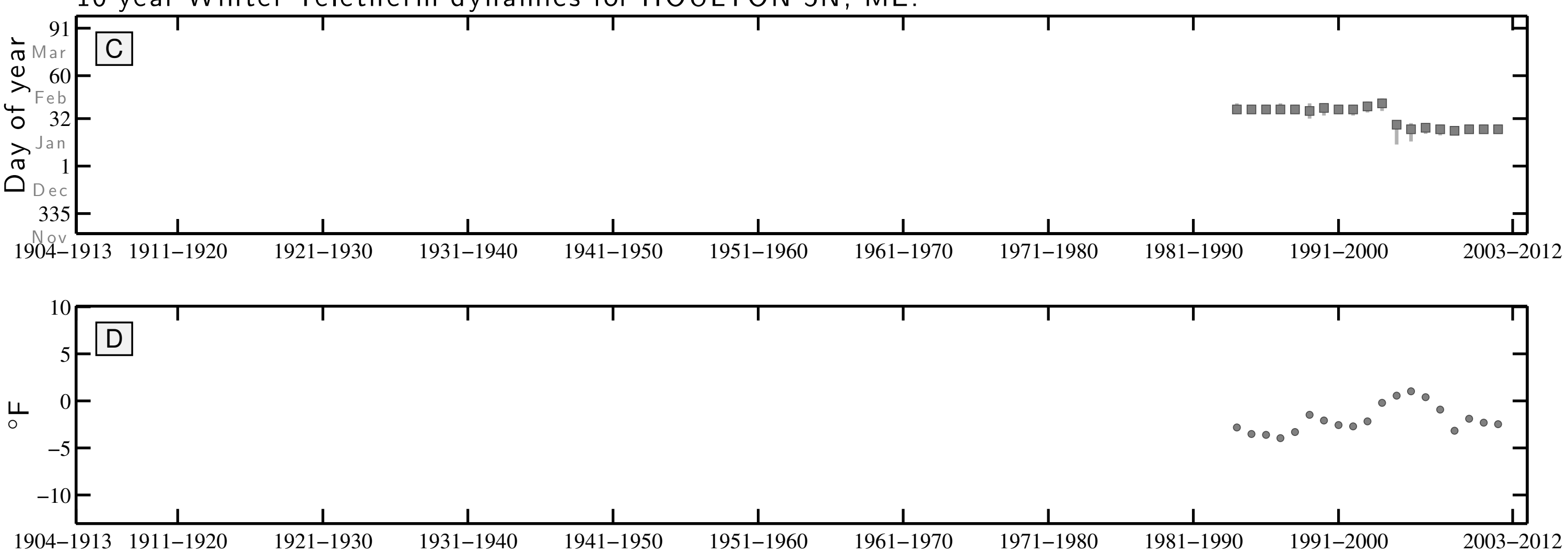
10 year Summer Teletherm dynamics for CUMBERLAND 2, MD:
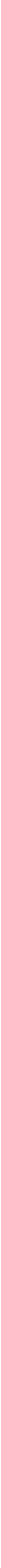
10 year Summer Teletherm dynamics for DENTON 2 E, MD:
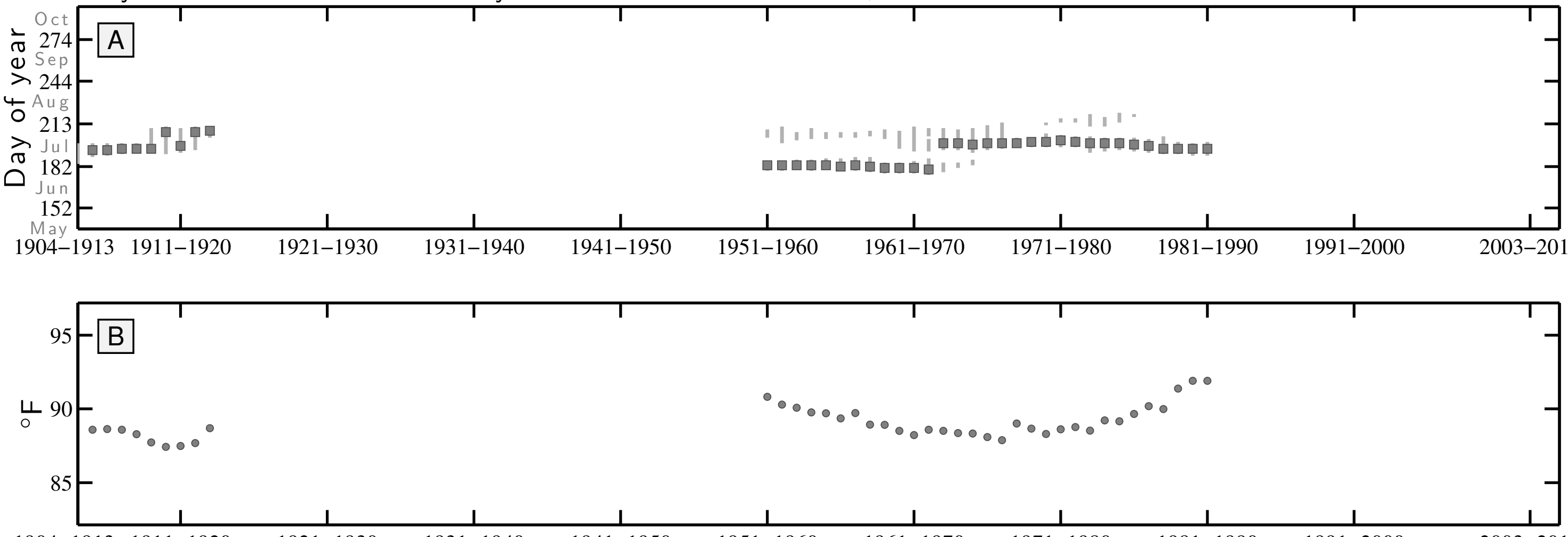

1904-1913 1911-1920

10 year Winter Teletherm dynamics for DENTON 2 E, MD:
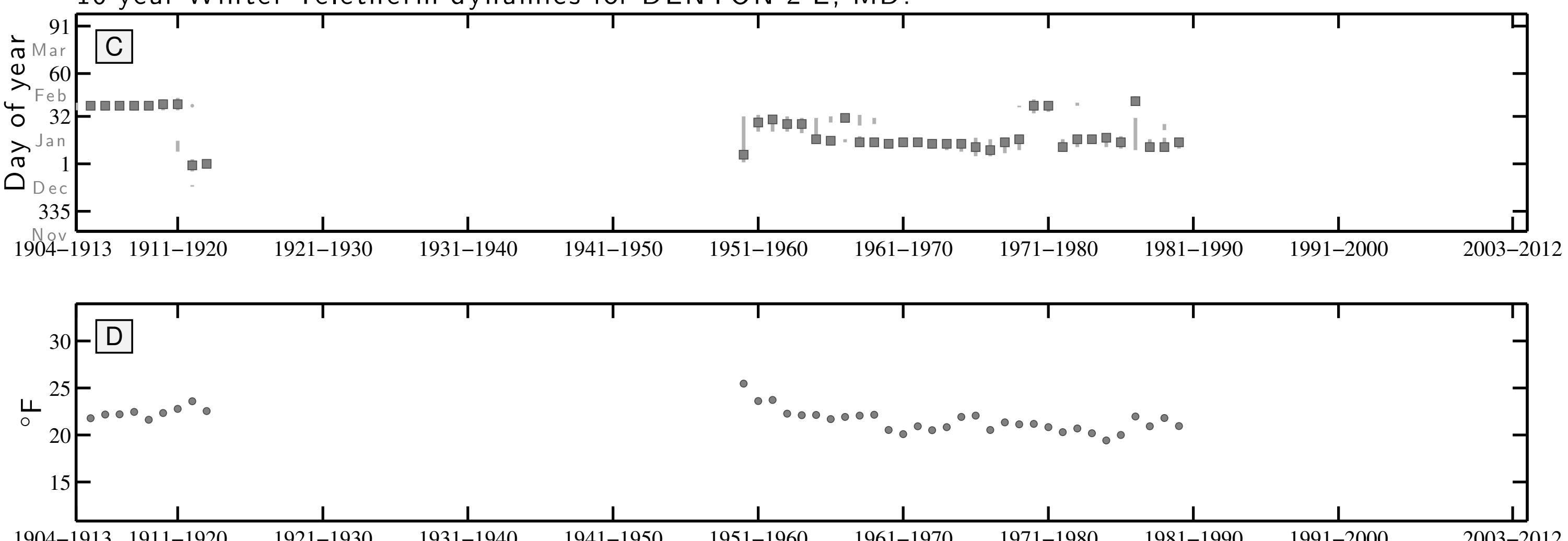
10 year Summer Teletherm dynamics for MD SCI CTR BALTIMORE, MD:
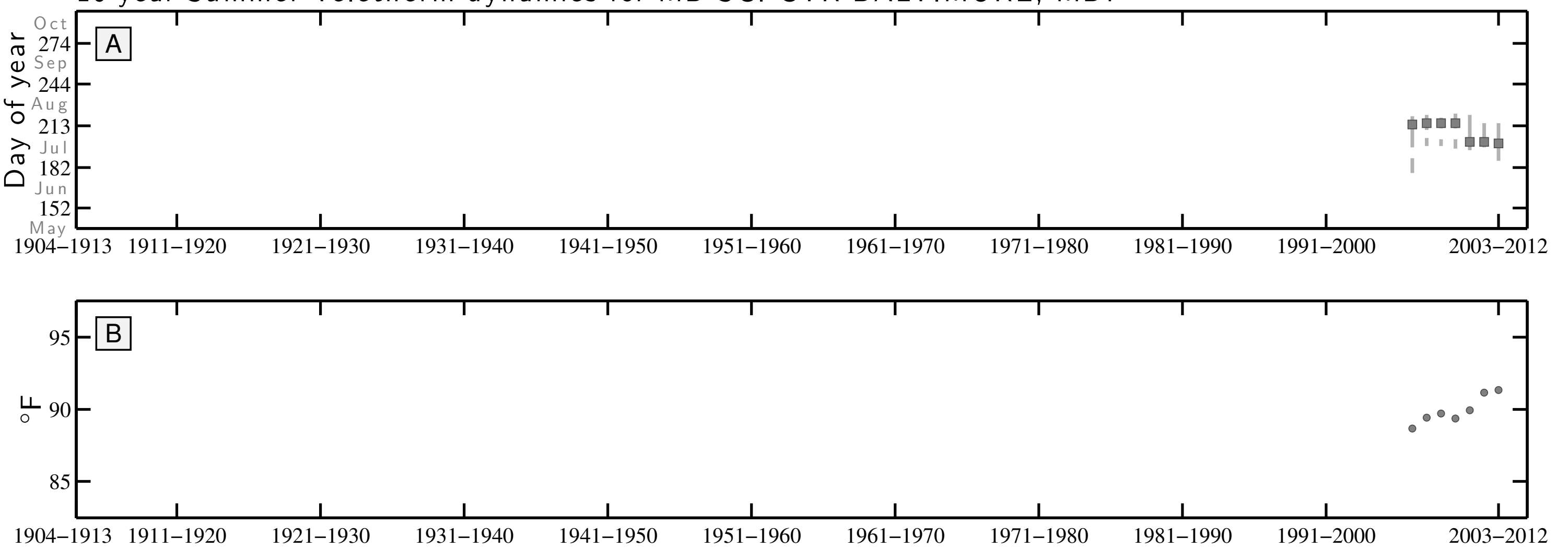

10 year Winter Teletherm dynamics for MD SCI CTR BALTIMORE, MD:
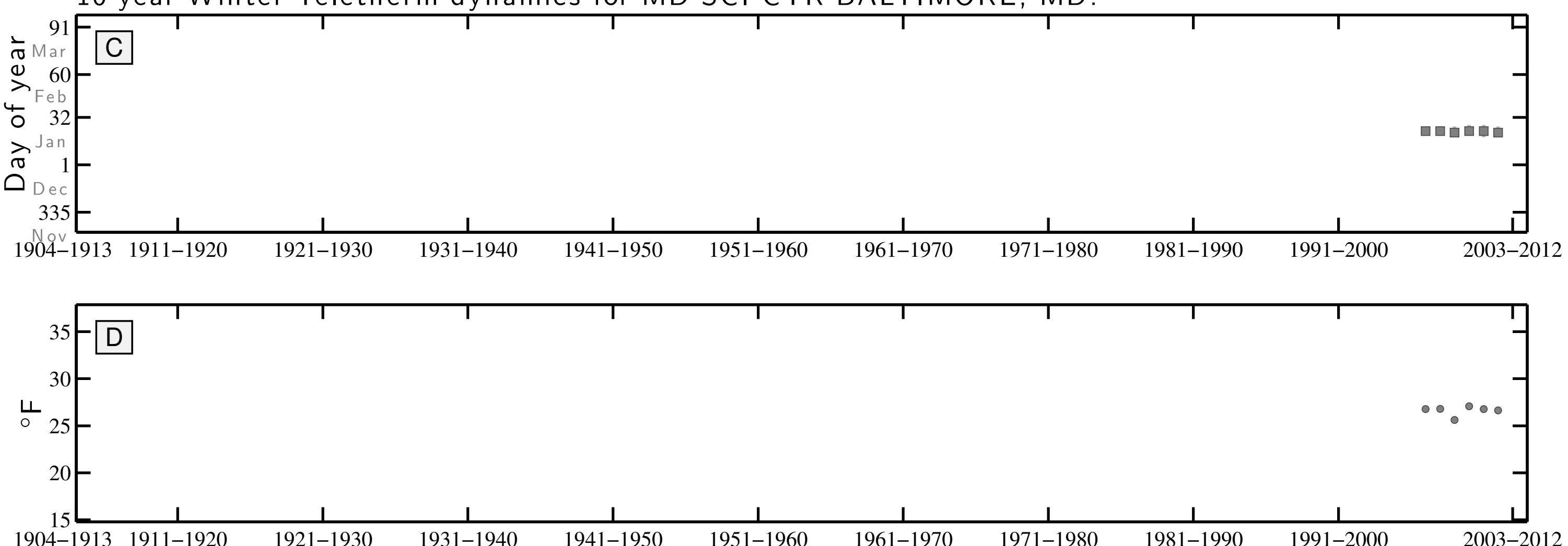
10 year Summer Teletherm dynamics for OAKLAND 1 SE, MD:

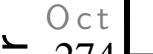

A

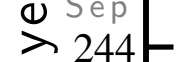

4 Aug

O $213-$

त Jul

$182-$

$152-$

152

|

|| || || | | -
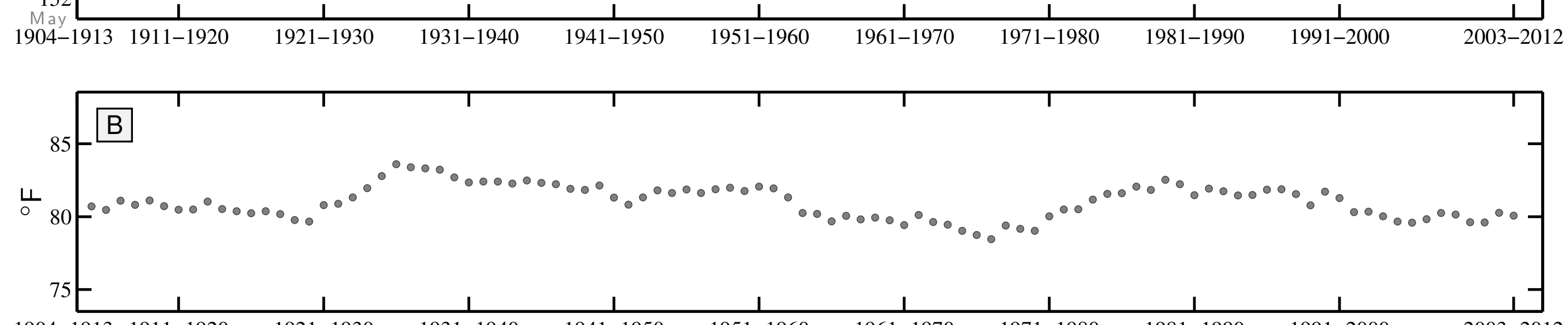

1904-1913 1911-1920

1921-1930

1931-1940

$1941-1950$

1951-1960

1961-1970

1971-1980

1981-1990

$1991-2000$

$2003-2012$

10 year Winter Teletherm dynamics for OAKLAND 1 SE, MD:

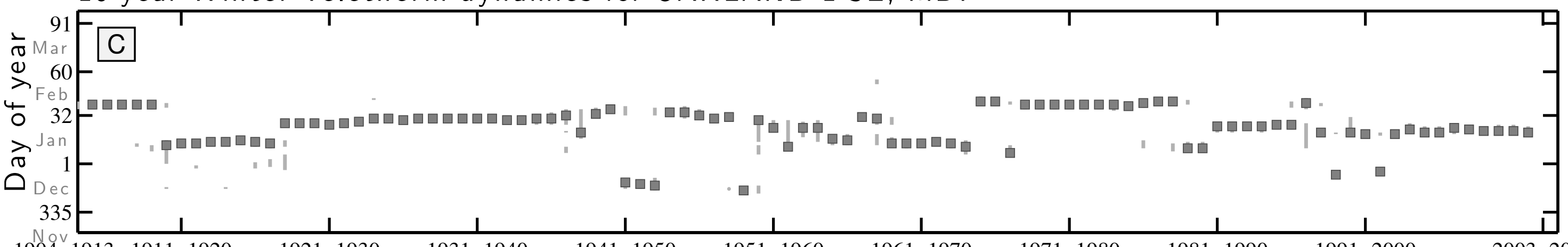

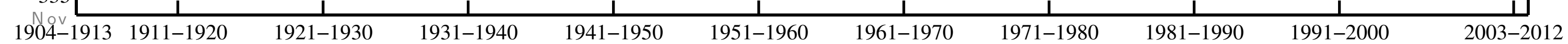

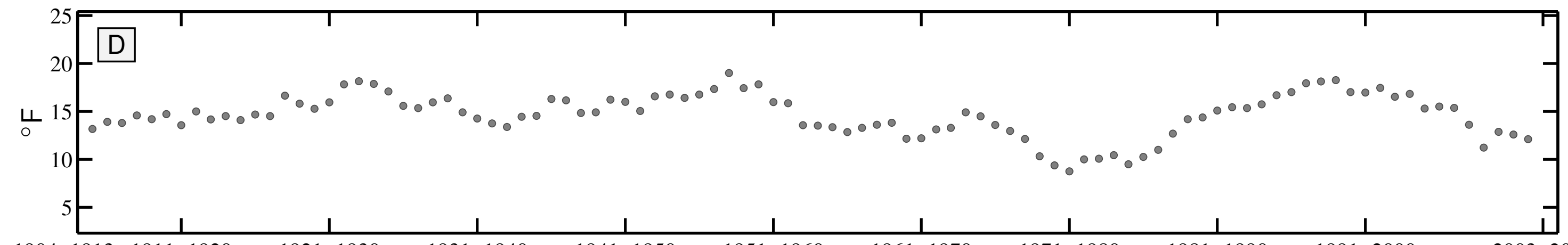


10 year Summer Teletherm dynamics for OWINGS FERRY LANDING, MD:

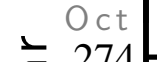

$274-A$

(1) Sep

$>244$

응

213
$\mathrm{Jul}$

\% 182

Jun
152

152

1904-1913 _ _

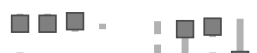

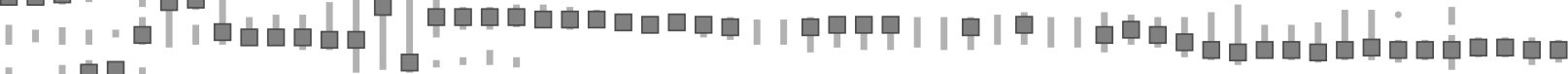

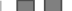
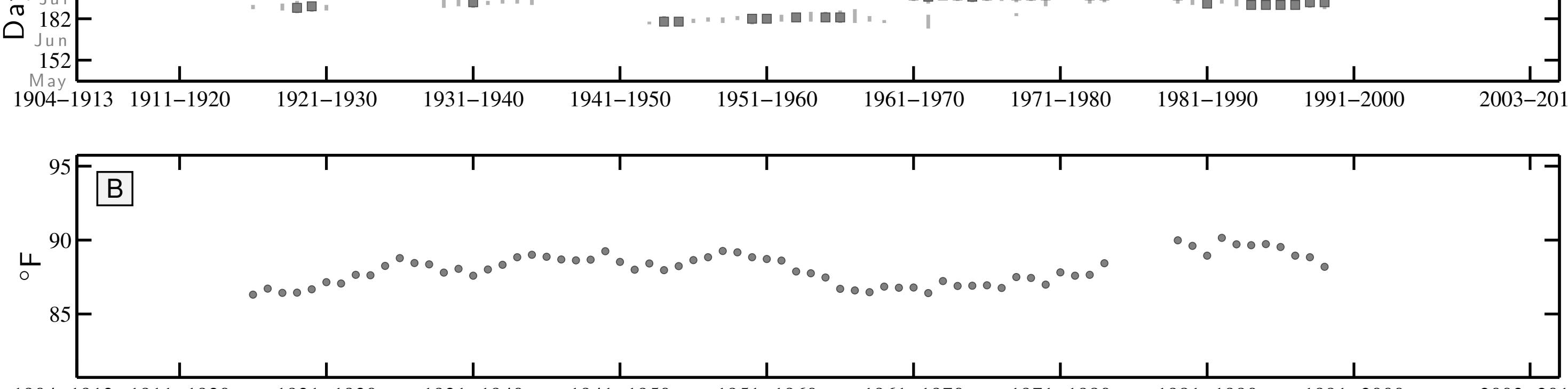

10 year Winter Teletherm dynamics for OWINGS FERRY LANDING, MD:

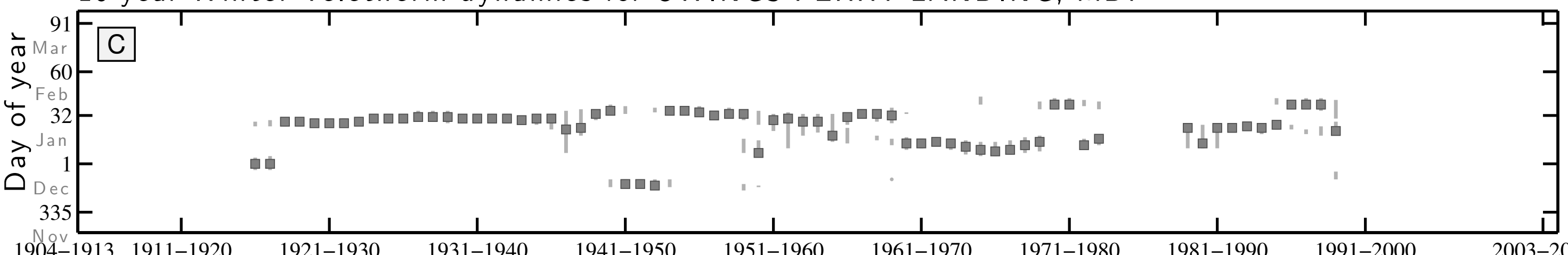

1904-1913 1911-1920

$1921-1930$

$1931-1940$

$1941-1950$

$1951-1960$

$1961-1970$

$1971-1980$

$1981-1990$

$1991-2000$

2003-2012

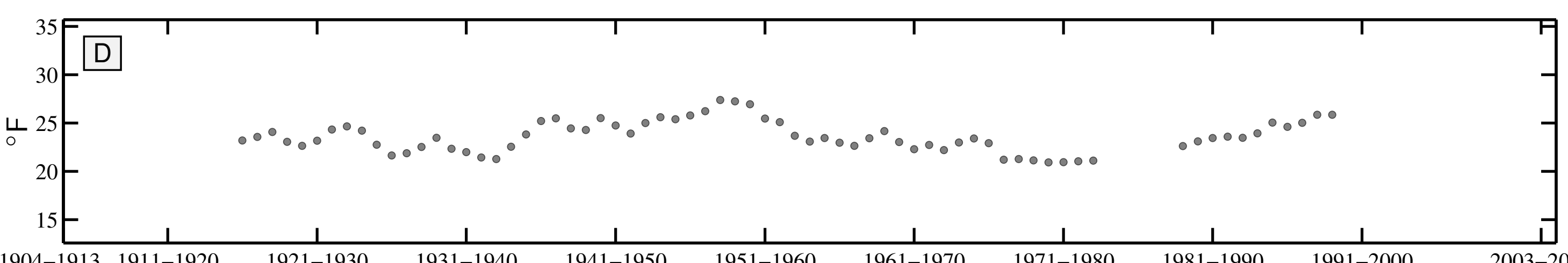


10 year Summer Teletherm dynamics for SALISBURY, MD:

$\div 274-\mathrm{A}$

(1) Sep

$>244-$

$4 \mathrm{Aug}$

$213-$

$182-$

$152-$

. " . | | | 무무

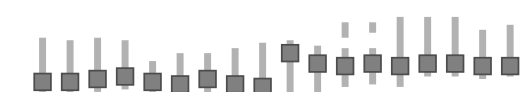

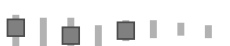

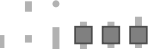

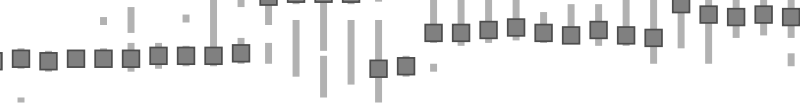

$-1920$

$1921-1930$

$1931-1940$

$\frac{1}{1941-1950}$

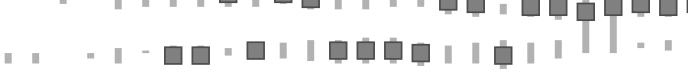

॥

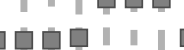

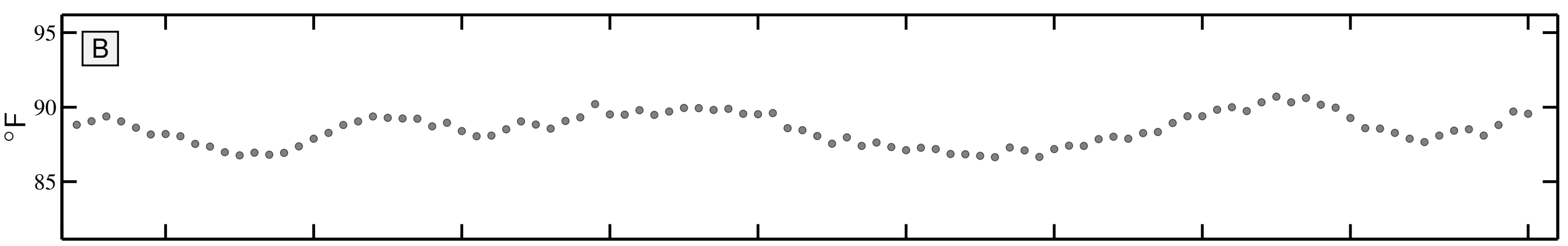

$1904-1913$ 1911-1920

$1921-1930$

$1931-1940$

$1941-1950$

$1951-1960$

$1961-1970$

$1971-1980$

$1981-1990$

$1991-2000$

2003-2012

10 year Winter Teletherm dynamics for SALISBURY, MD:

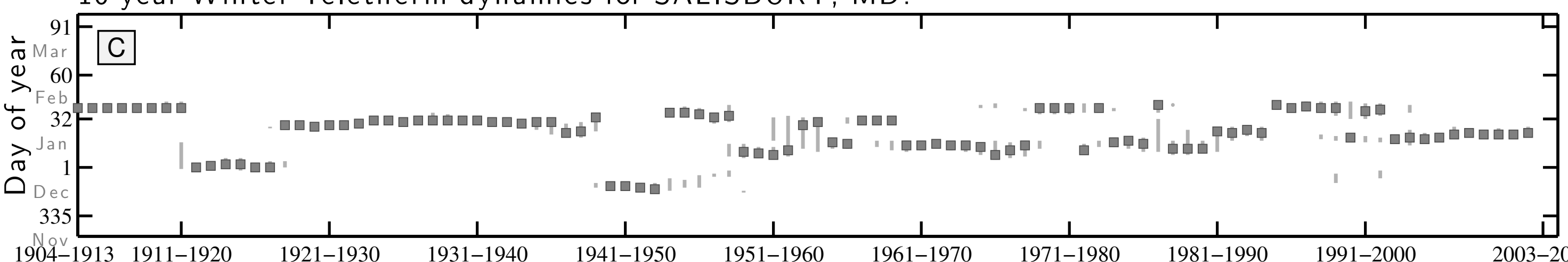

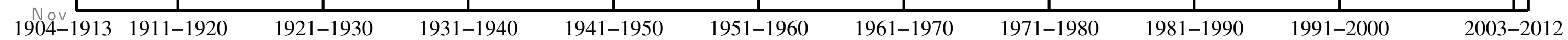

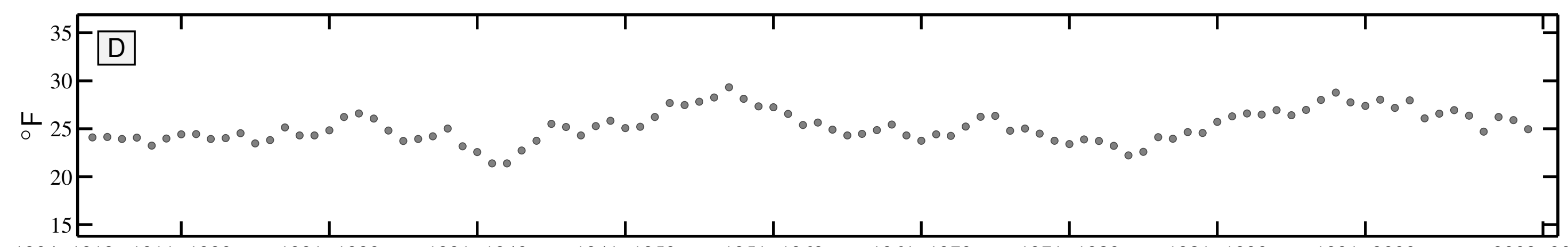


10 year Summer Teletherm dynamics for BLUE HILL, MA:

$=$ Oct

\% $274-A$

(1) Sep

$>244$

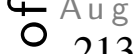

$213-$

$\vec{\sigma}_{1 \mathrm{u}} \mathrm{u}$

182

152

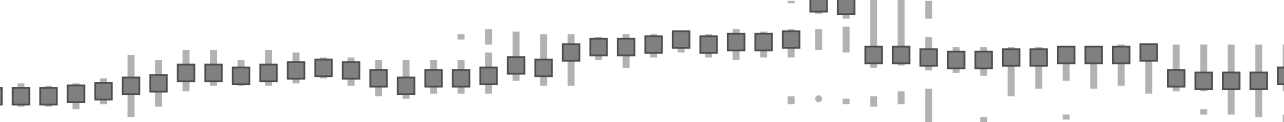

May

$-1920$

$\frac{1}{1921-1930}$

1931-1940

$\frac{1}{1941-1950}$

$\frac{1}{1951-1960}$

1961-1970

$\frac{1}{1971-1980}$

1981-1990

1991-2000

2003-2012

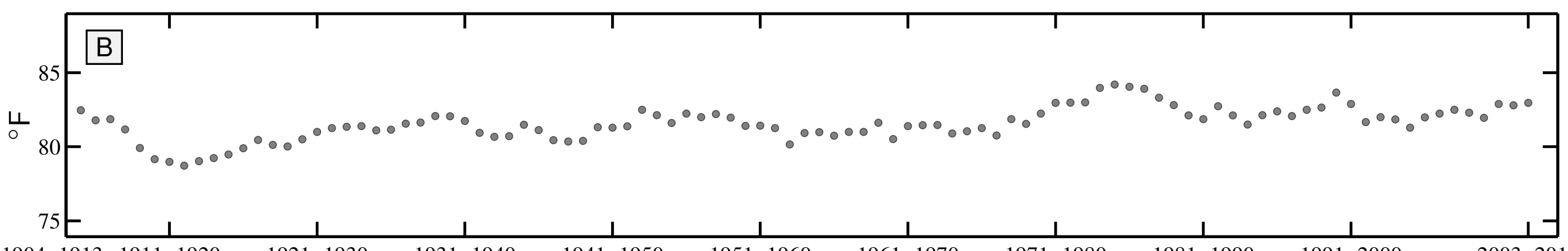

1904-1913 1911-1920

$1921-1930$

$1931-1940$

$1941-1950$

$1951-1960$

$1961-1970$

$1971-1980$

$1981-1990$

$1991-2000$

2003-2012

10 year Winter Teletherm dynamics for BLUE HILL, MA:

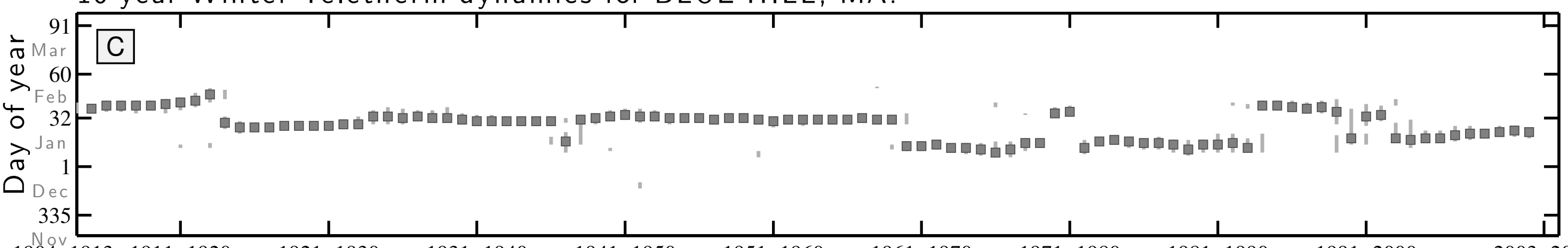

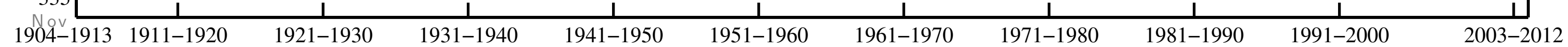

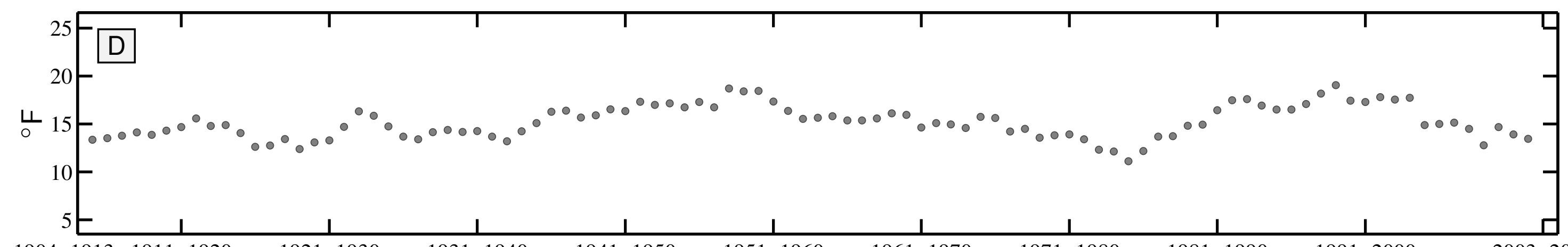


10 year Summer Teletherm dynamics for GREAT BARRINGTON 5 SW, MA:
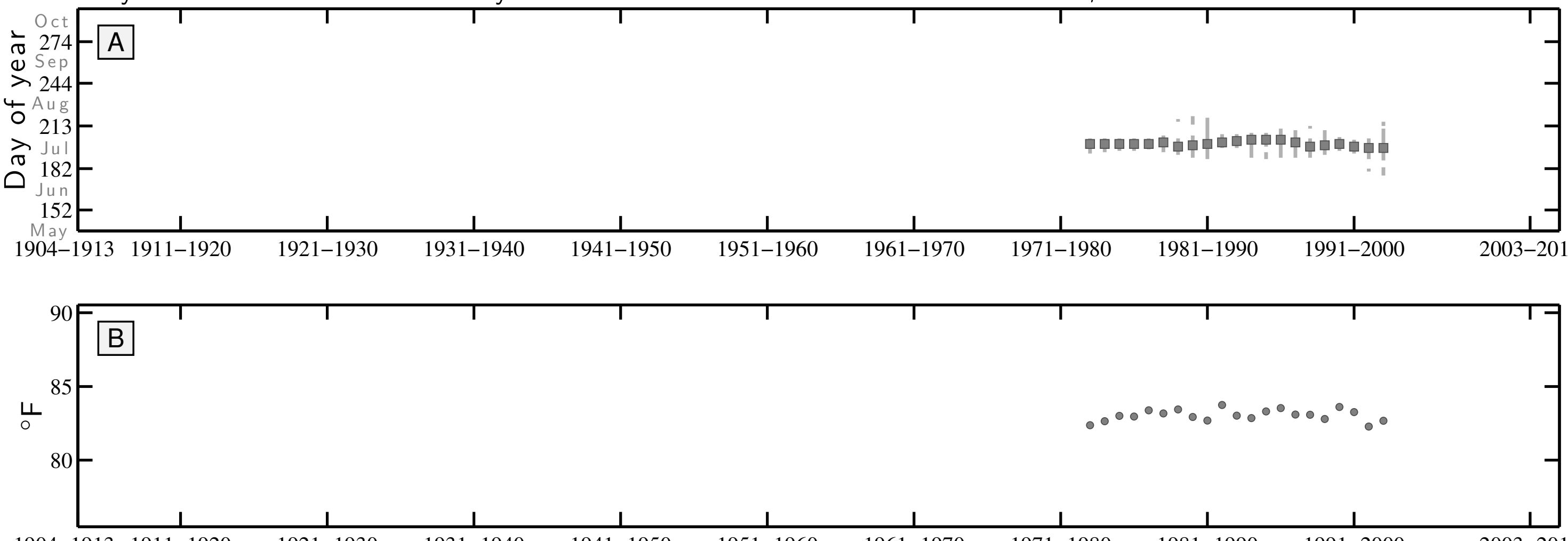

10 year Winter Teletherm dynamics for GREAT BARRINGTON 5 SW, MA:
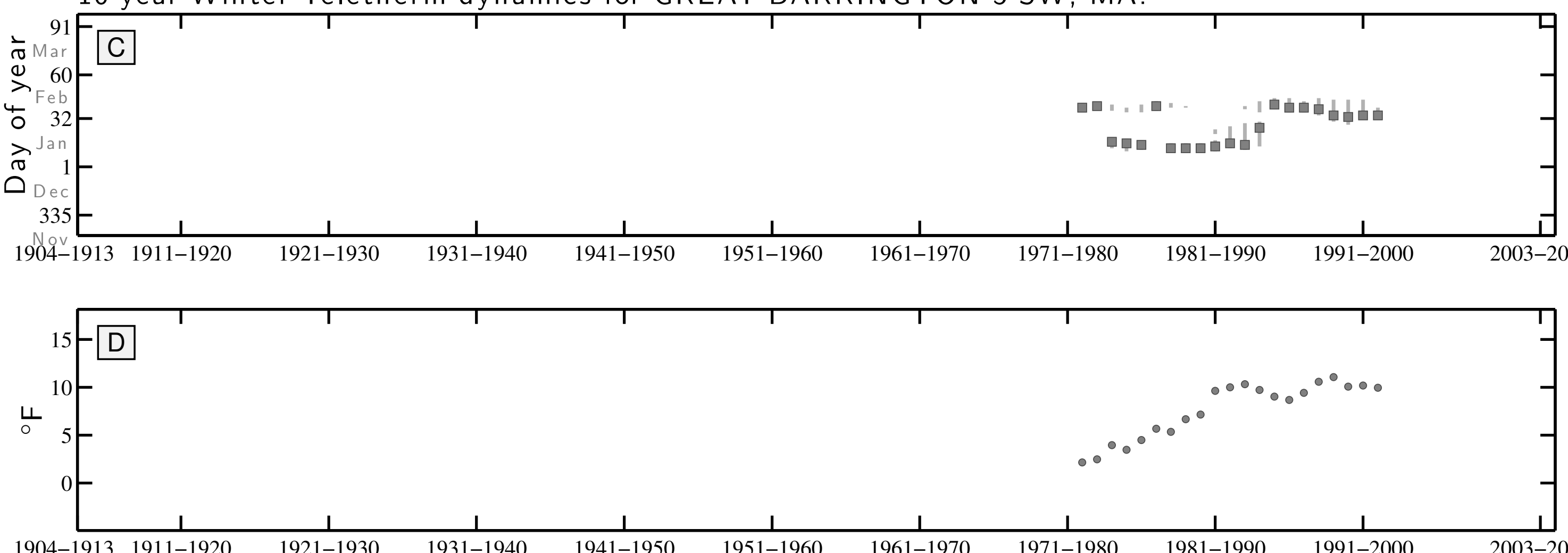
10 year Summer Teletherm dynamics for NEW BEDFORD, MA:

$\div$ Oct 274

\& Sep

$>244-$

4 Aug

$213-$

๙ 182

$\mathrm{J} u \mathrm{n}$
152

$152-1$ 10

คㅁㅁㅁषㅁ

| || คㅁㅁㅁㅁㅁำ

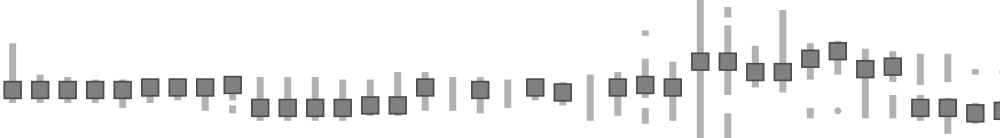

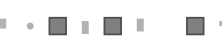

1904-1913 1911-1920

$1921-1930$

$1931-1940$

$\frac{1}{1941-1950}$

$1951-1960$

$1961-1970$

$\frac{1}{1971-1980}$

$981-1990$

991-2000

2003-201

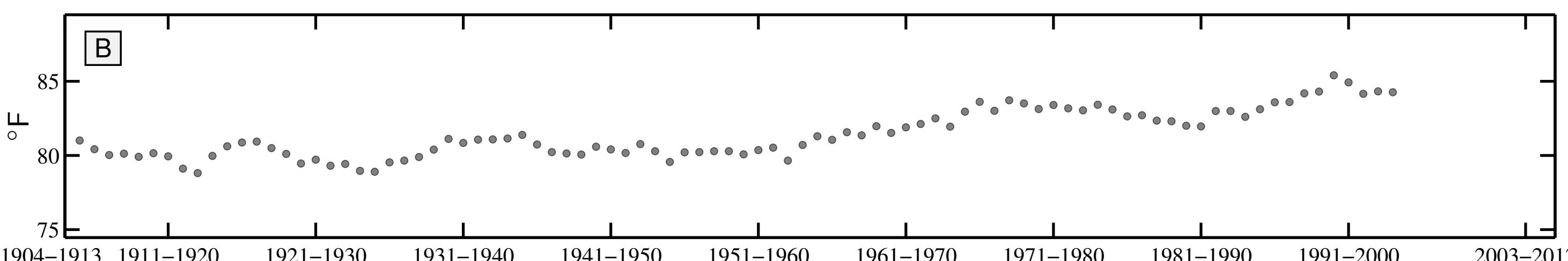

1904-1913 1911-1920

1921-1930

1931-1940

1941-1950

1951-1960

1961-1970

1971-1980

1981-1990

991-2000

2003-2012

10 year Winter Teletherm dynamics for NEW BEDFORD, MA:
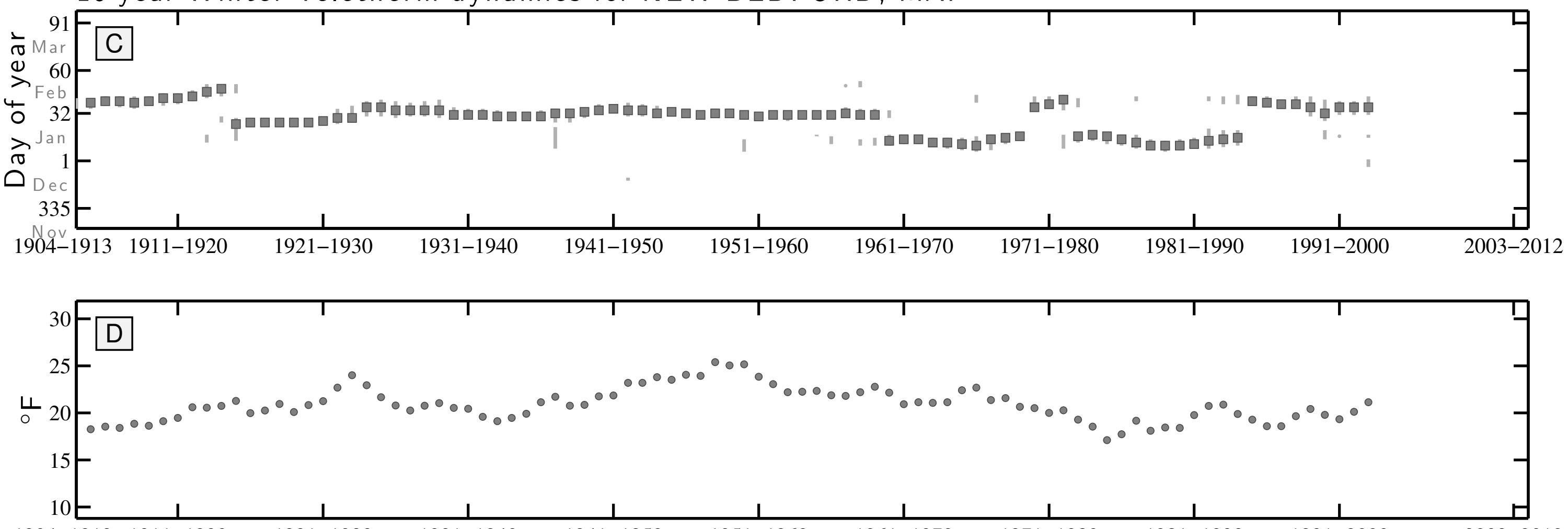
10 year Summer Teletherm dynamics for PLYMOUTH-KINGSTON, MA:

$\div \quad \frac{\mathrm{ct}}{\mathrm{c}} 274$

(1) Sep

$>244$

4 $\mathrm{Aug}$

$\gtrsim \mathrm{Ju}$

○ 182

Jun
152

152 -

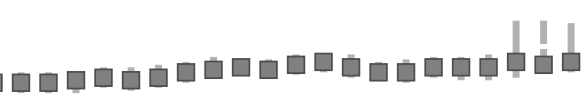

무미

1904-1913 1911-1920

$1921-1930$

$\frac{1}{1931-1940}$

$\frac{1}{1941-1950} \frac{1}{1951-1960}$

961-1970

$\frac{1}{1971-1980}$

1981-1990

1991-2000

2003-201

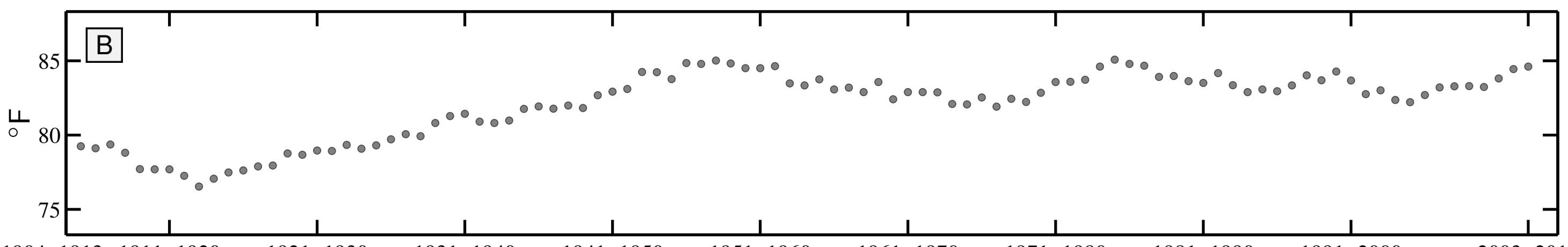

$1904-1913$ 1911-1920

$1921-1930$

$1931-1940$

$1941-1950$

$1951-1960$

$1961-1970$

$1971-1980$

$1981-1990$

$1991-2000$

$2003-2012$

10 year Winter Teletherm dynamics for PLYMOUTH-KINGSTON, MA:

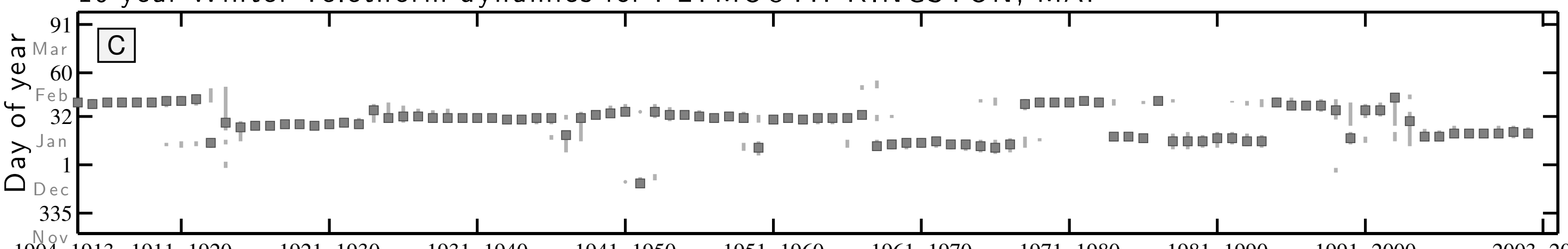

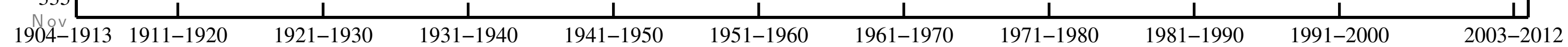

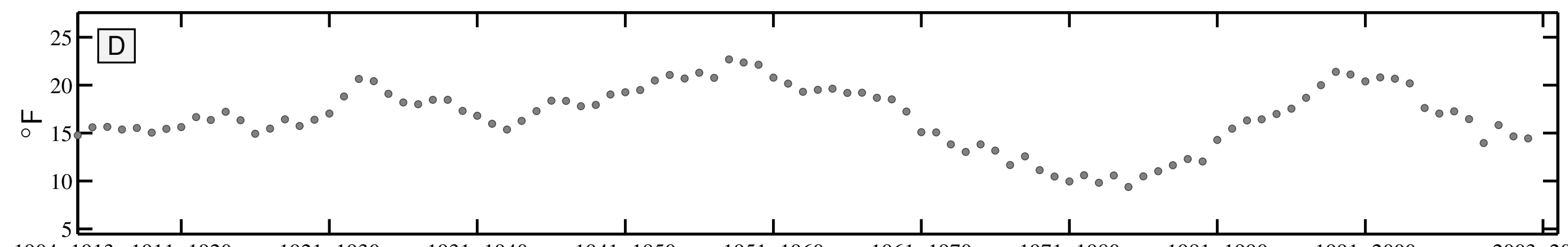


10 year Summer Teletherm dynamics for PROVINCETOWN, MA:
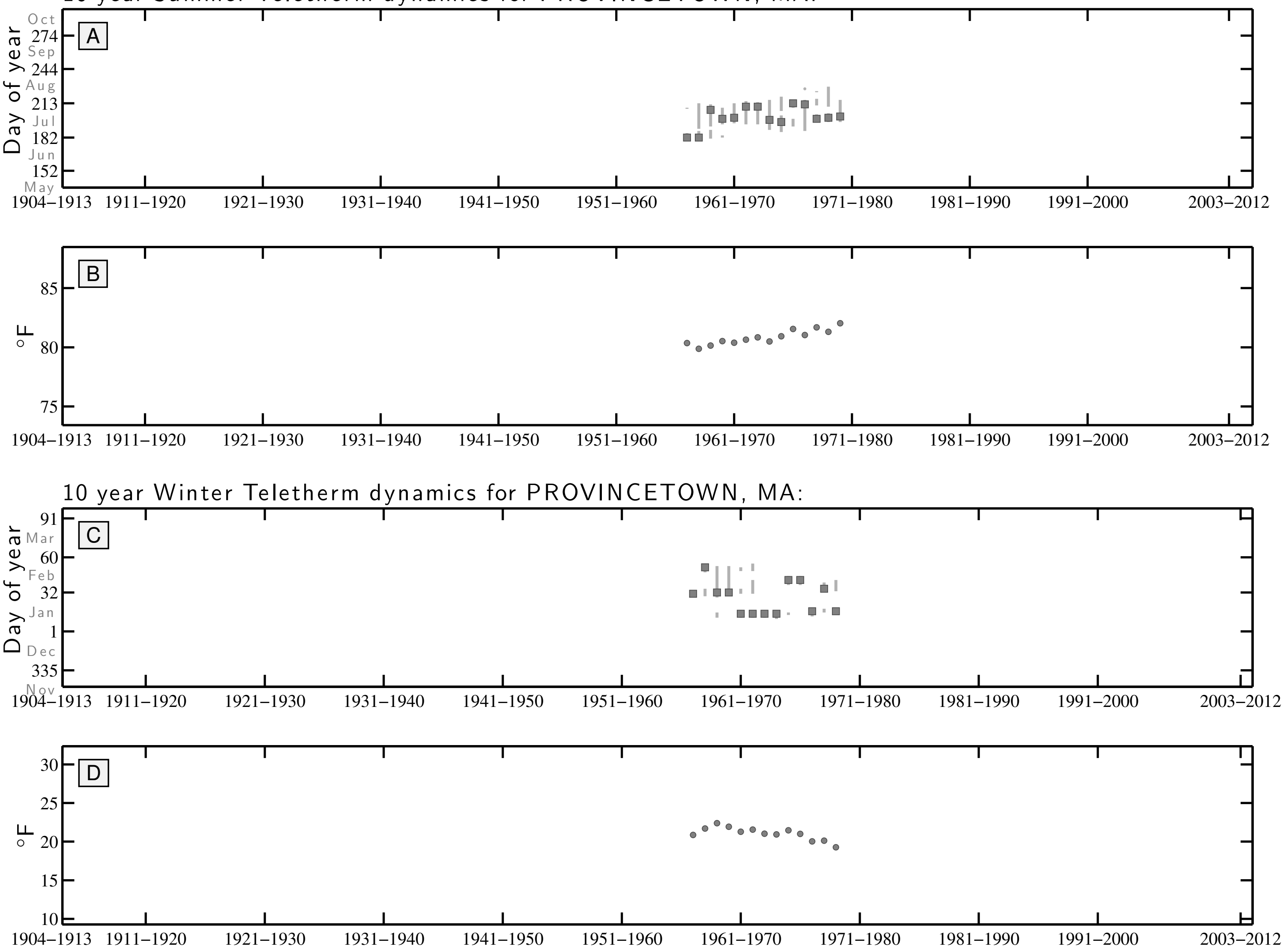
10 year Summer Teletherm dynamics for ALLEGAN 5NE, MI:

$+\mathrm{Oct}$

\% $274-A$

(1) Sep

$>244$

O A g g

$\gtrsim \begin{gathered}213 \\ \mathrm{Jul}\end{gathered}$

\% 182

$\mathrm{J} u \mathrm{n}$
152

152

1904-1913 1911-1920

|

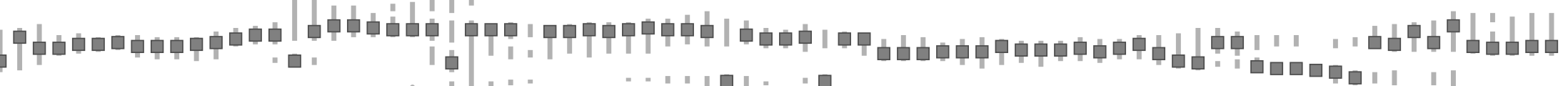

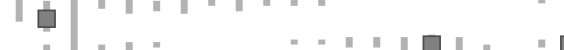

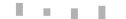

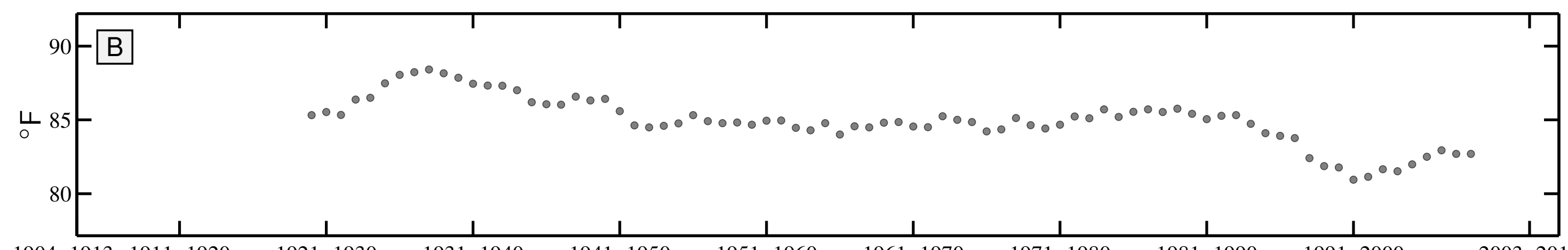

1904-1913 1911-1920

1921-1930

1931-1940

$1941-1950$

$1951-1960$

$1961-1970$

$1971-1980$

1981-1990

1991-2000

2003-2012

10 year Winter Teletherm dynamics for ALLEGAN 5NE, MI:

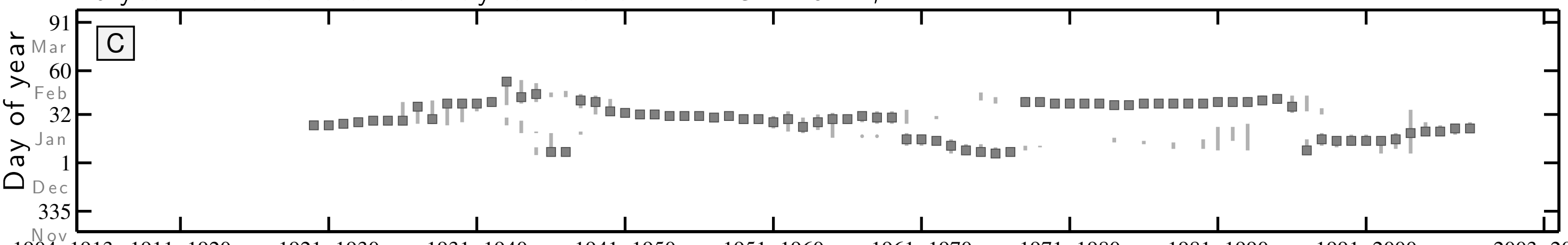

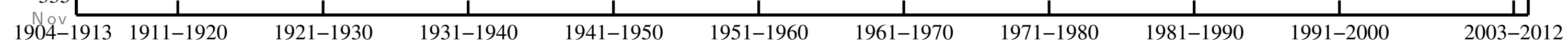

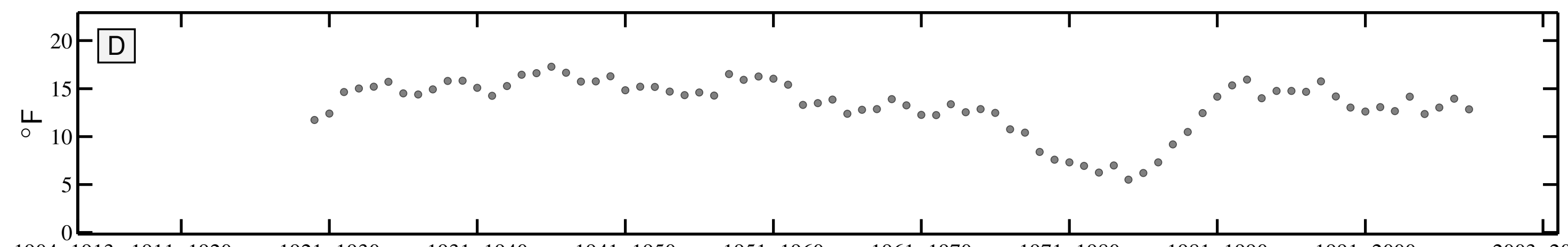


10 year Summer Teletherm dynamics for ALMA, MI:

Oct

A

(1) Sep

244

○ 213

$\gtrsim \mathrm{Ju}$

182

152

뭄=

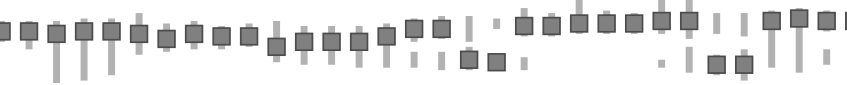

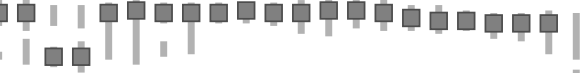

- " I |

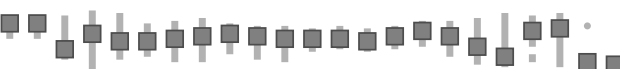

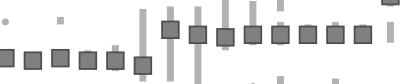

$\frac{1}{1-1940}$

$\frac{1}{1941-1950}$

$\frac{1}{1951-1960}$

$\frac{1}{961-1970}$

$1971-1980$

$\frac{1}{1981-1990}$

991-2000

2003-201

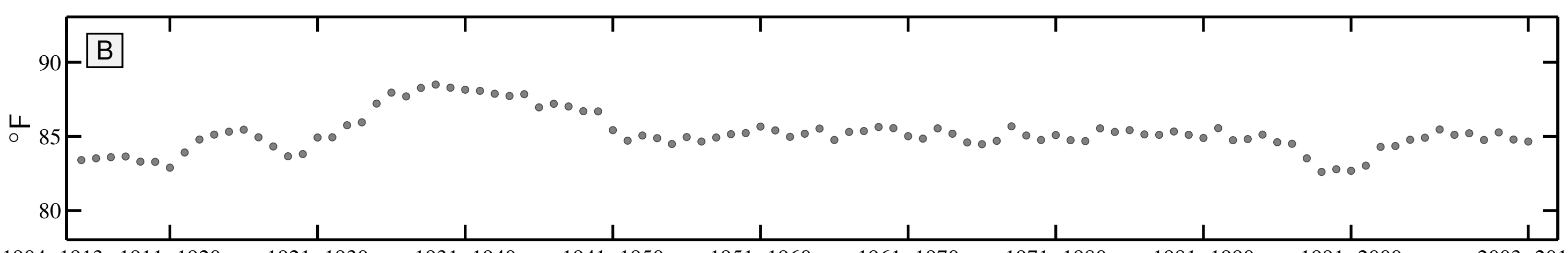

1904-1913 1911-1920

1921-1930

1931-1940

1941-1950

1951-1960

1961-1970

1971-1980

$1981-1990$

1991-2000

2003-2012

10 year Winter Teletherm dynamics for ALMA, MI:

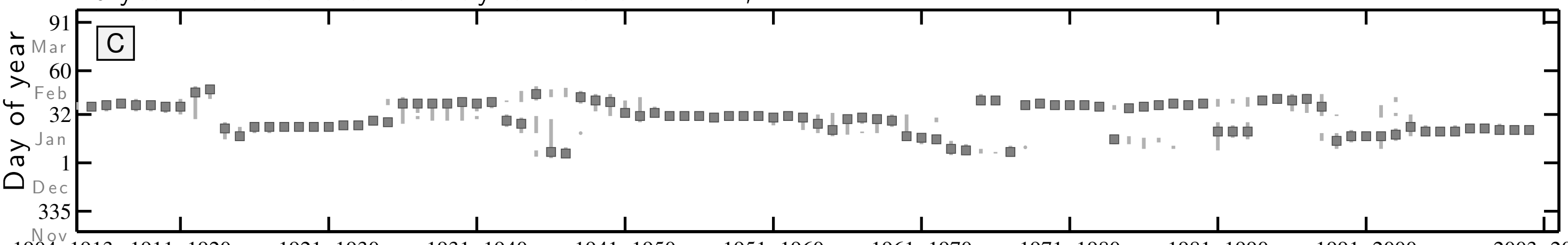

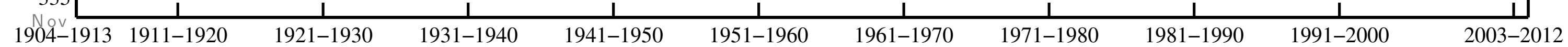

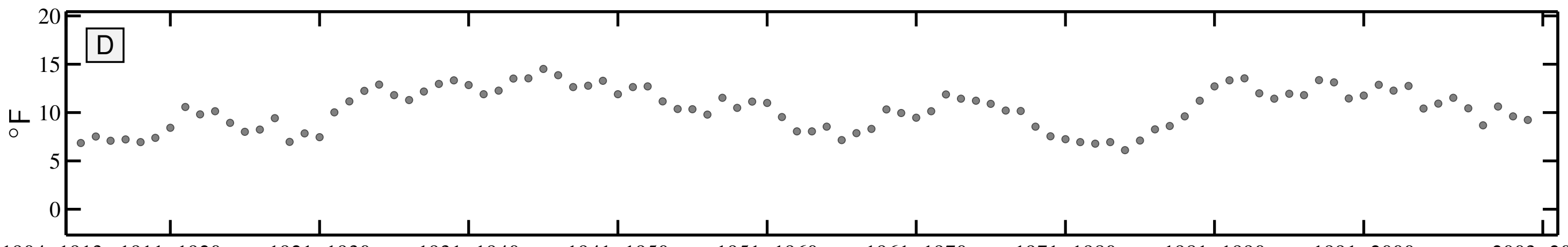


10 year Summer Teletherm dynamics for BIG RAPIDS WTR WKS, MI:

$\div$ Oct 274

Sep

(1) Sep

$>244$

4 A $\mathrm{Ag}$

213

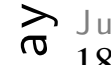

$\overbrace{\substack{u n \\ 1}}^{182}$

$152-$

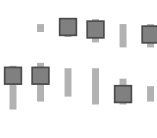

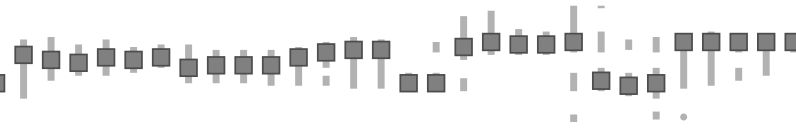

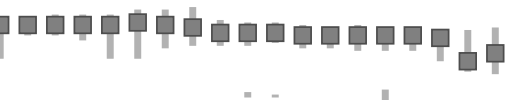

| ॥ | | | | = |

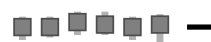
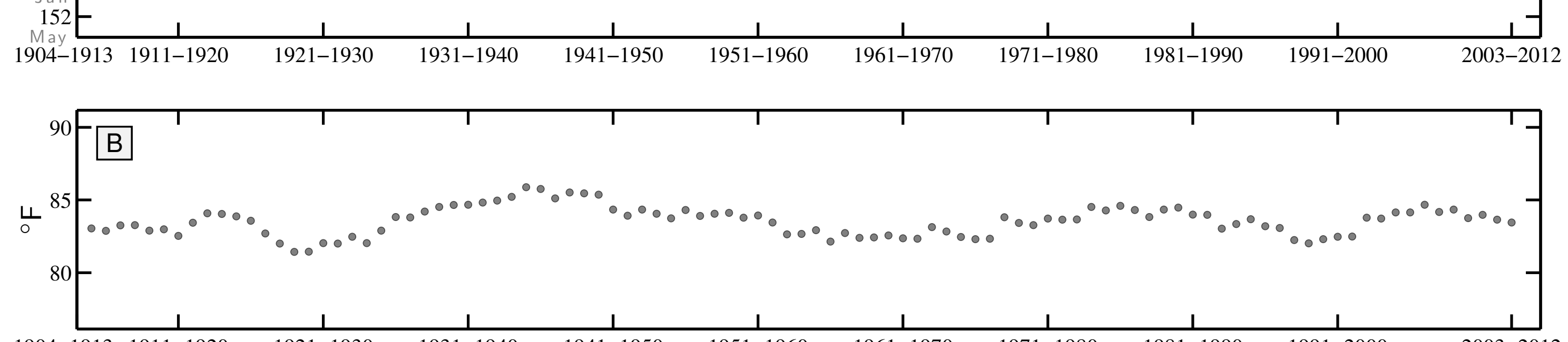

1904-1913 1911-1920

1921-1930

1931-1940

1941-1950

1951-1960

1961-1970

$1971-1980$

$1981-1990$

$1991-2000$

2003-2012

10 year Winter Teletherm dynamics for BIG RAPIDS WTR WKS, MI:

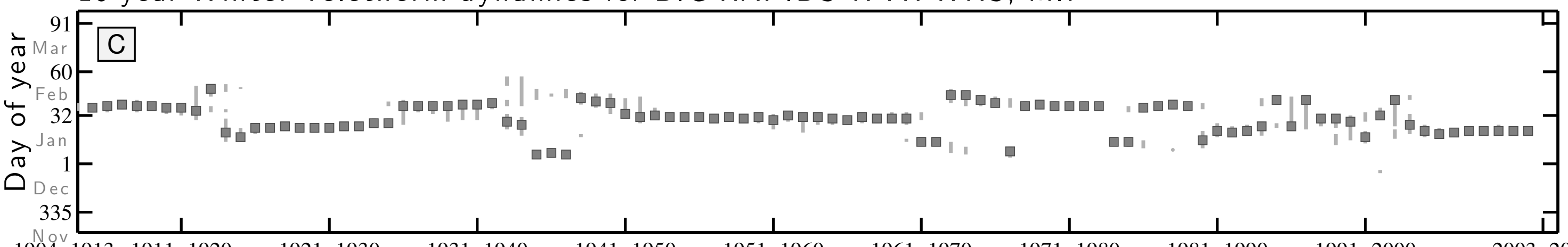

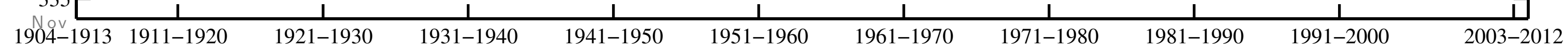

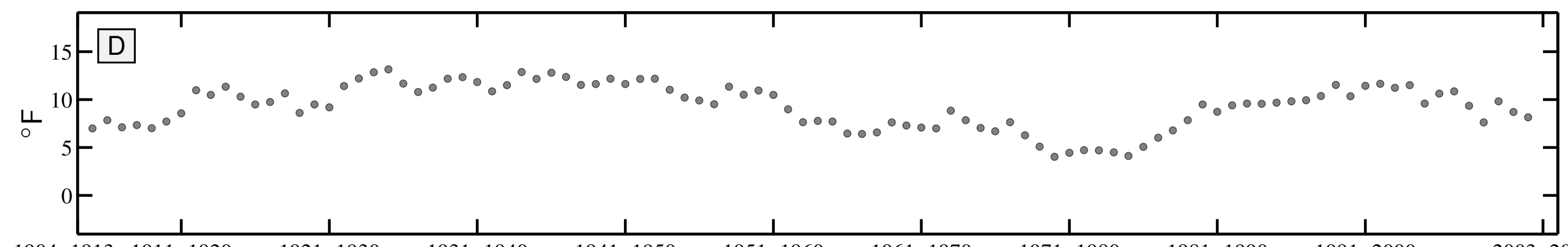


10 year Summer Teletherm dynamics for CHAMPION VAN RIPER PK, MI:
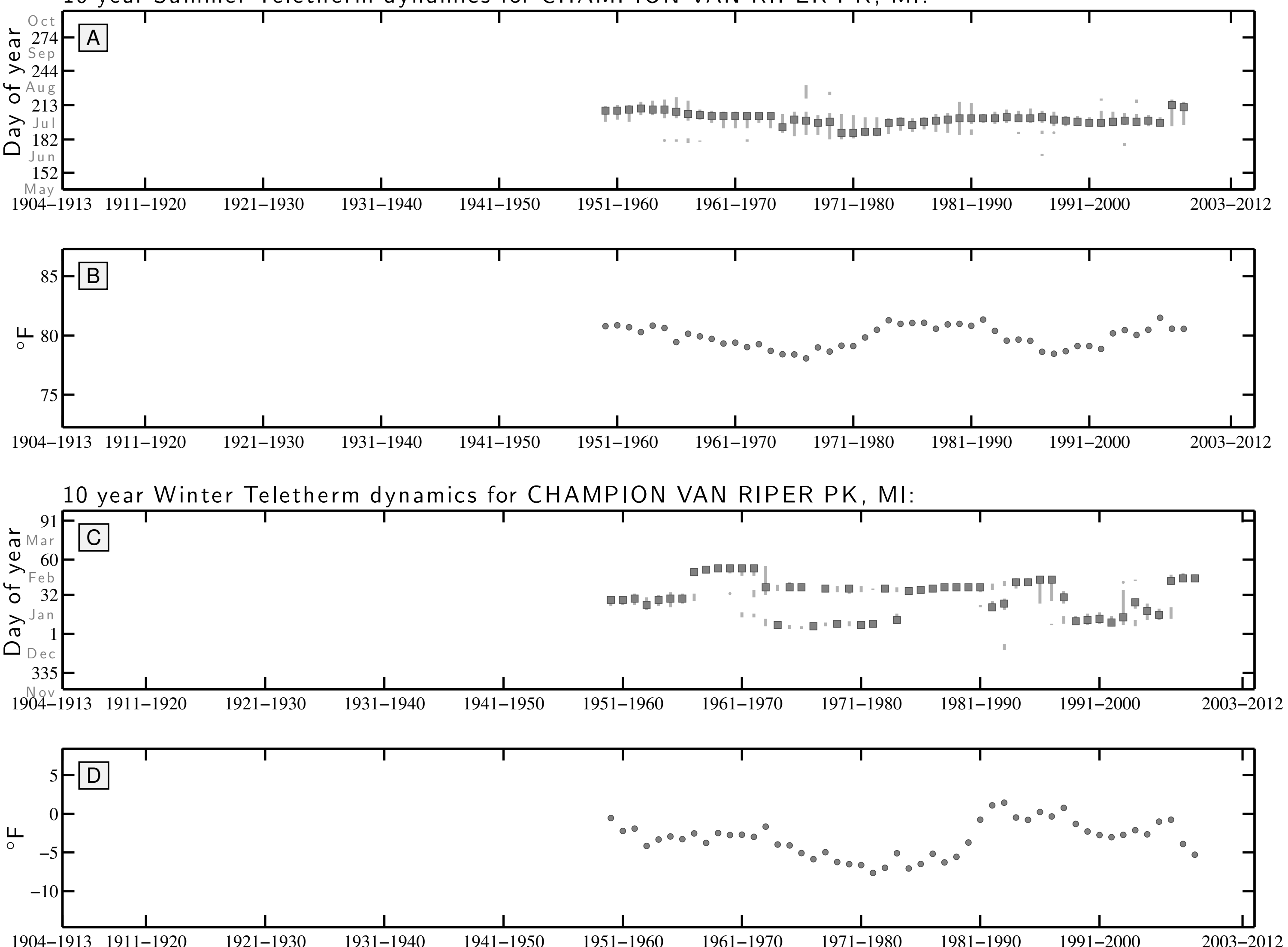

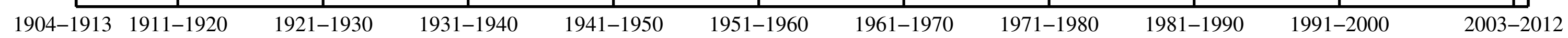


10 year Summer Teletherm dynamics for CHEBOYGAN, MI:

$\div \quad 0 \mathrm{ct} A$

(t)

(1)
$>$ 244

4 Aug

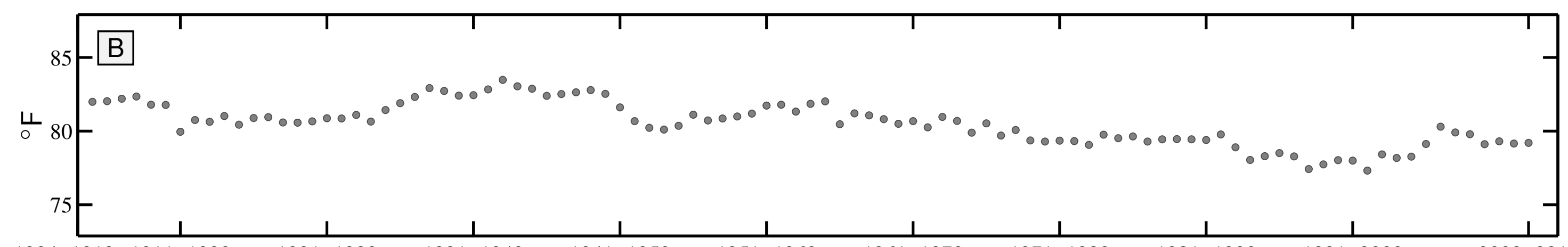

10 year Winter Teletherm dynamics for CHEBOYGAN, MI:
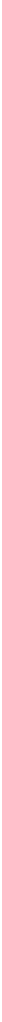
10 year Summer Teletherm dynamics for COLDWATER ST SCHOOL, MI:

$-O c t$

\% $274-A$

(1) Sep

$>244$

O Aug

213 - " ㄴ

(182

$182-$

152

May

1904-1913 1911-1920

1921-1930

1931-1940

$\frac{1}{1941-1950}$

1951-1960

1961-1970

1971-1980

$\frac{1}{1981-1990}$

1991-2000

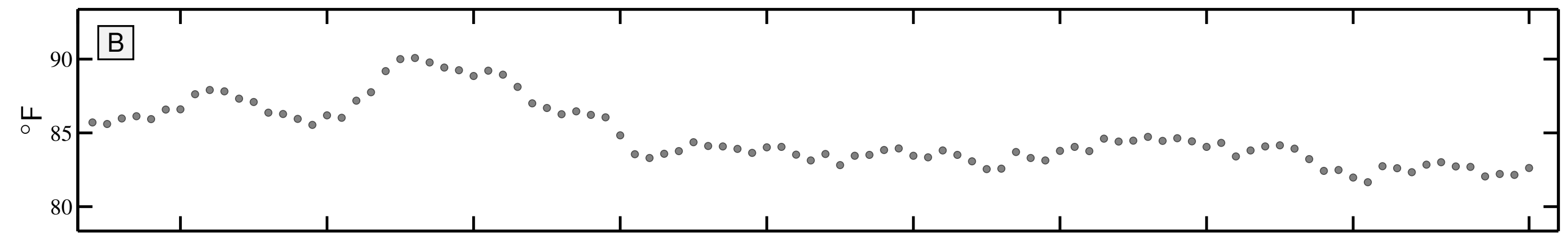

-

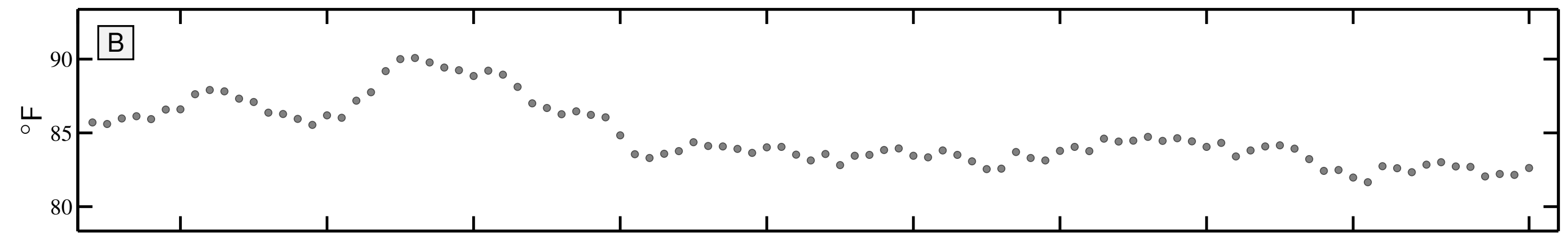

1

1904-1913 1911-1920

1921-1930

1931-1940

1941-1950

1951-1960

1961-1970

1971-1980

1981-1990

1991-2000

2003-2012

10 year Winter Teletherm dynamics for COLDWATER ST SCHOOL, MI:

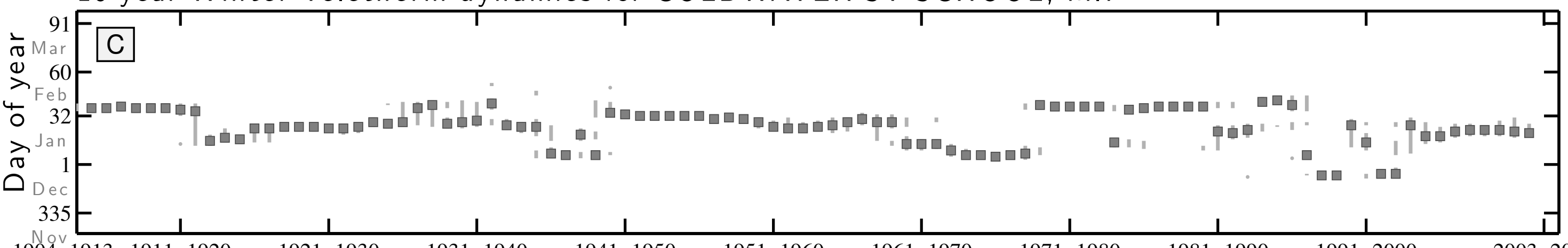

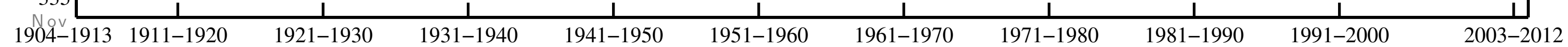

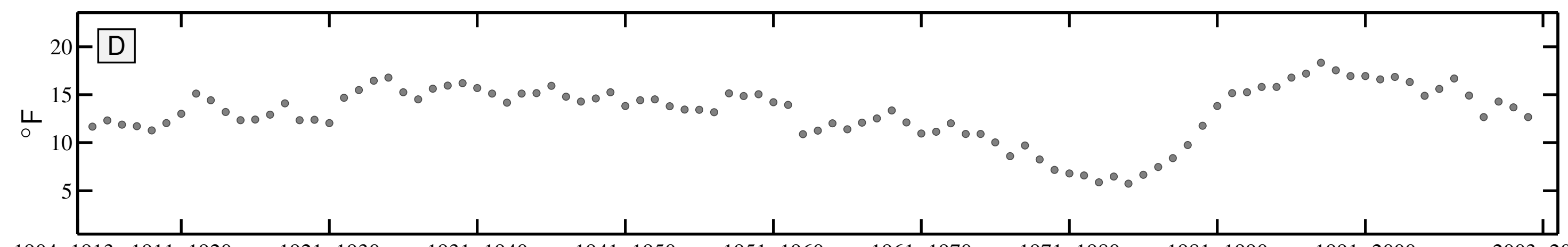


10 year Summer Teletherm dynamics for FAYETTE 4 SW, MI:

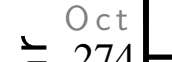

( $274-A$

(1) Sep

$>244$

O A g g

त Jul

$\overbrace{}^{\circ} 182$

$152-$

15ay

1904-1913 1911-1920

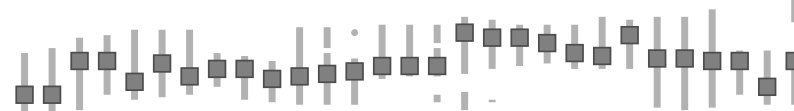

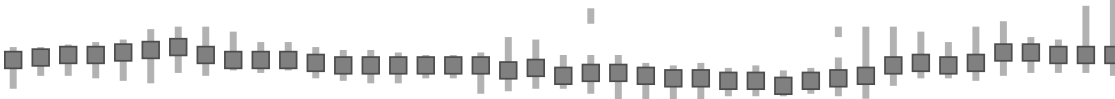

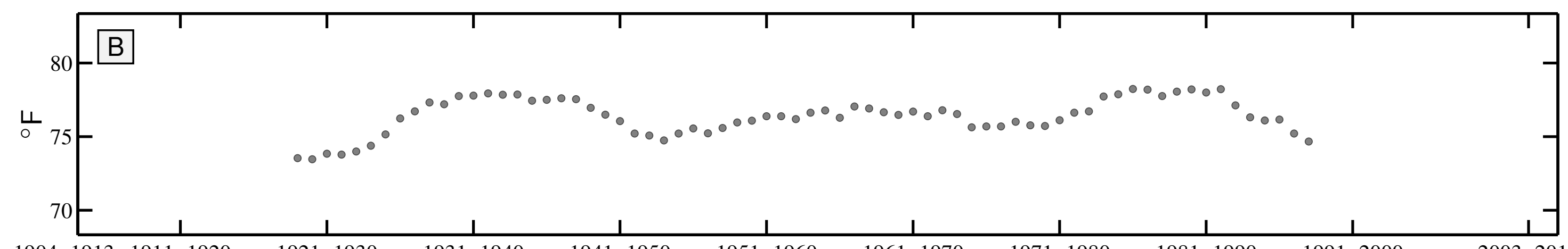

$1904-1913$ 1911-1920

$1921-1930$

$1931-1940$

$1941-1950$

$1951-1960$

$1961-1970$

$1971-1980$

$1981-1990$

$1991-2000$

$2003-2012$

10 year Winter Teletherm dynamics for FAYETTE 4 SW, MI:

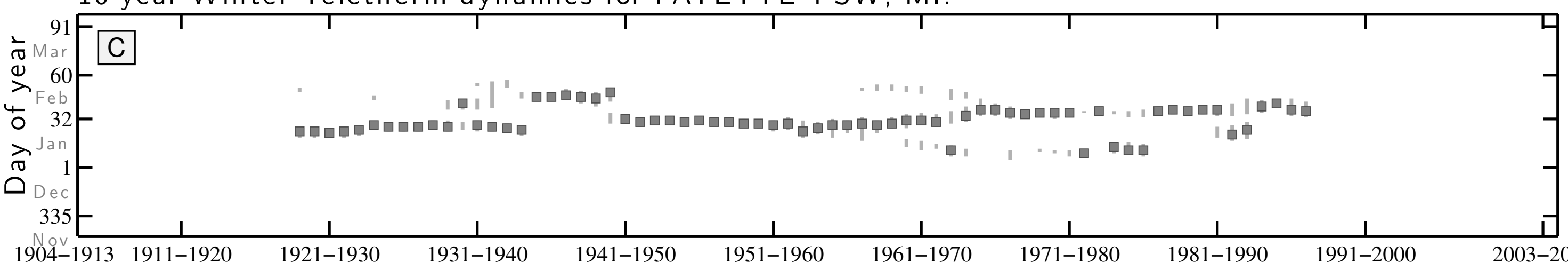

1904-1913 1911-192

$1921-1930$

$1931-1940$

$1941-1950$

$1951-1960$

1961-1970

1971-1980

1981-1990

003-2012

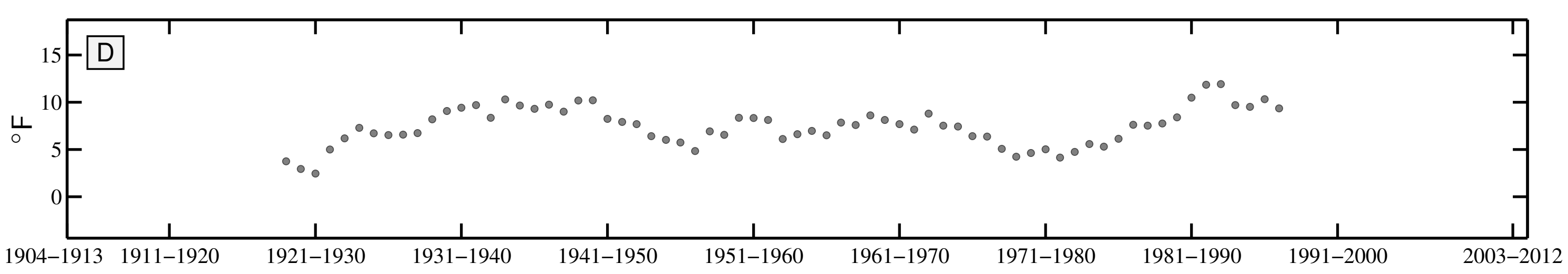


10 year Summer Teletherm dynamics for HILLSDALE, MI:

Oct

A

(1) Sep

$>244$

○ $213-$

त्र Jul ㅁㅁㅁ.

182

$152-$

-

1

1921-1930

1931-1940

$\frac{1}{1941-1950}$

1951-1960

961-1970

$\frac{1}{1971-1980}$

$1981-1990$

1991-2000

1 1 .

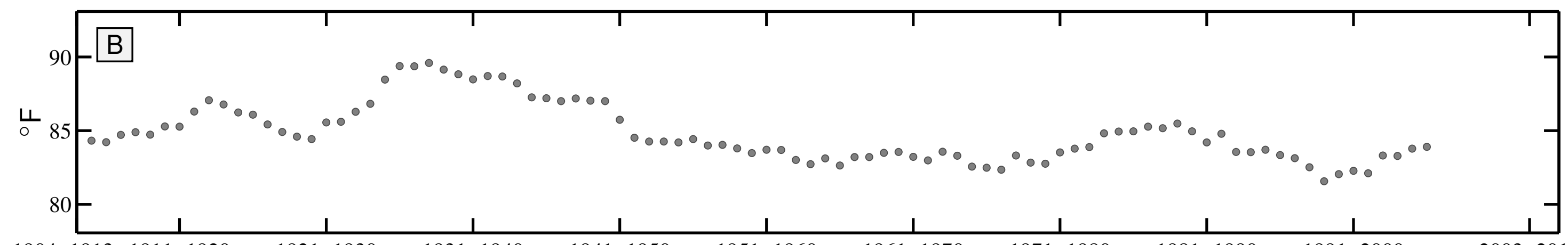

1904-1913 1911-1920 1921-1930

$1931-1940$

$1941-1950$

$1951-1960$

$1961-1970$

$1971-1980$

$1981-1990$

$1991-2000$

$2003-2012$

10 year Winter Teletherm dynamics for HILLSDALE, MI:

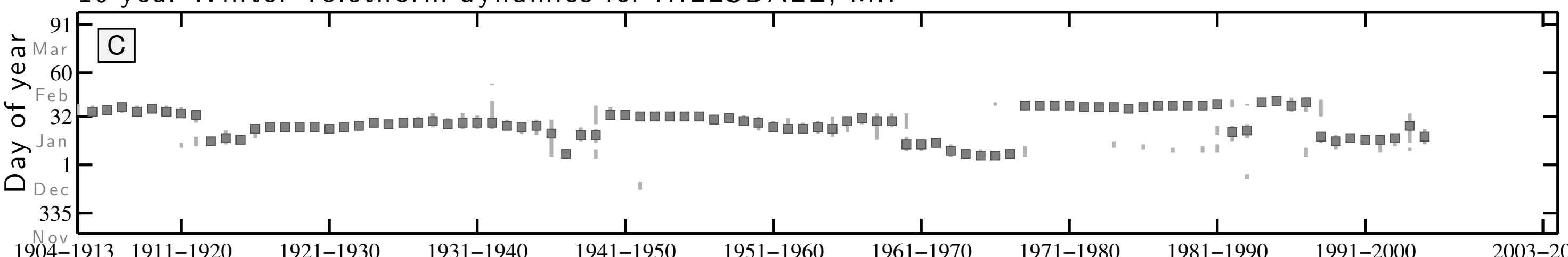

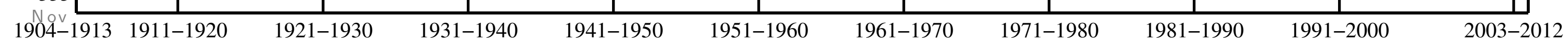

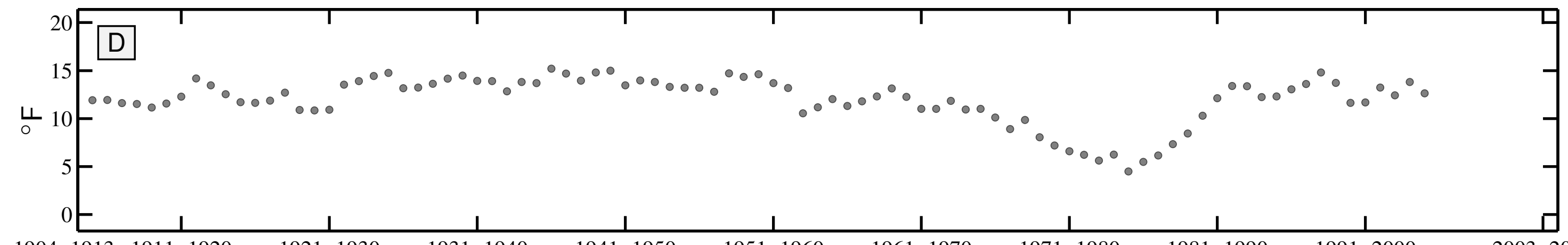


10 year Summer Teletherm dynamics for IRON MT KINGSFORD WWTP, MI:

Oct

त $274-A$

(1) Sep

$>244-$

4 A g g

$>\quad 213$

ণ 182 .

$152-$

May $\square$

1904-1913 1911-1920

1921-1930

1931-1940

1941-1950

1951-1960

$\frac{1}{1970}$

$\frac{1}{1971-1980}$

$\frac{1}{1981-1990}$

1991-2000

2003-2012

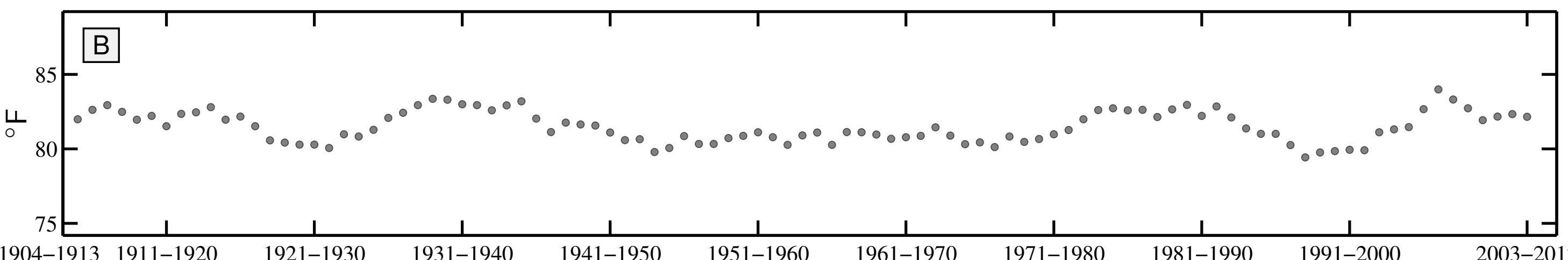

1904-1913 1911-1920

$1921-1930$

$1931-1940$

$1941-1950$

$1951-1960$

$1961-1970$

$1971-1980$

$1981-1990$

2003-2012

10 year Winter Teletherm dynamics for IRON MT KINGSFORD WWTP, MI:

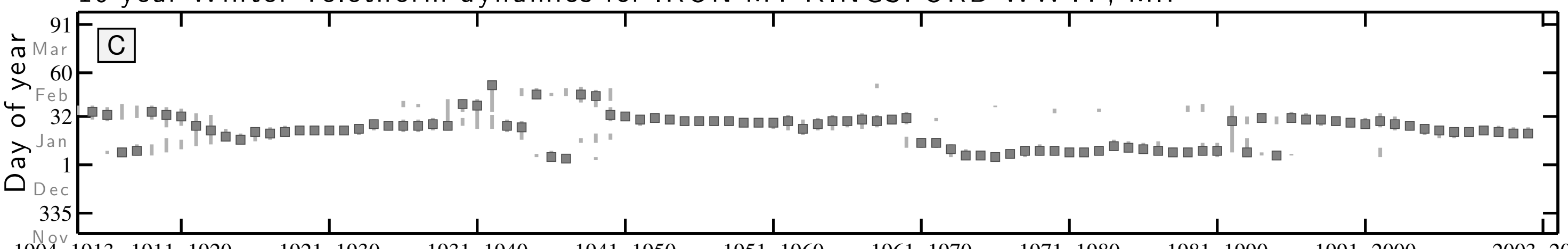

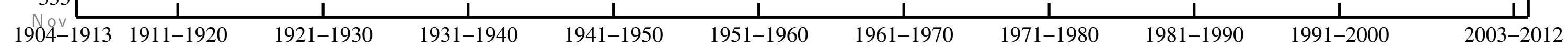

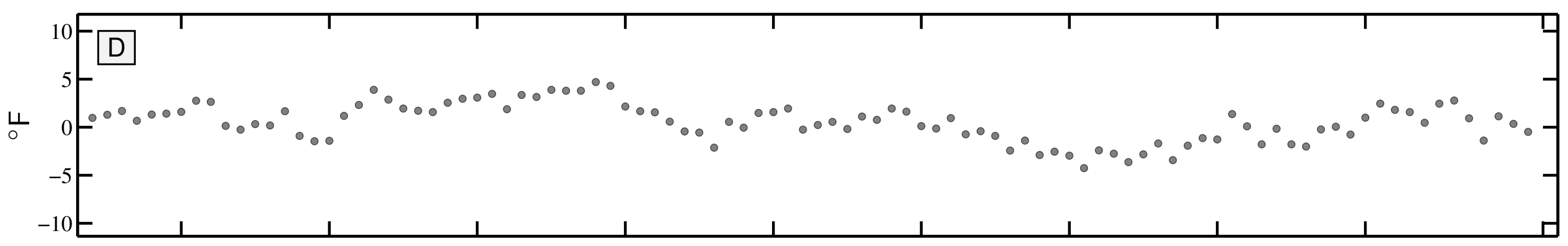


10 year Summer Teletherm dynamics for KALAMAZOO STATE HOSP, MI:

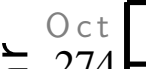

A

(1) Sep

- 244

O 213

$\gtrsim \mathrm{J}$

$\overbrace{}^{\circ} 182$

$152-$

May

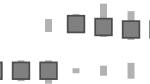

1904-1913 1911-1920

$\frac{1}{1921-1930}$

$1931-1940$

1941-1950

$\frac{1}{1951-1960}$

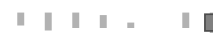

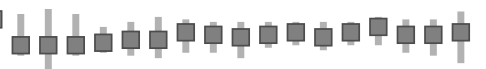

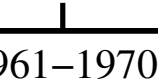

971-1980
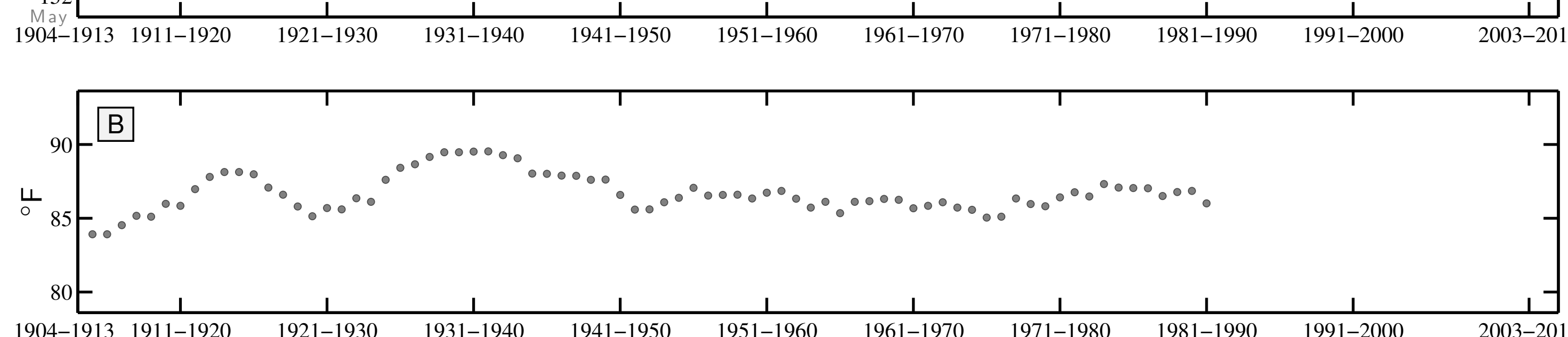

10 year Winter Teletherm dynamics for KALAMAZOO STATE HOSP, MI:

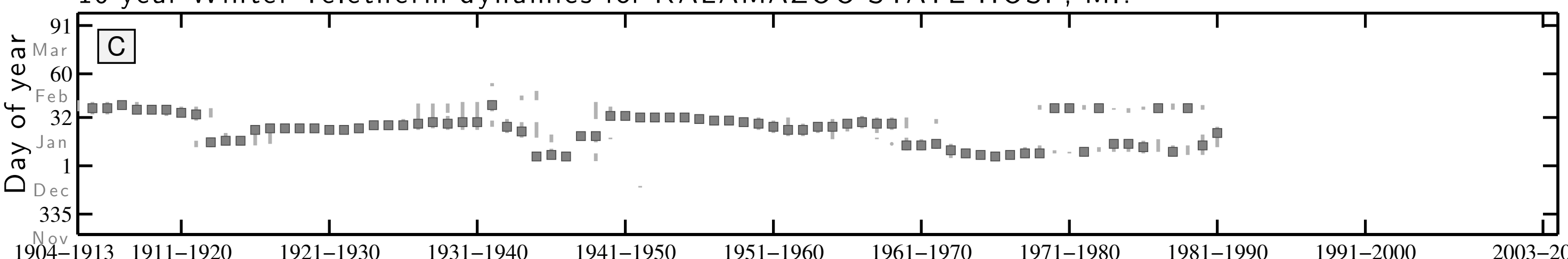

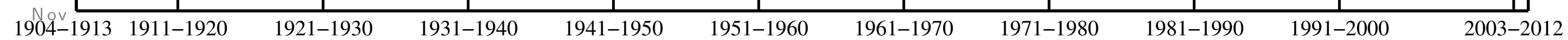

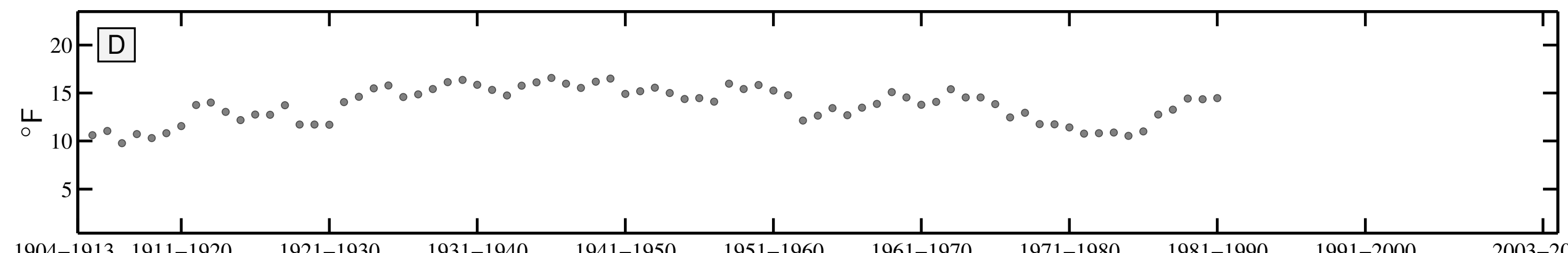


10 year Summer Teletherm dynamics for MIDLAND, MI:
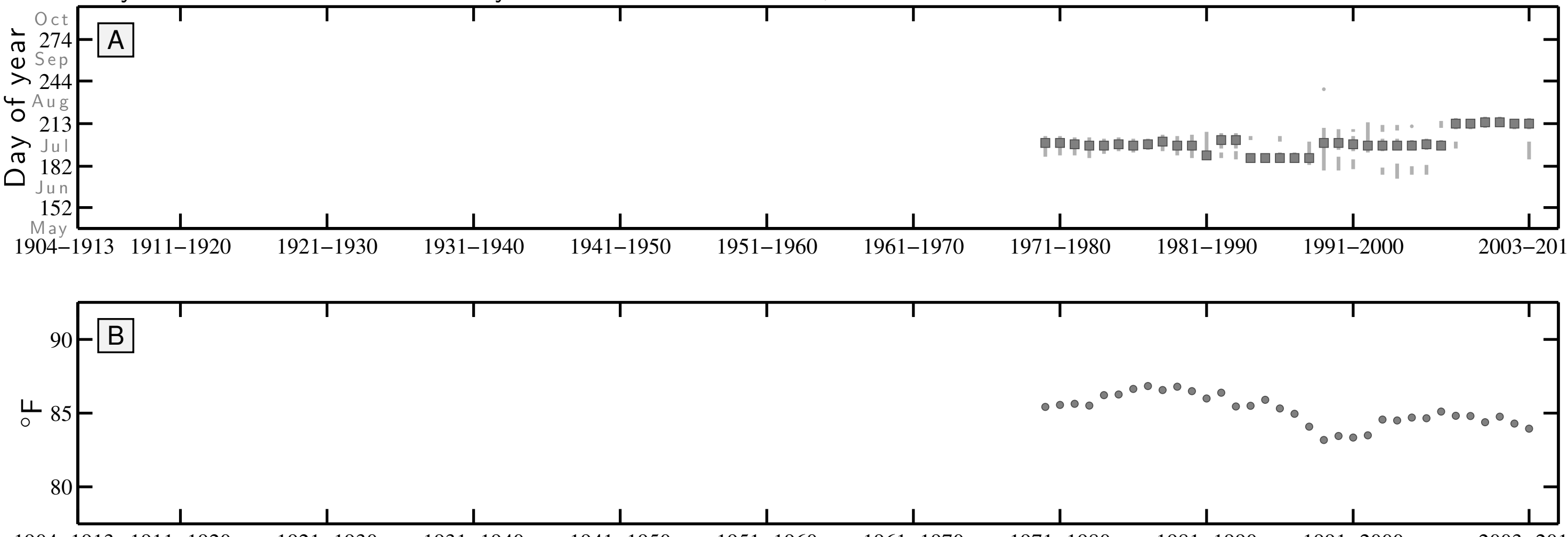

1904-1913 1911-1920

1921-1930

1931-1940

1941-1950

1951-1960

1961-1970

1971-1980

1981-1990

1991-2000

2003-2012

10 year Winter Teletherm dynamics for MIDLAND, MI:

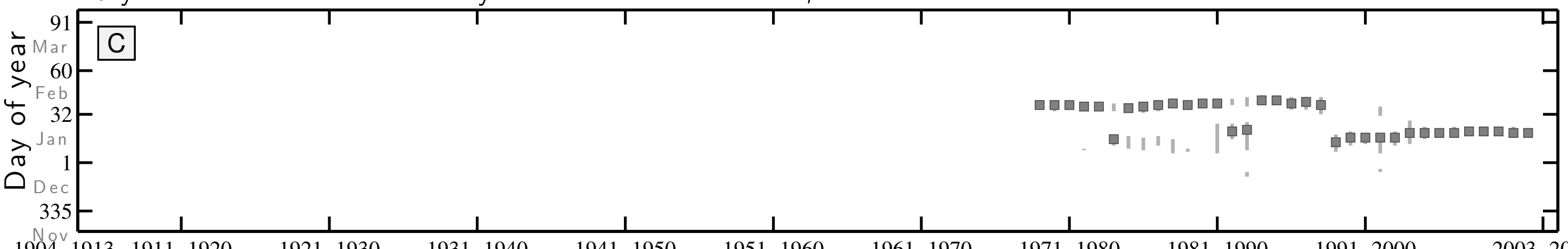

1904-1913 1911-1920

1921-1930

1931-1940

1941-1950

1951-1960

1961-1970

1971-1980

1981-1990

1991-2000

003-2012

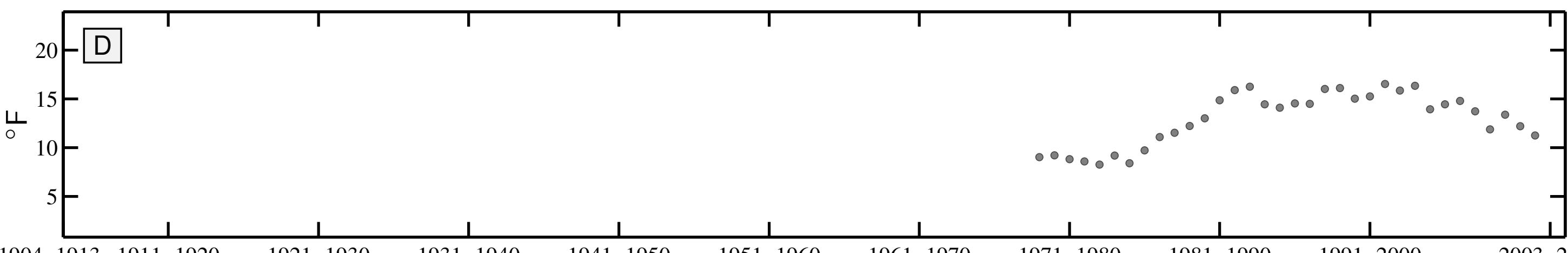


10 year Summer Teletherm dynamics for MT PLEASANT UNIV, MI:
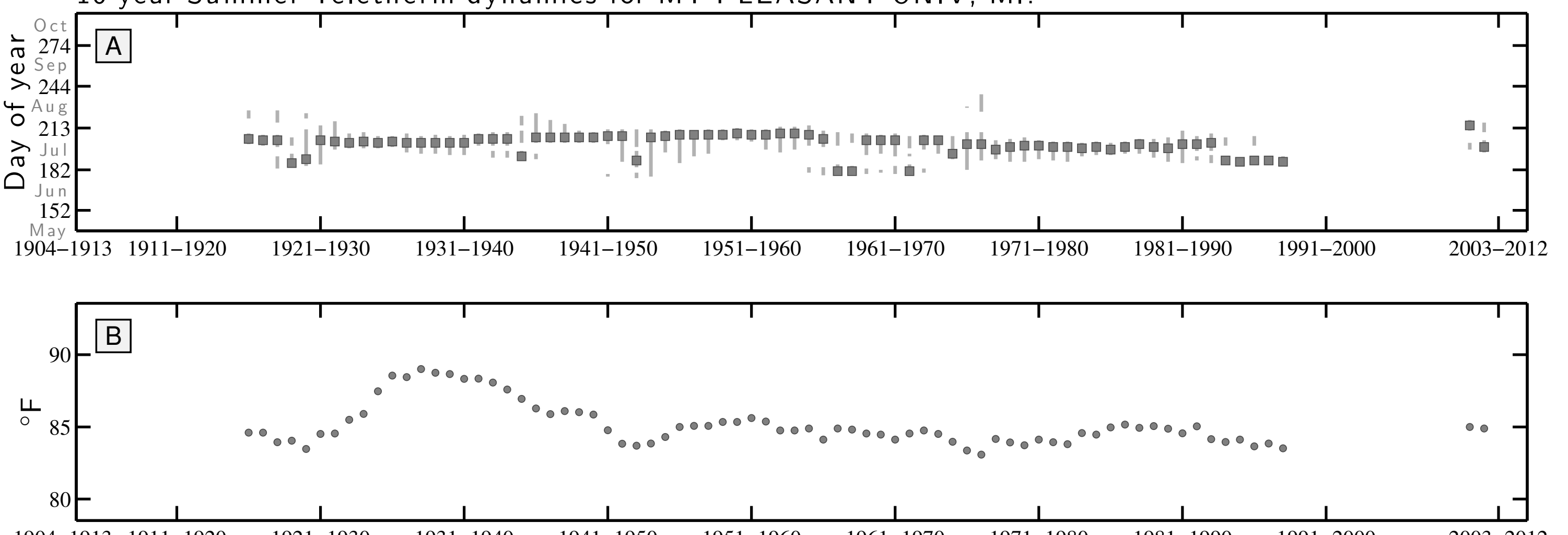

1904-1913 1911-1920

1921-1930

1931-1940

1941-1950

$1951-1960$

1961-1970

1971-1980

1981-1990

1991-2000

$2003-2012$

10 year Winter Teletherm dynamics for MT PLEASANT UNIV, MI:
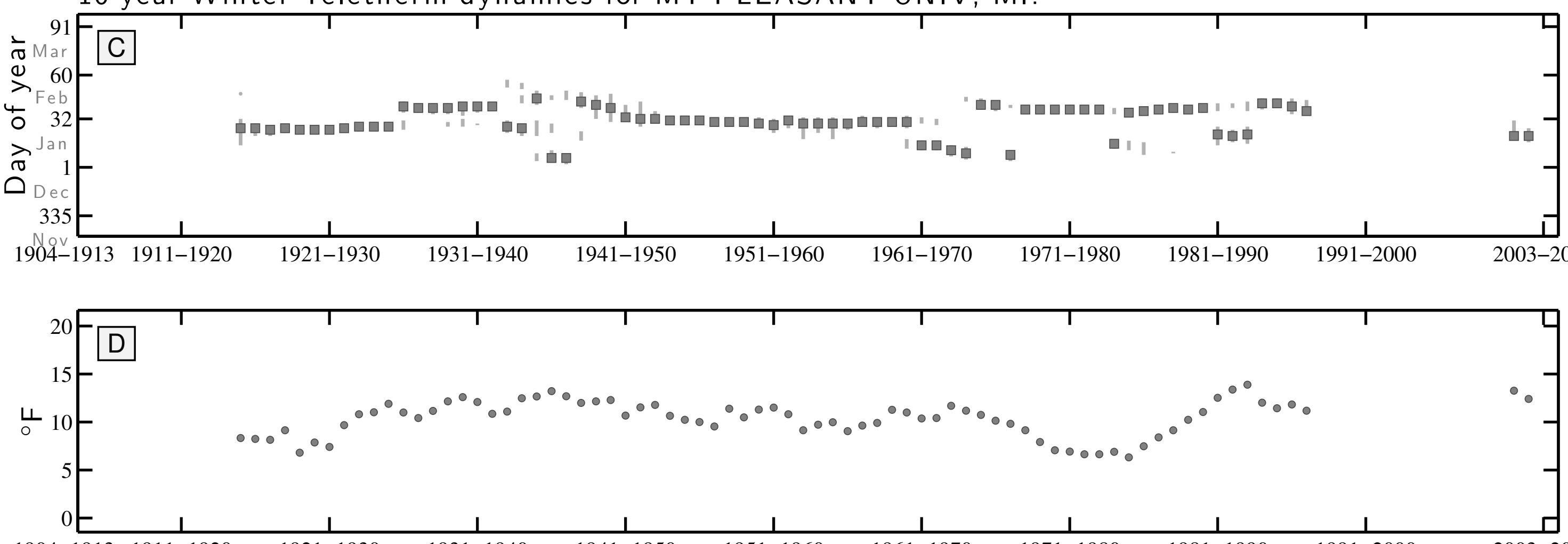
10 year Summer Teletherm dynamics for NEWBERRY 3S, MI:

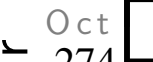

A

(1) Sep

$>244$

$213-$

$\gtrsim \mathrm{Ju}$

$\overbrace{}^{\sigma} 182$

152

ロ ॥ |

152

$1904-1913$ 1911-1920

1921-1930

1931-1940

1941-1950

1951-1960

1961-1970

1971-1980

1981-1990

$1991-2000$

2003-2012

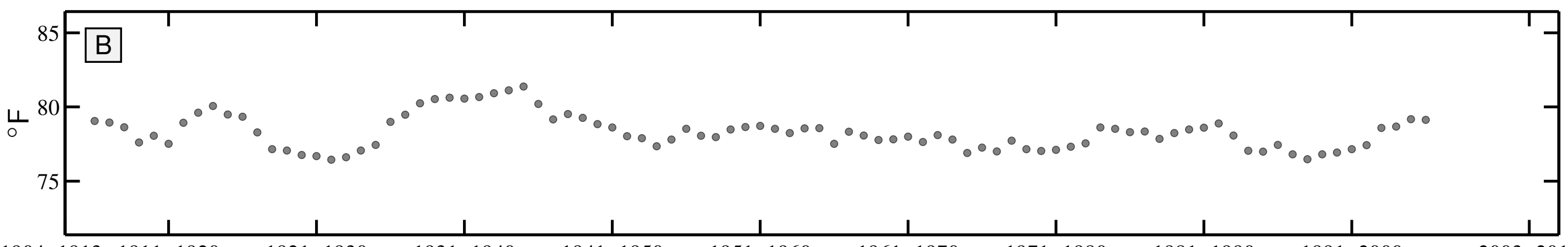

$1904-1913$ 1911-1920

$1921-1930$

$1931-1940$

$1941-1950$

$1951-1960$

$1961-1970$

$1971-1980$

$1981-1990$

$1991-2000$

2003-2012

10 year Winter Teletherm dynamics for NEWBERRY 3S, MI:

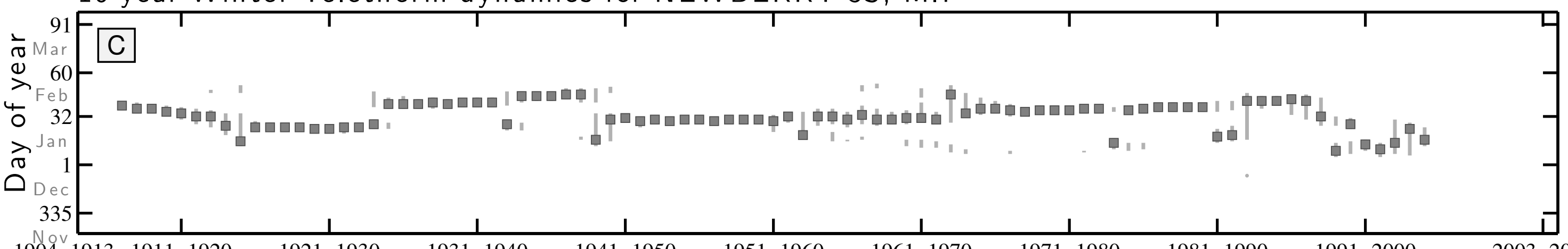

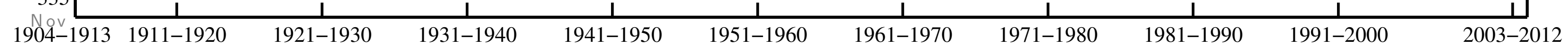

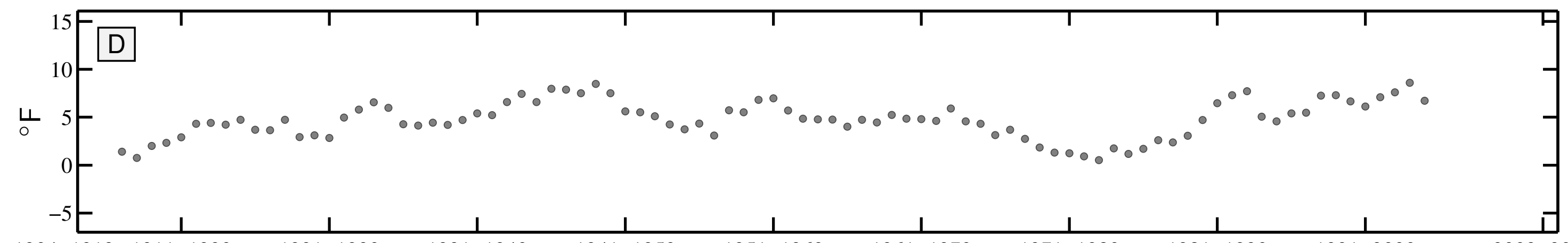


10 year Summer Teletherm dynamics for ALBERT LEA 3 SE, MN:

$+\quad 274$

(1) Sep

$>244-$

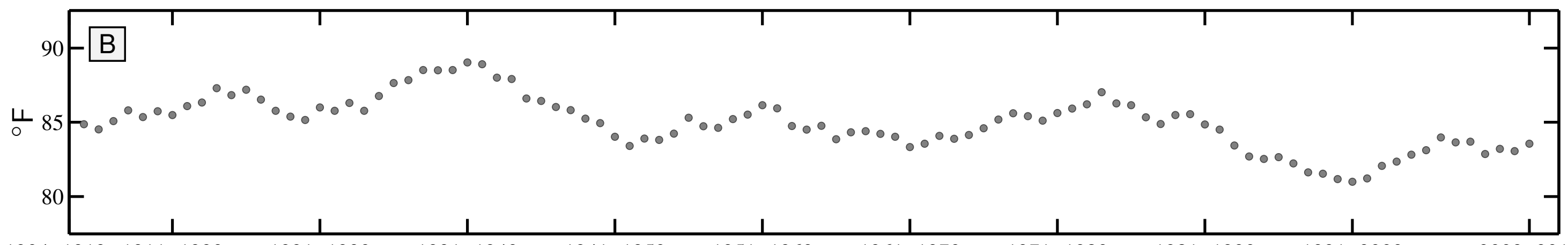

1904-1913 1911-1920

10 year Winter Teletherm dynamics for ALBERT LEA 3 SE, MN:
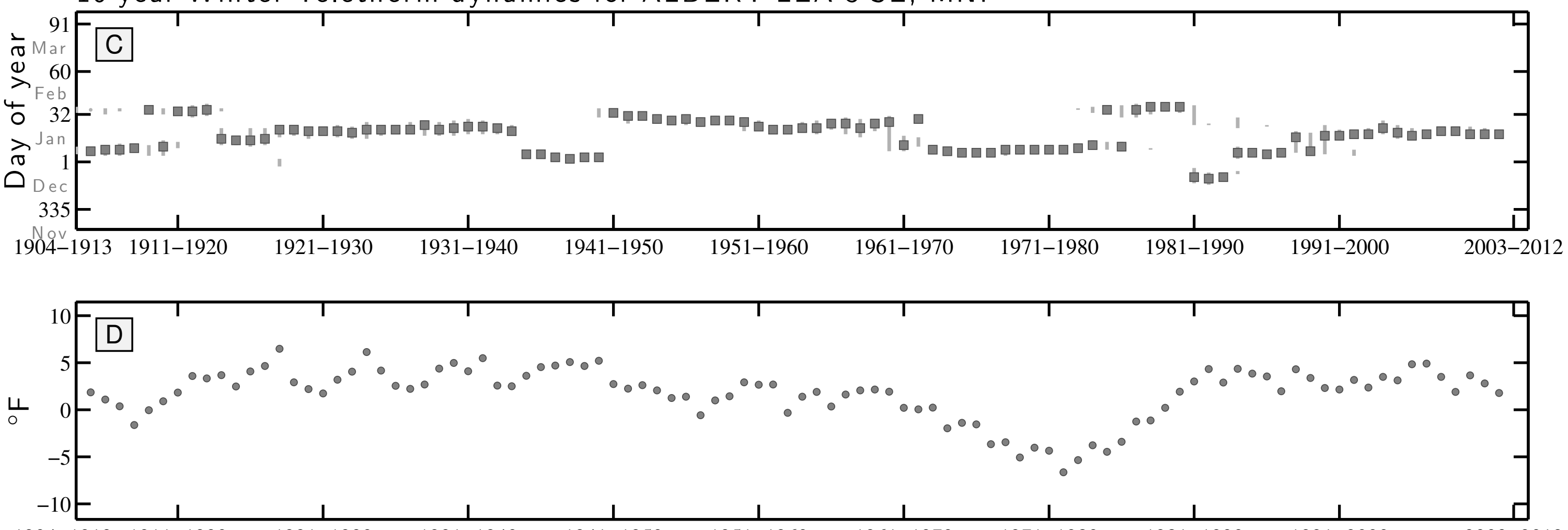
10 year Summer Teletherm dynamics for ARGYLE, MN:

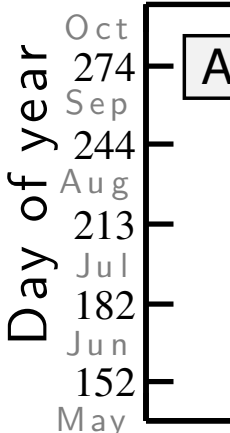
$A$

1904-1913 1911-1920

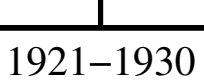

$1931-1940$

$1941-1950$

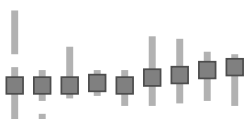
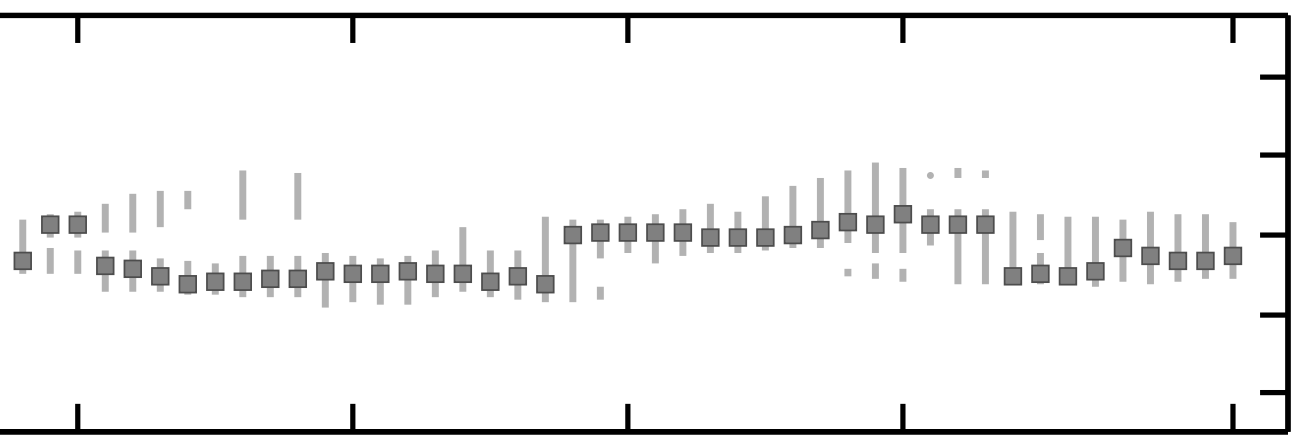

$1951-1960$

$1961-1970$

1971-1980

$1981-1990$

$1991-2000$

2003-2012

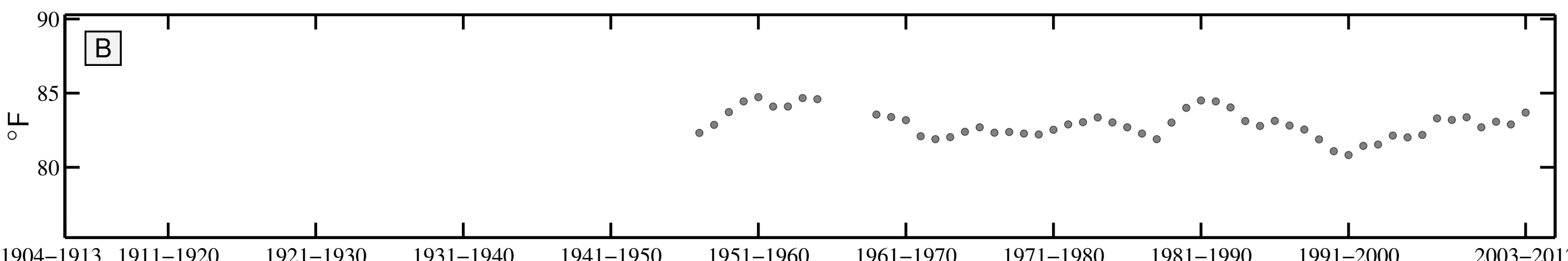

10 year Winter Teletherm dynamics for ARGYLE, MN:
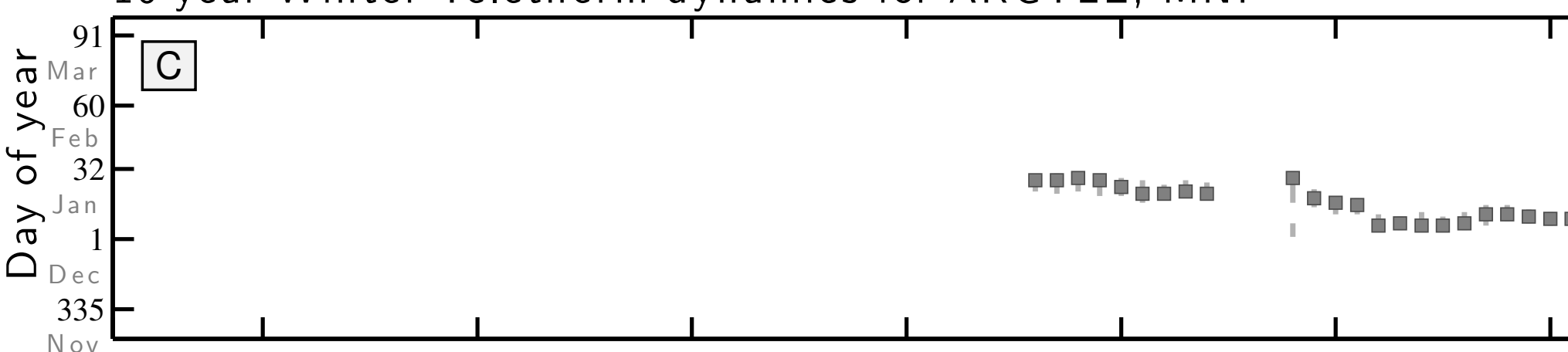

1

1981-1990

1991-2000

2003-2012

1904-1913 1911-1920

1921-1930

1931-1940

$\frac{1}{1941-1950}$

1951-1960

1961-1970

1971-1980
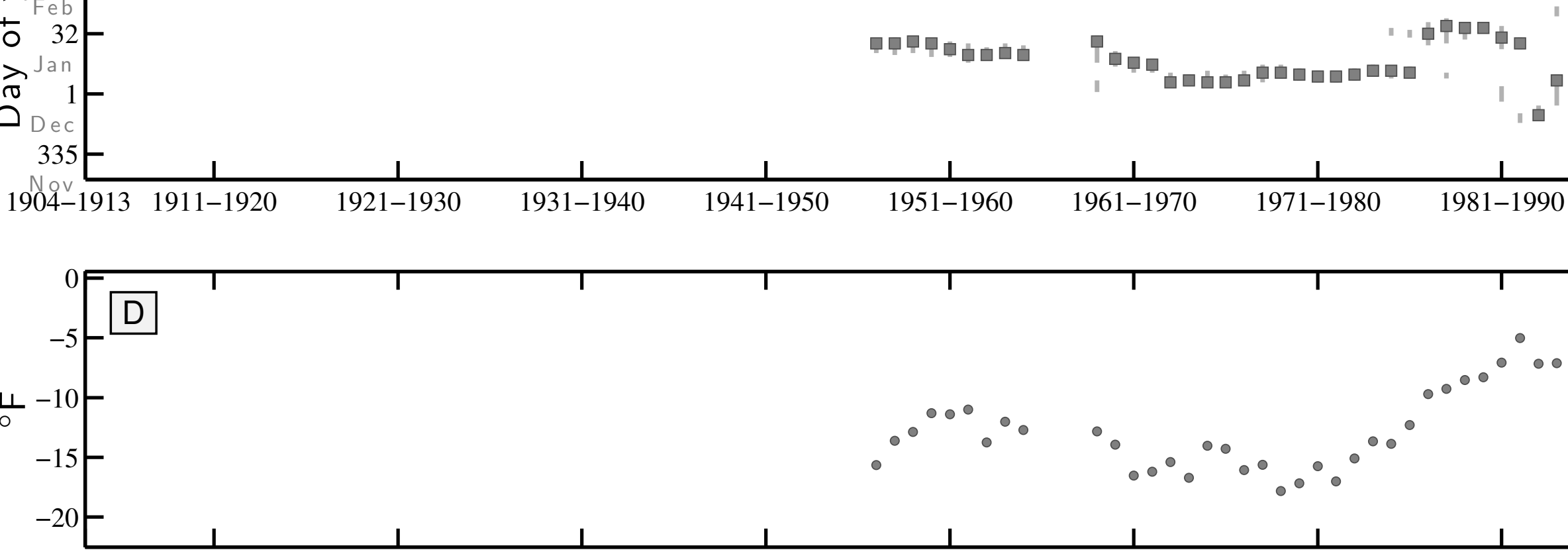

1991-2000

2003-2012 
10 year Summer Teletherm dynamics for CHASKA, MN:
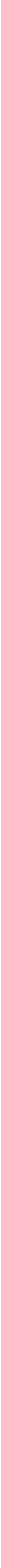
10 year Summer Teletherm dynamics for DETROIT LAKES 1 NNE, MN:

$\div 274-\mathrm{A}$

(1) Sep

$>244-$

잉.

त Jul

$\overparen{\nabla} 182$

$152-$

May $\square$

1904-1913 1911-1920

$\frac{1}{1921-1930}$

$\frac{1}{1931-1940}$

1941-1950

1951-1960

$\frac{1}{1961-1970}$

$\frac{1}{1971-1980}$

$\frac{1}{981-1990}$

$\frac{1}{1991-2000}$

2003-2012

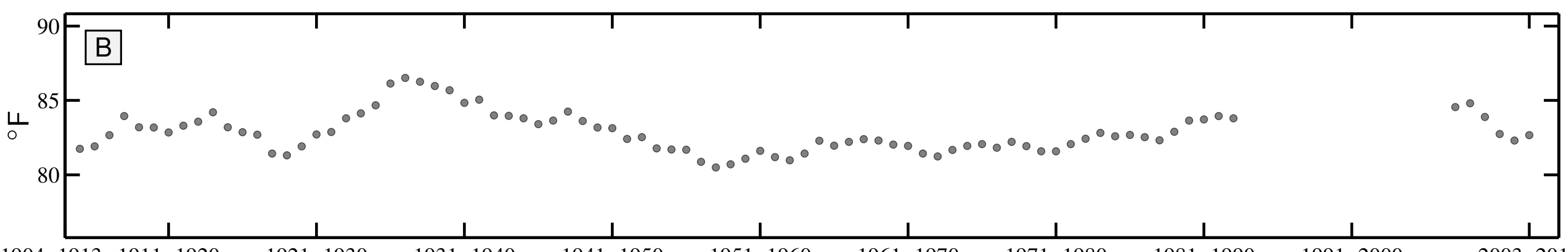

$1904-1913$ 1911-1920

$1921-1930$

$1931-1940$

$1941-1950$

$1951-1960$

$1961-1970$

$1971-1980$

$1981-1990$

$1991-2000$

$2003-2012$

10 year Winter Teletherm dynamics for DETROIT LAKES 1 NNE, MN:

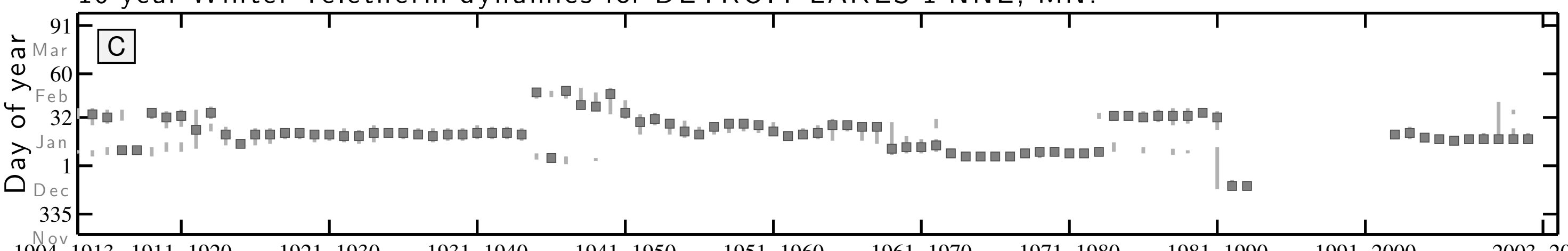

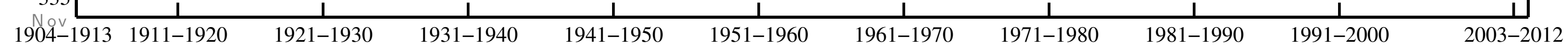

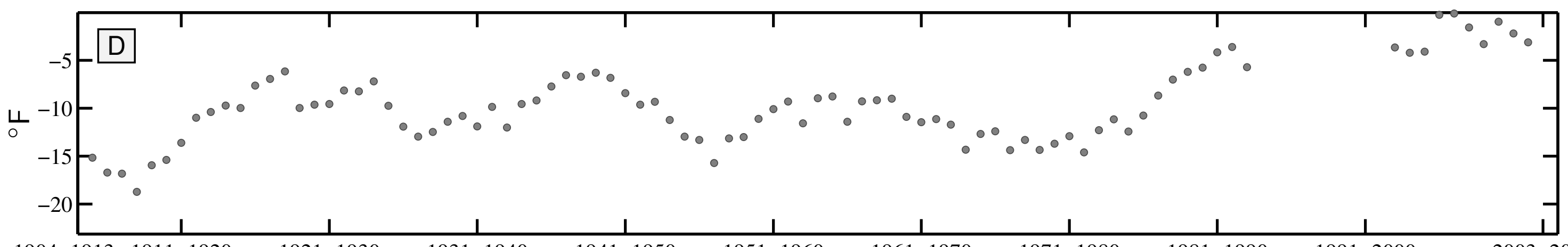


10 year Summer Teletherm dynamics for EVELETH WWTP, MN:
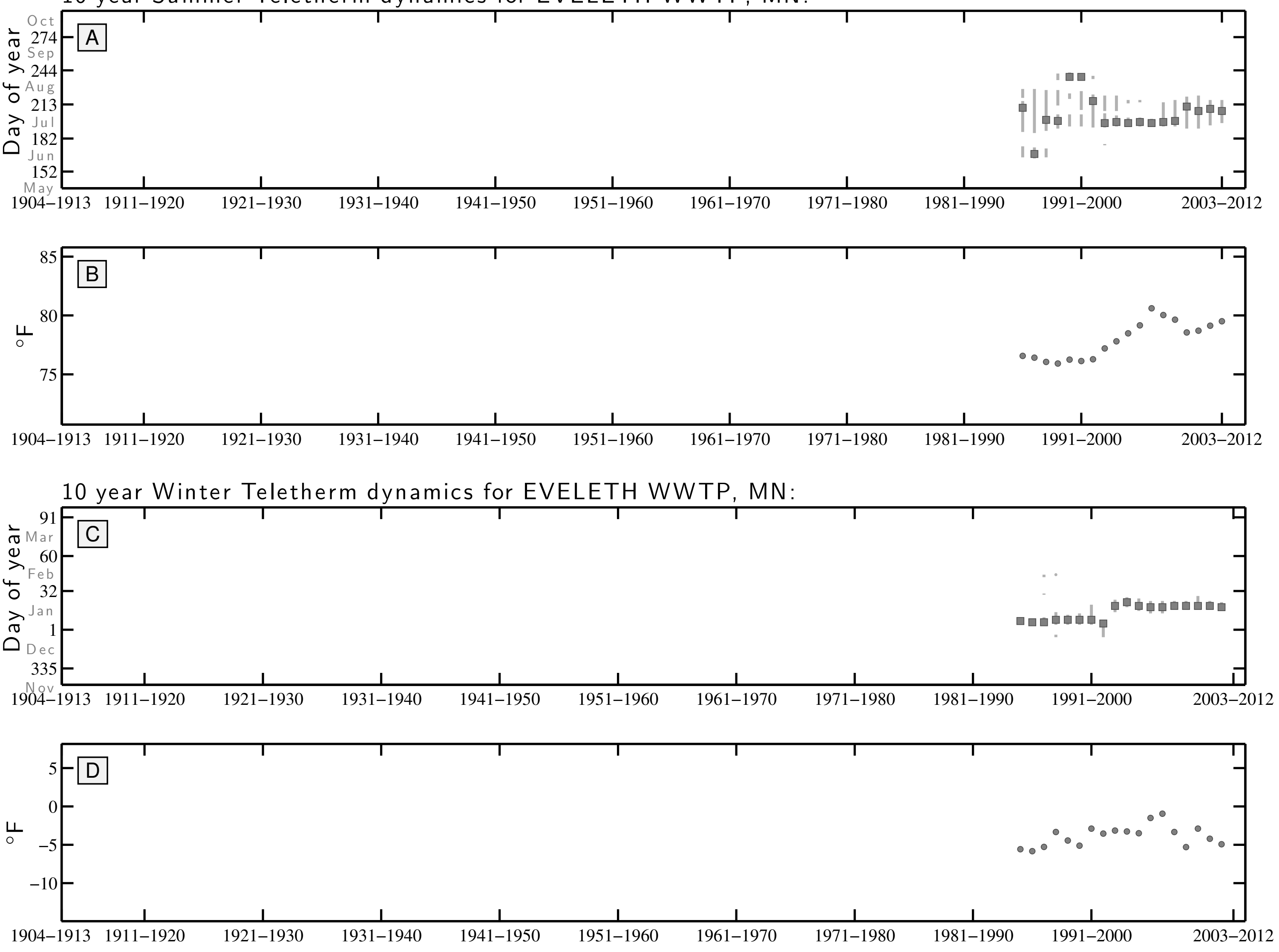
10 year Summer Teletherm dynamics for FOSSTON $1 \mathrm{E}, \mathrm{MN}$ :

$\div 274-A$

(t)

(1) Sep

244
+ Aug

$\begin{array}{ll}0 & 213 \\ > & \text { Jul }\end{array}$

ซ 182

Jun

152

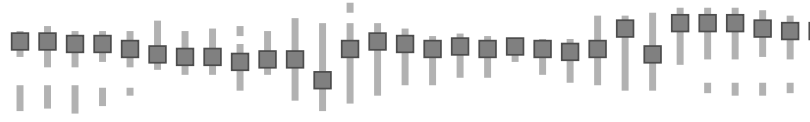

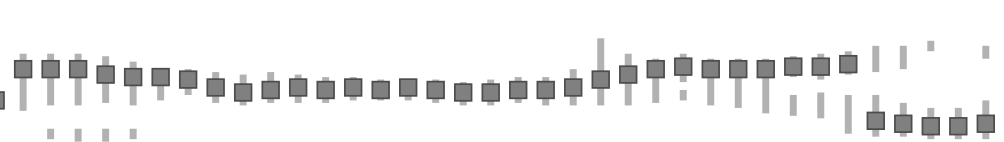

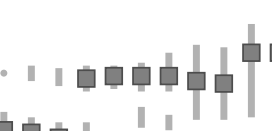

| ㅁㅁ | | |

$1904-1913 \quad 1911-1920$

$\frac{1}{1921-1930}$

$\frac{1}{1-1940}$

$\frac{1}{1941-1950}$

$\frac{1}{1951-1960}$

$\frac{1}{1961-1970}$

$\frac{1}{1971-1980}$

$\frac{1}{1981-1990}$

1991-2000

2003-2012

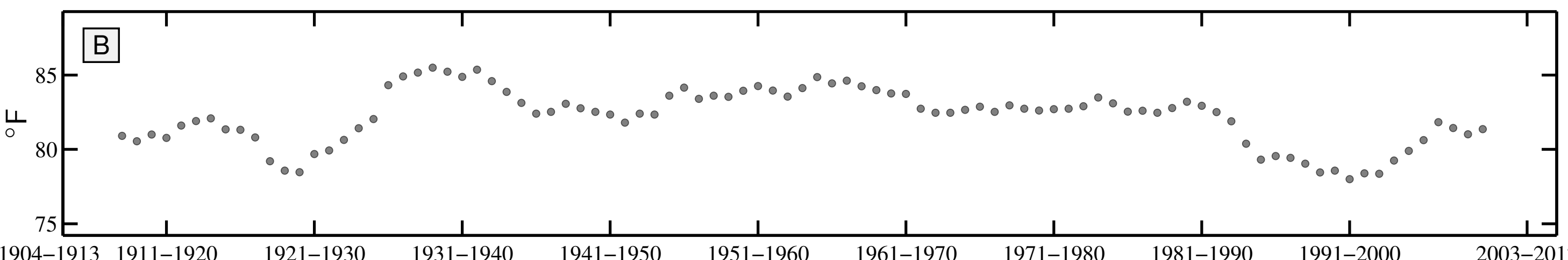

1904-1913 1911-1920

$1921-1930$

$1931-1940$

$1941-1950$

$1951-1960$

$1961-1970$

$1971-1980$

1981-1990

991-2000

$2003-2012$

10 year Winter Teletherm dynamics for FOSSTON $1 \mathrm{E}, \mathrm{MN}$ :

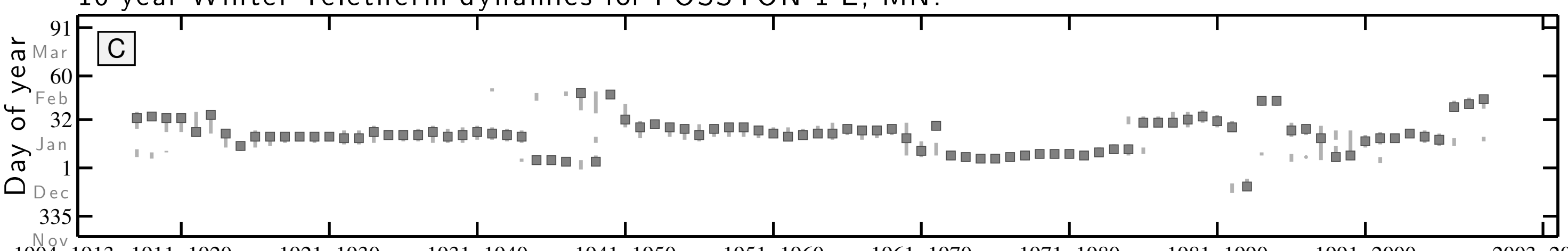

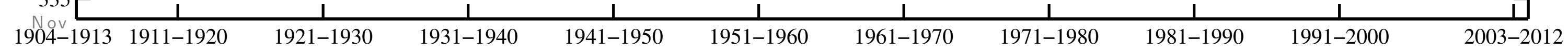

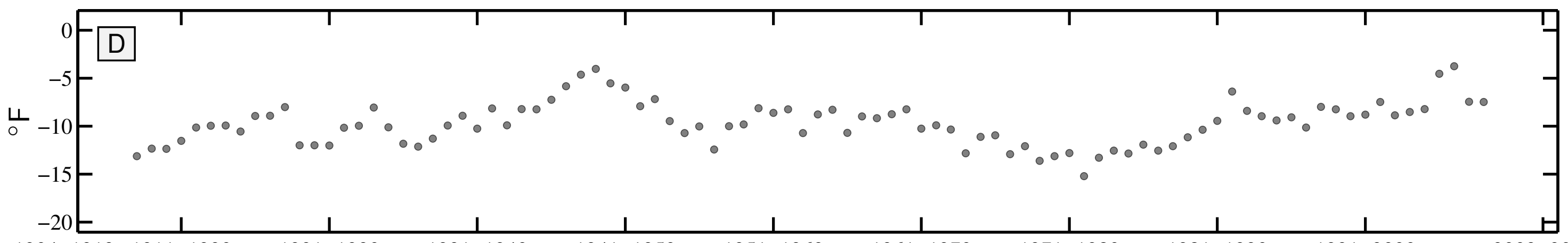


10 year Summer Teletherm dynamics for NEW ULM 2 SE, MN:

$0 c t$
0

A

(1) Sep

$244-$

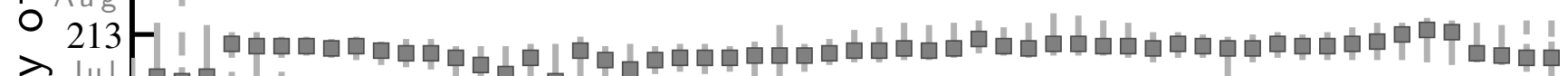

ते Jul

182
Jun

$152-$

May 1

$1904-1913 \quad 1911-1920$

$\frac{1}{1921-1930}$

1931-1940

$\frac{1}{1941-1950}$

1951-1960

$\frac{1}{1961-1970}$

$\frac{1}{1971-1980}$

$\frac{1}{1981-1990}$

991-2000

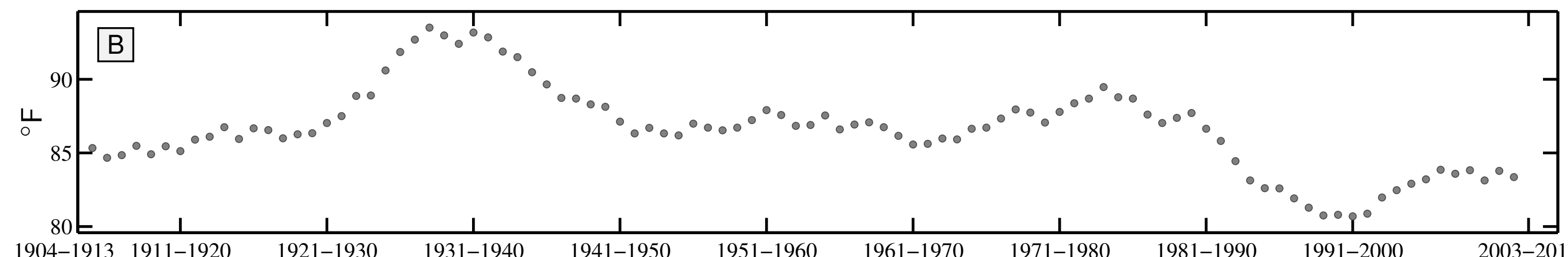

1904-1913 1911-1920

10 year Winter Teletherm dynamics for NEW ULM 2 SE, MN:

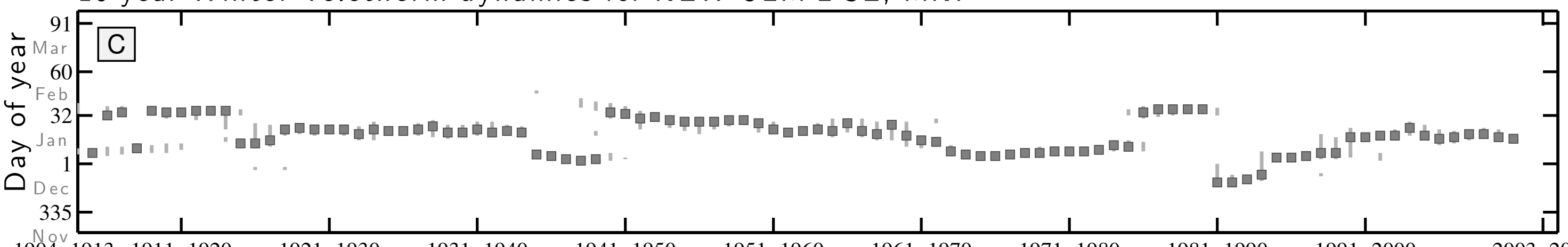

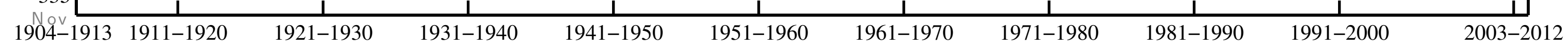

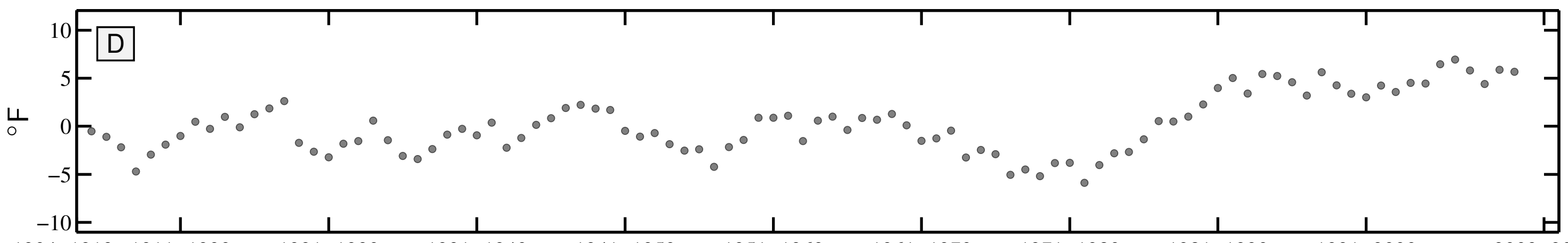


10 year Summer Teletherm dynamics for PINE RIVER DAM, MN:

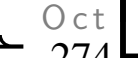

A 274

(1) Sep

$>244$

O 213

$\gtrsim \mathrm{Ju}$

$\overbrace{}^{\sigma} 182$

152 -

152

1904-1913 1911-1920

1921-1930

1931-1940

1941-1950

$\frac{1}{1951-1960}$

1961-1970

$\frac{1}{1971-1980}$

1981-1990

991-2000

2003-2012

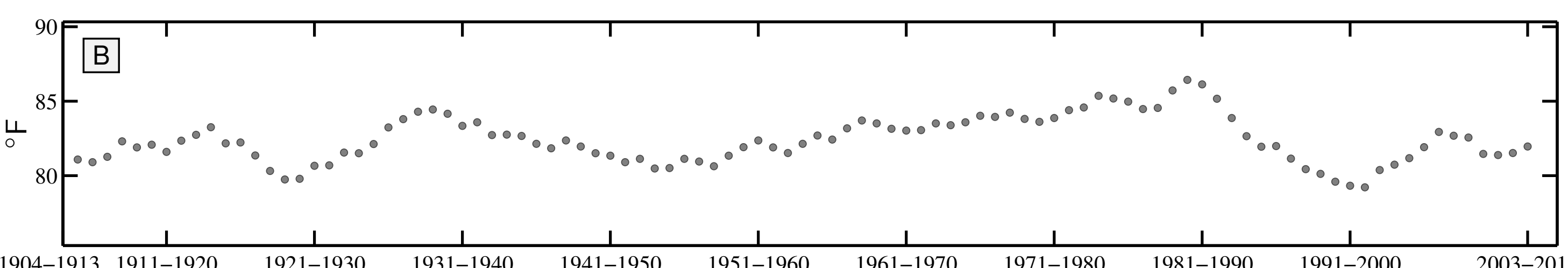

$1904-1913$ 1911-1920

$1921-1930$

$1931-1940$

$1941-1950$

$1951-1960$

$1961-1970$

$1971-1980$

$1981-1990$

$1991-2000$

2003-2012

10 year Winter Teletherm dynamics for PINE RIVER DAM, MN:

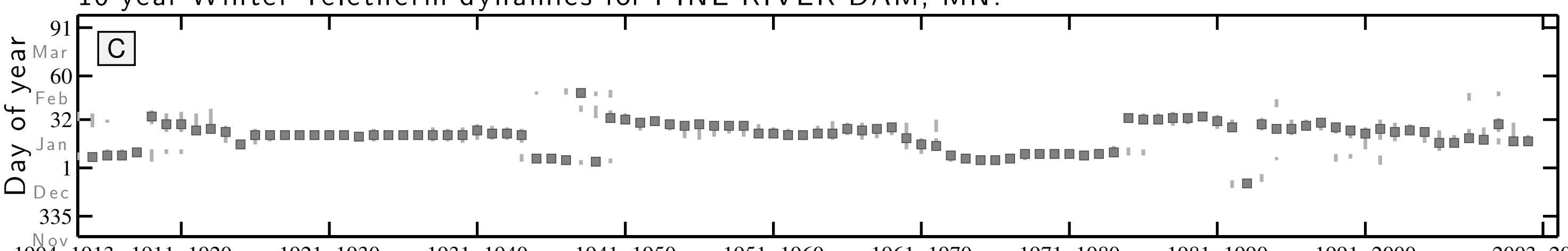

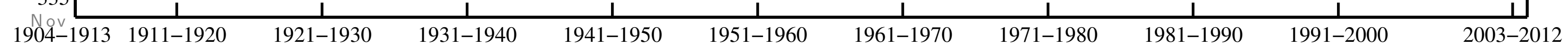

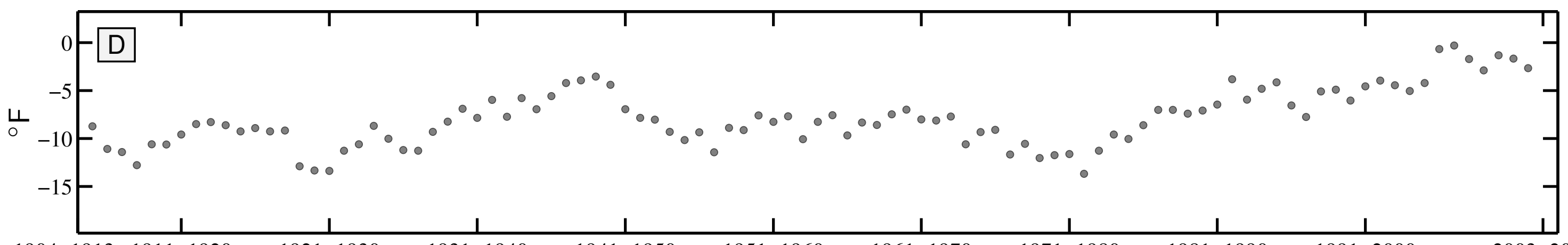


10 year Summer Teletherm dynamics for SANDY LAKE DAM LIBBY, MN:

Oct

A

(1) Sep

244

○ 213

त $\mathrm{Ju}$

ठ 182

152 -

May

1904-1913 1911-1920

1921-1930

1931-1940

1941-1950

1951-1960

1961-1970

1971-1980

1981-1990

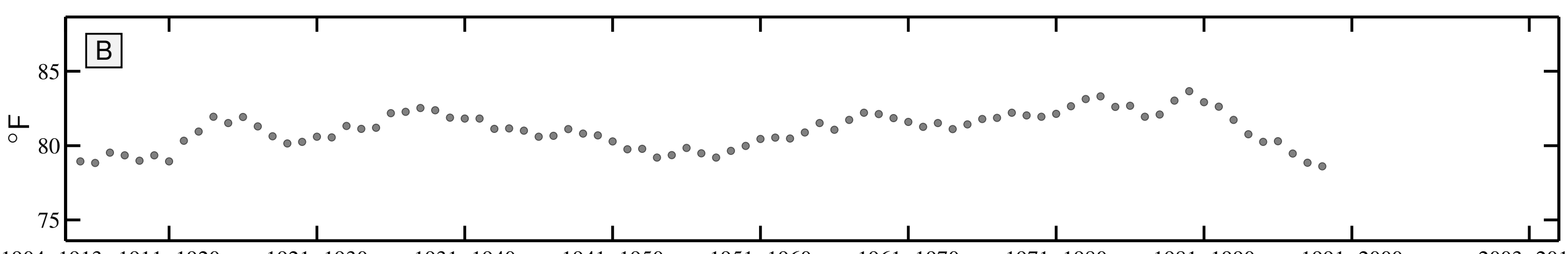

1904-1913 1911-1920

1921-1930

1931-1940

1941-1950

1951-1960

1961-1970

1971-1980

1981-1990

1991-2000

2003-2012

10 year Winter Teletherm dynamics for SANDY LAKE DAM LIBBY, MN:

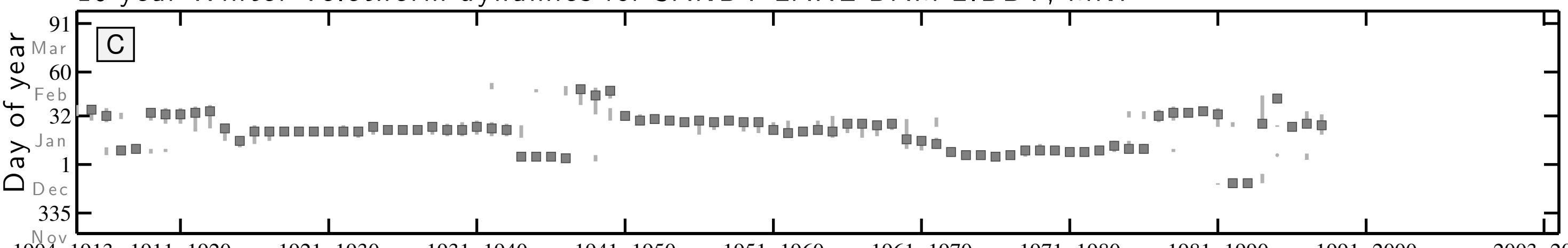

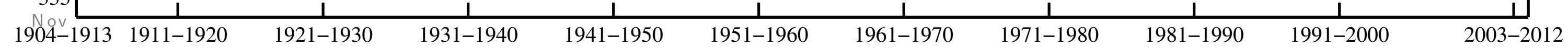

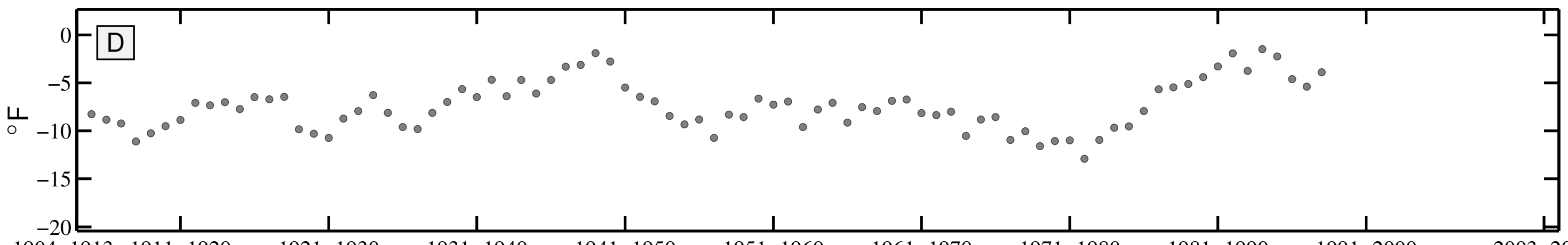


10 year Summer Teletherm dynamics for BATESVILLE 2 SW, MS:

- Oct

(1) Sep A

$>244$

岁 Aug

$\vec{\sigma}$

18

$\mathrm{Jun}$

$152-$

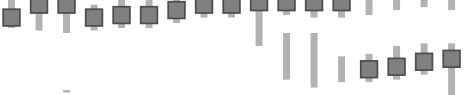

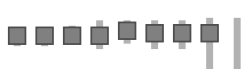

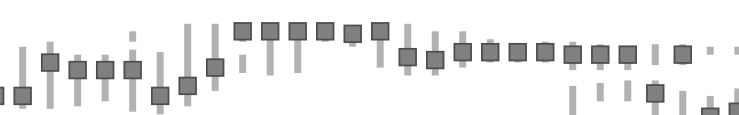

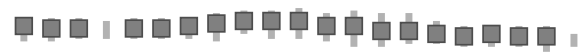

1904-1913 1911-1920

$\frac{1}{1921-1930}$

$1931-1940$

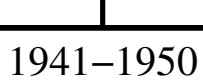

$\frac{1}{1951-1960}$

$1961-1970$

$1971-1980$

$1981-1990$

991-2000

2003-2012

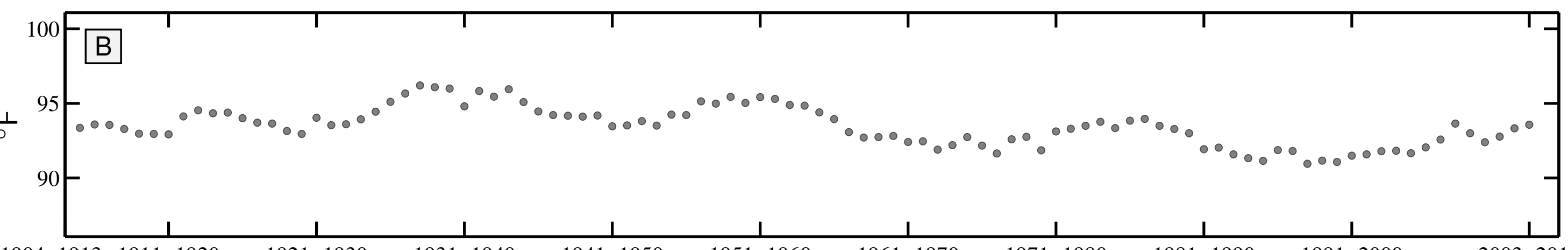

1904-1913 1911-1920

1921-1930

$1931-1940$

1941-1950

1951-1960

1961-1970

1971-1980

$1981-1990$

$1991-2000$

2003-2012

10 year Winter Teletherm dynamics for BATESVILLE 2 SW, MS:

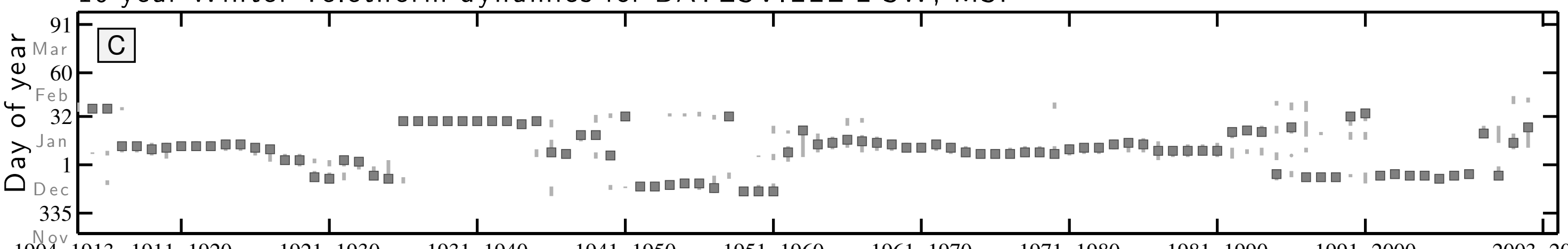

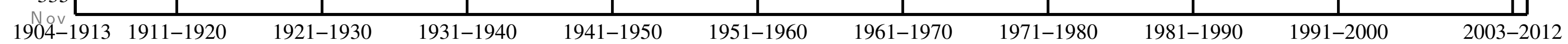

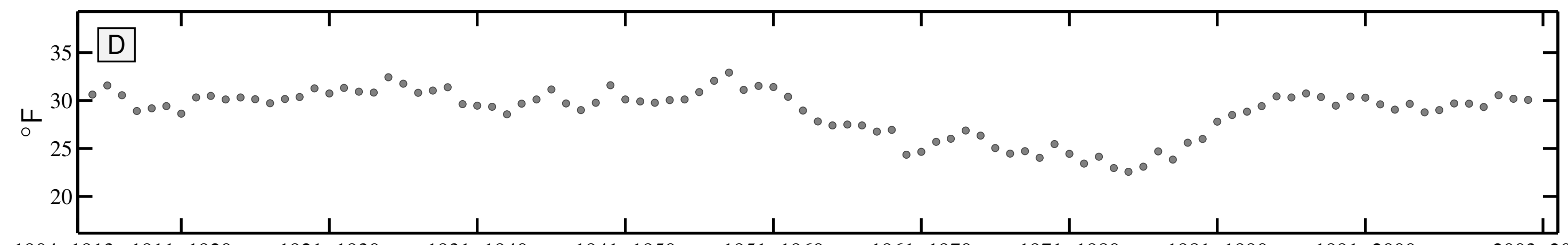


10 year Summer Teletherm dynamics for PORT GIBSON 1 NE, MS:

$\stackrel{0}{\circ} 274$

(1) Sep

$>244-\square \square \square$

213-

त

$\overbrace{\substack{\mathrm{Jun} \\ 1}}^{\mathrm{J}}$

$152-$

152

1904-1913 1911-1920

$\frac{1}{1921-1930}$

$\frac{1}{1931-1940}$

$\frac{1}{1941-1950}$

1951-1960

$\frac{1}{1961-1970}$

971-1980

981-1990

991-2000

2003-2012

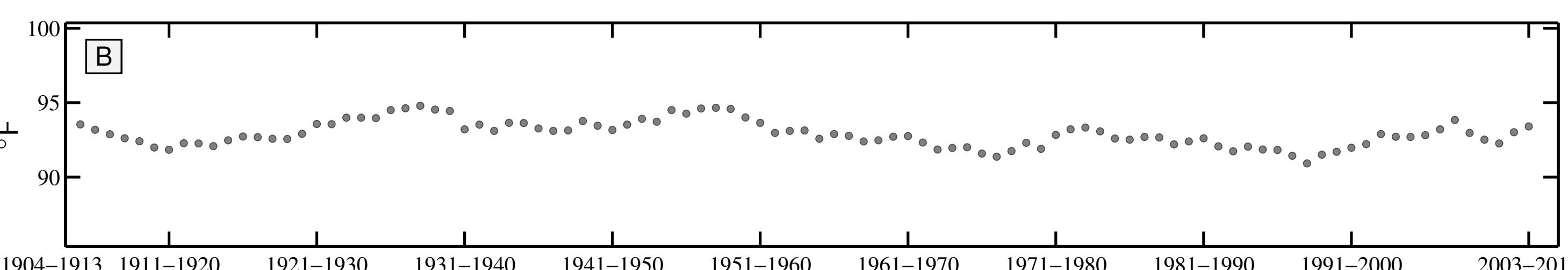

$1904-1913$ 1911-1920

$1921-1930$

$1931-1940$

$1941-1950$

$1951-1960$

$1961-1970$

$1971-1980$

$1981-1990$

$1991-2000$

2003-2012

10 year Winter Teletherm dynamics for PORT GIBSON 1 NE, MS:

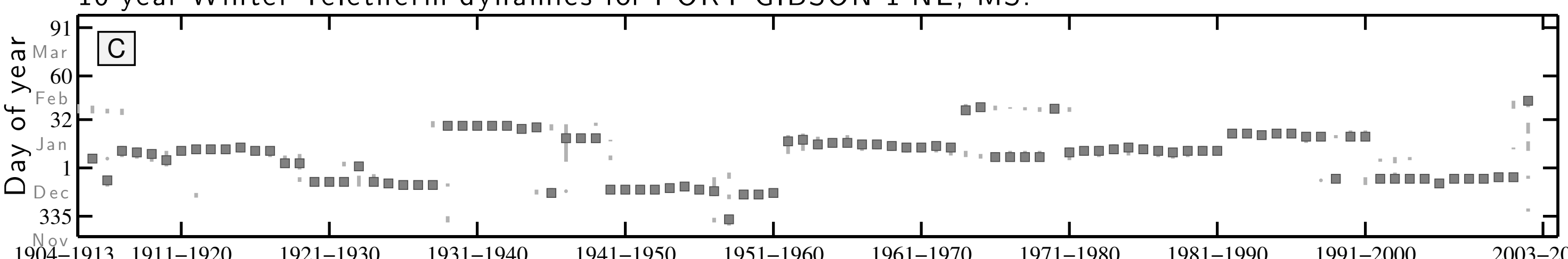

1904-1913 1911-1920

$1921-1930$

$1931-1940$

$1941-1950$

$1951-1960$

1961-1970

1971-1980

1981-1990

991-2000

003-2012

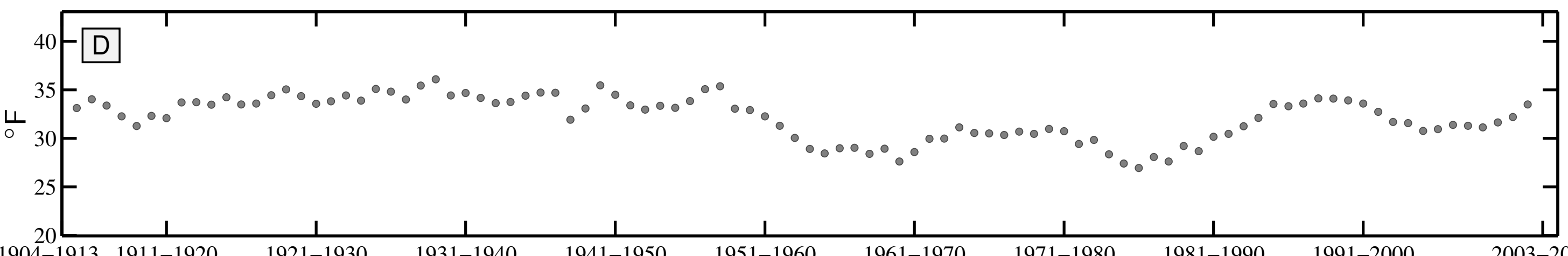


10 year Summer Teletherm dynamics for BOWLING GREEN $1 \mathrm{E}, \mathrm{MO}$ :
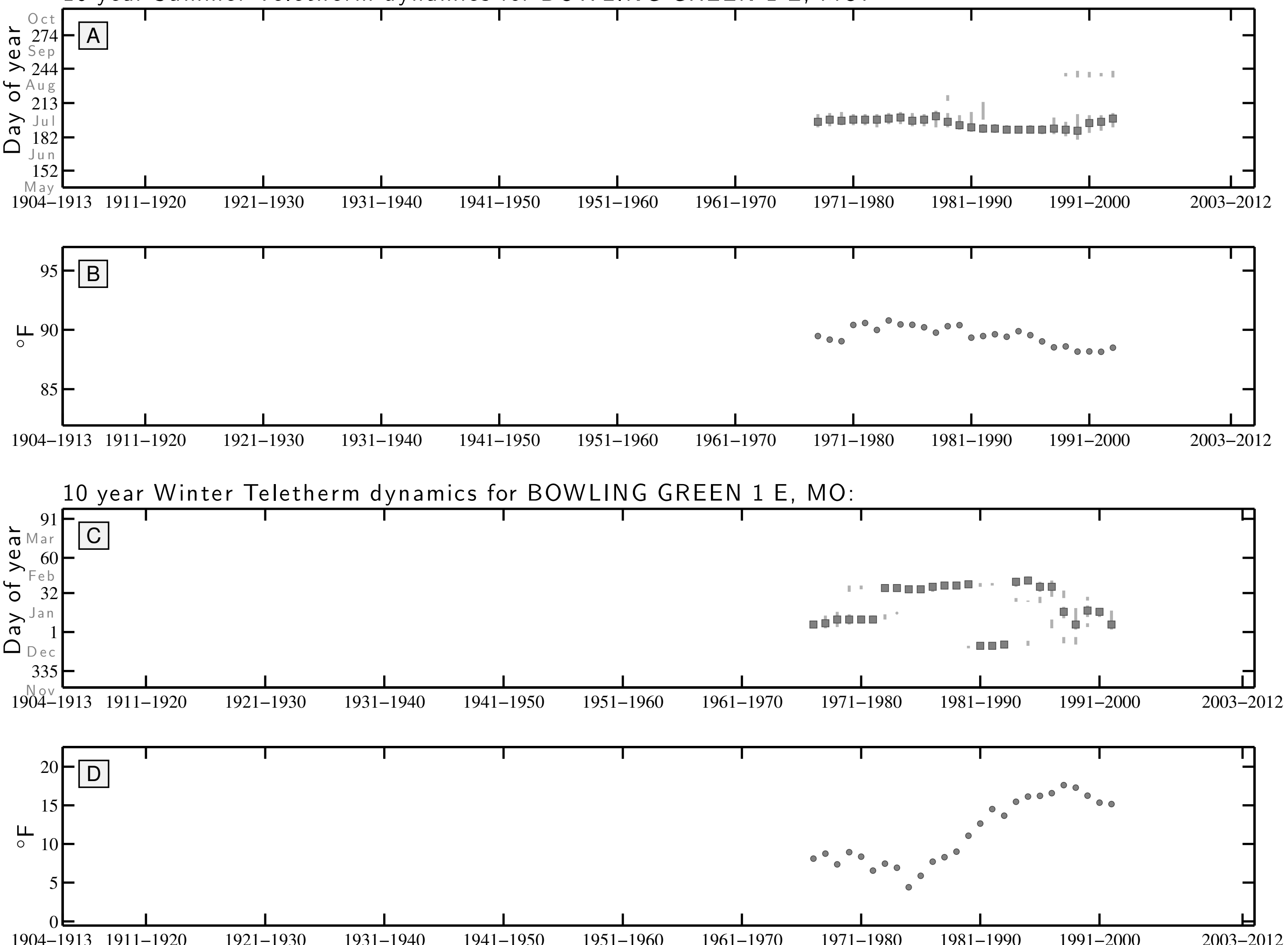

$\begin{array}{llllllllll}1904-1913 & 1911-1920 & 1921-1930 & 1931-1940 & 1941-1950 & 1951-1960 & 1961-1970 & 1971-1980 & 1981-1990 & 1991-2000\end{array}$


10 year Summer Teletherm dynamics for CONCEPTION, MO:

$\div$ Oct 274

Sep

$\stackrel{\text { U Sep }}{>} 244$

4 Aug $\square \square \square \square$

○ 213 - 13

त $\mathrm{Ju}$

○ $182-$

$152-$

May —

1904-1913 1911-1920

$\frac{1}{1921-1930}$

$\frac{1}{1931-1940}$

$\frac{1}{1941-1950}$

$\frac{1}{1951-1960}$

$\frac{1}{1961-1970}$

$\frac{1}{1971-1980}$

$\frac{1}{1981-1990}$

991-2000

2003-2012

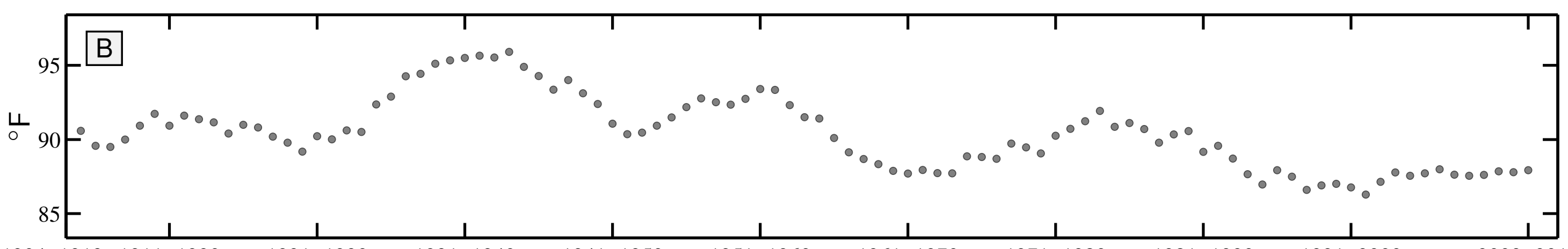

1904-1913 1911-1920

$1921-1930$

$1931-1940$

$1941-1950$

$1951-1960$

$1961-1970$

$1971-1980$

$1981-1990$

$1991-2000$

$2003-2012$

10 year Winter Teletherm dynamics for CONCEPTION, MO:

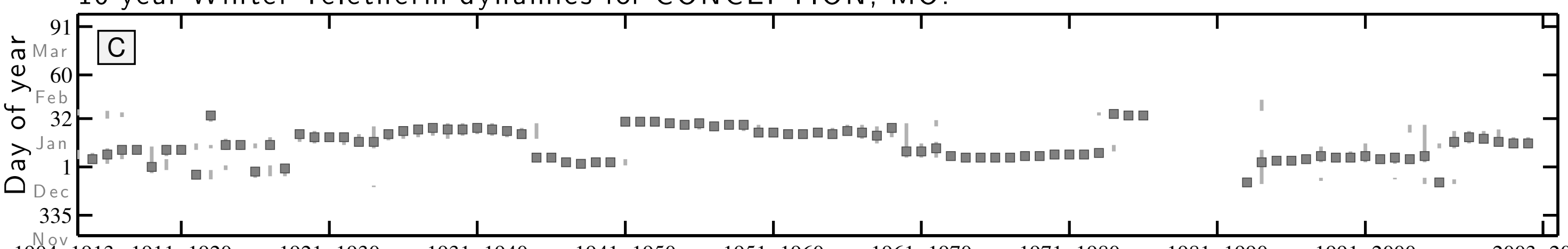

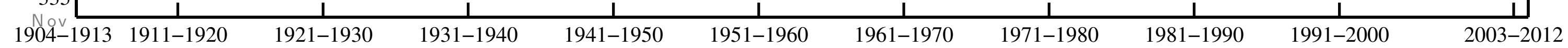

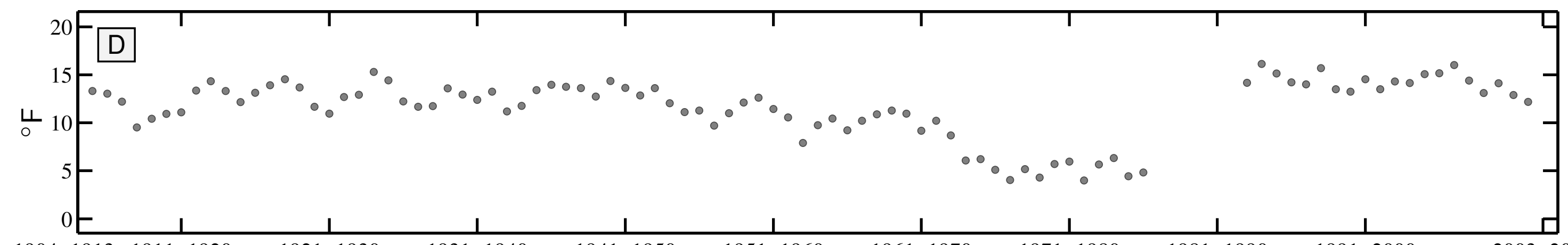


10 year Summer Teletherm dynamics for JEFFERSON CITY WTP, MO:

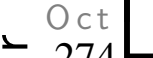

A

$>244-$

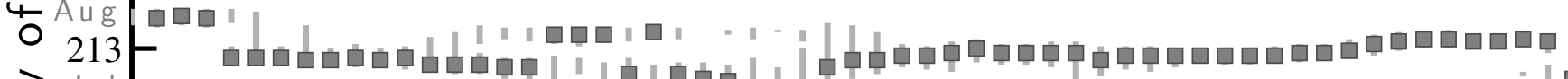

ते Ju

$\overbrace{}^{\circ} 182$

$152-$

152

1904-1913 1911-1920

$\frac{1}{1921-1930}$

$\frac{1}{1931-1940}$

$\frac{1}{1941-1950}$

$\frac{1}{1951-1960}$

$\frac{1}{1961-1970}$

1971-1980

$\frac{1}{1981-1990}$

1991-2000

2003-2012

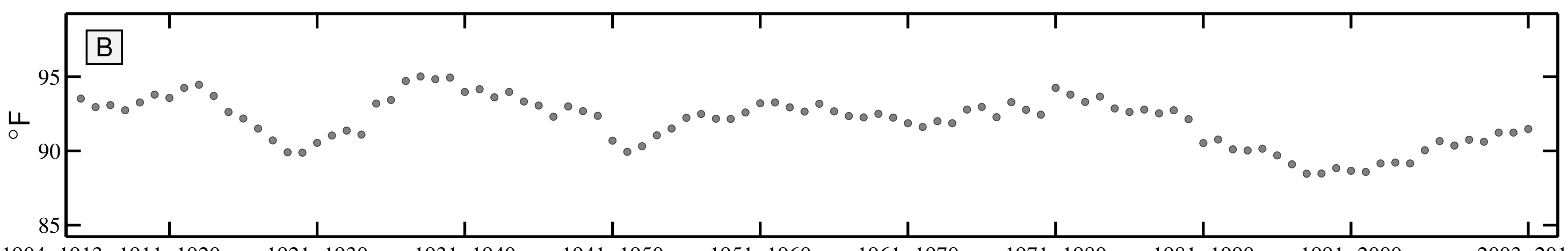

1904-1913 1911-1920

$1921-1930$

$1931-1940$

$1941-1950$

$1951-1960$

$1961-1970$

$1971-1980$

$1981-1990$

$1991-2000$

$2003-2012$

10 year Winter Teletherm dynamics for JEFFERSON CITY WTP, MO:

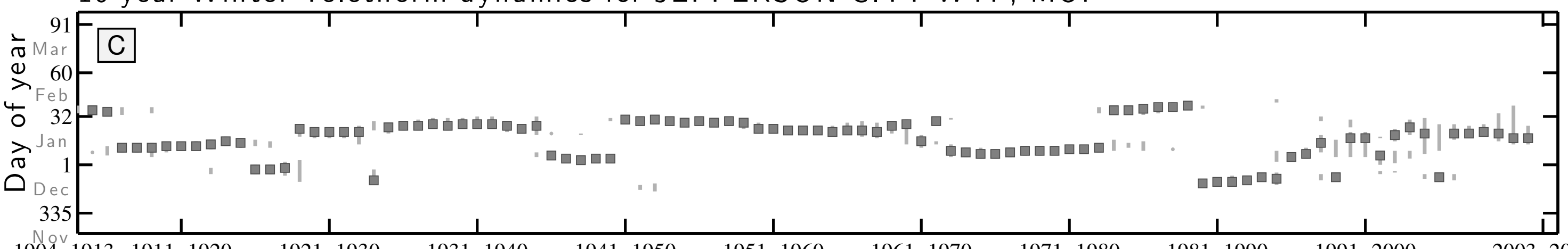

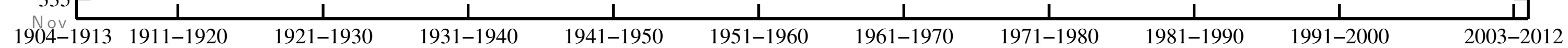

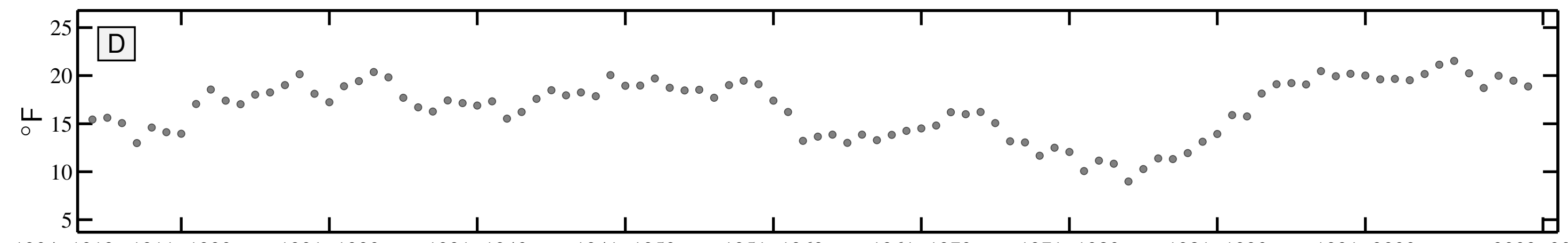


10 year Summer Teletherm dynamics for LEXINGTON 3E, MO:

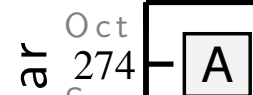

(1) Sep

ᄂ Aug 244

213

$213-$
Jul

$\overbrace{}^{\circ} 182-$

$152-$

|

lay

1904-1913 1911-1920

$\frac{1}{1921-1930}$

$\frac{1}{1931-1940}$

$\frac{1}{1941-1950}$

$\frac{1}{1951-1960}$

$\frac{1}{1961-1970}$

$\frac{1}{971-1980}$

$\frac{1}{981-1990}$

991-2000

2003-2012

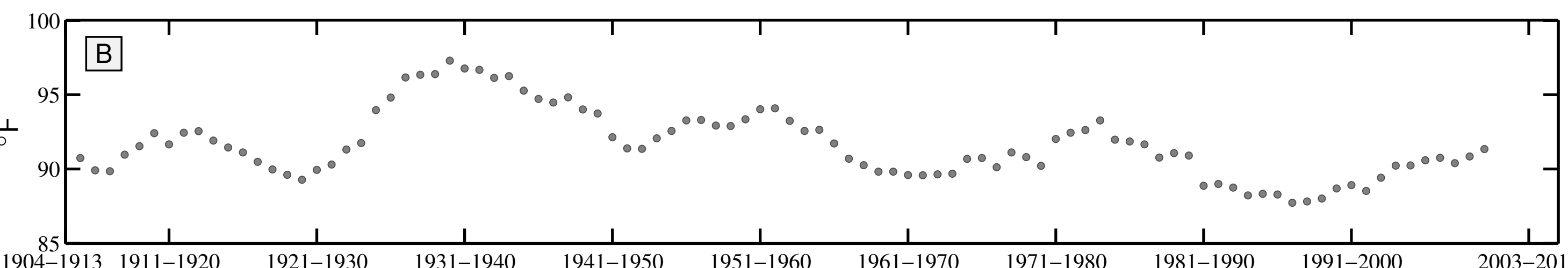

1904-1913 1911-1920

1921-1930

1931-1940

$1941-1950$

1951-1960

1961-1970

1971-1980

$1981-1990$

991-2000

$2003-2012$

10 year Winter Teletherm dynamics for LEXINGTON 3E, MO:

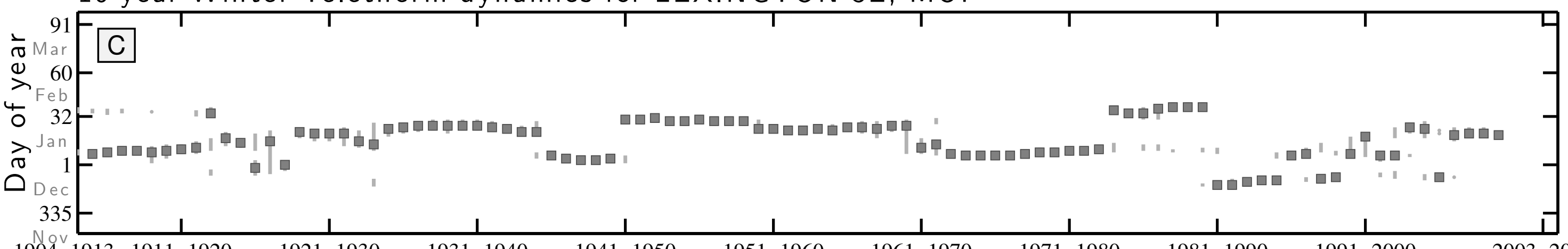

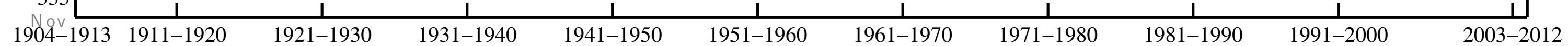

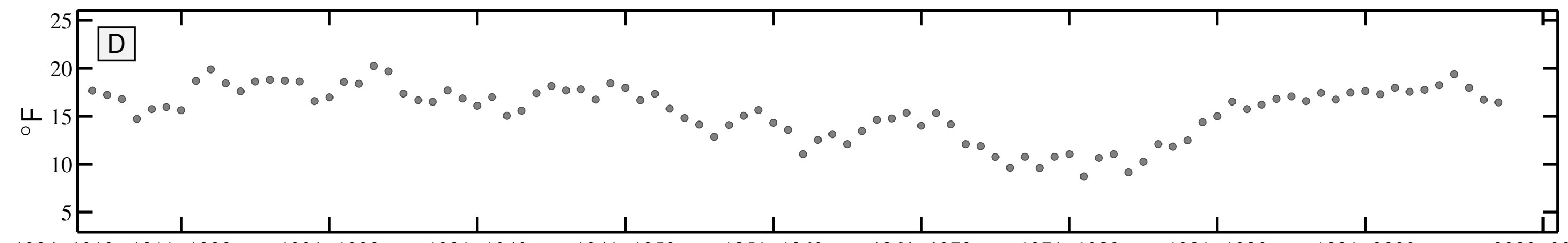


10 year Summer Teletherm dynamics for MARBLE HILL, MO:
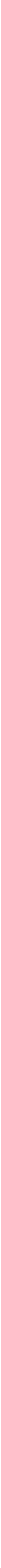

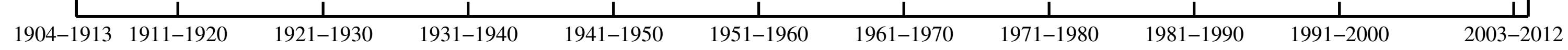


10 year Summer Teletherm dynamics for MEXICO, MO:
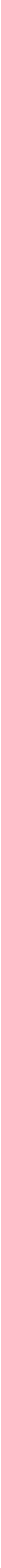

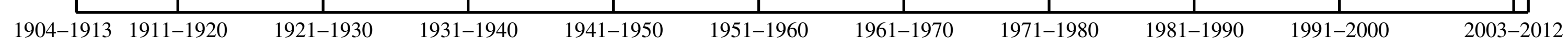




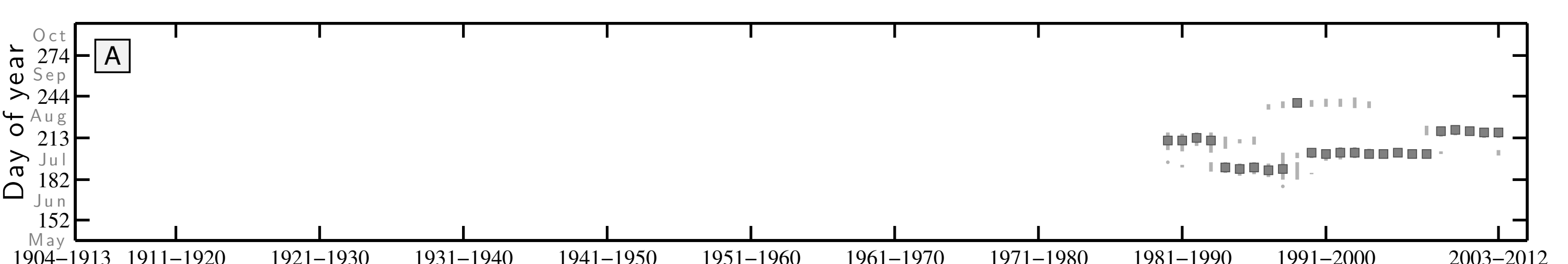

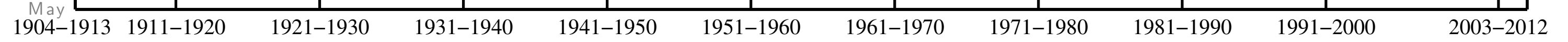
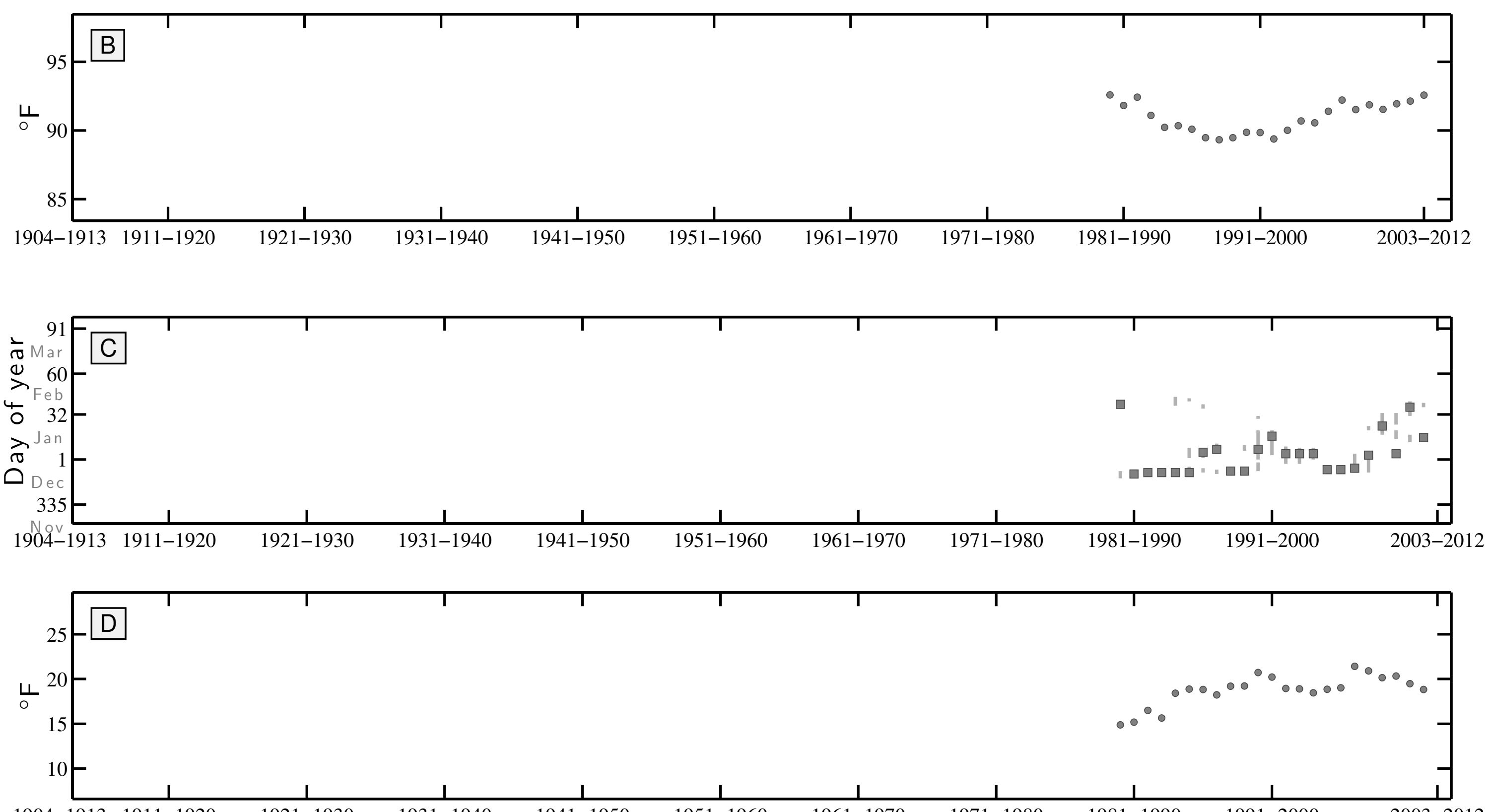

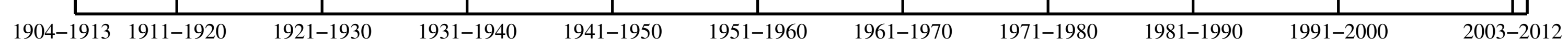


10 year Summer Teletherm dynamics for DILLON WMCE, MT:

$\div 274-A$

(a)

(1) Sep

$>244-$

范 Aug 213

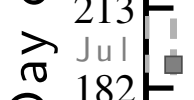

$\mathrm{J} u \mathrm{n}$

152

1904-1913 1911-1920

$\frac{1}{1921-1930}$

1931-1940

1941-1950

951-1960

1961-1970

$\frac{1}{971-1980}$

$\frac{1}{1981-1990}$

1991-2000

2003-2012

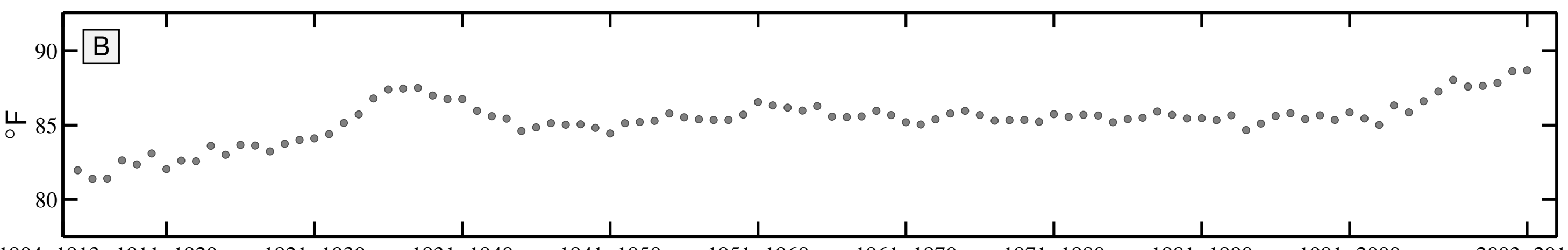

$1904-1913$ 1911-1920

$1921-1930$

$1931-1940$

$1941-1950$

$1951-1960$

$1961-1970$

$1971-1980$

$1981-1990$

$1991-2000$

$2003-2012$

10 year Winter Teletherm dynamics for DILLON WMCE, MT:

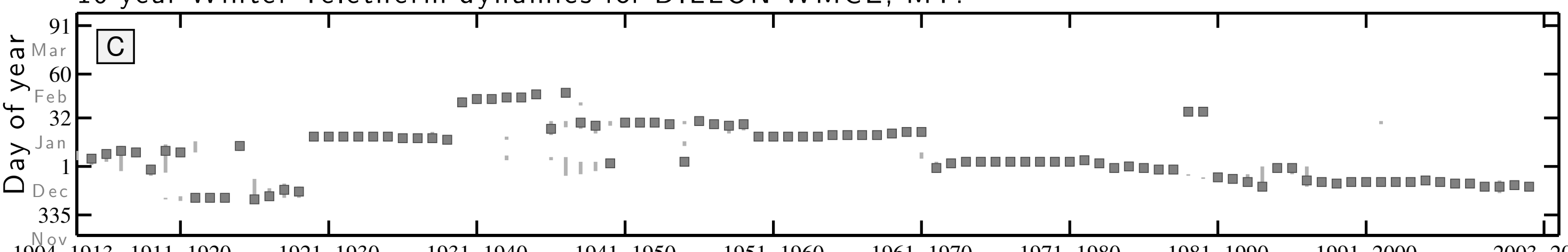

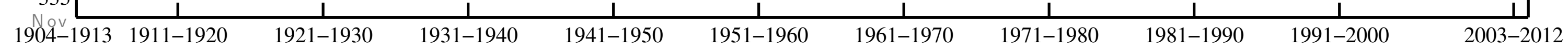

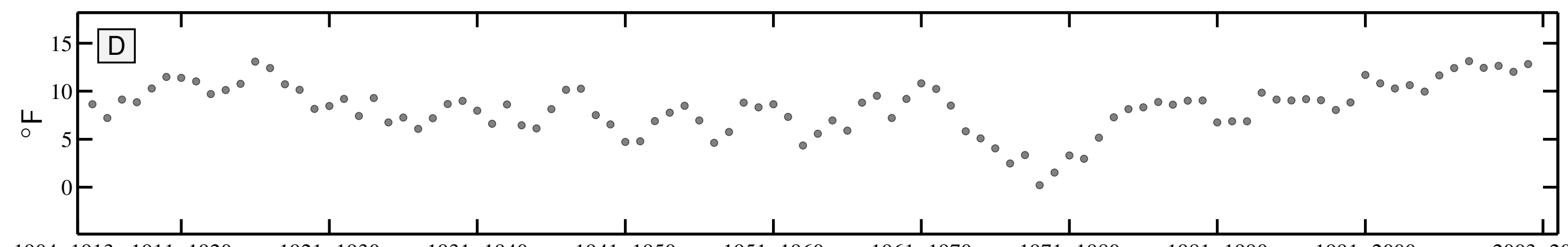


10 year Summer Teletherm dynamics for FORKS 4 NNE, MT:

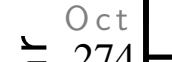

274- A

(1) Sep

$>244-$

능

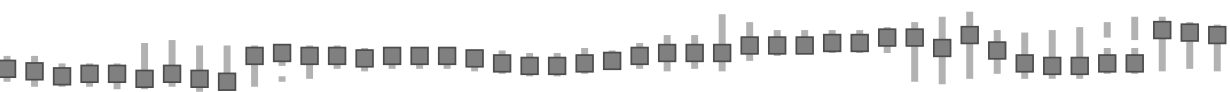

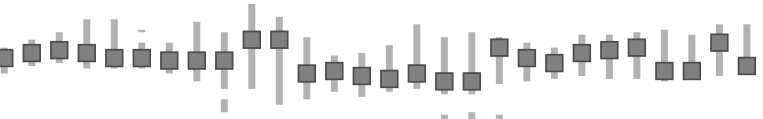

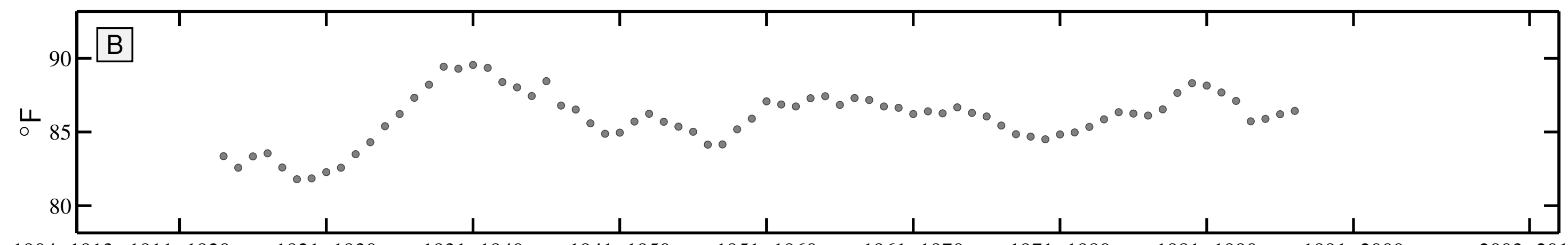

10 year Winter Teletherm dynamics for FORKS 4 NNE, MT:

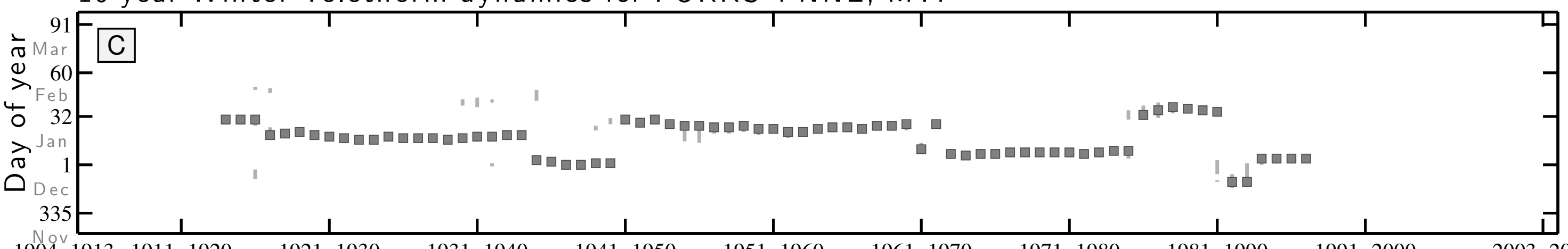

1904-1913 1911-192

1921-1930

1931-1940

1941-1950

1951-1960

1961-1970

1971-1980

1981-1990

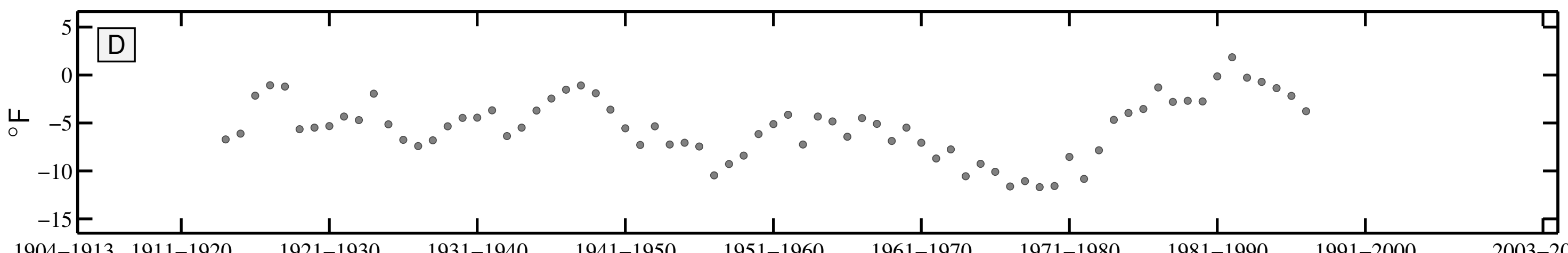


10 year Summer Teletherm dynamics for LIBBY 1 NE RS, MT:

$\therefore c t$

A

(1) Sep

$>244-$

- Aug
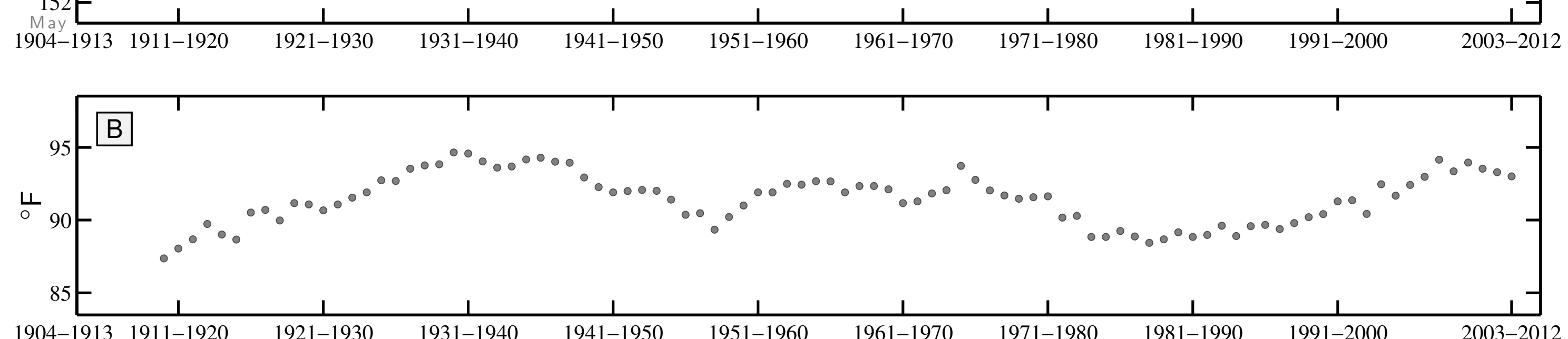

1904-1913 1911-1920

$1921-1930$

$1931-1940$

$1941-1950$

$1951-1960$

$1961-1970$

$1971-1980$

$1981-1990$

$1991-2000$

$2003-2012$

10 year Winter Teletherm dynamics for LIBBY 1 NE RS, MT:

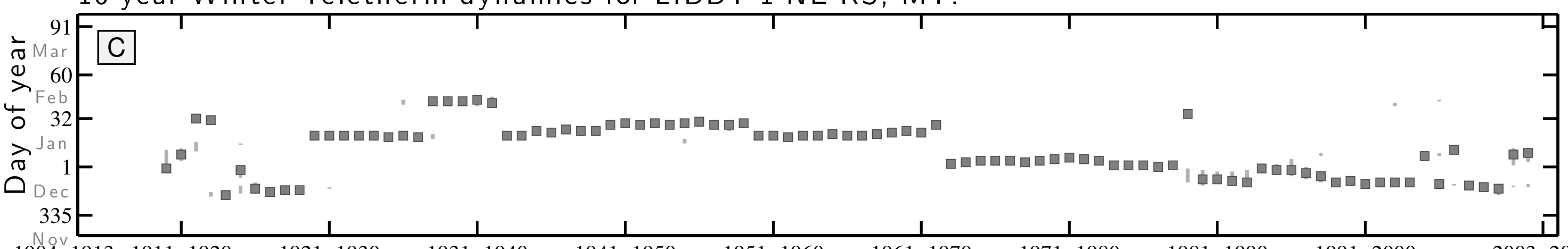

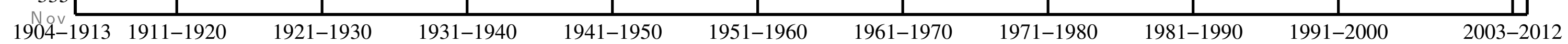

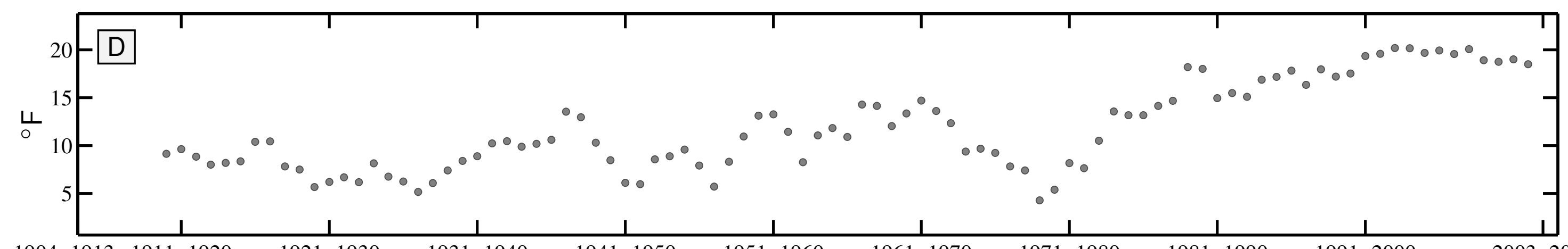


10 year Summer Teletherm dynamics for NORRIS MADISON PH, MT:

$\div$ Oct 274

更

(1) Sep

$>244-$

4 A u

\ Jul

$\overbrace{}^{\circ} 182-$

$152-$

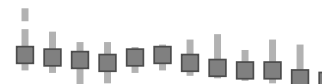

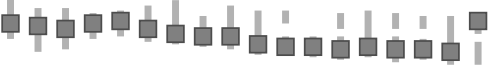

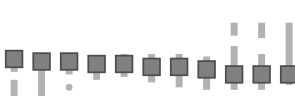

|

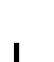

$-1920$

$\frac{1}{1921-1930}$

$\frac{1}{1931-1940}$

$\frac{1}{1941-1950}$

1951-1960

$\frac{1}{1961-1970}$

$\frac{1}{1971-1980}$

$\frac{1}{1981-1990}$

1991-2000

2003-2012

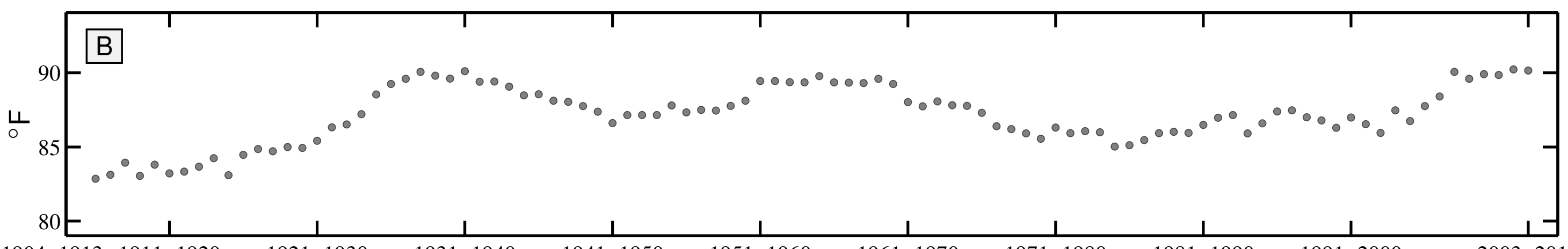

1904-1913 1911-1920

1921-1930

1931-1940

1941-1950

1951-1960

1961-1970

1971-1980

1981-1990

1991-2000

2003-2012

10 year Winter Teletherm dynamics for NORRIS MADISON PH, MT:
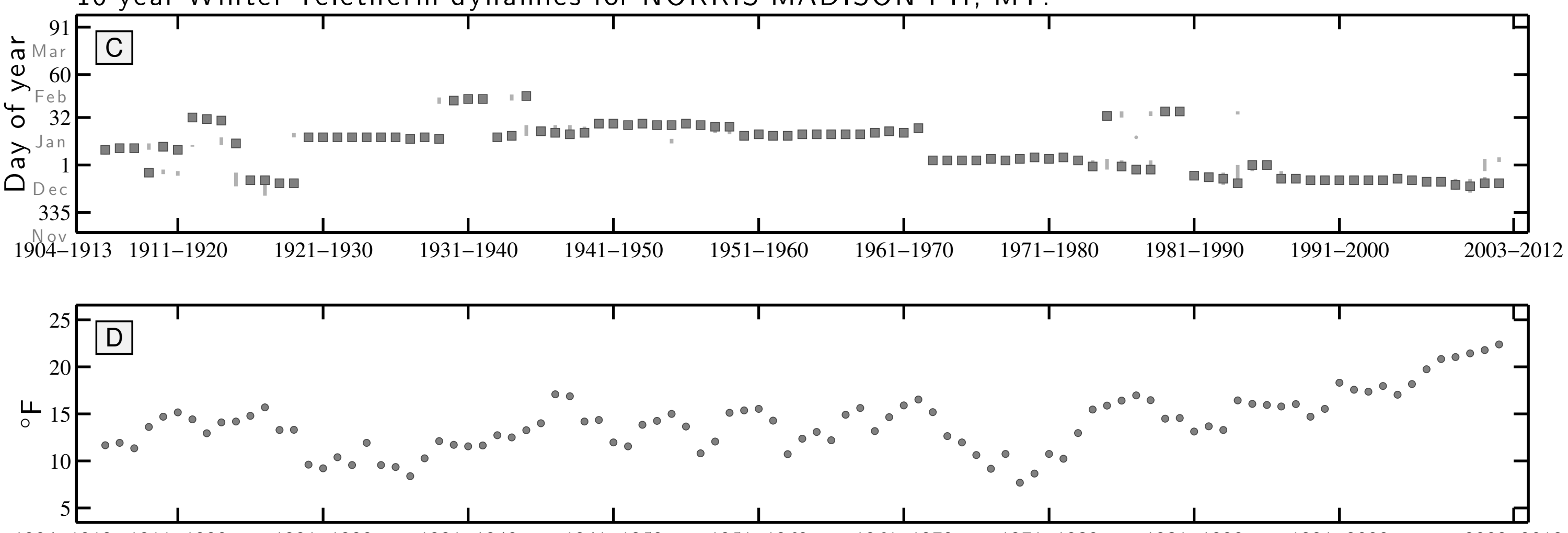
10 year Summer Teletherm dynamics for PHILIPSBURG RS, MT:
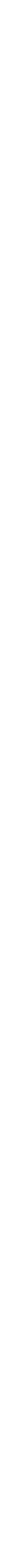

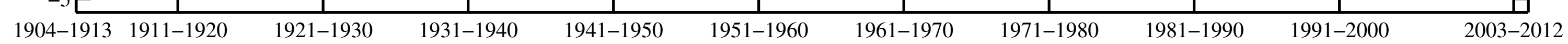


10 year Summer Teletherm dynamics for ATKINSON 3SW, NE:
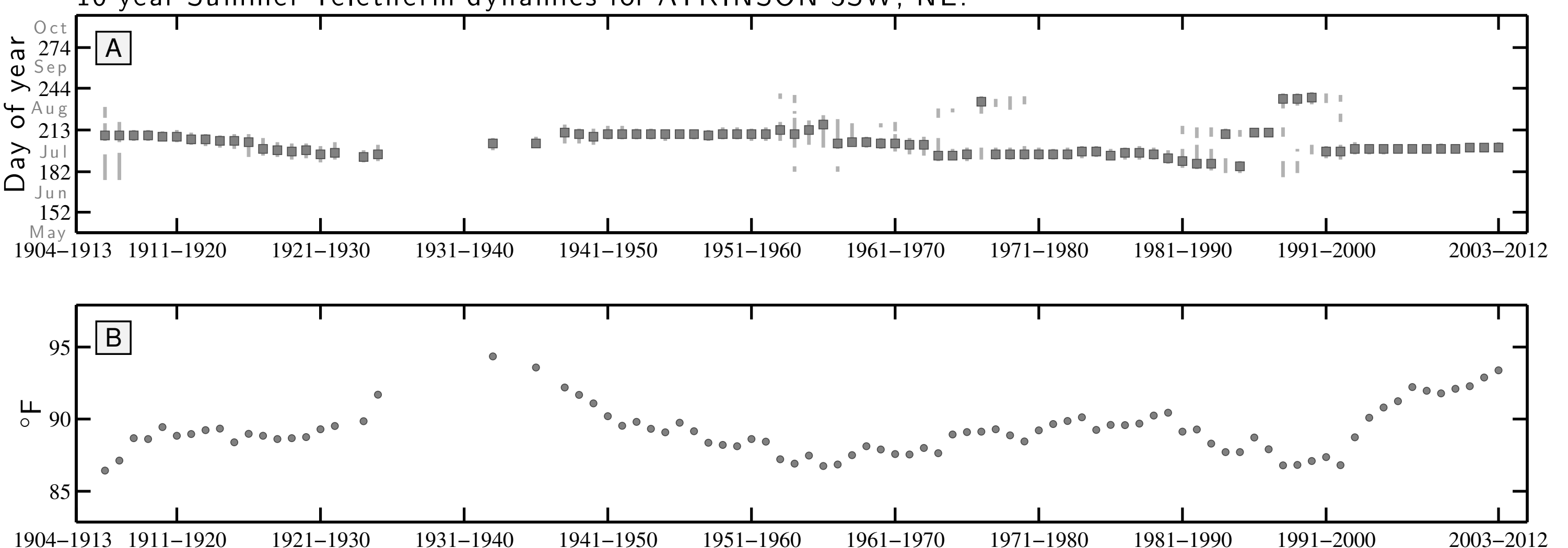

1931-1940

1941-1950

1951-1960

1961-1970

1971-1980

1981-1990

1991-2000

2003-2012

10 year Winter Teletherm dynamics for ATKINSON 3SW, NE:

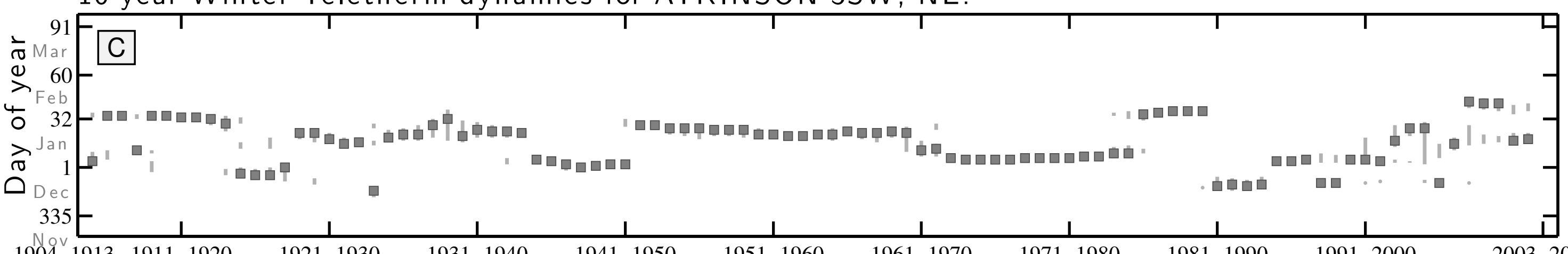

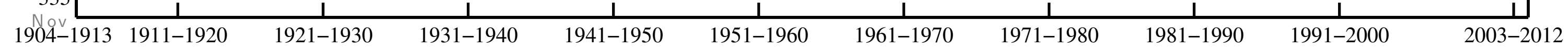

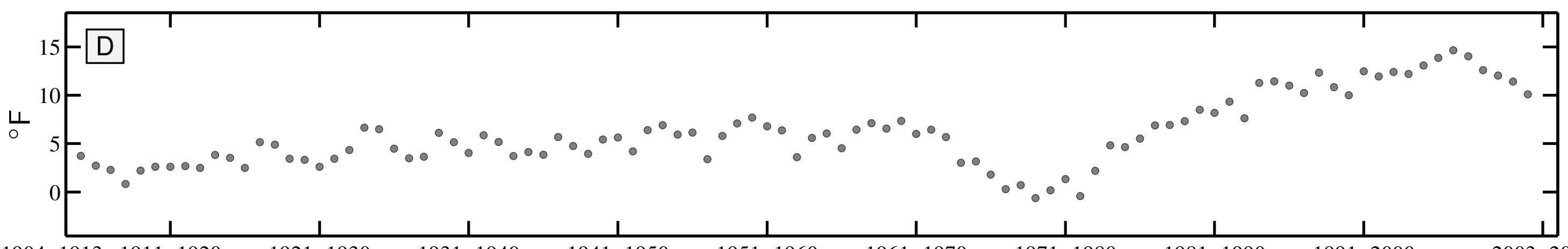


10 year Summer Teletherm dynamics for FAIRMONT, NE:

$\div$ Oct 274

(1) Sep

$>244-$

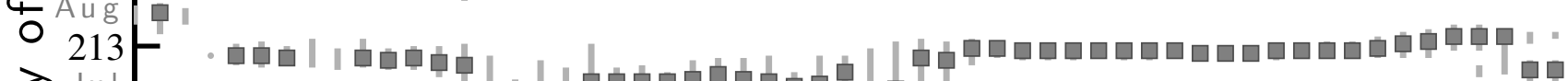

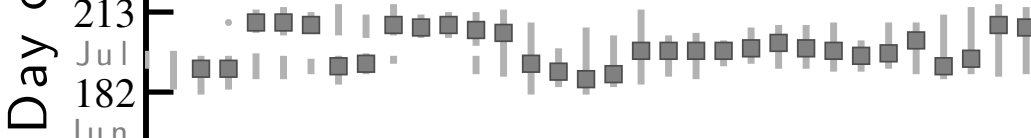

Jun

$152-$

1904-1913 1911-1920

$1921-1930$

$\frac{1}{1931-1940}$

$1941-1950$

$1951-1960$

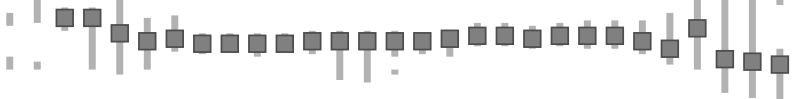
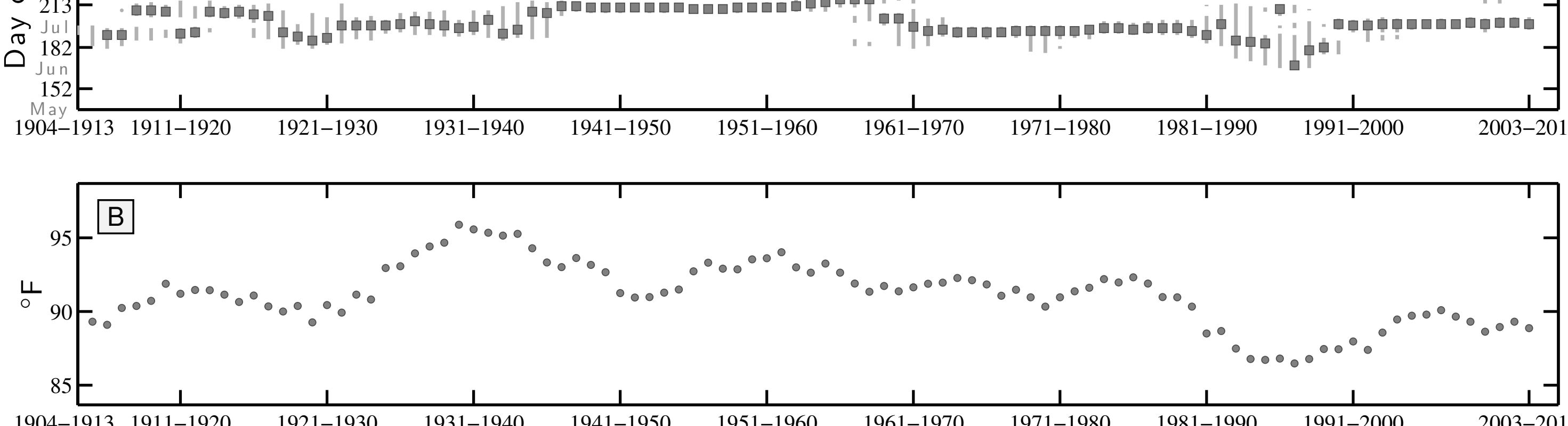

1904-1913 1911-1920

1921-1930

1931-1940

1941-1950

1951-1960

1961-1970

1971-1980

1981-1990

1991-2000

2003-2012

10 year Winter Teletherm dynamics for FAIRMONT, NE:

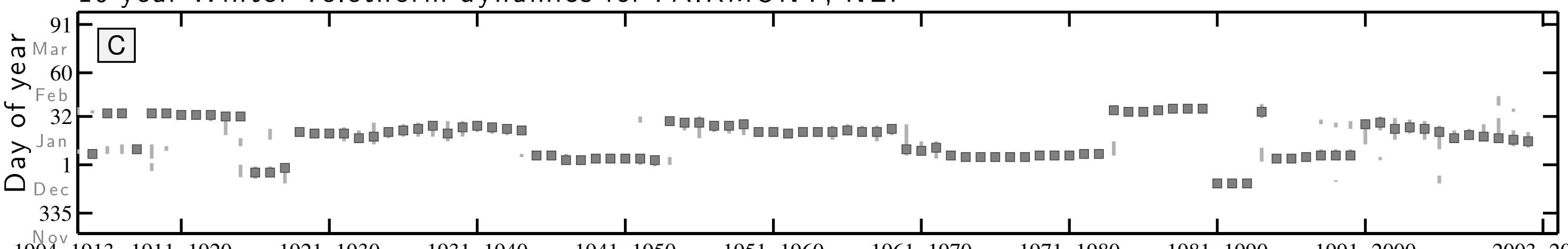

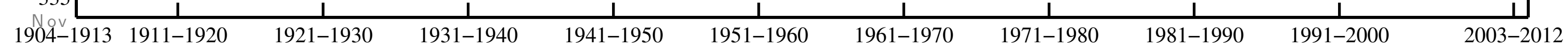

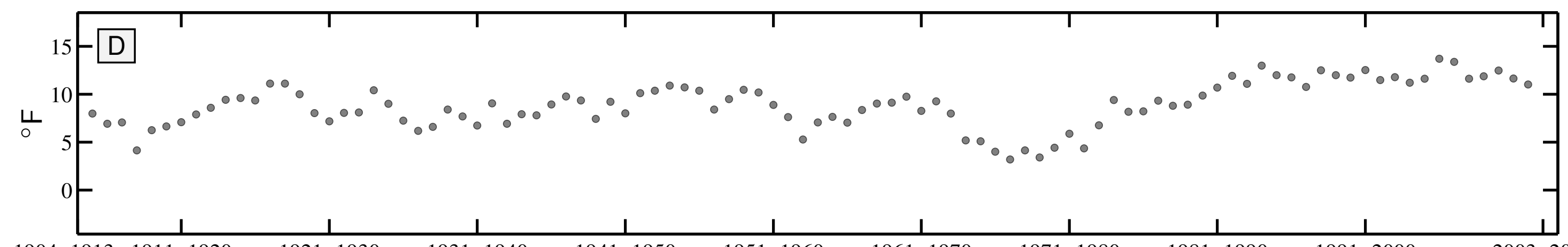


10 year Summer Teletherm dynamics for GENEVA, NE:

$\frac{\mathrm{O}}{\mathrm{\sigma}} 274 \mathrm{At}$

(1) Sep

$>244-$

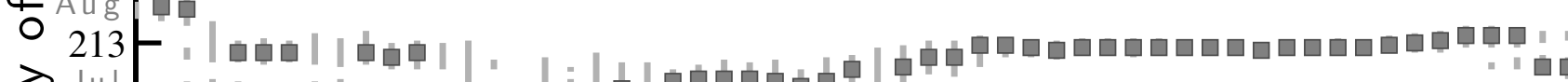

|

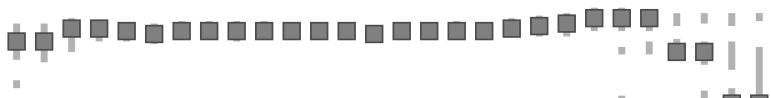

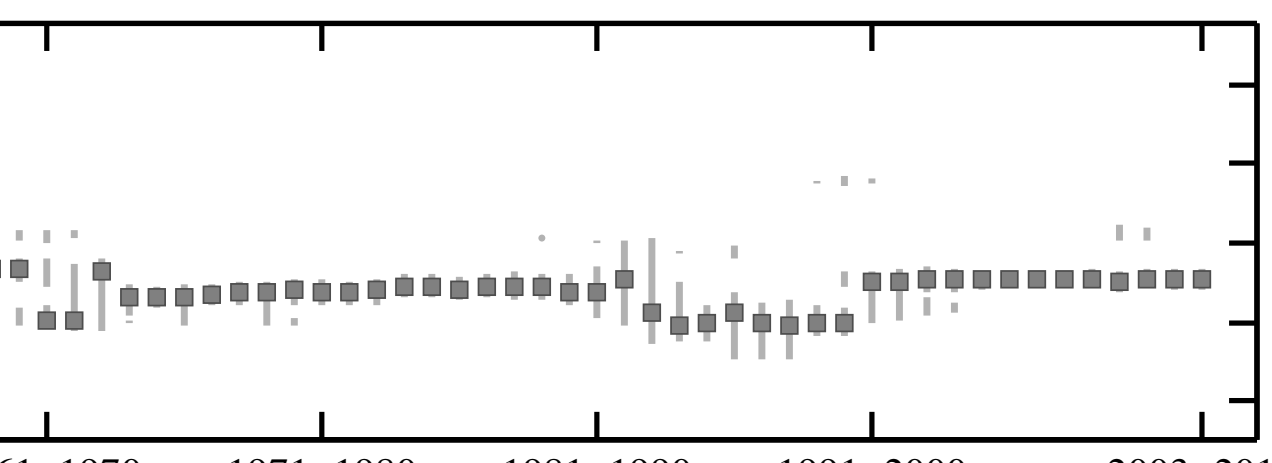

May $\frac{1}{1913-1911-1920}$

$1921-1930$

$1931-1940$

$1941-1950$

$1951-1960$

$1961-1970$

$1971-1980$

$1981-1990$

$1991-2000$

2003-2012

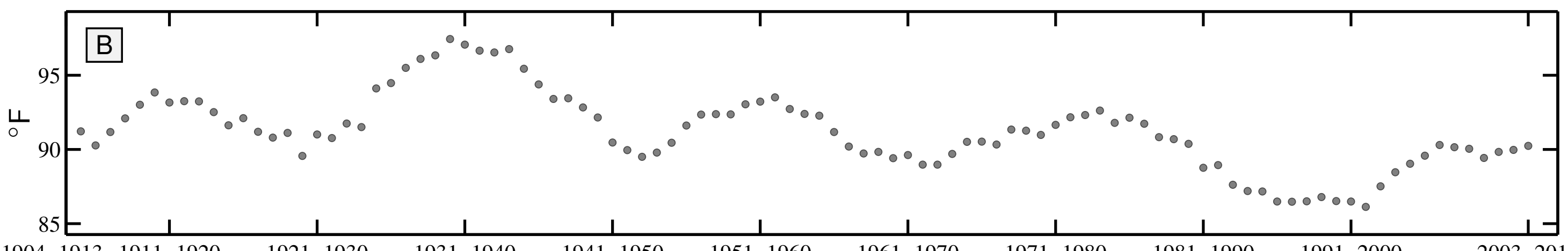

1904-1913 1911-1920

$1921-1930$

$1931-1940$

$1941-1950$

$1951-1960$

$1961-1970$

$1971-1980$

$1981-1990$

$1991-2000$

$2003-2012$

10 year Winter Teletherm dynamics for GENEVA, NE:

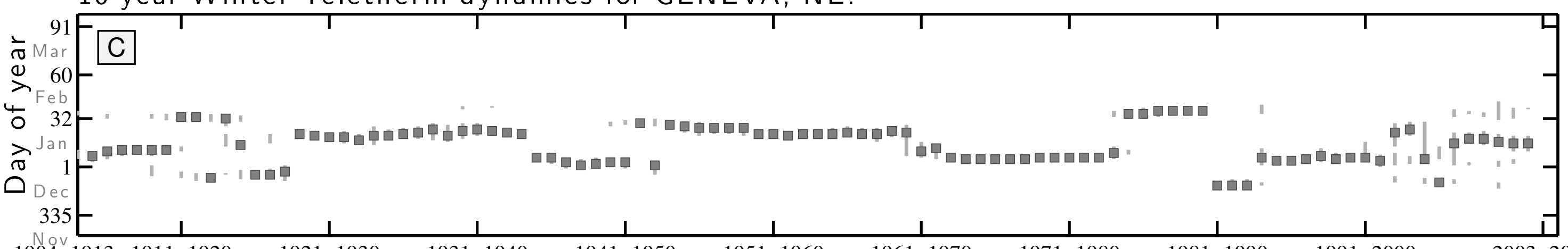

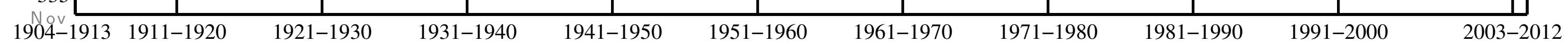

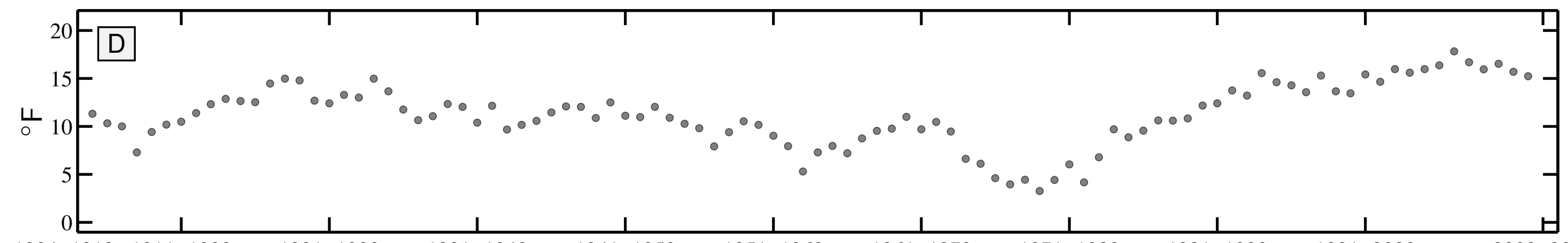


10 year Summer Teletherm dynamics for GOTHENBURG, NE:

$\sim$ Oct

\% $274-\mathrm{A}$

(1) Sep

$>244-$

O 213

त Jul

(182

$152-$

152

1904-1913 1911-1920

1921-1930

1931-1940

$\frac{1}{1941-1950}$

1951-1960

$\frac{1}{1961-1970}$

$\frac{1}{971-1980}$

$\frac{1}{1981-1990}$

1991-2000

2003-2012

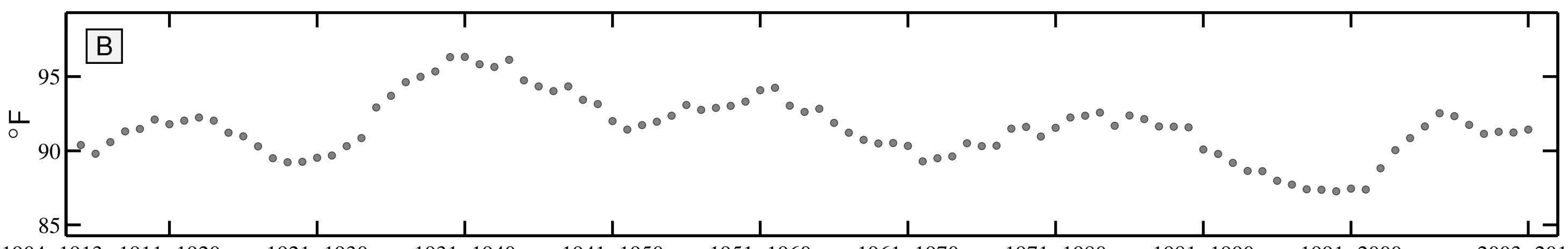

1904-1913 1911-1920

$1921-1930$

$1931-1940$

$1941-1950$

$1951-1960$

$1961-1970$

$1971-1980$

$1981-1990$

$1991-2000$

2003-2012

10 year Winter Teletherm dynamics for GOTHENBURG, NE:

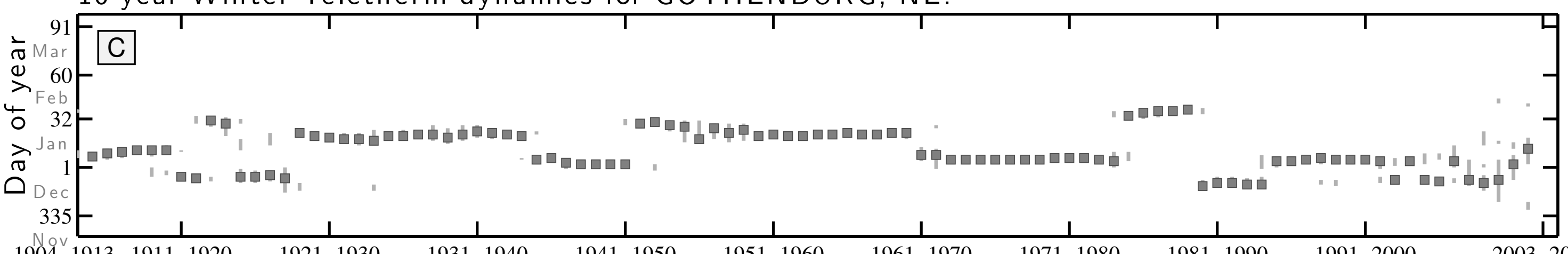

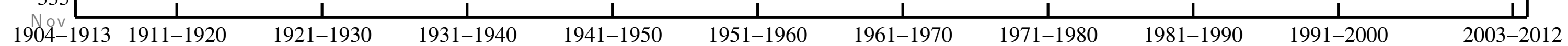

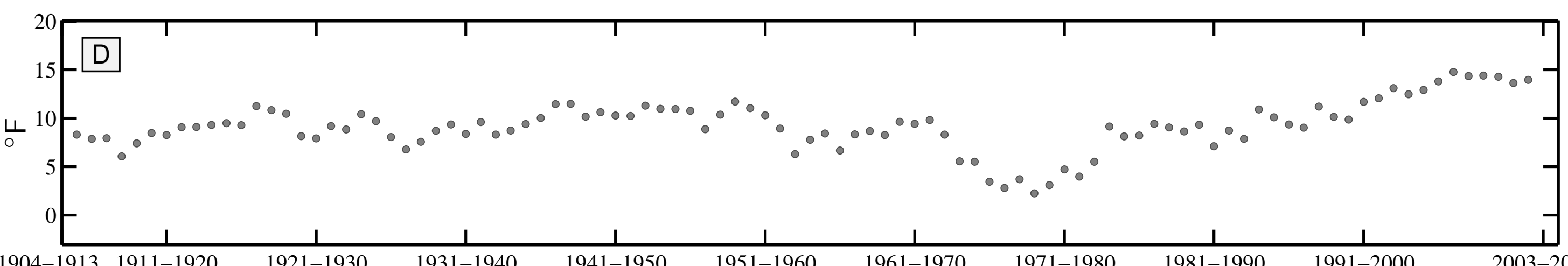


10 year Summer Teletherm dynamics for HARRISON, NE:
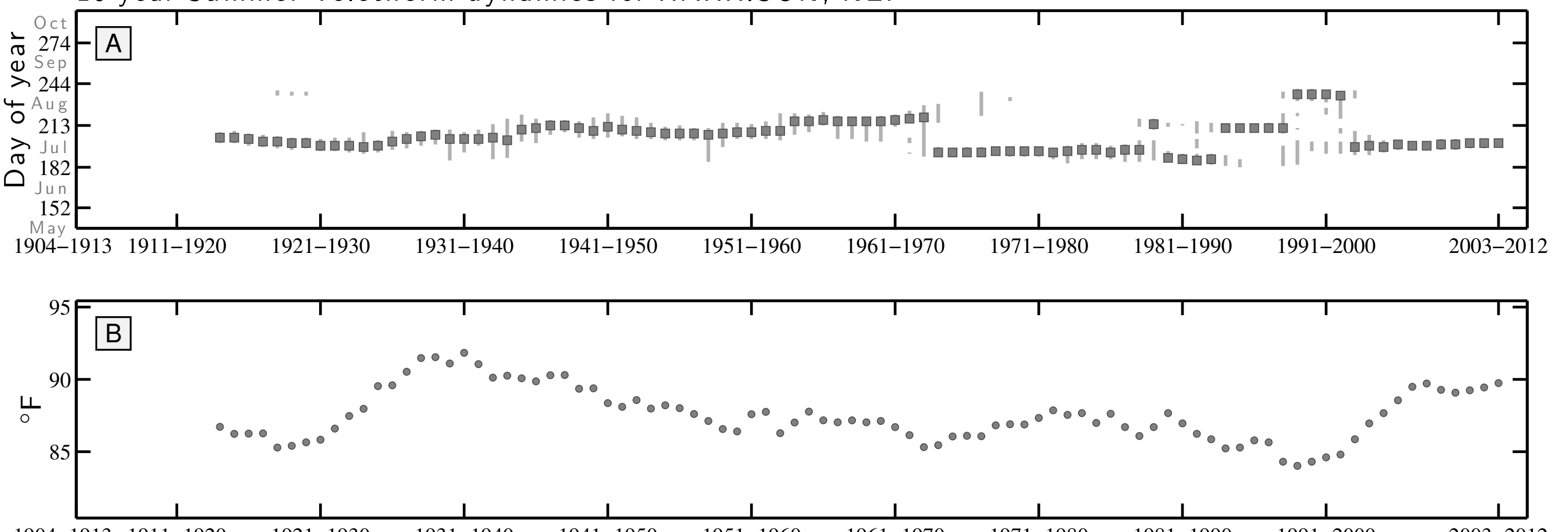

10 year Winter Teletherm dynamics for HARRISON, NE:
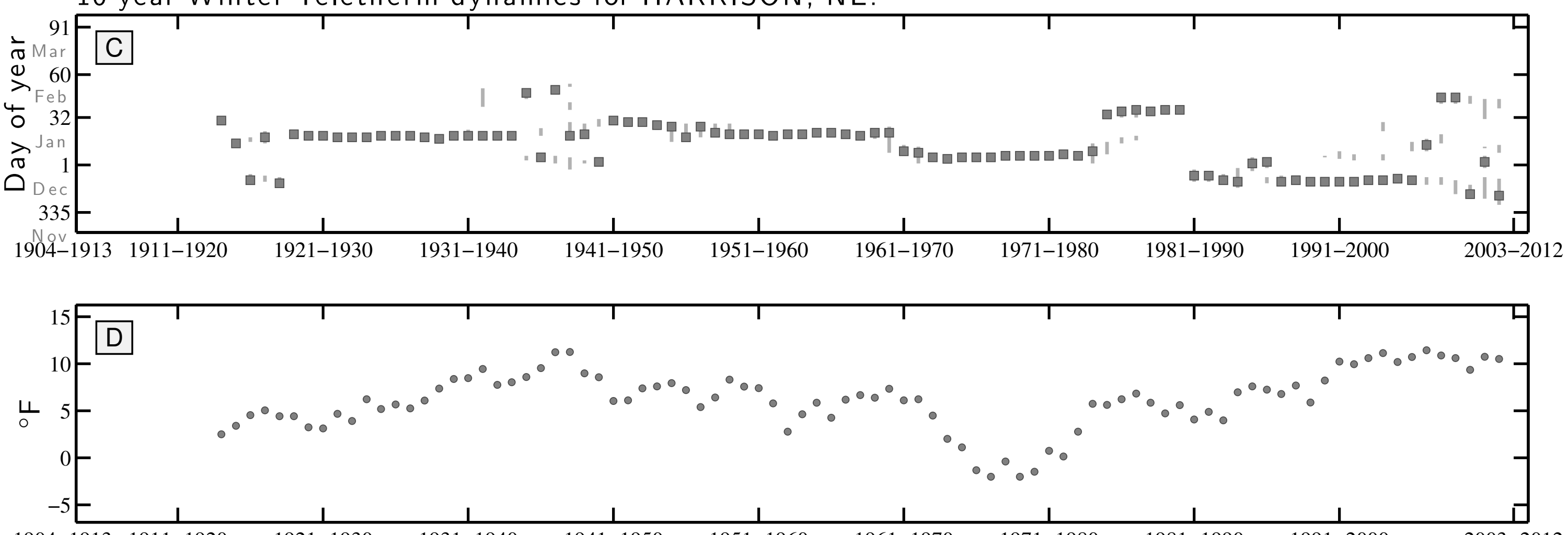
10 year Summer Teletherm dynamics for NORTH LOUP, NE:
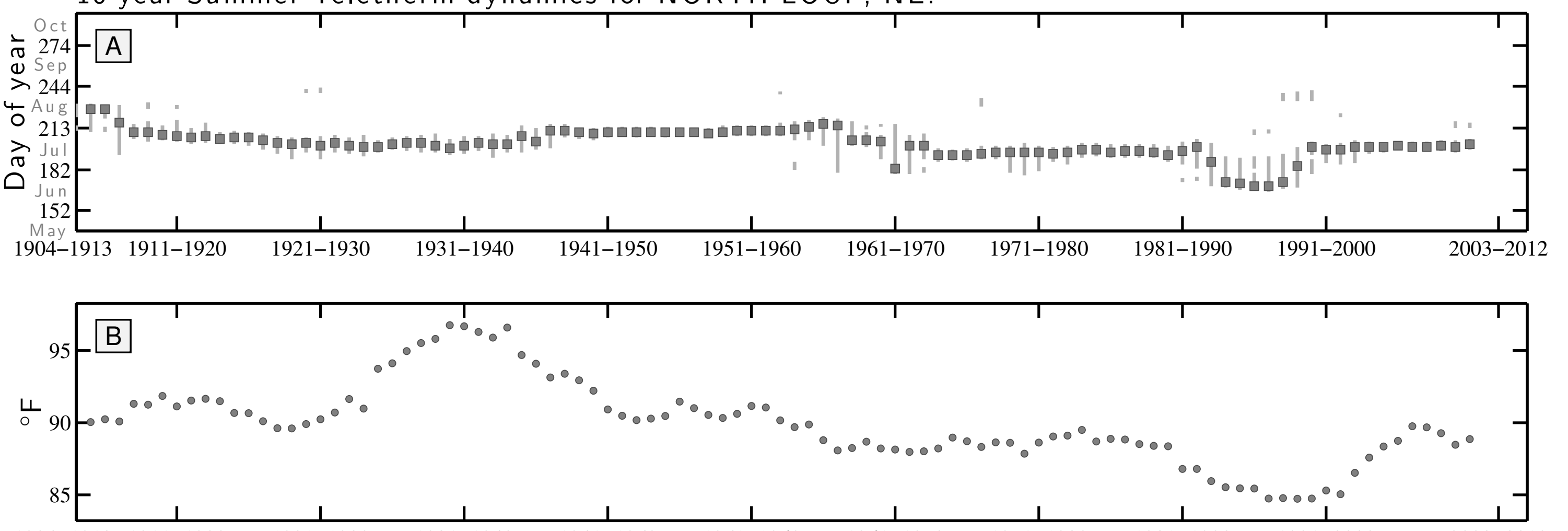

$1904-1913 \quad 1911-1920 \quad 1921-1930$

$1931-1940$

1941-1950

$1951-1960$

$1961-1970$

$1971-1980$

1981-1990

1991-2000

2003-2012

10 year Winter Teletherm dynamics for NORTH LOUP, NE:

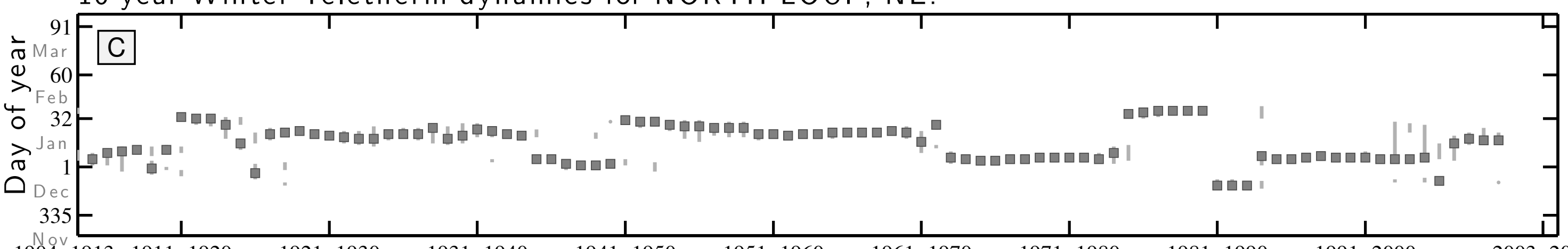

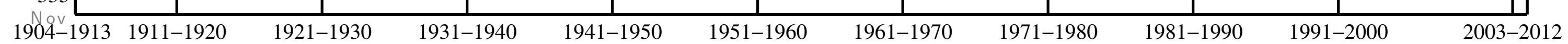

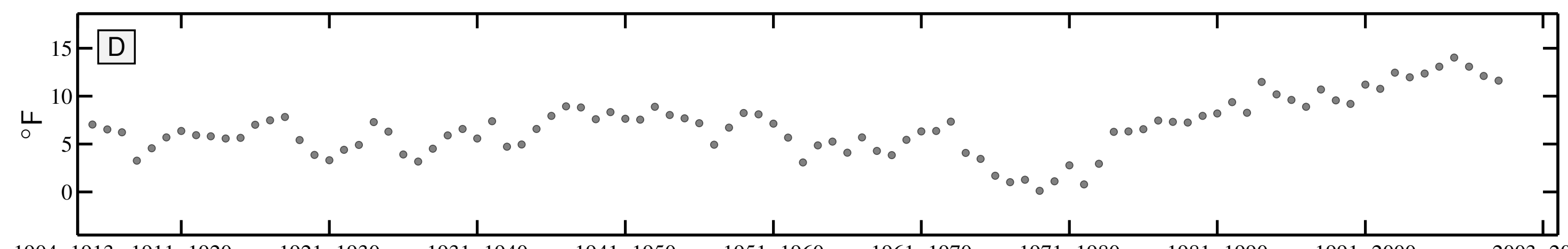

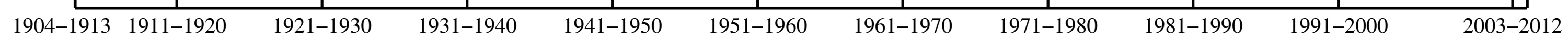


10 year Summer Teletherm dynamics for STAPLETON 5W, NE:
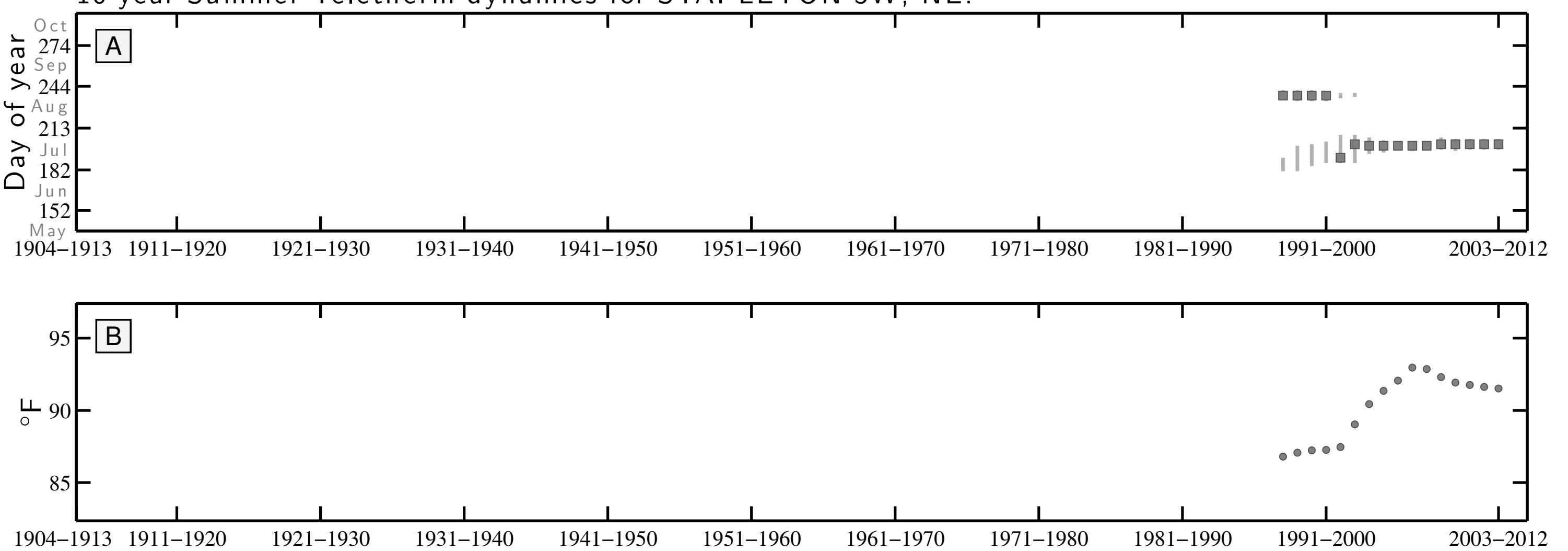

10 year Winter Teletherm dynamics for STAPLETON 5W, NE:
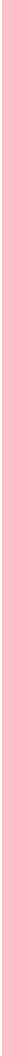
10 year Summer Teletherm dynamics for WAKEFIELD, NE:
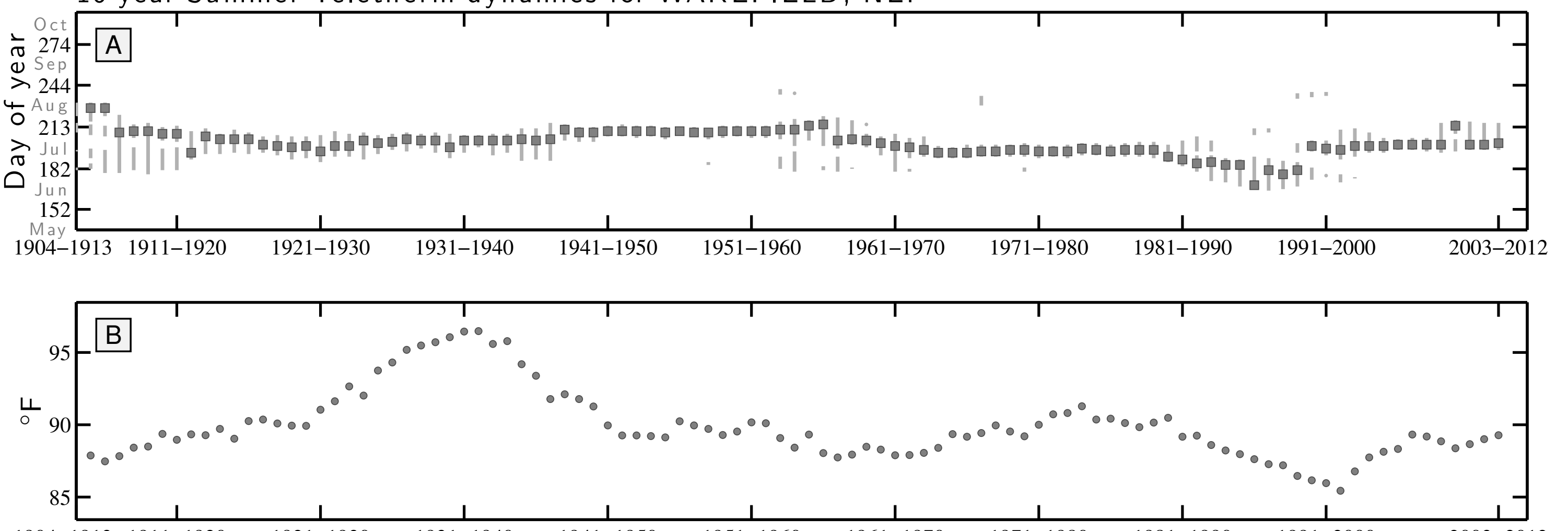

$1904-1913$ 1911-1920 1921-1930

1931-1940

1941-1950

1951-1960

1961-1970

1971-1980

1981-1990

1991-2000

2003-2012

10 year Winter Teletherm dynamics for WAKEFIELD, NE:

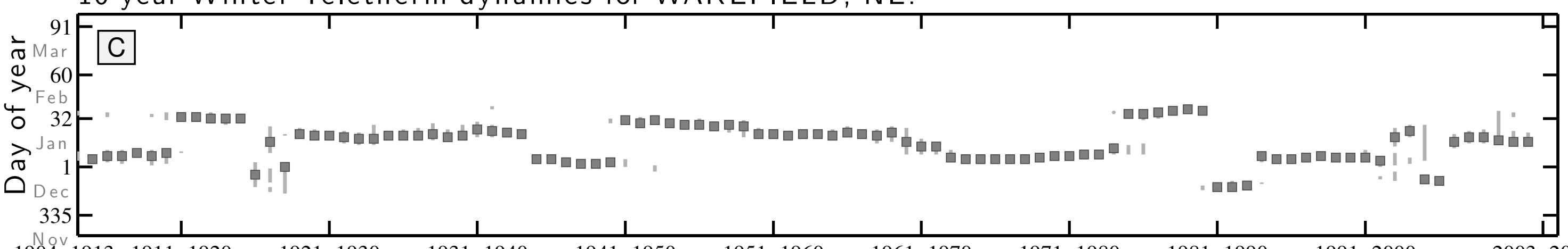

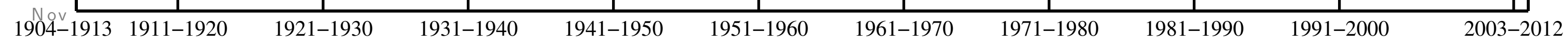

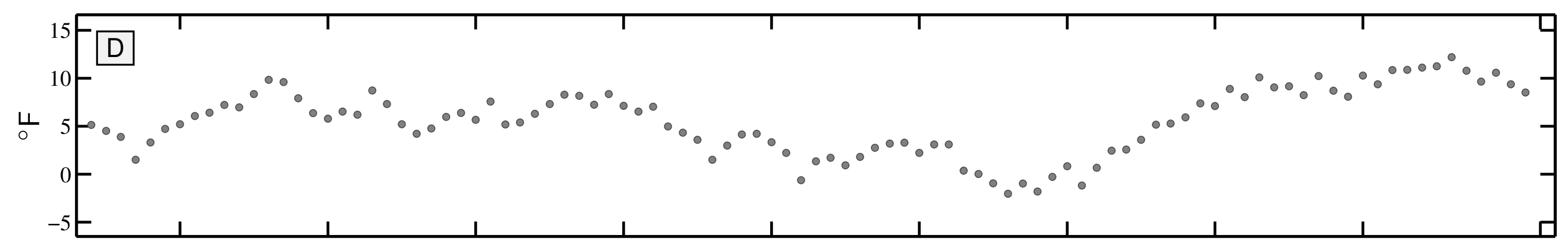

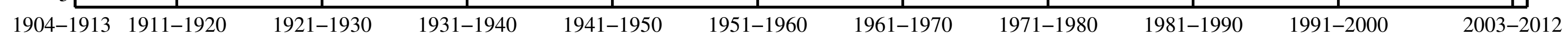




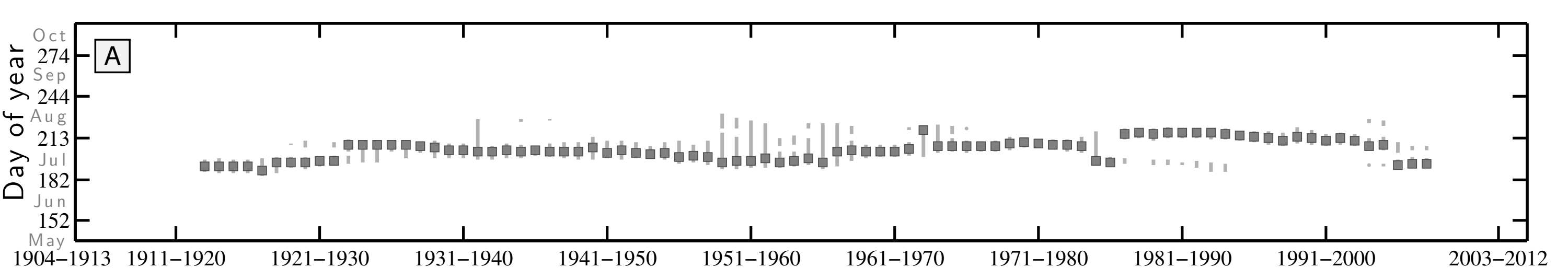

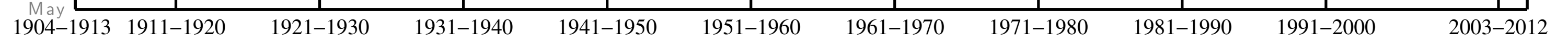
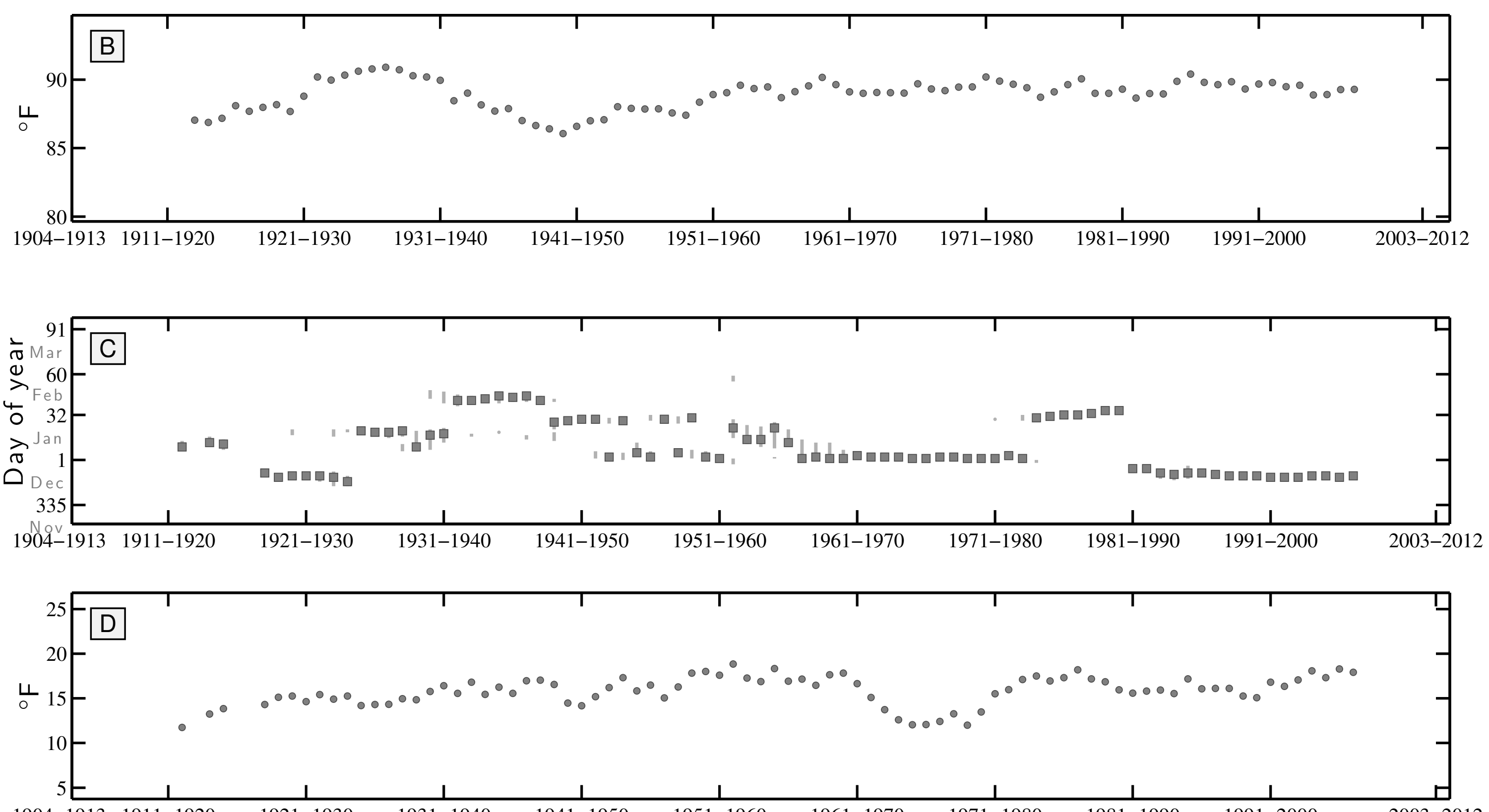

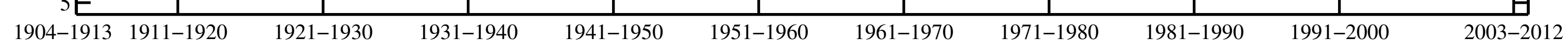


10 year Summer Teletherm dynamics for BATTLE MOUNTAIN 4SE, NV:
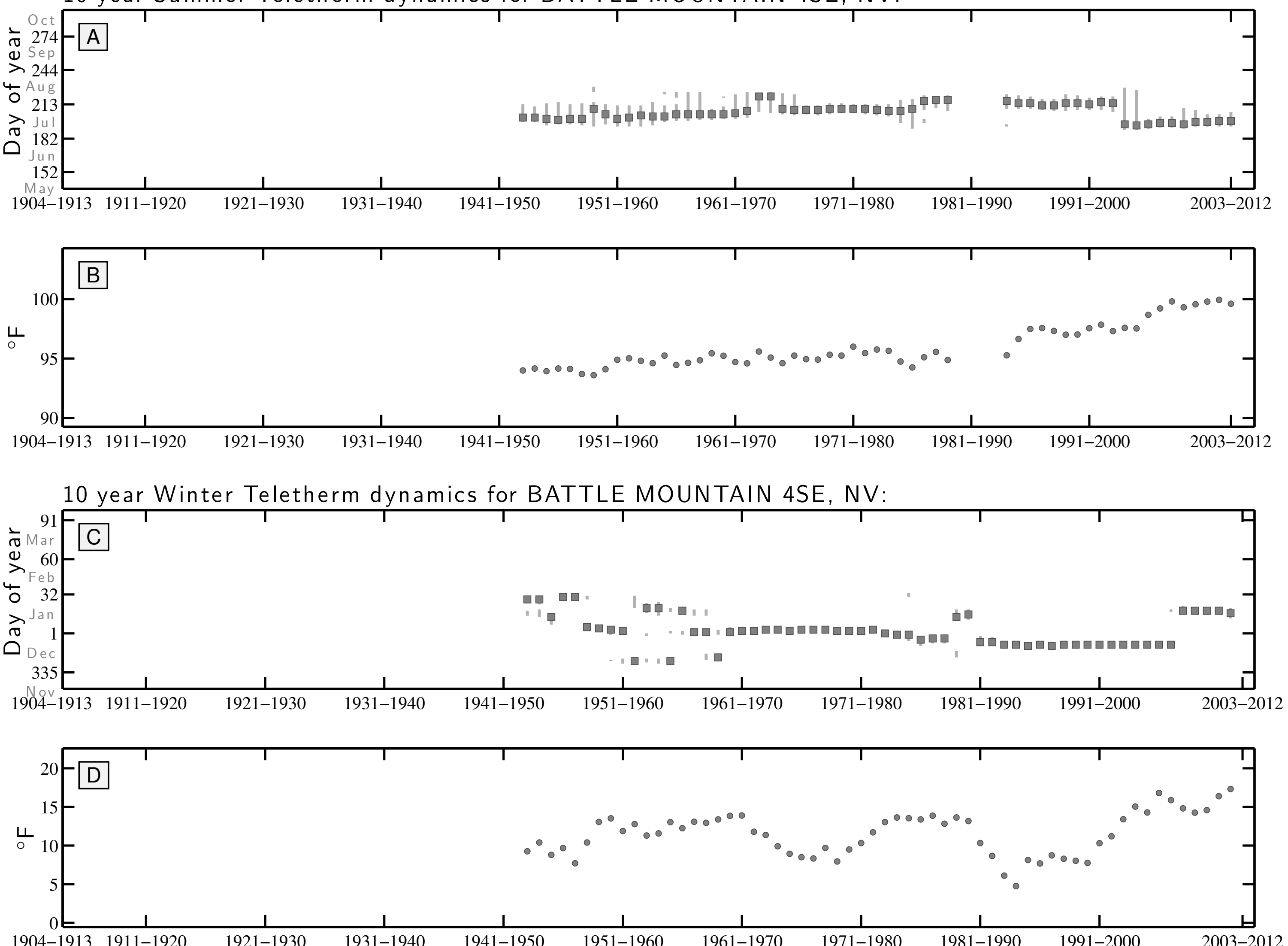

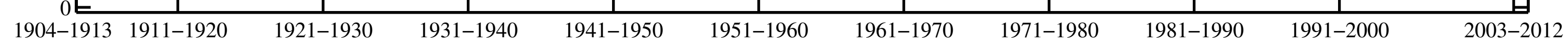


10 year Summer Teletherm dynamics for BOULDER CITY, NV:
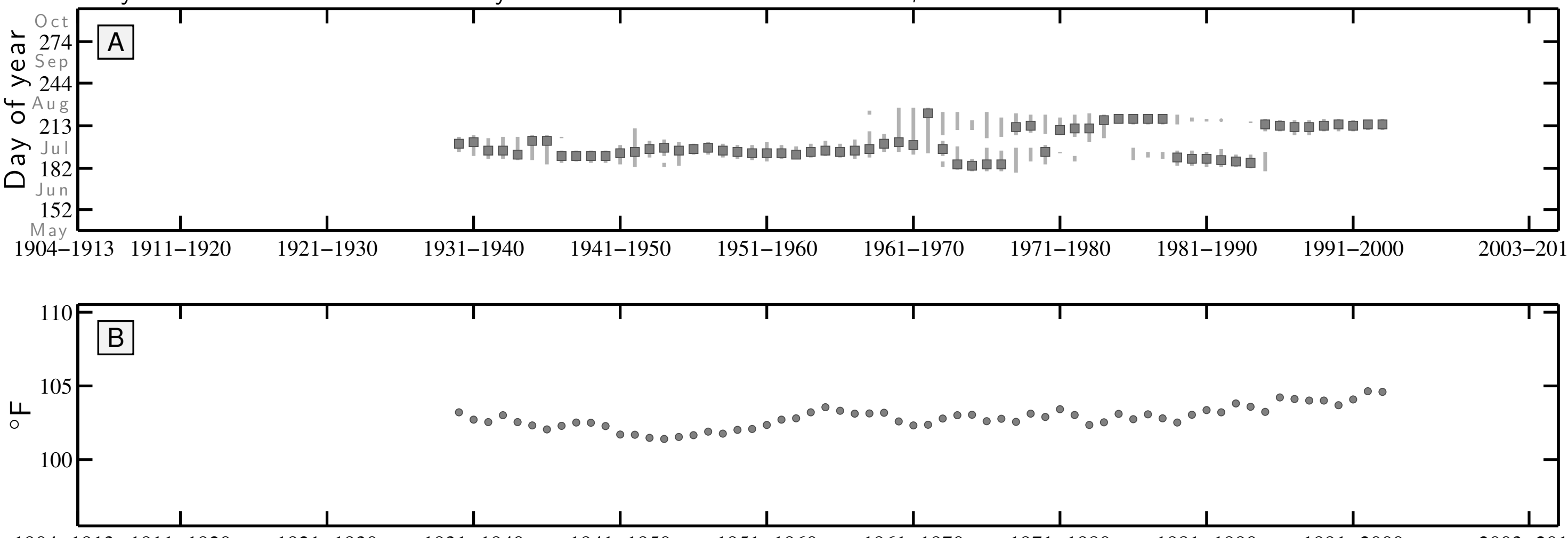

10 year Winter Teletherm dynamics for BOULDER CITY, NV:

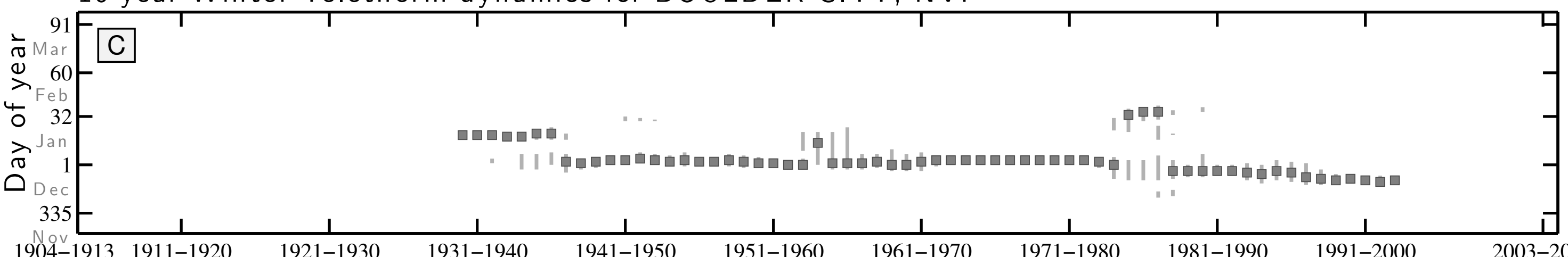

1904-1913 1911-1920

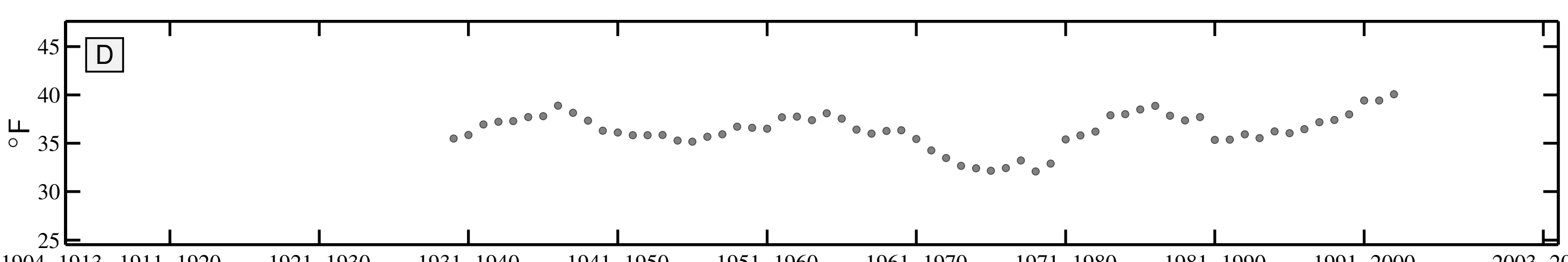


10 year Summer Teletherm dynamics for FALLON EXP STN, NV:
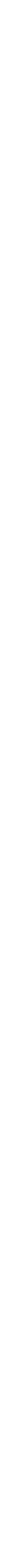

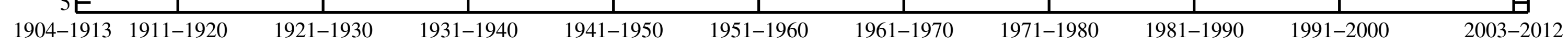


10 year Summer Teletherm dynamics for WELLS, NV:
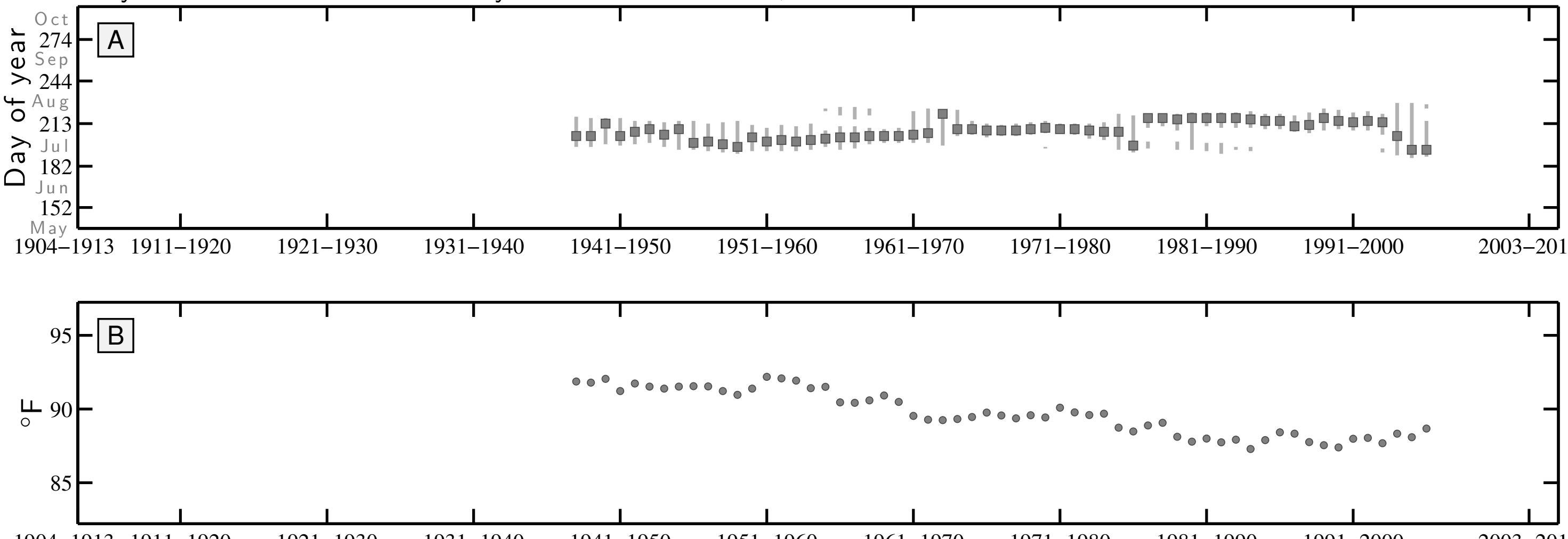

10 year Winter Teletherm dynamics for WELLS, NV:

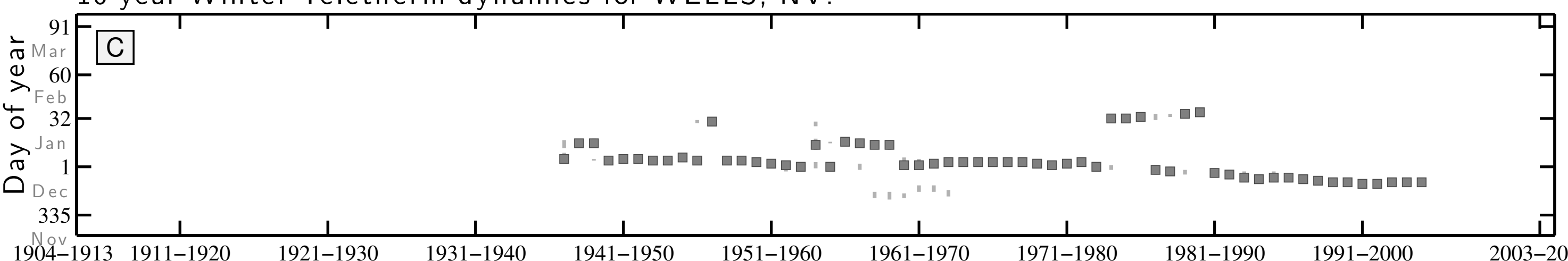

1904-1913 1911-192

1921-1930

1931-1940

1941-1950

951-1960

1961-1970

1971-1980

981-1990
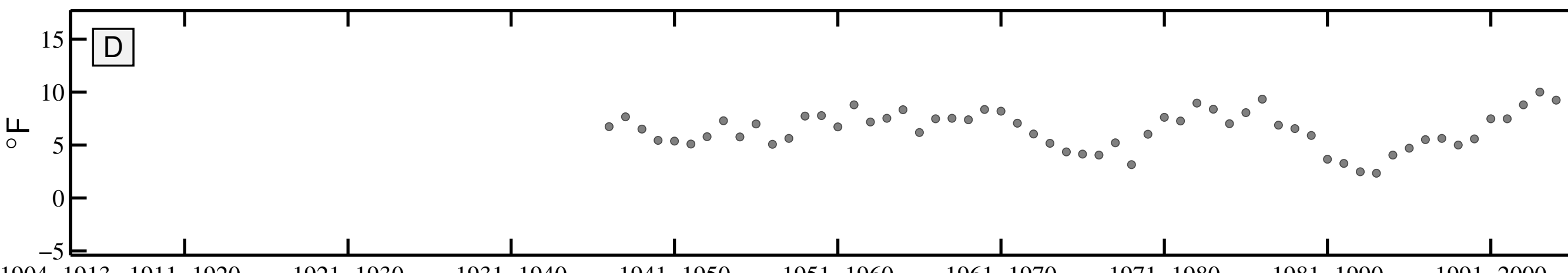
10 year Summer Teletherm dynamics for BETHLEHEM 2, NH:
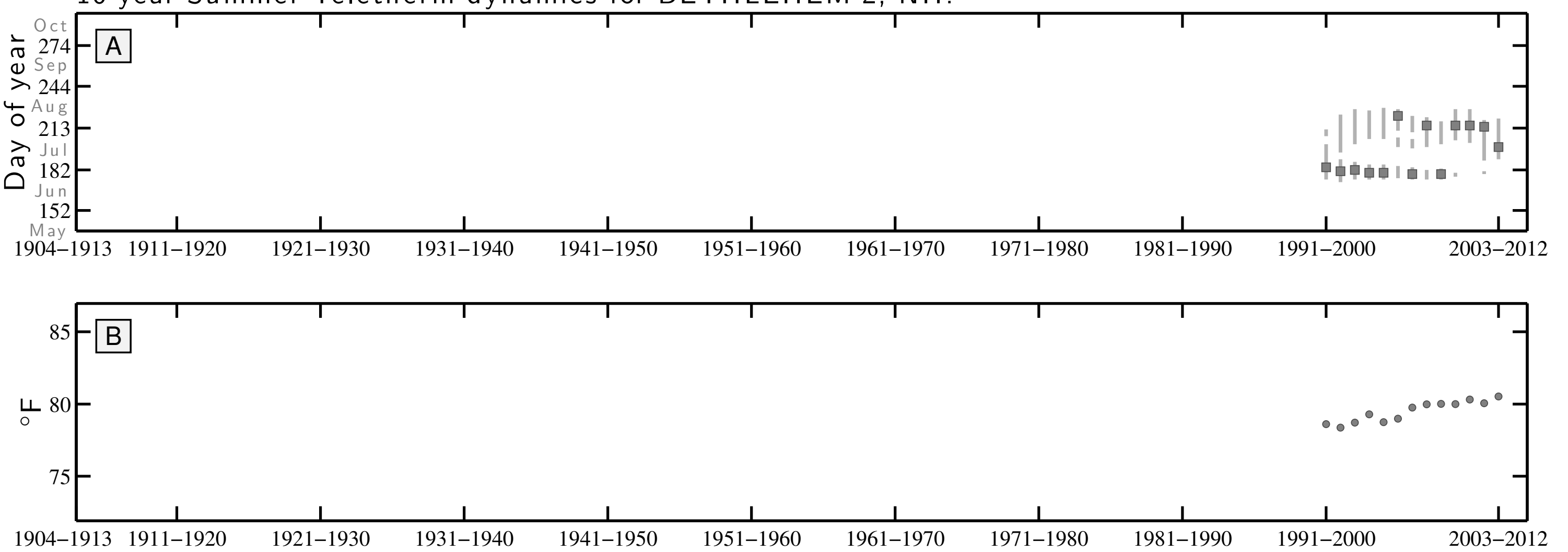

10 year Winter Teletherm dynamics for BETHLEHEM 2, NH:
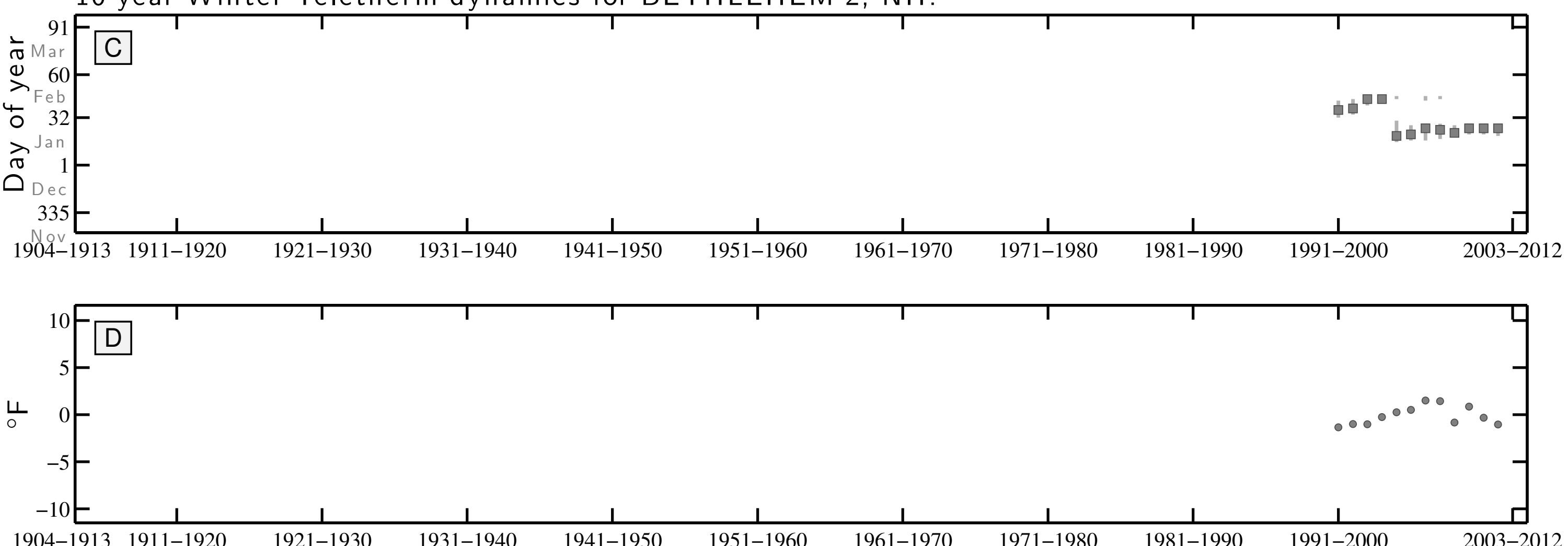

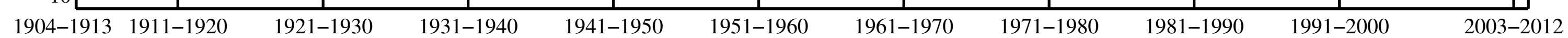


10 year Summer Teletherm dynamics for ATLANTIC CITY, NJ:

Oct

A

(1) Sep

$>244-$

O 213 -

$\gtrsim \mathrm{J}$

\% $182-$

Jun

152 -

| 口曰"

04-1913 1911-1920

$\frac{1}{1921-1930}$

1931-1940

$\frac{1}{1941-1950}$

$\frac{1}{1951-1960}$

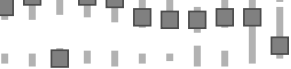

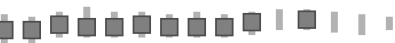

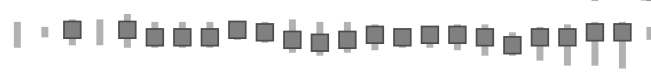

\ロ・ロロ日ロロ

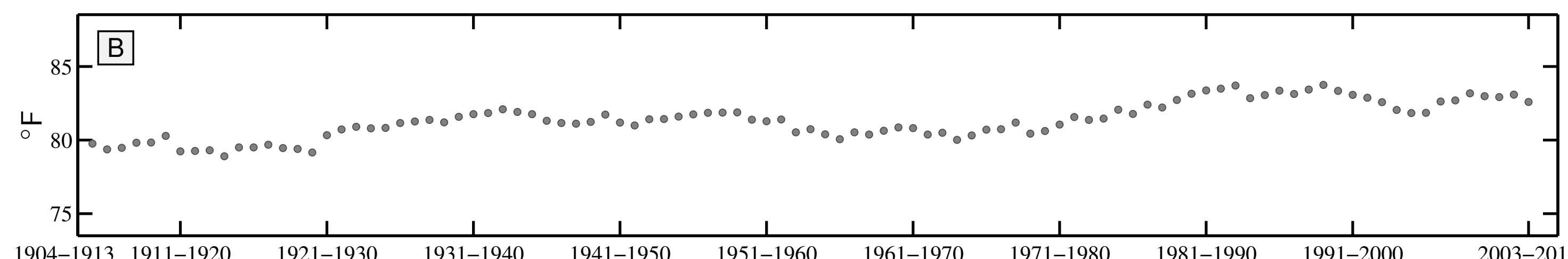

$1904-1913$ 1911-1920

1921-1930

1931-1940

$1941-1950$

$1951-1960$

$1961-1970$

1971-1980

981-1990

991-2000

2003-2012

10 year Winter Teletherm dynamics for ATLANTIC CITY, NJ:

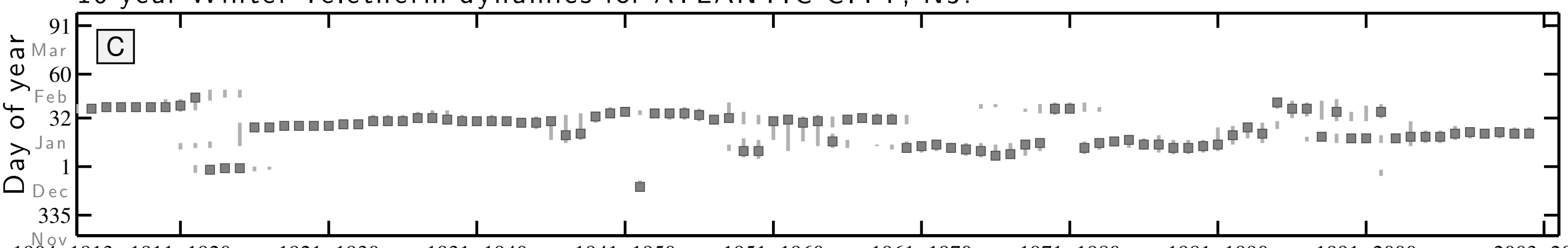

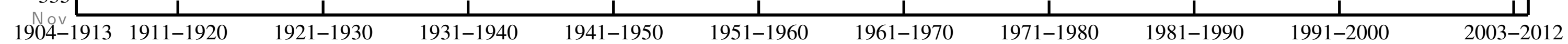

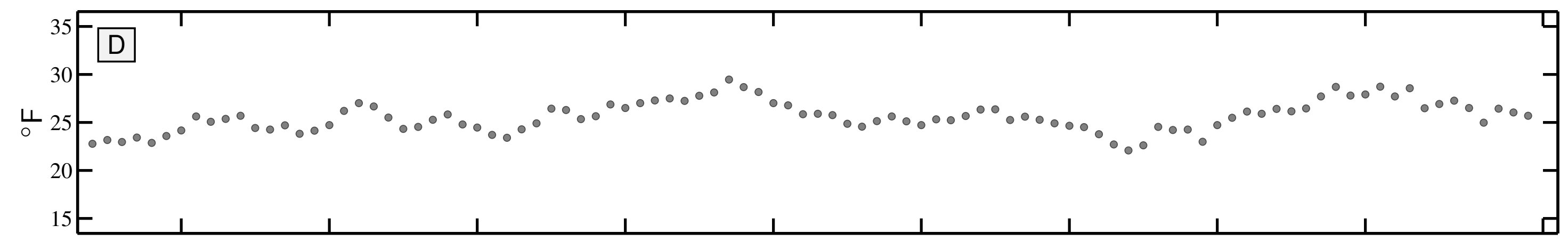


10 year Summer Teletherm dynamics for NEW BRUNSWICK 3 SE, NJ:
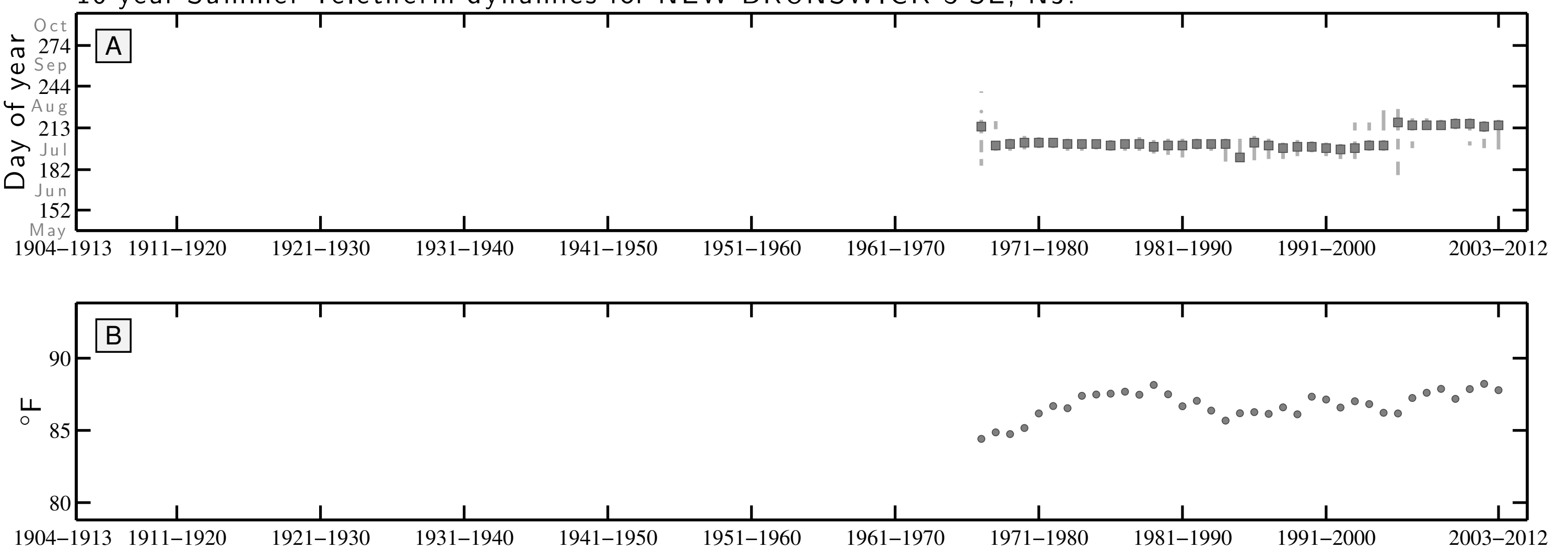

10 year Winter Teletherm dynamics for NEW BRUNSWICK 3 SE, NJ:
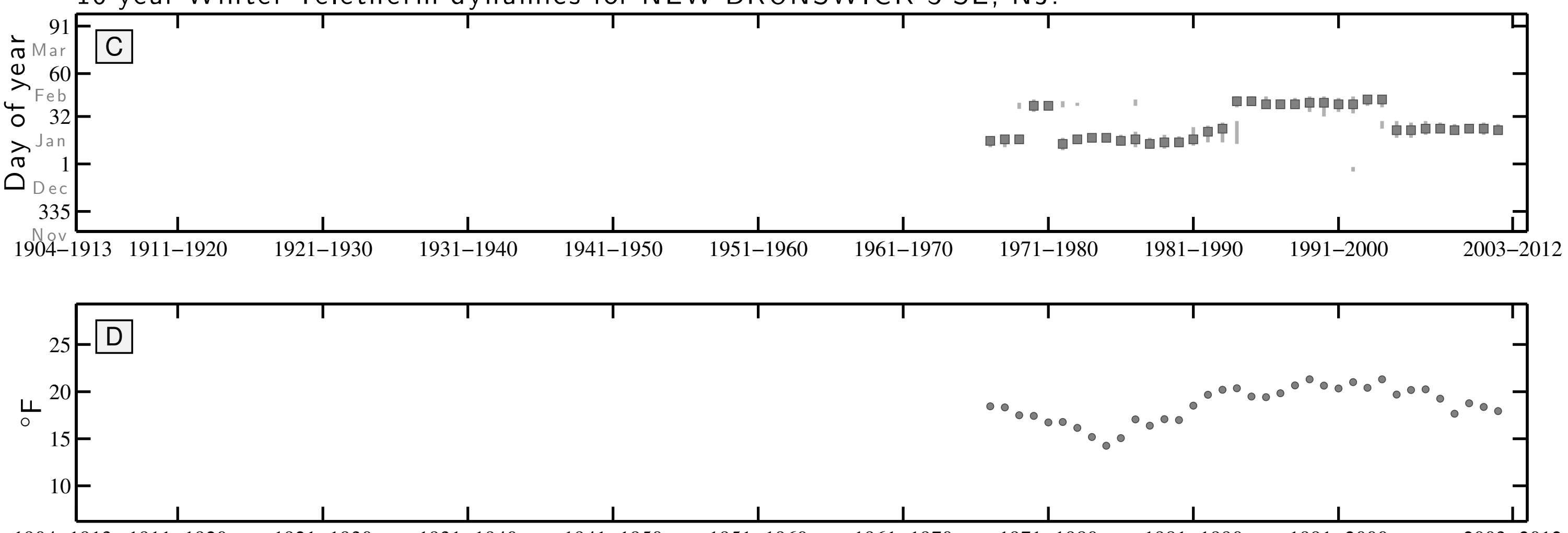

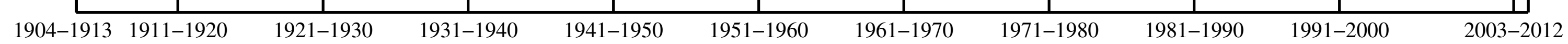


10 year Summer Teletherm dynamics for PLAINFIELD, NJ:

$\div$ Oct 274

(1) Sep

$>244-$

O 213

(

$182-$

$152-$

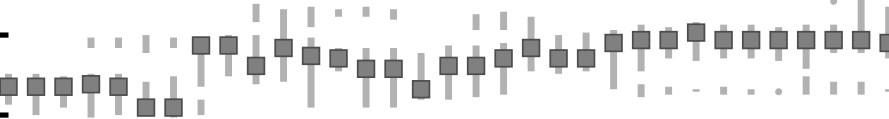

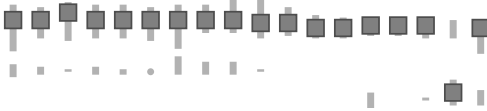

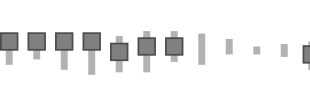

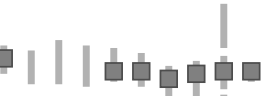

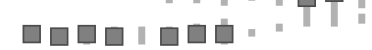

|| | 中ตव

-

$\frac{1}{1921-1930}$

$1931-1940$

$1941-1950$

$1951-1960$

$1961-1970$

$1971-1980$

$\frac{1}{981-1990}$

991-2000

$2003-2012$

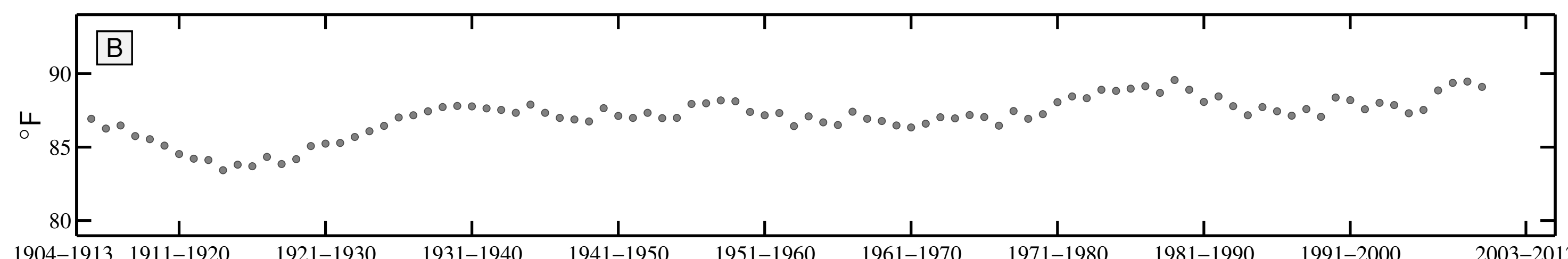

10 year Winter Teletherm dynamics for PLAINFIELD, NJ:

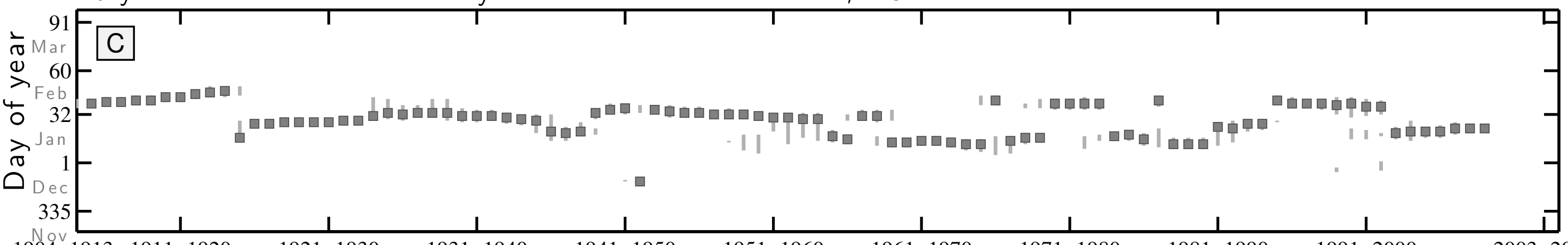

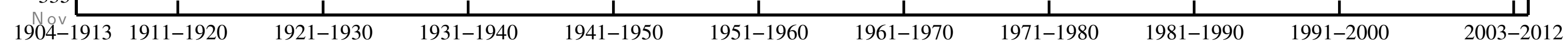

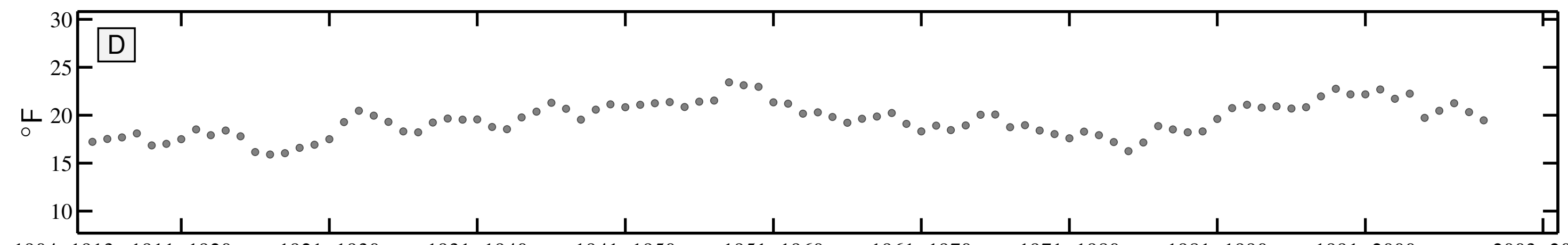


10 year Summer Teletherm dynamics for TOMS RIVER, NJ:
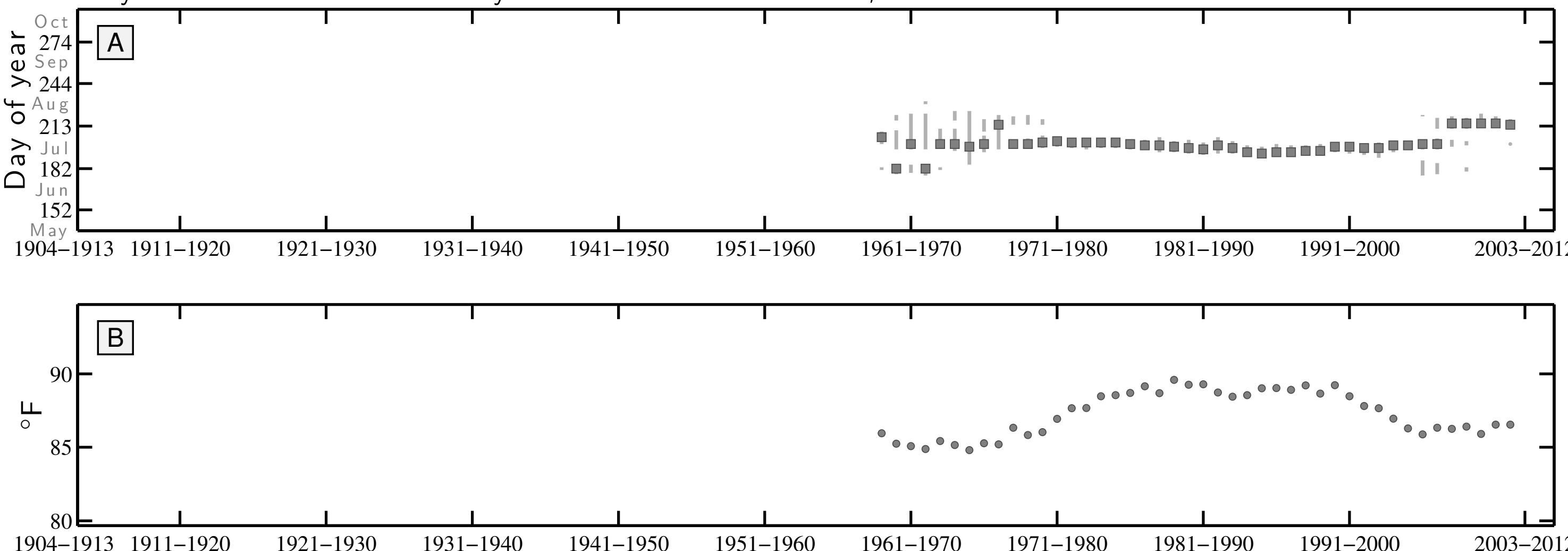

10 year Winter Teletherm dynamics for TOMS RIVER, NJ:
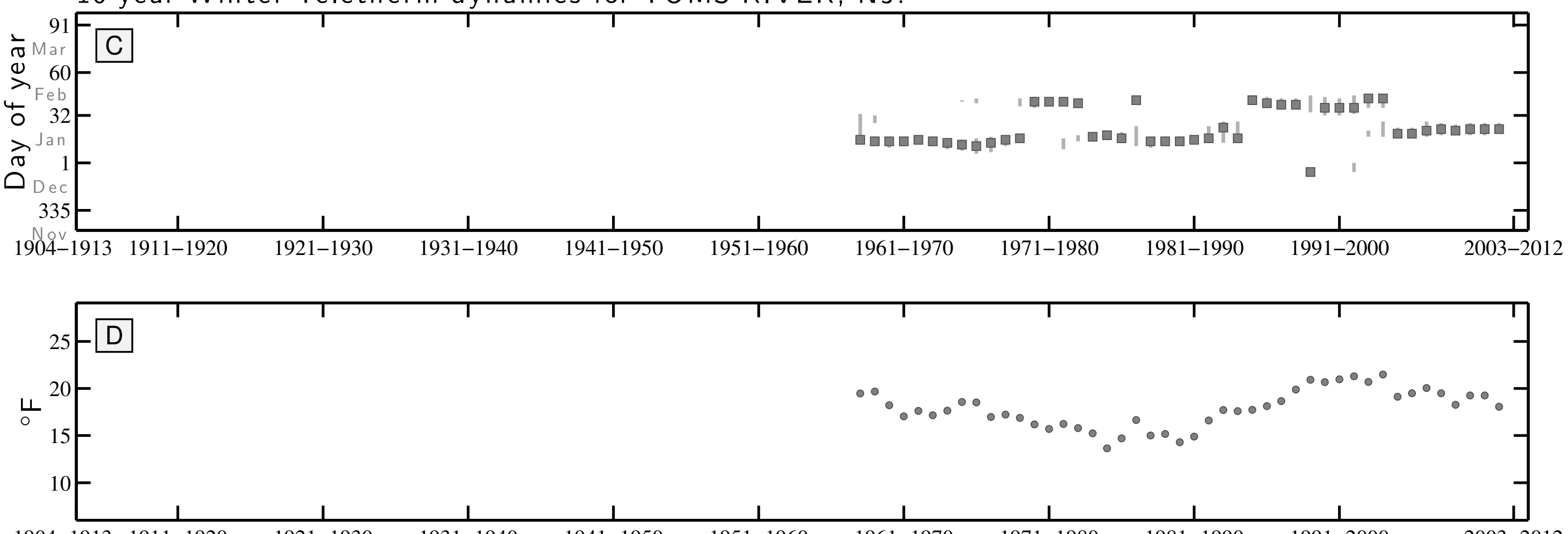
10 year Summer Teletherm dynamics for CIMARRON 4 SW, NM:

$\div$ Oct 274

(a)

(1) Sep

$>244-$

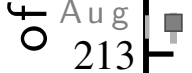

$213-=1$

(

$\bigcirc 182$

$152-$

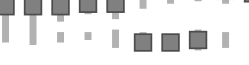

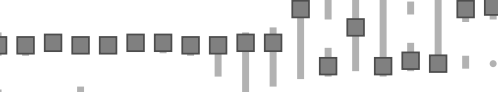

டாロロロロロロロロ

1904-1913 1911-1920

$\frac{1}{1921-1930}$

$1931-1940$

$1941-1950$

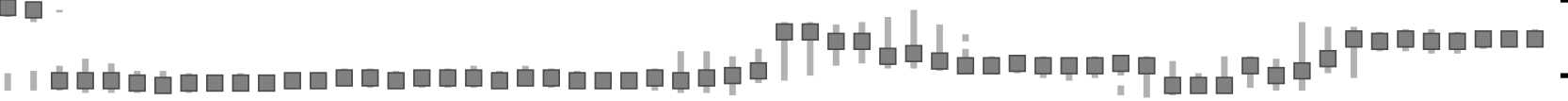

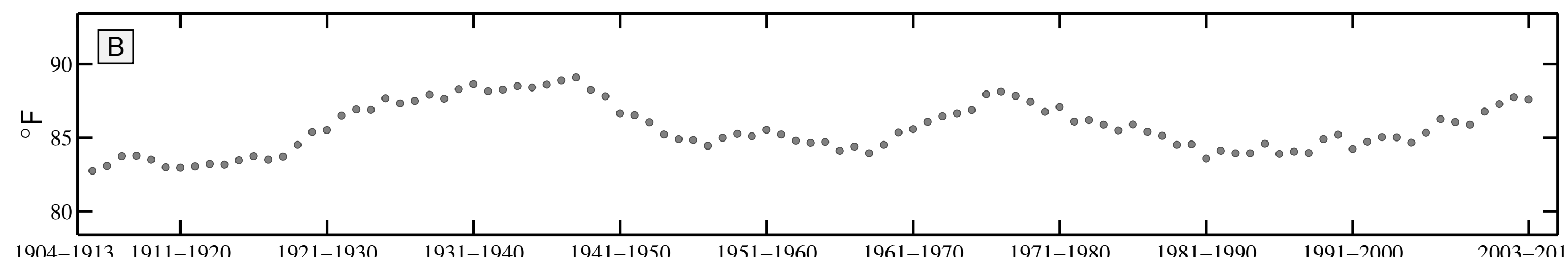

$1904-19131911-1920$

$1921-1930$

1931-1940

1941-1950

1951-1960

1961-1970

$1971-1980$

$1981-1990$

$1991-2000$

$2003-2012$

10 year Winter Teletherm dynamics for CIMARRON 4 SW, NM:

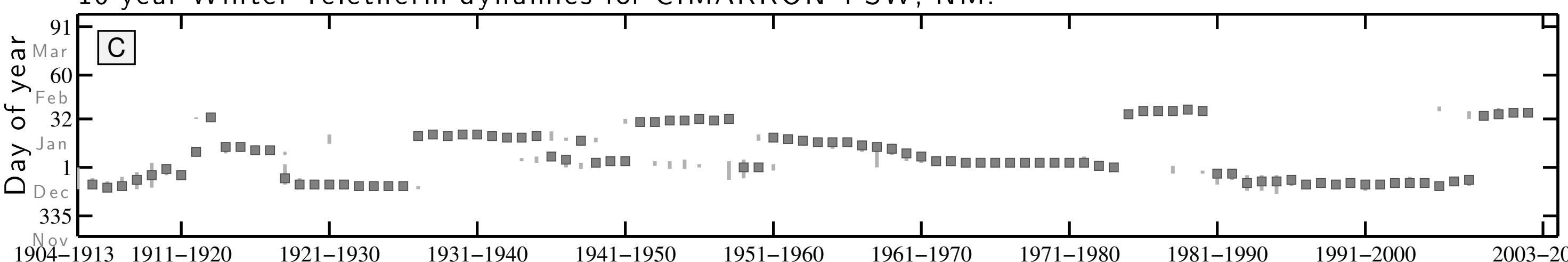

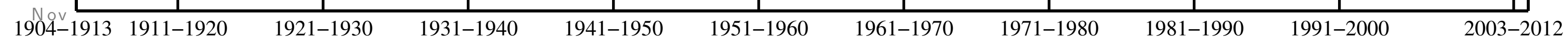

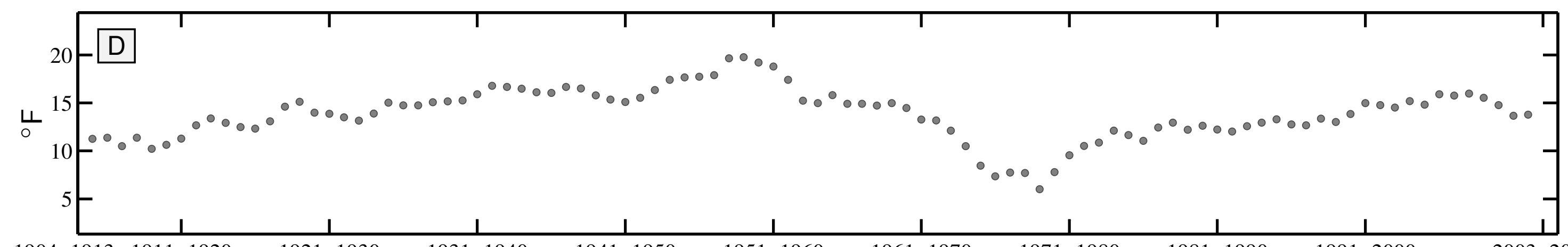


10 year Summer Teletherm dynamics for ELEPHANT BUTTE DAM, NM:

$\div 274-A$

Sep

(1) Sep

$>244$

4 Aug

ㄱ Jul

182

○un $\begin{gathered}182 \\ \text { Jun }\end{gathered}$

$152-$

| | |

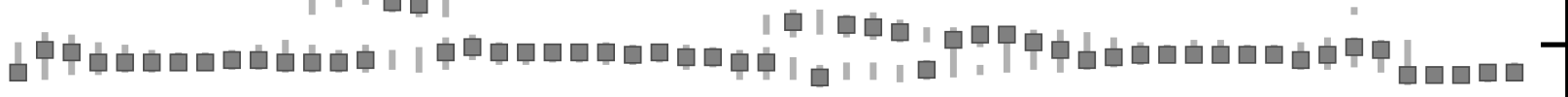

May

$1904-19131911-1920$

$\frac{1}{1921-1930}$

$1931-1940$

$\frac{1}{1941-1950}$

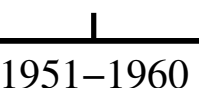

$\frac{1}{1961-1970}$

1971-1980

$\frac{1}{1981-1990}$

1991-2000

2003-2012

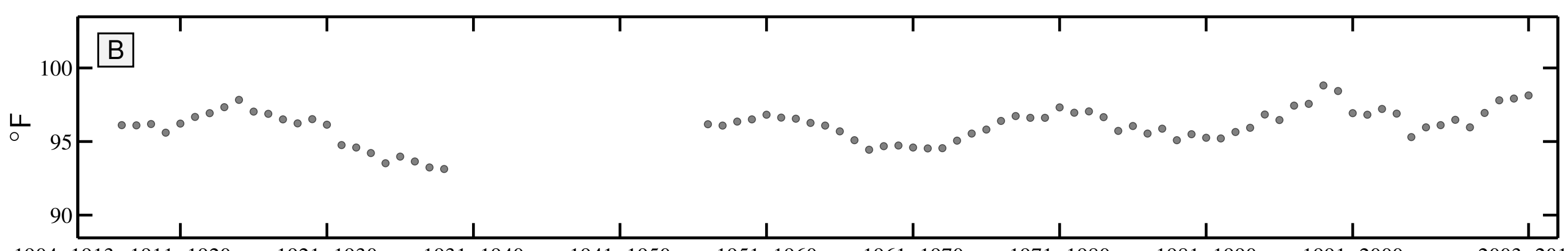

1904-1913 1911-1920

$1921-1930$

$1931-1940$

$1941-1950$

1951-1960

$1961-1970$

1971-1980

$1981-1990$

$1991-2000$

$2003-2012$

10 year Winter Teletherm dynamics for ELEPHANT BUTTE DAM, NM:

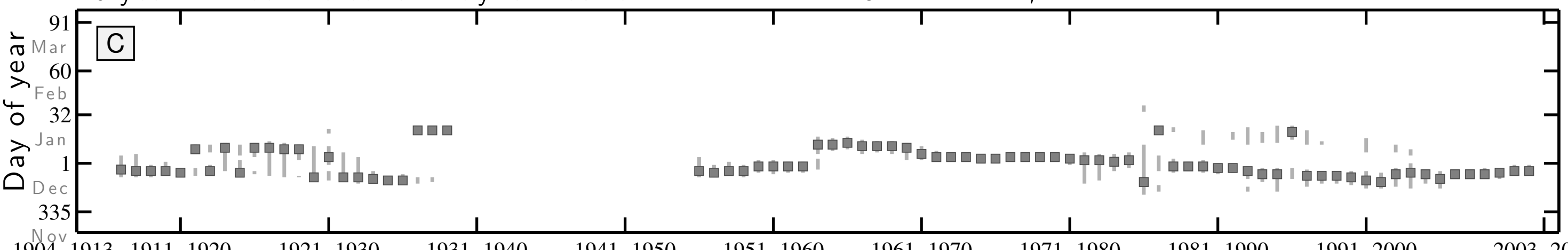

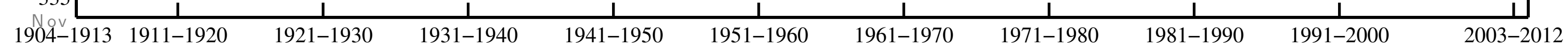

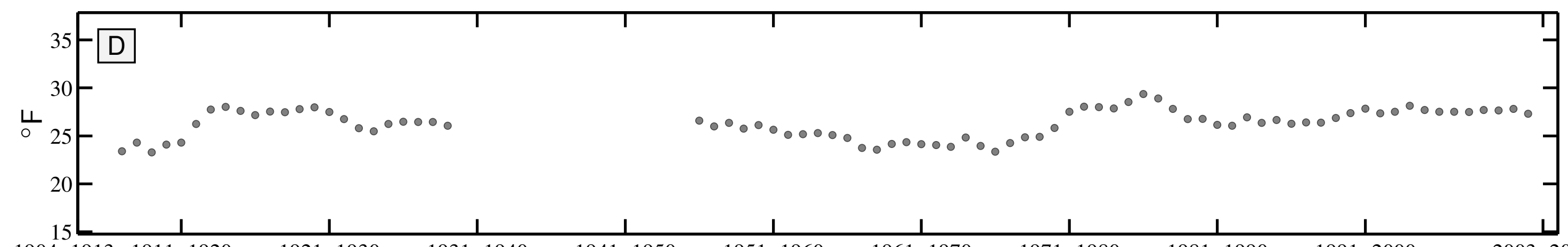


10 year Summer Teletherm dynamics for FT BAYARD, NM:

$\div$ Oct 274

Sep

(1) Sep

- Aug

○ 213

$\gtrsim \mathrm{Jul}$

182

$152-$

May $\square$

1904-1913 1911-1920

$\frac{1}{1921-1930}$

무

뭄 =

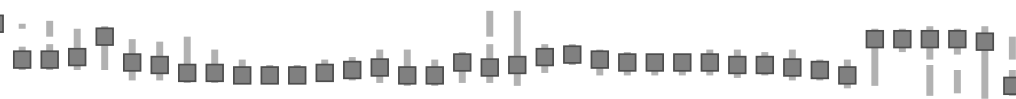

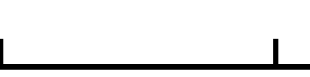

1

$1961-1970$

$1971-1980$

$1981-1990$

$1991-2000$

2003-2012

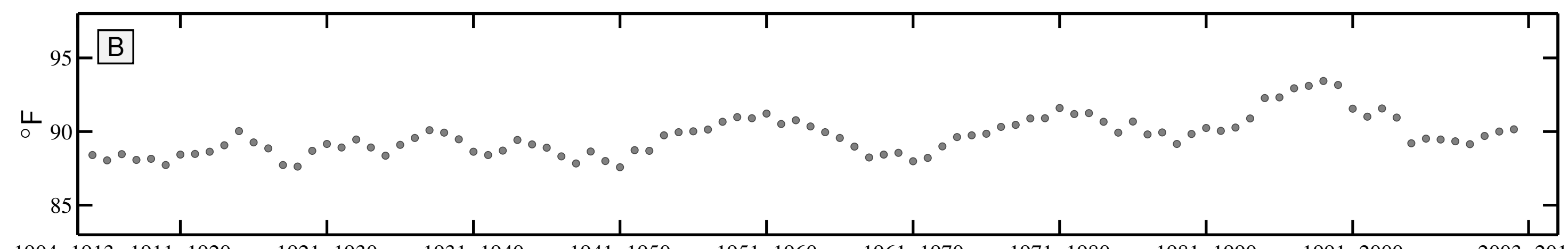

1904-1913 1911-1920

1921-1930

1931-1940

1941-1950

1951-1960

1961-1970

1971-1980

1981-1990

1991-2000

2003-2012

10 year Winter Teletherm dynamics for FT BAYARD, NM:
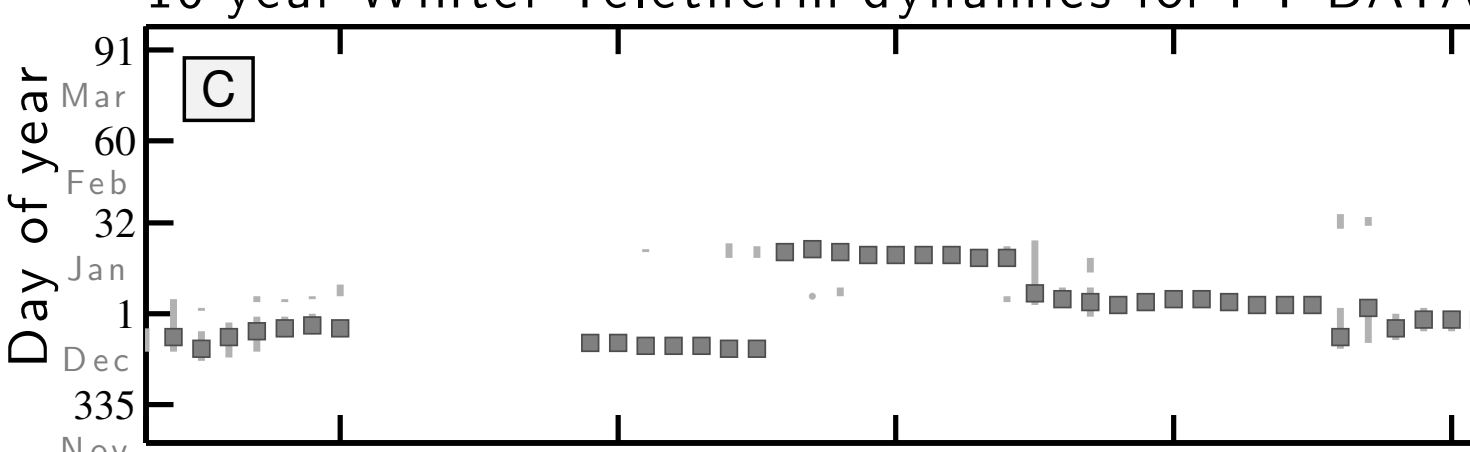

\section{ARD, NM:}


10 year Summer Teletherm dynamics for GAGE, NM:

$\div$ Oct 274

Sep

(1) Sep

$\longrightarrow 244=$

$213-$

त $\mathrm{Ju}$

○ 182

$152-$

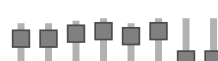

(1)

पㅁํ

1 ।

1904-1913 1911

$1921-1930$

1931-1940

1941-1950

$\frac{1}{1951-1960}$

$1961-1970$

1971-1980

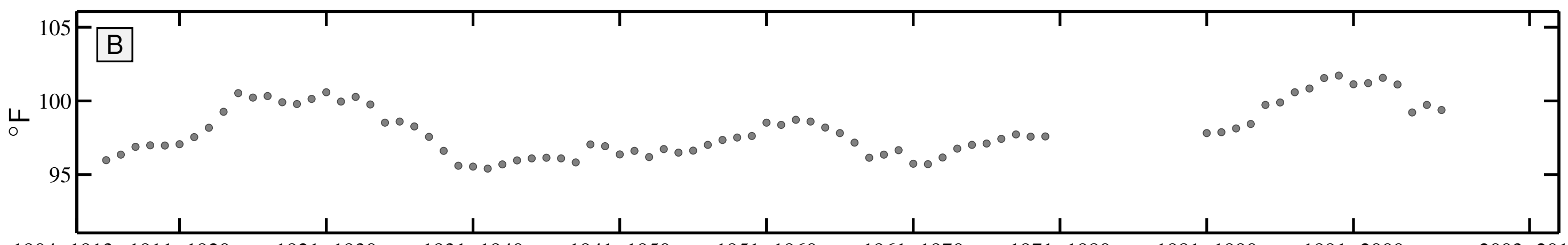

1904-1913 1911-1920

$1921-1930$

$1931-1940$

1941-1950

1951-1960

$1961-1970$

$1971-1980$

$1981-1990$

$1991-2000$

2003-2012

10 year Winter Teletherm dynamics for GAGE, NM:

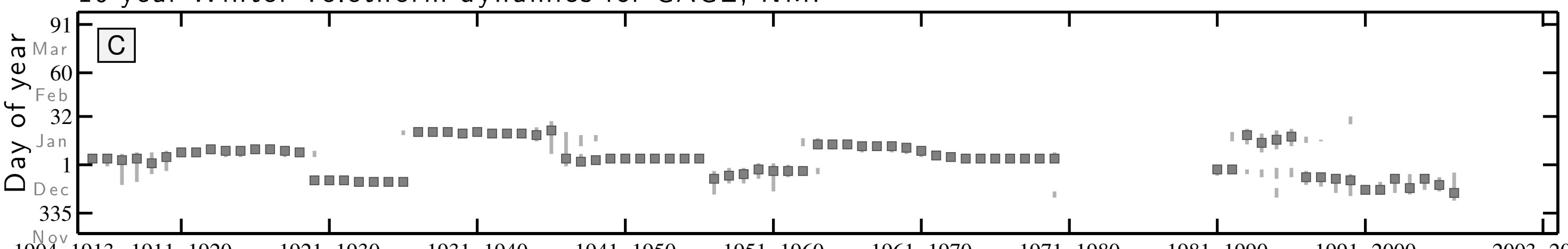

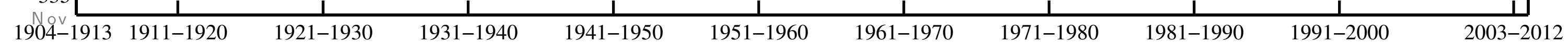

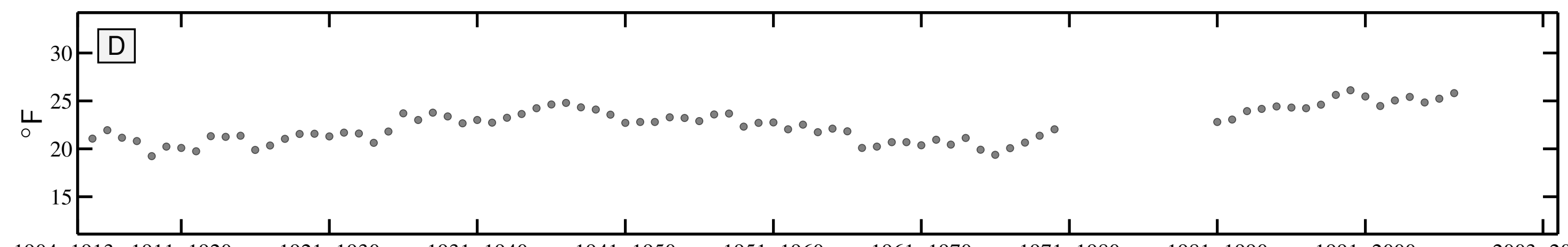


10 year Summer Teletherm dynamics for JEMEZ SPRINGS, NM:

Oct

政 274

o S P

244

O

$213-$

त 182

182

$152-$

| -

| " "

Q

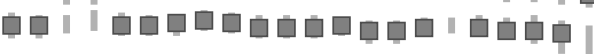

ㅁ =

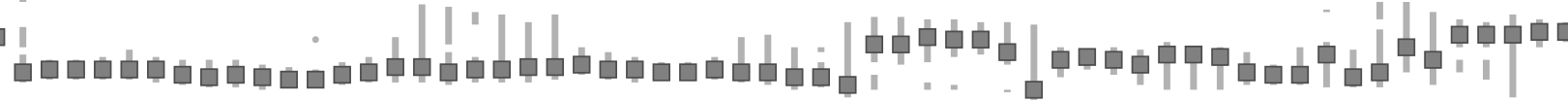

$1904-1913 \quad 1911-1920$

$1921-1930$

$1931-1940$

$\frac{1}{1941-1950}$

$1951-1960$

1961-1970

$\frac{1}{1971-1980}$

$\frac{1}{191-1990}$

991-2000

2003-2012

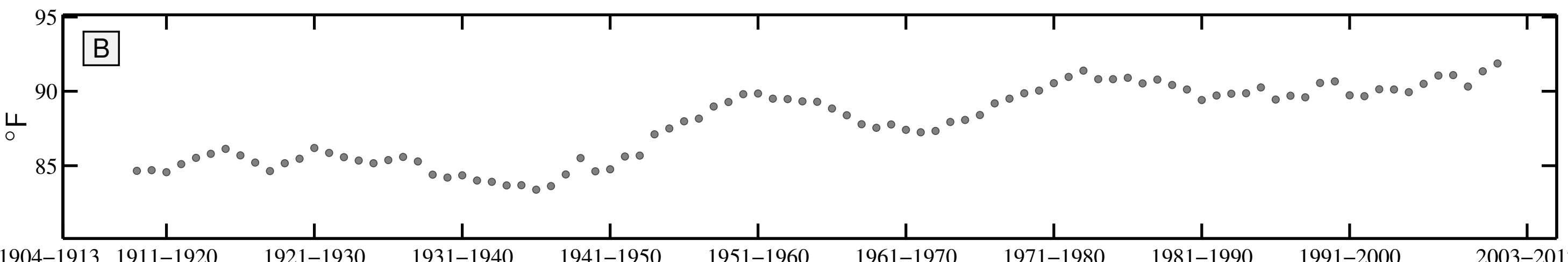

1904-1913 1911-1920

1921-1930

1931-1940

1941-1950

1951-1960

1961-1970

1971-1980

1981-1990

1991-2000

2003-2012

10 year Winter Teletherm dynamics for JEMEZ SPRINGS, NM:

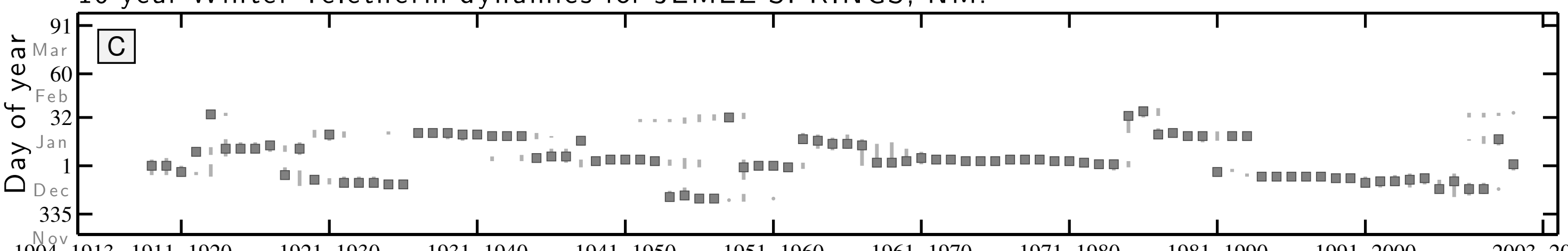

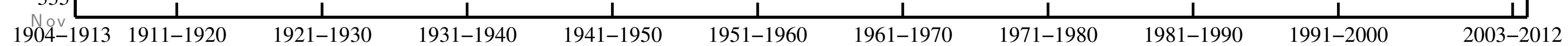

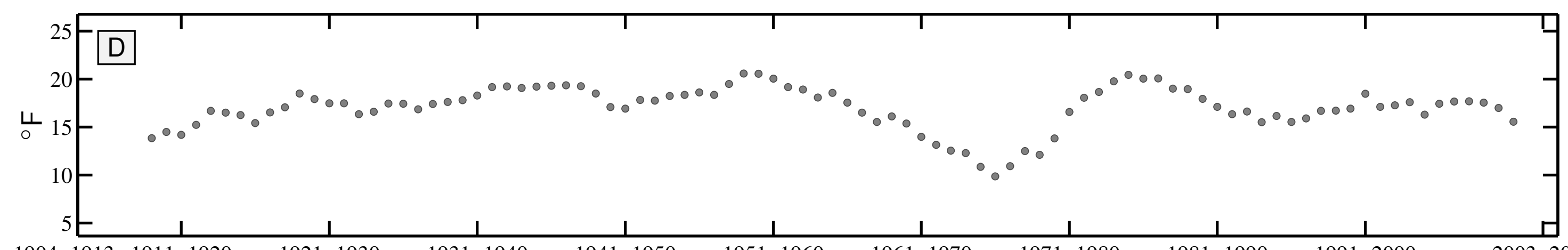


10 year Summer Teletherm dynamics for JORNADA EXP RANGE, NM:
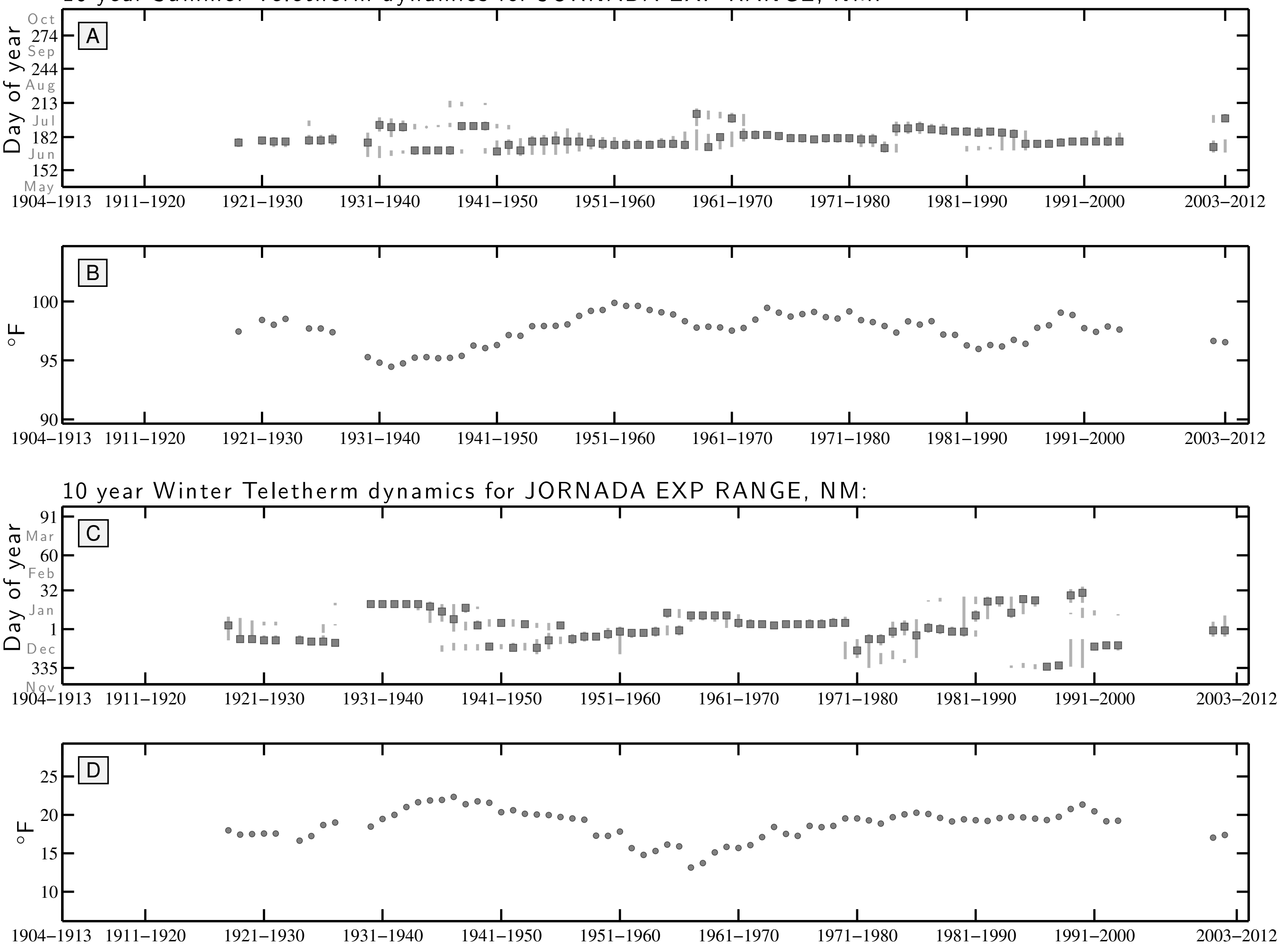
10 year Summer Teletherm dynamics for MTN PARK, NM:

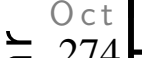

A

(1) Sep

$>244$

O 213

$\gtrsim \mathrm{Jul}$

(182

Jun
152

152

1904-1913 1911-1920

$\frac{1}{1921-1930}$

的田

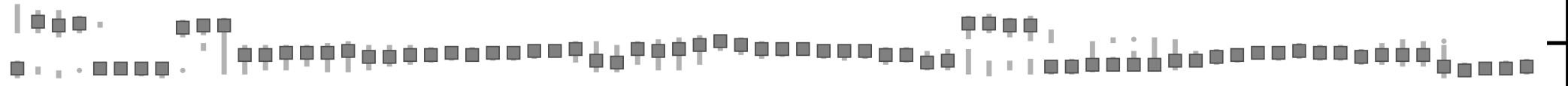

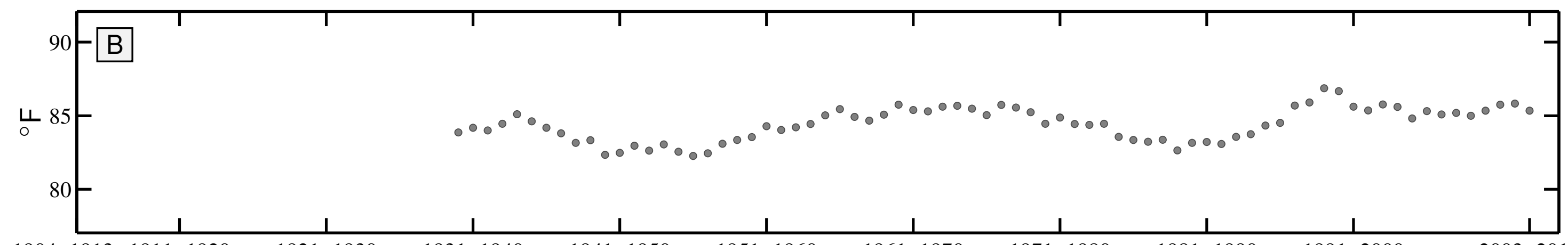

$1904-1913$ 1911-1920

$1921-1930$

$1931-1940$

$1941-1950$

$1951-1960$

$1961-1970$

$1971-1980$

$1981-1990$

$1991-2000$

2003-2012

10 year Winter Teletherm dynamics for MTN PARK, NM:

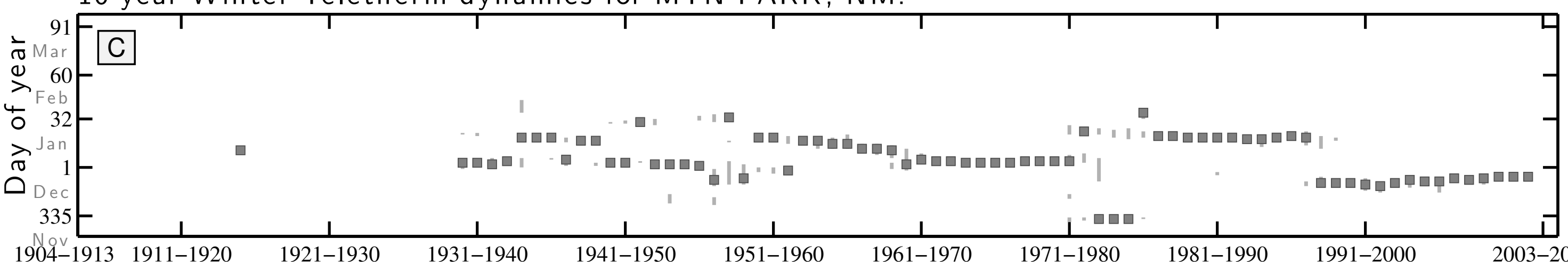

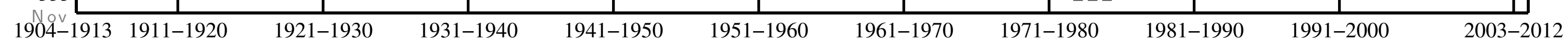

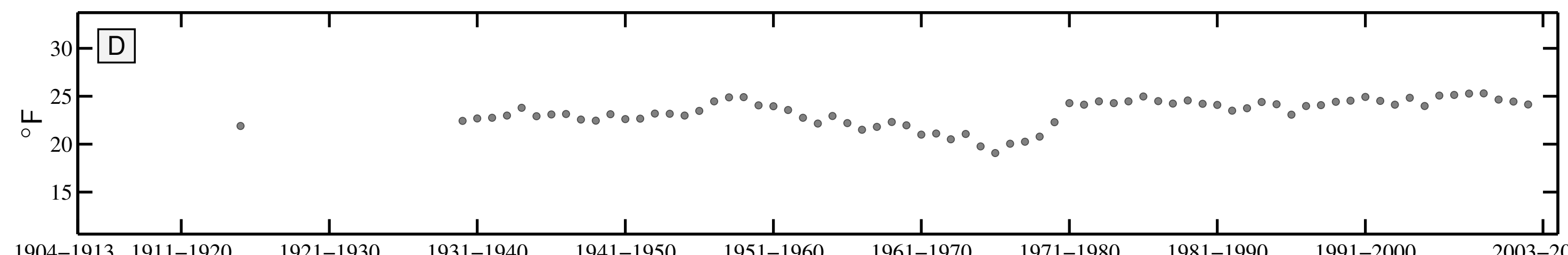


10 year Summer Teletherm dynamics for RED RIVER, NM:

$\div$ Oct 274

\& Sep

$>244$

O 213 -

$\gtrsim \mathrm{Jul}$

$\overbrace{}^{\circ} 182-$

152

May $\square$

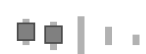

| ||

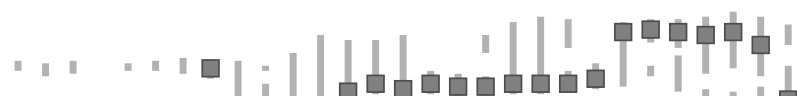

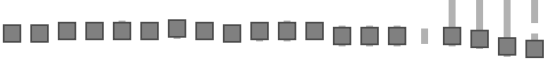

1904-1913 1911-1920

$\frac{1}{1921-1930}$

$\frac{1}{1931-1940}$

$\frac{1}{1941-1950}$

$\frac{1}{1951-1960}$

$\frac{1}{1961-1970}$

$\frac{1}{1971-1980}$

1981-1990

1991-2000

2003-2012

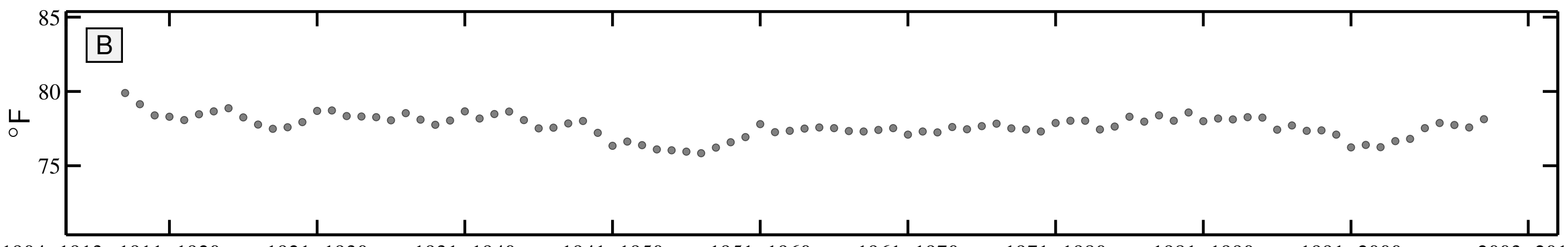

1904-1913 1911-1920

$1921-1930$

$1931-1940$

$1941-1950$

$1951-1960$

$1961-1970$

$1971-1980$

$1981-1990$

$1991-2000$

2003-2012

10 year Winter Teletherm dynamics for RED RIVER, NM:

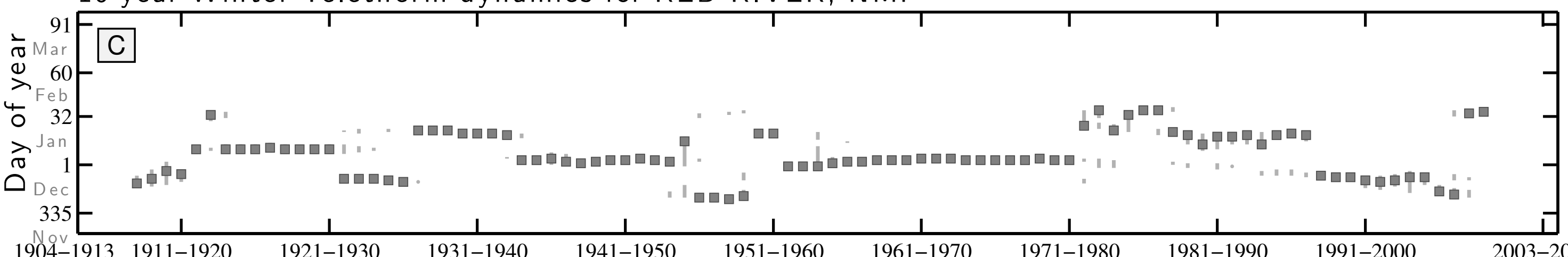

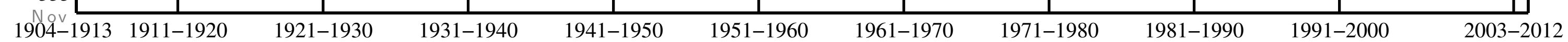

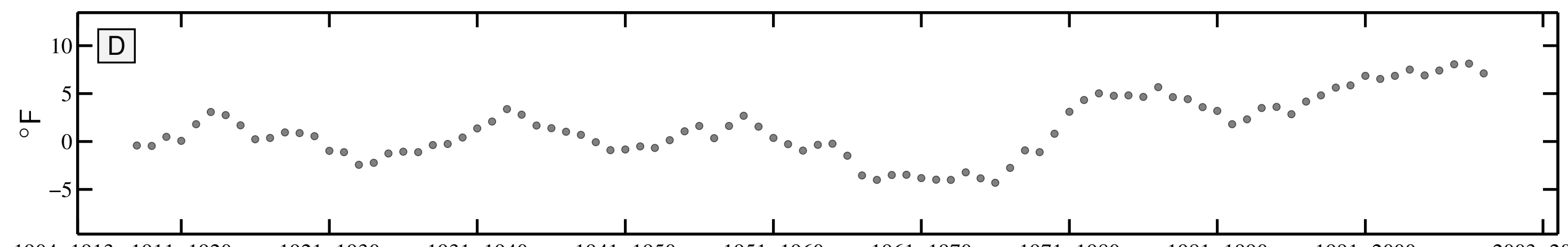


10 year Summer Teletherm dynamics for SPRINGER, NM:

$-O c t$

A

(1) Sep

$>244-$

( $\mathrm{Aug}$

$>\mathrm{Jul}$

182

152

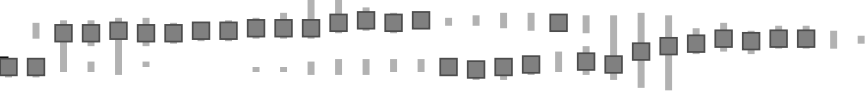

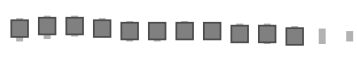

1904-1913 1911-1920

$1921-1930$

1931-1940

$\frac{1}{1941-1950}$

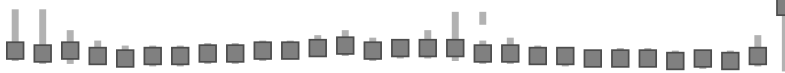

中吊

的

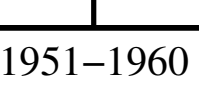

$1961-1970$

$\frac{1}{1971-1980}$

981-1990

$1991-2000$

2003-2012

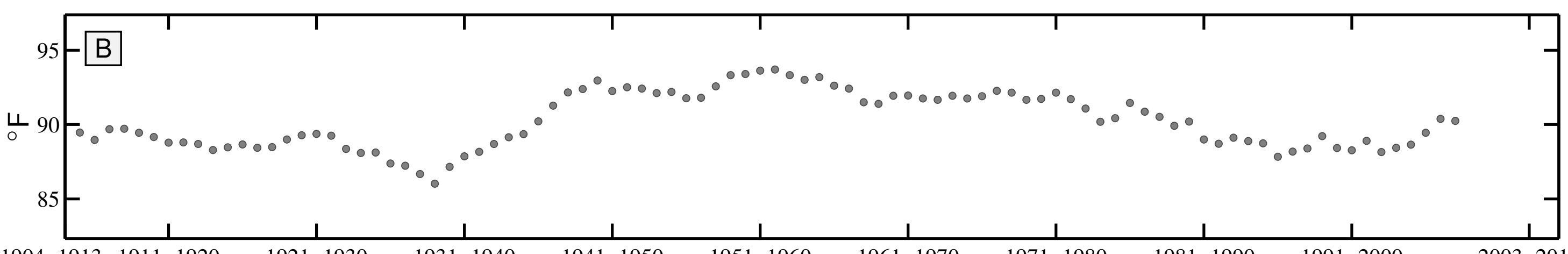

1904-1913 1911-1920

1921-1930

1931-1940

1941-1950

1951-1960

1961-1970

1971-1980

1981-1990

1991-2000

2003-2012

10 year Winter Teletherm dynamics for SPRINGER, NM:

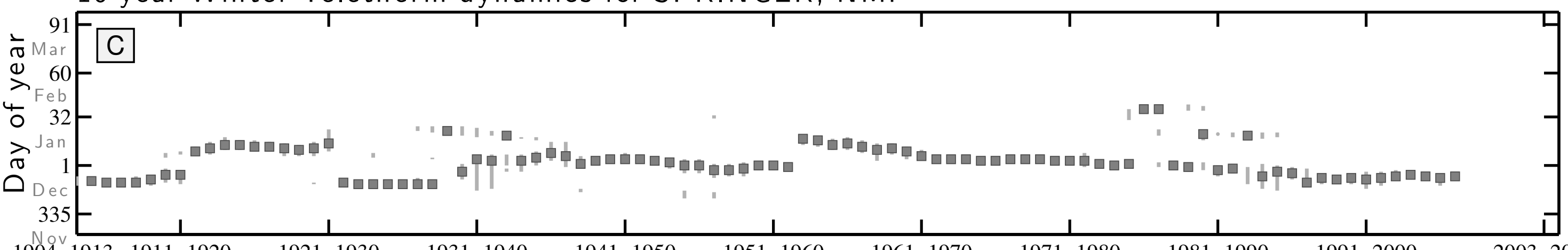

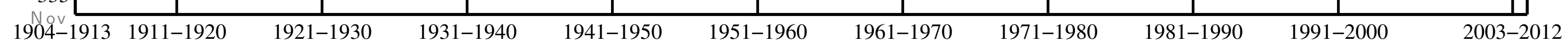

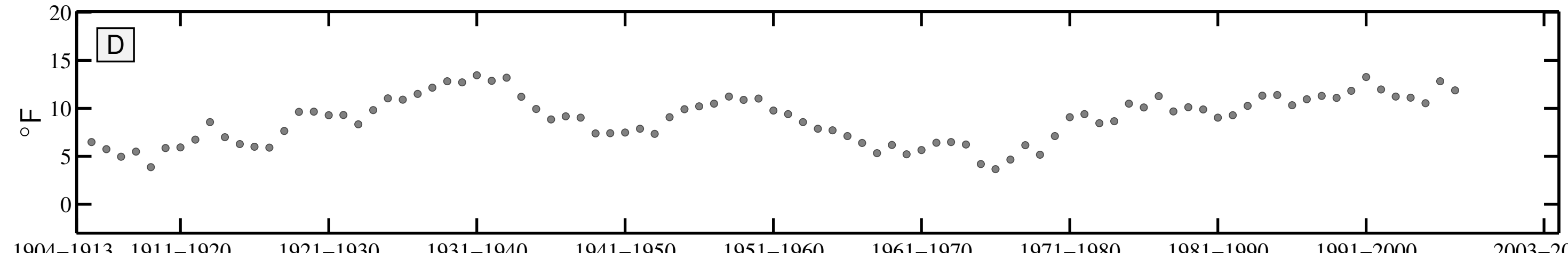


10 year Summer Teletherm dynamics for BUFFALO NIAGARA INTL, NY:

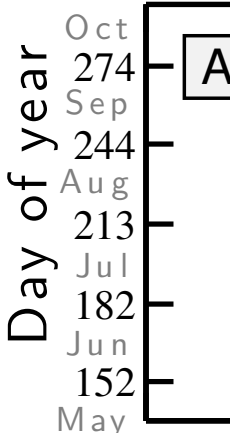
$A$

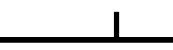

1904-1913 1911-1920

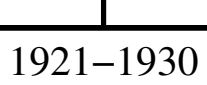

$\frac{1}{1931-1940}$

\section{|| I I 1}

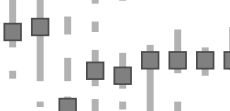

I

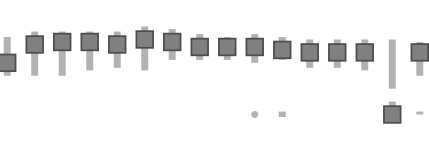

1941-1950

1951-1960

1961-1970

1971-1980

981-1990

991-2000

2003-201

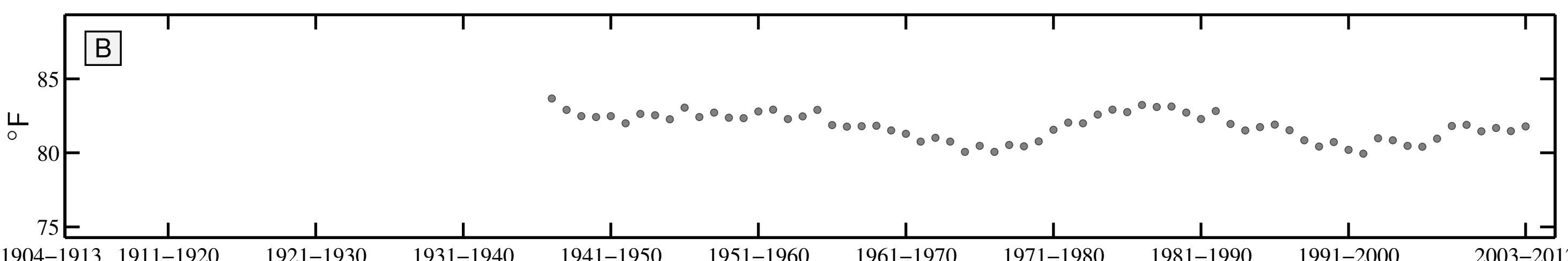

10 year Winter Teletherm dynamics for BUFFALO NIAGARA INTL, NY:
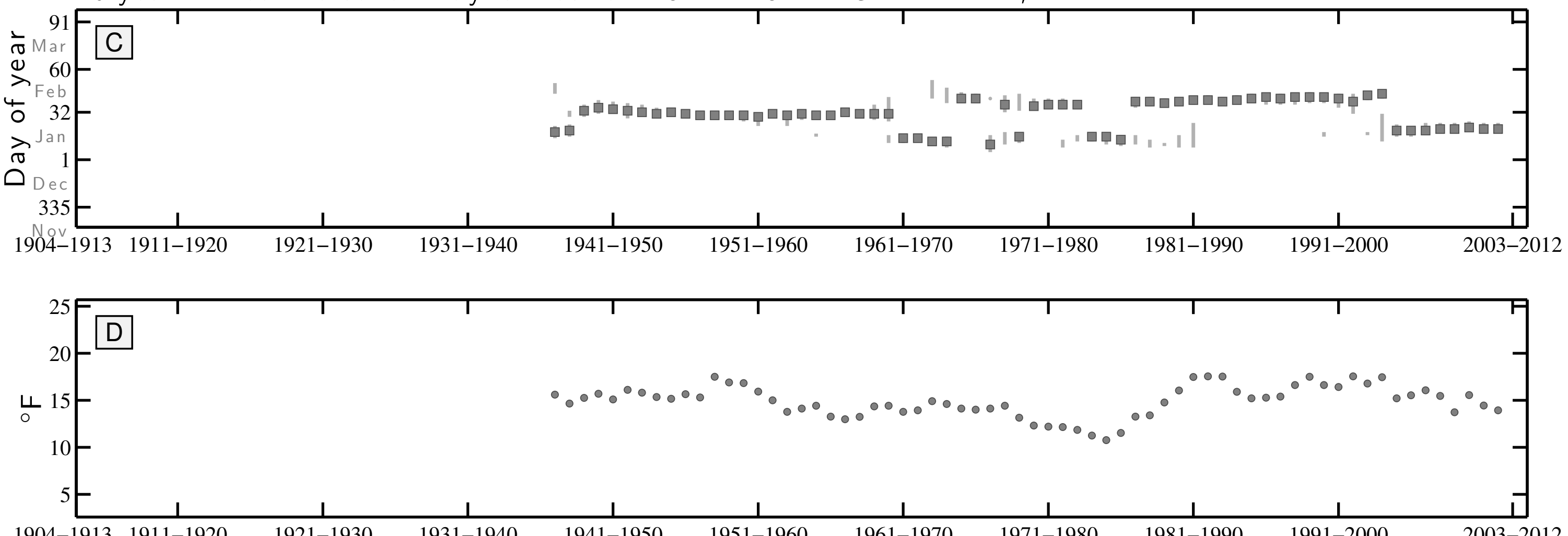
10 year Summer Teletherm dynamics for INDIAN LAKE 2SW, NY:

$+\mathrm{Oct}$

A

(1) Sep

$>244$

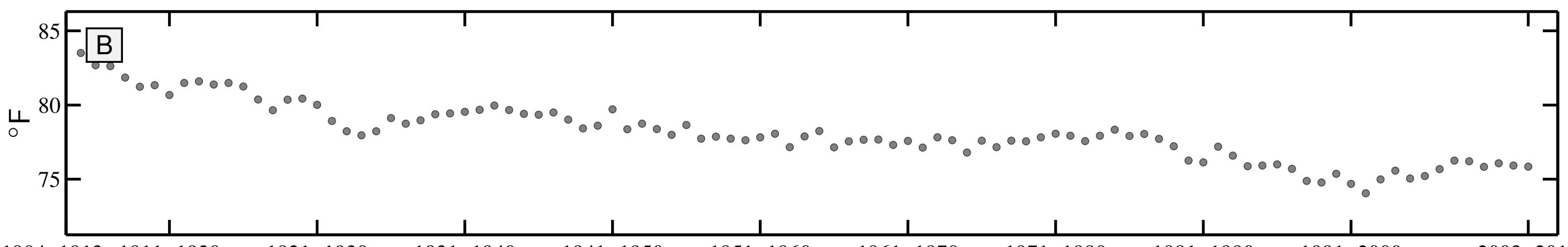

10 year Winter Teletherm dynamics for INDIAN LAKE 2SW, NY:

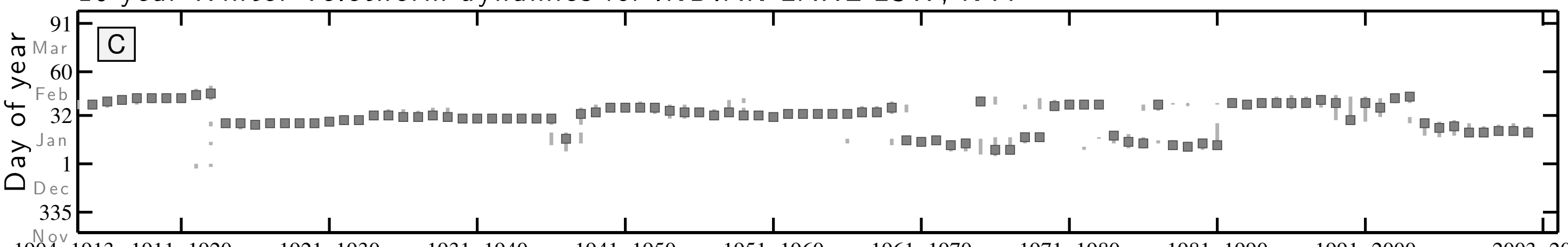

1904-1913 1911-1920

$1921-1930$

$1931-1940$

$1941-1950$

$1951-1960$

$1961-1970$

$1971-1980$

1981-1990

$1991-2000$

2003-2012

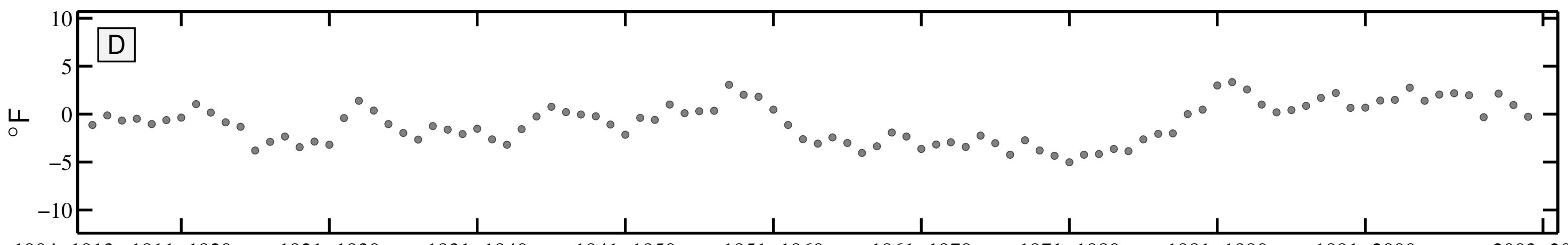


10 year Summer Teletherm dynamics for LOCKPORT 3 S, NY:

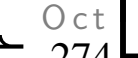

A

(1) Sep

$-\mathrm{A} 4 \mathrm{~g}$

○ 213 - | | " " " "

\% 18

$152-$

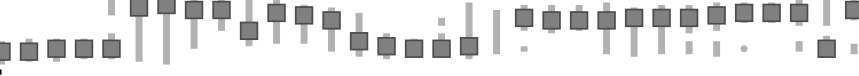

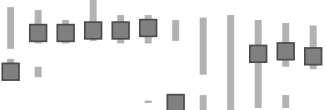

|

$1904-1913$ 1911-1920

$\frac{1}{1921-1930}$

1931-1940

1941-1950

$\frac{1}{1-1960}$

1961-1970

$\frac{1}{1971-1980}$

$\frac{1}{1981-1990}$

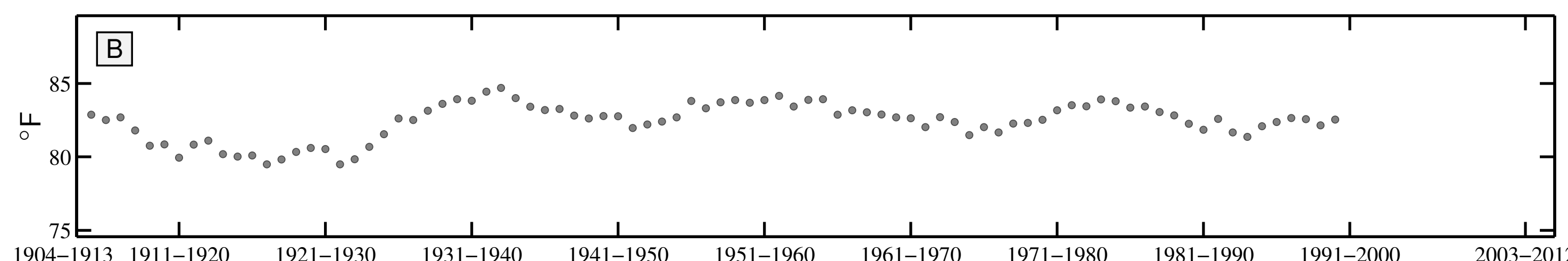

10 year Winter Teletherm dynamics for LOCKPORT $3 \mathrm{~S}, \mathrm{NY}$ :

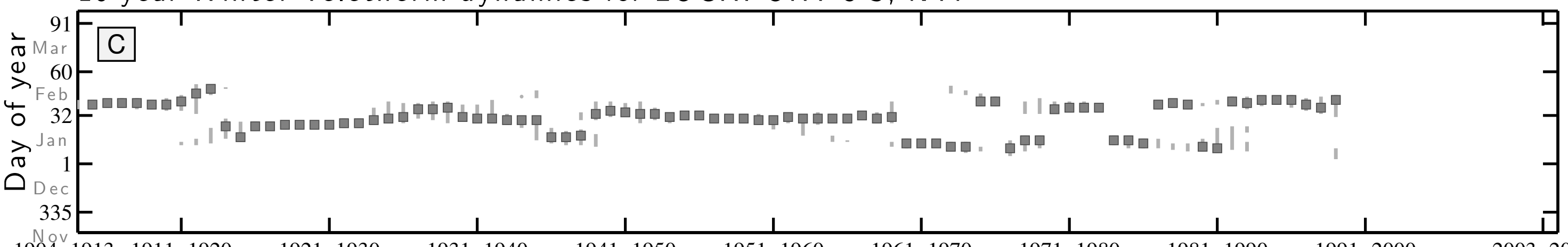

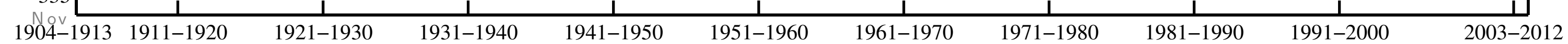

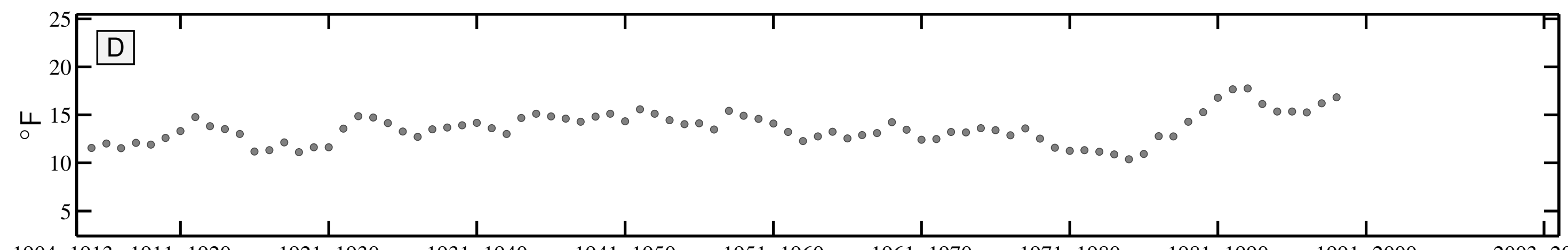


10 year Summer Teletherm dynamics for LOWVILLE, NY:

$\mathrm{Oct}$

( $274-A$

(1) Sep

$244-$

O $213-$

21

\% 182

Jun
152
$M$ ay

$1904-19131911-1920$

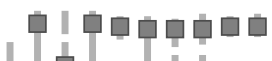

|

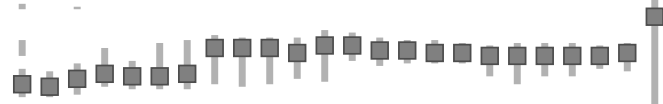

पा - I (n)

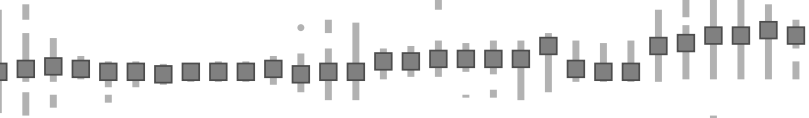

| | " -
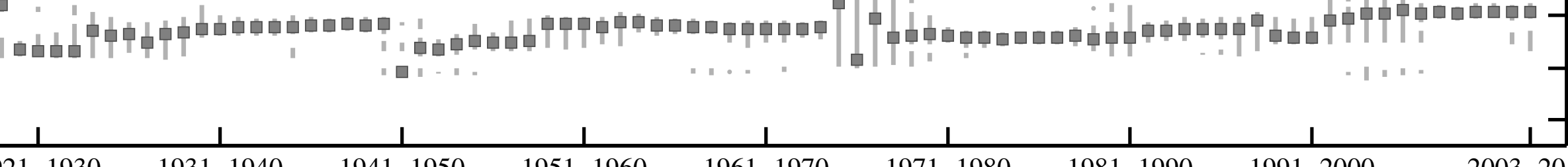

1931-1940

$\frac{1}{1941-1950}$

$1951-1960$

961-1970

$1971-1980$

981-1990

$1991-2000$

2003-2012

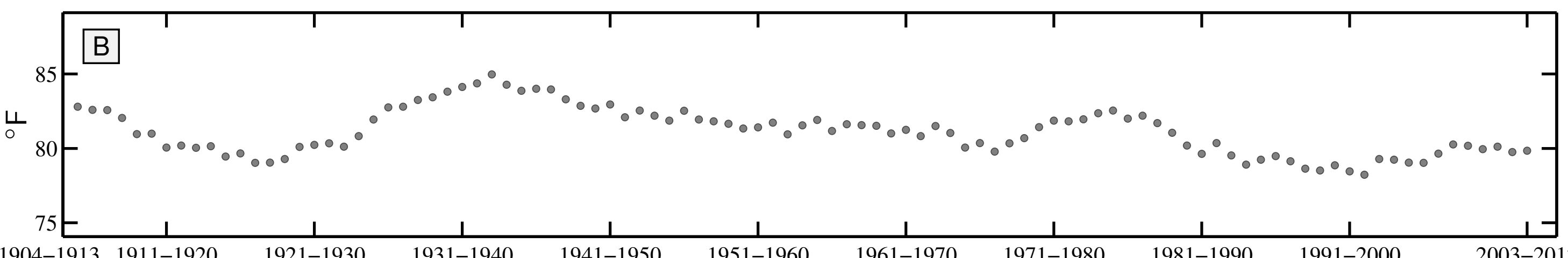

1904-1913 1911-1920

$1921-1930$

$1931-1940$

$1941-1950$

$1951-1960$

$1961-1970$

$1971-1980$

$1981-1990$

$1991-2000$

2003-2012

10 year Winter Teletherm dynamics for LOWVILLE, NY:

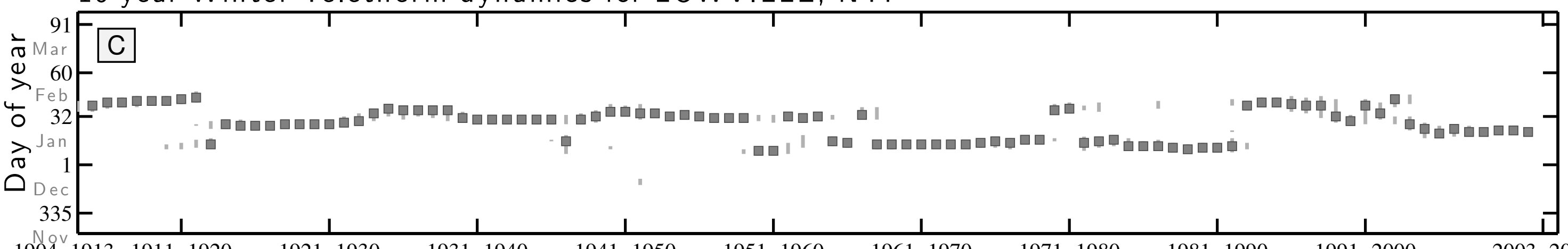

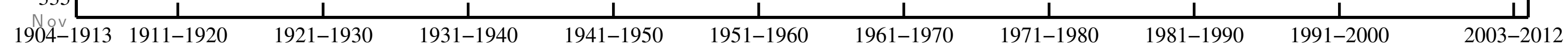

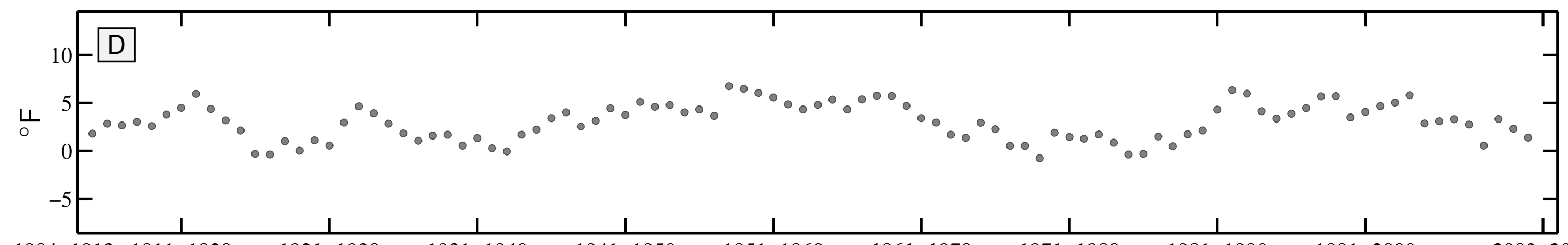


10 year Summer Teletherm dynamics for MARYLAND 9 SW, NY:
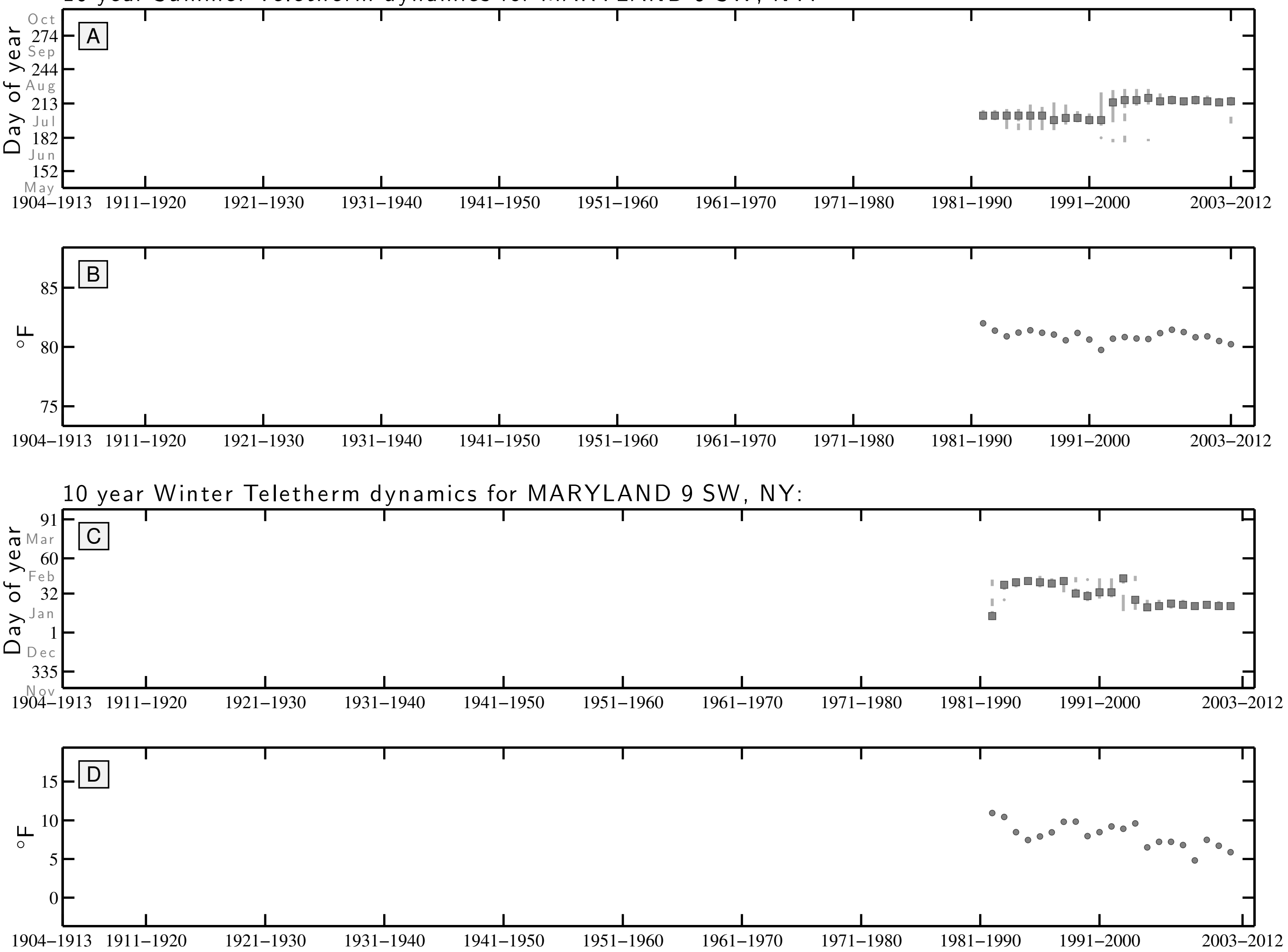
10 year Summer Teletherm dynamics for PORT JERVIS, NY:

Oct

A

(1) Sep

244

O 213

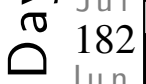

$152-$

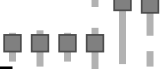

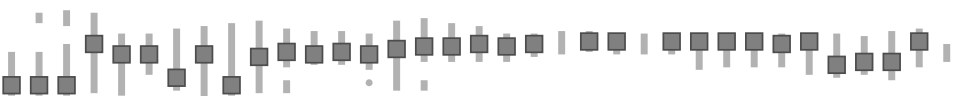

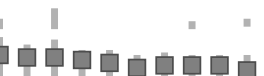

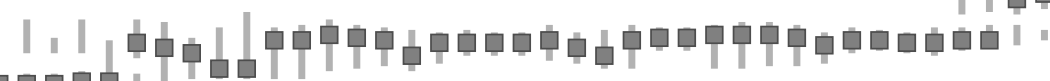

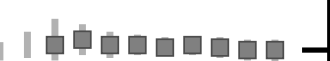
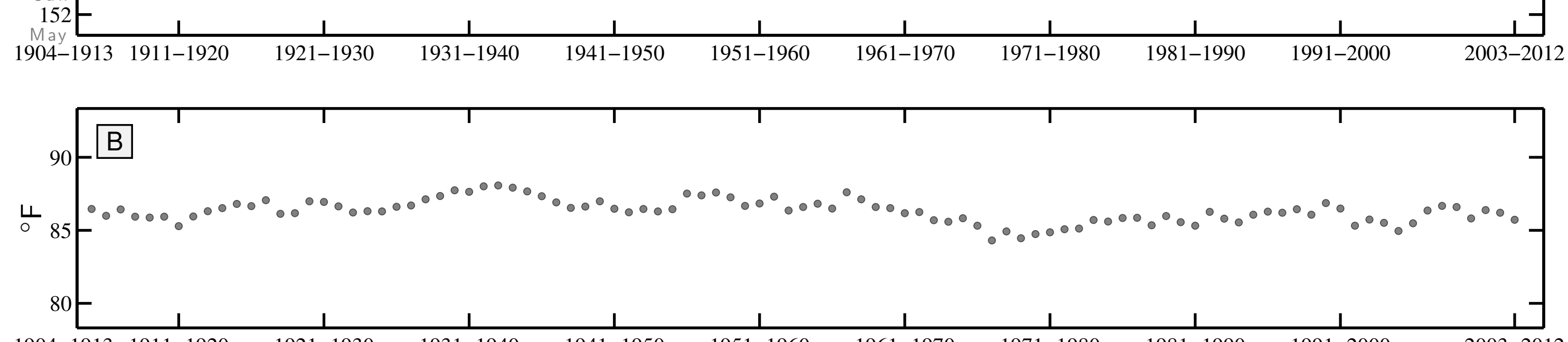

1904-1913 1911-1920

$1921-1930$

$1931-1940$

$1941-1950$

$1951-1960$

$1961-1970$

$1971-1980$

$1981-1990$

$1991-2000$

2003-2012

10 year Winter Teletherm dynamics for PORT JERVIS, NY:

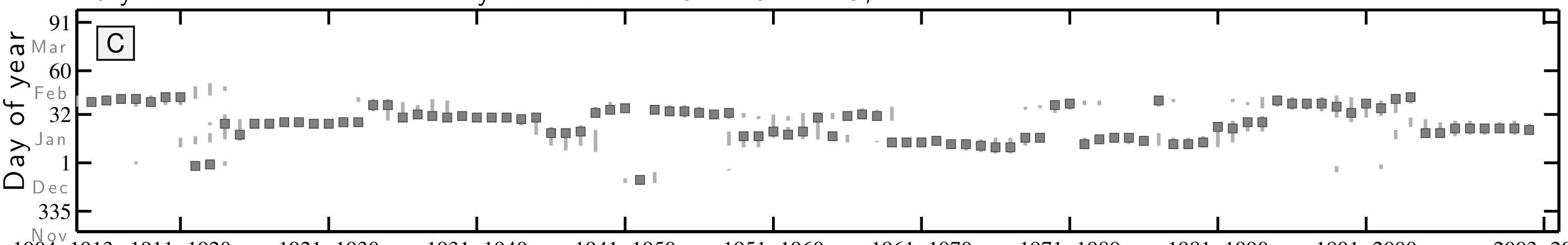

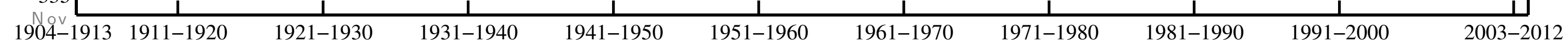

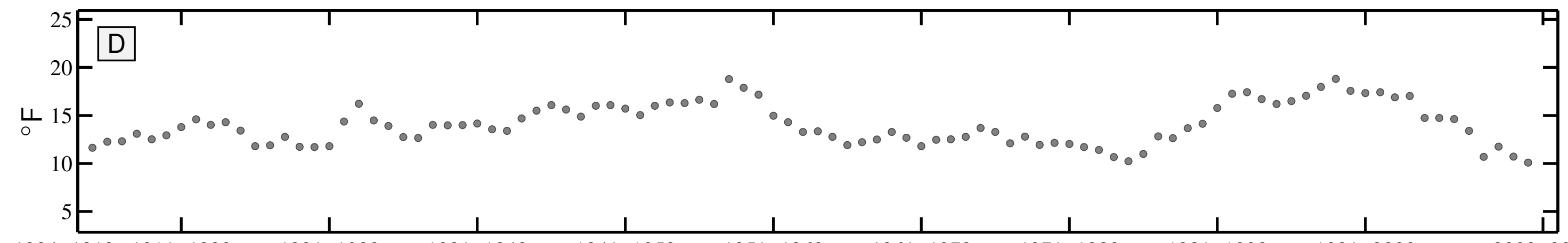


10 year Summer Teletherm dynamics for SARATOGA SPRINGS 4 SW, NY:
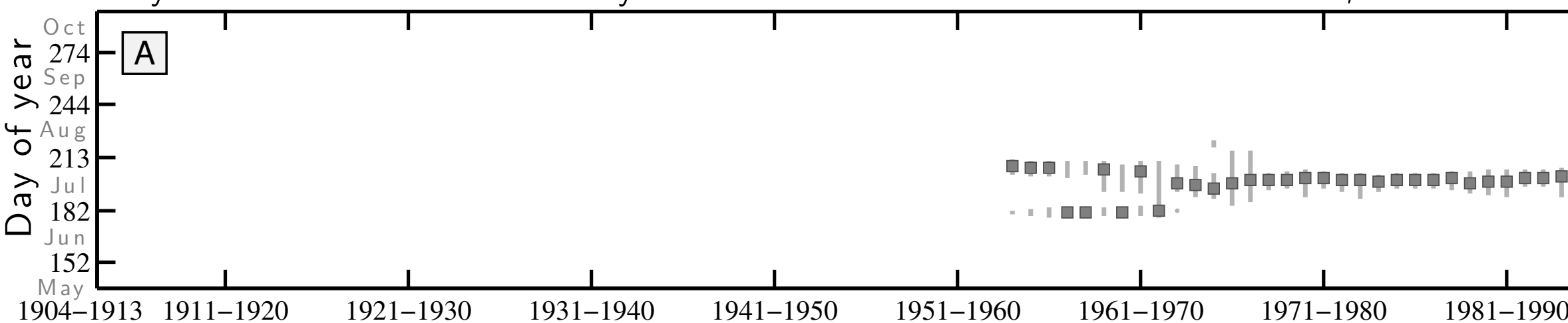

$1991-2000$

2003-2012

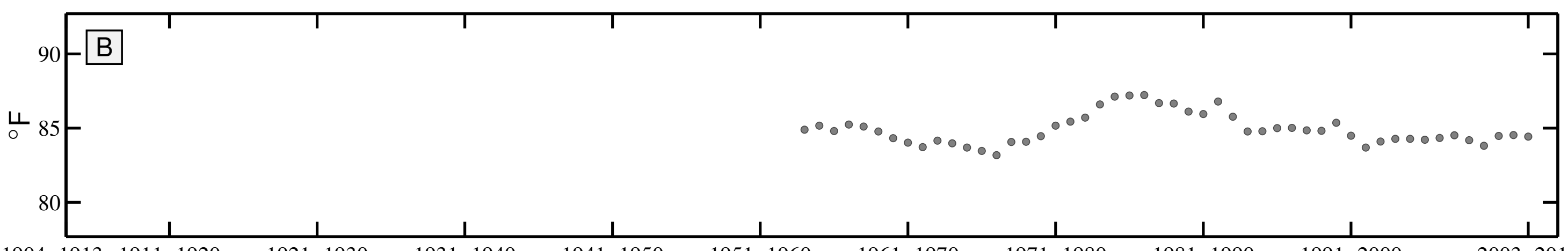

10 year Winter Teletherm dynamics for SARATOGA SPRINGS 4 SW, NY:

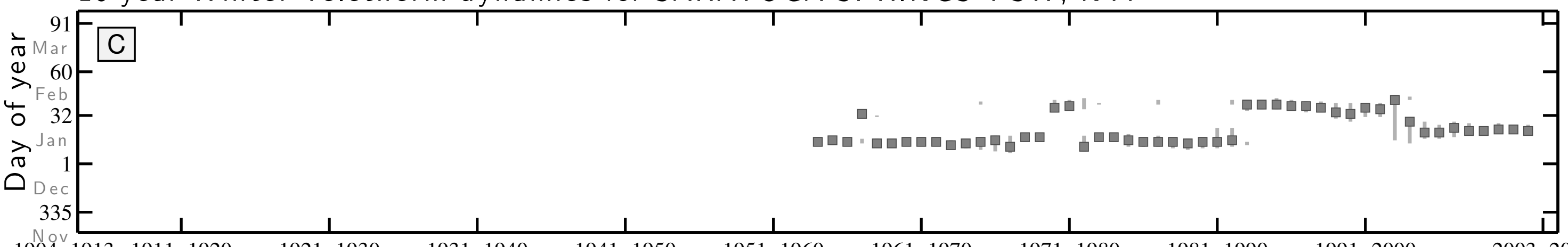

1904-1913 1911-192(

1921-1930

1931-1940

1941-1950

1951-1960

1961-1970

1971-1980

1981-1990

1991-2000

2003-2012

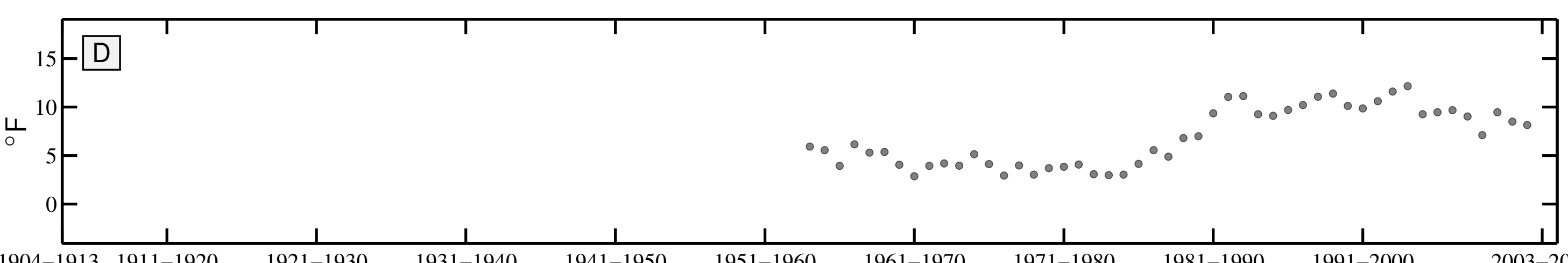




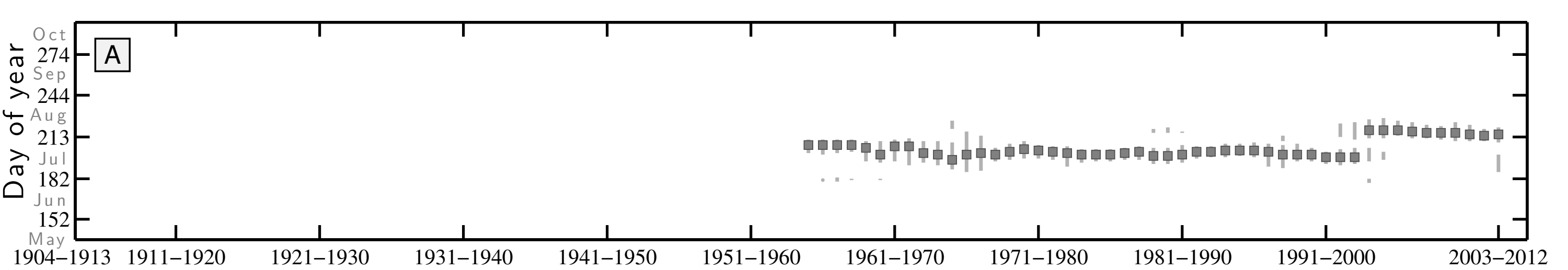

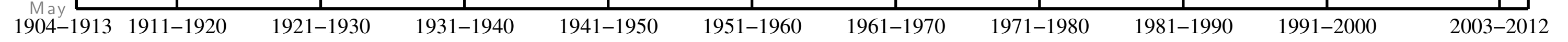
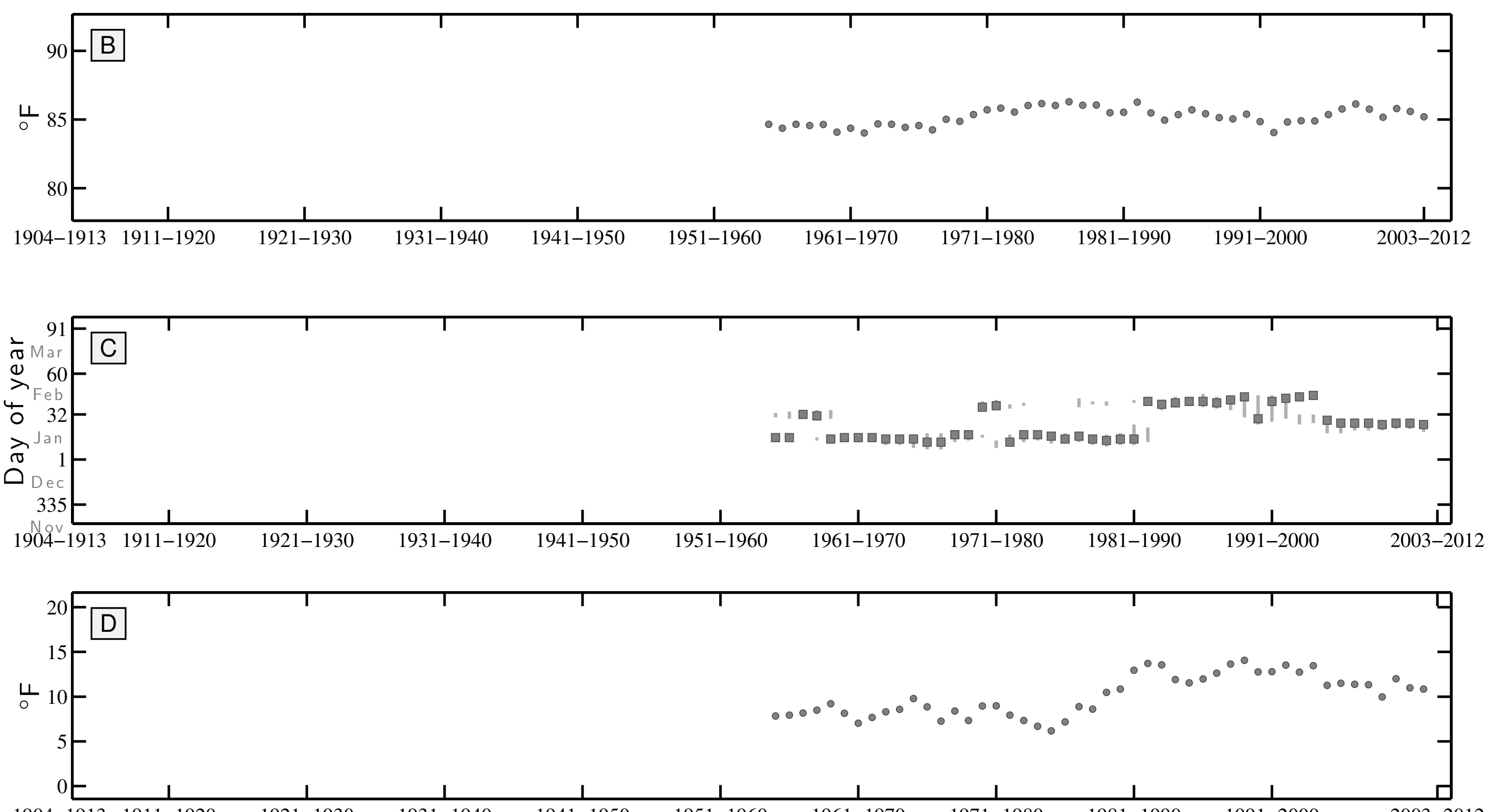

$\begin{array}{lllllllllll}1904-1913 & 1911-1920 & 1921-1930 & 1931-1940 & 1941-1950 & 1951-1960 & 1961-1970 & 1971-1980 & 1981-1990 & 1991-2000 & 2003-2012\end{array}$


10 year Summer Teletherm dynamics for UTICA FAA AP, NY:

Oct

A

(1) Sep

$>244$

$\gtrsim \mathrm{Ju}$

$182-$

152 -

1

1904-1913 1911-1920

1921-1930

1931-1940

$\frac{1}{1941-1950}$

$\frac{1}{1951-1960}$

1961-1970

1971-1980

$1981-1990$

1991-2000

2003-2012

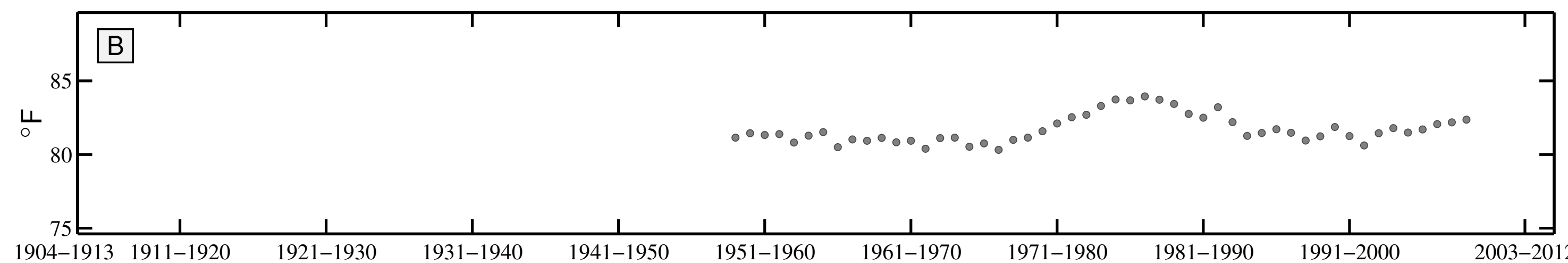

10 year Winter Teletherm dynamics for UTICA FAA AP, NY:
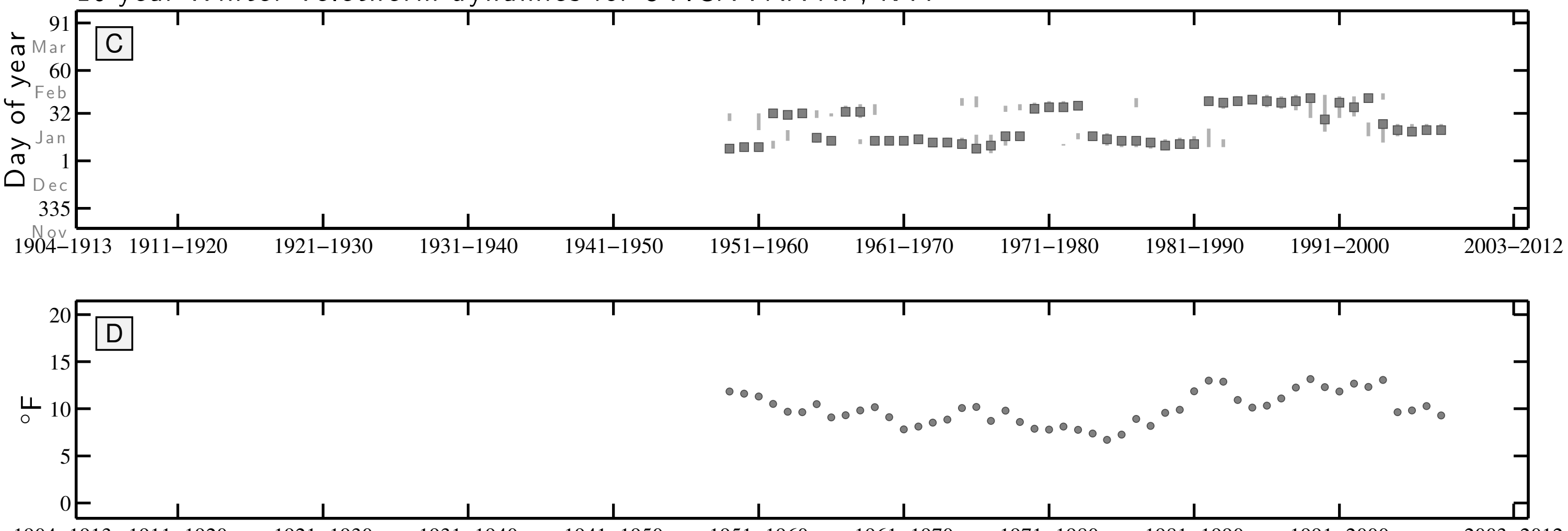
10 year Summer Teletherm dynamics for WALES, NY:
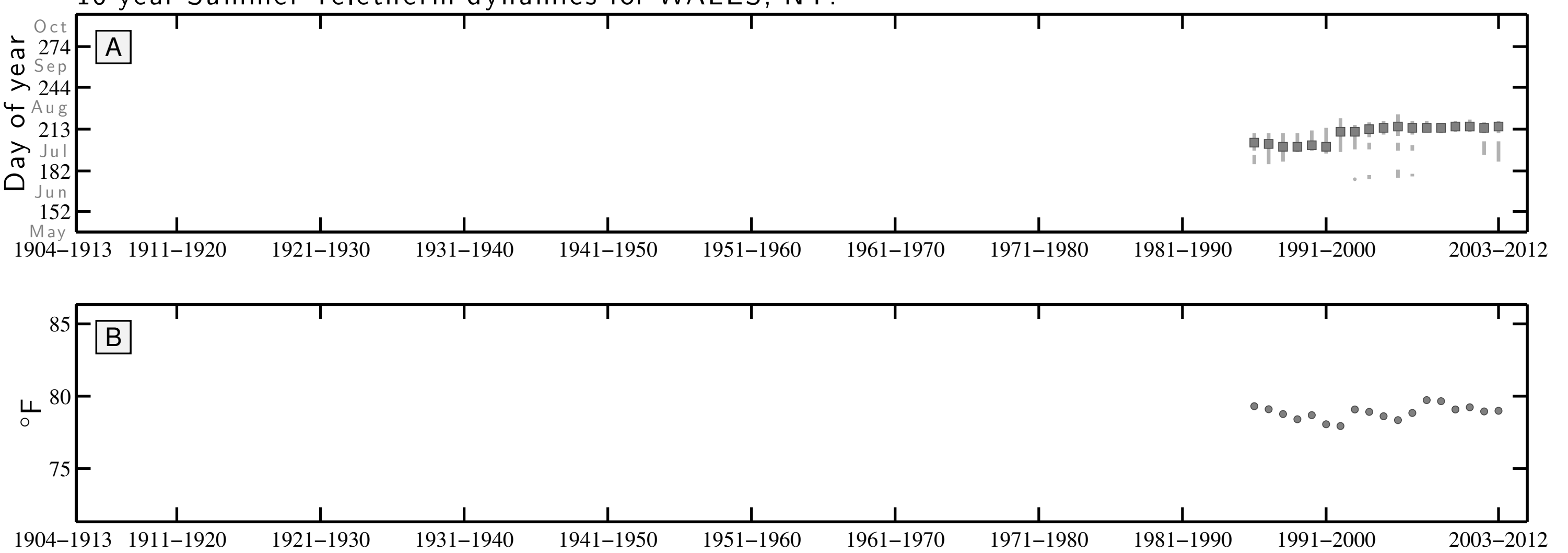

10 year Winter Teletherm dynamics for WALES, NY:
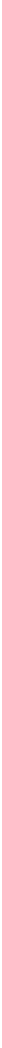

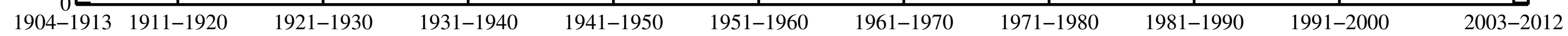


10 year Summer Teletherm dynamics for CAPE HATTERAS AP, NC:
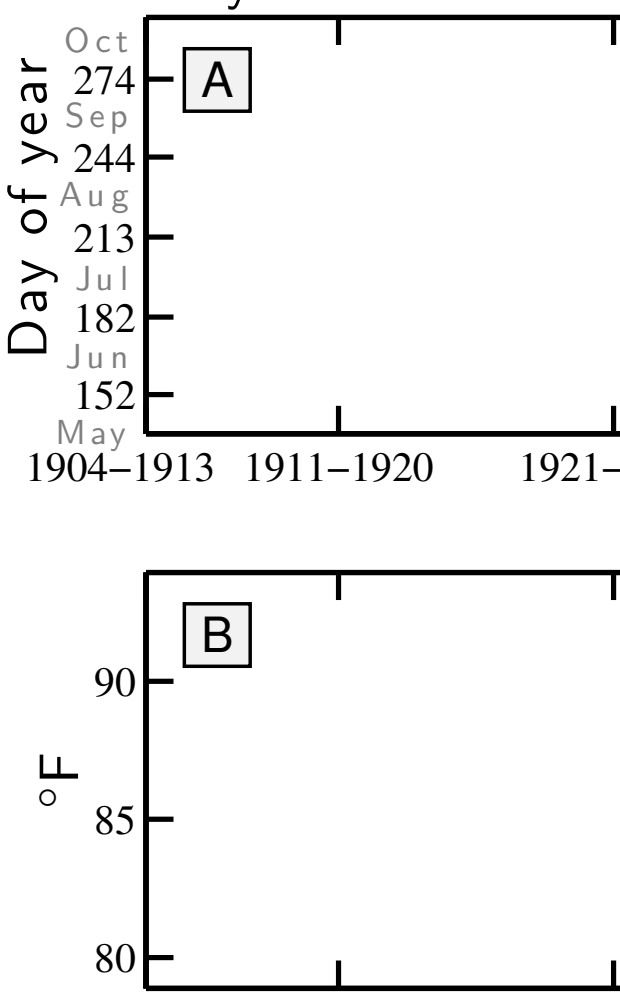

$1904-1913$ 1911-1920
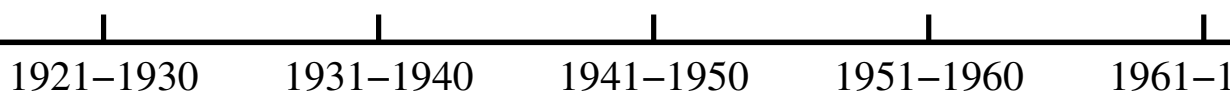

10 year Winter Teletherm dynamics for CAPE HATTERAS AP, NC:

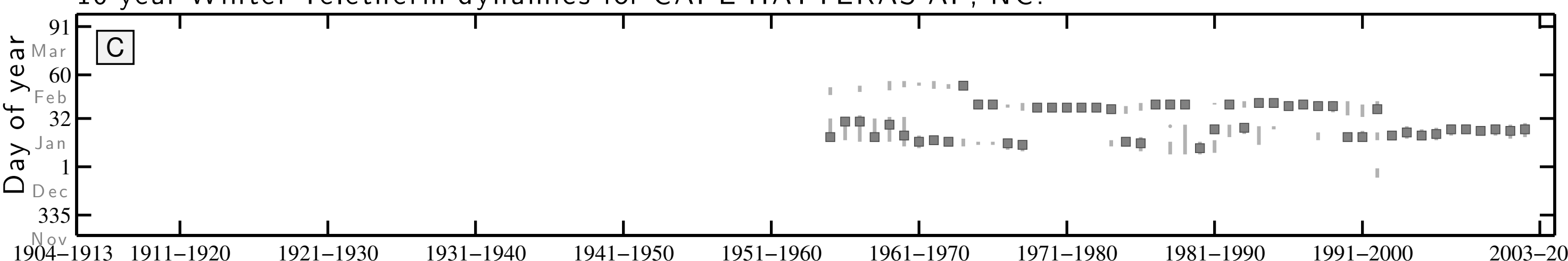

1904-1913 1911-192

$1921-1930$

$1931-1940$

1941-1950

1951-1960

1961-1970

1971-1980

1981-1990

1991-2000

2003-2012

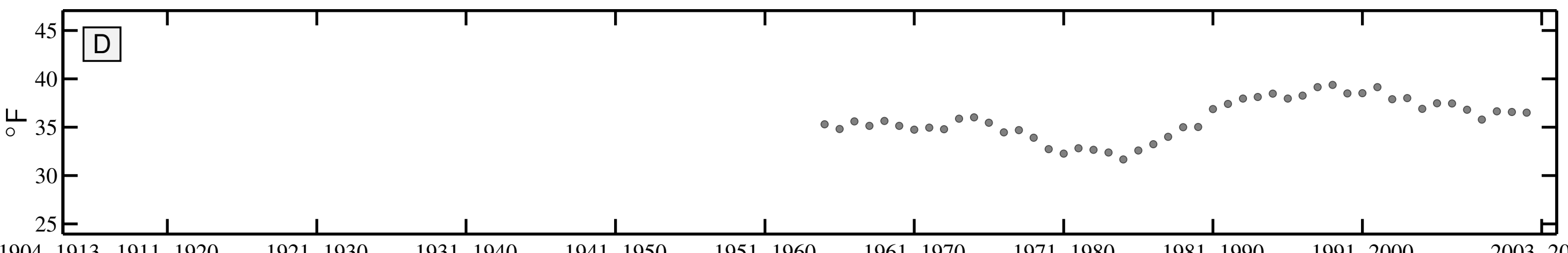


10 year Summer Teletherm dynamics for FAYETTEVILLE PWC, NC:

$\div$ Oct 274

(1) Sep

$>244-$

213 - ㅁㅁ

$\gtrsim \mathrm{Ju}$

182

$152-$

15ay

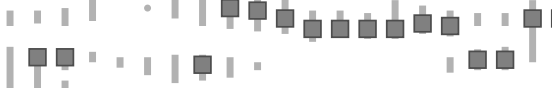
|| || "

प $\square$

$-1920$

$\frac{1}{1921-1930}$

$\frac{1}{1931-1940}$

$\frac{1}{1941-1950}$

$\frac{1}{1951-1960}$

$1961-1970$

1971-1980

$\frac{1}{981-1990}$

1991-2000

2003-201

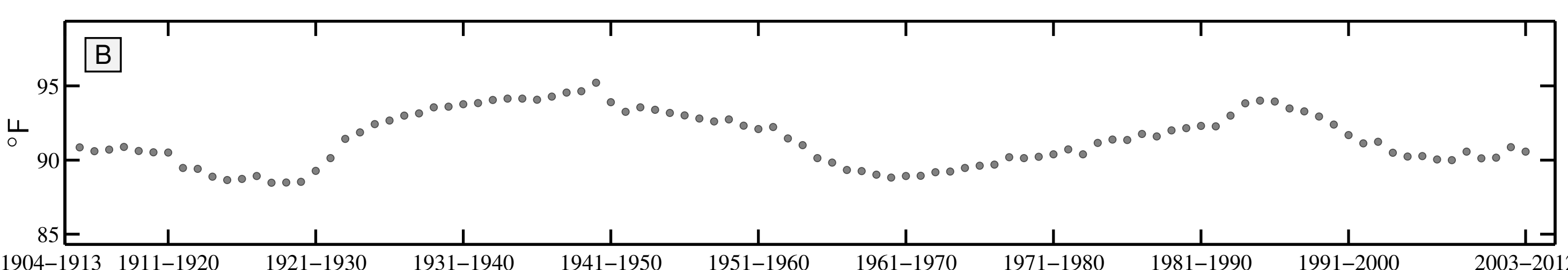

1904-1913 1911-1920

1921-1930

1931-1940

1941-1950

1951-1960

1961-1970

1971-1980

1981-1990

$1991-2000$

$2003-2012$

10 year Winter Teletherm dynamics for FAYETTEVILLE PWC, NC:

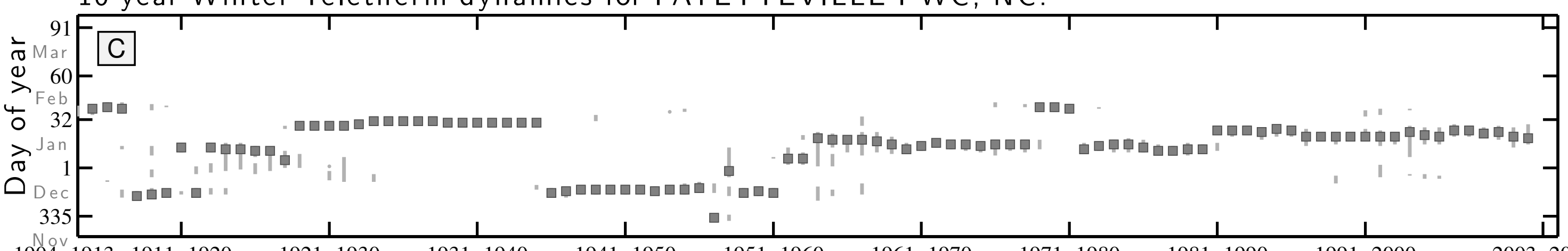

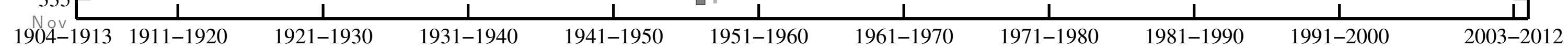

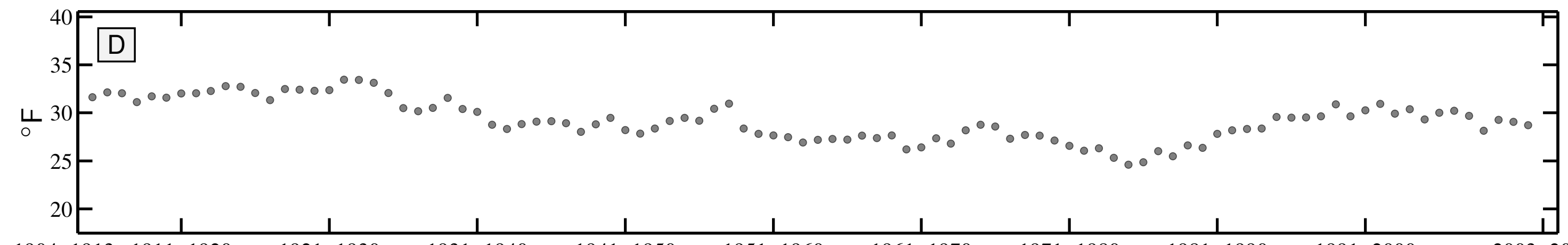


10 year Summer Teletherm dynamics for MARION 2 NW, NC:

$\div 2 \mathrm{ct} A$

$\stackrel{2}{>} 244$

4 Aug

213

$>213-$

$\overbrace{}^{\circ} 182$

$152-$

May

1904-1913 1911-1920

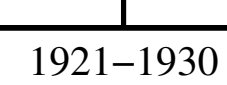

$\frac{1}{1931-1940}$

1941-1950

1951-1960

1961-1970

$\frac{1}{1971-1980}$

1981-1990

$1991-2000$

$\square$

뭄ㅁㅁำ

$\square$

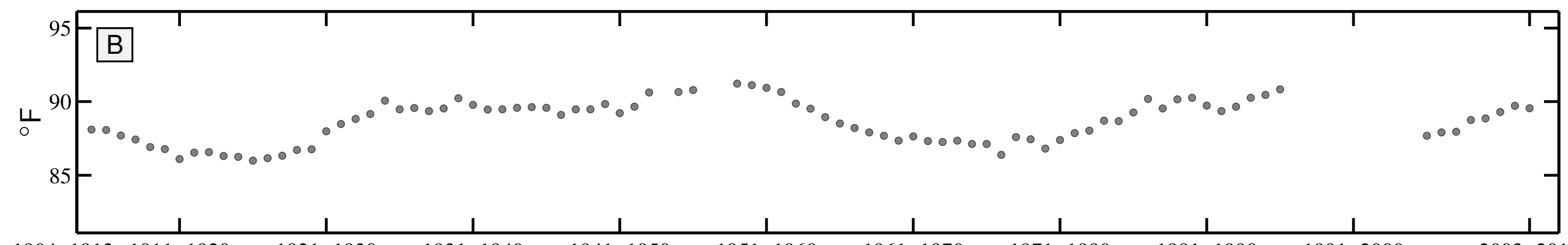

1904-1913 1911-1920

1921-1930

1931-1940

$1941-1950$

$1951-1960$

1961-1970

$1971-1980$

$1981-1990$

$1991-2000$

2003-2012

10 year Winter Teletherm dynamics for MARION 2 NW, NC:

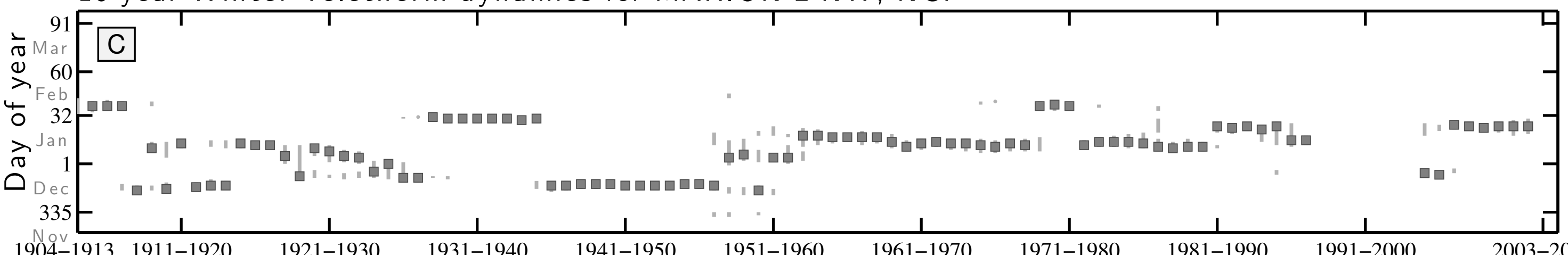

1904-1913 $1911-1920 \quad 1921-1930 \quad 1931-1940$

$1941-1950$

$1951-1960$

1961-1970

1971-1980

1981-1990

2003-2012

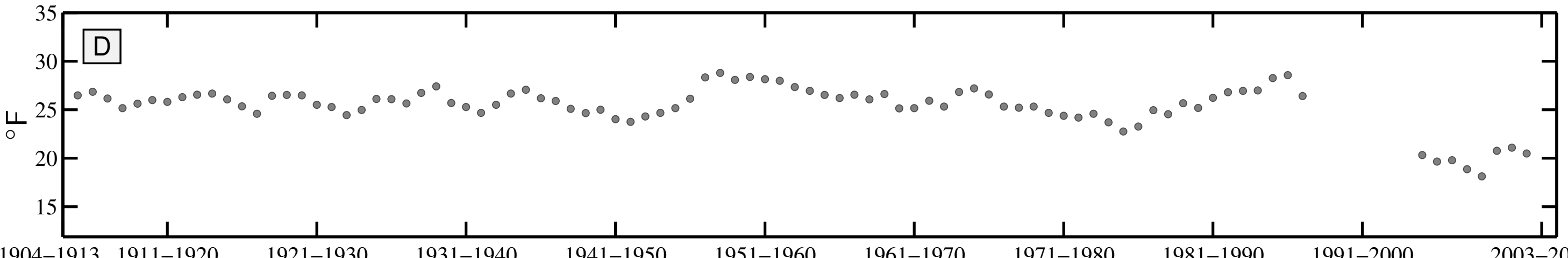


10 year Summer Teletherm dynamics for WILSON 3 SW, NC:
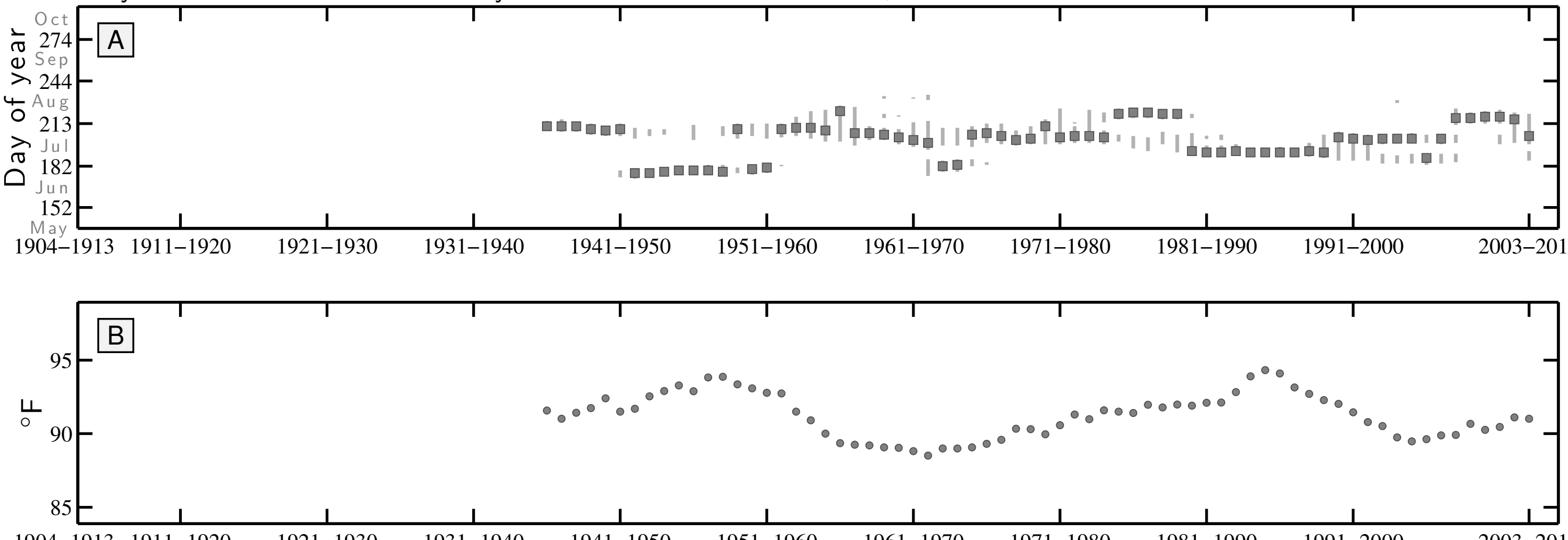

10 year Winter Teletherm dynamics for WILSON 3 SW, NC:
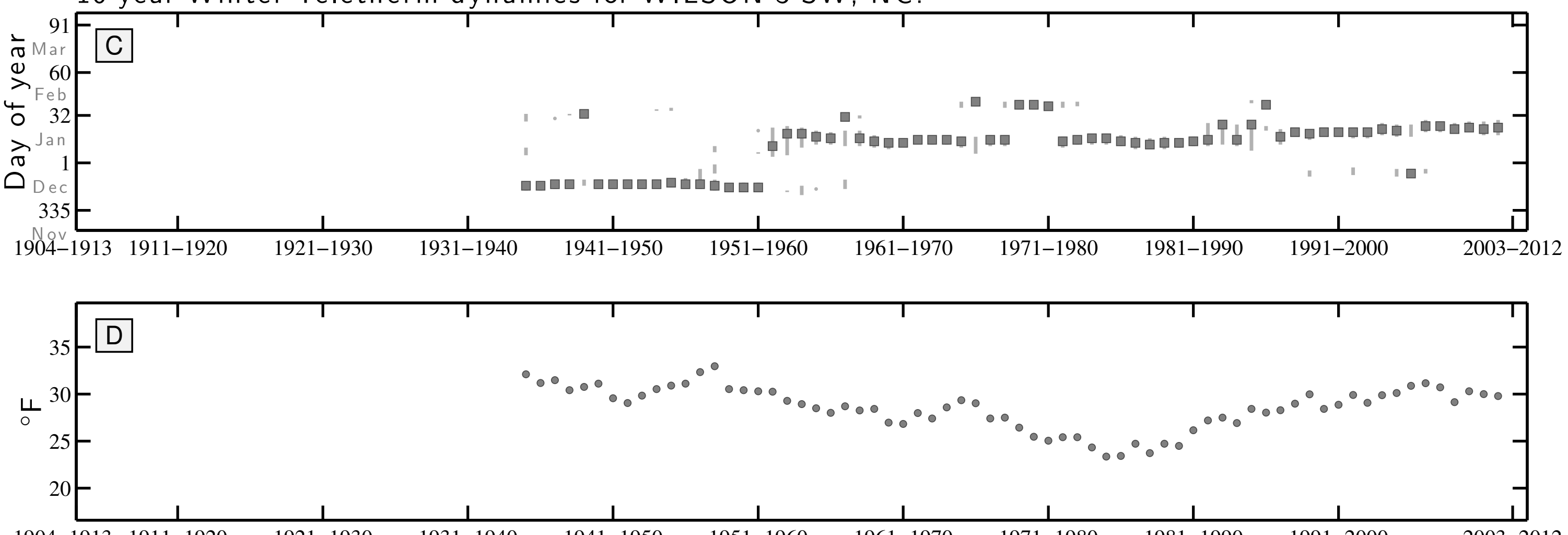
10 year Summer Teletherm dynamics for DUNN CENTER 1E, ND:
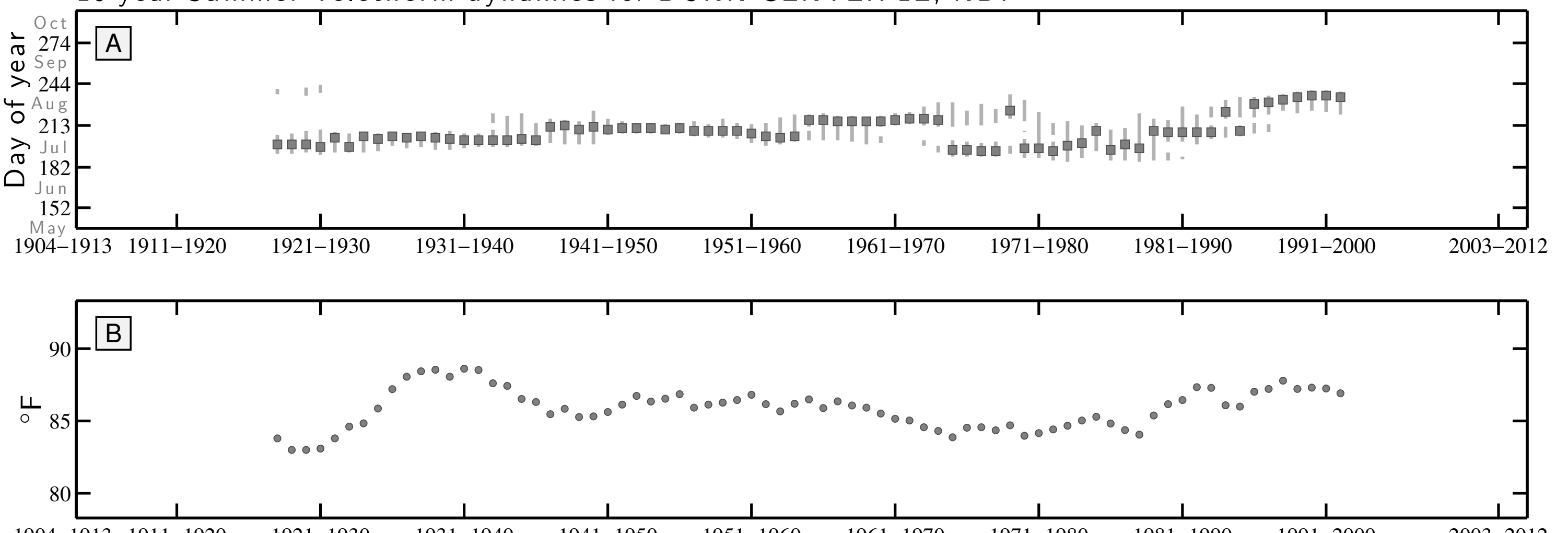

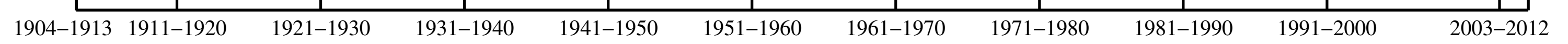

10 year Winter Teletherm dynamics for DUNN CENTER 1E, ND:
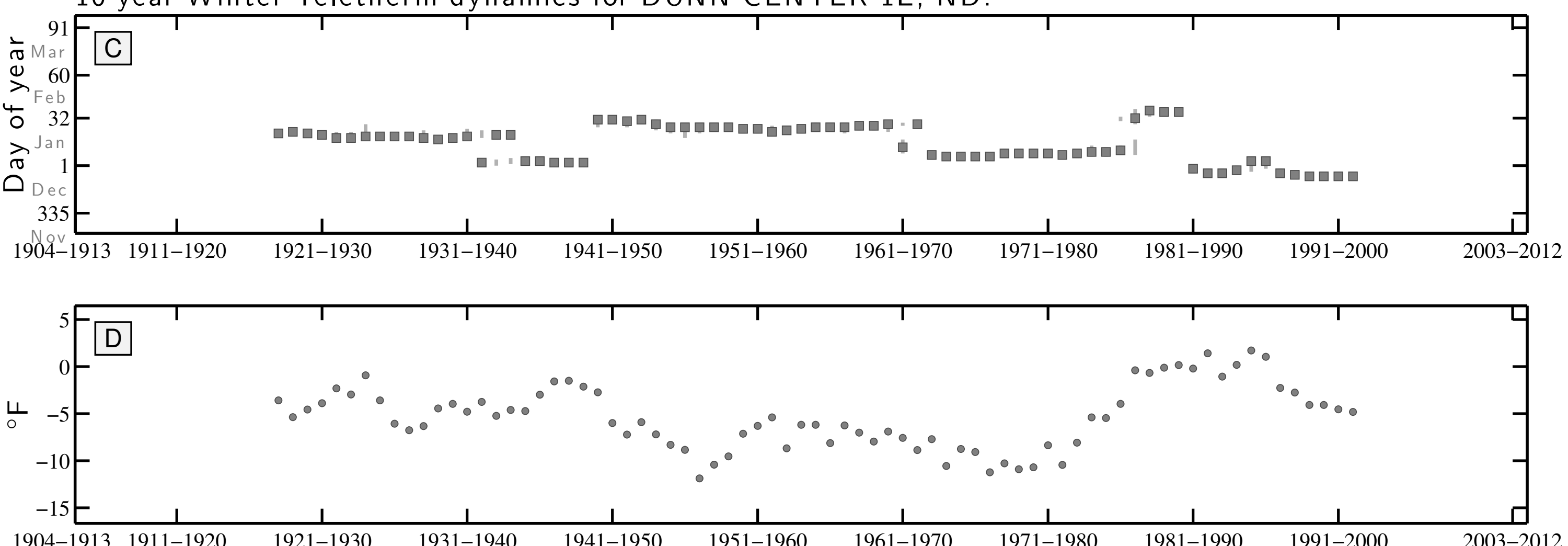

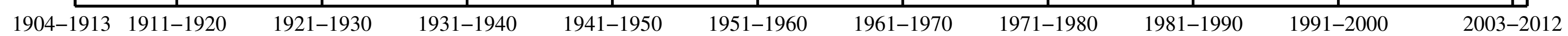


10 year Summer Teletherm dynamics for FULLERTON 1 ESE, ND:

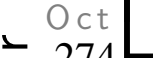

$274-A$

(1) Sep

$>244-$

4 Aug

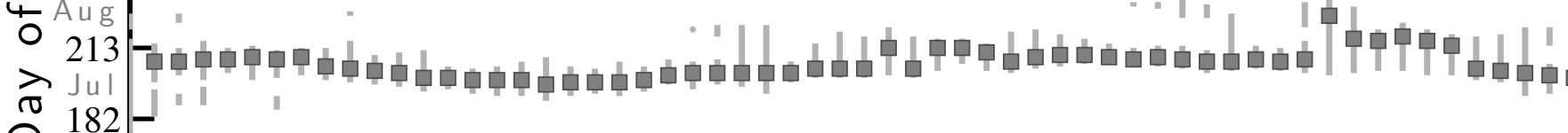

Jun

$\underset{1904-1913 \quad 1911-1920}{\operatorname{May}}$

$\frac{1}{1921-1930}$

$\frac{1}{1931-1940}$

$\frac{1}{1941-1950}$

$\frac{1}{1951-1960}$

$\frac{1}{961-1970}$

$\frac{1}{971-1980}$

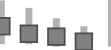

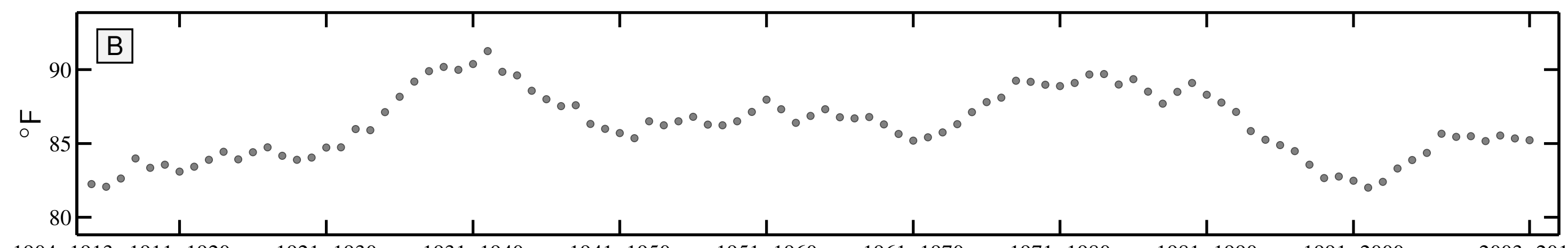

1904-1913 1911-1920

1921-1930

1931-1940

1941-1950

1951-1960

1961-1970

1971-1980

1981-1990

1991-2000

2003-2012

10 year Winter Teletherm dynamics for FULLERTON 1 ESE, ND:

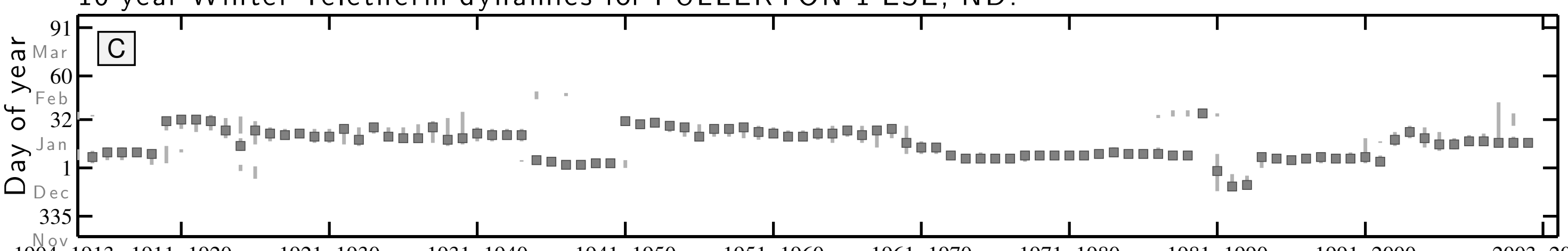

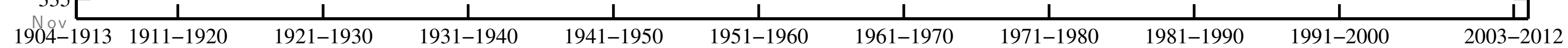

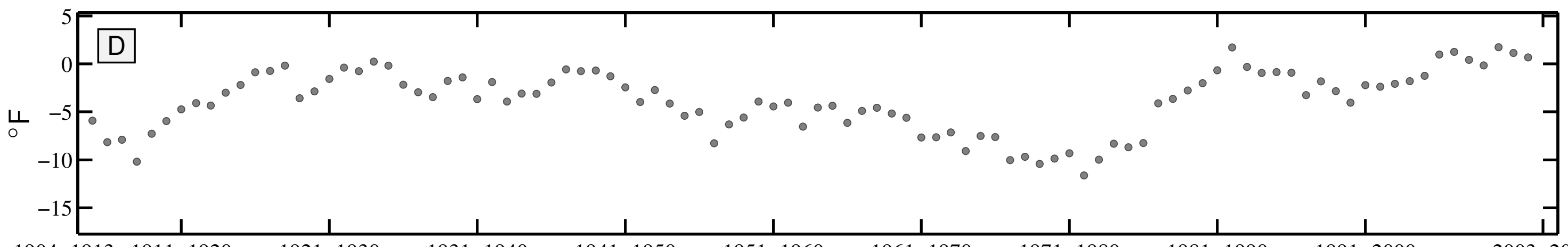


10 year Summer Teletherm dynamics for GRAND FORKS UNIV NWS, ND:

$\div \quad 0 \mathrm{ct}$

(1) Sep

$>244-$

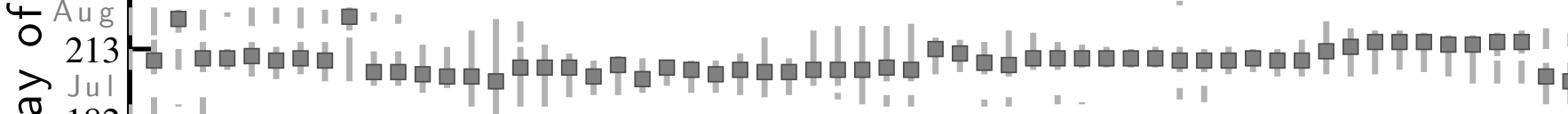

182

$152-$

May

1904-1913 1911-1920

$\frac{1}{1921-1930}$

$\frac{1}{1931-1940}$

$\frac{1}{1941-1950}$

951-1960

$\frac{1}{1961-1970}$

$1971-1980$

981-1990

991-2000

2003-201

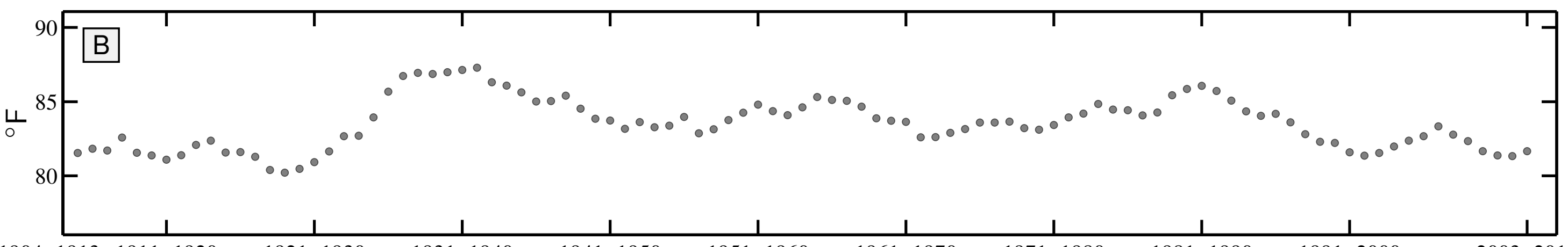

1904-1913 1911-1920 1921-1930

1931-1940

1941-1950

1951-1960

1961-1970

1971-1980

1981-1990

1991-2000

2003-2012

10 year Winter Teletherm dynamics for GRAND FORKS UNIV NWS, ND:

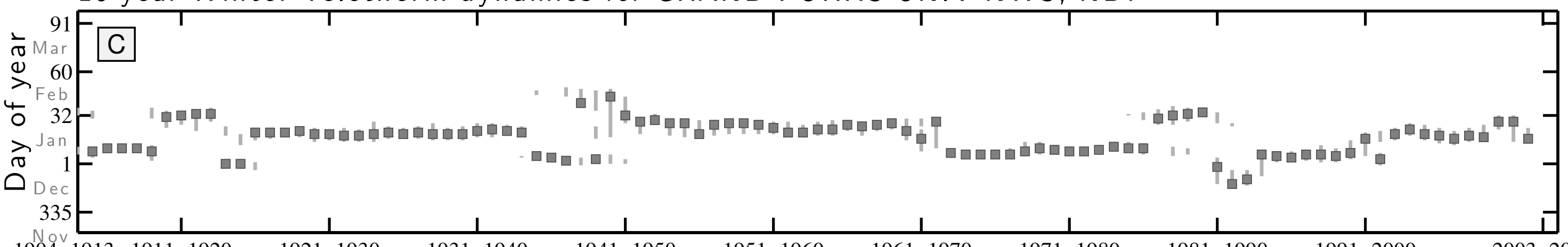

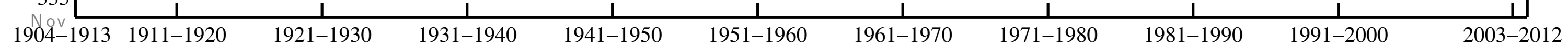

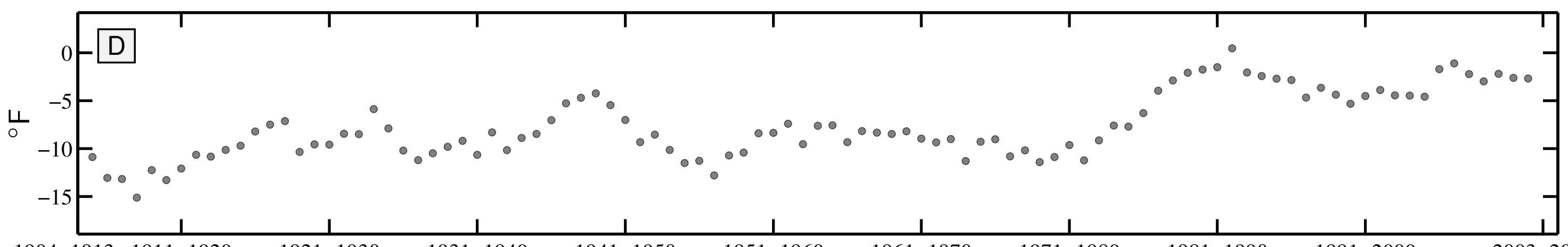


10 year Summer Teletherm dynamics for JAMESTOWN STATE HOSP, ND:

Oct

( 274 A

(1) Sep

$>244-$

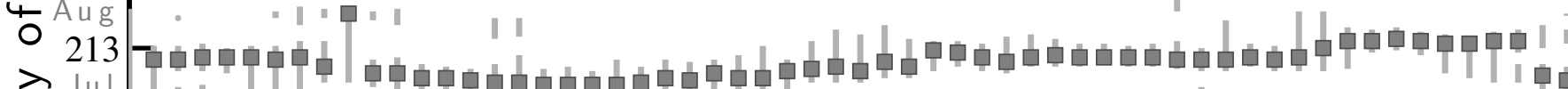

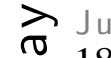

$\overbrace{}^{\circ} 182=$

$152-$

May

1904-1913 1911-1920

1921-1930

1931-1940

$\frac{1}{1941-1950}$

1951-1960

$\frac{1}{1961-1970}$

1971-1980

1981-1990

991-2000

2003-2012

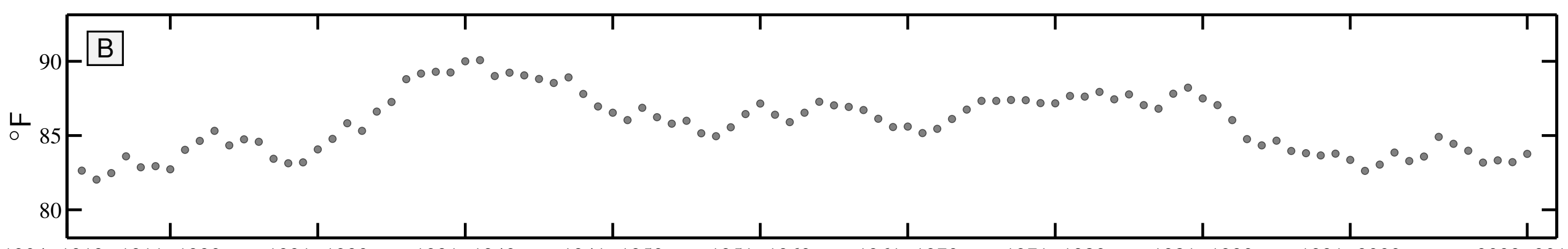

1904-1913 1911-1920

1921-1930

1931-1940

1941-1950

1951-1960

1961-1970

1971-1980

1981-1990

1991-2000

2003-2012

10 year Winter Teletherm dynamics for JAMESTOWN STATE HOSP, ND:

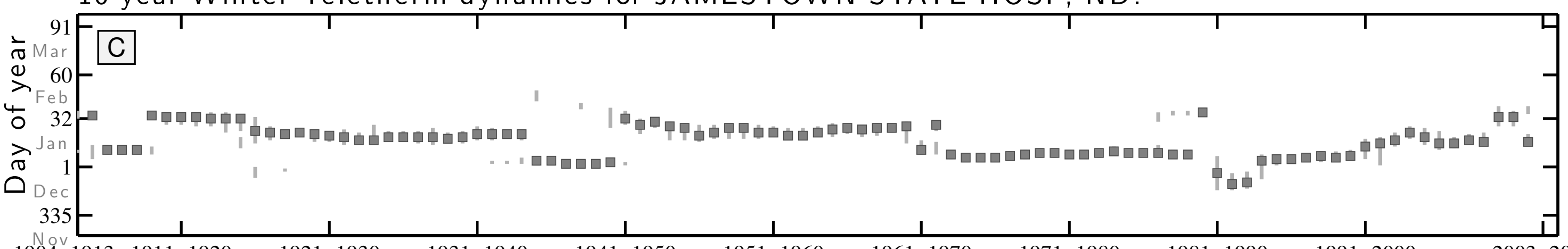

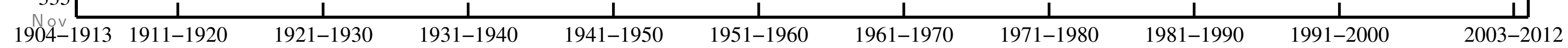

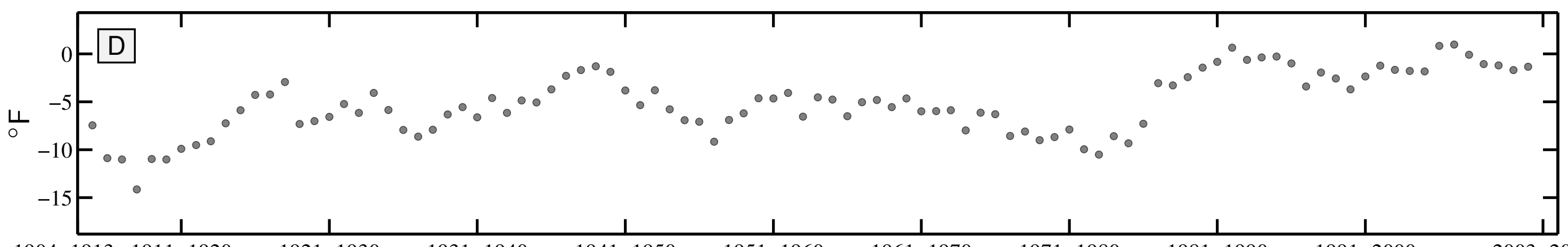


10 year Summer Teletherm dynamics for LISBON, ND:

$\mathrm{Oct}$

A

(1) Sep

$>244-$

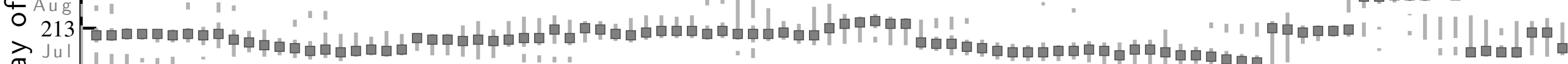

182

152

May

1904-1913 1911-1920

1921-1930

1931-1940

$\frac{1}{1941-1950}$

1951-1960

$\frac{1}{1970}$

$\frac{1}{971-1980}$

1981-1990

$\frac{1}{991-2000}$
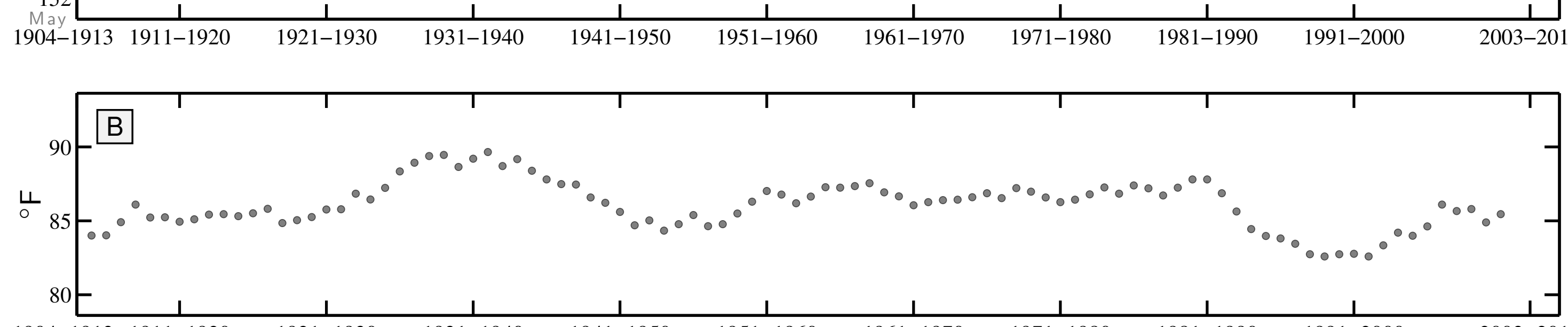

1904-1913 1911-1920

$1921-1930$

$1931-1940$

$1941-1950$

$1951-1960$

$1961-1970$

$1971-1980$

$1981-1990$

$1991-2000$

2003-2012

10 year Winter Teletherm dynamics for LISBON, ND:

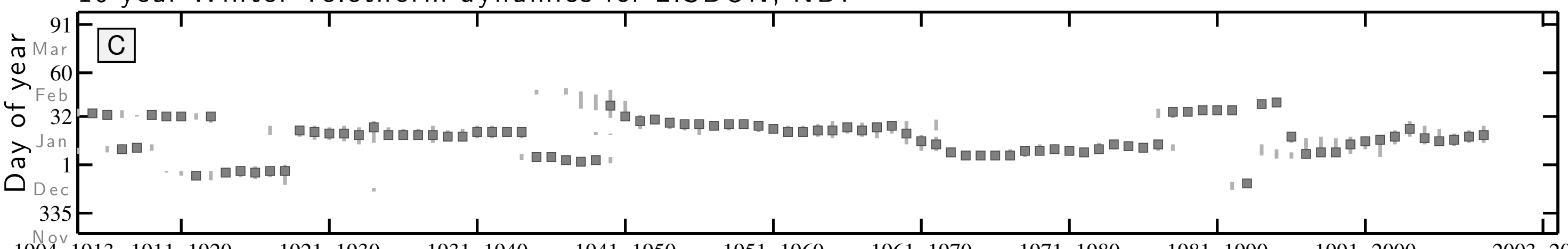

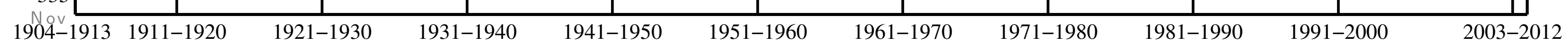

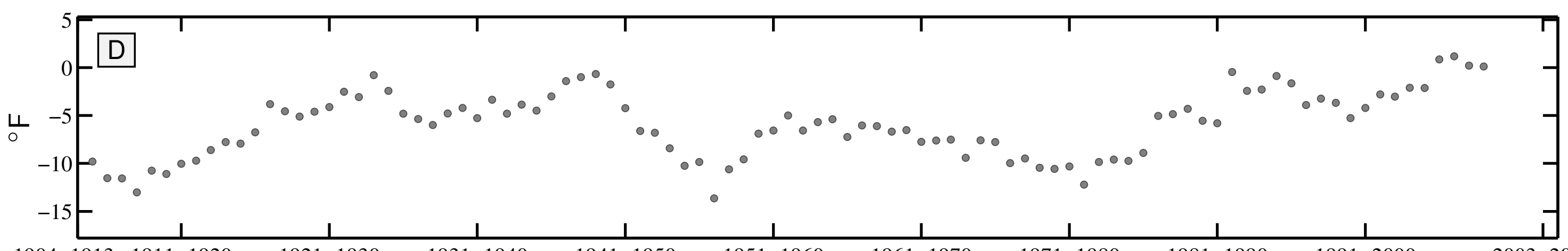


10 year Summer Teletherm dynamics for NAPOLEON, ND:

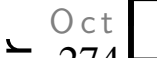

\% $274-A$

(1) Sep

$>244-$

$4 \mathrm{Aug}$

$\gtrsim \mathrm{Ju}$

$\overbrace{}^{\circ} 182$

$152-$

May —

1904-1913 1911-1920

$\frac{1}{1921-1930}$

$\frac{1}{1931-1940}$

$\frac{1}{1941-1950}$

1951-1960

$\frac{1}{961-1970}$

$\frac{1}{1971-1980}$

1981-1990

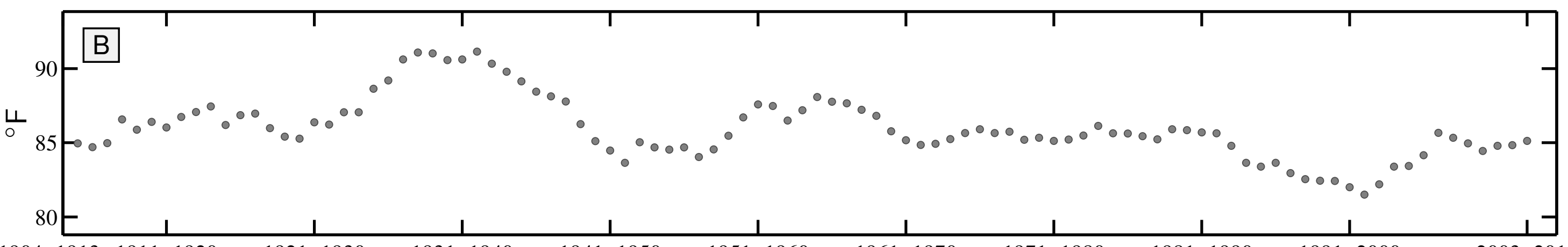

1904-1913 1911-1920

$1921-1930$

$1931-1940$

$1941-1950$

$1951-1960$

$1961-1970$

$1971-1980$

$1981-1990$

$1991-2000$

2003-2012

10 year Winter Teletherm dynamics for NAPOLEON, ND:

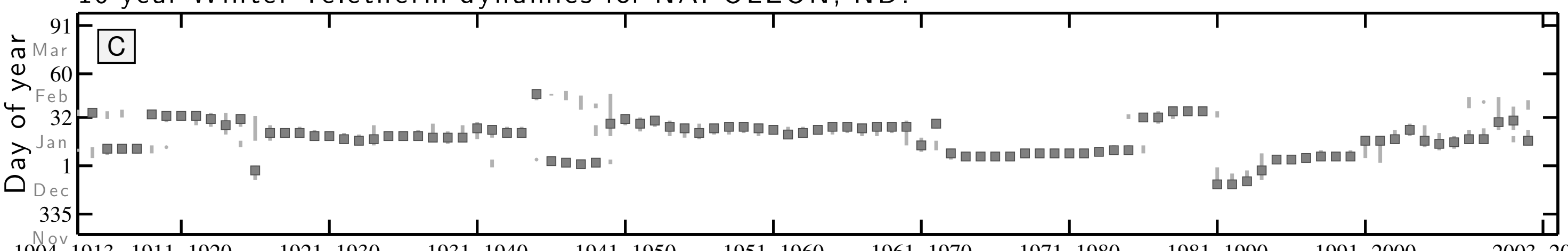

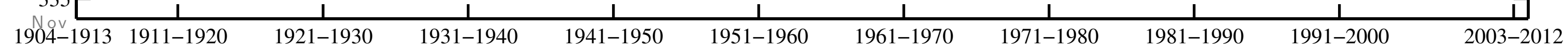

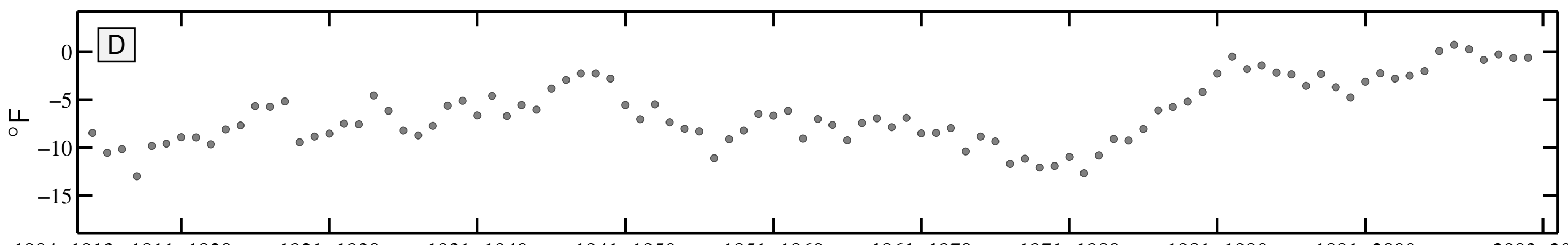


10 year Summer Teletherm dynamics for WAHPETON 3 N, ND:
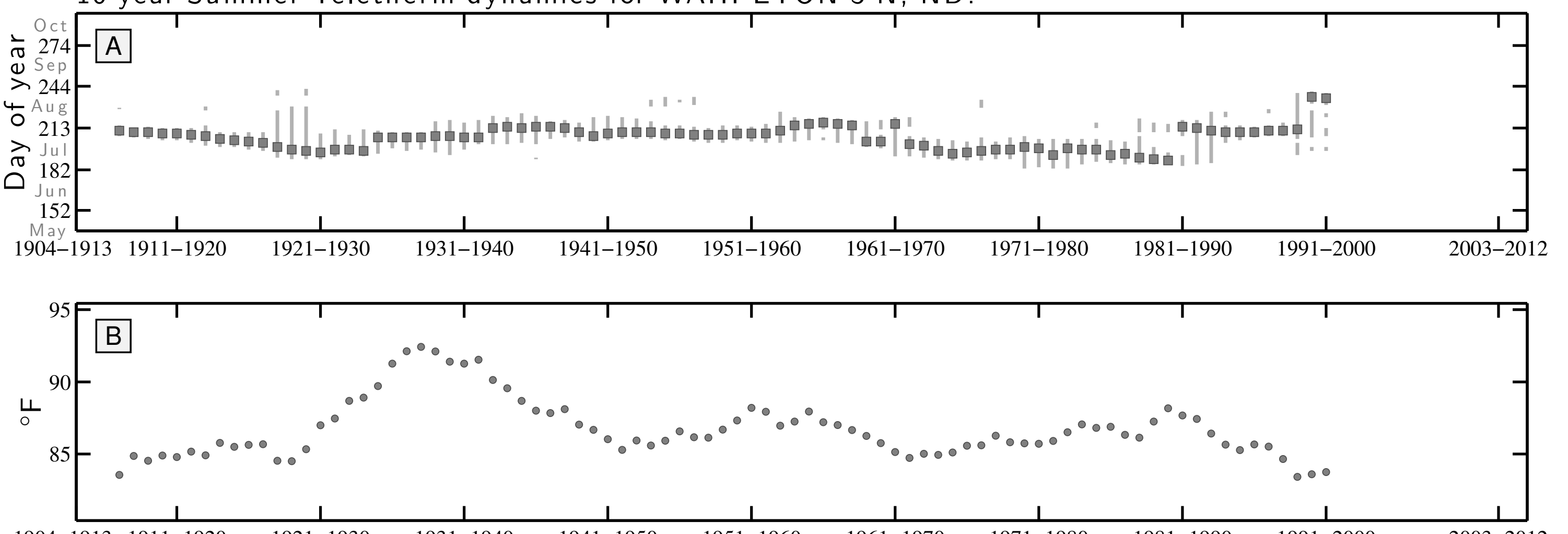

$1904-1913$ 1911-1920 1921-1930

$1931-1940$

$1941-1950$

$1951-1960$

$1961-1970$

1971-1980

$1981-1990$

$1991-2000$

2003-2012

10 year Winter Teletherm dynamics for WAHPETON 3 N, ND:

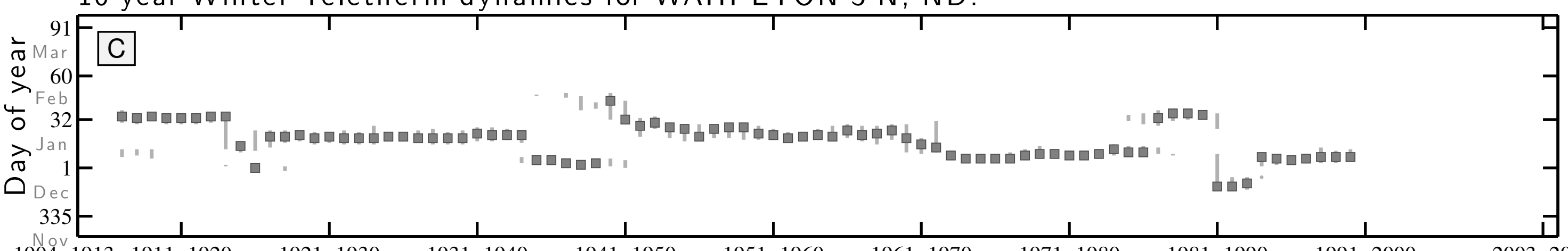

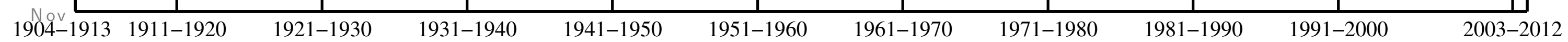

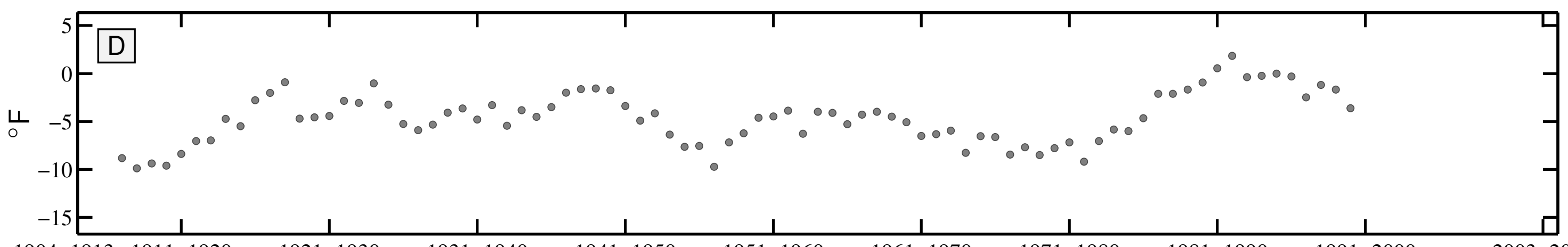

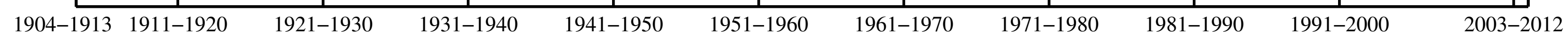


10 year Summer Teletherm dynamics for CADIZ, OH:

$\therefore \mathrm{Oct}$

$\frac{\pi}{2} 274$

(1) Sep

$>244-$

응

$213-$

$\begin{array}{cc}213 \\ \vec{\sigma} & J u l \\ 0 & 182\end{array}$

$152-$

May

1904-1913 1911-1920

$\frac{1}{1921-1930}$

1931-1940

$\frac{1}{1941-1950}$

$\frac{1}{1951-1960}$

1961-1970

$\frac{1}{1971-1980}$

$\frac{1}{1981-1990}$

1991-2000

2003-2012

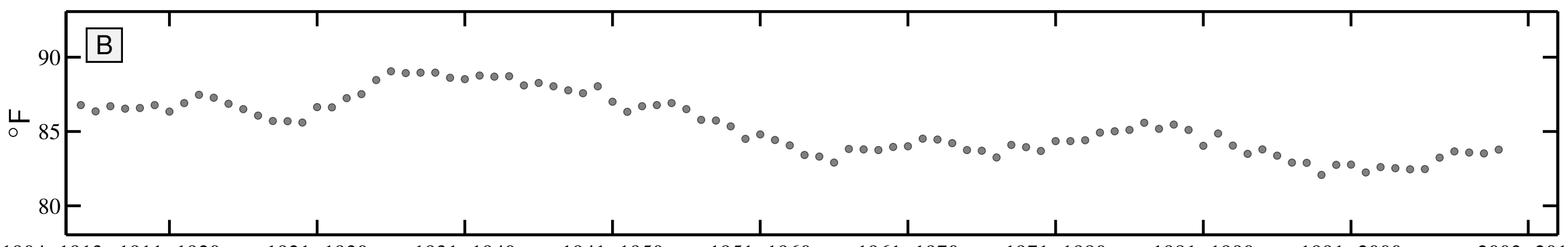

1904-1913 1911-1920

$1921-1930$

$1931-1940$

$1941-1950$

1951-1960

$1961-1970$

$1971-1980$

$1981-1990$

$1991-2000$

2003-2012

10 year Winter Teletherm dynamics for CADIZ, OH:

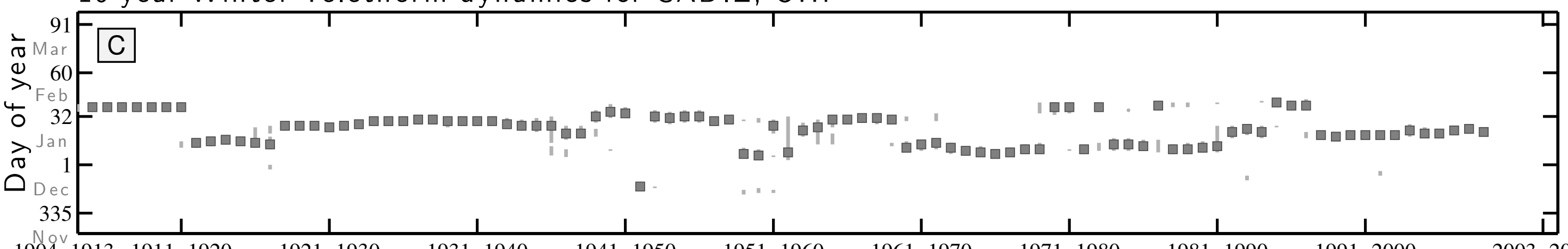

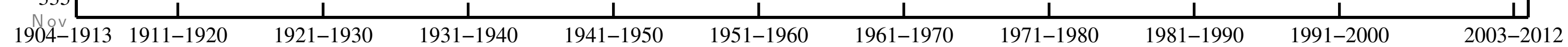

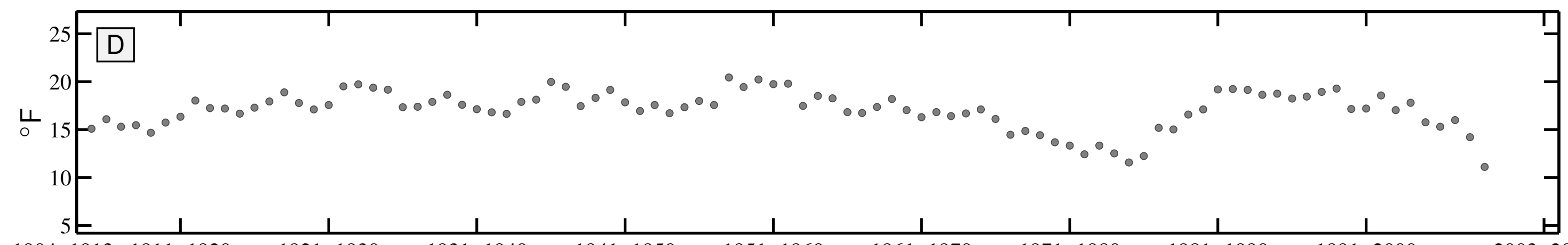


10 year Summer Teletherm dynamics for DELAWARE, OH:

$\div$ Oct

\% $274-A$

(1) Sep

$>244$

萑 Aug

$213-$

182

152

152

1904-1913 1911-1920

$\frac{1}{1921-1930} \quad \frac{1}{1931-1940}$

$\frac{1}{1941-1950}$

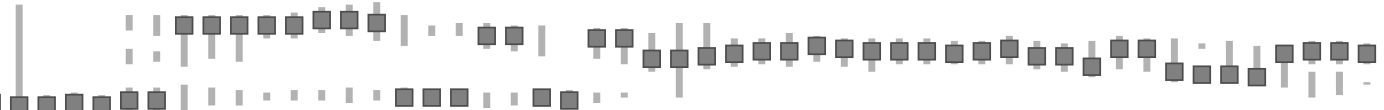

" | "

$1961-1970$

$1971-1980$

$1981-1990$

991-2000

2003-2012

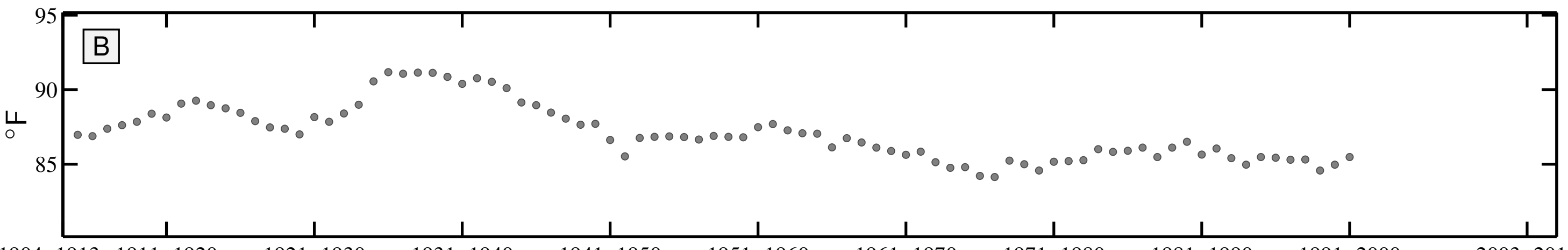

$1904-1913$ 1911-1920

$1921-1930$

$1931-1940$

$1941-1950$

$1951-1960$

$1961-1970$

$1971-1980$

$1981-1990$

$1991-2000$

2003-2012

10 year Winter Teletherm dynamics for DELAWARE, OH:

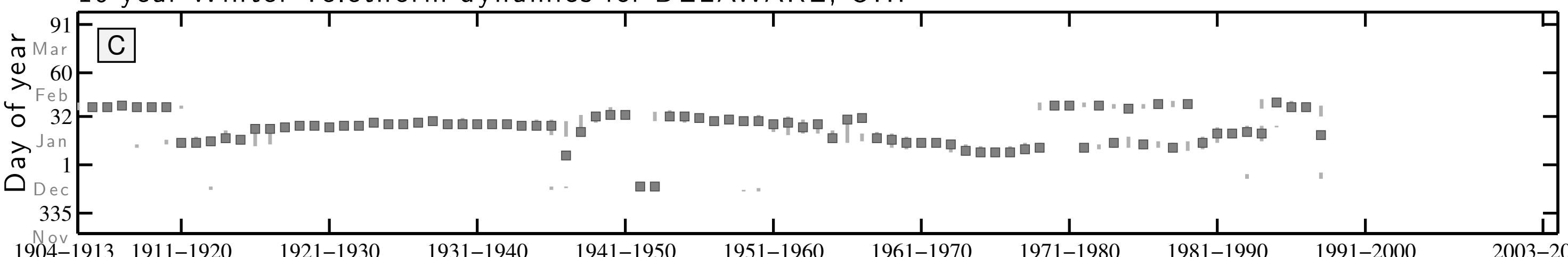

$1904-1913 \quad 1911-1920$

$1921-1930$

1931-1940

1941-1950

1951-1960

1961-1970

1971-1980

1981-1990

1991-2000

2003-2012

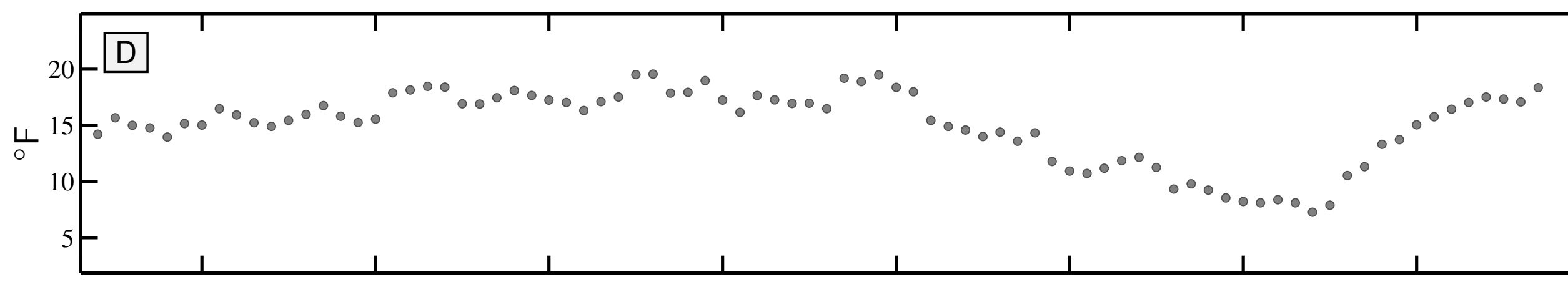


10 year Summer Teletherm dynamics for HIRAM, OH:

Oct

A

(1) Sep

$>244-$

\% Aug

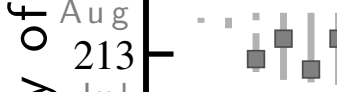

Jul

$\bigcirc 182-$

152 -

152 -

1904-1913 1911-1920

$\frac{1}{1921-1930}$

1931-1940

$\frac{1}{1941-1950}$

1951-1960

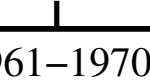

: I

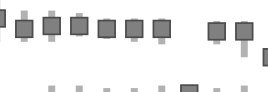

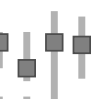
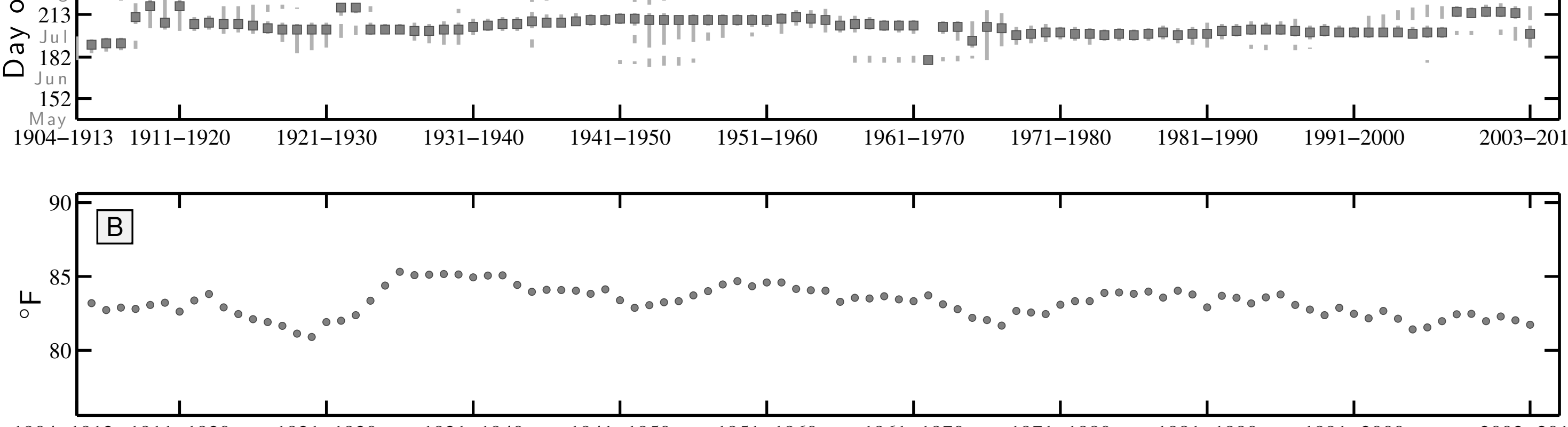

1904-1913 1911-1920

$1921-1930$

$1931-1940$

1941-1950

$1951-1960$

$1961-1970$

$1971-1980$

$1981-1990$

$1991-2000$

2003-2012

10 year Winter Teletherm dynamics for HIRAM, OH:

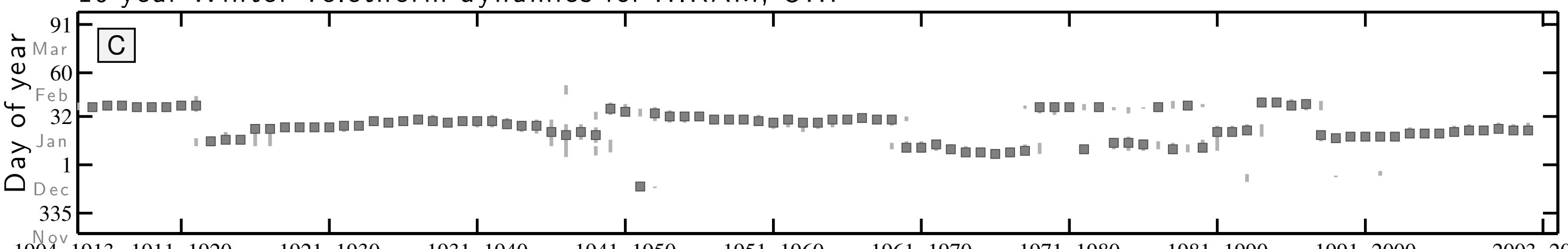

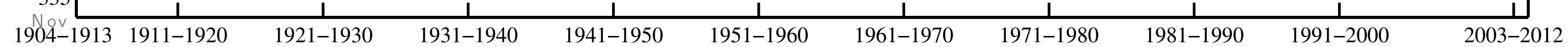

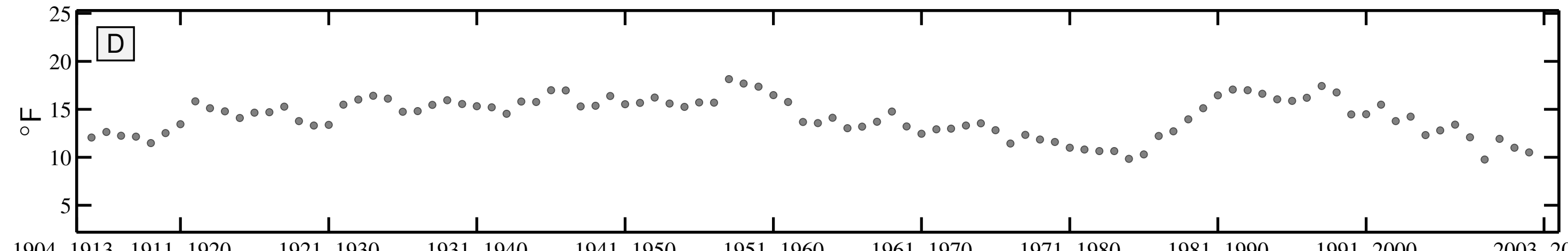


10 year Summer Teletherm dynamics for KENTON, OH:

$-\mathrm{Cct}$

(1) Sep

$>244-$

O A g g

$\underset{\mathrm{Jul}}{213}-$

क 182

152

1904-1913 1911-1920

| | "

I I

" | . -

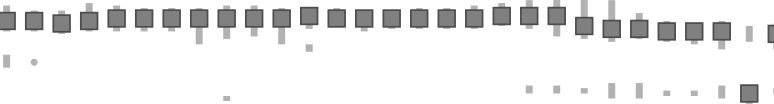

प中中田
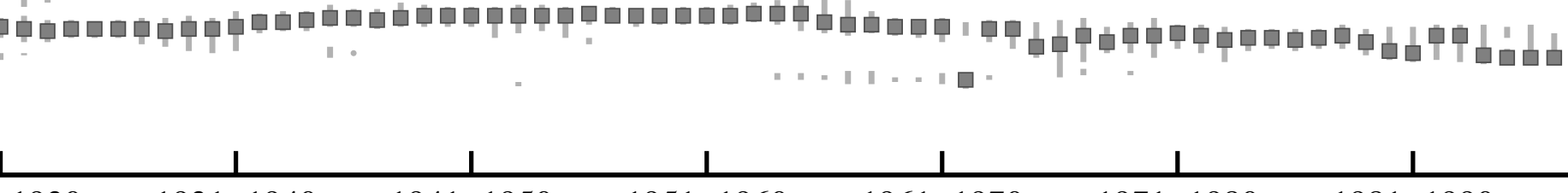

$\frac{1}{1-1940}$

1941-1950

$\frac{1}{1951-1960}$

$\frac{1}{1961-1970}$

1971-1980

$\frac{1}{1981-1990}$

1991-2000

| เ แ เ |

뭄ㅁำ

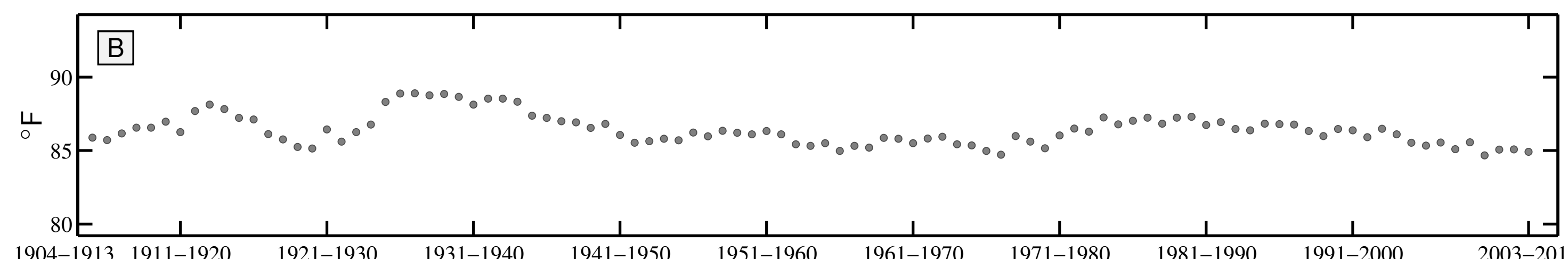

1904-1913 1911-1920

1921-1930

1931-1940

1941-1950

1951-1960

1961-1970

1971-1980

981-1990

003-2012

10 year Winter Teletherm dynamics for KENTON, OH:

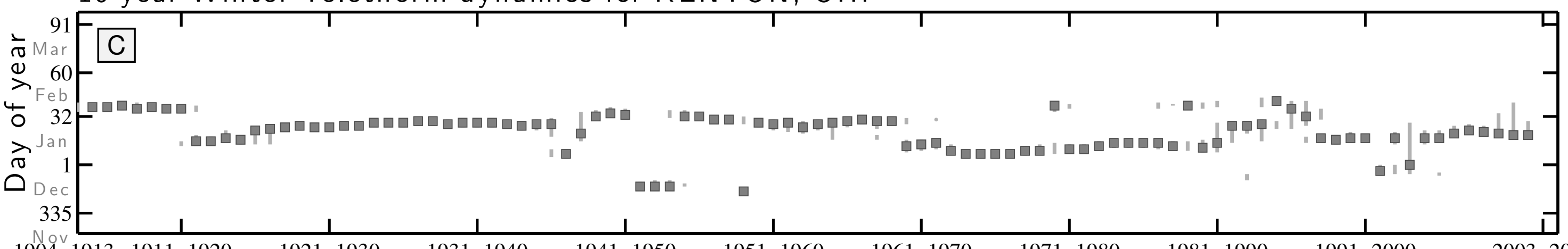

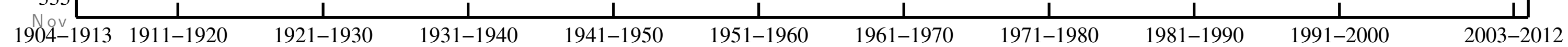

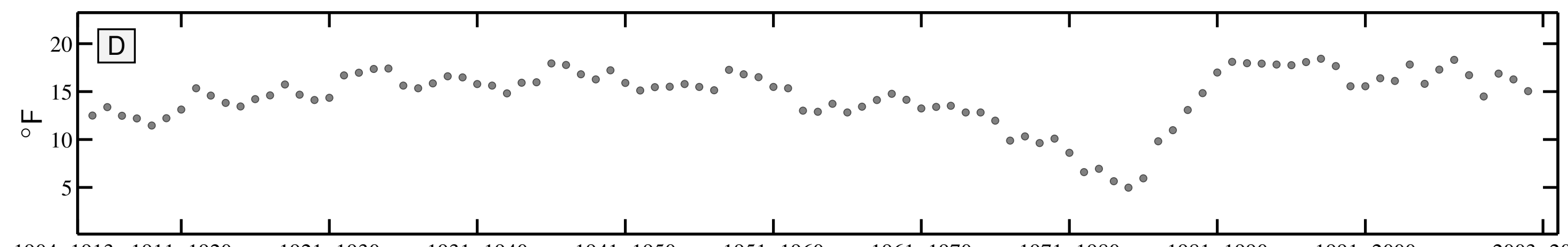


10 year Summer Teletherm dynamics for MC CONNELLSVILLE LK 7, OH:

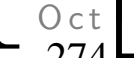

\% $274-A$

(1) Sep

$>244$

응

त $\mathrm{Ju}$

ก 182

152

152

1904-1913 1911-1920

$\frac{1}{1921-1930}$

1931-1940

$\frac{1}{1941-1950}$

1951-1960

पा

| | |

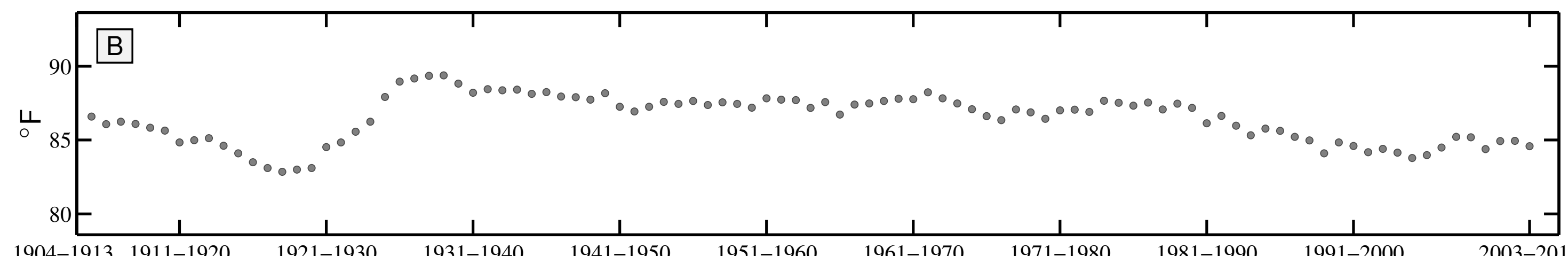

1904-1913 1911-1920

1921-1930

1931-1940

1941-1950

1951-1960

1961-1970

1971-1980

1981-1990

1991-2000

2003-2012

10 year Winter Teletherm dynamics for MC CONNELLSVILLE LK 7, OH:

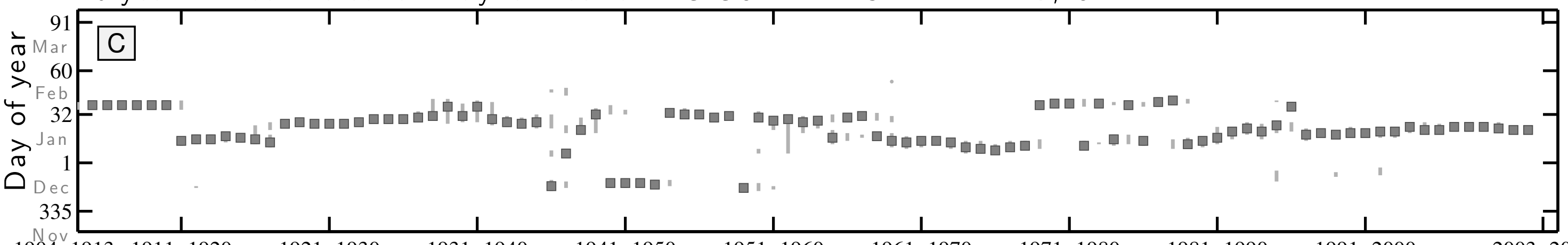

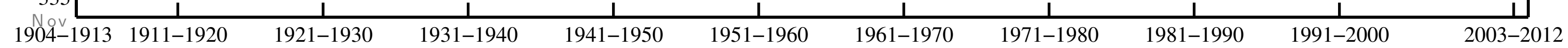

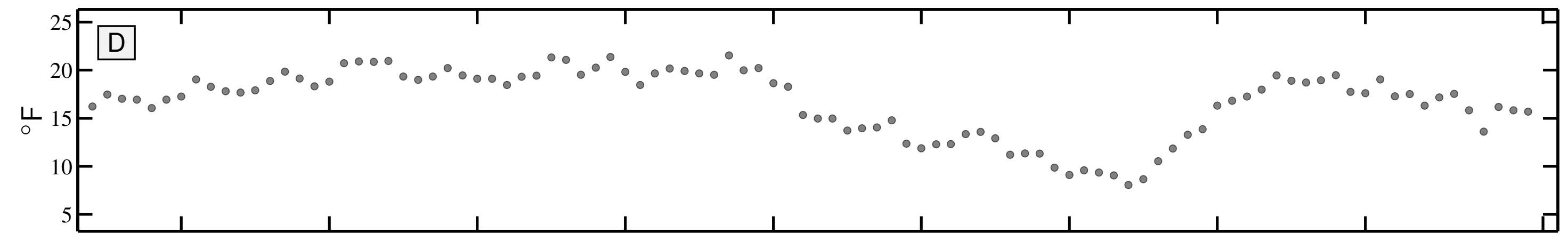


10 year Summer Teletherm dynamics for MILLERSBURG, OH:

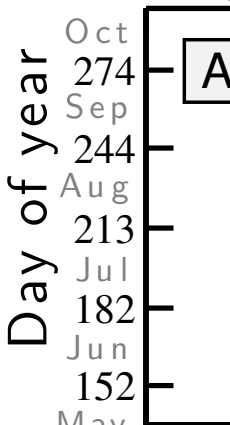
$A$
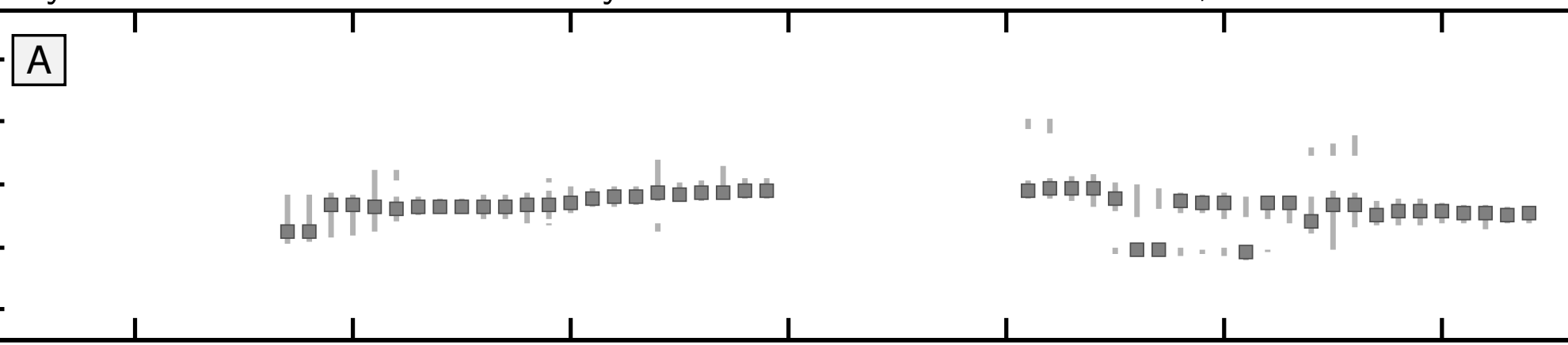

$$
\text { May }
$$

पाT

1904-1913 1911-1920

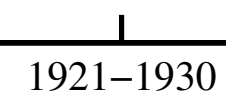

1931-1940

1941-1950

$\frac{1}{1951-1960}$

$\frac{1}{1961-1970}$

1971-1980

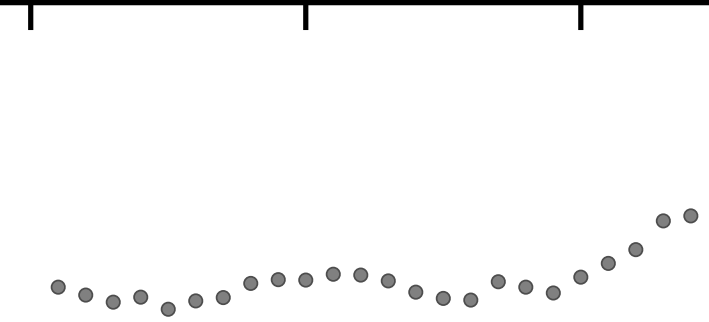

$\stackrel{9}{\circ}{ }_{85}^{90-}$

B

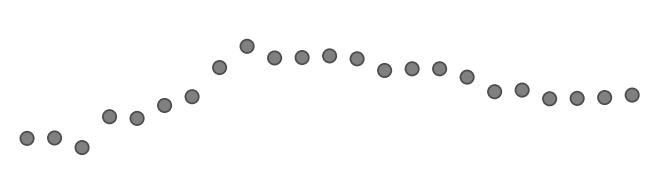

1904-1913 1911-1920

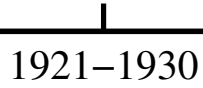

$\frac{1}{1931-1940}$

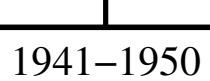

1951-1960

1961-1970

1971-1980

1981-1990

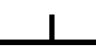

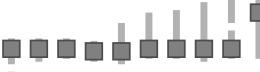

|| || | | | -

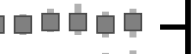

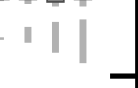

1

$\frac{1}{991-2000}$

2003-2012

10 year Winter Teletherm dynamics for MILLERSBURG, OH:

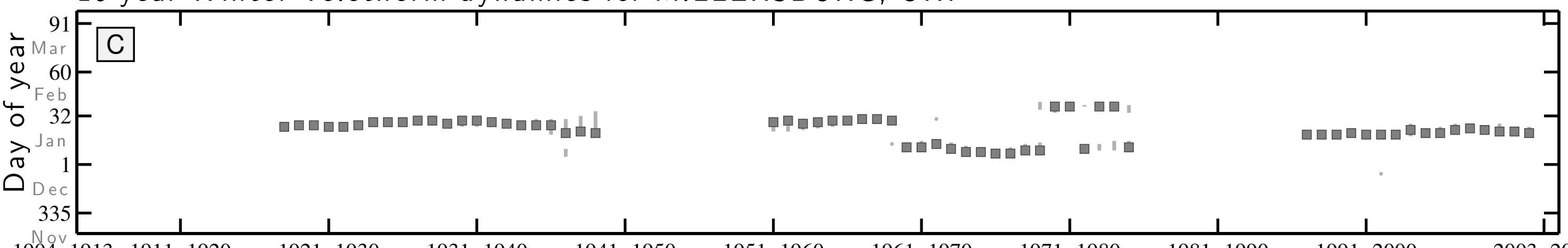

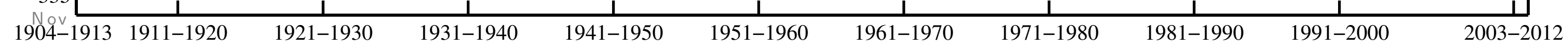

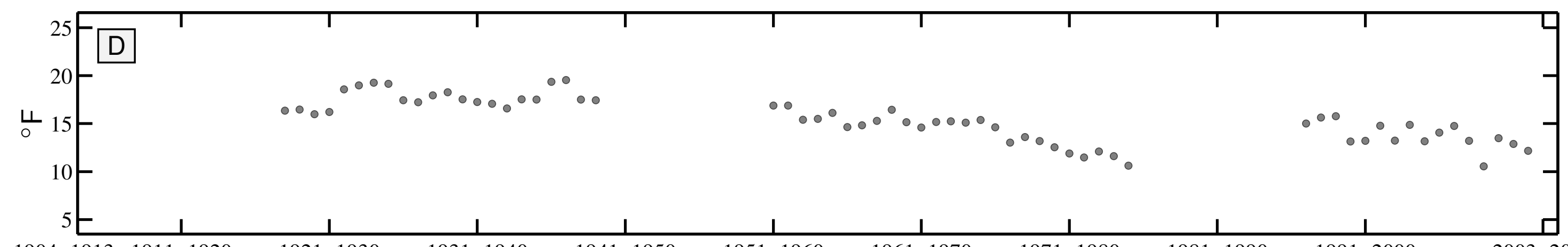


10 year Summer Teletherm dynamics for PHILO $3 \mathrm{SW}, \mathrm{OH}$ :

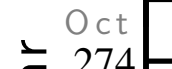

A

(1) Sep

$>244-$

$4 \mathrm{Aug}$

213 - 213 由ा

T Jul $\square \square \square$

$182-$

$152-$

-

1

|

1 1

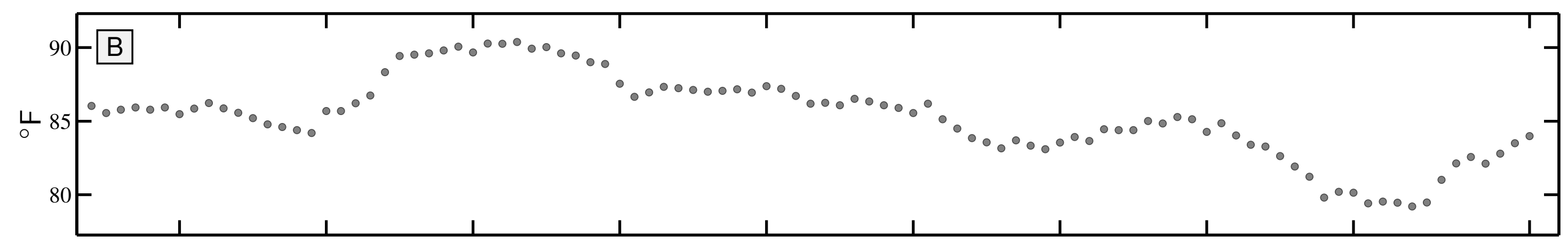

1904-1913 1911-1920

1921-1930

$1931-1940$

$1941-1950$

1951-1960

$1961-1970$

$1971-1980$

1981-1990

$1991-2000$

2003-2012

10 year Winter Teletherm dynamics for PHILO $3 \mathrm{SW}, \mathrm{OH}$ :

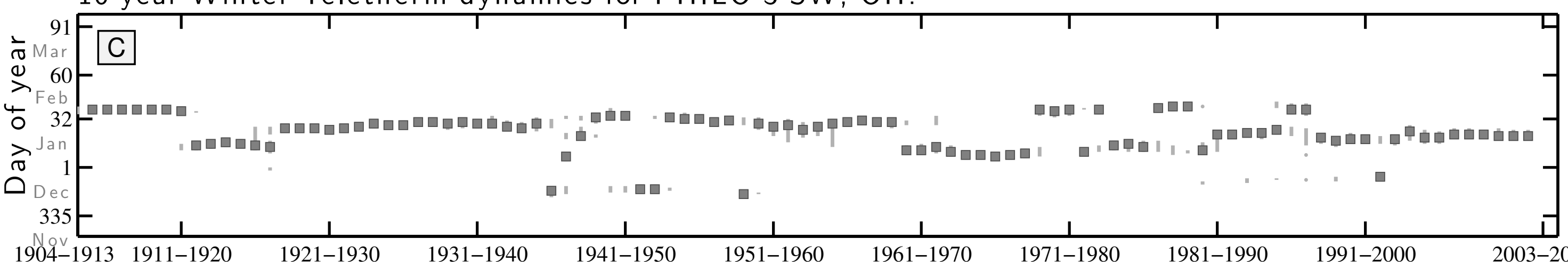

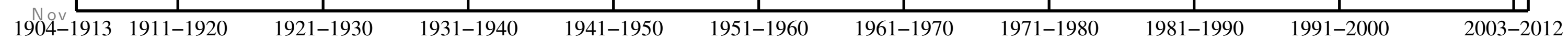

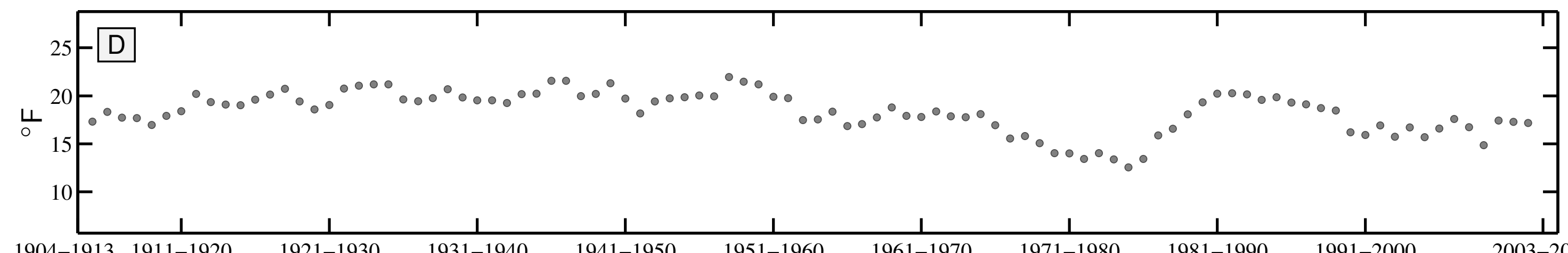


10 year Summer Teletherm dynamics for TIFFIN, OH:

$\mathrm{Oct}$

A

$>244-$

4 A $\mathrm{Ag}$

$213-$

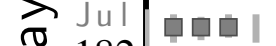

182

$152-$

1

1904-1913 1911-1920

$\frac{1}{1921-1930}$

1931-1940

$\frac{1}{1941-1950}$

$\frac{1}{1951-1960}$

$\frac{1}{1961-1970}$

1971-1980

$\frac{1}{1981-1990}$

991-2000

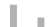

| || เดแ|| | -

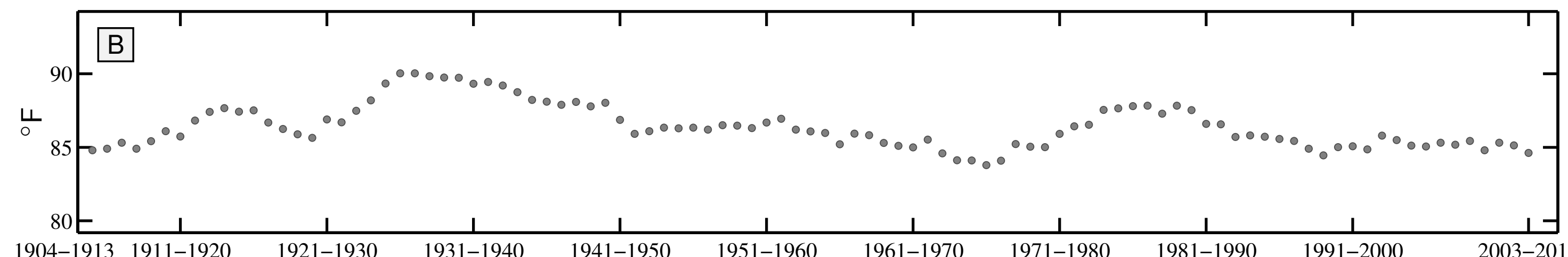

1904-1913 1911-1920

$1921-1930$

1931-1940

$1941-1950$

$1951-1960$

$1961-1970$

1971-1980

$1981-1990$

991-2000

2003-2012

10 year Winter Teletherm dynamics for TIFFIN, OH:

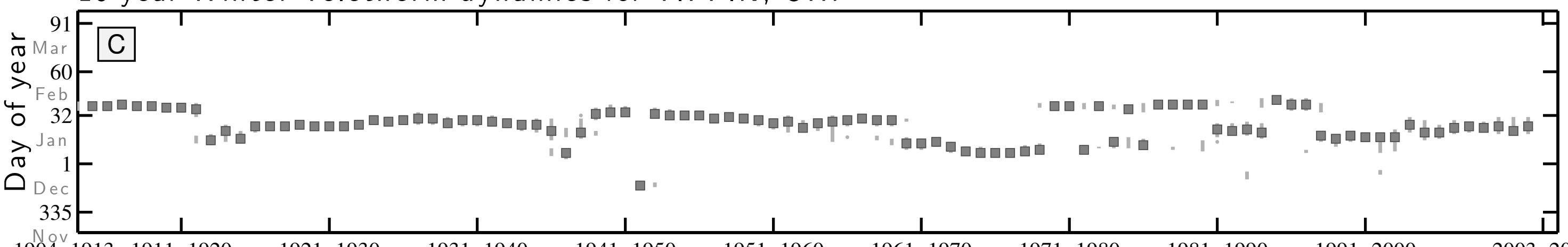

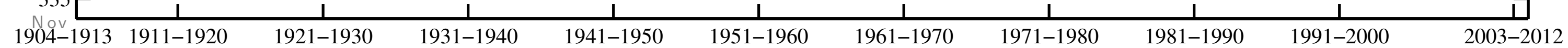

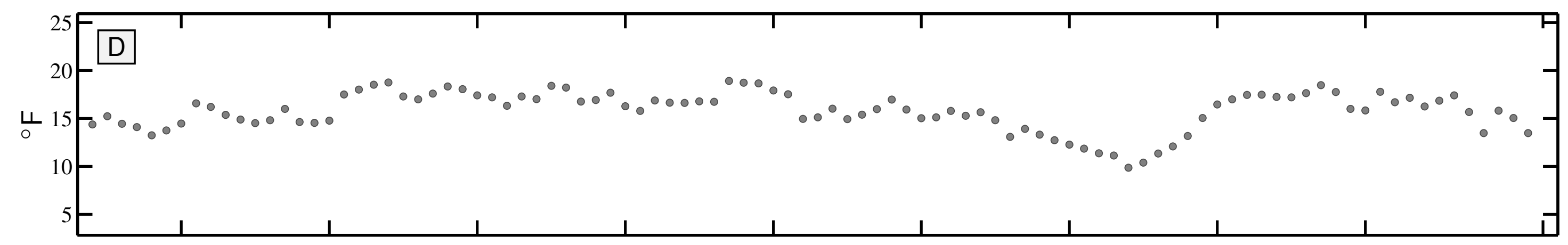


10 year Summer Teletherm dynamics for WARREN $3 \mathrm{~S}, \mathrm{OH}$ :

$\div$ Oct 274

(l)

$\stackrel{2}{>} 244$

$4 \mathrm{Aug}$

$213-$

त Jul $\square \square \square$

$\overbrace{}^{\circ} 182$

$152-$

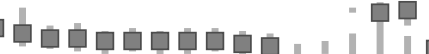

1 व

| | | |

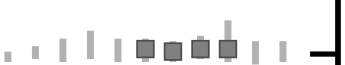

1 1

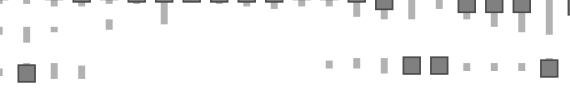

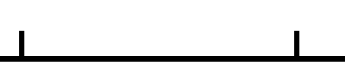

$-1960$

$\frac{1}{1961-1970}$

$\frac{1}{1971-1980}$

$\frac{1}{1981-1990}$

991-2000

.

1904-1913 1911-1920

$1931-1940$

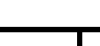

T

1

1

$+2$

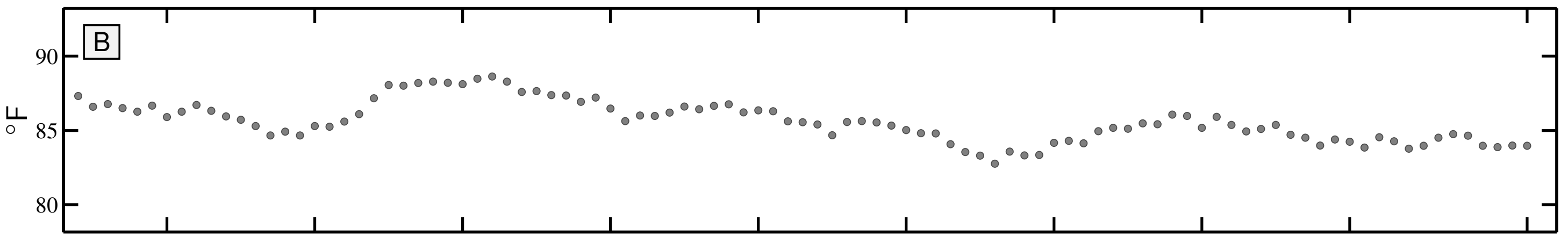

1904-1913 1911-1920 1921-1930

1931-1940

$1941-1950$

1951-1960

$1961-1970$

1971-1980

$1981-1990$

$1991-2000$

2003-2012

10 year Winter Teletherm dynamics for WARREN $3 \mathrm{~S}, \mathrm{OH}$ :

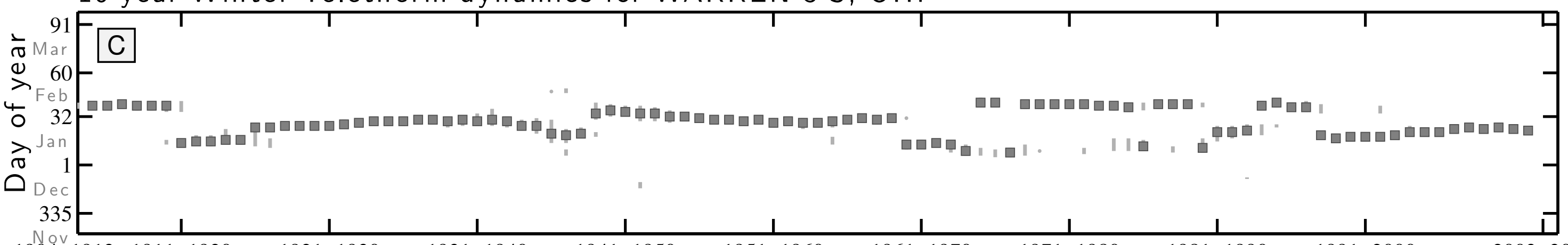

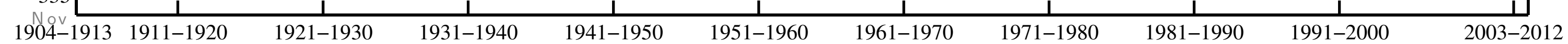

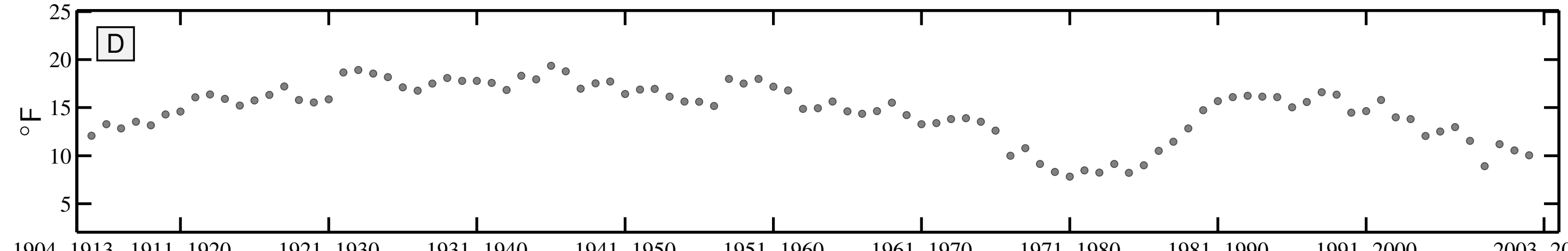


10 year Summer Teletherm dynamics for DURANT, OK:
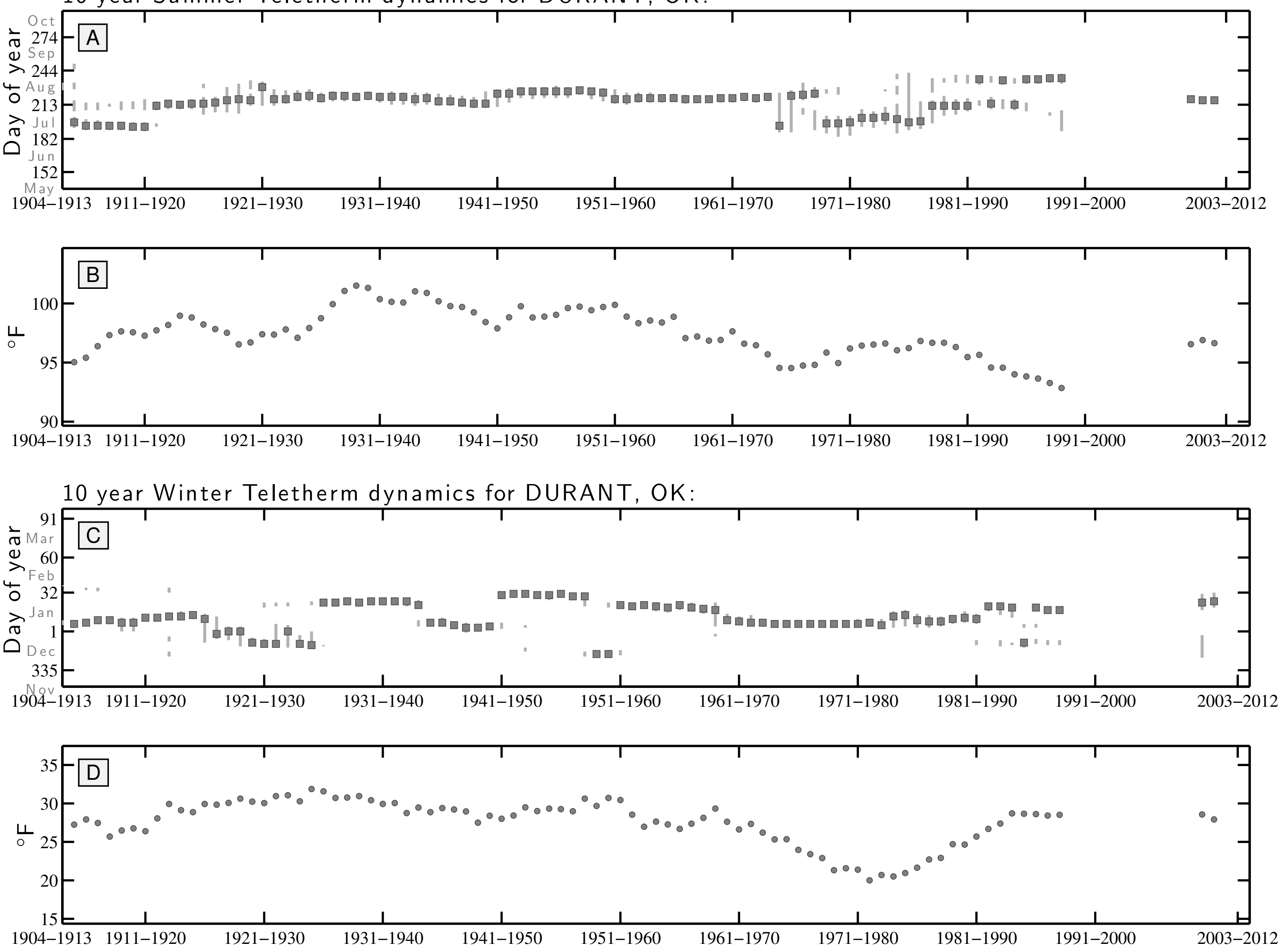
10 year Summer Teletherm dynamics for GEARY, OK:
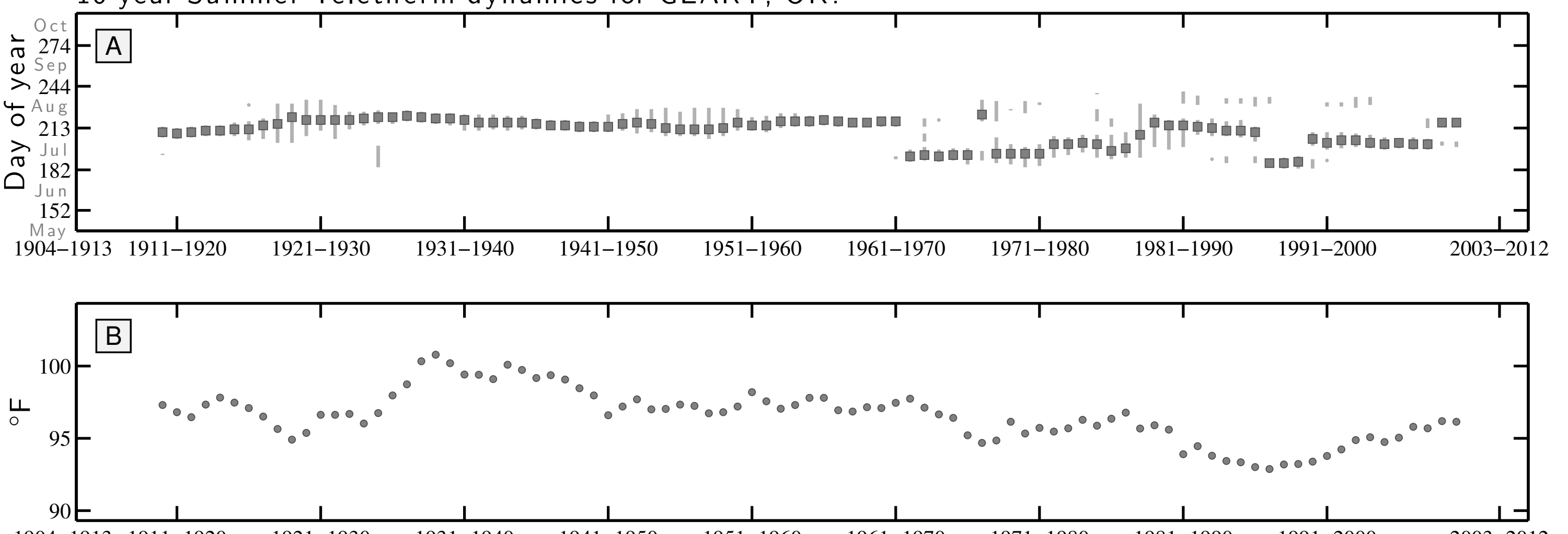

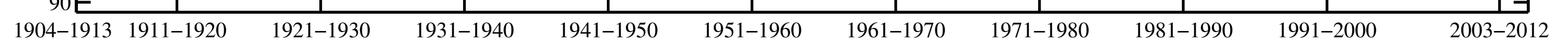
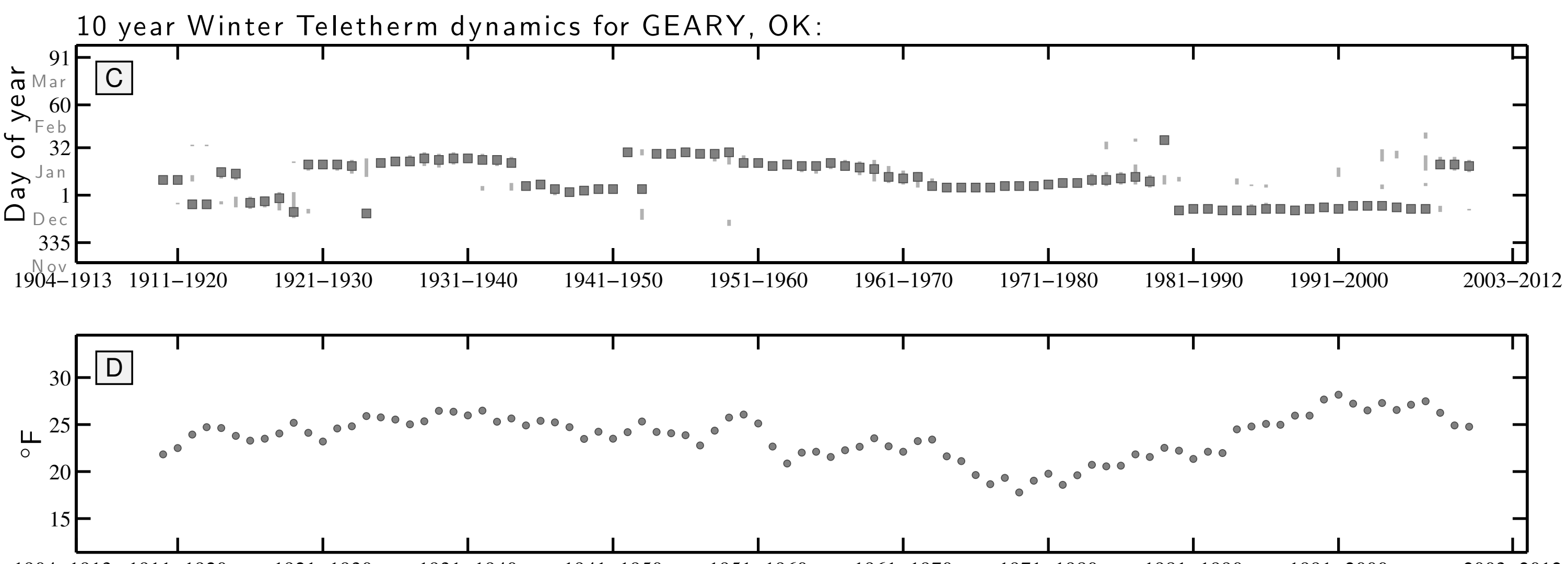

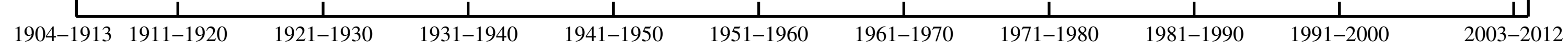


10 year Summer Teletherm dynamics for GUTHRIE 5S, OK:

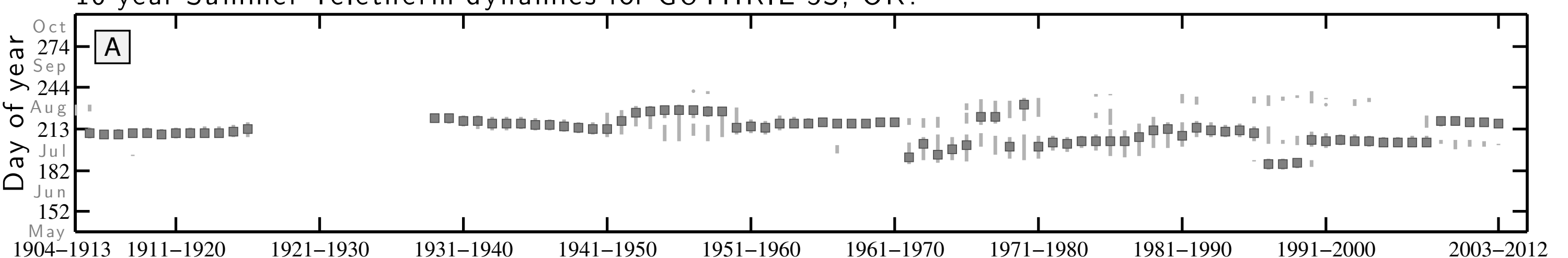

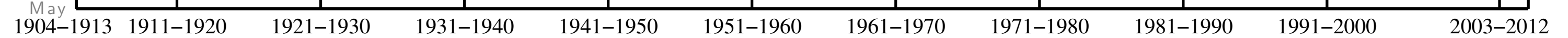

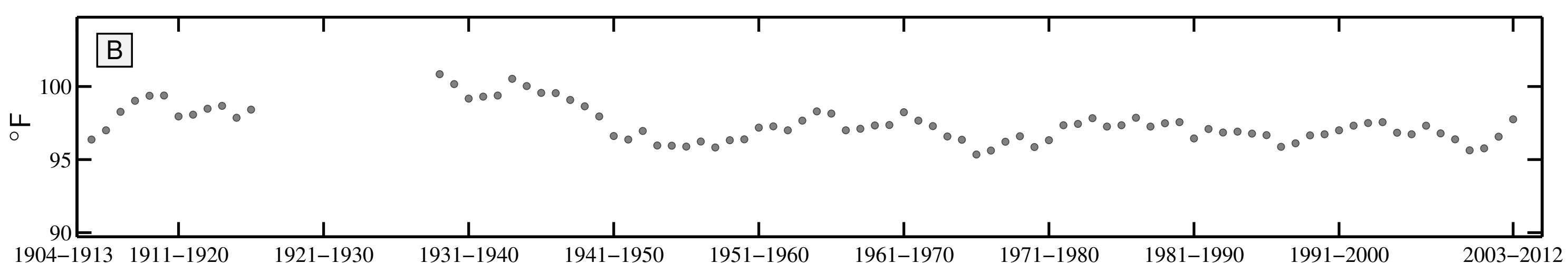

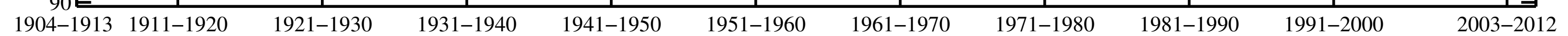

10 year Winter Teletherm dynamics for GUTHRIE 5S, OK:

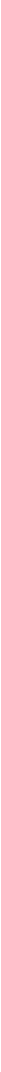


10 year Summer Teletherm dynamics for JEFFERSON, OK:
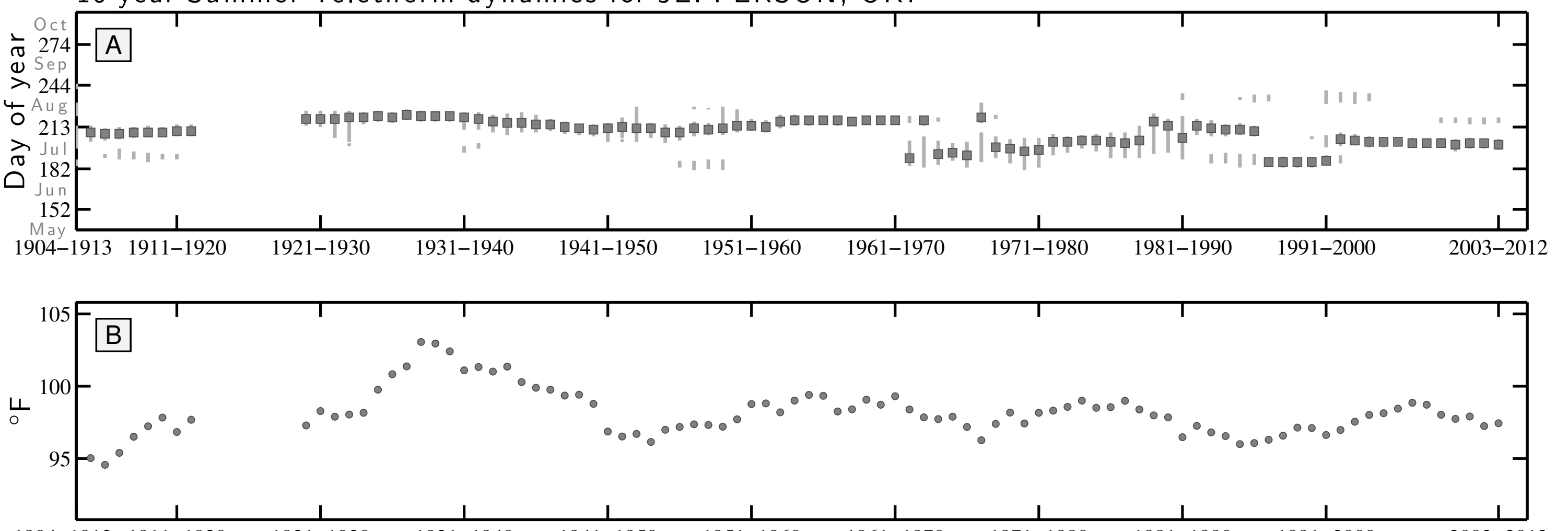

1904-1913 1911-1920

$1921-1930$

1931-1940

$1941-1950$

$1951-1960$

1961-1970

1971-1980

1981-1990

$1991-2000$

$2003-2012$

10 year Winter Teletherm dynamics for JEFFERSON, OK:

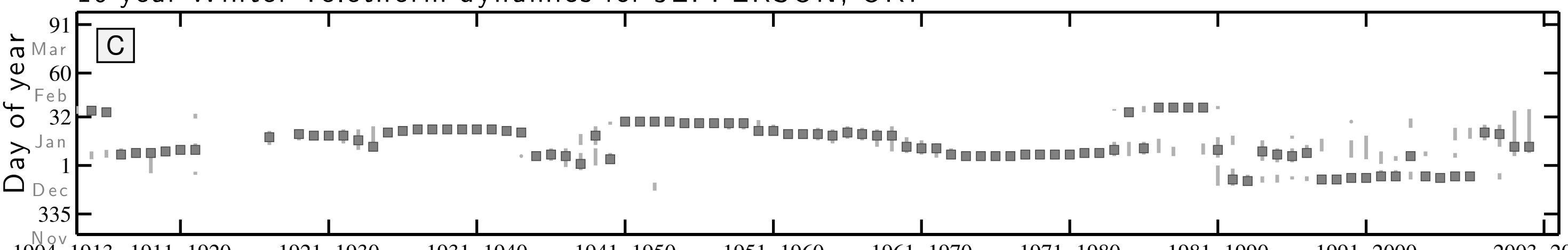

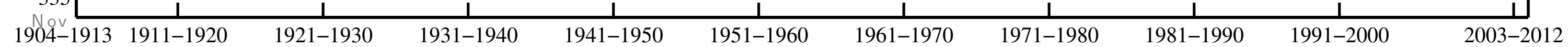

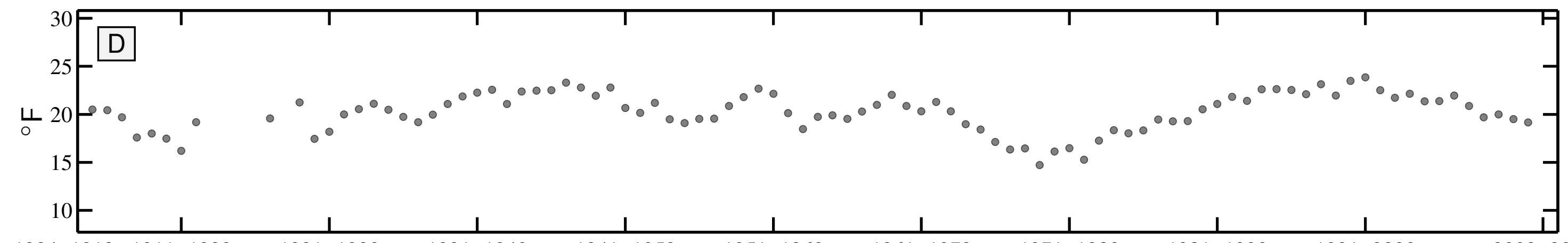


10 year Summer Teletherm dynamics for TAHLEQUAH, OK:
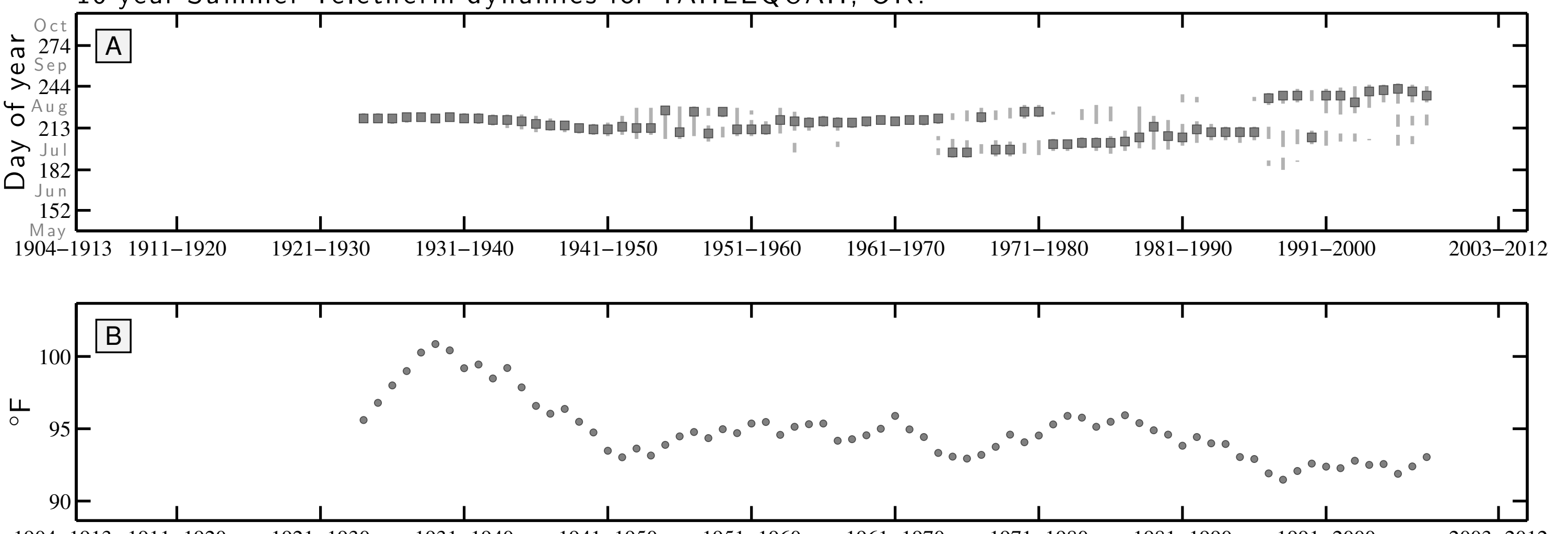

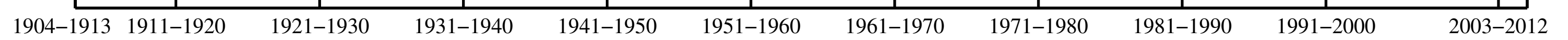
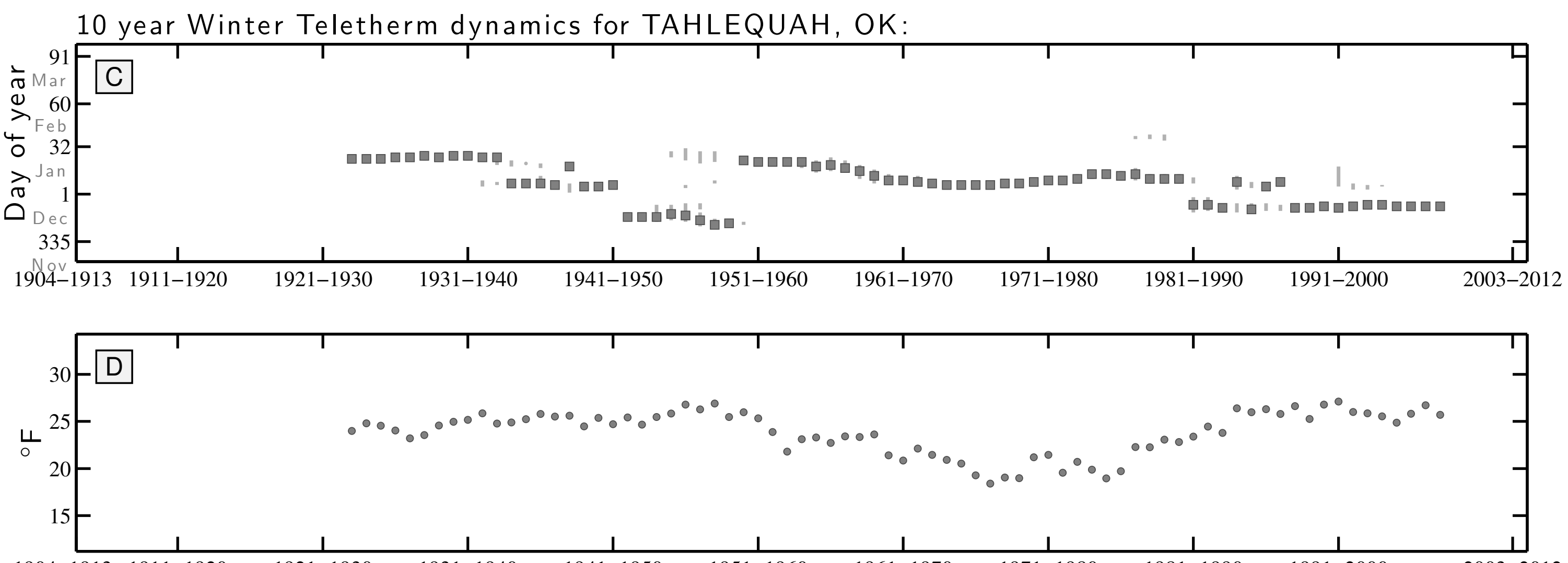

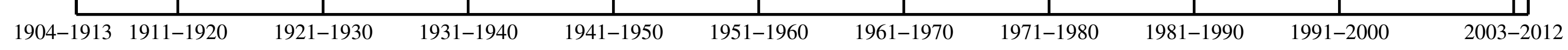


10 year Summer Teletherm dynamics for CONDON, OR:

$-\mathrm{ct}$

A

(1) Sep

$>244-$

( $\mathrm{Alg}$

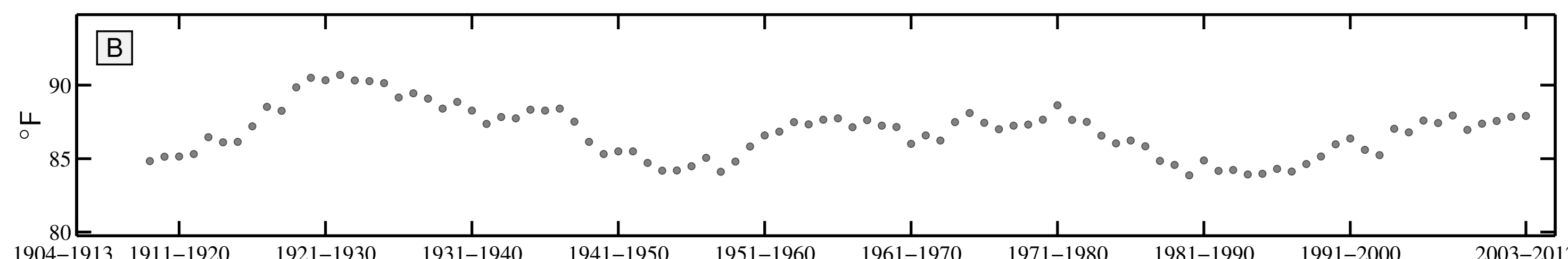

10 year Winter Teletherm dynamics for CONDON, OR:

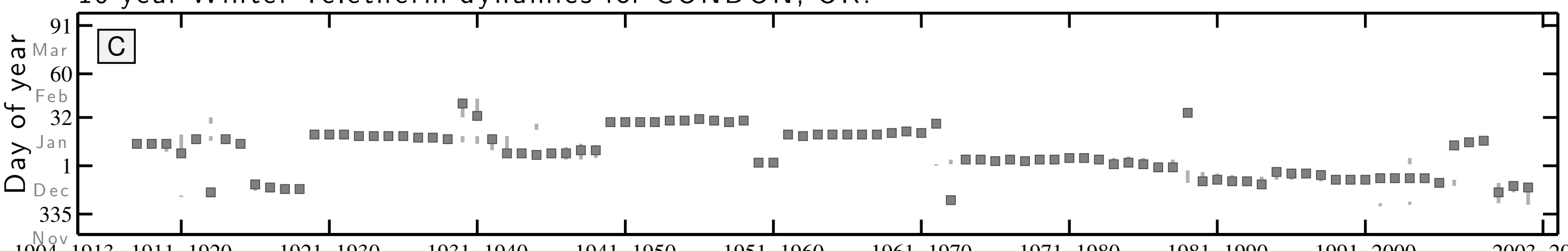

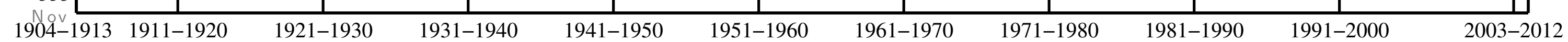

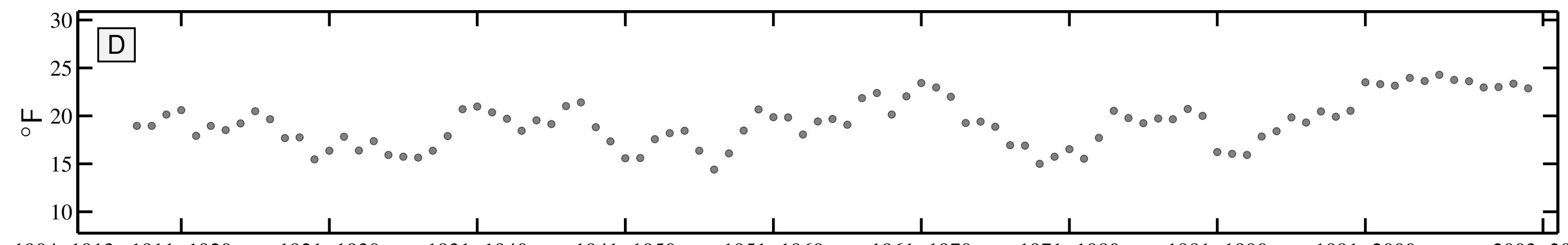


10 year Summer Teletherm dynamics for FREMONT 5 NW, OR:

$-O c t$

$274-A$

(1) Sep

$>244-$

응

213
$\mathrm{Jul}$

\% 182

Jun
152

152

1904-1913 1911-1920

" | |

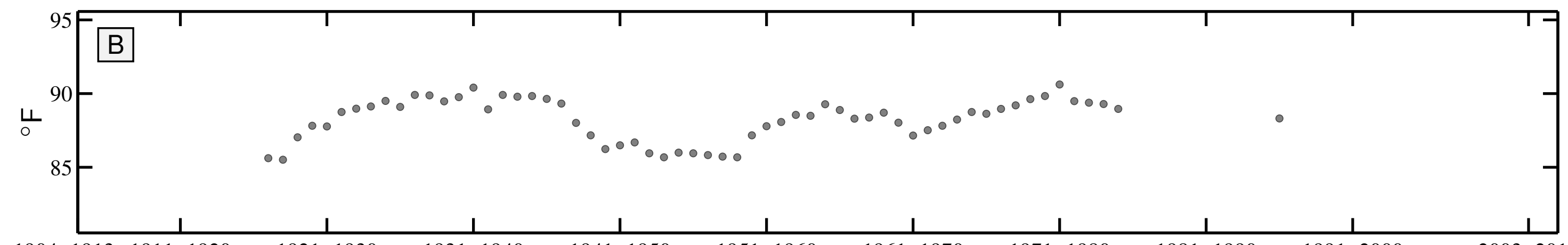

1904-1913 1911-1920

1921-1930

1931-1940

1941-1950

1951-1960

1961-1970

1971-1980

1981-1990

1991-2000

2003-2012

10 year Winter Teletherm dynamics for FREMONT 5 NW, OR:

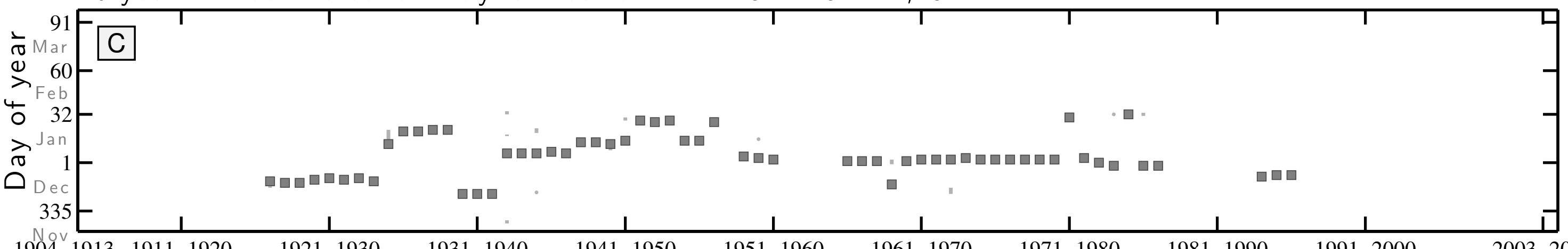

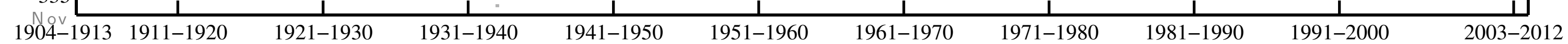

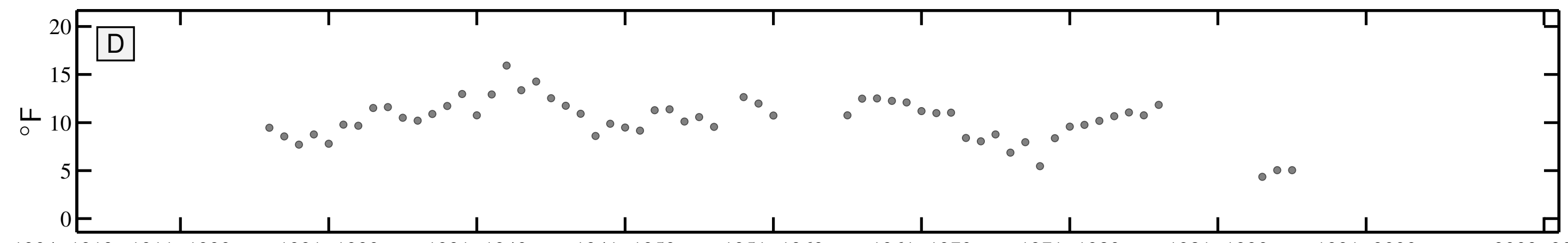


10 year Summer Teletherm dynamics for GRANTS PASS, OR:

$\mathrm{Oct}$

( $274-A$

(1) Sep

$>244-$

$213-$

त Jul $\square \square \square \square$

ก $182-$

$152-$

152 -

$\frac{1}{1-1920}$

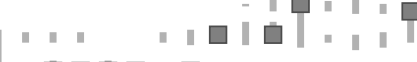

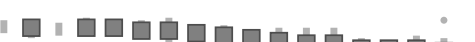

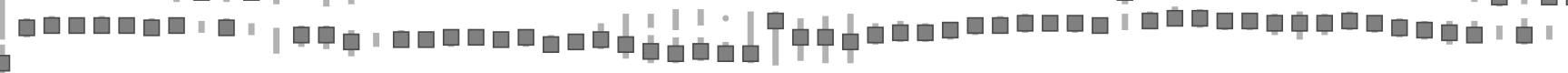

由日中
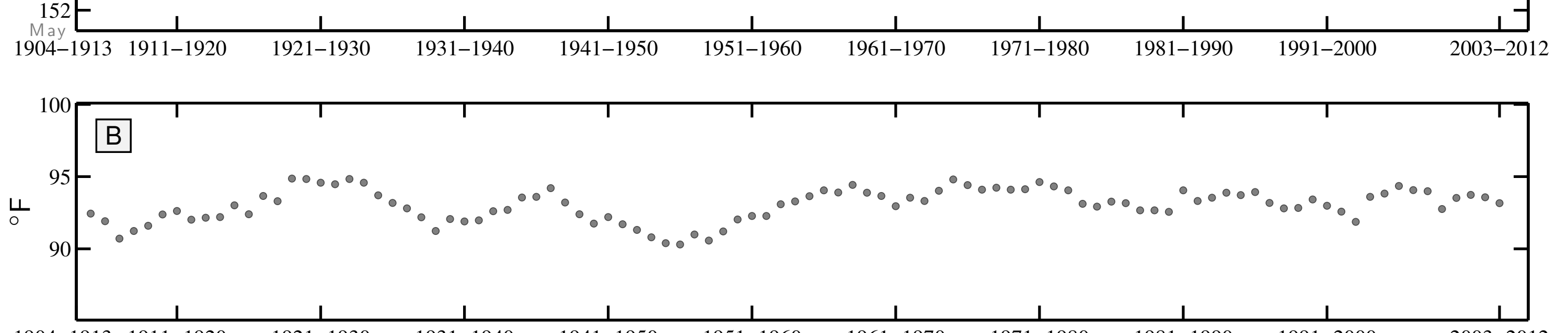

$1904-1913$ 1911-1920

$1921-1930$

$1931-1940$

$1941-1950$

1951-1960

1961-1970

1971-1980

1981-1990

$1991-2000$

2003-2012

10 year Winter Teletherm dynamics for GRANTS PASS, OR:

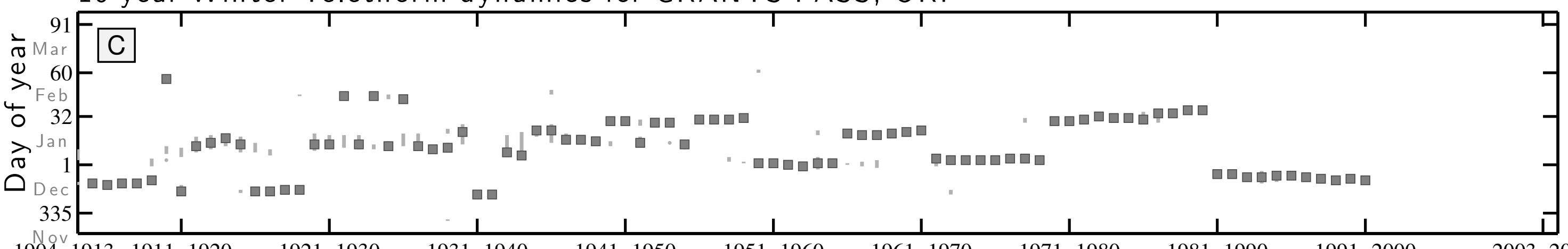

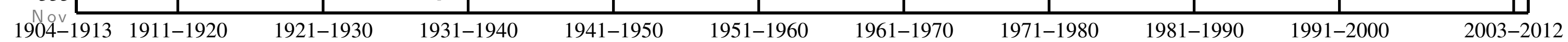

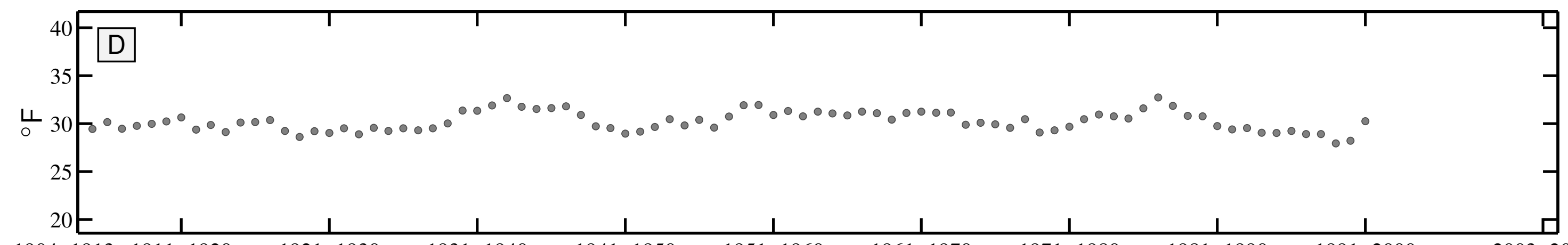


10 year Summer Teletherm dynamics for HEPPNER, OR:
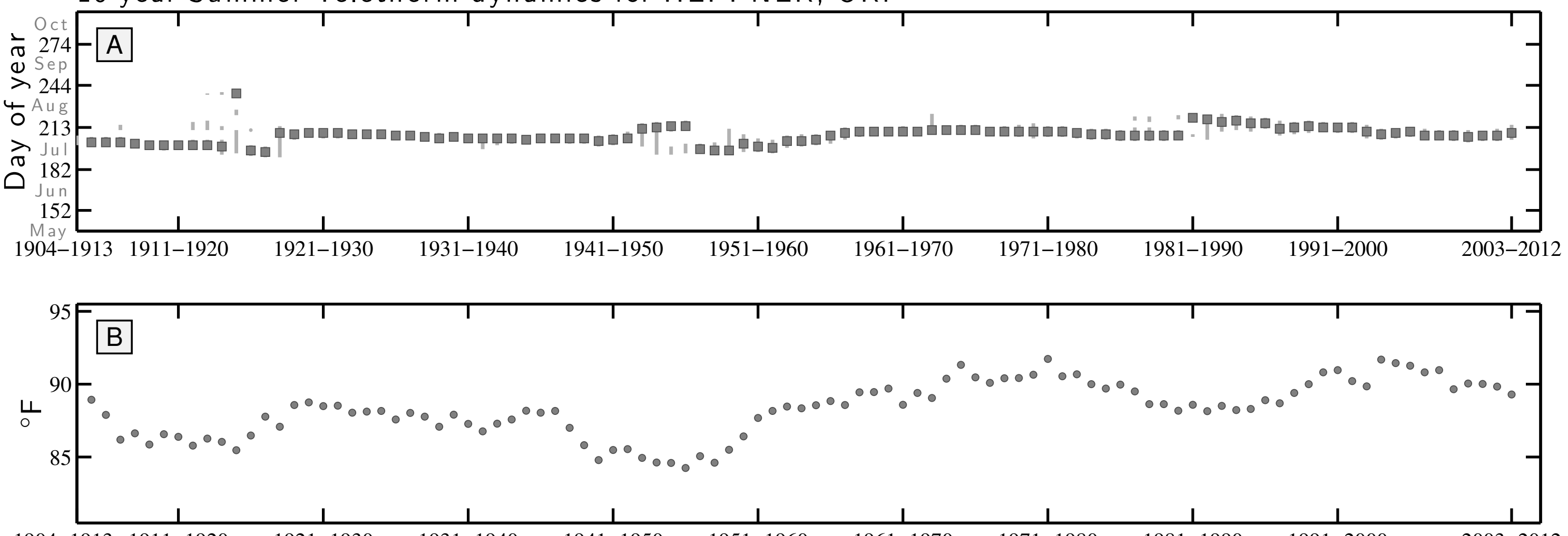

10 year Winter Teletherm dynamics for HEPPNER, OR:
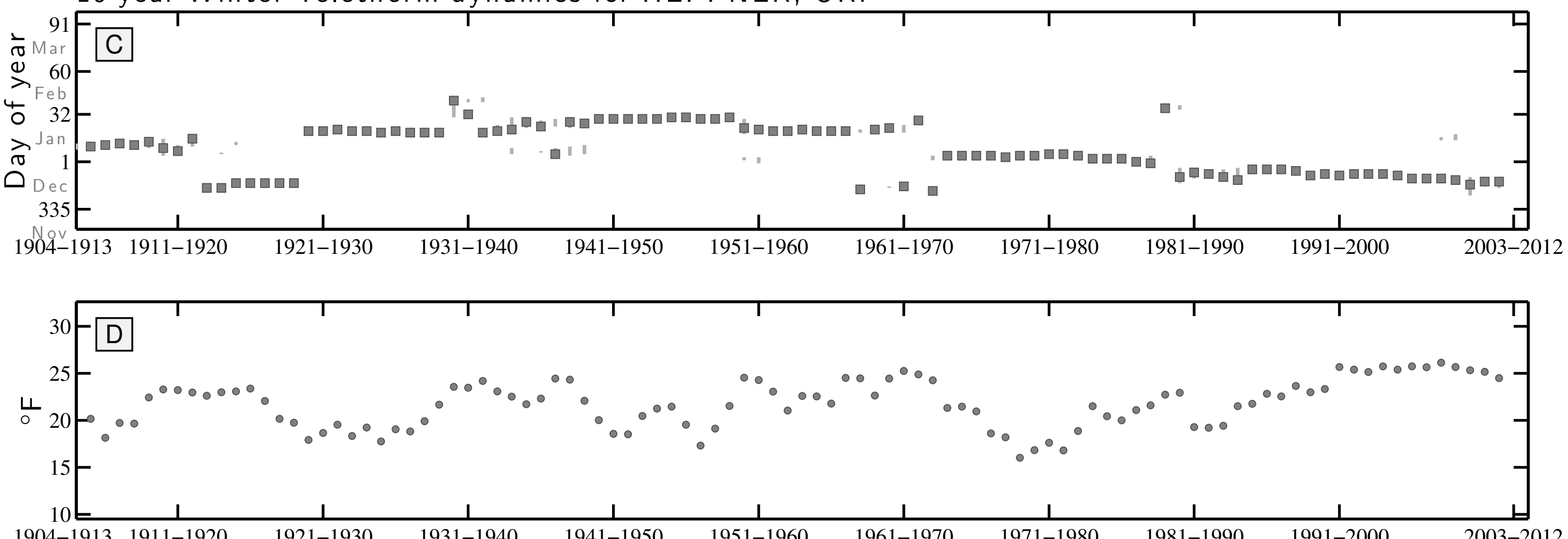
10 year Summer Teletherm dynamics for KLAMATH FALLS 2 SSW, OR:

- Oct

\% $274-A$

(1) Sep

$>244$

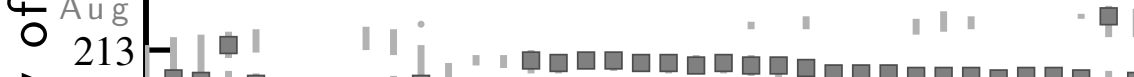

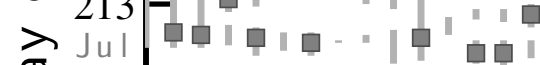

○ 182

152

May

1904-1913 1911-1920

$1921-1930$

$1931-1940$

$1941-1950$

$1951-1960$

961-1970

$1971-1980$

$\frac{1}{1981-1990}$

1

1991-2000

2003-2012

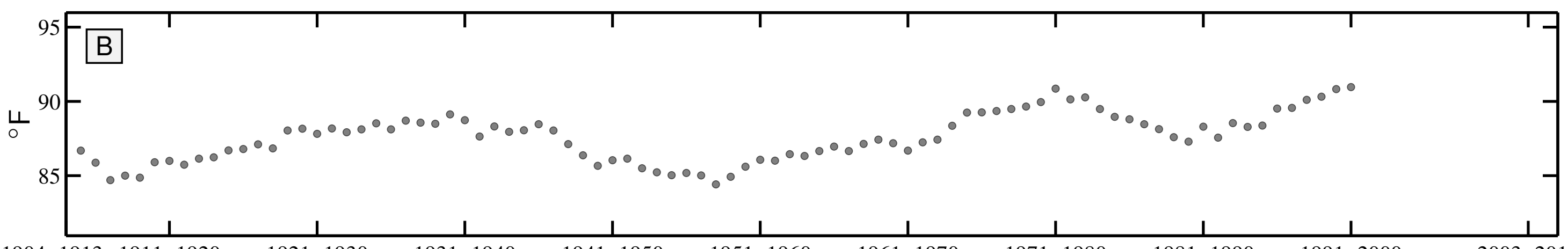

1904-1913 1911-1920

1921-1930

1931-1940

1941-1950

1951-1960

1961-1970

1971-1980

1981-1990

1991-2000

2003-2012

10 year Winter Teletherm dynamics for KLAMATH FALLS 2 SSW, OR:

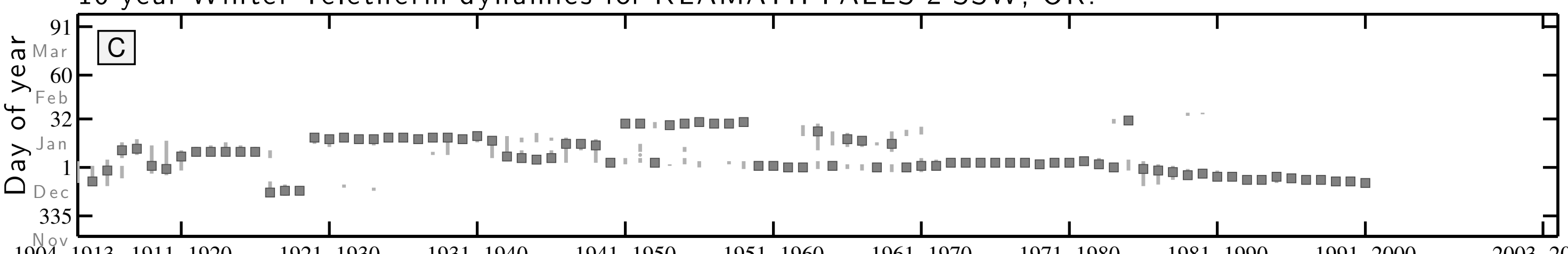

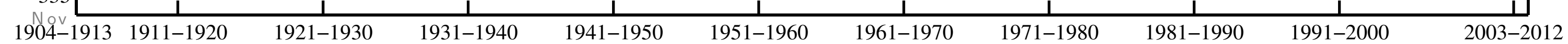

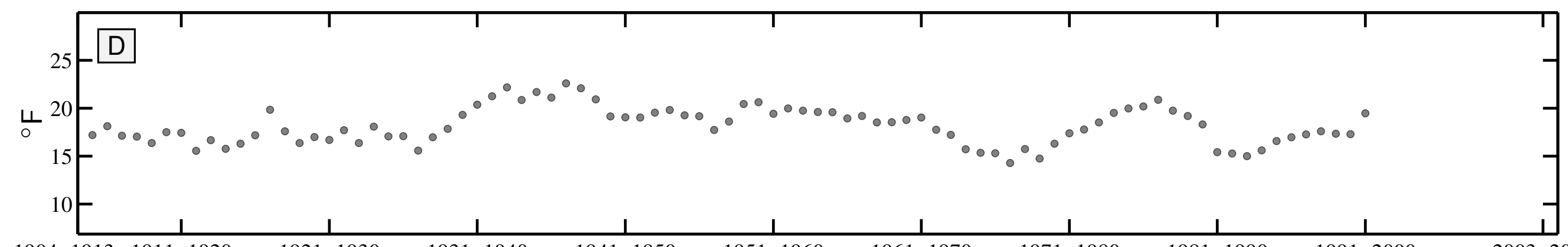


10 year Summer Teletherm dynamics for LAKEVIEW 2 NNW, OR:
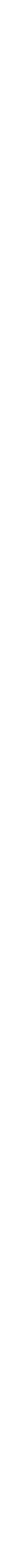

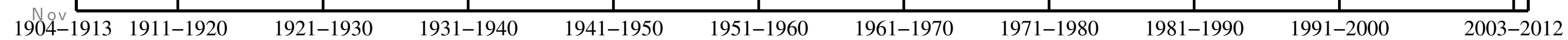

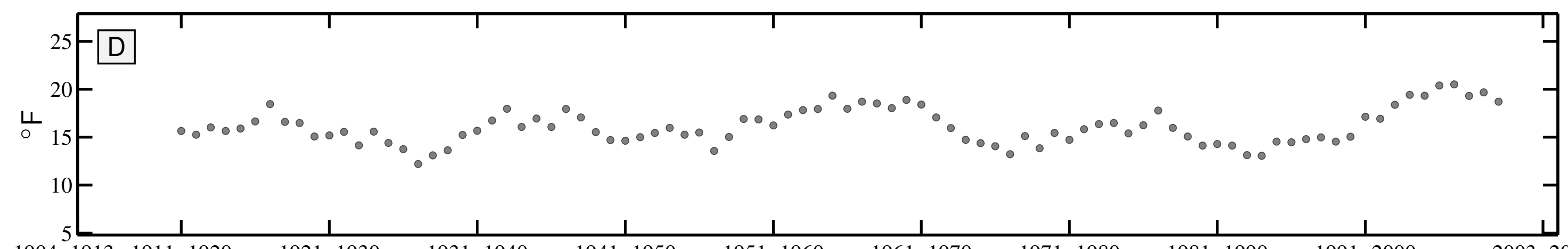

\begin{tabular}{|c|c|c|c|c|c|}
\hline $1904-1913 \quad 1911-1920$ & $1921-1930$ & $1931-1940$ & $1941-1950$ & $1951-1960$ & $1961-1970$ \\
\hline
\end{tabular}


10 year Summer Teletherm dynamics for MALHEUR REFUGE HQ, OR:

$-O c t$

\% $274-A$

(1) Sep

Aug

○ 213

ब 182

Jun

$152-$

1

1904-1913 1911-1920

$\frac{1}{1921-1930}$

1931-1940

1941-1950

1951-1960

$\frac{1}{1961-1970}$

$\frac{1}{1971-1980}$

1981-1990

1

1991-2000

2003-2012

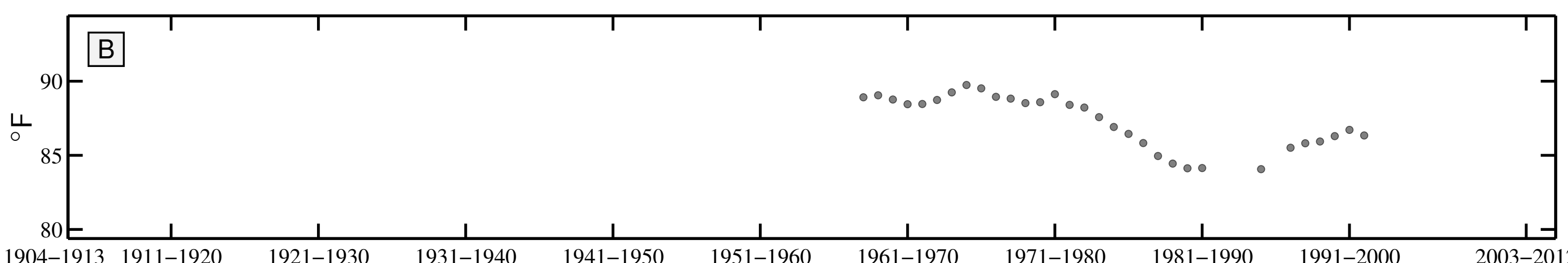

1904-1913 1911-1920

1971-1980

1981-1990

991-2000

2003-2012

10 year Winter Teletherm dynamics for MALHEUR REFUGE HQ, OR:
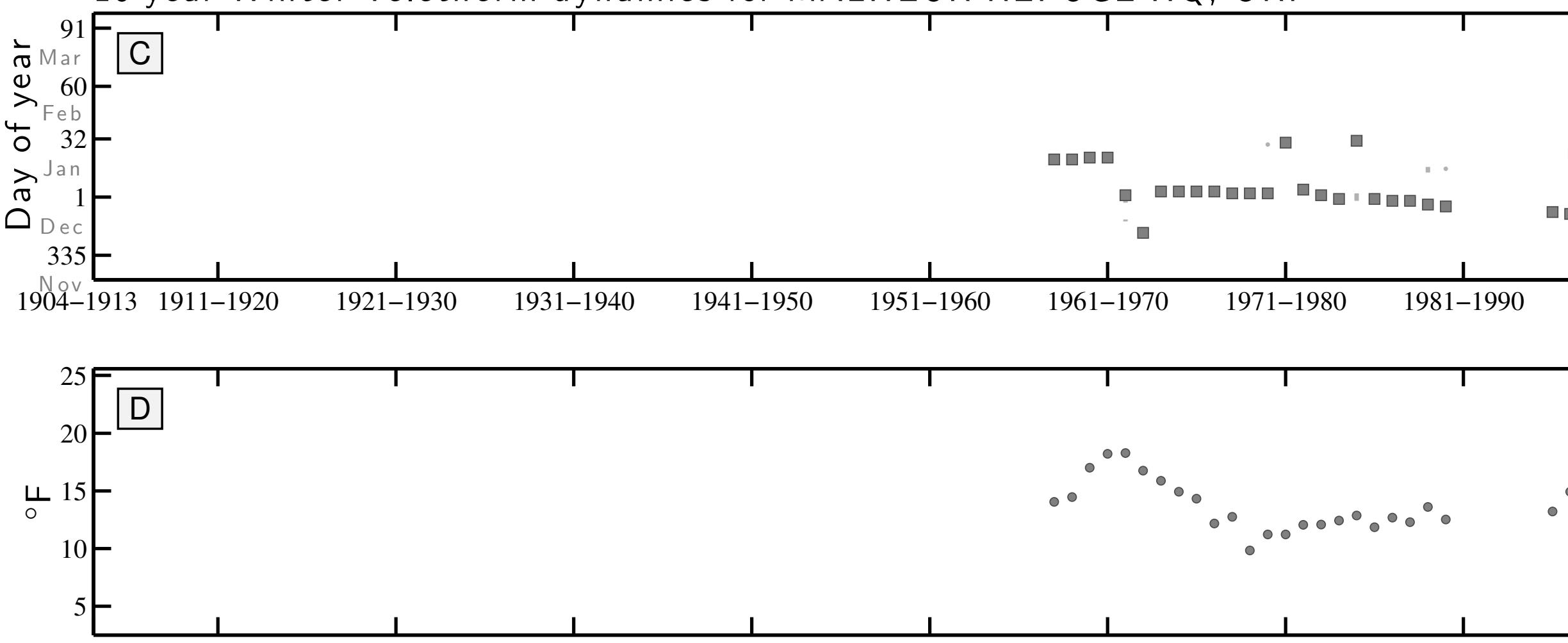

1921-1930 
10 year Summer Teletherm dynamics for PRINEVILLE, OR:

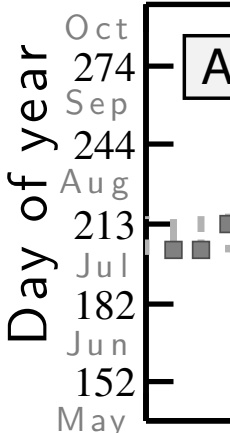
A

- $\square \square \square \quad \square \square \square$

I

। | $\square \square$ "

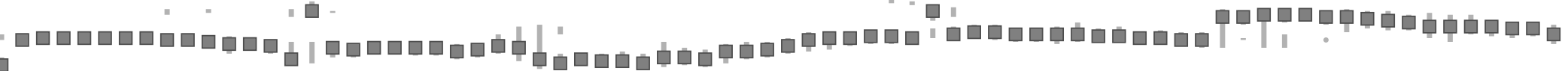

$152-$

1

$\frac{1}{1921-1930}$

1931-1940

1941-1950

1951-1960

$\frac{1}{1961-1970}$

$\frac{1}{1971-1980}$

$\frac{1}{1981-1990}$

991-2000

2003-201

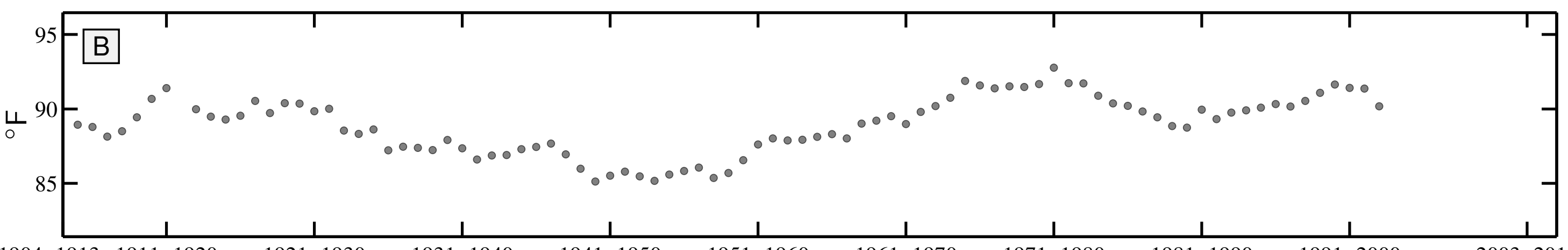

1904-1913 1911-1920

1921-1930

1931-1940

1941-1950

1951-1960

1961-1970

1971-1980

1981-1990

1991-2000

2003-2012

10 year Winter Teletherm dynamics for PRINEVILLE, OR:

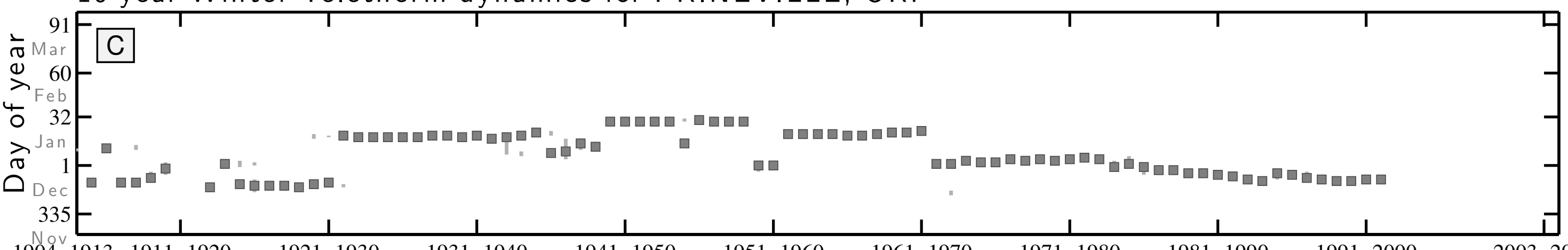

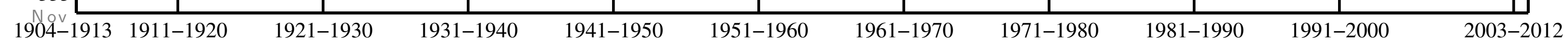

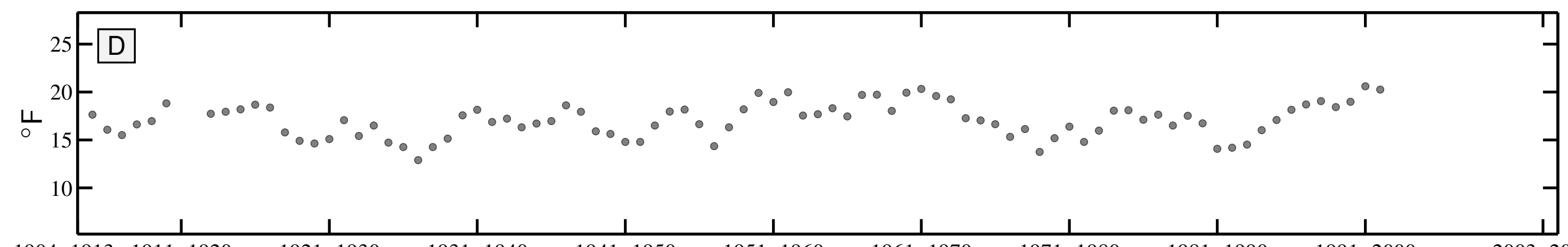


10 year Summer Teletherm dynamics for ALLENTOWN AP, PA:
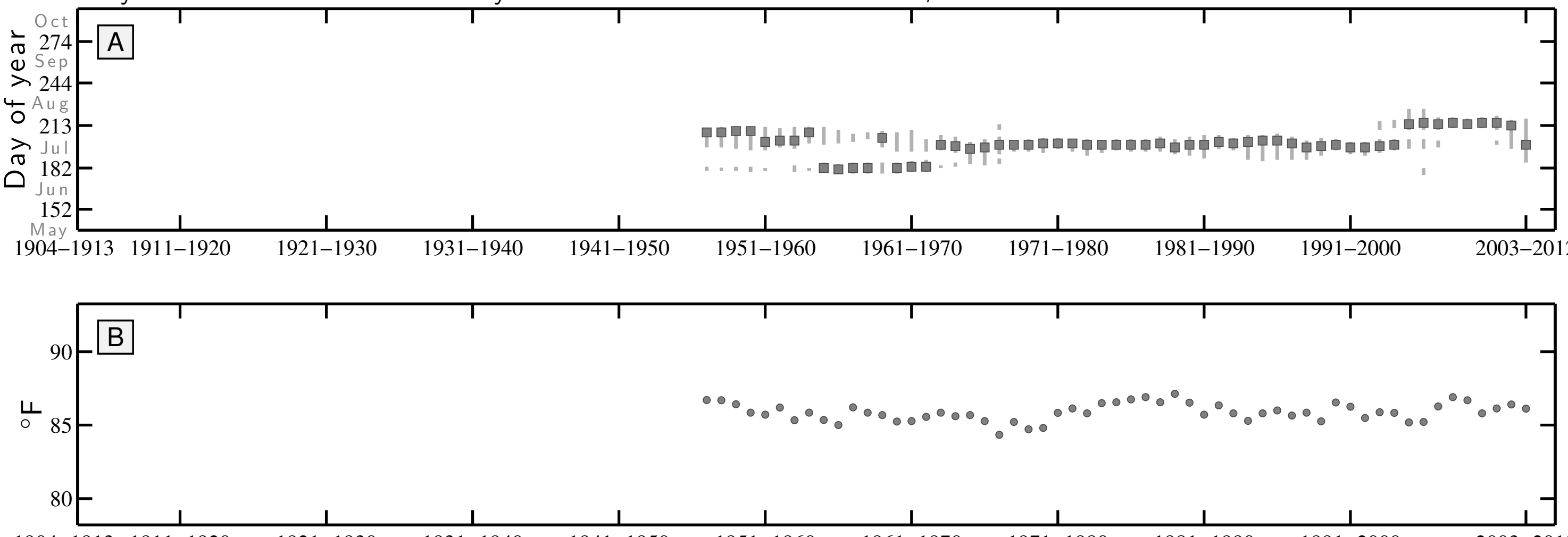

1904-1913 1911-1920

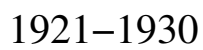

1931-1940

1941-1950

1951-1960

1961-1970

1971-1980

1981-1990

1991-2000

2003-2012

10 year Winter Teletherm dynamics for ALLENTOWN AP, PA:

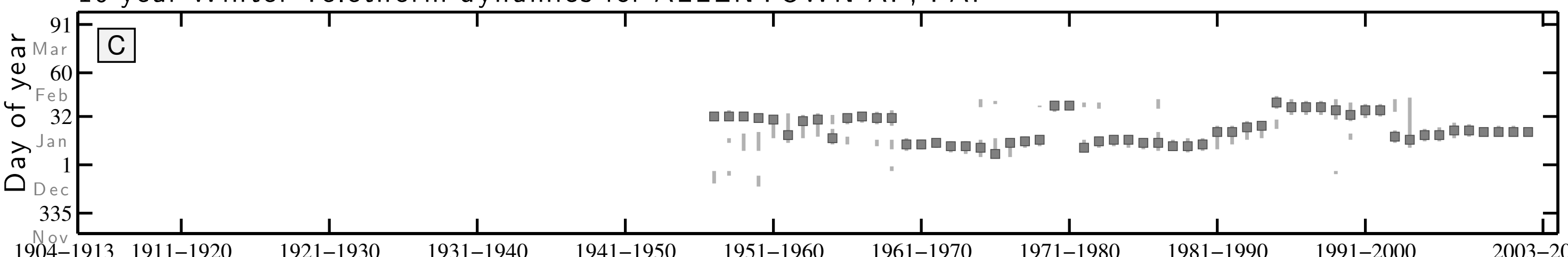

1904-1913 1911-1920

1921-1930

1931-1940

1941-1950

1951-1960

1961-1970

1971-1980

1981-1990

1991-2000

003-2012

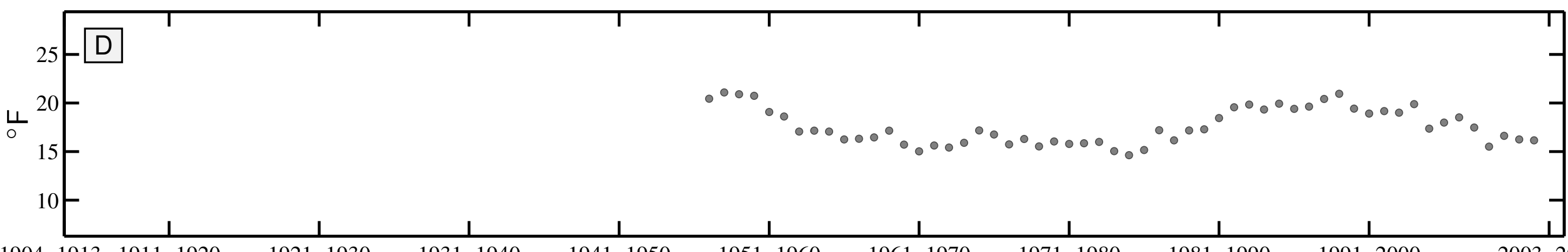


10 year Summer Teletherm dynamics for PLEASANT MT $1 \mathrm{~W}, \mathrm{PA}$ :
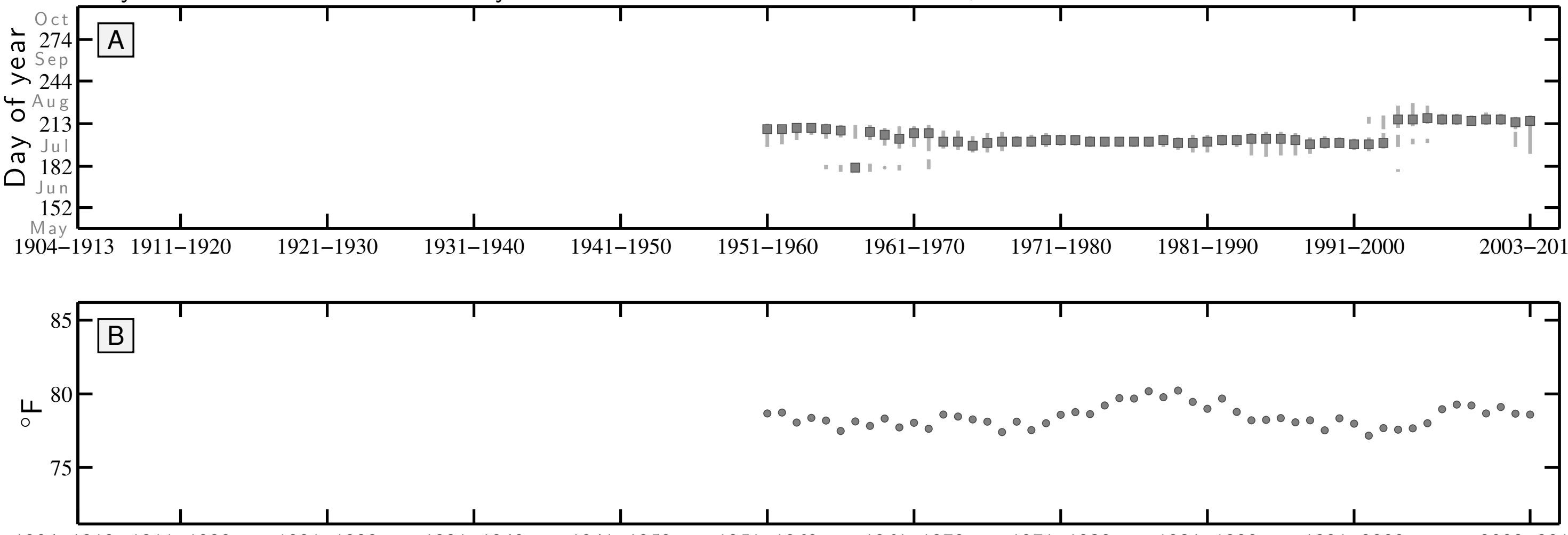

\section{0 year Winter Teletherm dynamics for PLEASANT MT 1 W, PA:}

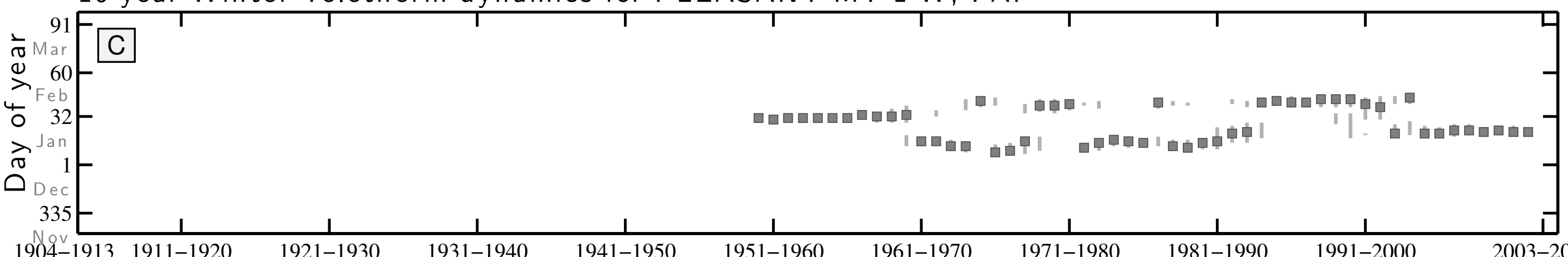

1904-1913 1911-1920

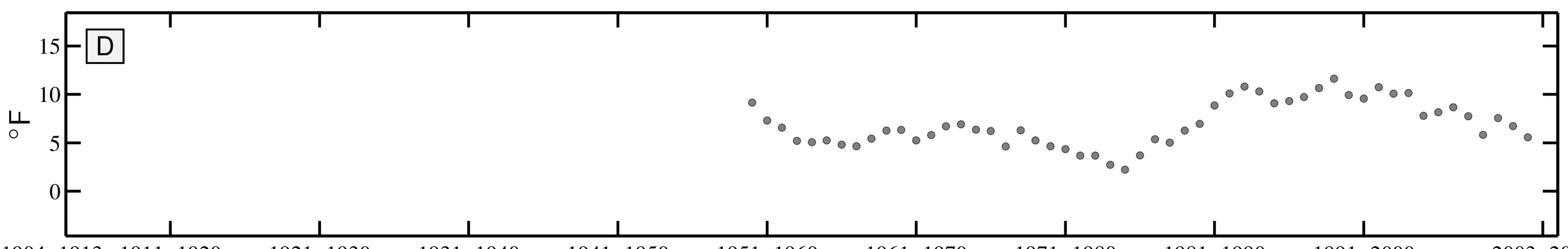


10 year Summer Teletherm dynamics for STATE COLLEGE, PA:

$\div$ Oct 274

(1) Sep

$>244-$

$4 \mathrm{Aug}$

$213-$

ते Ju

$\overbrace{}^{\circ} 182$

$152-$

$\square \square \square \square \square$

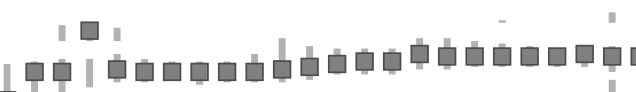

शी

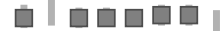

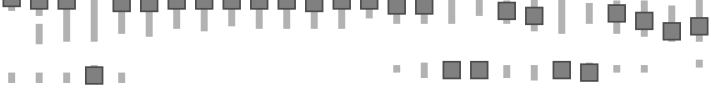

| 무뭄फ |
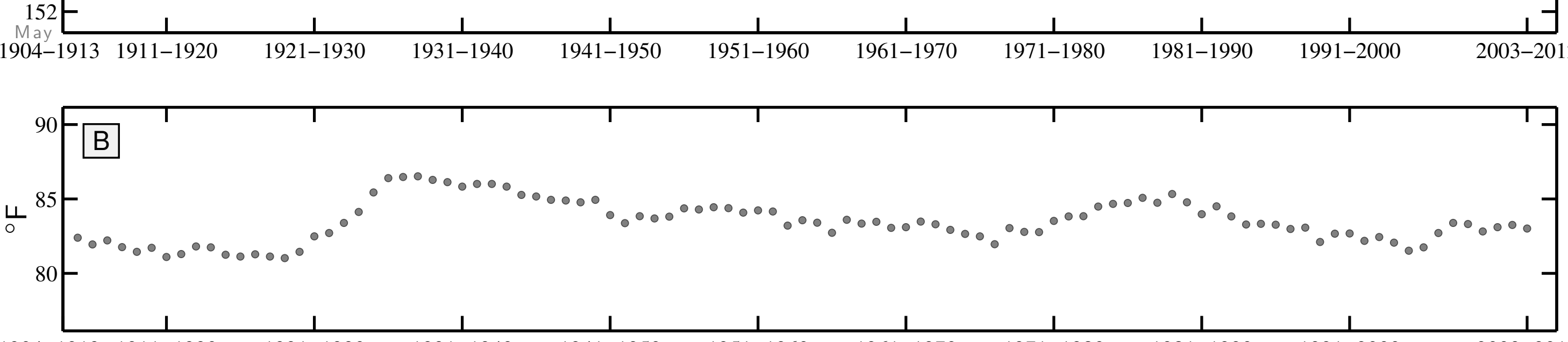

10 year Winter Teletherm dynamics for STATE COLLEGE, PA:

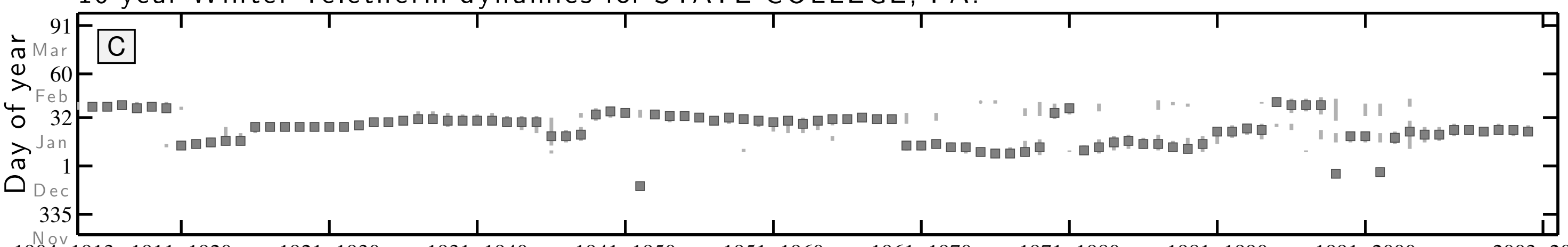

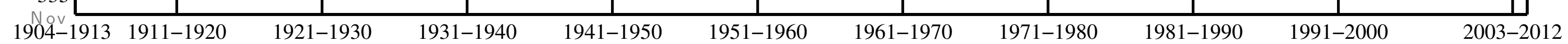

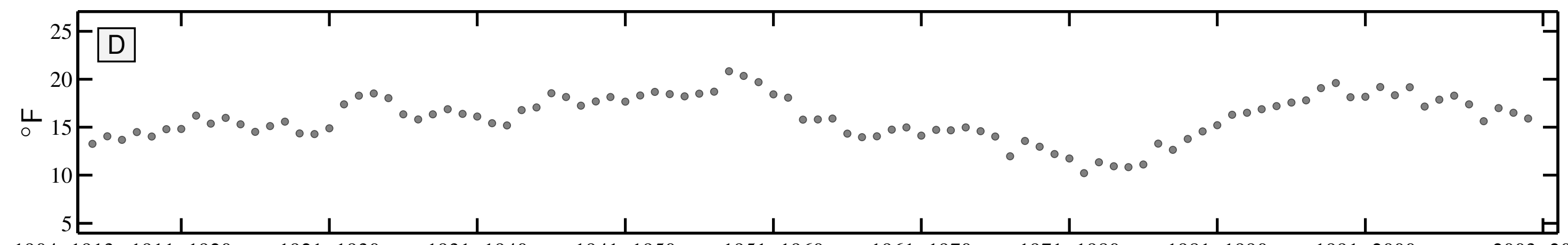


10 year Summer Teletherm dynamics for WARREN, PA:

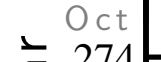

A

(1) Sep

244

○ 213 -

入 Jul

\% 18

$152-$

1904-1913 1911-1920

$1921-1930$

1931-1940

1941-1950

1951-1960

$\frac{1}{1961-1970}$

1971-1980

1981-1990

1991-2000

2003-2012

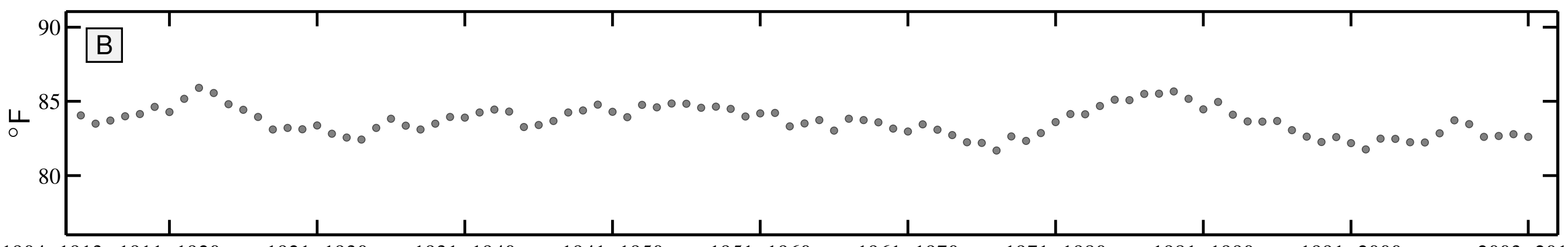

1904-1913 1911-1920

$1921-1930$

$1931-1940$

$1941-1950$

$1951-1960$

$1961-1970$

$1971-1980$

$1981-1990$

$1991-2000$

2003-2012

10 year Winter Teletherm dynamics for WARREN, PA:

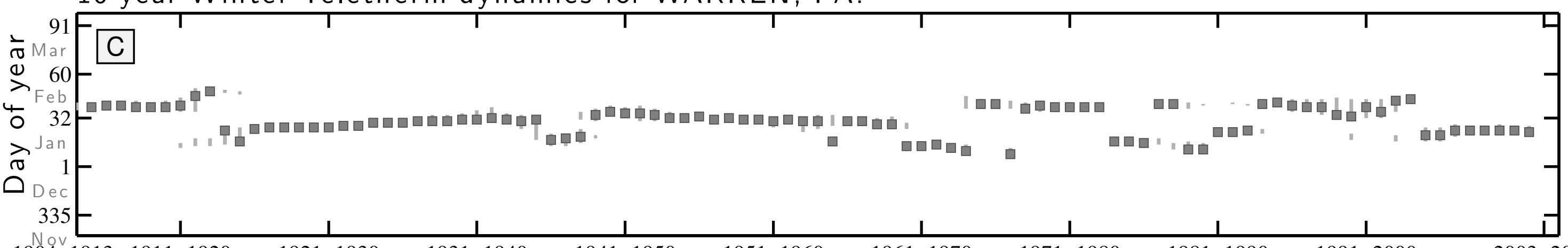

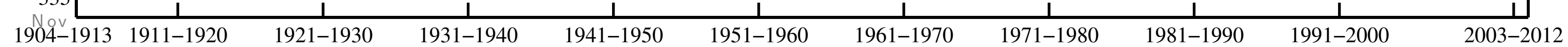

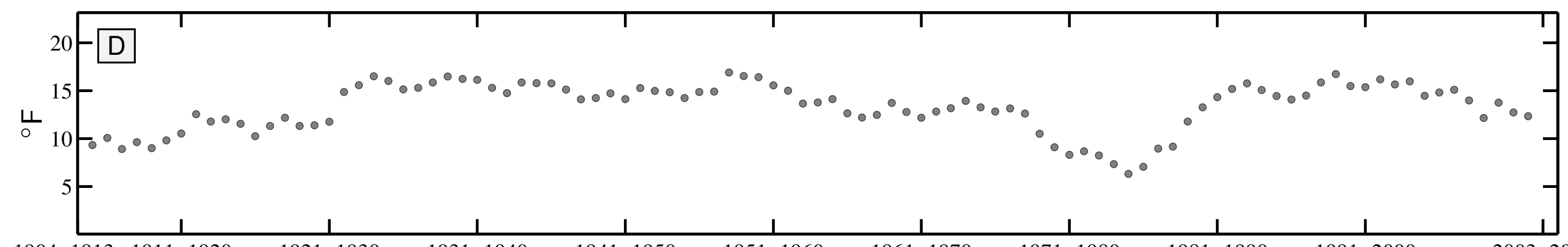


10 year Summer Teletherm dynamics for BLACKVILLE 3 W, SC:

Oct

(ब $274-A$

(1) Sep

$>244-$

4 Aug

$213-$

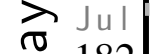

$\bigcirc$ Jun

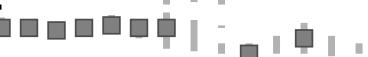

$152-$

May _ _ _ _

|| || ||

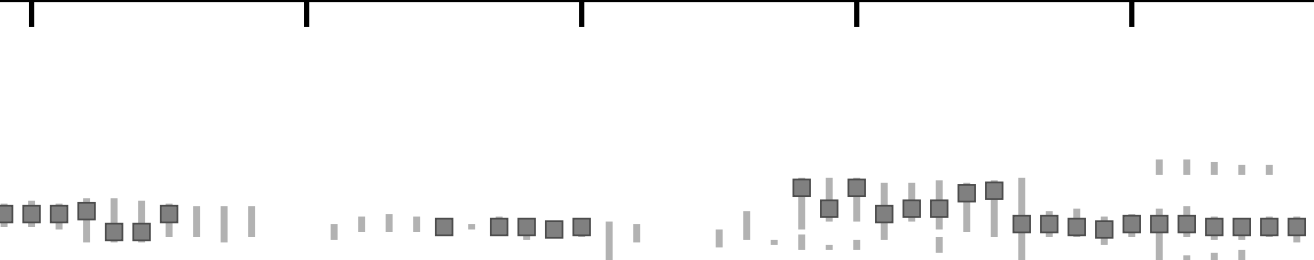

1904-1913 1911-1920

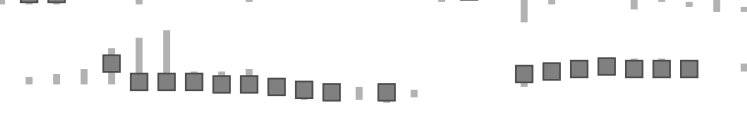

$\frac{1}{1921-1930}$

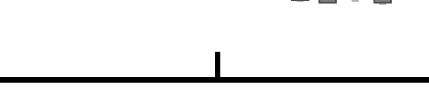

$\frac{1}{1941-1950}$

$1951-1960$

$\frac{1}{1961-1970}$

$\frac{1}{1971-1980}$

$1981-1990$

$1991-2000$

2003-201

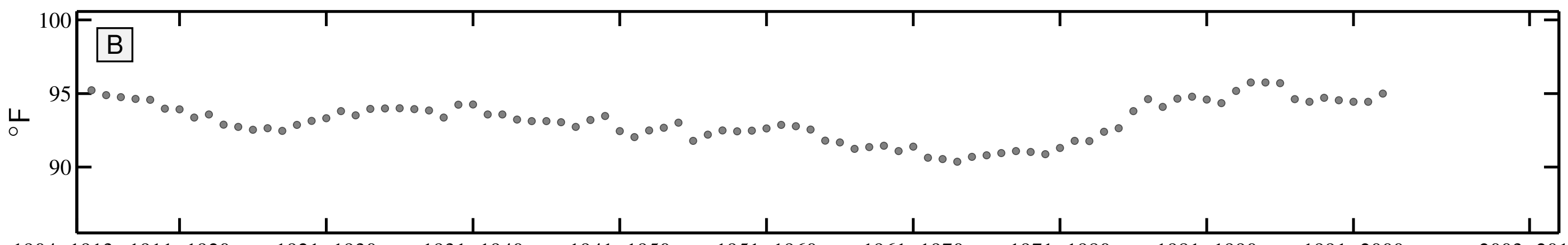

1904-1913 1911-1920

1921-1930

1931-1940

1941-1950

1951-1960

1961-1970

1971-1980

1981-1990

1991-2000

2003-2012

10 year Winter Teletherm dynamics for BLACKVILLE 3 W, SC:

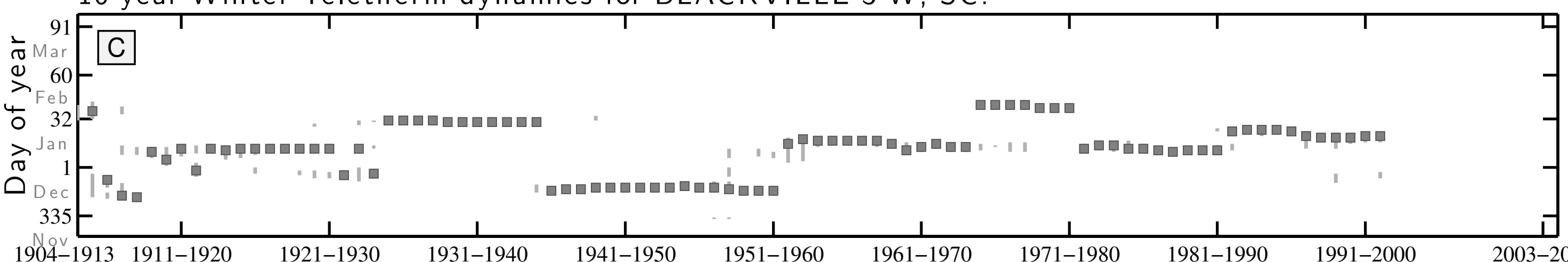

1904-1913 1911-1920 1921-193

1931-1940

1941-1950

1951-1960

1961-1970

1971-1980

1981-1990

003-2012

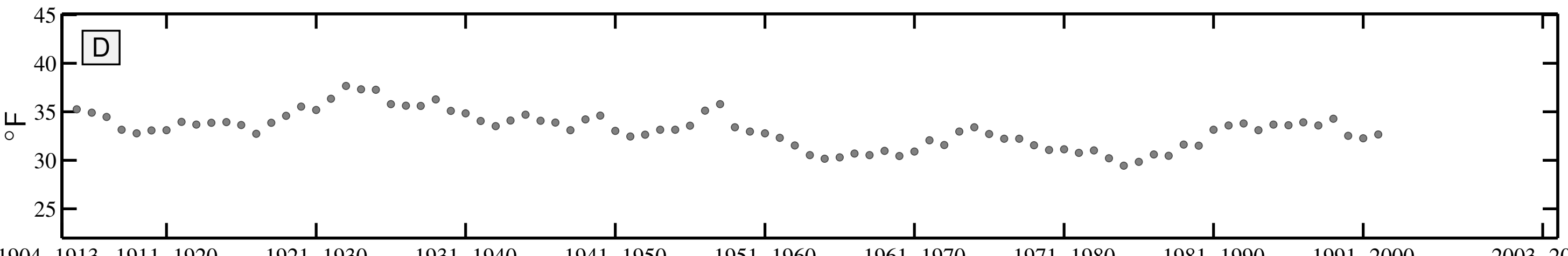


10 year Summer Teletherm dynamics for CLEMSON UNIV, SC:

$+\mathrm{Oct}$

$274-A$

(1) Sep

$>244$

O A g g

$>\mathrm{Jul}$

\% 182

Jun
152

152 -

1904-1913 1911-1920

$\frac{1}{1921-1930}$

\section{ㅁำロロ・}

| | || || - | - 믐ㅁ | |

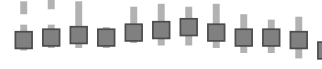

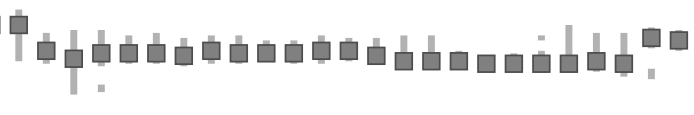

†口

무무

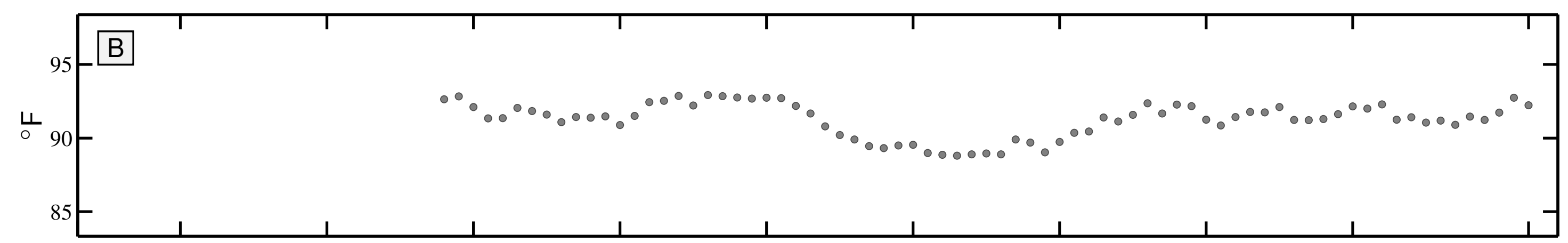

1904-1913 1911-1920

1921-1930

1931-1940

1941-1950

1951-1960

1961-1970

1971-1980

1981-1990

1991-2000

2003-2012

10 year Winter Teletherm dynamics for CLEMSON UNIV, SC:

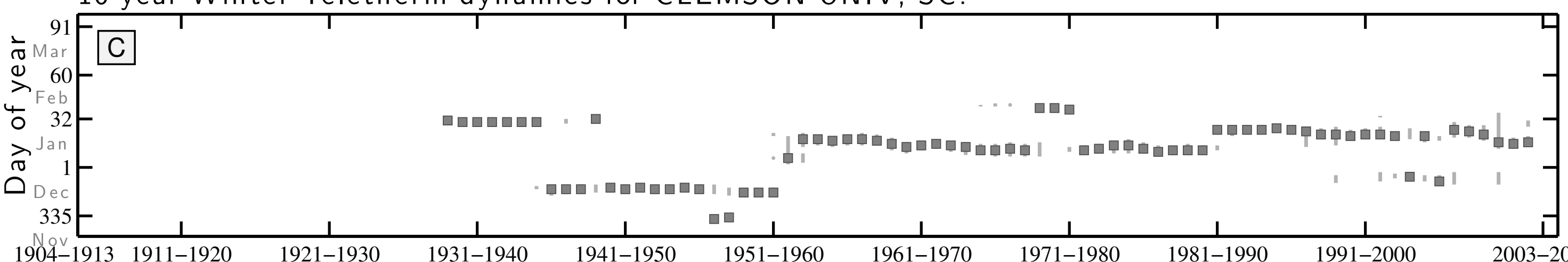

1904-1913 1911-192

1921-1930

$1931-1940$

$1941-1950$

$1951-1960$

$1961-1970$

$1971-1980$

$1981-1990$

991-2000

2003-2012

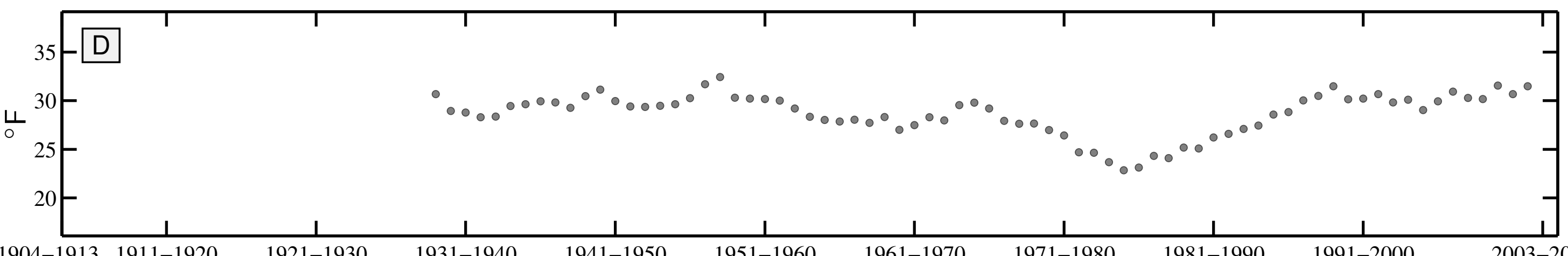


10 year Summer Teletherm dynamics for GREENWOOD, SC:

$\therefore \mathrm{Oct}$

A

(1) Sep

$>244-$

$213-$

त $\mathrm{Jul}$

$\overbrace{\substack{\mathrm{J} u \mathrm{n} \\ 1}}^{\infty}$

$152-$

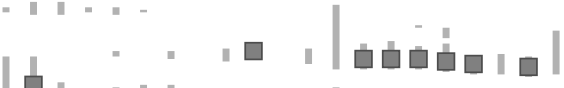

|| - || || ||

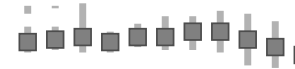

1904-1913 1911-1920

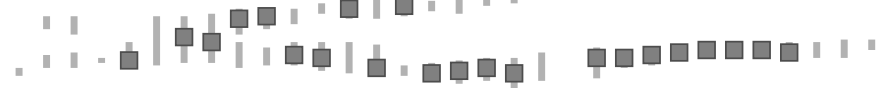

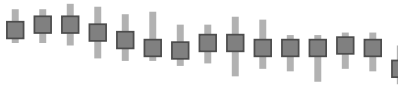

| || || || || ||

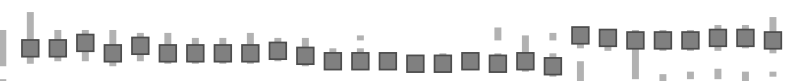

1

$\frac{1}{1951-1960}$

$1961-1970$

$1971-1980$

$\frac{1}{1981-1990}$

991-2000

2003-201

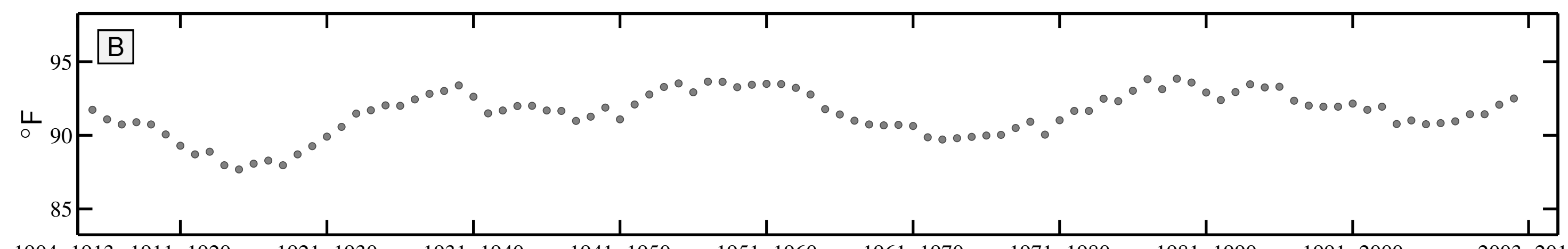

1904-1913 1911-1920

1921-1930

1931-1940

1941-1950

1951-1960

1961-1970

1971-1980

1981-1990

1991-2000

2003-2012

10 year Winter Teletherm dynamics for GREENWOOD, SC:

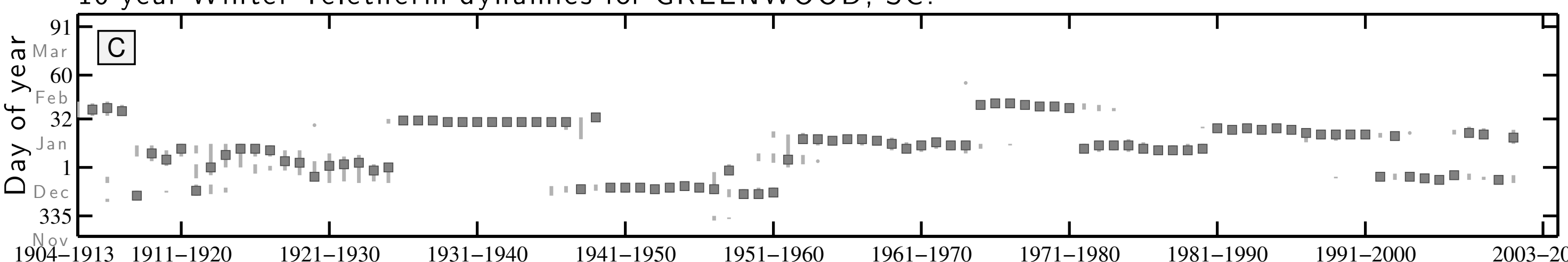

1904-1913 1911-1920 1921-1930 1931-194

$1941-1950$

$1951-1960$

$1961-1970$

$1971-1980$

$1981-1990$

991-2000

$2003-2012$

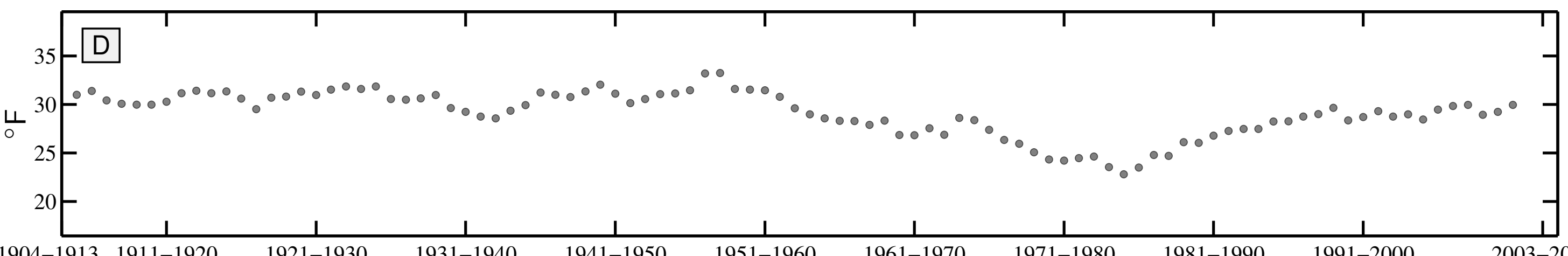


10 year Summer Teletherm dynamics for ORANGEBURG 2, SC:
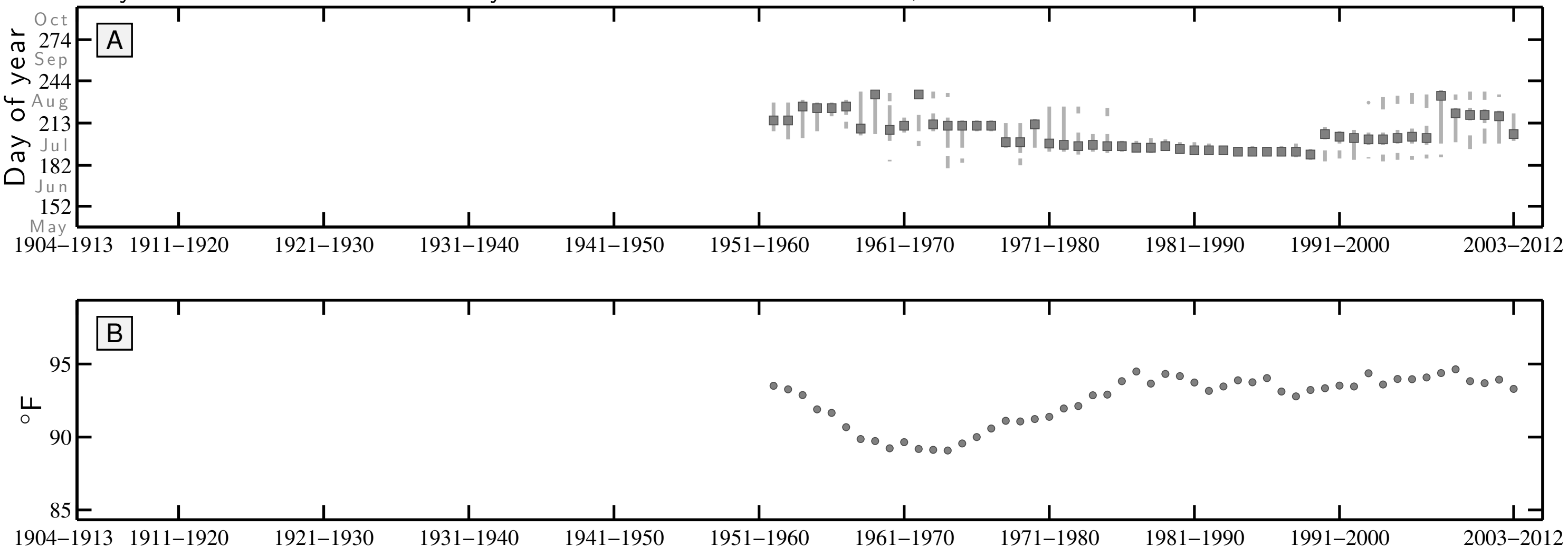

10 year Winter Teletherm dynamics for ORANGEBURG 2, SC:

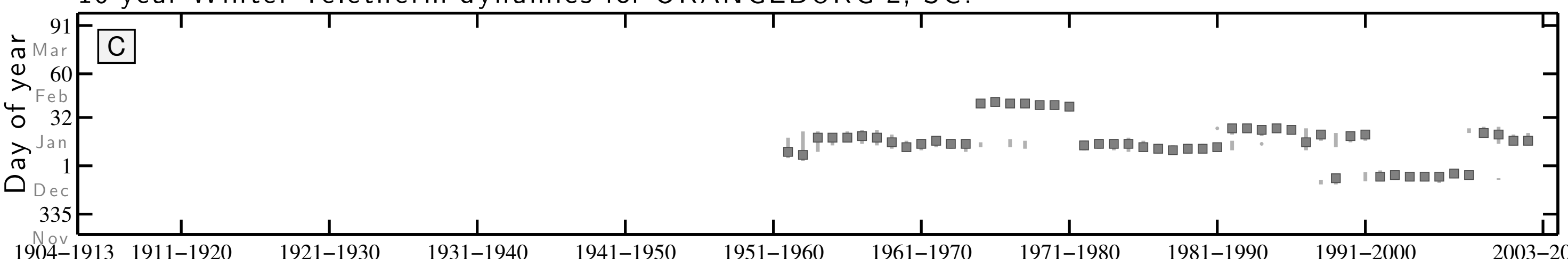

1904-1913 1911-192(

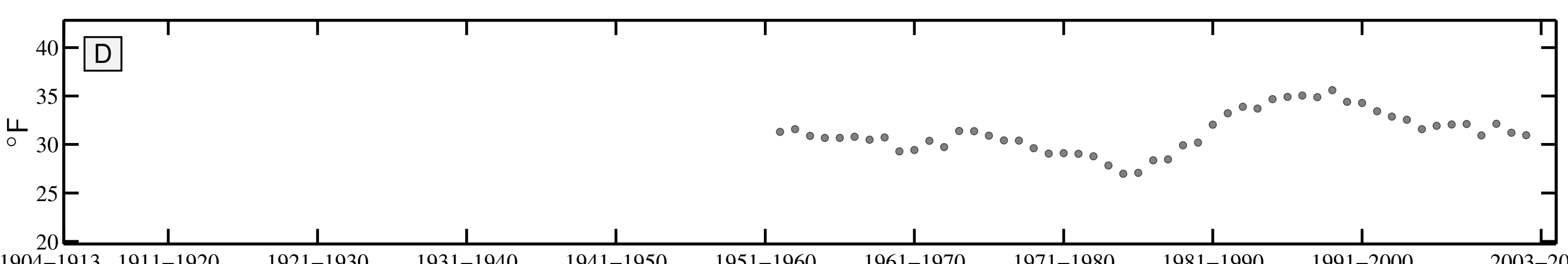


10 year Summer Teletherm dynamics for SANTUCK, SC:

$\div 274$

Sep

$\stackrel{\text { Sep }}{>} 244$

4 Aug

O $213-$

$\gtrsim$ Jul

$182-$

$152-$

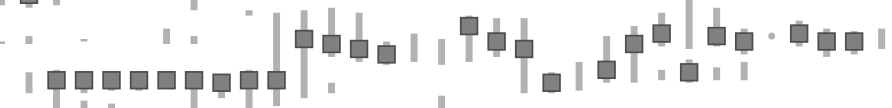

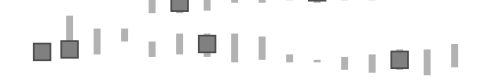

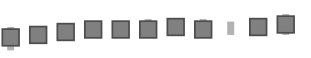

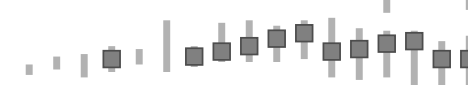

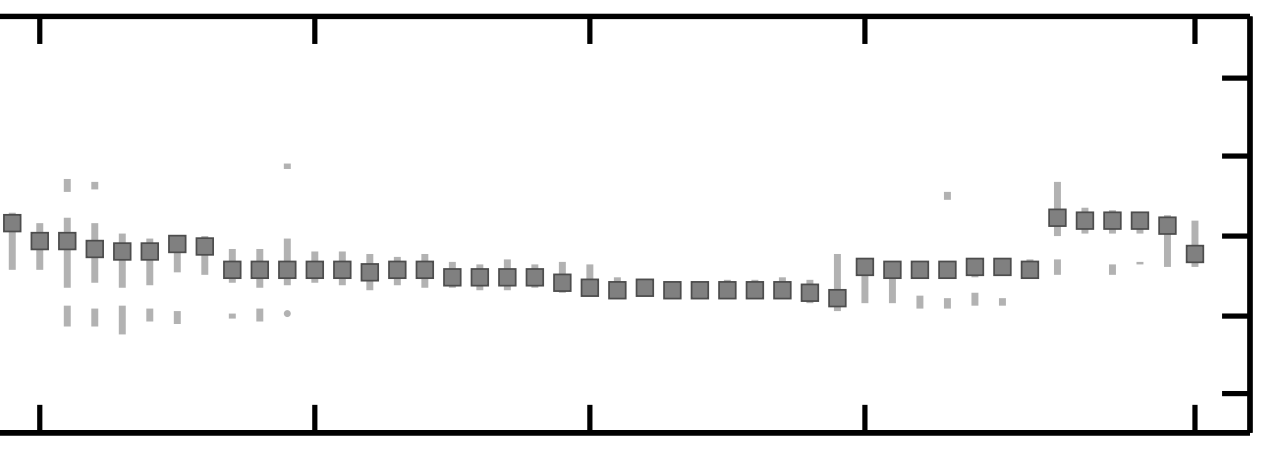

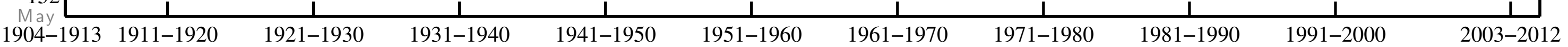

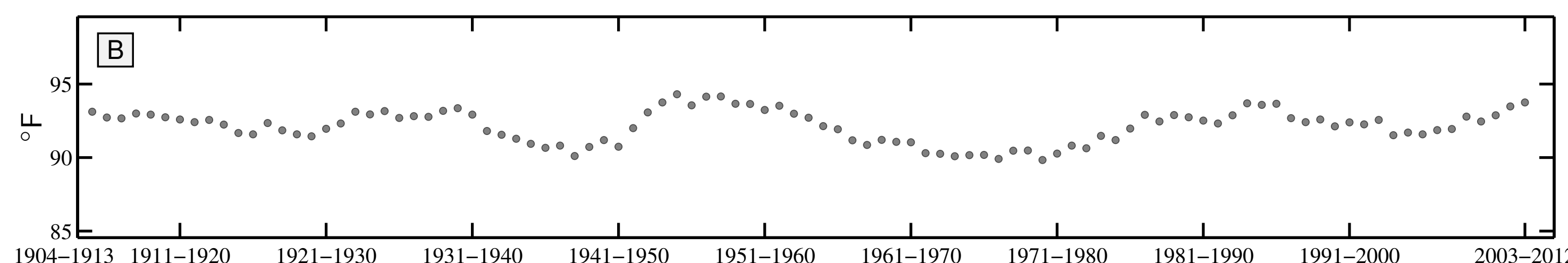

1904-1913 1911-192

1921-1930

1931-1940

1941-1950

1951-1960

1961-1970

1971-1980

1981-1990

1991-2000

2003-2012

10 year Winter Teletherm dynamics for SANTUCK, SC:

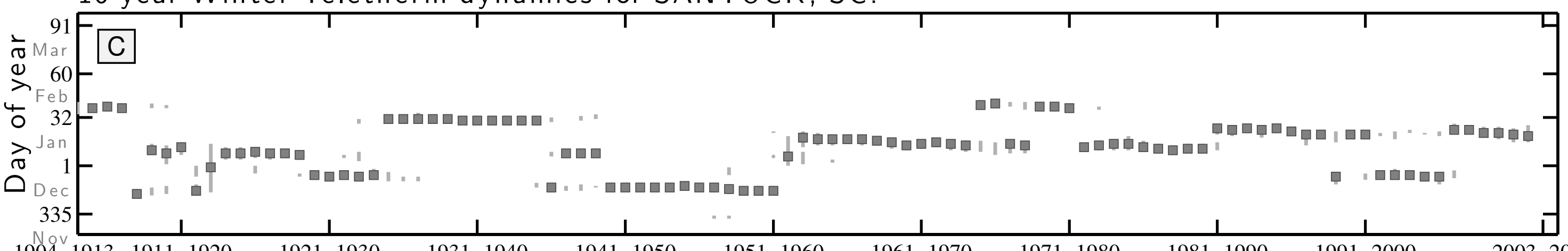

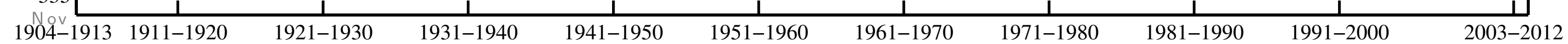

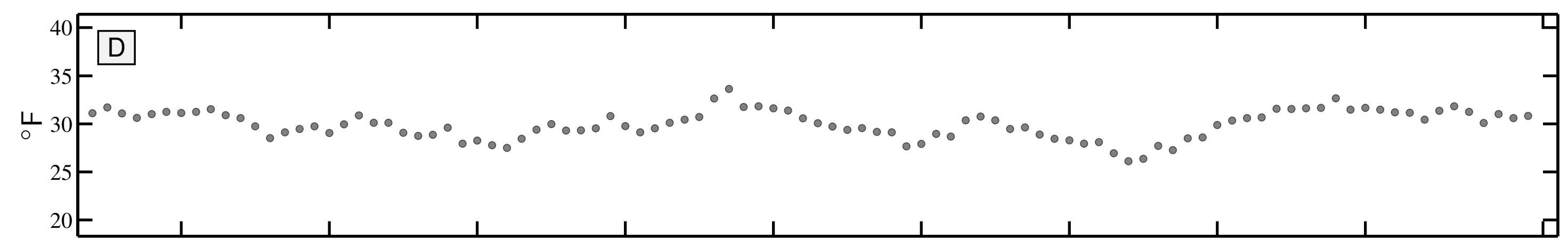


10 year Summer Teletherm dynamics for SUMMERVILLE 4W, SC:

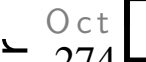

$\div 274-\mathrm{A}$

(1) Sep

$>244-$

O 213

त Jul

$\bigcirc \underset{\mathrm{Jun}}{182}$

| || || || | | ||

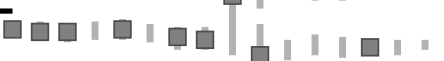

152

1

$\frac{1}{1921-1931}$

ㅁำロロロ " ロ "

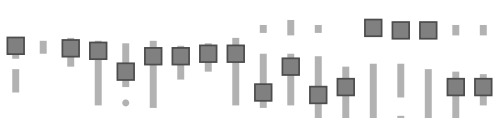
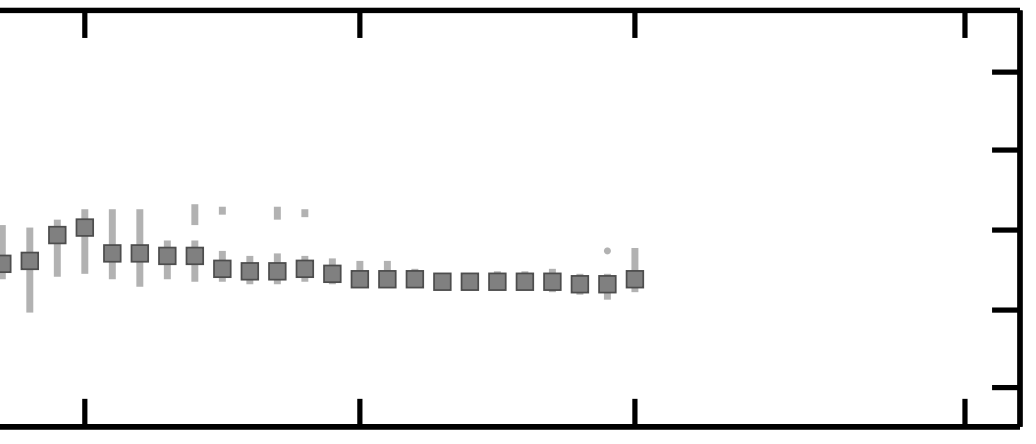

1904-1913 1911-1920

$\frac{1}{1931-1940}$

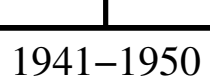

1951-1960

1961-1970

$\frac{1}{1971-1980}$

$\frac{1}{981-1990}$

991-2000

2003-2012

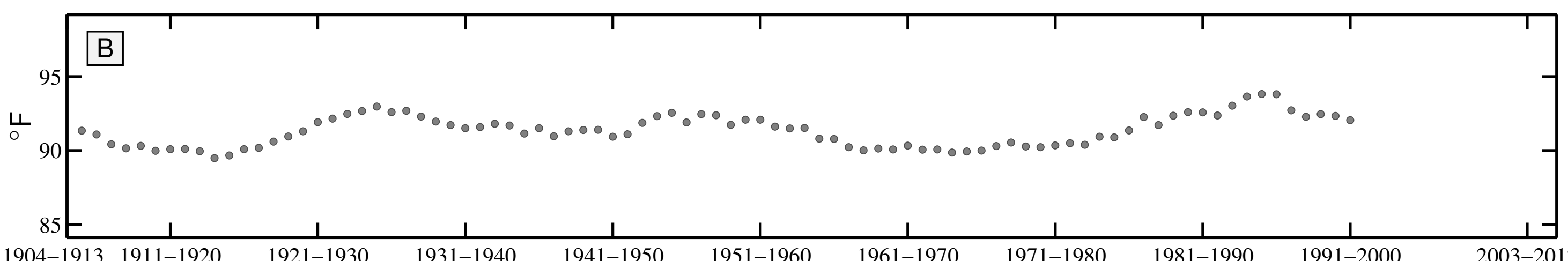

10 year Winter Teletherm dynamics for SUMMERVILLE 4W, SC:

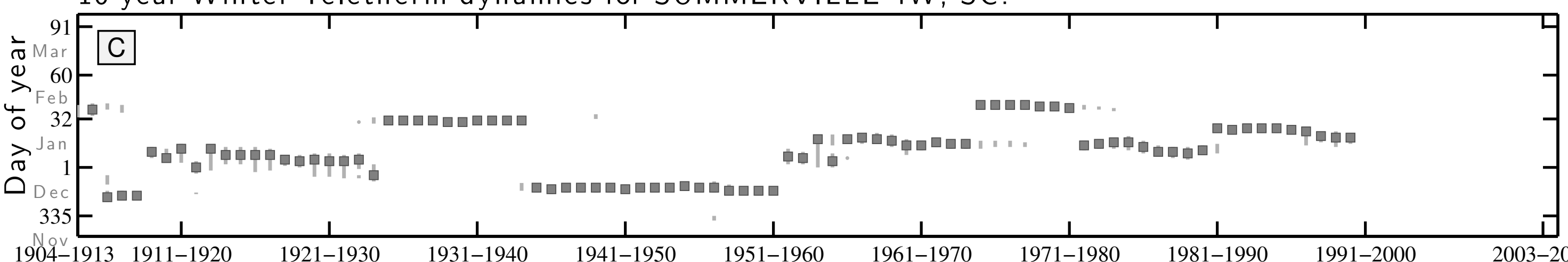

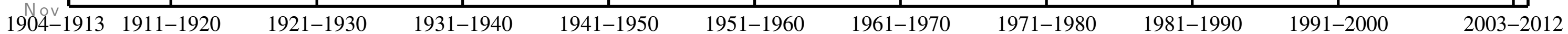

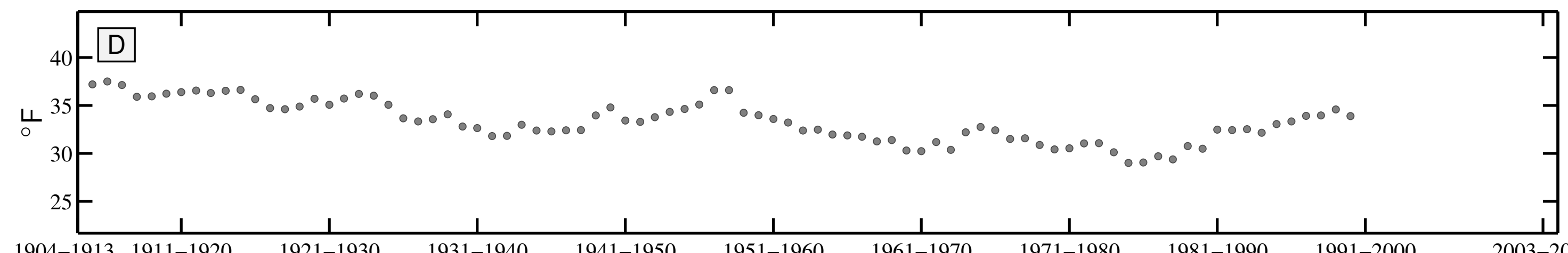


10 year Summer Teletherm dynamics for WINNSBORO, SC:

$+\mathrm{Oct}$

( $274-A$

(1) Sep

$>244-$

( $\mathrm{Aug}$

$\lambda \begin{gathered}213- \\ \mathrm{Jul}\end{gathered}$

\% 182

$\mathrm{Jun}$

$152-$

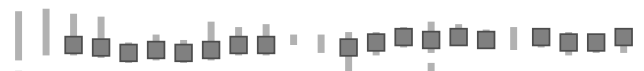
| | | | = - = | | म

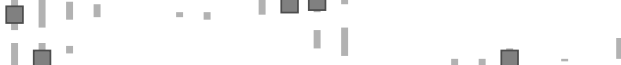

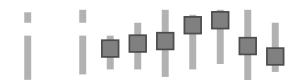

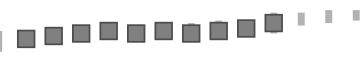

\begin{tabular}{lccc} 
& $\mathrm{L}$ & $\mathrm{L}$ & $\mathrm{L}$ \\
\hline 1930 & $1931-1940$ & $1941-1950$ & $1951-1960$
\end{tabular}

$1961-1970$

$1971-1980$

$1981-1990$

991-2000

2003-2012

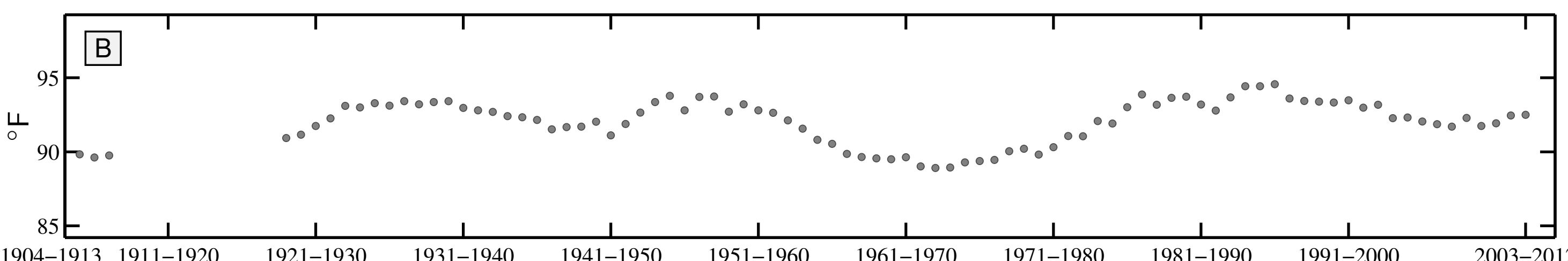

1904-1913 1911-1920

1921-1930

1961-1970

1971-1980

1981-1990

991-2000

2003-2012

10 year Winter Teletherm dynamics for WINNSBORO, SC:

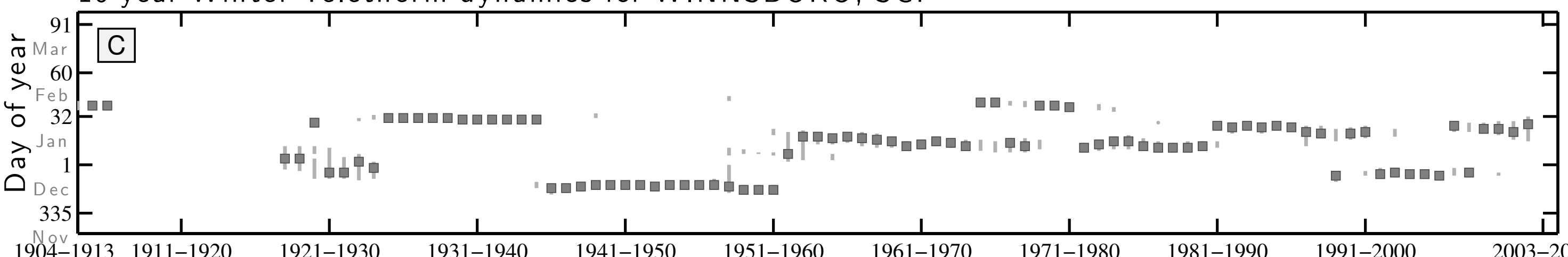

1904-1913 1911-1920

1921-1930 1931-1940

1941-1950

1951-1960

961-1970

$1971-1980$

1981-1990

$1991-2000$

2003-2012

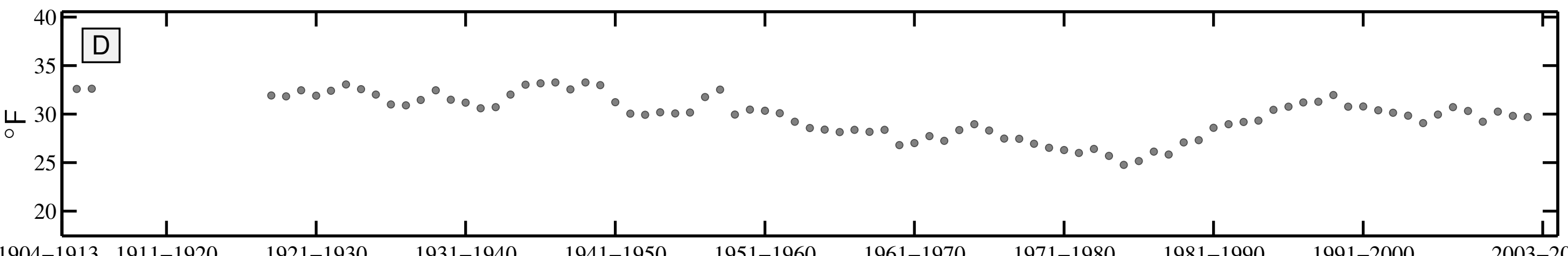


10 year Summer Teletherm dynamics for WINTHROP UNIV, SC:

$+\mathrm{Oct}$

A

(1) Sep

$>244$

○ 213

$>$ Jul

ก 182

$\mathrm{Jun}$

$152-$

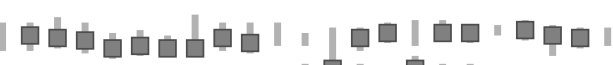

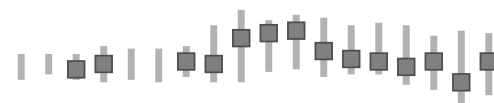

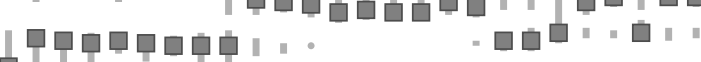

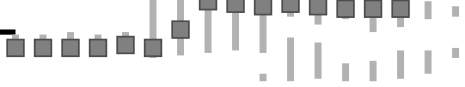

|| || - | | | || || | | |

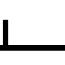

$\frac{1}{1921-1930}$

$\frac{1}{1931-1940}$

$\frac{1}{1941-1950}$

$1951-1960$

$1961-1970$

971-1980

$\frac{1}{981-1990}$

991-2000

2003-201

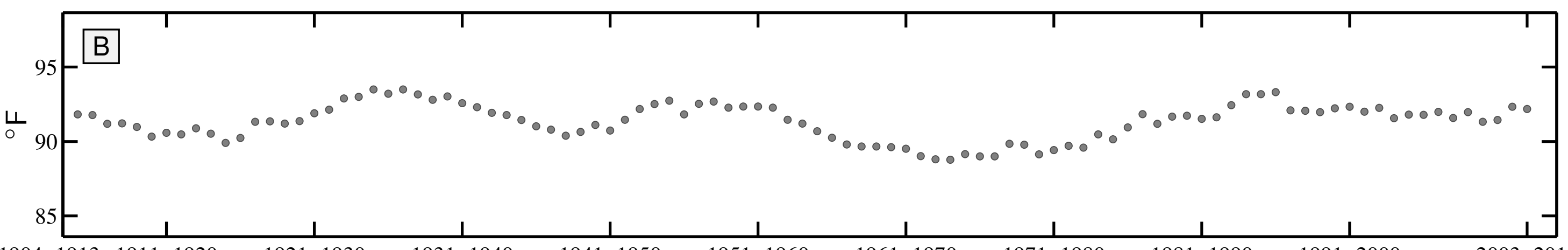

1904-1913 1911-1920

1921-1930

1931-1940

1941-1950

1951-1960

1961-1970

1971-1980

1981-1990

1991-2000

2003-2012

10 year Winter Teletherm dynamics for WINTHROP UNIV, SC:

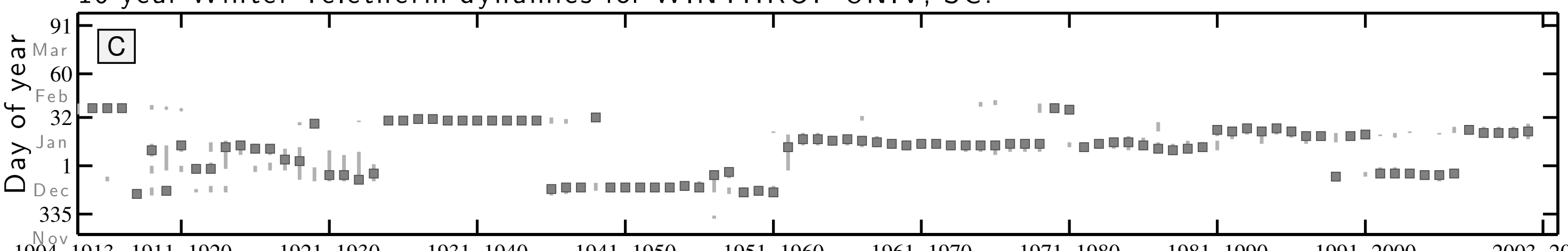

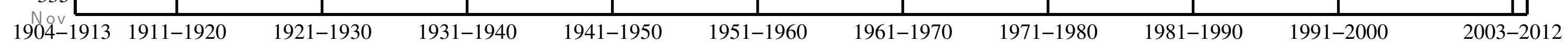

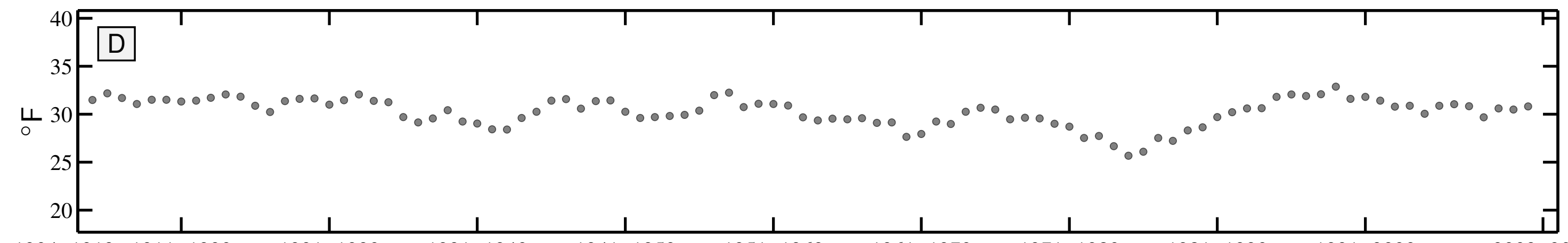


10 year Summer Teletherm dynamics for FAULKTON 1 NW, SD:
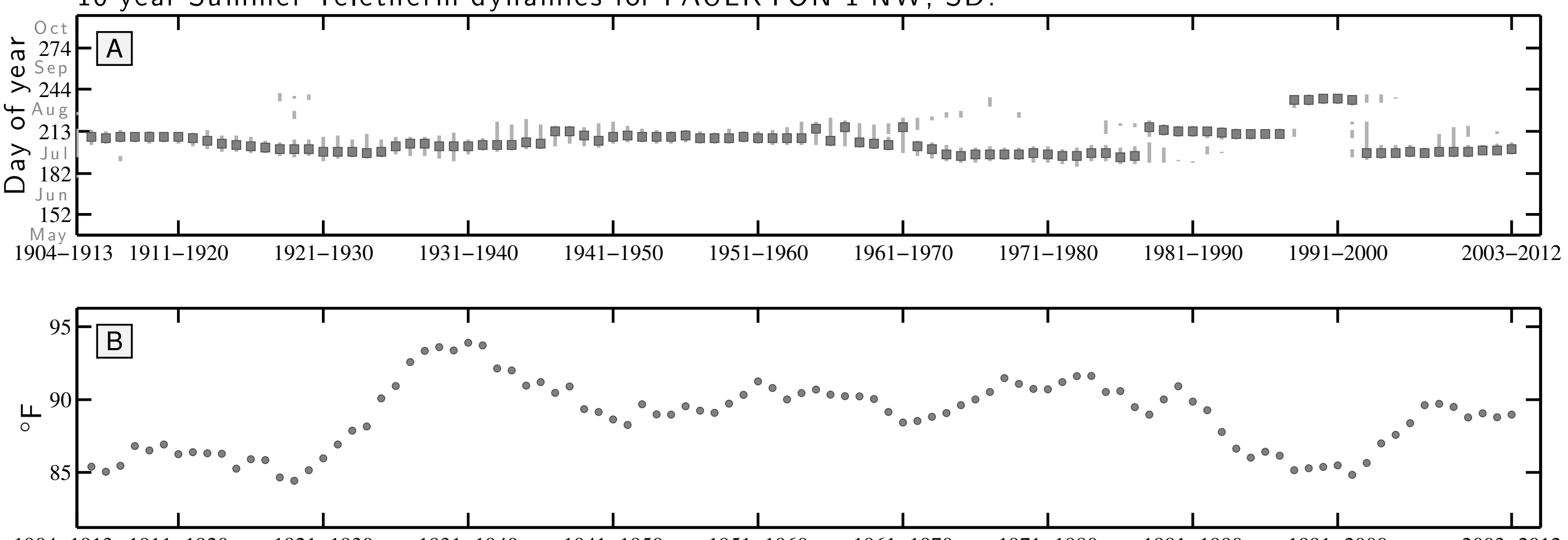

$1904-1913 \quad 1911-1920 \quad 1921-1930$

1931-1940

1941-1950

$1951-1960$

$1961-1970$

1971-1980

1981-1990

1991-2000

2003-2012

10 year Winter Teletherm dynamics for FAULKTON 1 NW, SD:

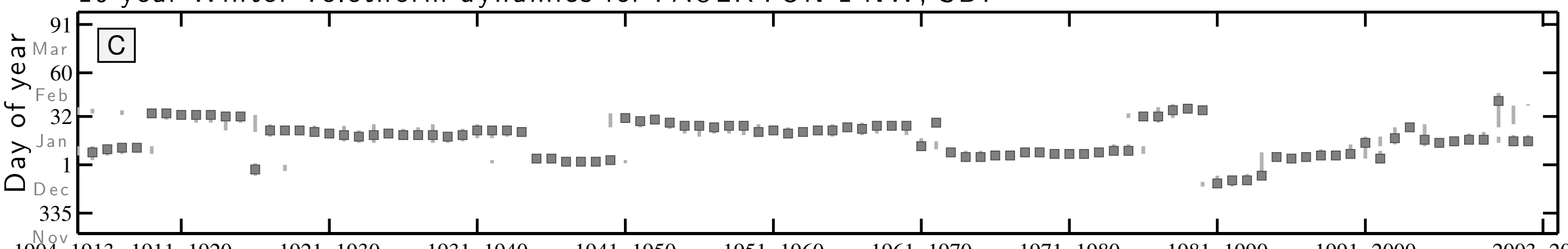

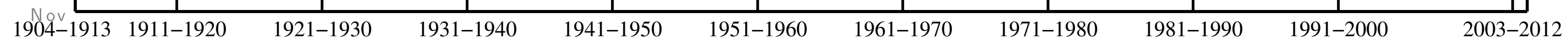

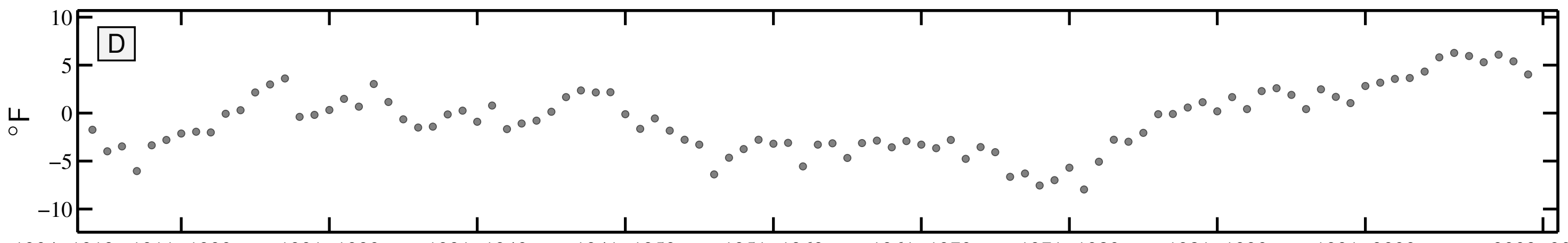

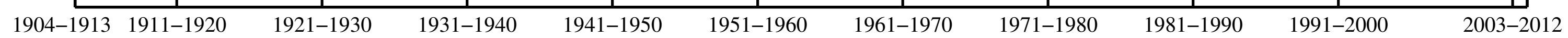


10 year Summer Teletherm dynamics for FORESTBURG 3 NE, SD:

Oct

$274-A$

(1) Sep

$>244-$

4 Aug

213 - I |

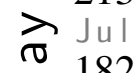

182

152 -

May — _

1904-1913 1911-1920

1921-1930

1931-1940

$\frac{1}{1941-1950}$

1951-1960

961-1970

$\frac{1}{971-1980}$

981-1990

991-2000

2003-2012

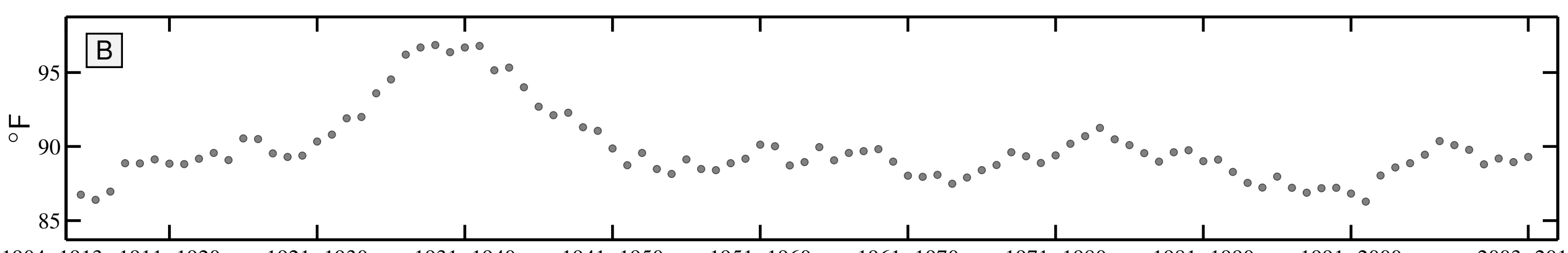

$1904-1913$ 1911-1920

$1921-1930$

$1931-1940$

$1941-1950$

$1951-1960$

$1961-1970$

$1971-1980$

$1981-1990$

$1991-2000$

$2003-2012$

10 year Winter Teletherm dynamics for FORESTBURG 3 NE, SD:

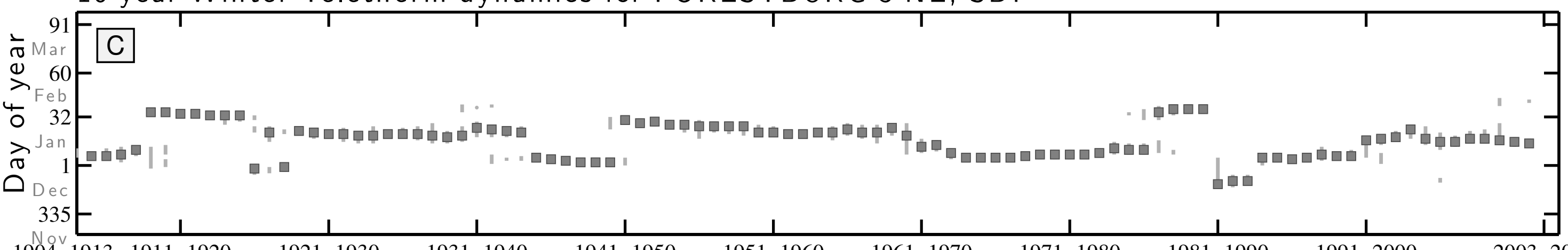

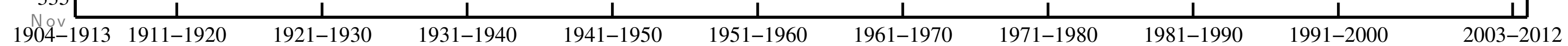

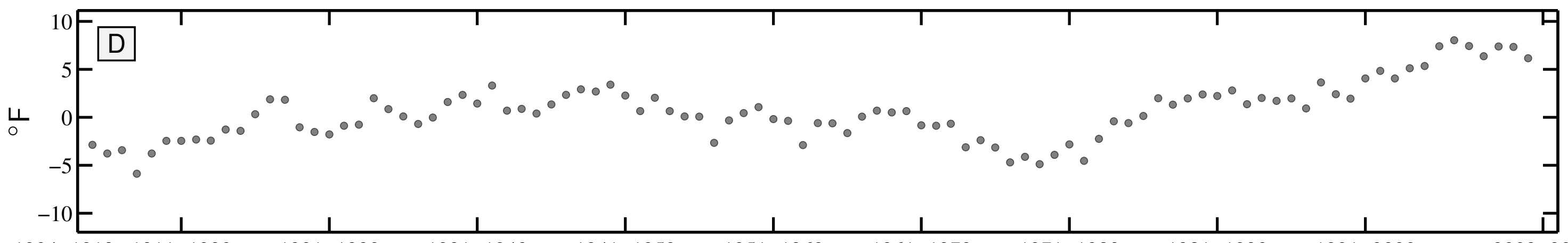

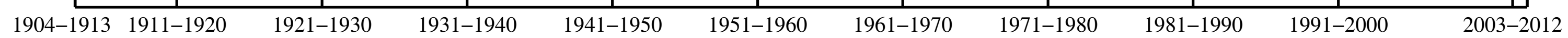


10 year Summer Teletherm dynamics for HOWARD, SD:
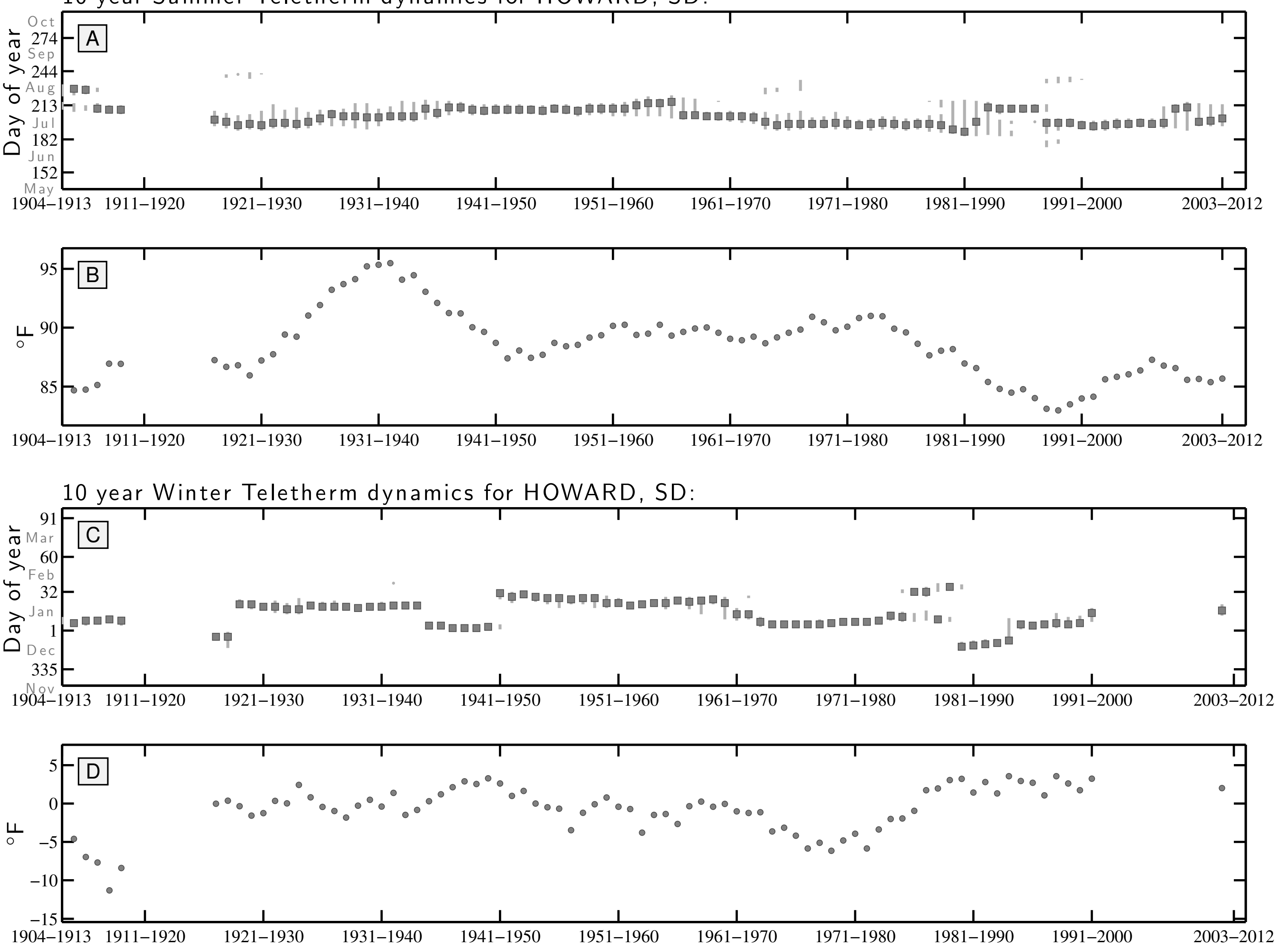
10 year Summer Teletherm dynamics for MILBANK 4 NW, SD:

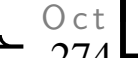

274 A

(1) Sep

$244-$

213 -

ते

$\overbrace{}^{\circ} 182$

$152-$

(19)

1904-1913 1911-1920

1921-1930

$1931-1940$

1941-1950

$\frac{1}{1951-1960}$

1961-1970

$\frac{1}{971-1980}$

$\frac{1}{981-1990}$

991-2000

Whith

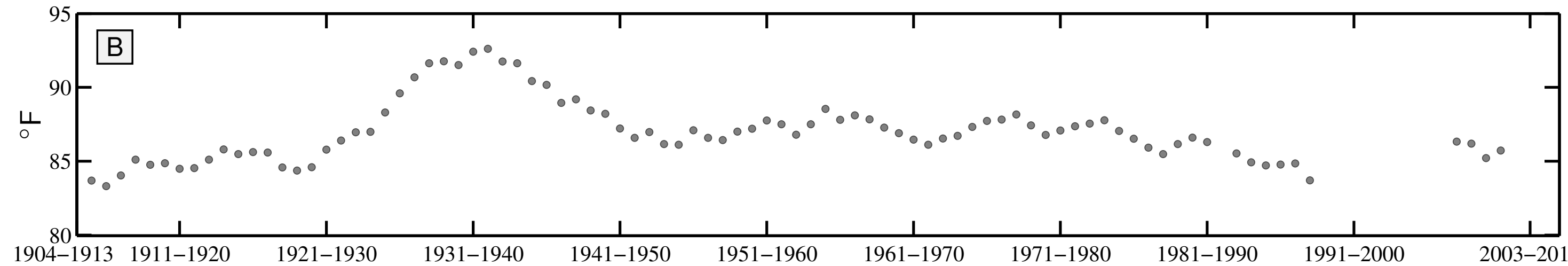

1904-1913 1911-1920

$1931-1940$

$1941-1950$

1951-1960

$1961-1970$

$1971-1980$

$1981-1990$

$1991-2000$

2003-2012

10 year Winter Teletherm dynamics for MILBANK 4 NW, SD:

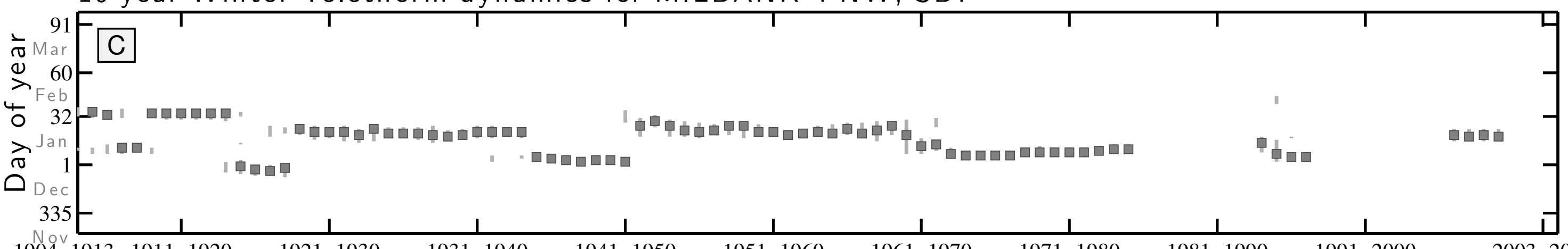

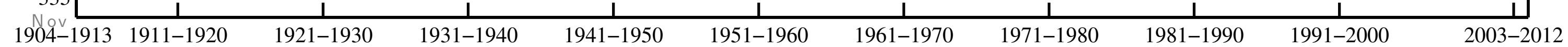

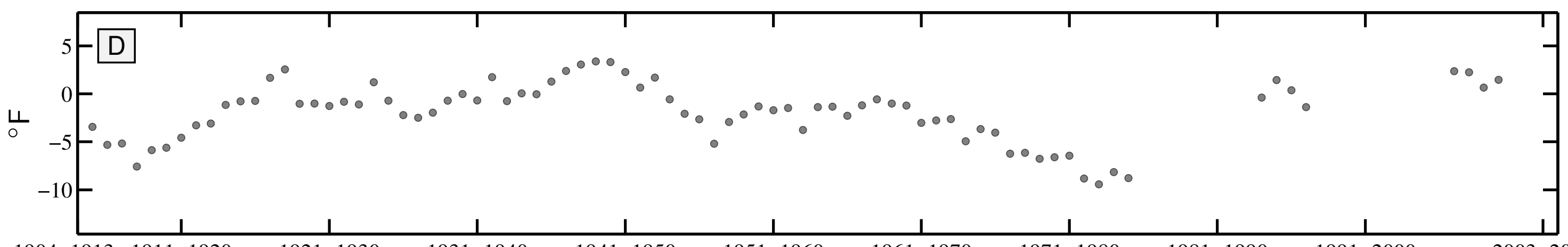


10 year Summer Teletherm dynamics for OAHE DAM, SD:
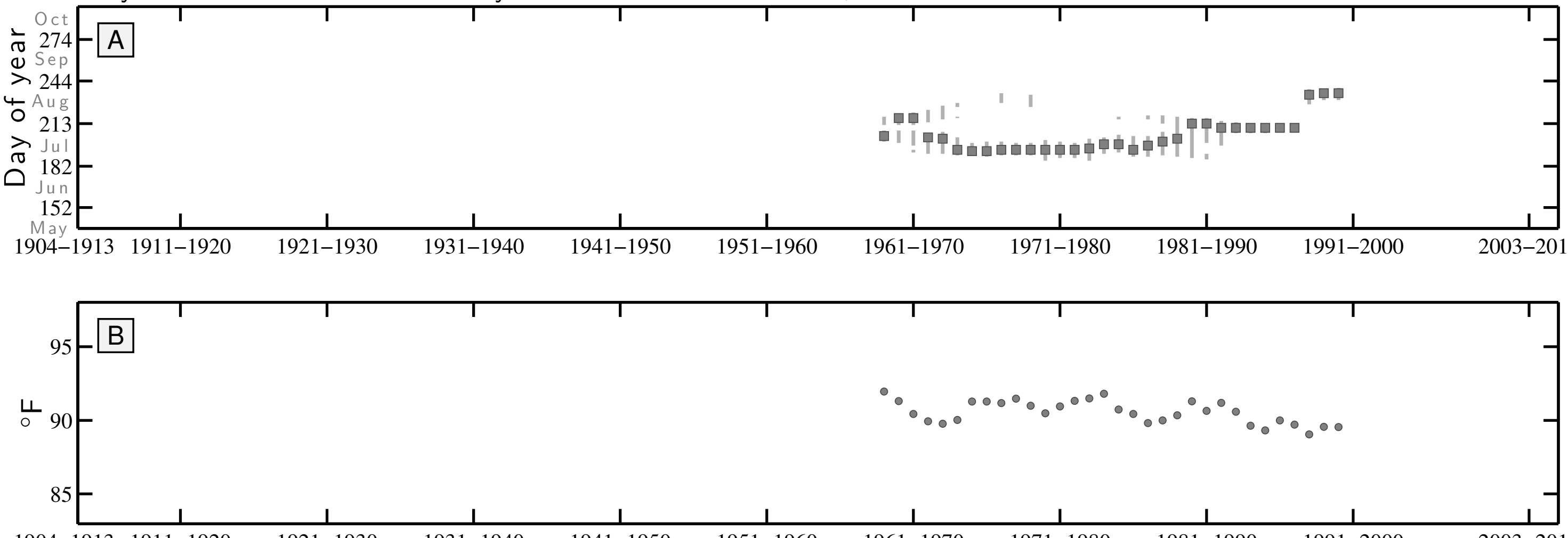

1904-1913 1911-1920

1921-1930

1931-1940

1941-1950

1951-1960

1961-1970

1971-1980

1981-1990

1991-2000

2003-2012

10 year Winter Teletherm dynamics for OAHE DAM, SD:

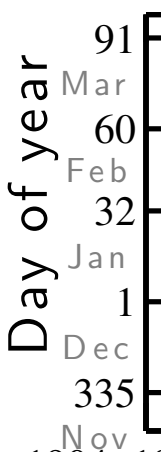

\section{I I I I I I I I} C
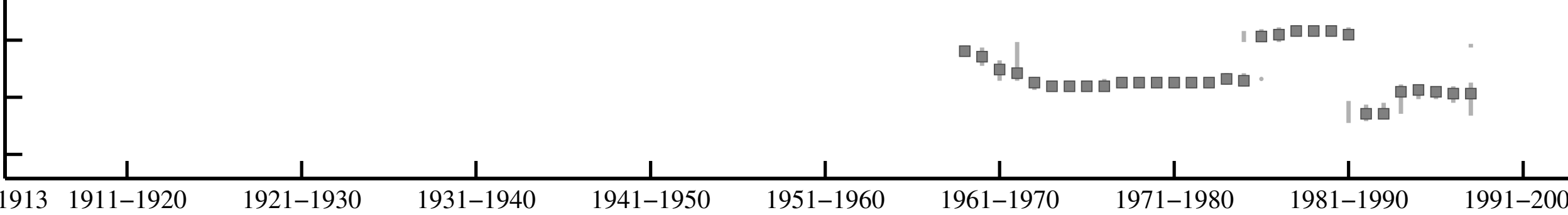

1

190
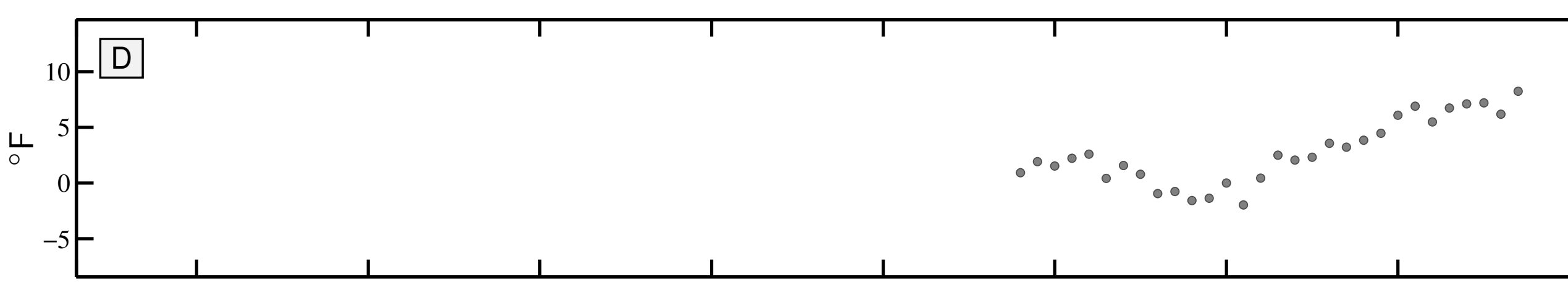
10 year Summer Teletherm dynamics for TULLAHOMA, TN:

$\frac{O}{\sigma} 274$

(s Sep

$>244-$

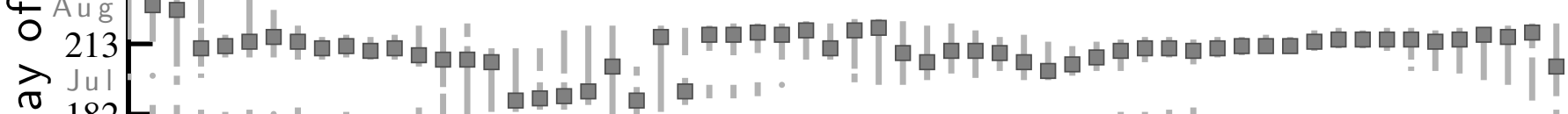

$\overbrace{}^{\infty} 182$

152

May

1904-1913 1911-1920

| 1 |

ㅁ | | |

$\frac{1}{1921-1930}$

$\frac{1}{1931-1940}$

$\frac{1}{1941-1950}$

$\frac{1}{1951-1960}$

$1961-1970$

$\frac{1}{971-1980}$

$\frac{1}{1981-1990}$

991-2000

2003-201

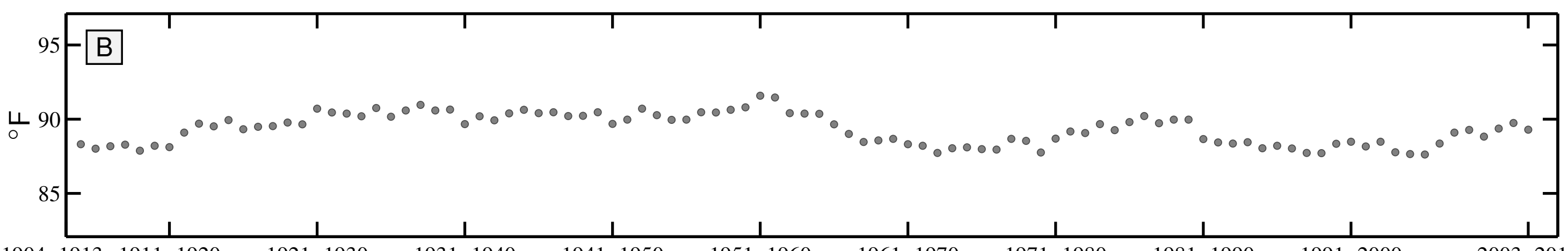

1904-1913 1911-1920

$1921-1930$

1931-1940

1941-1950

1951-1960

$1961-1970$

1971-1980

$1981-1990$

$1991-2000$

2003-2012

10 year Winter Teletherm dynamics for TULLAHOMA, TN:

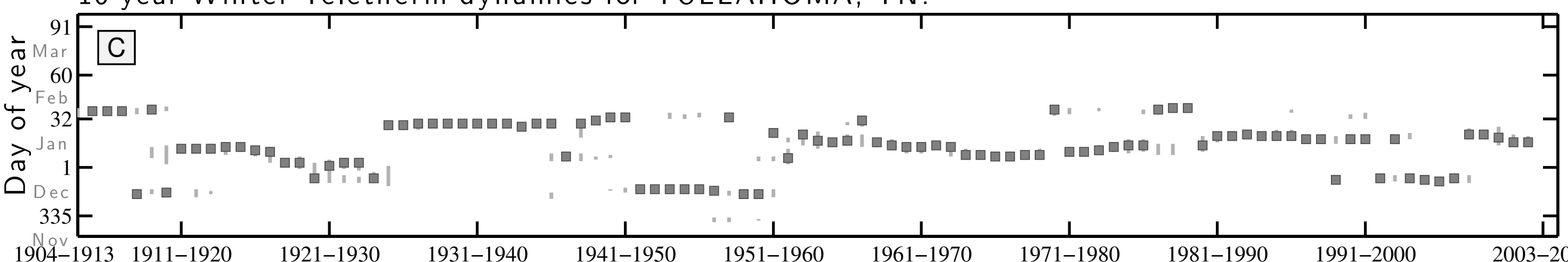

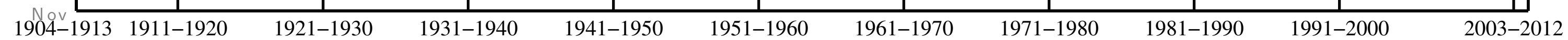

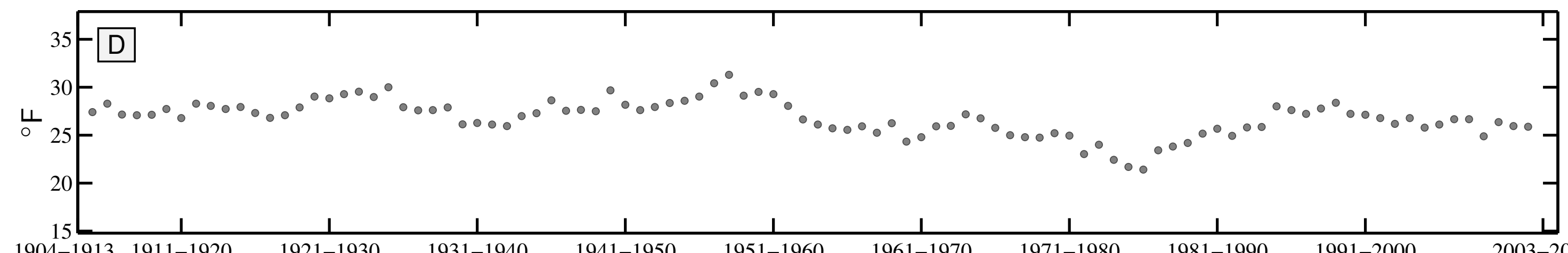


10 year Summer Teletherm dynamics for UNION CITY, TN:
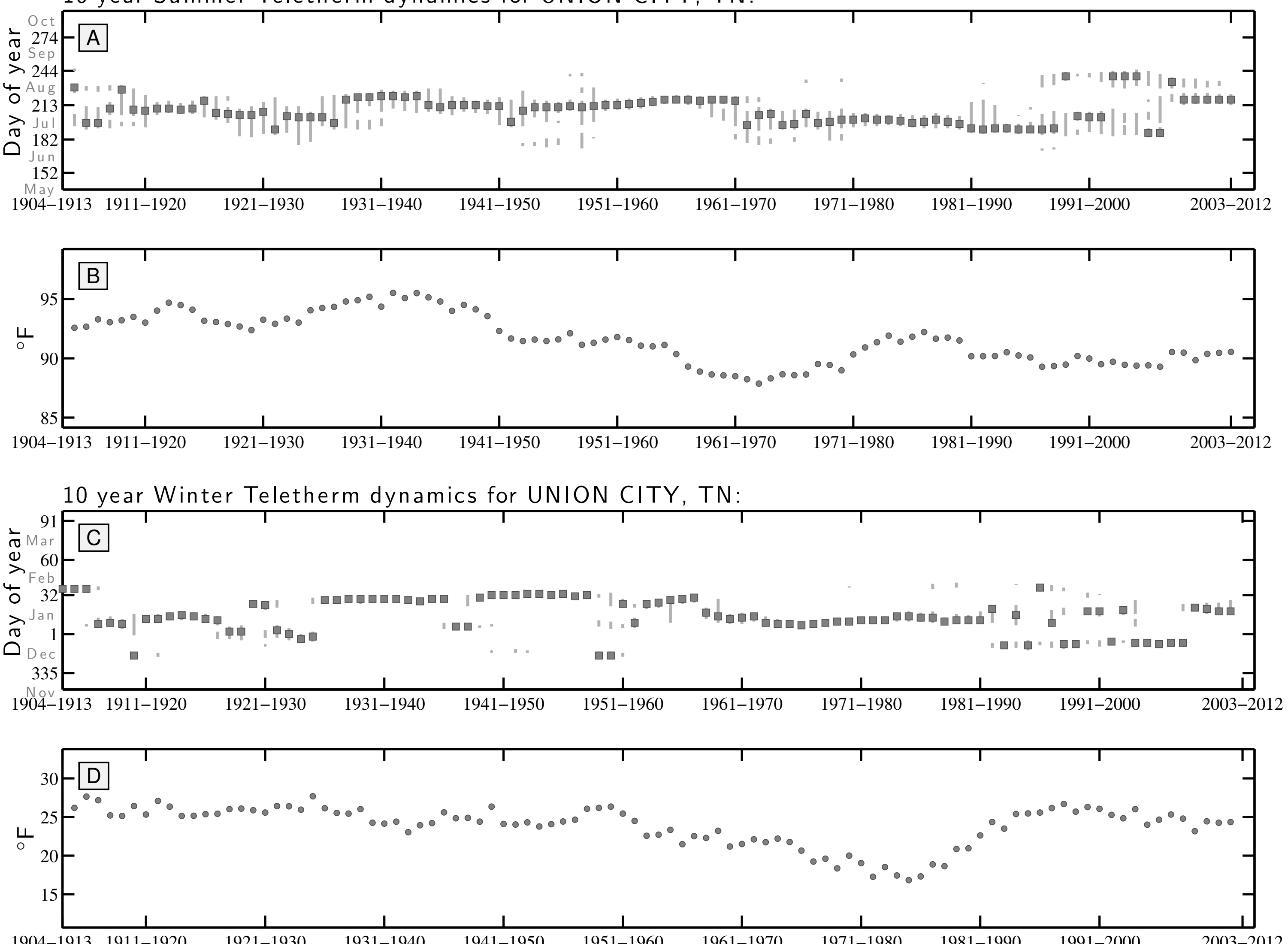

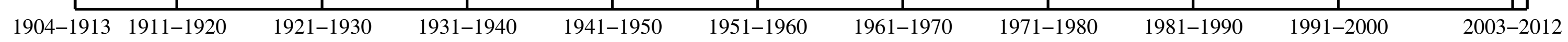


10 year Summer Teletherm dynamics for BOYS RANCH, TX:
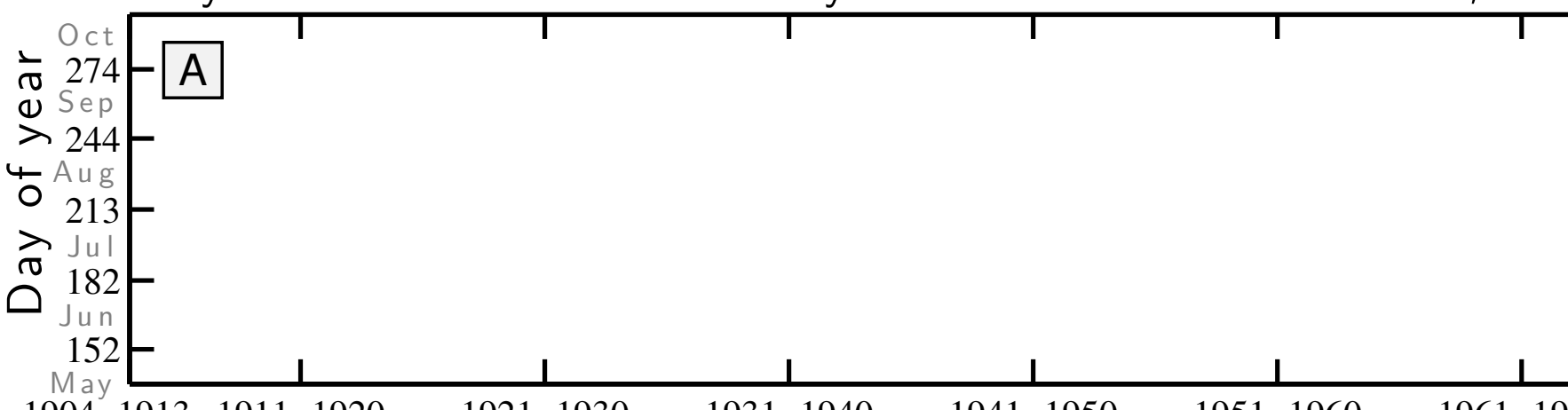

1961-1970

1971-1980

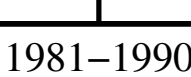

1991-2000

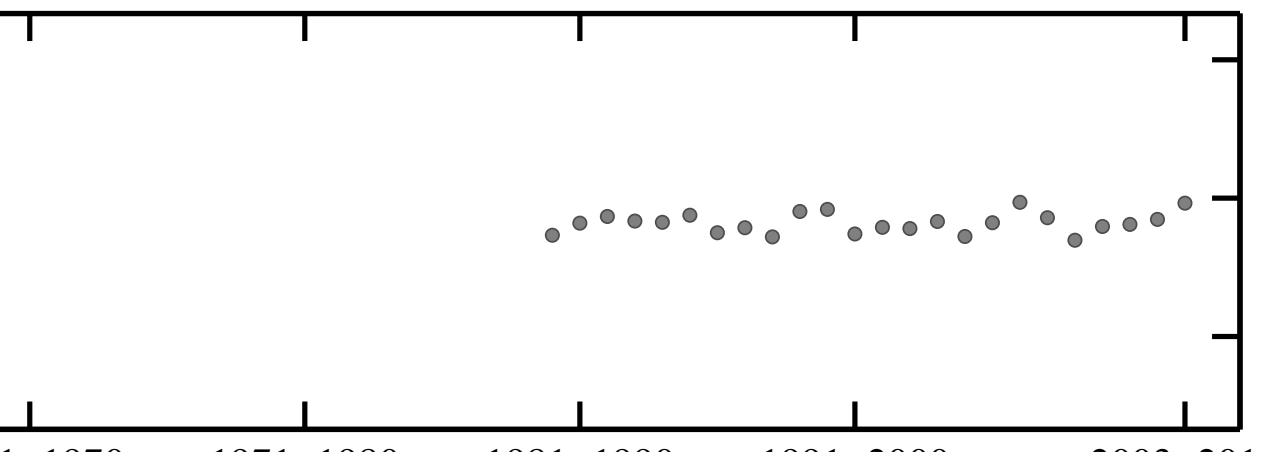

$90-$

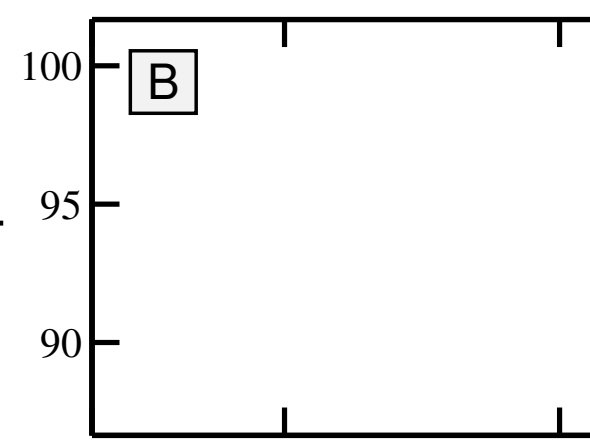

1904-1913 1911-1920

1921-1930

1931-1940

1941-1950

1951-1960

1961-1970

1971-1980

1981-1990

1991-2000

2003-2012

10 year Winter Teletherm dynamics for BOYS RANCH, TX:

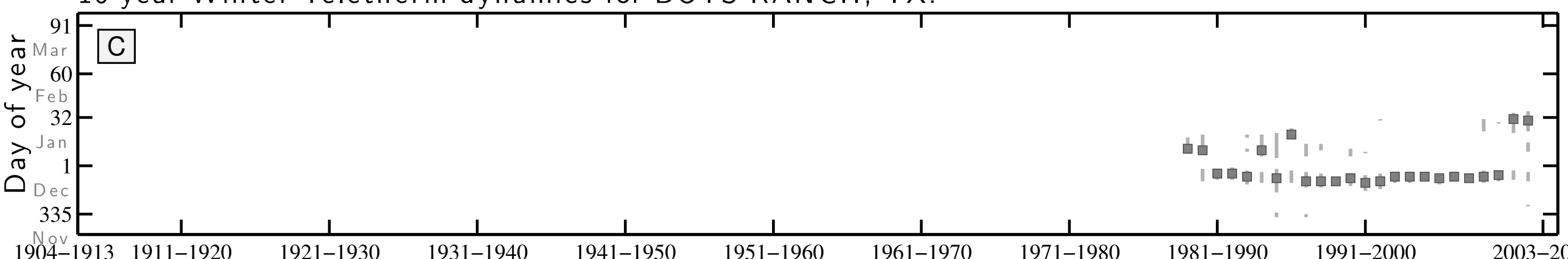

1904-1913 1911-192

1921-1930

1931-1940

1941-1950

1951-1960

1961-1970

1971-1980

1981-1990

1991-2000

2003-2012

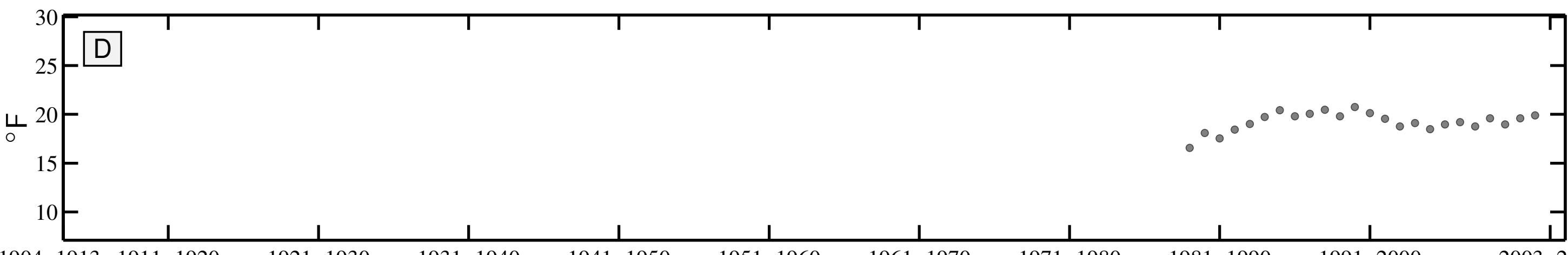


10 year Summer Teletherm dynamics for GAINESVILLE 5 ENE, TX:
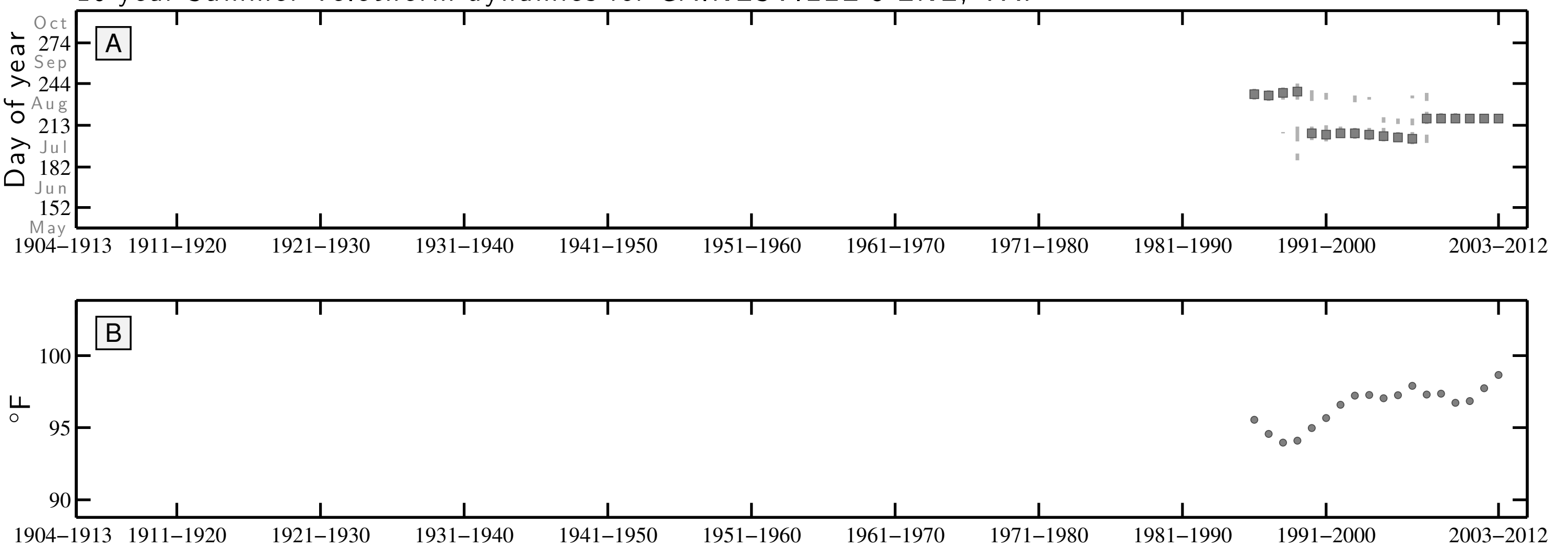

10 year Winter Teletherm dynamics for GAINESVILLE 5 ENE, TX:
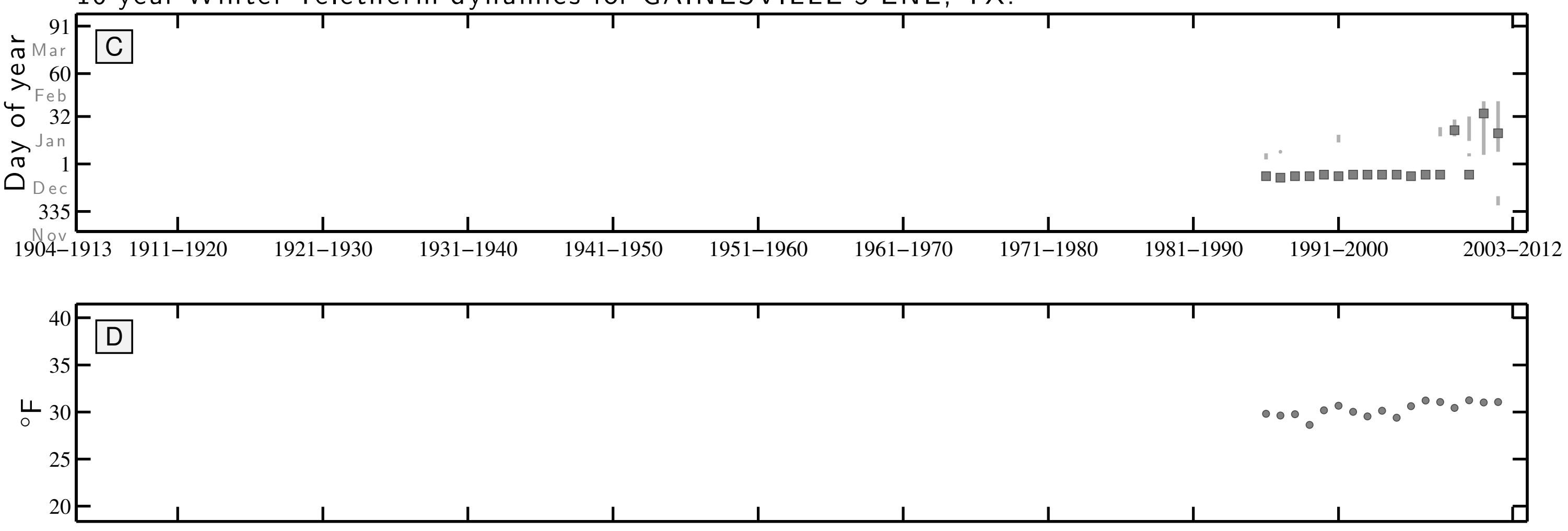

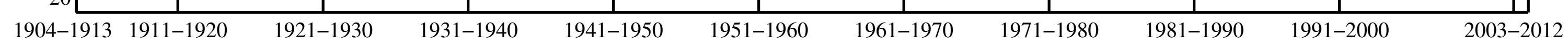



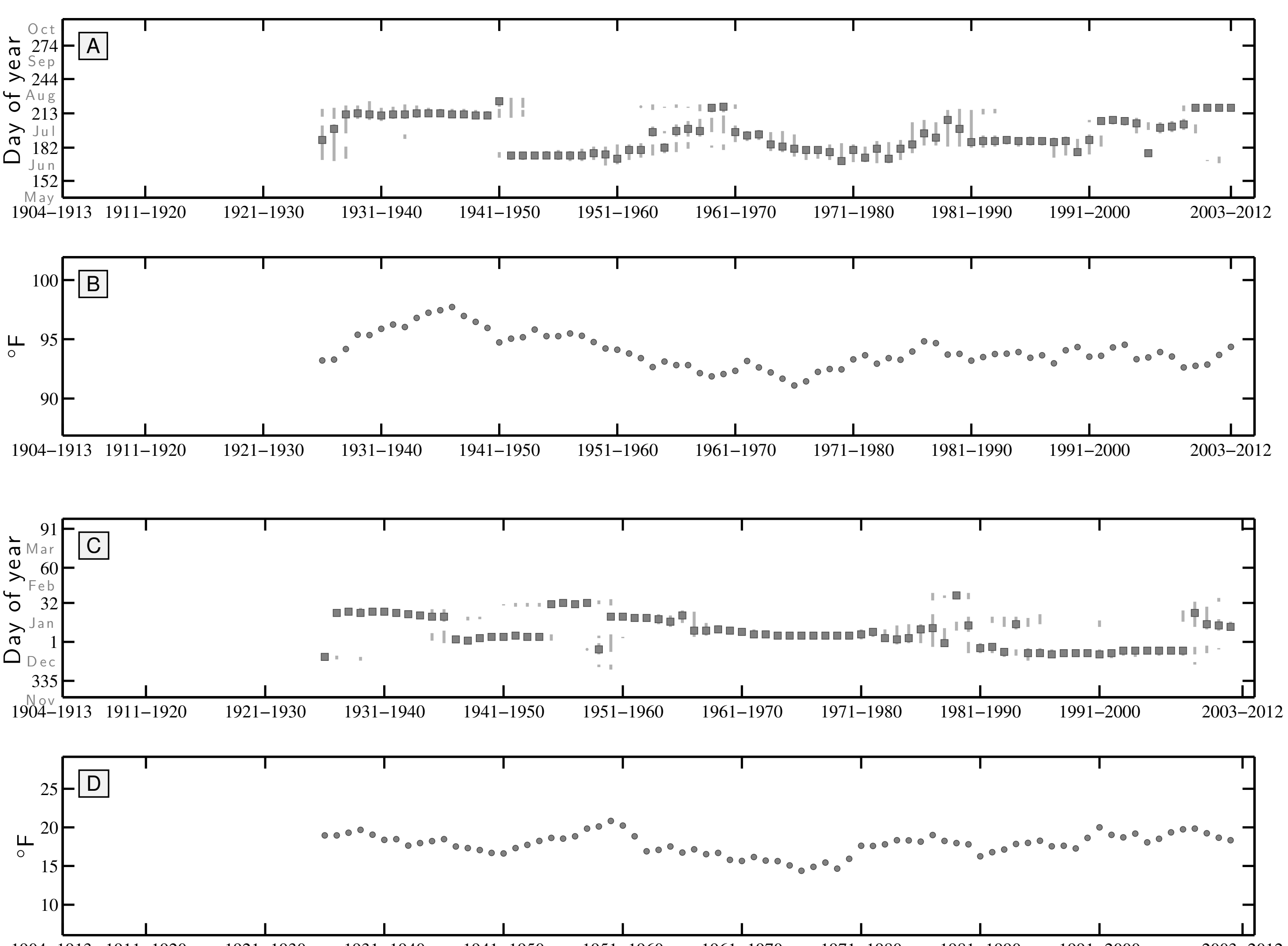

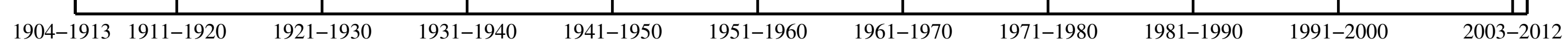


10 year Summer Teletherm dynamics for PARIS, TX:

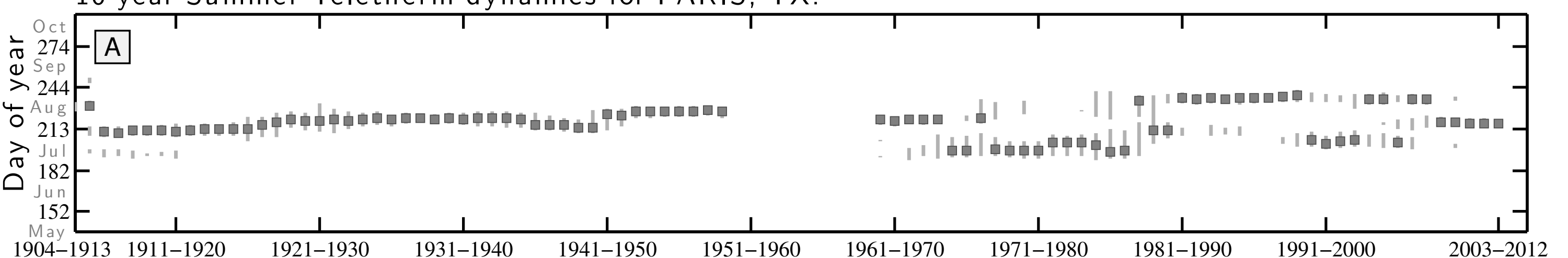

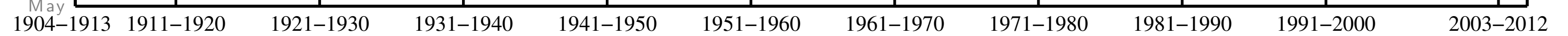
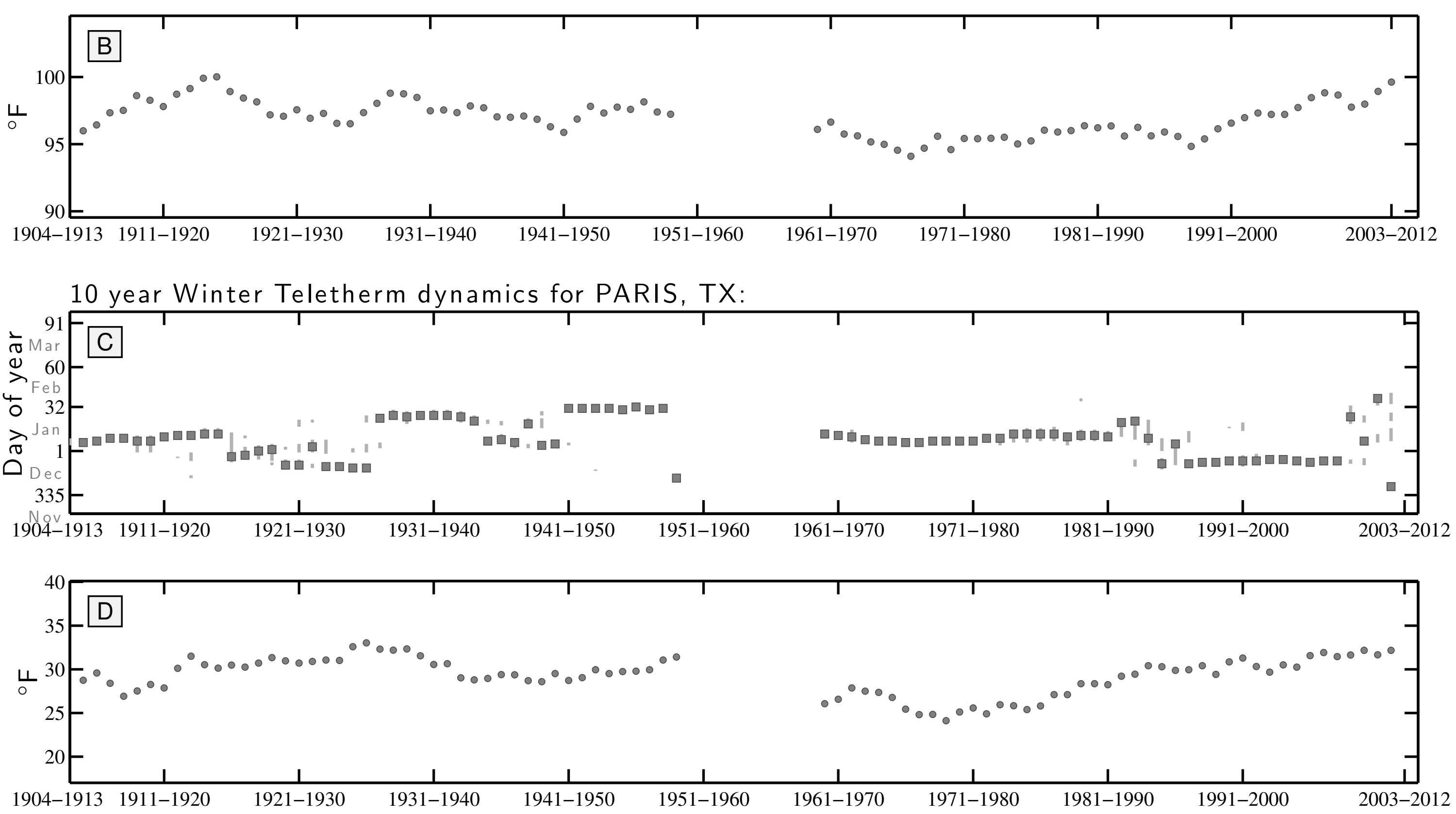
10 year Summer Teletherm dynamics for TEMPLE, TX:
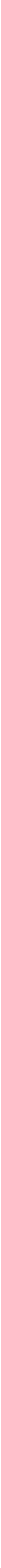

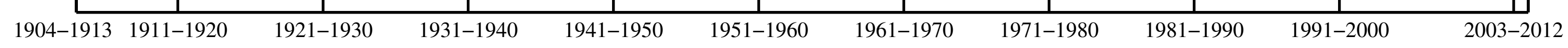


10 year Summer Teletherm dynamics for OGDEN PIONEER P H, UT:

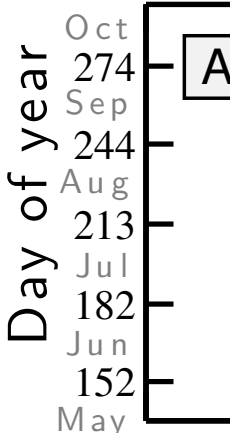
A

1904-1913 1911-1920

$\frac{1}{1921-1930}$

$\frac{1}{1931-1940}$

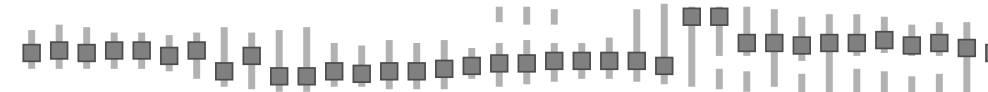

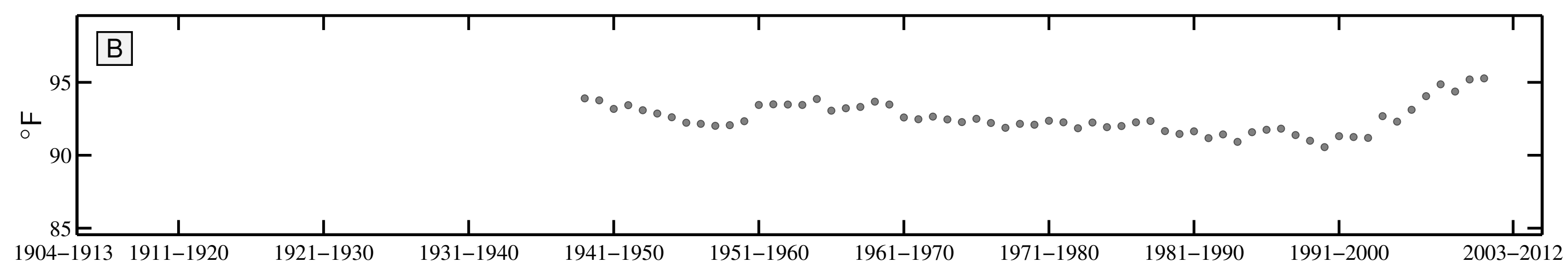

1904-1913 1911-1920

$1921-1930$

$1931-1940$

$1941-1950$

$1961-1970$

1971-1980

2003-2012

10 year Winter Teletherm dynamics for OGDEN PIONEER P H, UT:
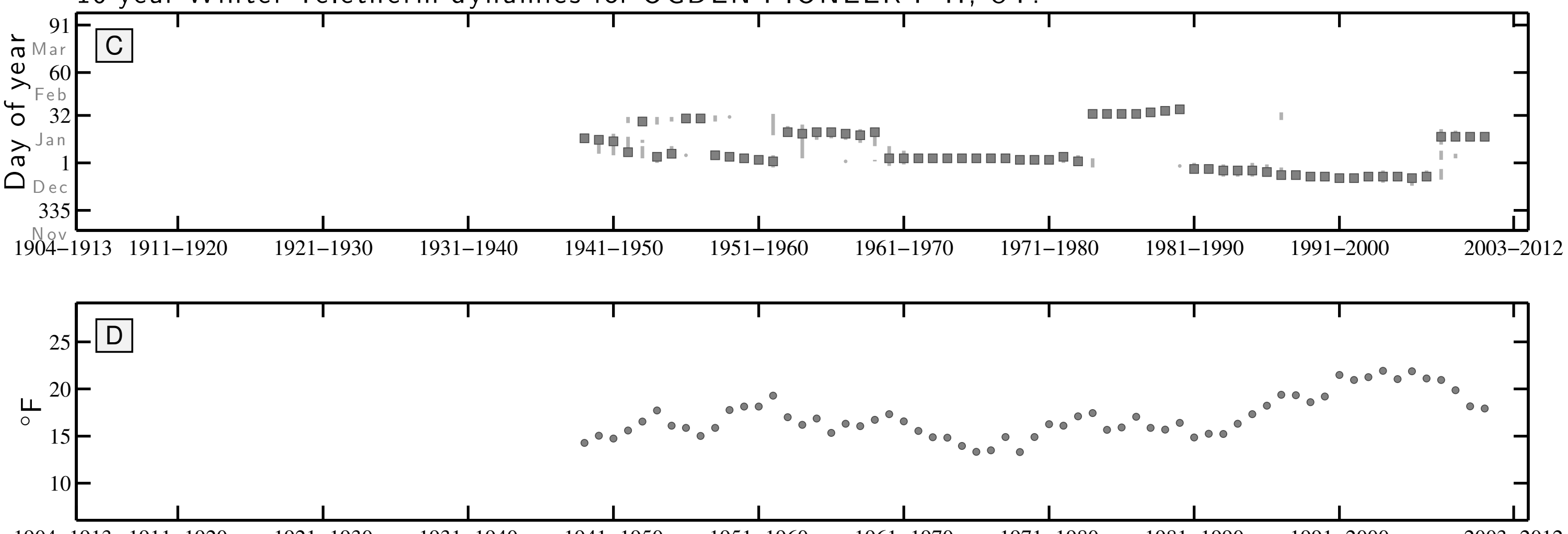
10 year Summer Teletherm dynamics for TOOELE, UT:

$\div 274-\mathrm{A}$

¿ Sep

$>244-$

○ $\begin{array}{r}\text { Aug } \\ 213\end{array}$

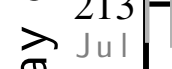

ס $182-$

$152-$

May ए

1904-1913 1911-1920

$\frac{1}{1921-1930}$

$\frac{1}{1931-1940}$

$\frac{1}{1941-1950}$

1951-1960

1961-1970

1971-1980

1981-1990

1991-2000

2003-2012

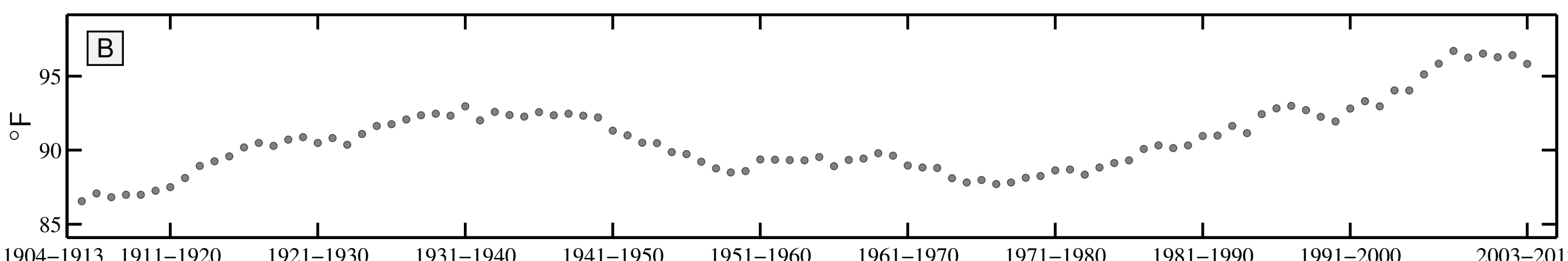

1904-1913 1911-1920

$1921-1930$

1931-1940

$1941-1950$

$1951-1960$

$1961-1970$

1971-1980

1981-1990

$1991-2000$

2003-2012

10 year Winter Teletherm dynamics for TOOELE, UT:

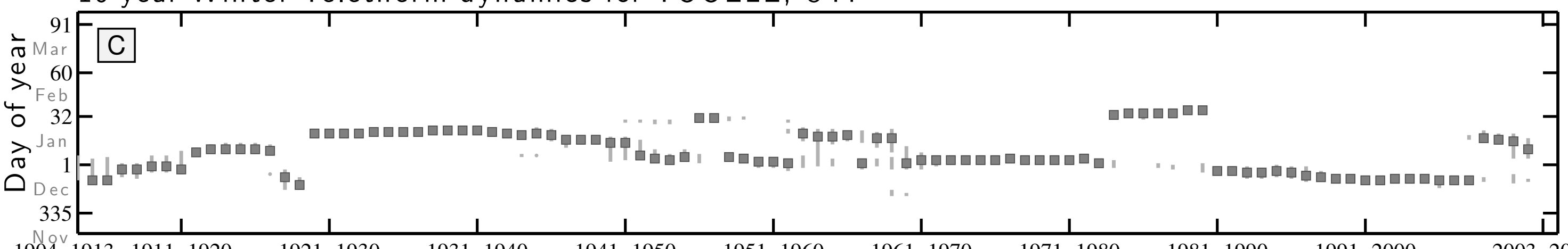

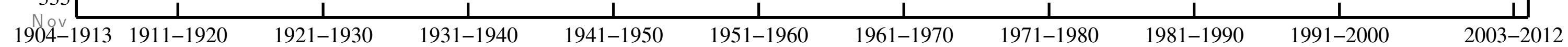

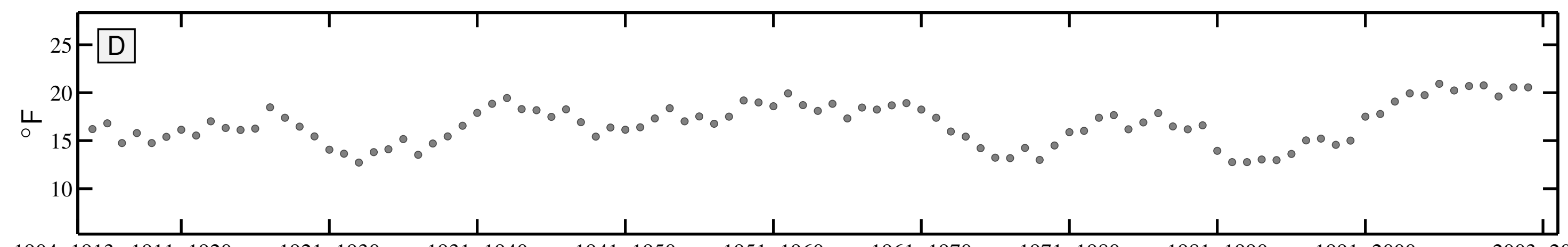


10 year Summer Teletherm dynamics for ENOSBURG FALLS, VT:

$=$ Oct

274 A

(1) Sep

- Aug

O 213

ते Jul

182

$152-$

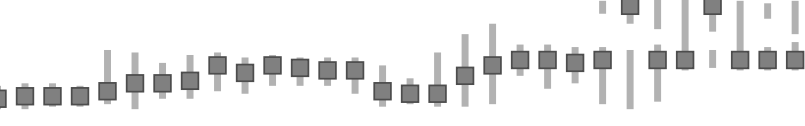

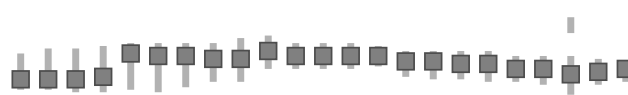

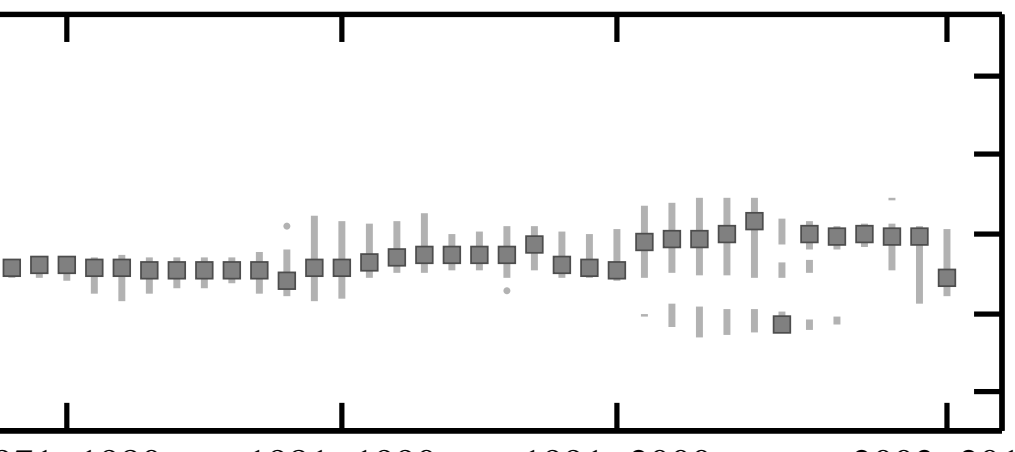

1904-1913 1911-1920 1921-1930 1931-1940

$1941-1950$

$1951-1960$

$1961-1970$

1971-1980

1981-1990

1991-2000

2003-2012

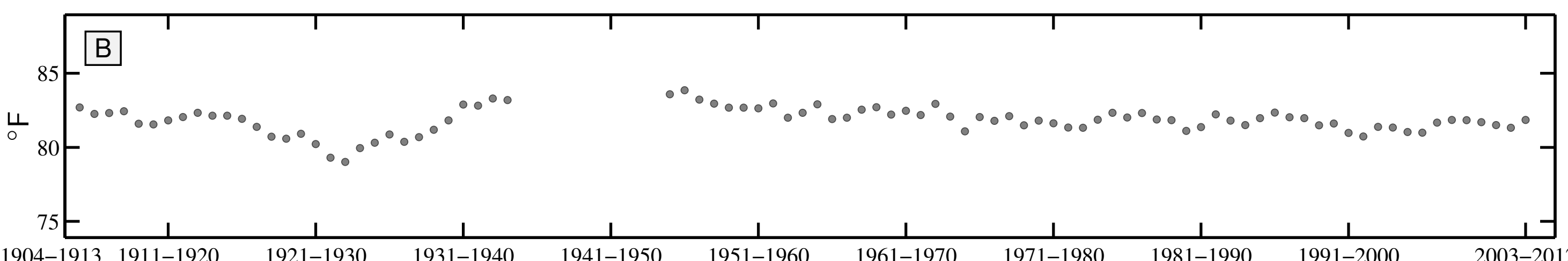

1904-1913 1911-1920

1921-1930

1931-1940

1941-1950

1951-1960

1961-1970

1971-1980

1981-1990

2003-2012

10 year Winter Teletherm dynamics for ENOSBURG FALLS, VT:

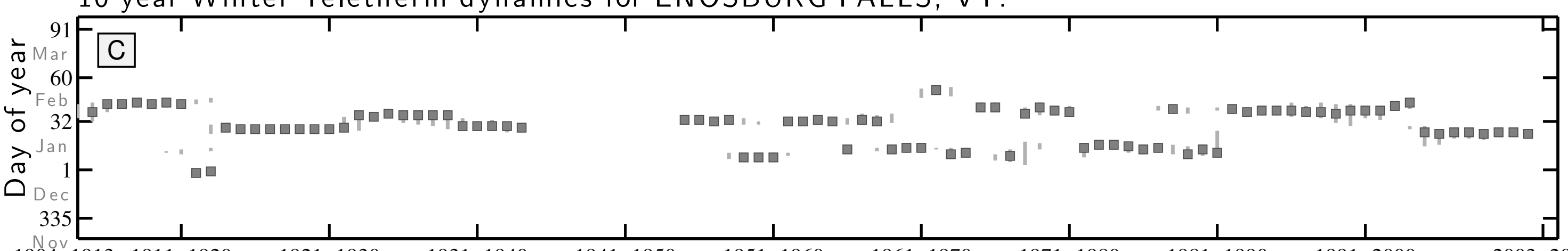

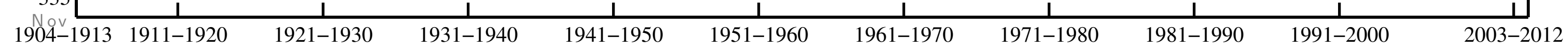

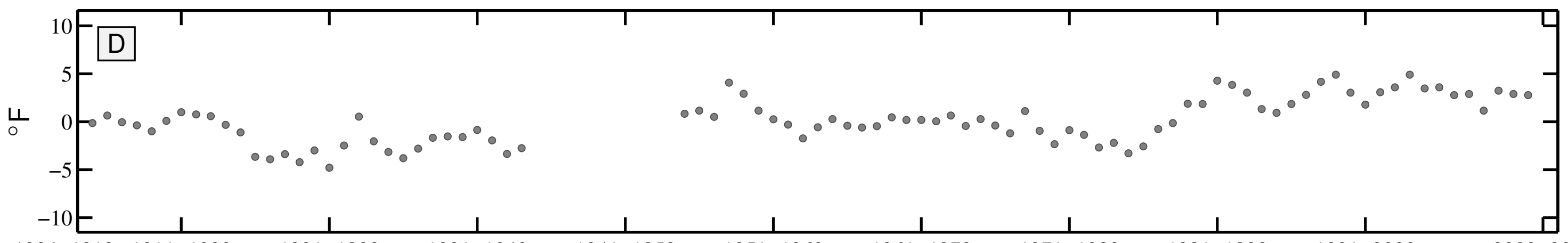

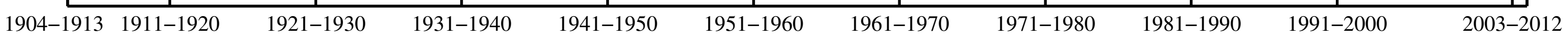


10 year Summer Teletherm dynamics for SOUTH HERO, VT:
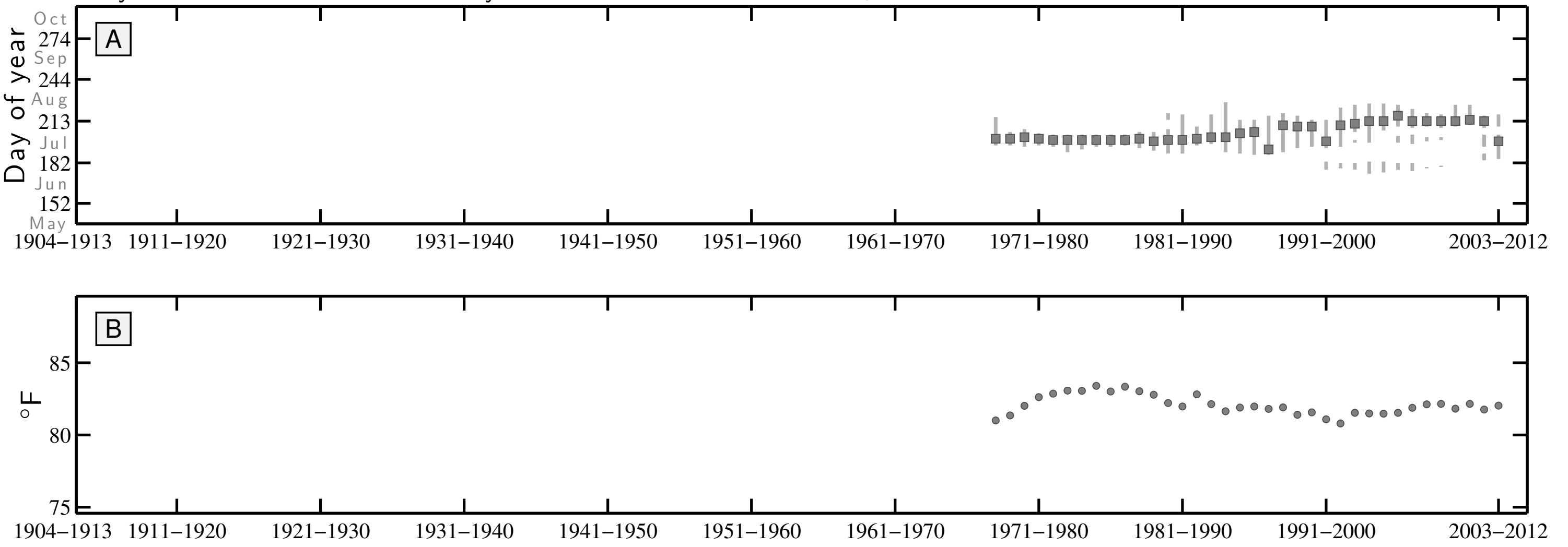

10 year Winter Teletherm dynamics for SOUTH HERO, VT:

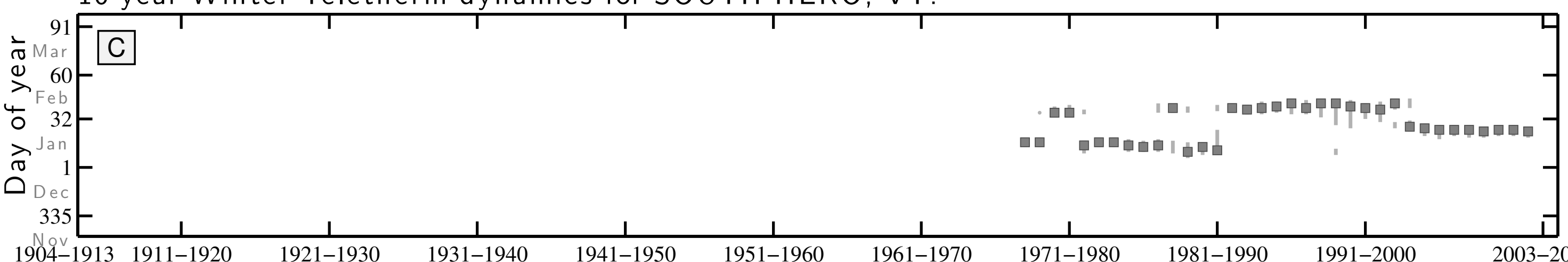

1904-1913 1911-192

$1921-1930$

$1931-1940$

$1941-1950$

$1951-1960$

$1961-1970$

$1971-1980$

$1981-1990$

$1991-2000$

2003-2012

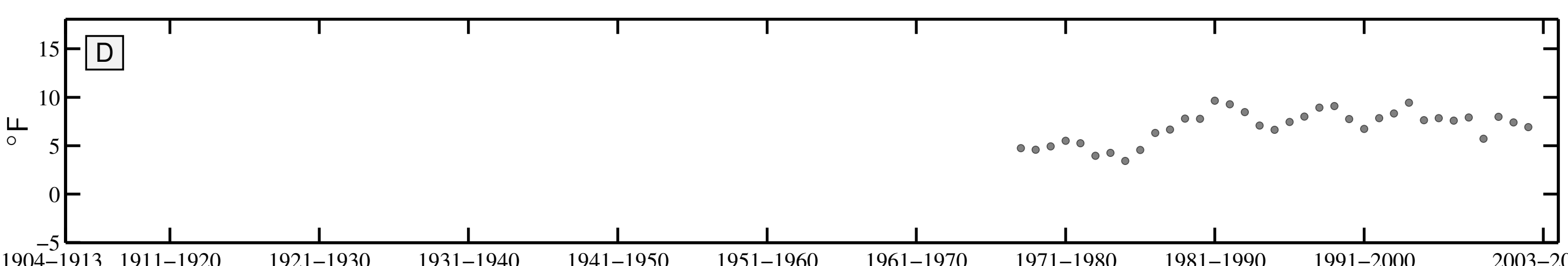


10 year Summer Teletherm dynamics for BREMO BLUFF, VA:
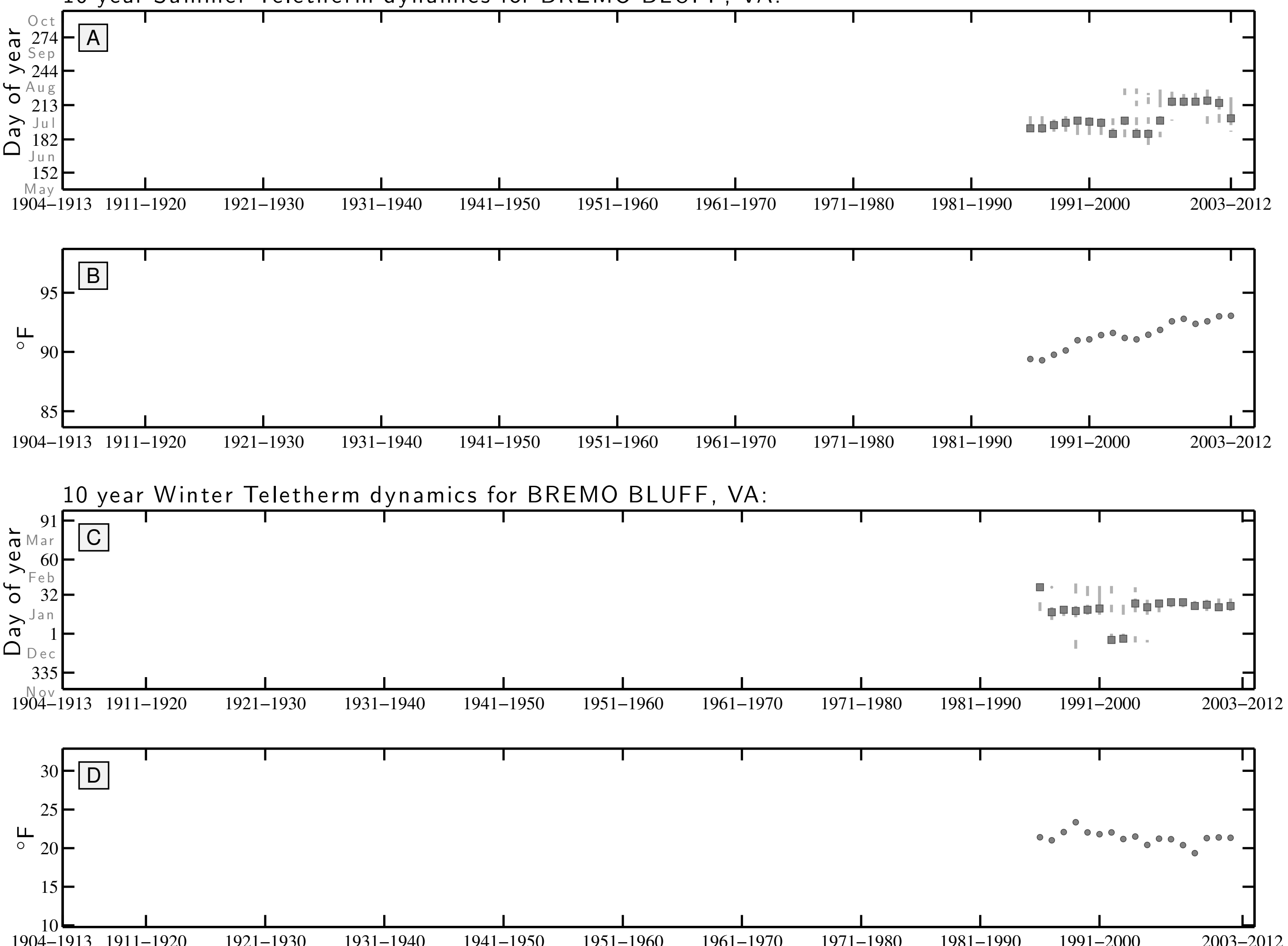
10 year Summer Teletherm dynamics for STAUNTON WATER TRMTMT PLT, VA:

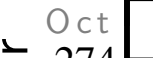

A

(1) Sep

244

○ $213-$

$\gtrsim \mathrm{Ju}$

○ 182

$152-$

May L

1904-1913 1911-1920

1921-1930

1931-1940

$\frac{1}{1941-1950}$

$\frac{1}{1951-1960}$

961-1970

$\frac{1}{1971-1980}$

1981-1990

1991-2000

2003-2012

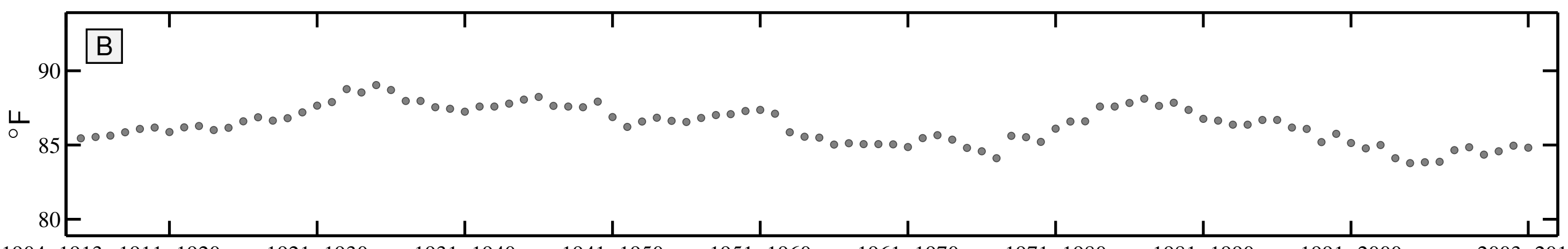

1904-1913 1911-1920

1921-1930

1931-1940

1941-1950

1951-1960

1961-1970

1971-1980

1981-1990

1991-2000

2003-2012

10 year Winter Teletherm dynamics for STAUNTON WATER TRMTMT PLT, VA:

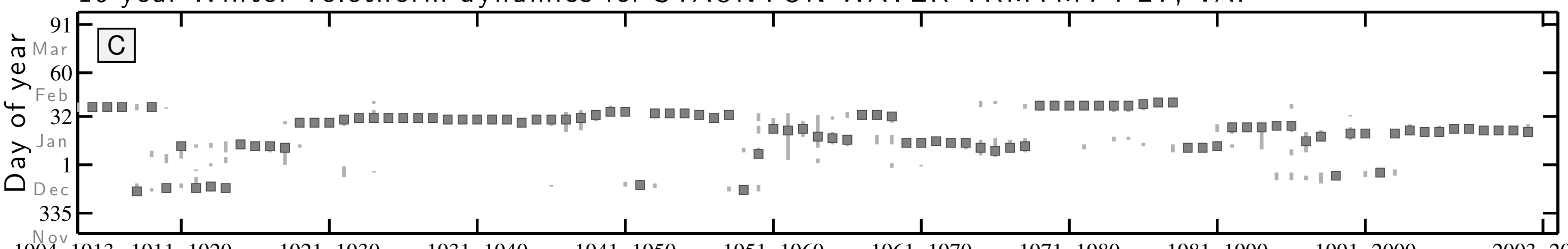

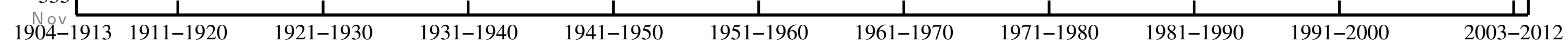

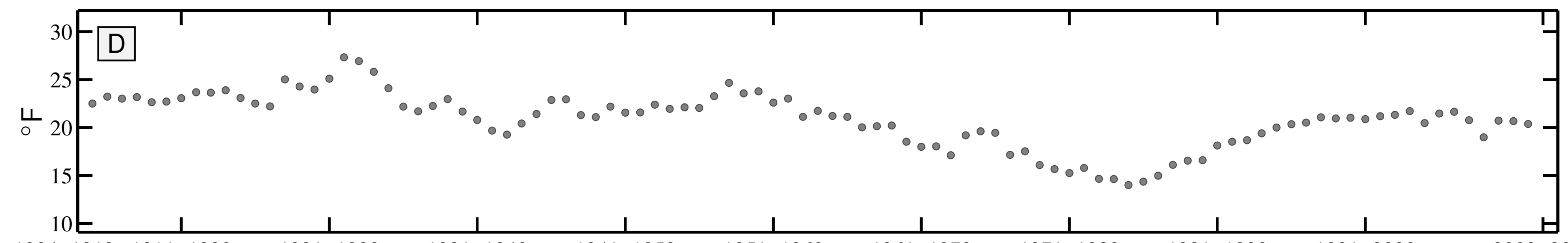


10 year Summer Teletherm dynamics for ABERDEEN, WA:
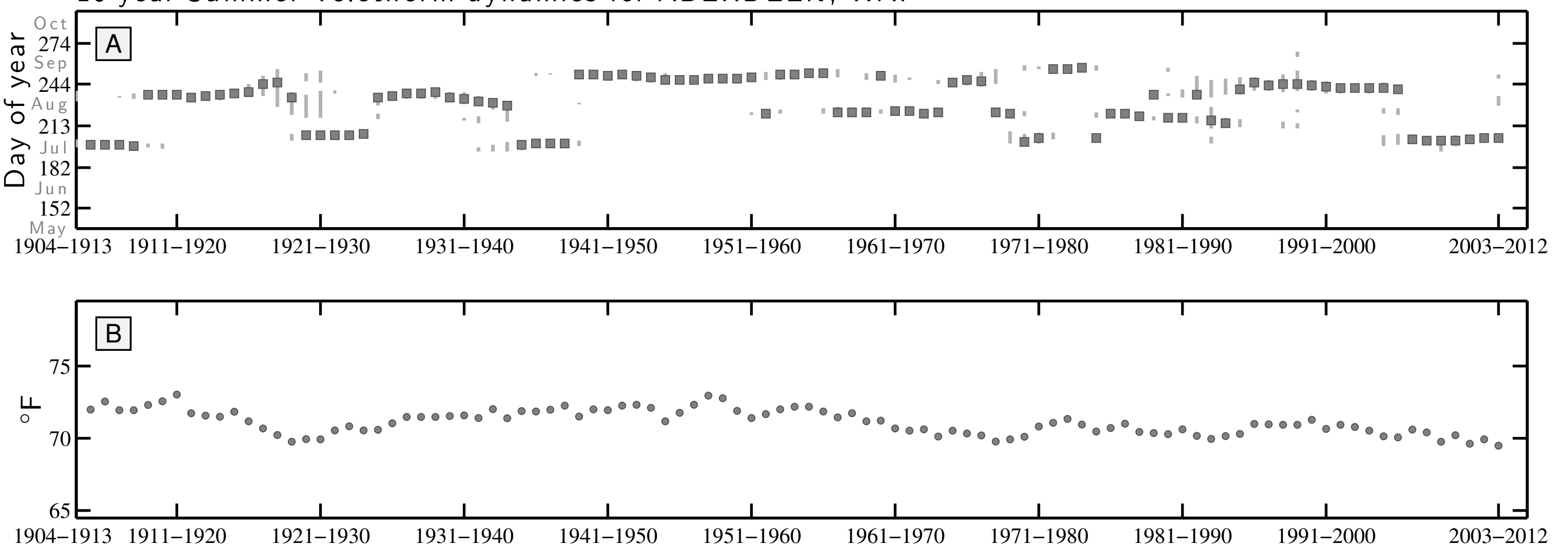

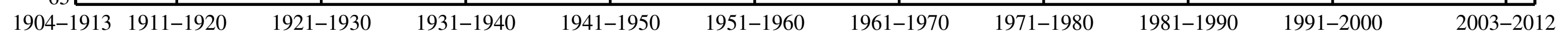

10 year Winter Teletherm dynamics for ABERDEEN, WA:

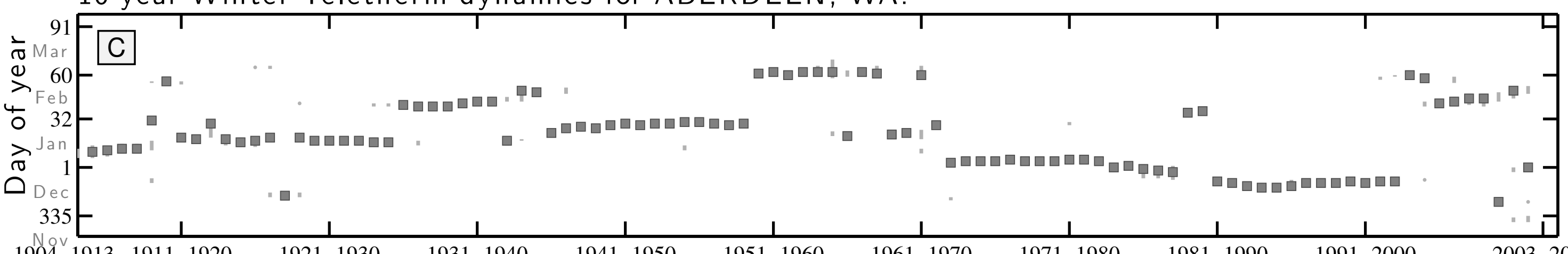

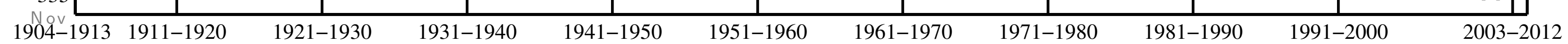

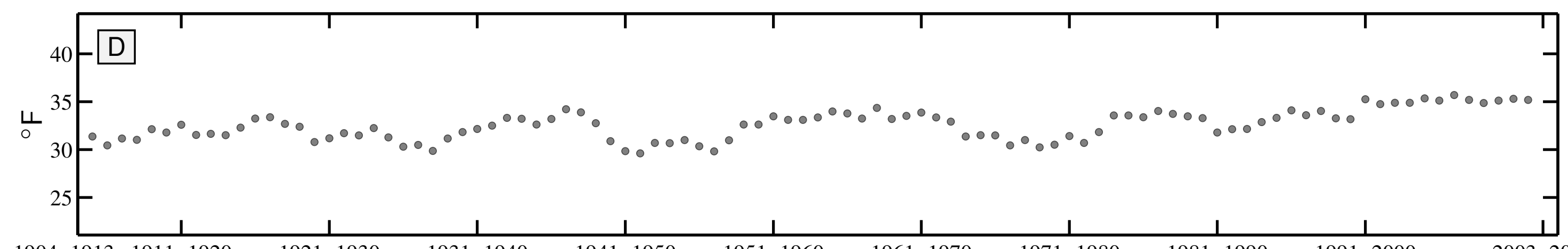

\begin{tabular}{|c|c|c|c|c|c|}
\hline 1 & \multicolumn{2}{|r|}{ 1931-1940 } & $1941-1950$ & $1951-1960$ & $1961-1970$ \\
\hline
\end{tabular}


10 year Summer Teletherm dynamics for COLVILLE, WA:
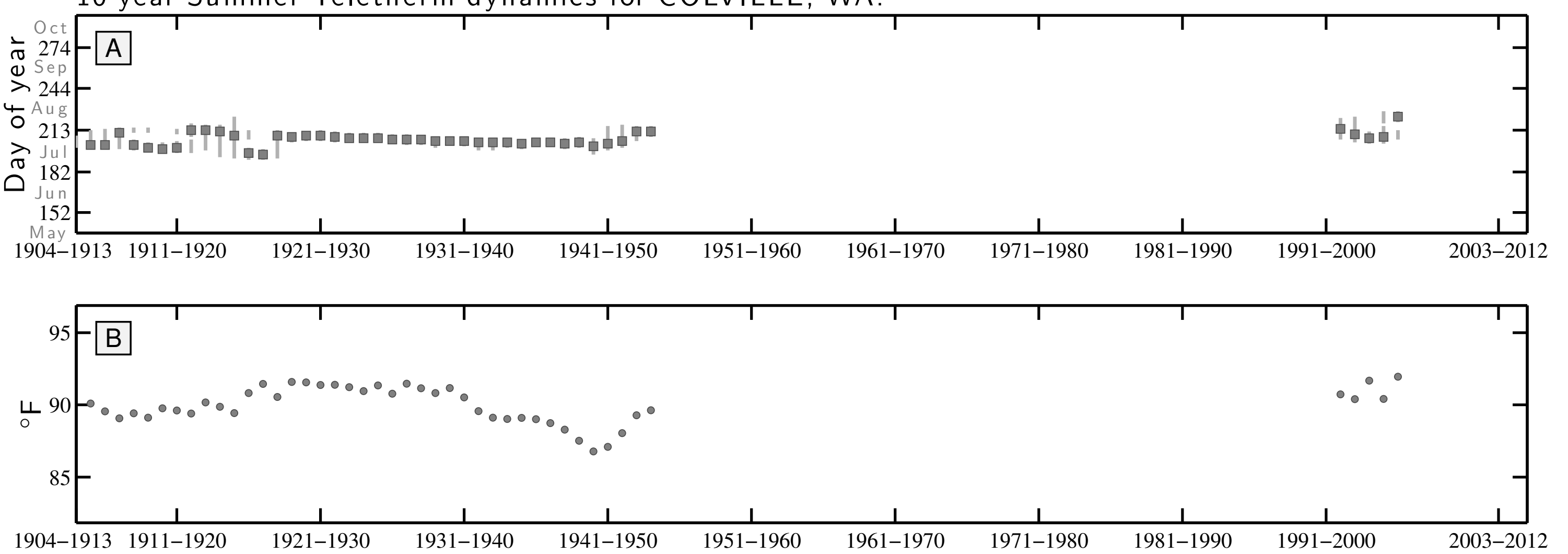

10 year Winter Teletherm dynamics for COLVILLE, WA:
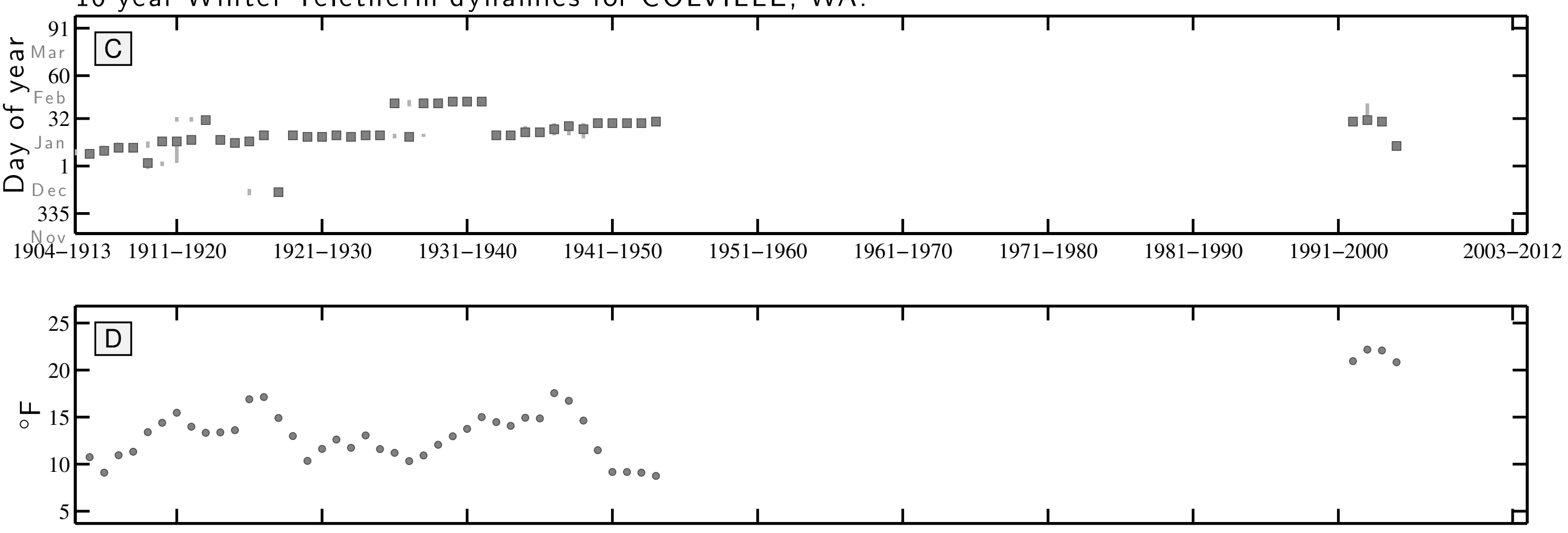

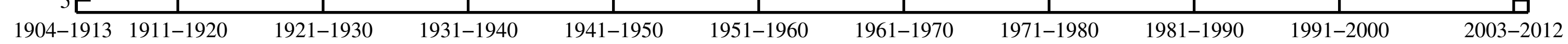


10 year Summer Teletherm dynamics for FORKS 1 E, WA:

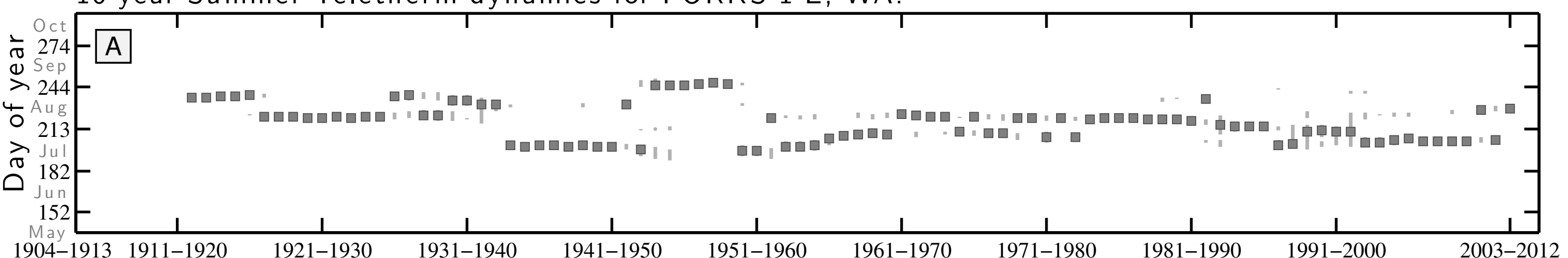

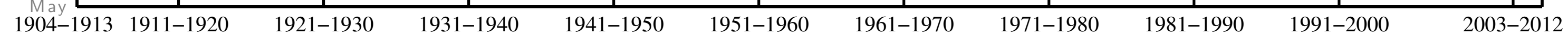

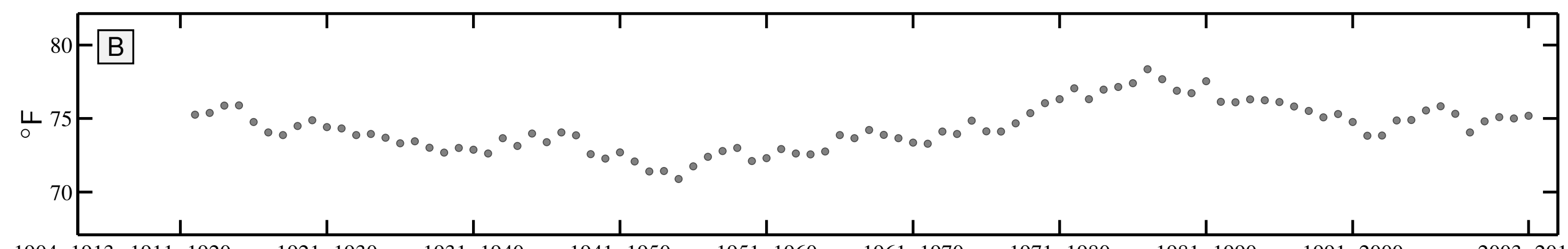

10 year Winter Teletherm dynamics for FORKS $1 \mathrm{E}$, WA:
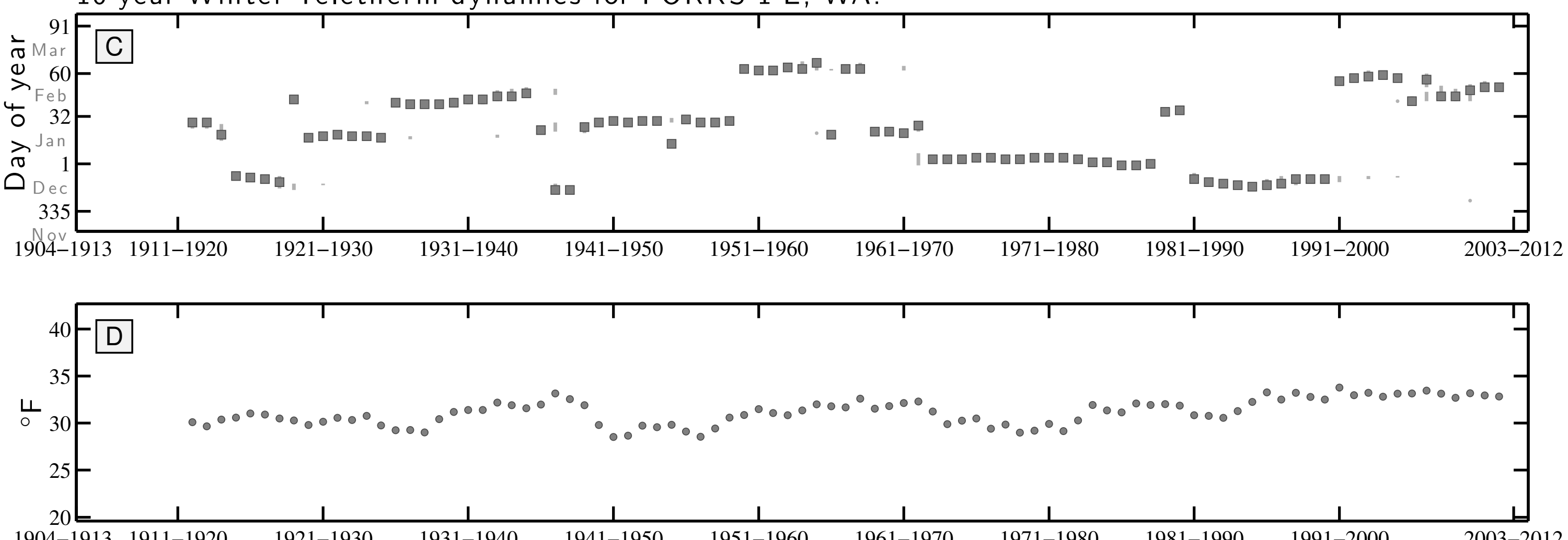
10 year Summer Teletherm dynamics for ODESSA, WA:

$\div 274$

(1) Sep

$>244-$

$4 \mathrm{Aug}$

$213-1$

\% $182-$

$\mathrm{J} u \mathrm{n}$
$152-$

May $\square$

1904-1913 1911-1920

$\frac{1}{1921-1930}$

1931-1940

1941-1950

$\frac{1}{1951-1960}$

$\frac{1}{1961-1970}$

$\frac{1}{1971-1980}$

$\frac{1}{1981-1990}$

1991-2000

2003-2012

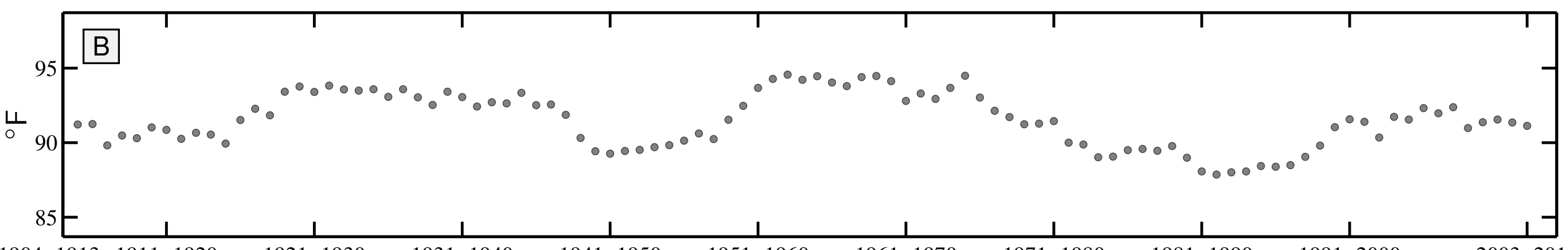

$1904-1913$ 1911-1920

$1921-1930$

$1931-1940$

$1941-1950$

$1951-1960$

$1961-1970$

$1971-1980$

$1981-1990$

$1991-2000$

2003-2012

10 year Winter Teletherm dynamics for ODESSA, WA:

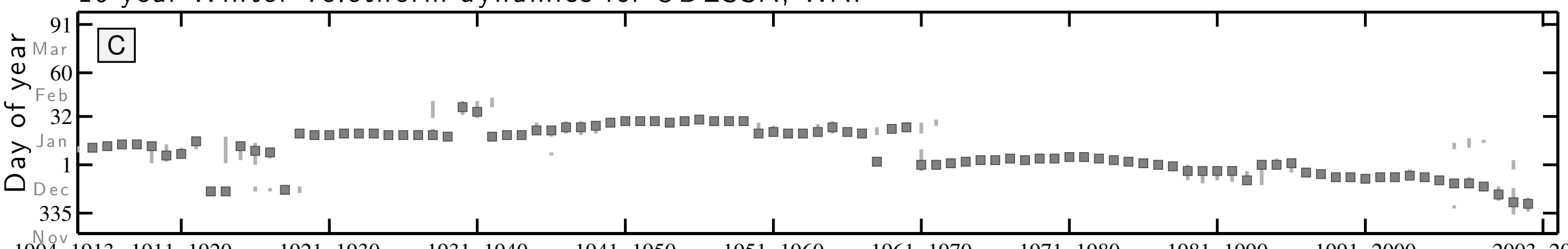

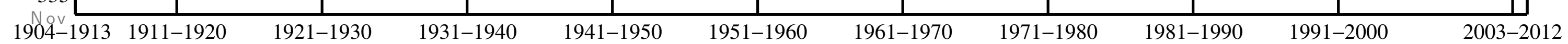

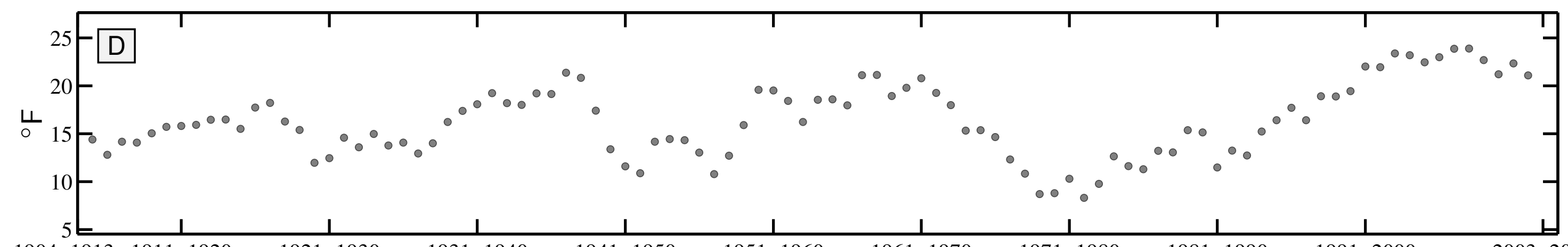


10 year Summer Teletherm dynamics for SPOKANE INTL AP, WA:

$\div$ Oct 274

(1) Sep

$>244-$

O Aug

213

\begin{tabular}{ll}
$\vec{\sigma}^{\circ}$ & $J u l$ \\
$\bigcirc$ & 182 \\
\hline &
\end{tabular}

$152-$

May —

미

1904-1913 1911-1920

$\frac{1}{1921-1930}$

1931-1940

$\frac{1}{1941-1950}$

$\frac{1}{1951-1960}$

$\frac{1}{1961-1970}$

$\frac{1}{1971-1980}$

$\frac{1}{1981-1990}$

1991-2000

2003-201

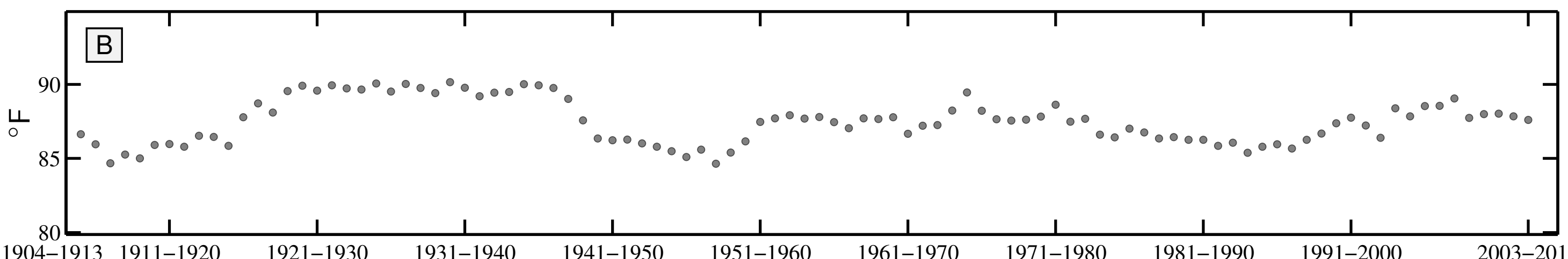

1904-1913 1911-1920

$1921-1930$

$1931-1940$

$1941-1950$

$1951-1960$

$1961-1970$

$1971-1980$

$1981-1990$

$1991-2000$

2003-2012

10 year Winter Teletherm dynamics for SPOKANE INTL AP, WA:

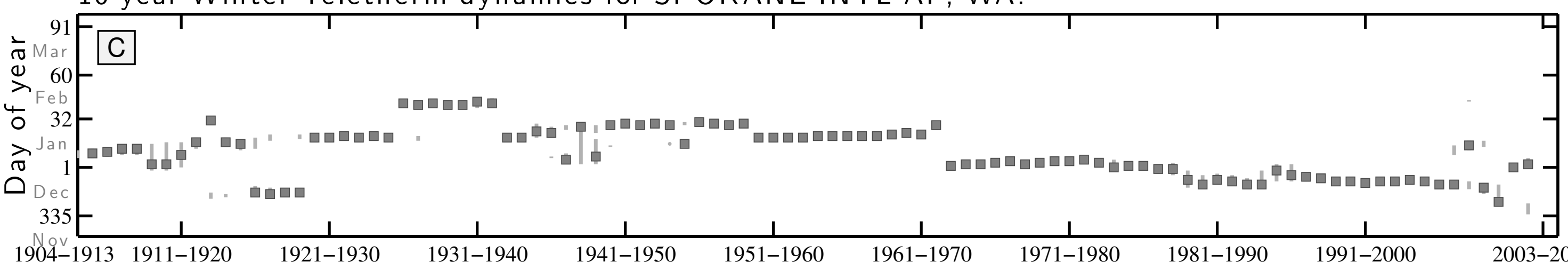

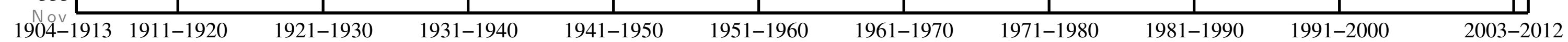

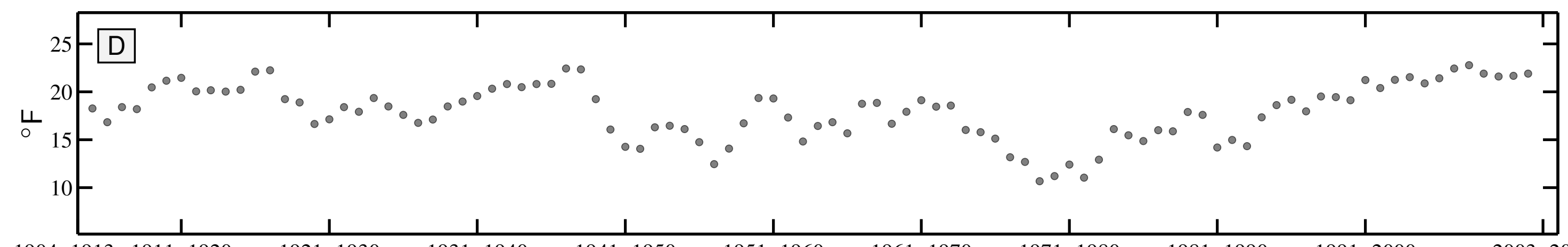


10 year Summer Teletherm dynamics for STEHEKIN 4 NW, WA:
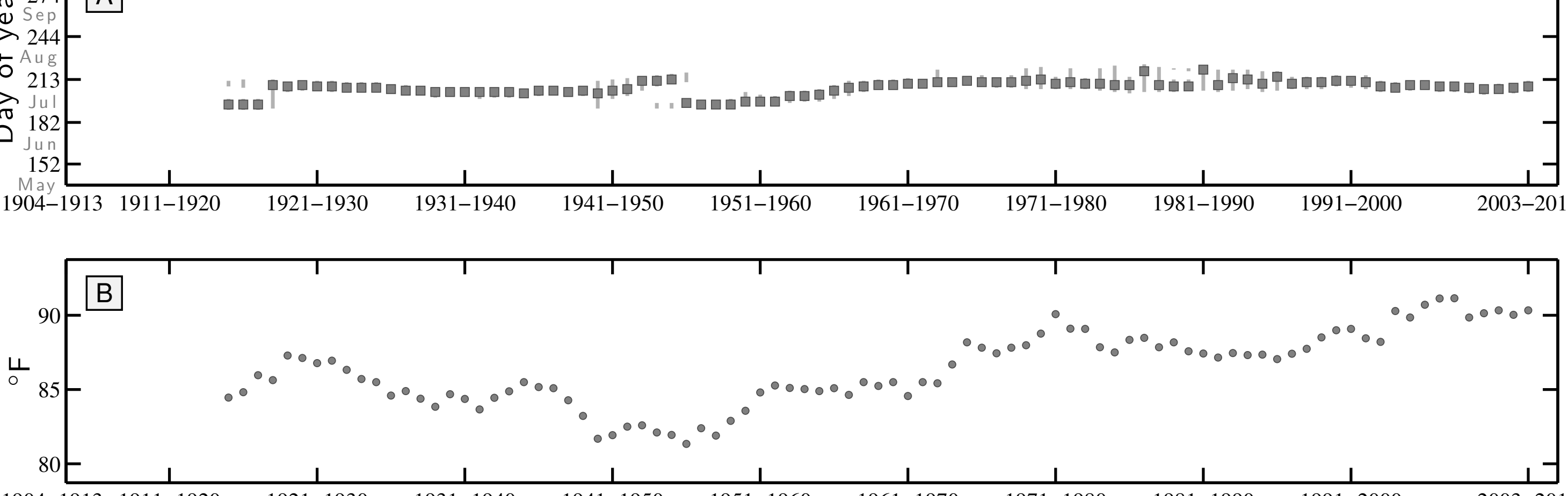

10 year Winter Teletherm dynamics for STEHEKIN 4 NW, WA:

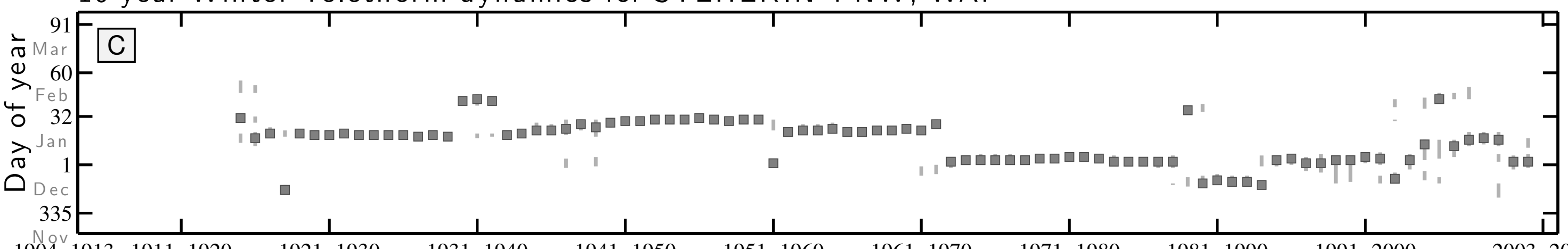

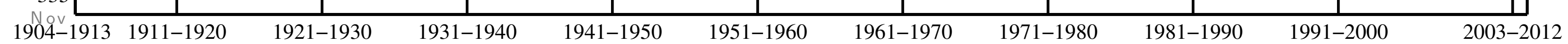

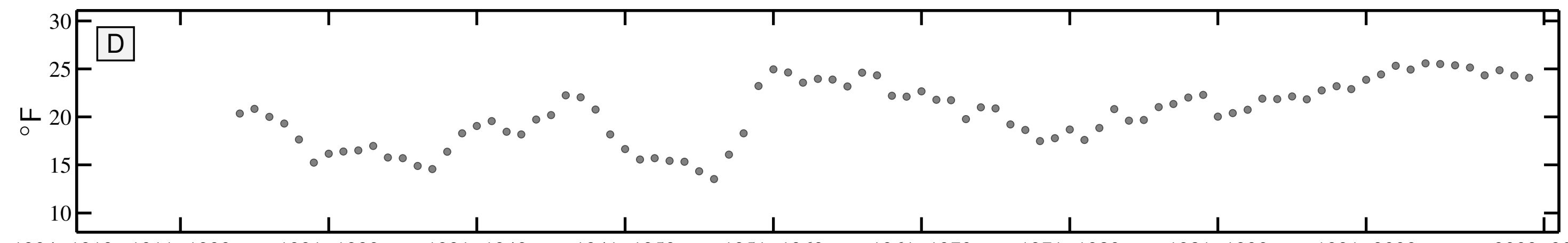


10 year Summer Teletherm dynamics for SUNNYSIDE, WA:

$\div$ Oct 274

Sep

(1) Sep

$>244-$

$4 \mathrm{Aug}$

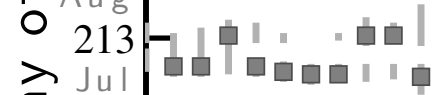

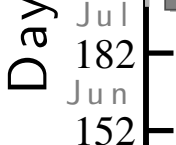

$152-$

1904-1913 1911-1920

$\frac{1}{1921-1930}$

1931-1940

1941-1950

$\frac{1}{1951-1960}$

$\frac{1}{191-1970}$

$\frac{1}{971-1980}$

$\frac{1}{981-1990}$

991-2000

2003-2012

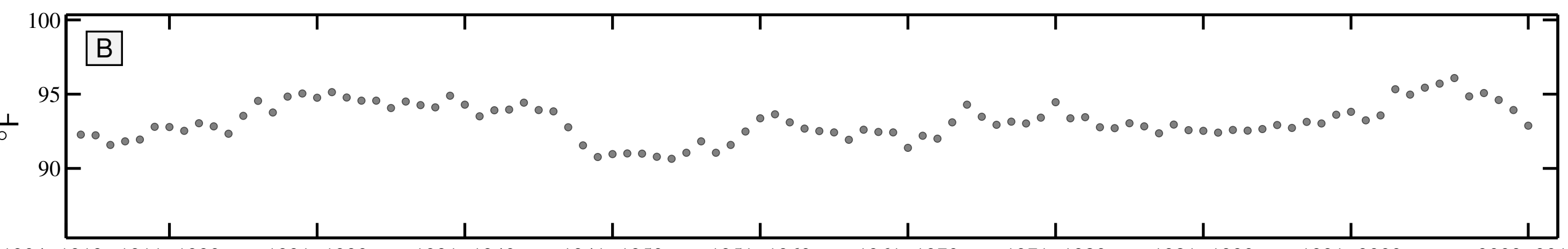

1904-1913 1911-1920

$1921-1930$

$1931-1940$

1941-1950

1951-1960

$1961-1970$

$1971-1980$

$1981-1990$

$1991-2000$

2003-2012

10 year Winter Teletherm dynamics for SUNNYSIDE, WA:

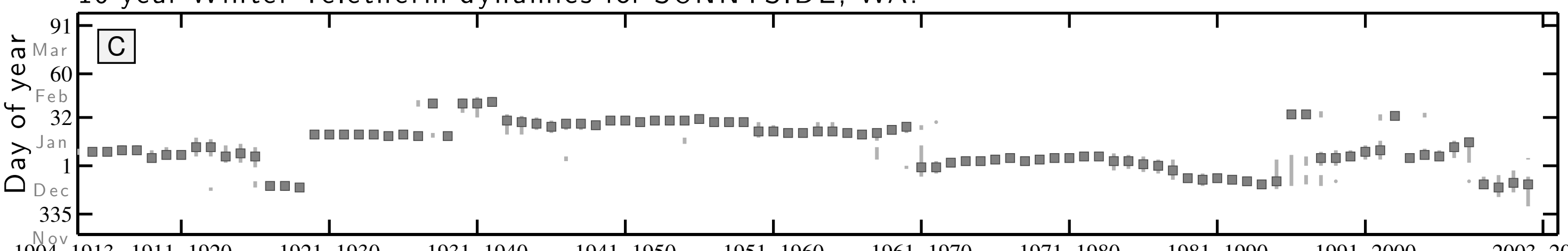

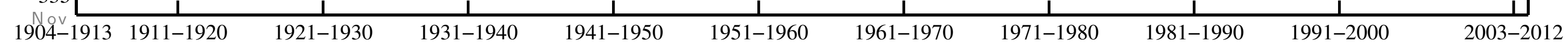

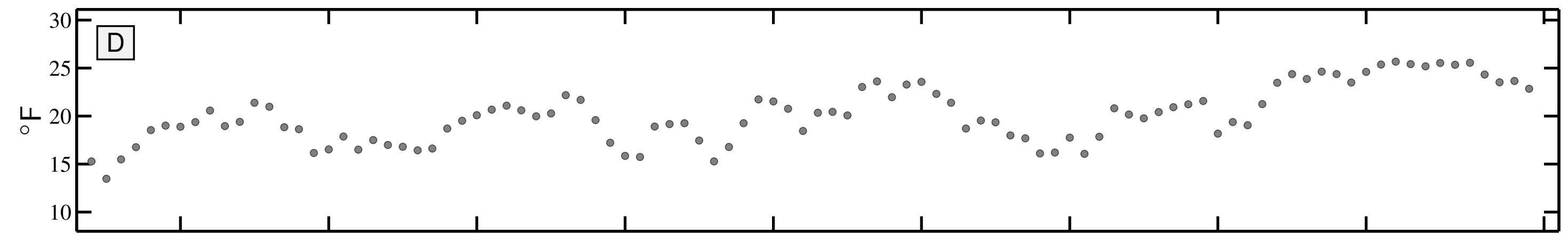


10 year Summer Teletherm dynamics for WILBUR, WA:
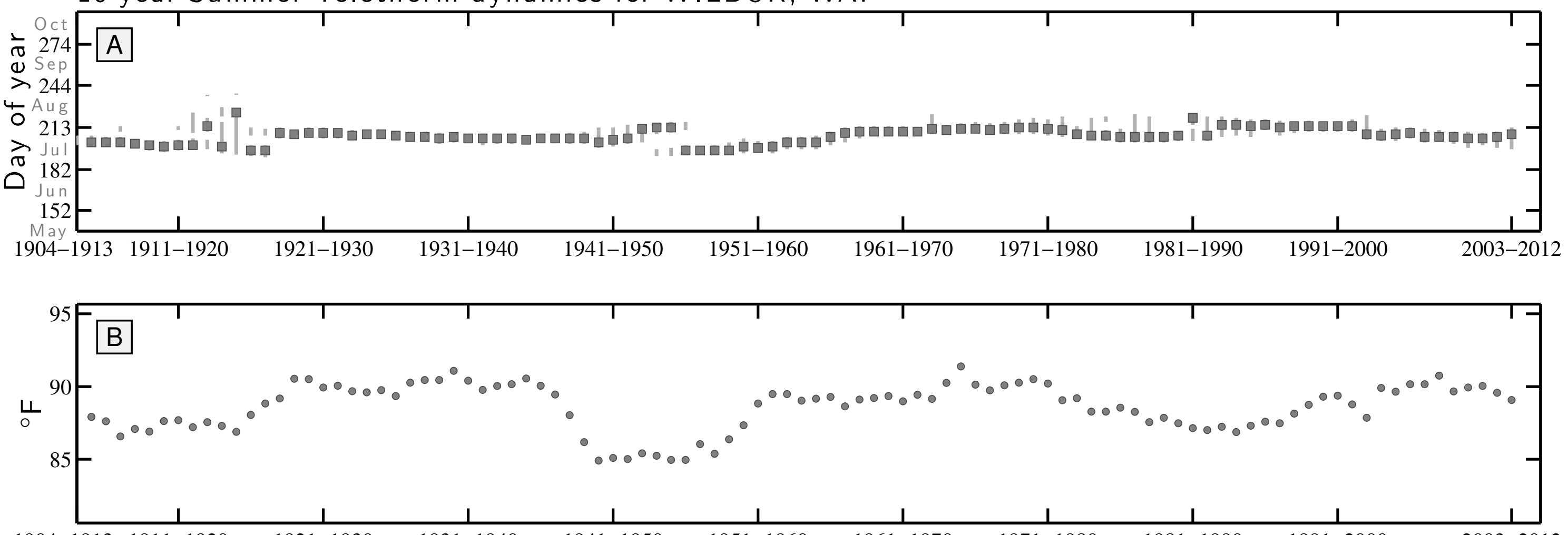

$1904-1913$ 1911-1920

$1921-1930$

1931-1940

$1941-1950$

$1951-1960$

1961-1970

$1971-1980$

$1981-1990$

$1991-2000$

2003-2012

10 year Winter Teletherm dynamics for WILBUR, WA:

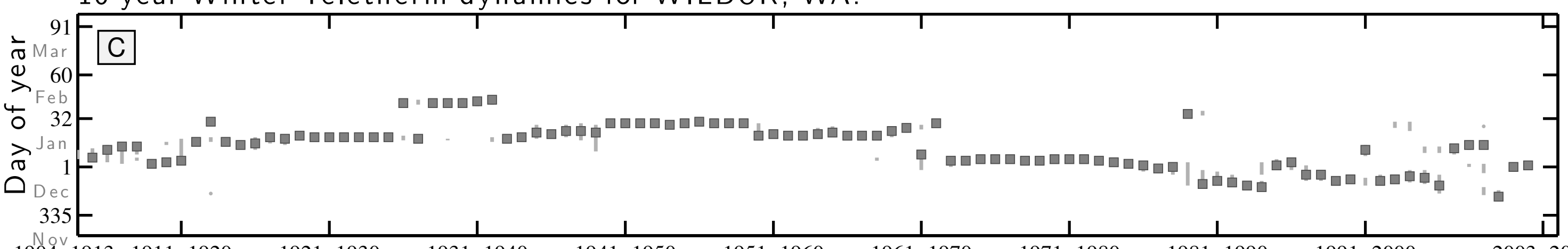

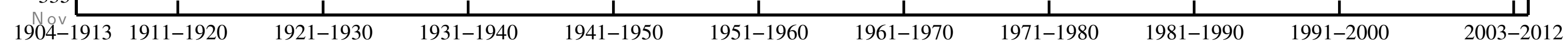

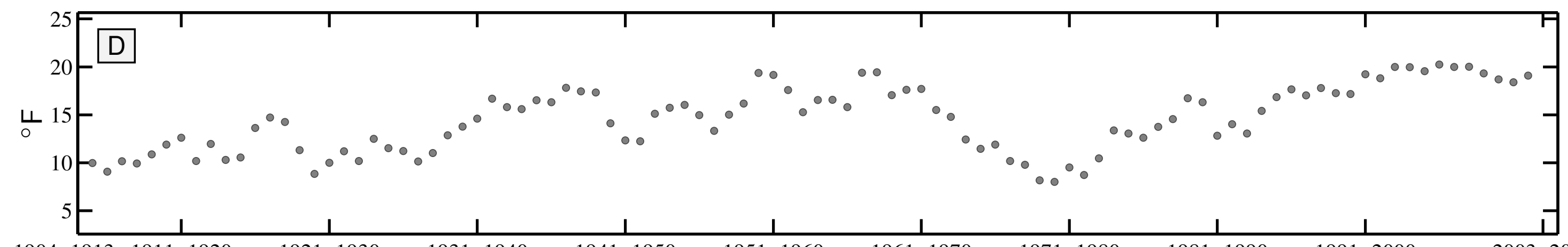


10 year Summer Teletherm dynamics for BUCKHANNON, WV:

$\mathrm{Oct}$

(274-A

(1) Sep

$>244-$

4 Aug

$\gtrsim \mathrm{Ju}$

$\overbrace{}^{\circ} 18$

$152-$

May $\square$

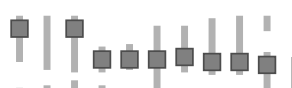

" I

1904-1913 1911-1920

1921-1930

1931-1940

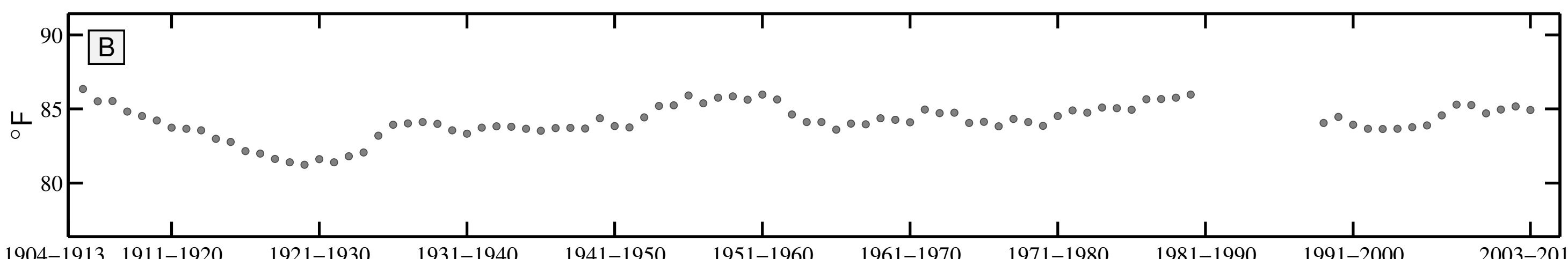

$1904-1913 \quad 1911-1920$

$1921-1930$

$1931-1940$

$1941-1950$

$1951-1960$

$1961-1970$

$1971-1980$

$1981-1990$

$1991-2000$

2003-2012

10 year Winter Teletherm dynamics for BUCKHANNON, WV:

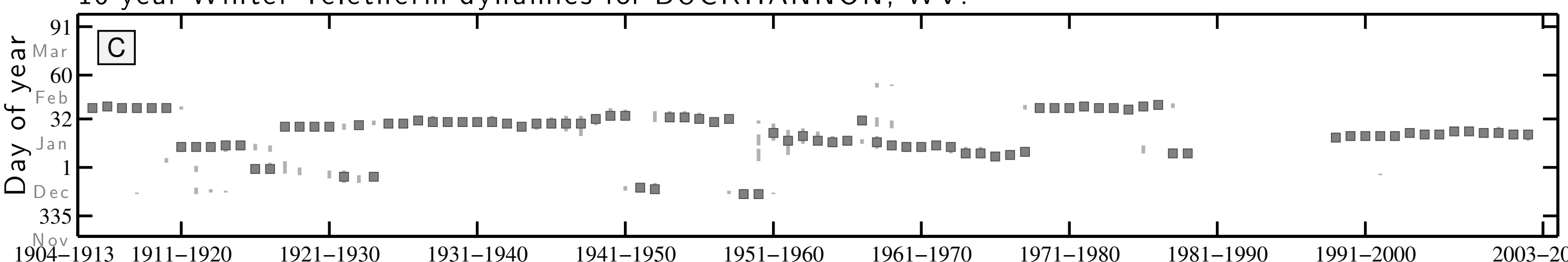

1904-1913 1911-1920

1921-1930

1931-1940

1941-1950

1951-1960

1961-1970

1971-1980

981-1990

991-2000

003-2012

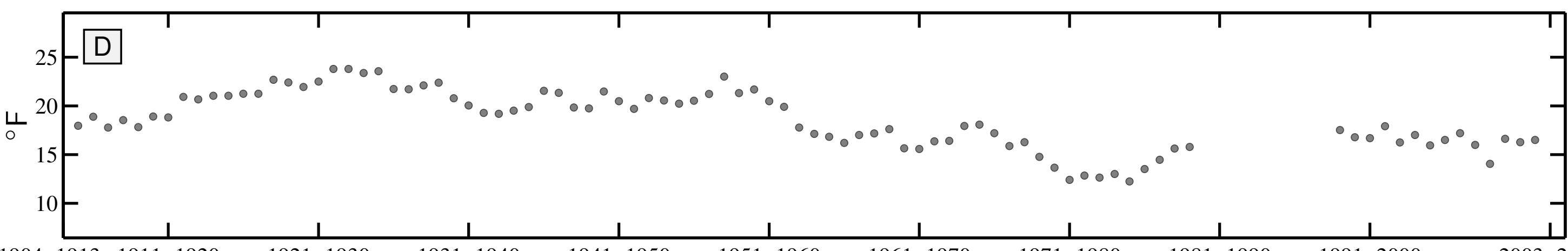


10 year Summer Teletherm dynamics for CAIRO, WV:
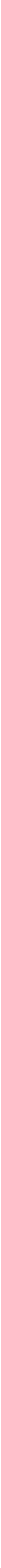
10 year Summer Teletherm dynamics for MARTINSBURG E WV RGNL, WV:

$+\mathrm{Oct}$

政

(1) Sep

$>244-$

응

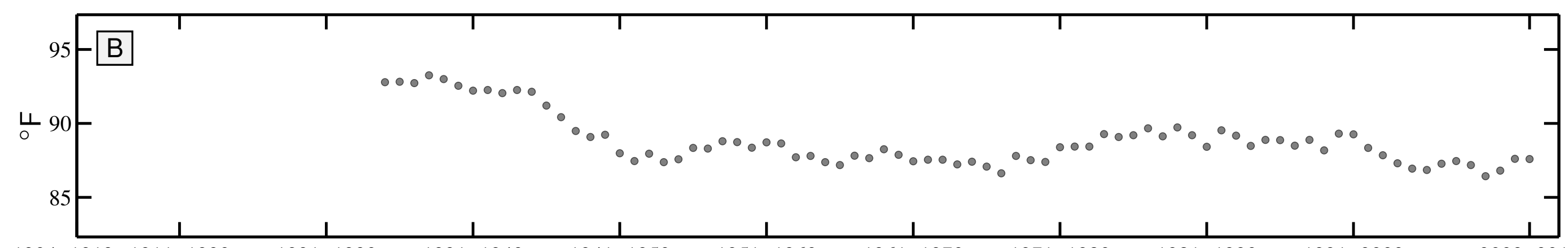

10 year Winter Teletherm dynamics for MARTINSBURG E WV RGNL, WV:
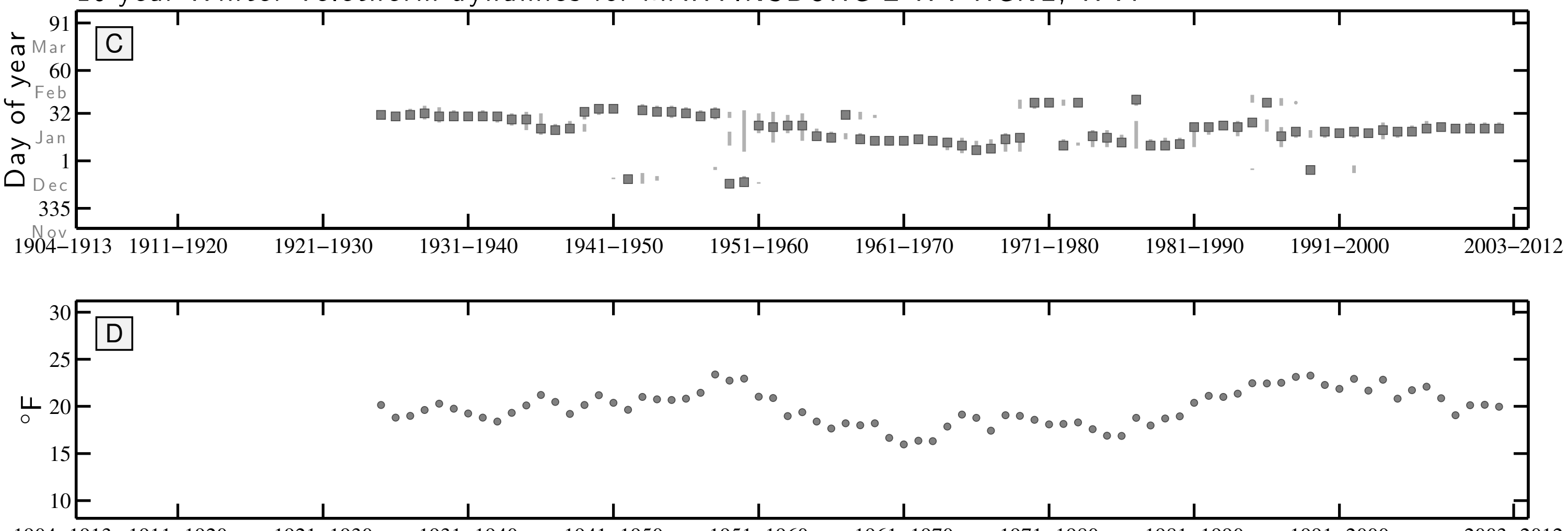
10 year Summer Teletherm dynamics for PICKENS 2 N, WV:
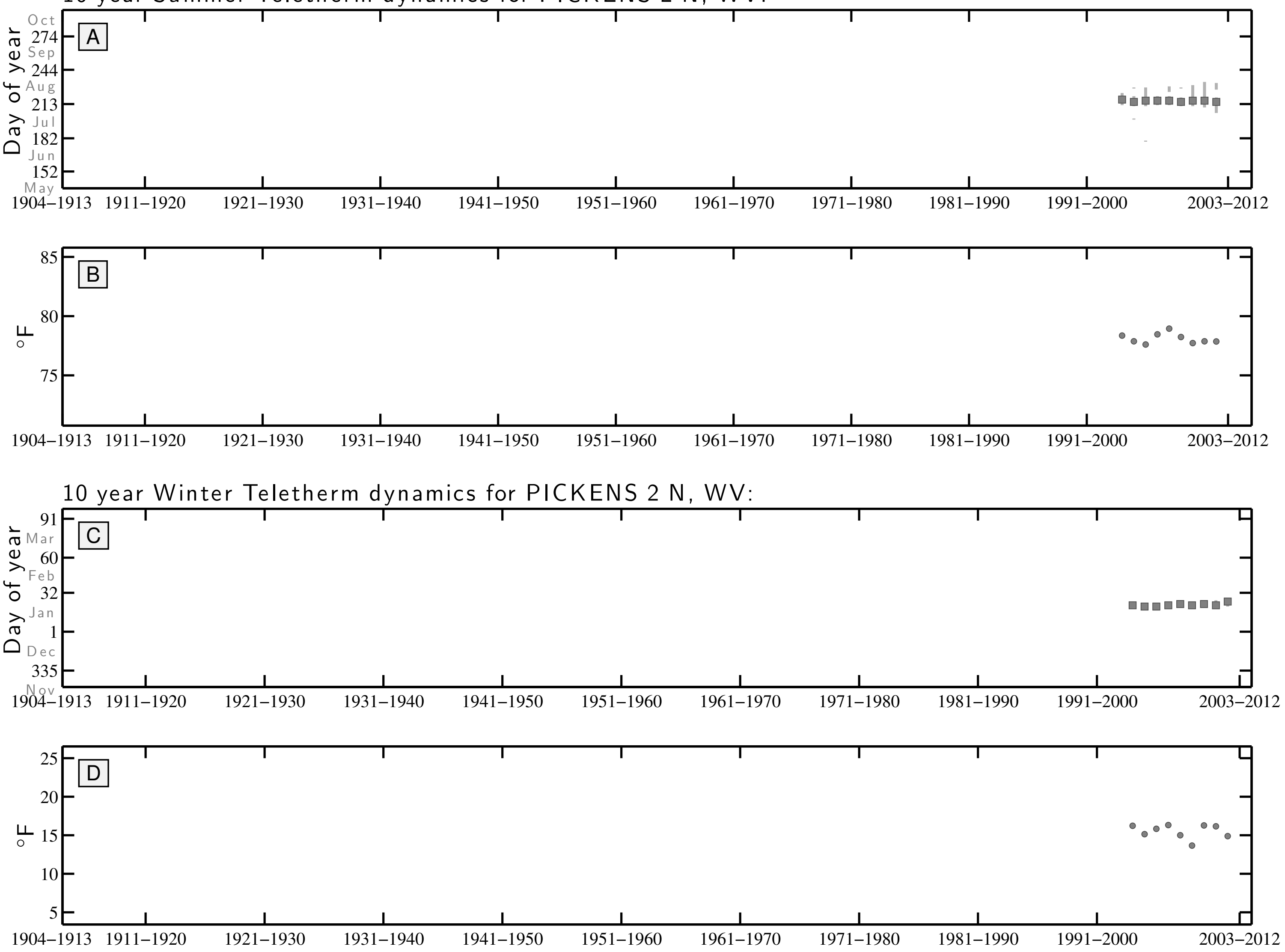
10 year Summer Teletherm dynamics for WILLIAMSON, WV:
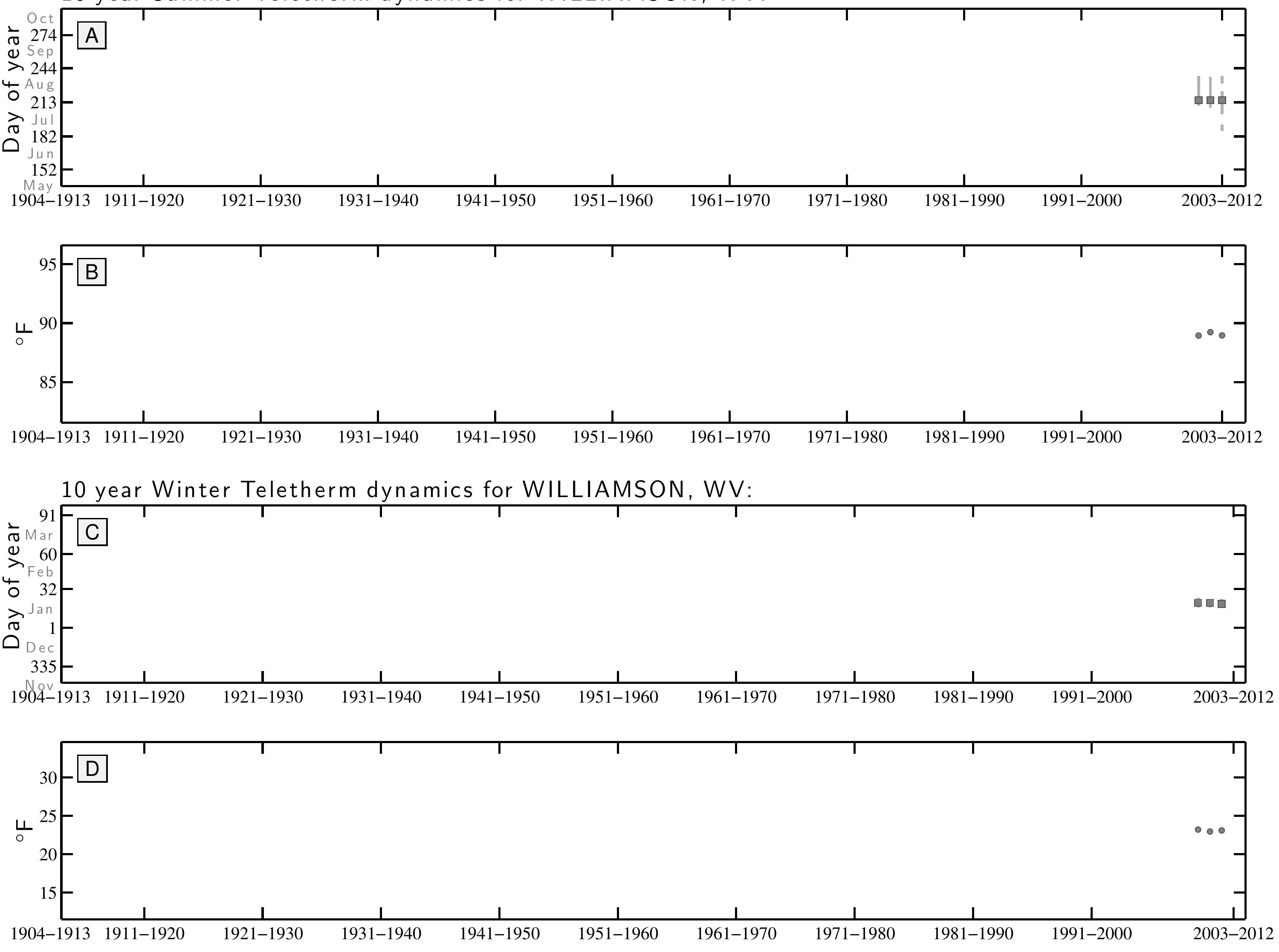
10 year Summer Teletherm dynamics for ASHLAND EXP FARM, WI:

$\div$ Oct 274

(a)

(1) Sep

$>244$

4 Aug

৯ Jul



152 -

May $\square$

1904-1913 1911-1920

$\frac{1}{1921-1930}$

1931-1940

1941-1950

1951-1960

1961-1970

1971-1980

$\frac{1}{1981-1990}$

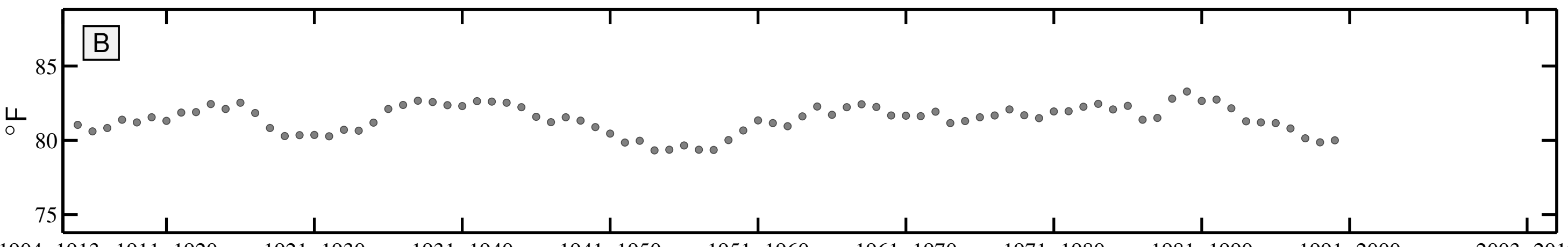

$1904-1913$ 1911-1920

$1921-1930$

$1931-1940$

$1941-1950$

$1951-1960$

$1961-1970$

$1971-1980$

$1981-1990$

$1991-2000$

$2003-2012$

10 year Winter Teletherm dynamics for ASHLAND EXP FARM, WI:

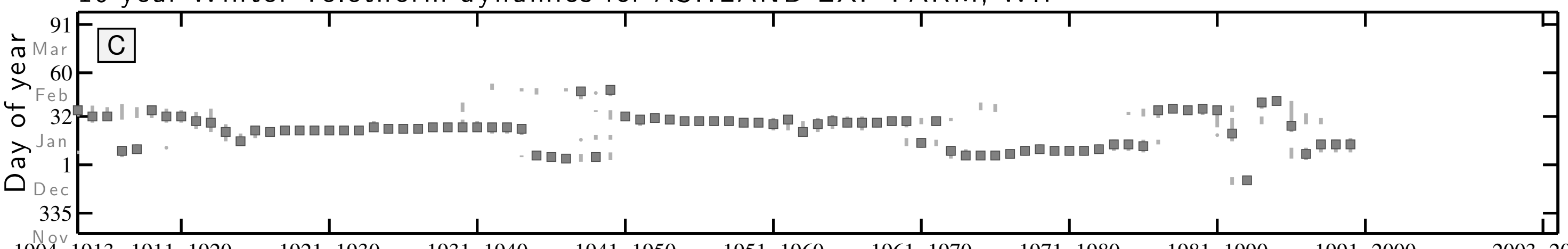

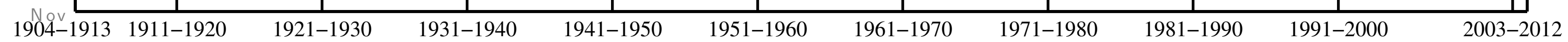

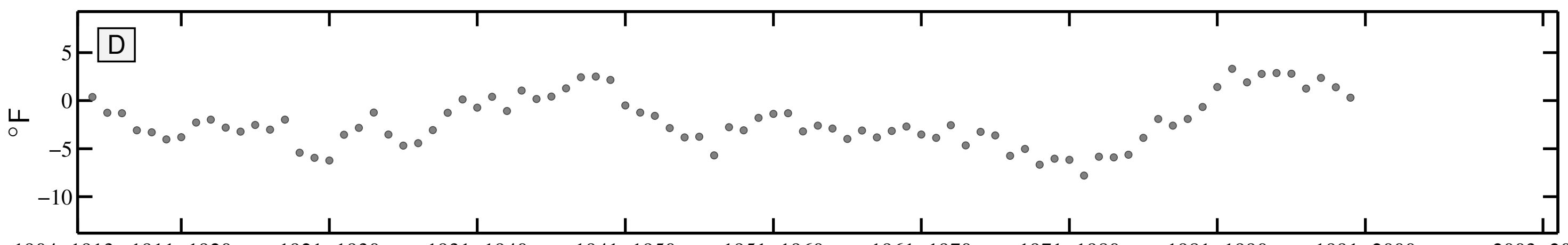

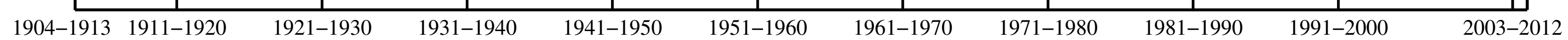


10 year Summer Teletherm dynamics for FOND DU LAC, WI:

Oct

A

(1) Sep

244

○ 213

त

182

$\bigcirc$

$152-$ 11

$\frac{1}{1-1920}$

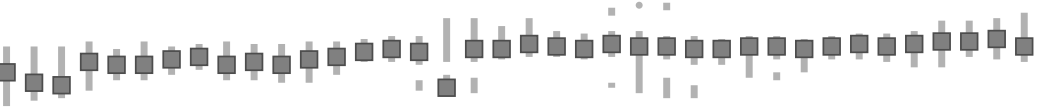

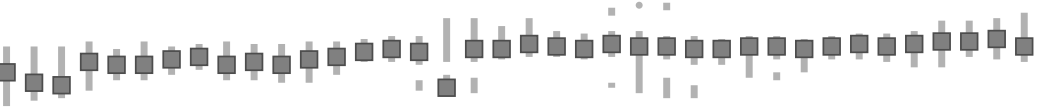

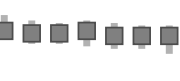

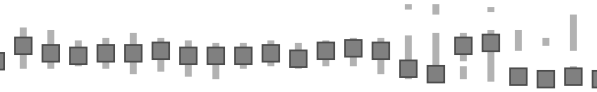

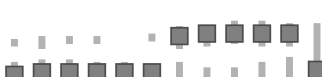
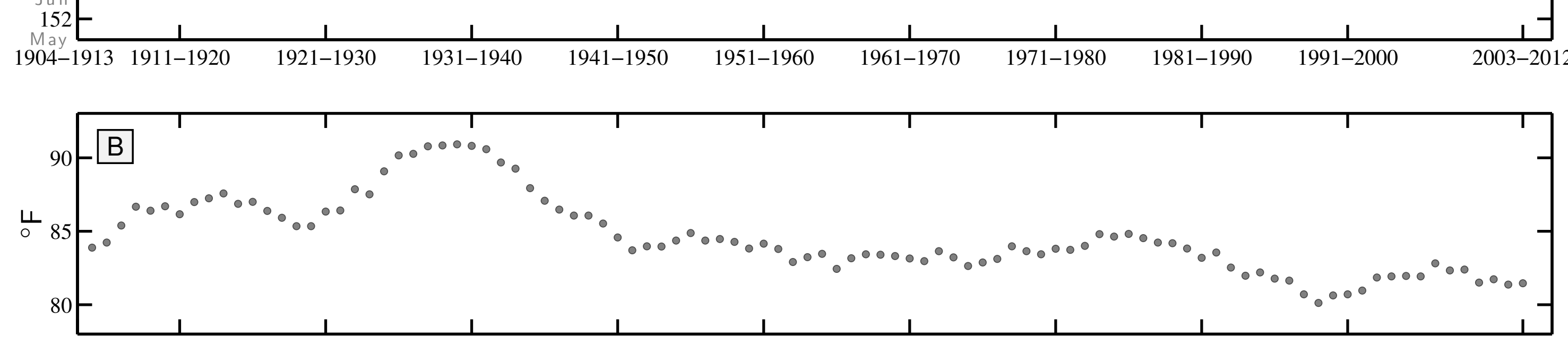

$1904-1913$ 1911-1920

$1921-1930$

$1931-1940$

$1941-1950$

$1951-1960$

$1961-1970$

$1971-1980$

$1981-1990$

$1991-2000$

2003-2012

10 year Winter Teletherm dynamics for FOND DU LAC, WI:

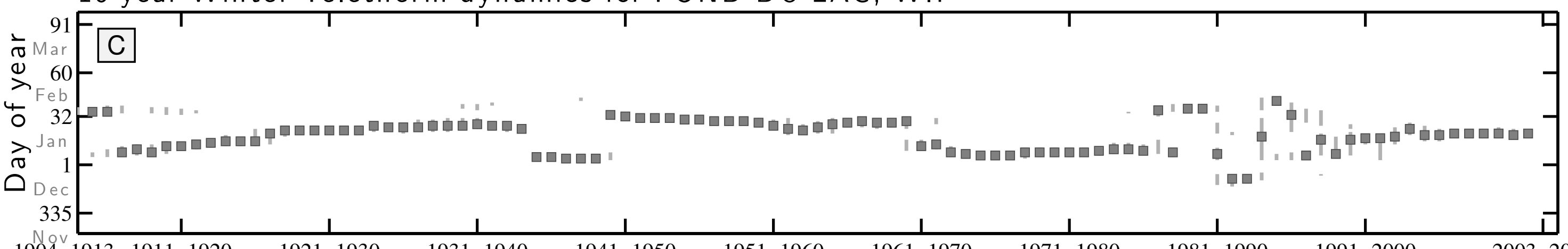

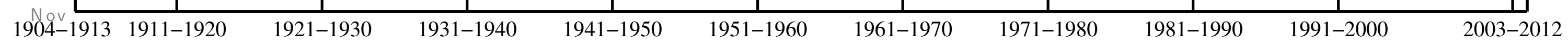

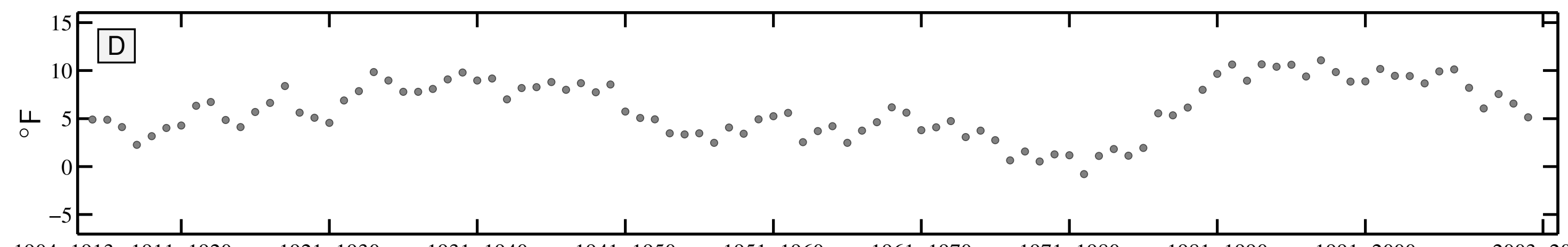


10 year Summer Teletherm dynamics for MANITOWOC, WI:

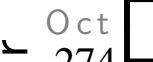

A

(1) Sep

$>244$

( $\mathrm{Aug}$

$\gtrsim \mathrm{J}$

$\overbrace{}^{\sigma} 182$

$152-$

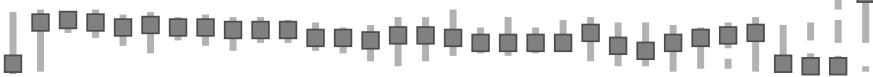

\section{| 中 | " "}

- || | " || || |

" เ | " " *

May

1904-1913 1911-1920

$1921-1930$

1931-1940

$\frac{1}{1941-1950}$

1951-1960

$\frac{1}{1961-1970}$

1971-1980

1981-1990

991-2000

2003-201

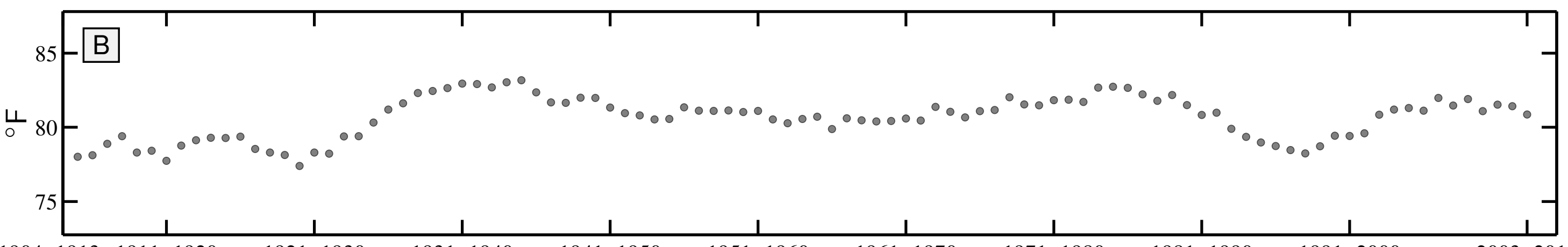

1904-1913 1911-1920

1921-1930

1931-1940

1941-1950

1951-1960

1961-1970

1971-1980

1981-1990

1991-2000

2003-2012

10 year Winter Teletherm dynamics for MANITOWOC, WI:

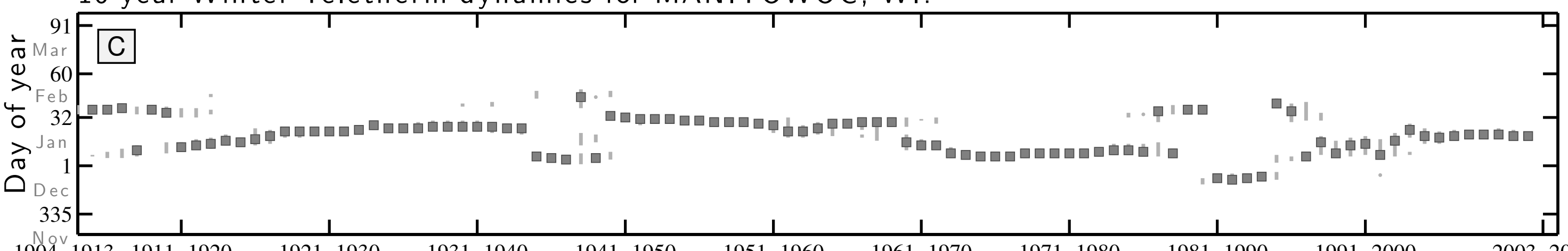

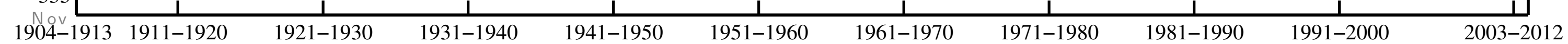

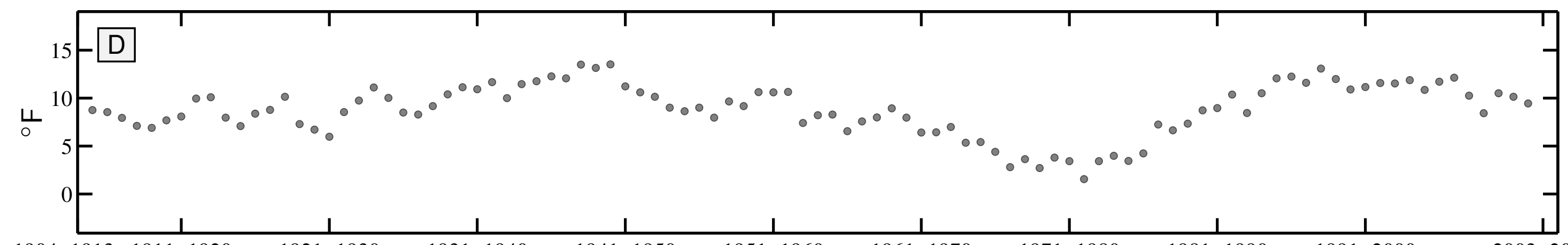


10 year Summer Teletherm dynamics for MEDFORD, WI:

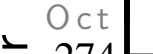

A

(1) Sep

$>244$

ᄂ Aug

$>\mathrm{Jul}$

त 182 .

Jun

$152-$

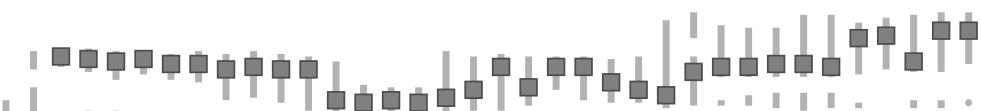

1904-1913 1911-1920

1921-1930

1931-1940

1941-1950

1951-1960

$\frac{1}{961-1970}$

971-1980

1981-1990

991-2000

2003-2012

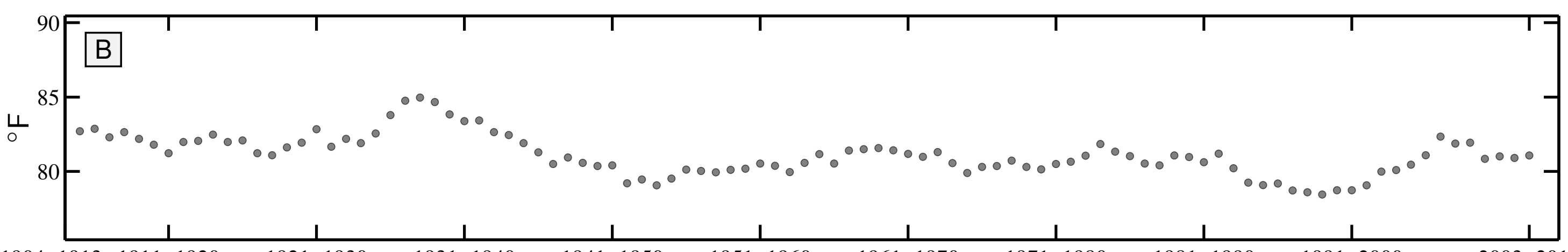

1904-1913 1911-1920

$1921-1930$

$1931-1940$

$1941-1950$

$1951-1960$

$1961-1970$

$1971-1980$

$1981-1990$

$1991-2000$

2003-2012

10 year Winter Teletherm dynamics for MEDFORD, WI:

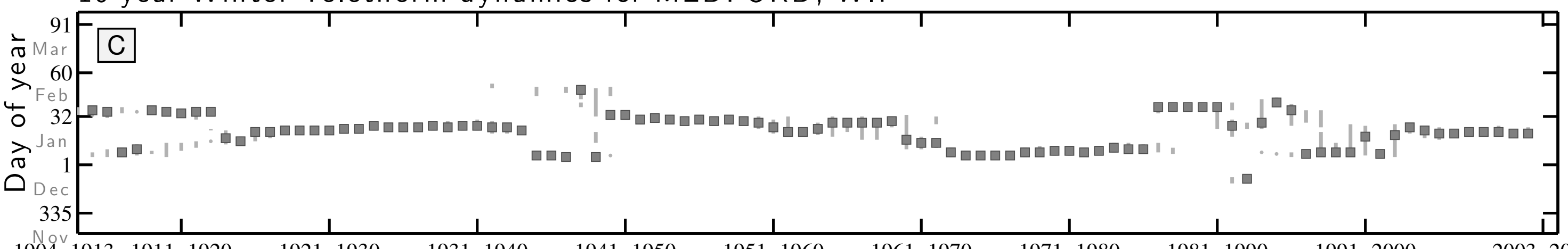

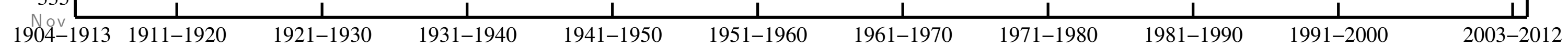

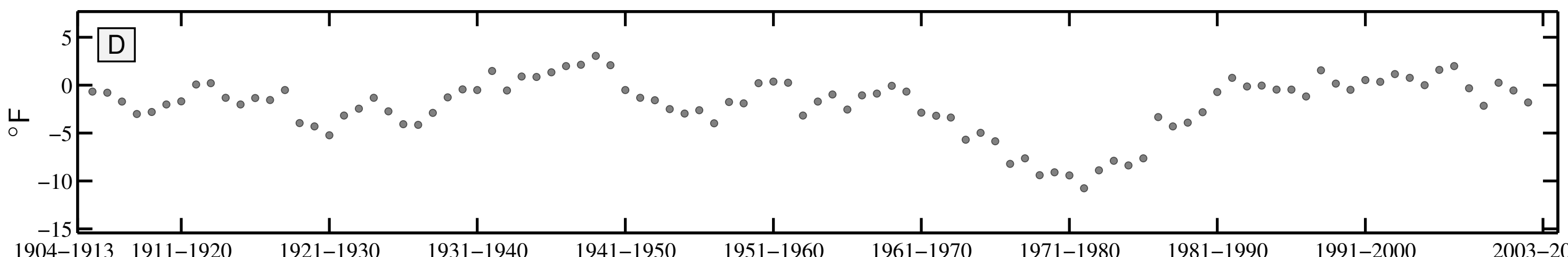


10 year Summer Teletherm dynamics for NEW LONDON, WI:

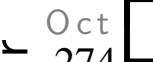

A

(1) Sep

244

O Aug

$\gtrsim \mathrm{Ju}$

182

152 -

1

1904-1913 1911-1920

$\frac{1}{1921-1930}$

1931-1940

1941-1950

1951-1960

$\frac{1}{1970}$

$\frac{1}{1971-1980}$

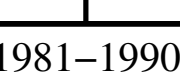

$\square$

ㅁㅁ | |

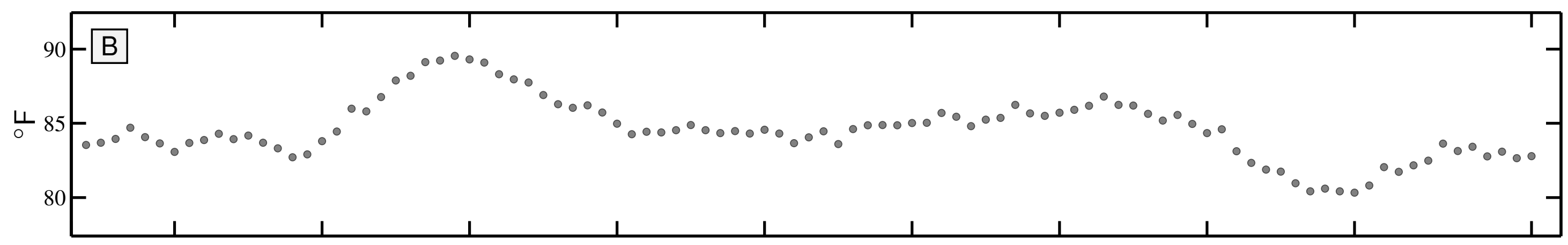

1904-1913 1911-1920

$1921-1930$

$1931-1940$

$1941-1950$

$1951-1960$

$1961-1970$

$1971-1980$

$1981-1990$

$1991-2000$

2003-2012

10 year Winter Teletherm dynamics for NEW LONDON, WI:

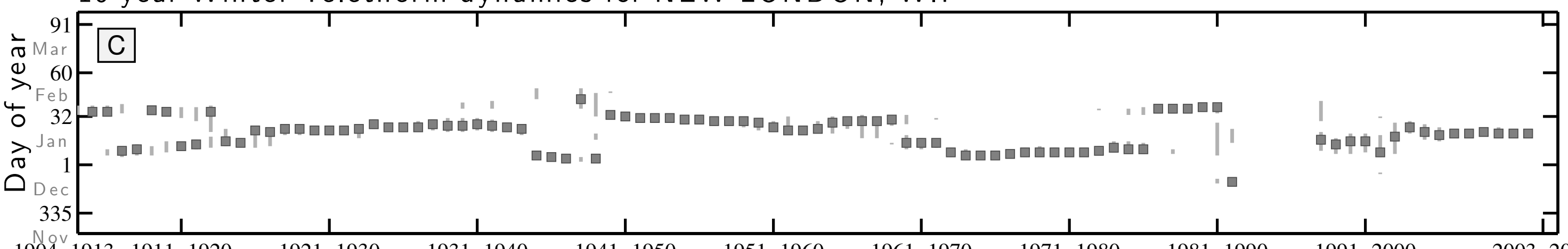

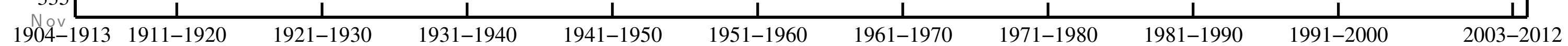

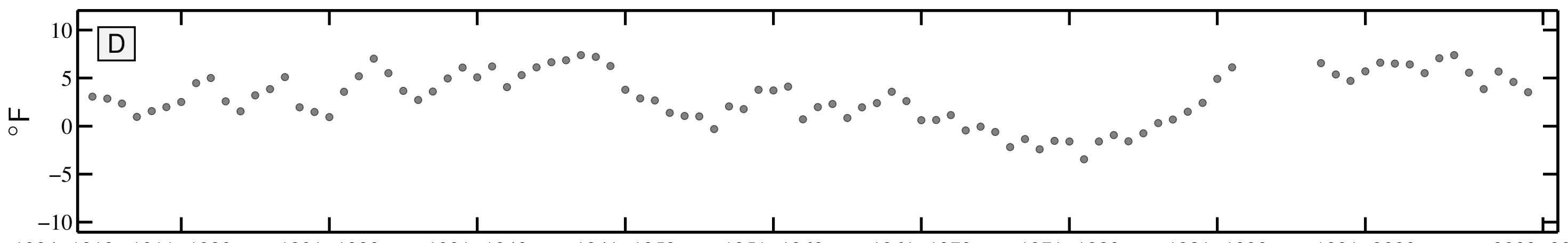


10 year Summer Teletherm dynamics for PRAIRIE DU CHIEN, WI:

$+\mathrm{Oct}$

A

(1) Sep

$>244$

- Aug

$\vec{\sigma}^{2} \mathrm{~J}$

$\overbrace{\substack{u n \\ 1}}^{182}$

$152-$

ㅁㅁ

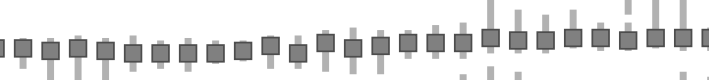

-

1904-1913 1911-1920

$1921-1930$

1931-1940

$1941-1950$

1951-1960

$1961-1970$

$\frac{1}{1971-1980}$

$\frac{1}{1981-1990}$

1991-2000

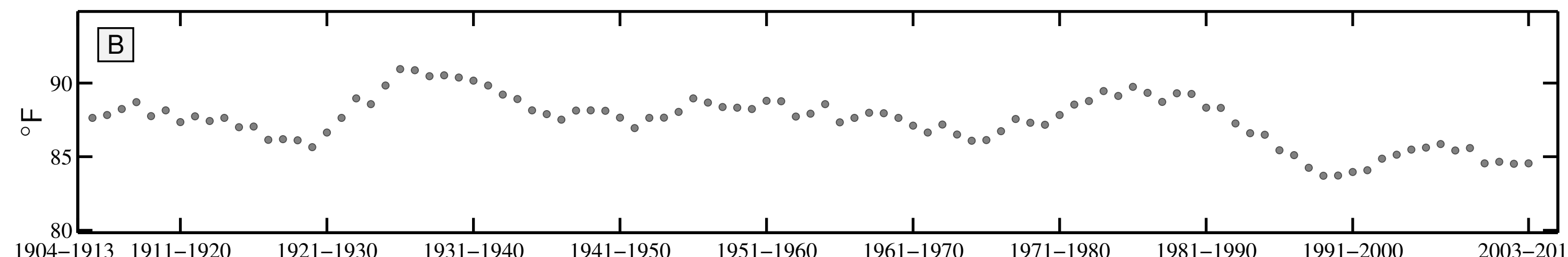

1904-1913 1911-1920

1921-1930

1931-1940

1941-1950

1951-1960

1961-1970

1991-2000

2003-2012

10 year Winter Teletherm dynamics for PRAIRIE DU CHIEN, WI:

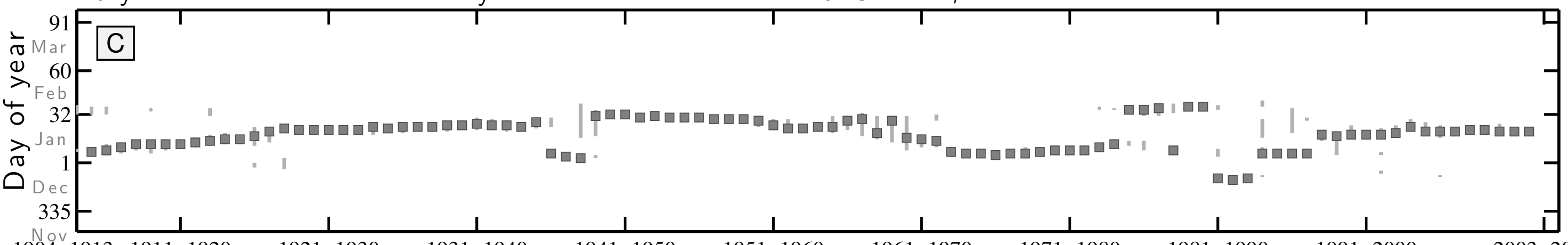

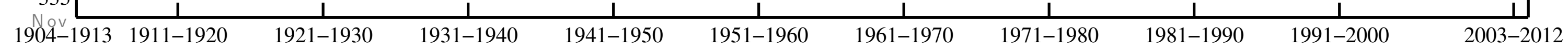

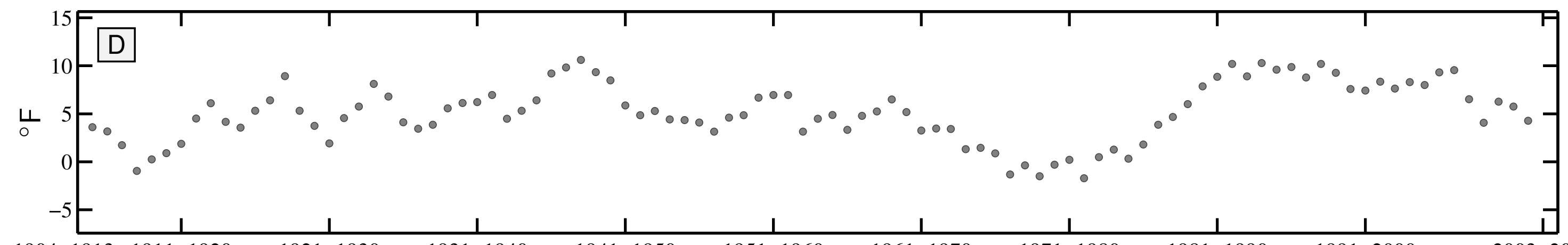


10 year Summer Teletherm dynamics for WATERTOWN, WI:

$\mathrm{Oct}$

- 274

(1) Sep

> 244

○ 213

त Ju

$\overbrace{}^{\circ} 182$

$152-$

-

1904-1913 1911-1920

$\frac{1}{1921-1930}$

1931-1940

$\frac{1}{1941-1950}$

1951-1960

$\frac{1}{1961-1970}$

$\frac{1}{1971-1980}$

$\frac{1}{1981-1990}$

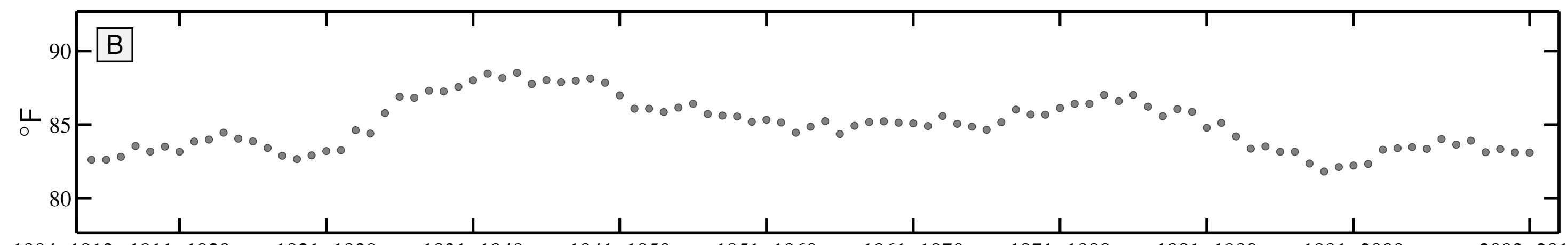

1904-1913 1911-1920

1921-1930

1931-1940

1941-1950

1951-1960

1961-1970

1971-1980

1981-1990

1991-2000

2003-2012

10 year Winter Teletherm dynamics for WATERTOWN, WI:

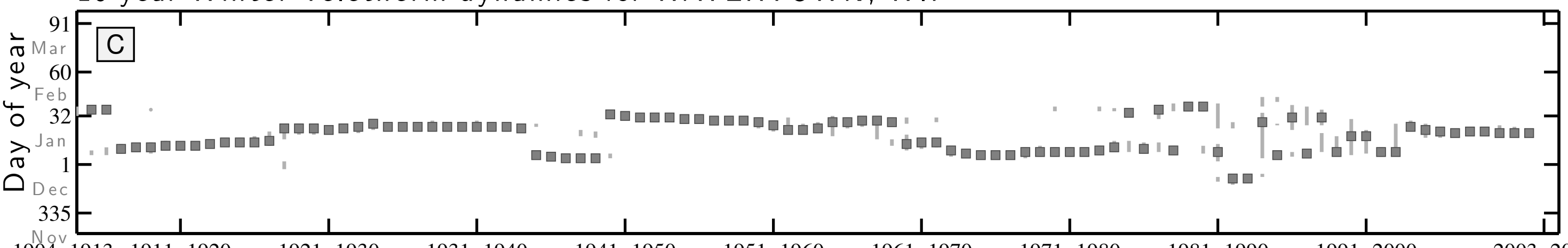

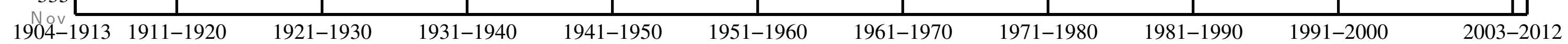

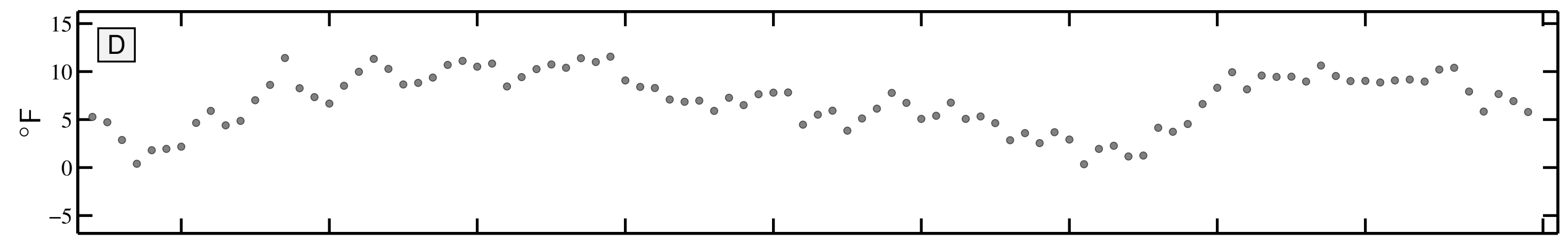

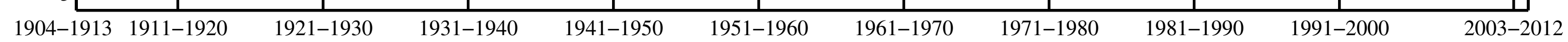


10 year Summer Teletherm dynamics for ALTA 1 NNW, WY:

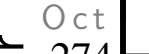

A

(1) Sep

$>244$

A A g

入 Jul

$\overbrace{}^{\pi} 182$

$\mathrm{Jun}$

$152-$

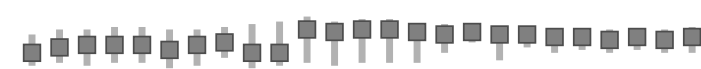

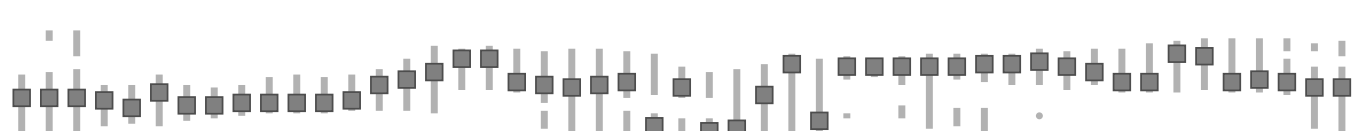

1904-1913 1911-1920 1921-1930 1931-1940

$\frac{1}{1951-1960}$

1961-1970

1971-1980

$1981-1990$

$991-2000$

2003-201

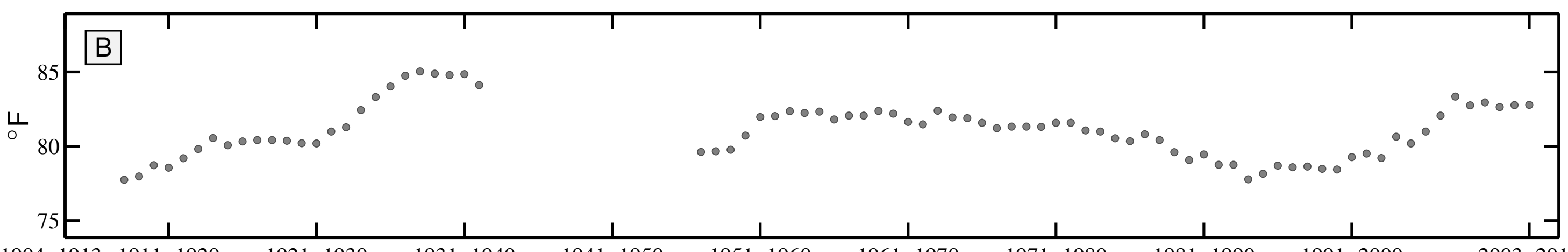

1904-1913 1911-1920

1921-1930

1931-1940

1941-1950

1951-1960

1961-1970

1971-1980

1981-1990

1991-2000

2003-2012

10 year Winter Teletherm dynamics for ALTA 1 NNW, WY:
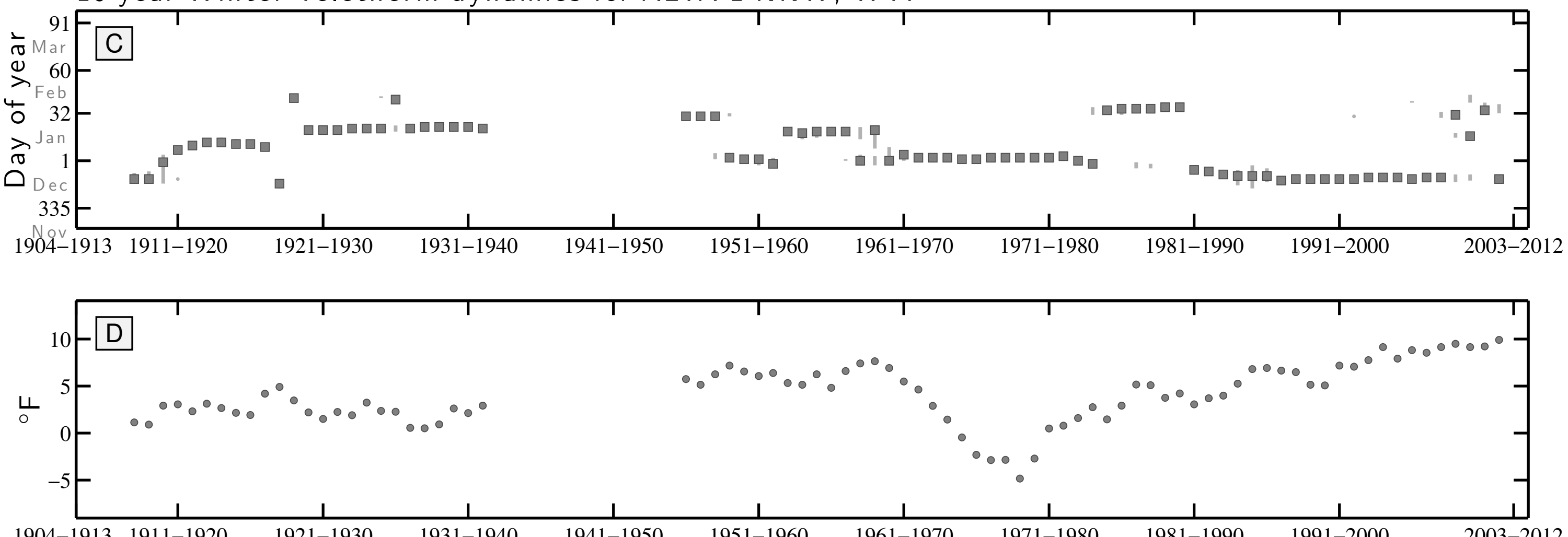

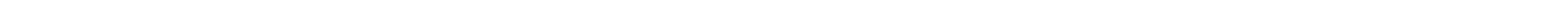
10 year Summer Teletherm dynamics for CHUGWATER, WY:

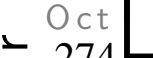

A

(1) Sep

$>244-$

\% Aug

$\gtrsim \mathrm{Jul}$

$182-$

$152-$

뭄ำ

中国

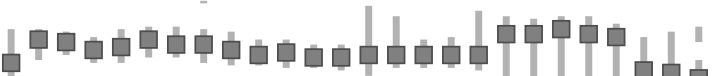

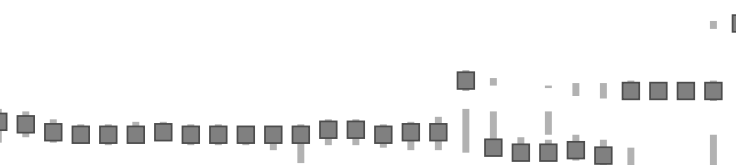

" ㅁ |

1

$\frac{1}{1-1940}$

$\frac{1}{1-1970}$

$\frac{1}{971-1980}$

1981-1990

$1991-2000$

$2003-2012$

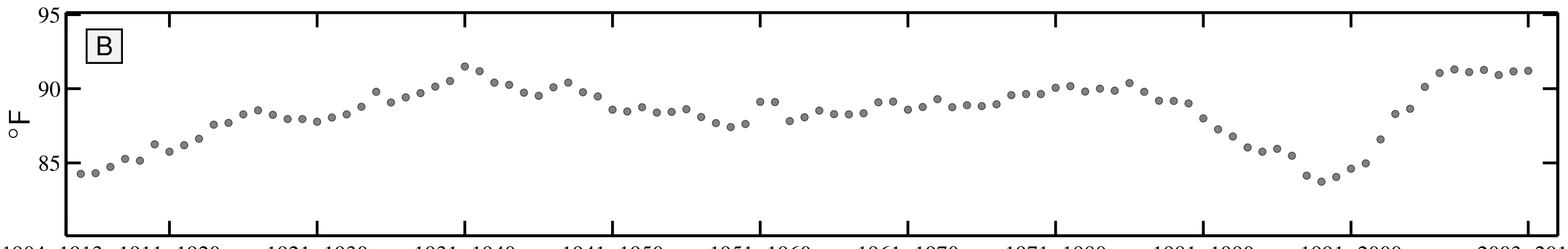

1904-1913 1911-1920

$1921-1930$

$1931-1940$

$1941-1950$

$1951-1960$

$1961-1970$

$1971-1980$

$1981-1990$

$1991-2000$

$2003-2012$

10 year Winter Teletherm dynamics for CHUGWATER, WY:

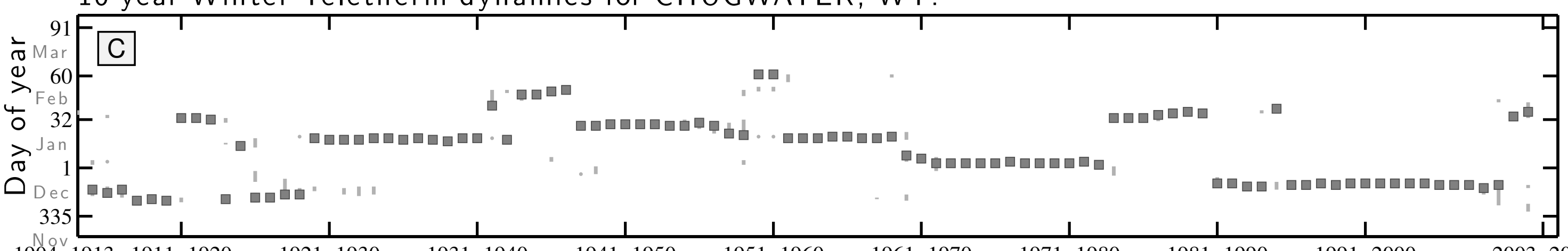

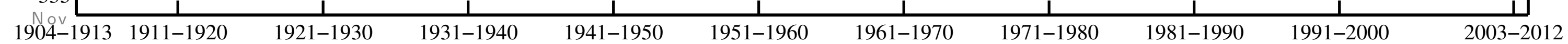

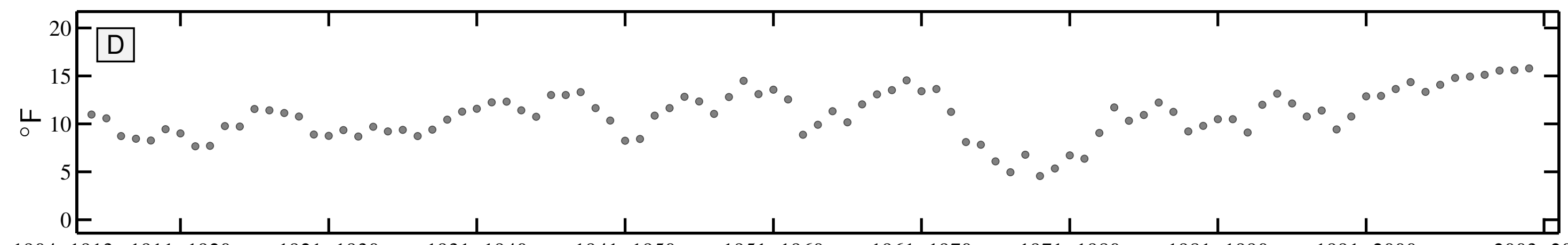


10 year Summer Teletherm dynamics for CODY, WY:
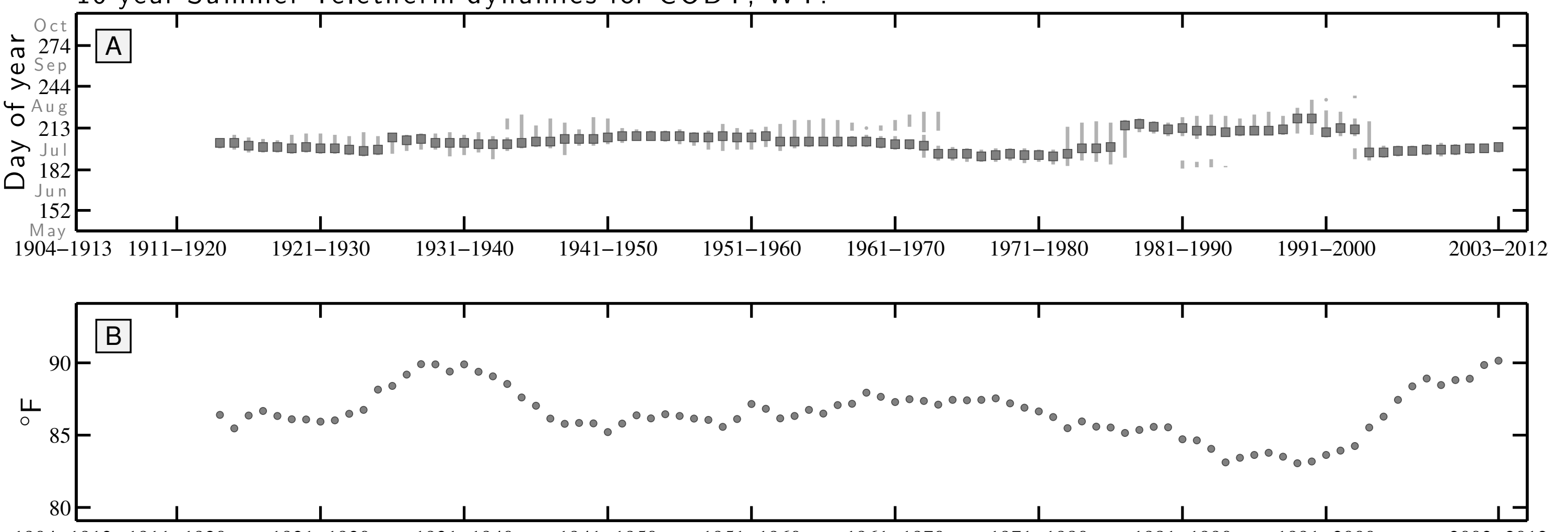

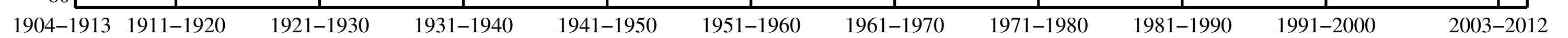
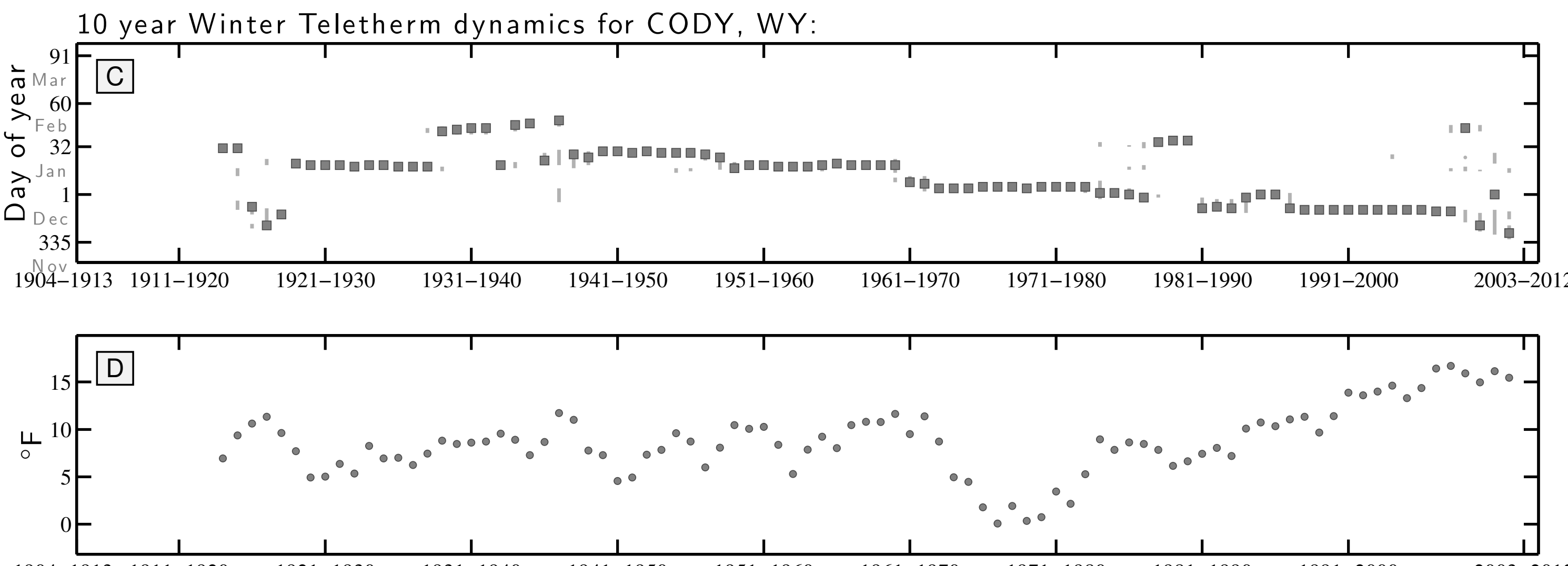

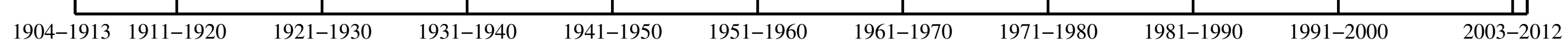


10 year Summer Teletherm dynamics for DIVERSION DAM, WY:
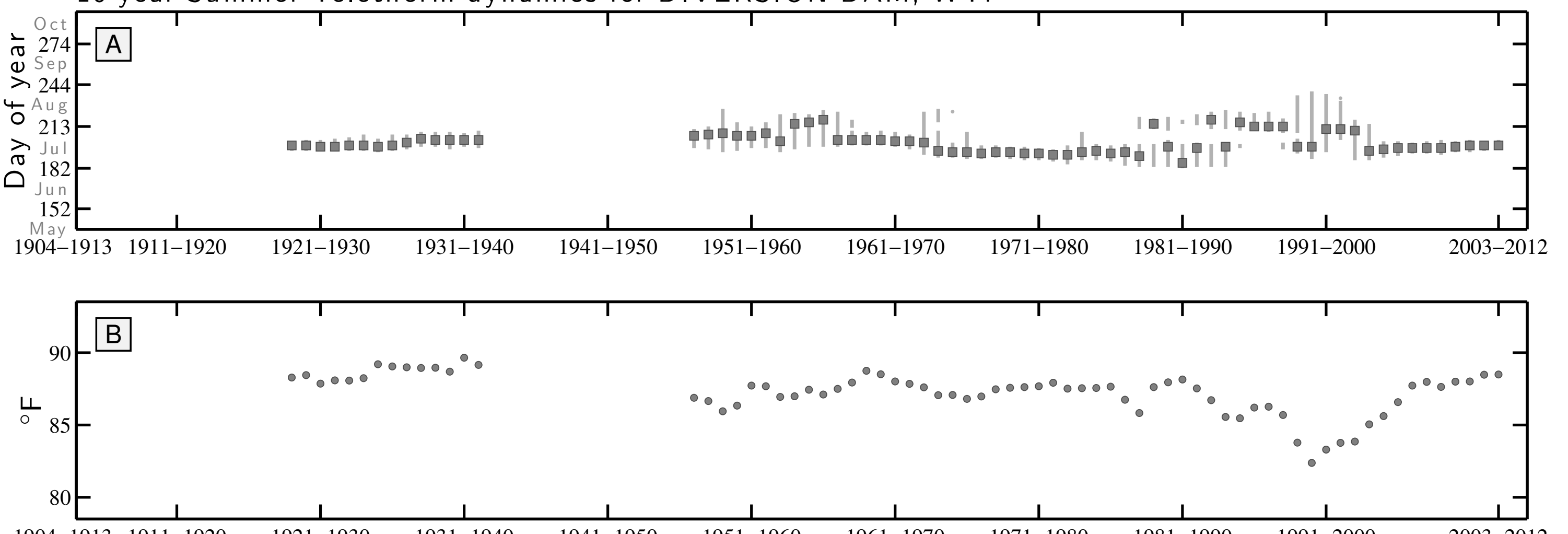

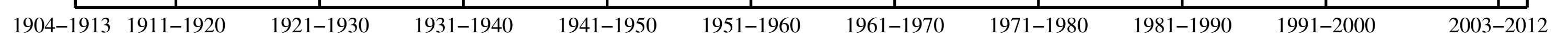

10 year Winter Teletherm dynamics for DIVERSION DAM, WY:

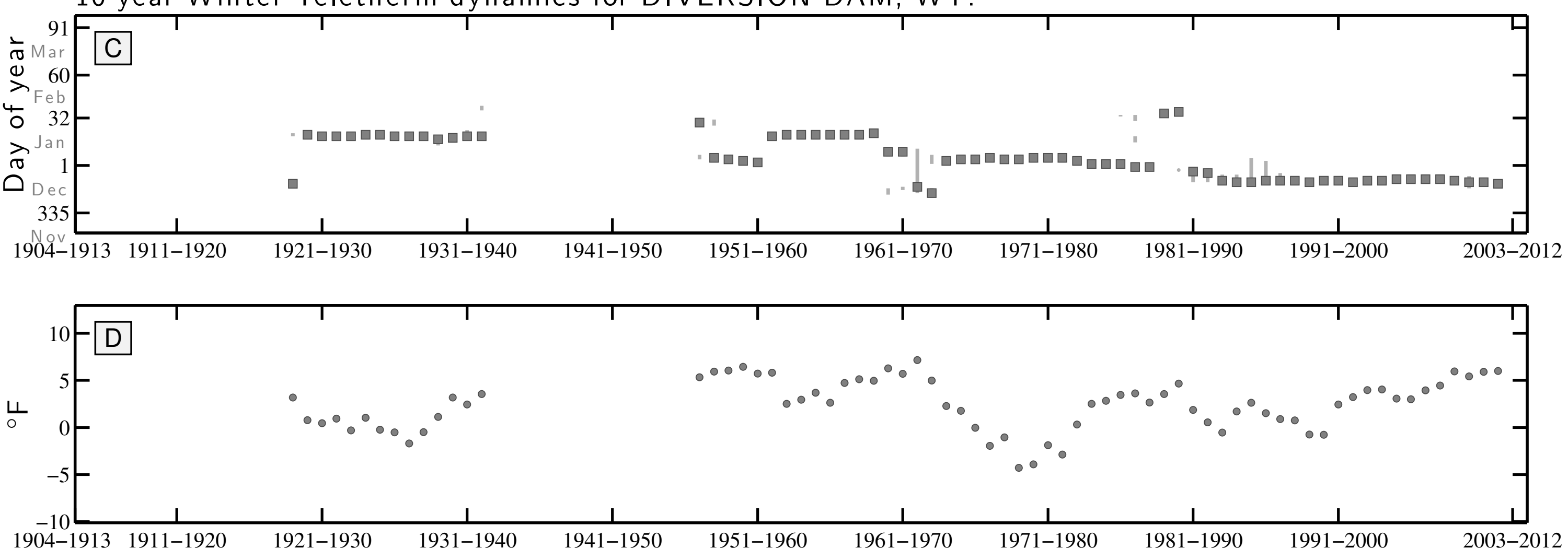


10 year Summer Teletherm dynamics for EVANSTON $1 \mathrm{E}$, WY:

$\div$ Oct 274

(1) Sep

$>244-$

$\begin{array}{rr}4 & A u g \\ 0 & 213\end{array}$

入 Jul

ก $182-$

152

152 -

1904-1913 1911-1920

1921-1930

$\frac{1}{1931-1940}$

1941-1950

1951-1960

1961-1970

1971-1980

981-1990

1991-2000

2003-2012

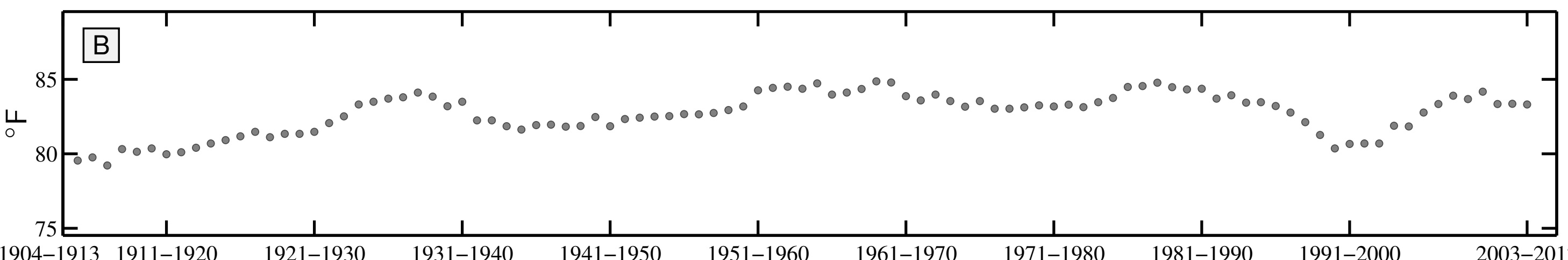

1904-1913 1911-1920

1921-1930

1931-1940

1941-1950

1951-1960

1961-1970

1971-1980

1981-1990

1991-2000

2003-2012

10 year Winter Teletherm dynamics for EVANSTON 1 E, WY:

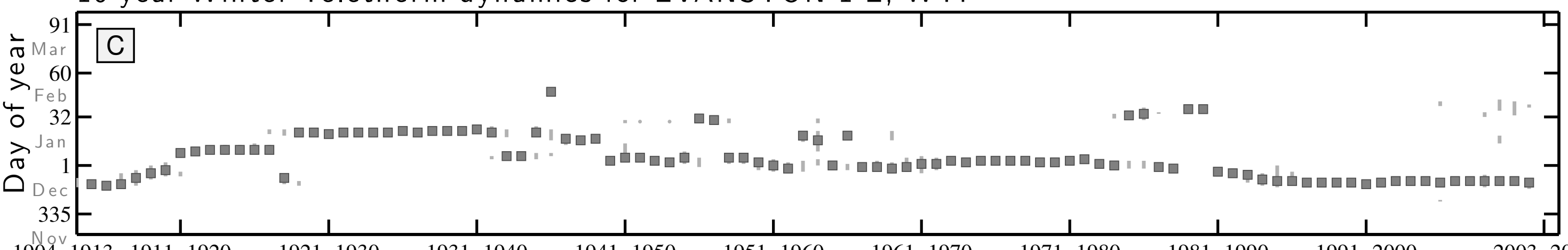

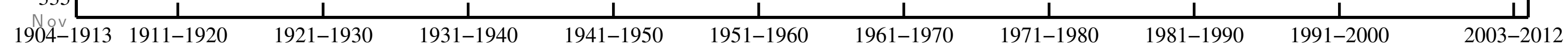

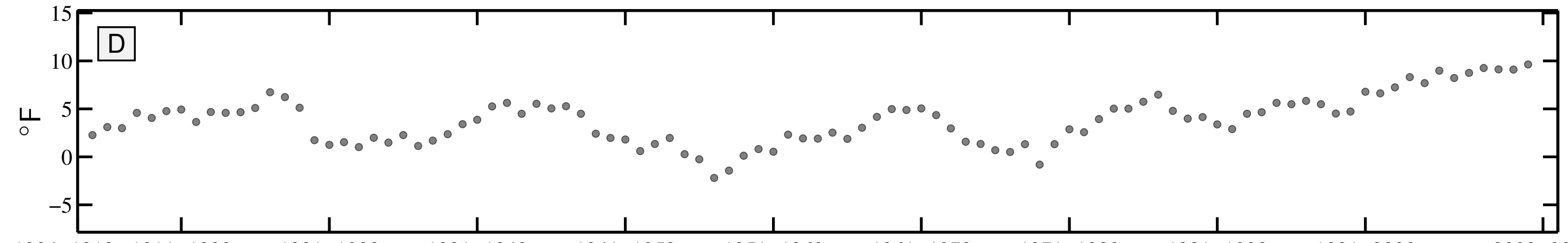

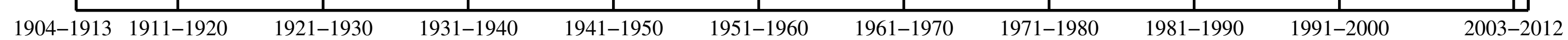


10 year Summer Teletherm dynamics for LARAMIE RGNL AP, WY:
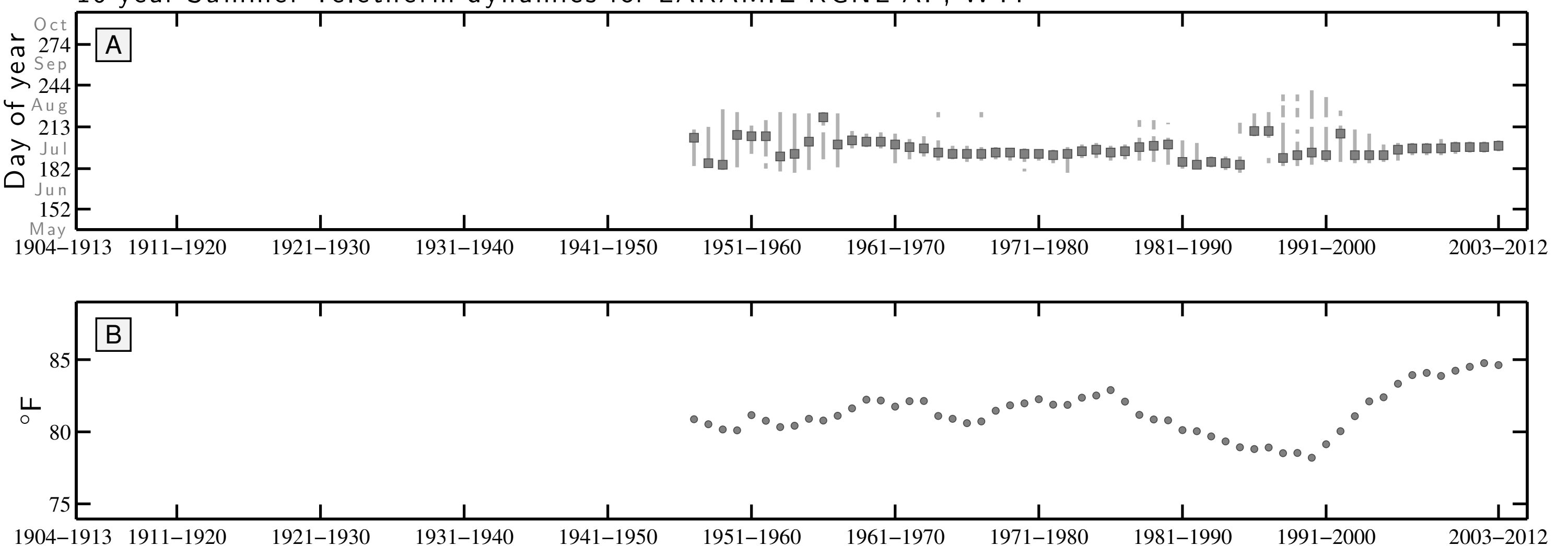

10 year Winter Teletherm dynamics for LARAMIE RGNL AP, WY:
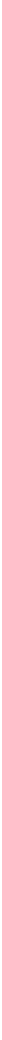

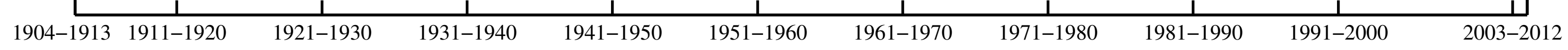


10 year Summer Teletherm dynamics for PAVILLION, WY:
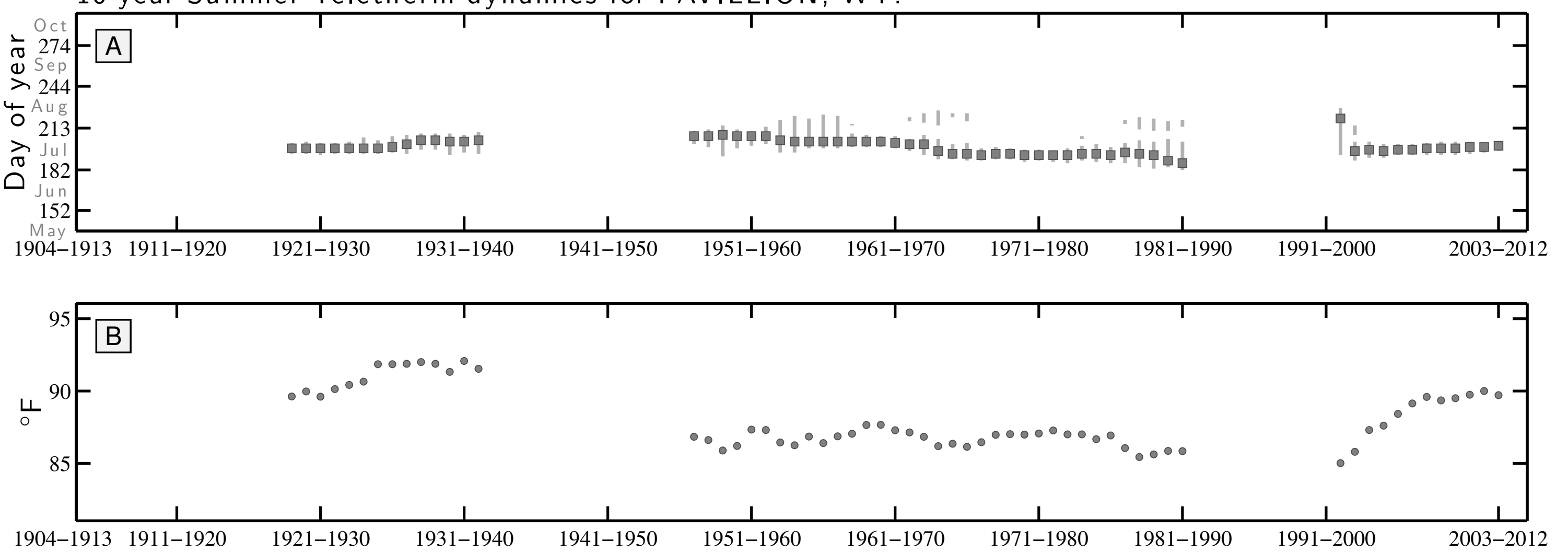

10 year Winter Teletherm dynamics for PAVILLION, WY:
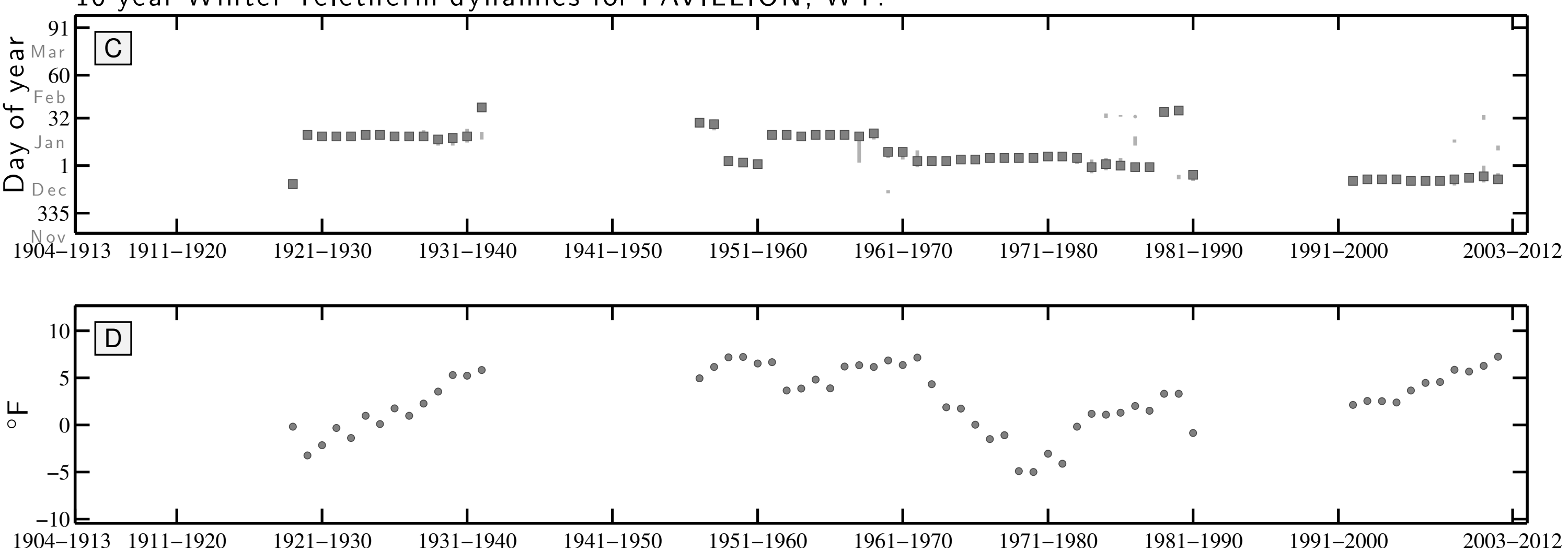
10 year Summer Teletherm dynamics for POWELL FLD STN, WY:
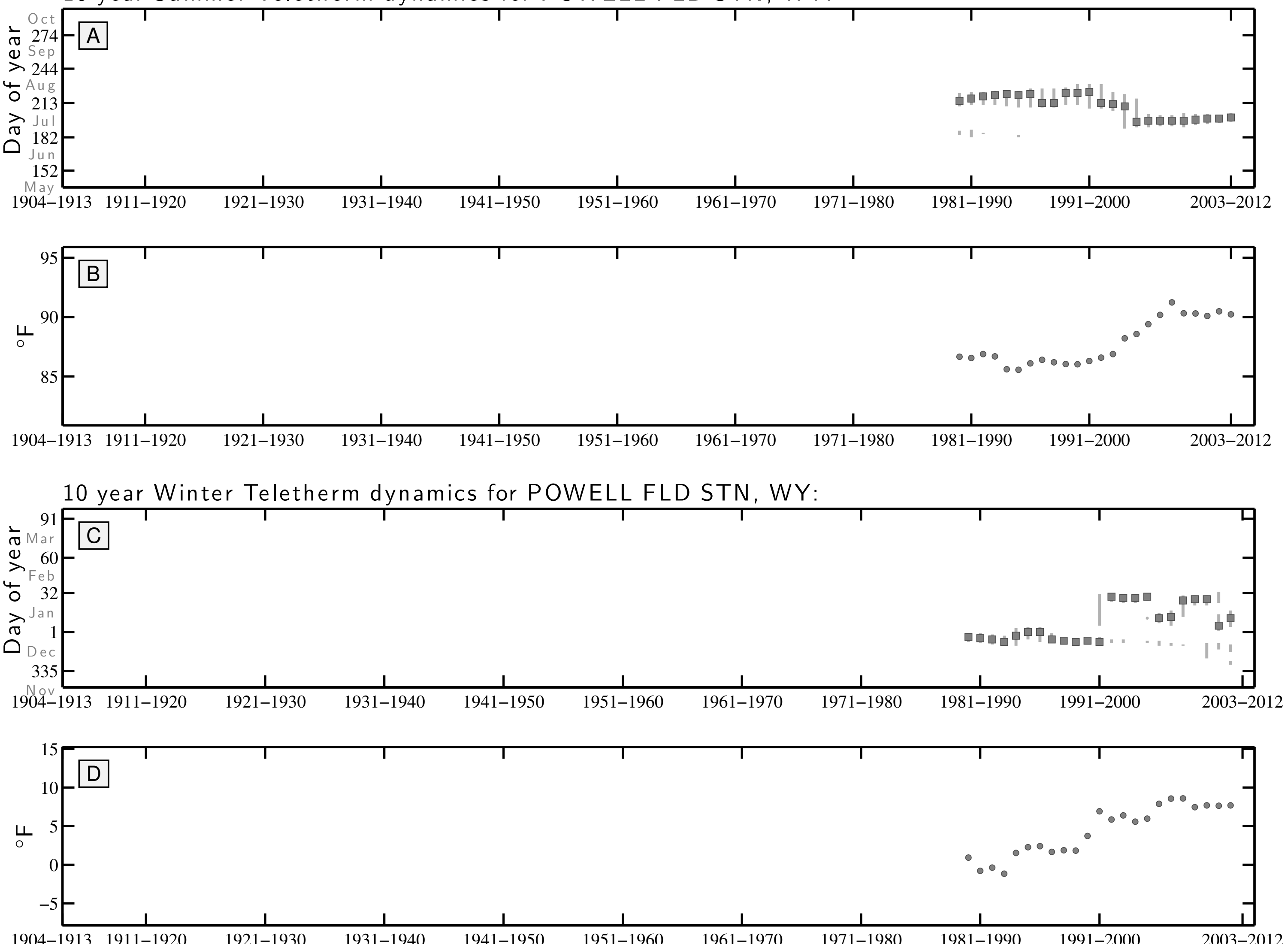

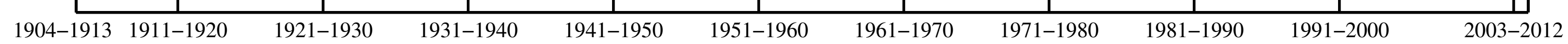


